GÖTTINGER SCHRIFTEN ZUR INTERNETFORSCHUNG

Hg.: S. Hagenhoff, D. Hogrefe, E. Mittler,

M. Schumann, G. Spindler, V. Wittke

\title{
Individualisierung und mobile Dienste am Beispiel der Medienbranche
}

Ansätze zum Schaffen von Kundenmehrwert

Christian Kaspar 



\title{
Individualisierung und mobile Dienste am Beispiel der Medienbranche - Ansätze zum Schaffen von Kundenmehrwert
}

\author{
Dissertation \\ zur Erlangung des wissenschaftlichen Doktorgrades der Wirt- \\ schaftswissenschaftlichen Fakultät der Georg-August-Universität \\ Göttingen
}

vorgelegt von

Dipl.-Kfm. Christian Markus Kaspar

aus München

Göttingen, 2005 

Individualisierung und mobile Dienste am Beispiel der Medienbranche

This work is licensed under the Creative Commons License 2.0 "by-nd", allowing you to download, distribute and print the document in a few copies for private or educational use, given that the document stays unchanged and the creator is mentioned. You are not allowed to sell copies of the free version. 
erschienen als Band 3 in der Reihe „Göttinger Schriften zur Internetforschung“ im Universitätsverlag Göttingen 2006 
Christian Kaspar

Individualisierung und mobile Dienste am Beispiel der Medienbranche

Ansätze zum Schaffen von Kundenmehrwert

Göttinger Schriften zur

Internetforschung, Band 3

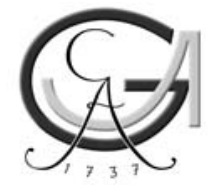

Universitätsverlag Göttingen 2006 


\section{Bibliographische Information der Deutschen Nationalbibliothek}

Die Deutsche Nationalbibliothek verzeichnet diese Publikation in der Deutschen Nationalbibliographie; detaillierte bibliographische Daten sind im Internet über $<$ http://dnb.ddb.de $>$ abrufbar

\section{Reihe}

Band 3 der Reihe „Göttinger Schriften zur Internetforschung“ in der qualitätsgeprüften Sparte des Universitätsverlags Göttingen.

Herausgeber der Reihe: Svenja Hagenhoff, Dieter Hogrefe, Elmar Mittler, Matthias Schumann, Gerald Spindler und Volker Wittke.

\section{Mitwirkung}

Entstanden im Rahmen des Forschungsprojekts „Mediaconomy“ der Georg-AugustUniversität Göttingen. Im Rahmen des Schwerpunktprogramms „Internetökonomie“ durch das BMBF gefördert. Sprecher: Prof. Dr. Matthias Schumann

GEFÖRDERT VOM

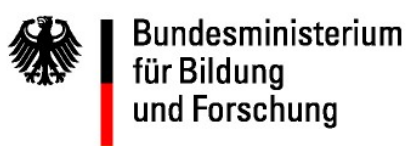

Dissertation zur Erlangung des wissenschaftlichen Doktorgrades der Wirtschaftswissenschaftlichen Fakultät der Georg-August-Universität Göttingen vorgelegt von Dipl.-Kfm. Christian Markus Kaspar aus München Göttingen, 2005

Dieses Buch ist auch als freie Onlineversion über die Homepage des Verlags sowie über den OPAC der Niedersächsischen Staats- und Universitätsbibliothek (http://www.sub.uni-goettingen.de) erreichbar und darf gelesen, heruntergeladen sowie als Privatkopie ausgedruckt werden. Es ist nicht gestattet, Kopien oder gedruckte Fassungen der freien Onlineversion zu veräußern.

(C) 2006 Universitätsverlag Göttingen

http://univerlag.uni-goettingen.de Umschlaggestaltung: Martin Kaspar und Margo Bargheer

ISSN 1863-0944

ISBN-10: 3-938616-53-9

ISBN-13: 978-3-938616-53-6 


\section{Geleitwort}

Zu den zentralen Zielen der Wirtschaftsinformatik gehören die sinnhafte Vollautomation betriebswirtschaftlicher Aufgaben und Entscheidungsprozesse sowie die optimale Deckung menschlicher Informationsbedarfe. Vor diesem Hintergrund finden aktuell insbesondere Softwarekonzepte zur automatischen Individualisierung von Güterangeboten und Mobilfunktechnologien als Distributionsmedien für Echtzeitinformationen im so genannten "mobilen Internet“ besondere Beachtung zur Bewältigung dieser Ziele. Diese zwei Themen, Individualisierung und mobiles Internet, bilden den Gegenstand der vorliegenden Arbeit von Herrn Kaspar. Die beiden Themen werden nicht nur isoliert dargestellt, sondern aus dem speziellen Blickwinkel der Medienbranche betrachtet. Herr Kaspar geht dabei der Frage nach, in wiefern Individualisierungsformen und mobile Dienste im Stande sind, den Kunden von Medienunternehmen speziell im Onlinegeschäft im Vergleich zu traditionellen Publikumsmedien einen Mehrwert zu stiften. Dieses geschieht vor dem Hintergrund aktueller Veränderungen in der Medienbranche. Im Zuge der Arbeit werden die Individualisierung von Mediengütern und das Inhalteangebot im mobilen Internet als Mehrwertstrategien im Rahmen grundsätzlicher strategischer Handlungsmöglichkeiten von Medienunternehmen eingeordnet. Darauf aufbauend werden diese zwei Strategien auf der Grundlage konzeptioneller Überlegungen und anhand empirischer Untersuchungen auf ihren Nutzenbeitrag aus Rezipientensicht hin bewertet.

Die Forschungstätigkeit von Herrn Kaspar am Institut für Wirtschaftsinformatik war eingebettet in das vom BMBF finanzierte Forschungsprojekt Mediaconomy der Universität Göttingen, in dem Beteiligte aus den Wirtschaftswissenschaften, der Informatik, der Soziologie, den Rechts- und den Bibliothekswissenschaften interdisziplinär zukünftige Handlungsmöglichkeiten für Medienunternehmen erforschen und gestalten. Die vorliegende Arbeit stellt vor diesem Hintergrund eine erste geschlossene Abhandlung der Themen Individualisierung und mobiles Internet aus dem Blickwinkel der Medienbranche dar. Sie betrachtet das Thema nicht nur monodisziplinär aus wirtschaftswissenschaftlicher Sicht, sondern interdisziplinär, unter Einbeziehung technologischer, rechtswissenschaftlicher und soziologischer Aspekte. Die Ergebnisse dieser Auseinandersetzung liefern zum einen einen wichtigen Beitrag für die wissenschaftliche Diskussion in der Wirtschaftsinformatik, insbesondere im noch jungen Gebiet der Internetökonomie. Zum anderen enthält die Arbeit konkrete praktische Gestaltungshinweise für individuelle oder mobile Inhalteangebote in der Medienbranche. Daher verdient die Arbeit ein hohes Maß an Aufmerksamkeit in Wissenschaft und Praxis. 



\section{Vorwort}

Die vorliegende Arbeit entstand im Zuge meiner Tätigkeit als wissenschaftlicher Mitarbeiter an der Abteilung 2 des Instituts für Wirtschaftsinformatik der Universität Göttingen. Die Arbeit wurde im Dezember 2005 von der Wirtschaftswissenschaftlichen Fakultät der Georg-August-Universität Göttingen als Dissertation angenommen. Geprägt durch mein kaufmännisches Studium an der Universität München war mein Forschungsinteresse am zugrunde liegenden Gegenstand der Individualisierung und des mobilen Internets ursprünglich vor allem betriebswirtschaftlicher Natur. Der endgültige, deutlich breiter gefächerte Charakter der Arbeit wurde jedoch darüber hinaus in positiver Weise durch die interdisziplinäre Natur der Forschungsarbeit im Forschungsbereich „Konvergente Märkte der Internetökonomie“ am Institut für Wirtschaftsinformatik sowie im Forschungsprojekt "Mediaconomy“ der Universität Göttingen beeinflusst. Ich möchte mich deshalb an dieser Stelle bei all denjenigen bedanken, die mich bei der Durchführung meiner Forschungsaufgaben und dem Anfertigen dieser Arbeit unterstützt haben:

Meinem Doktorvater, Herrn Prof. Dr. Matthias Schumann, möchte ich für die Gestaltungsfreiheit und Unterstützung während der Erstellung der Arbeit danken. Herrn Prof. Dr. Günter Silberer gebührt mein herzlicher Dank für die Erstellung des Zweitgutachtens. Herrn Prof. Dr. Klasen danke ich für die Übernahme des fachfremden Teils der Rigorosumsprüfung. Bei der Entwicklung und Implementierung der im Rahmen der Arbeit vorgestellten Individualisierungssysteme wurde ich sachkundig unterstützt durch meine studentischen Hilfskräfte Deno von Selasinsky, Dennis Wulf und Alexander Willner, wofür ich mich hiermit noch einmal besonders bedanken möchte. Maßgeblich an der Durchführung der Nutzerbefragung zur Akzeptanz dieser Systeme waren Frau Heidemarie Hanekop und Frau Carmen Lanfer beteiligt, denen für diesen Anteil an der vorliegenden Arbeit ebenfalls Dank gebührt. Für Ihre Unterstützung bei der Endkorrektur der Arbeit danke ich Herrn Dr. Frank Witzel, Frau Kirsten Hahne und Frau Julia Wandt. Weiterhin möchte ich mich bei meinen Kolleginnen und Kollegen bedanken, die mich nicht nur während der Dienstzeit in zahlreichen Gesprächen durch kreative, kompetente sowie kritische Hinweise und Anmerkungen unterstützt haben, und die darüber hinaus den Beweis angetreten sind, dass ein Bayer auch in Norddeutschland ein Stück Heimat finden kann. Mein besonderer Dank gebührt der Leiterin des Forschungsbereichs „Konvergente Märkte der Internetökonomie“ am Institut für Wirtschaftsinformatik, Frau Dr. Svenja Hagenhoff, die mit ihren konstruktiven Beiträgen wesentlich zum Gelingen dieser Arbeit beigetragen hat. 
Schließlich möchte ich meinen Eltern Dank sagen, nicht nur für ihr Interesse an meiner aktuellen beruflichen Entwicklung, sondern darüber hinaus dafür, dass sie mir die Freiheit gaben, meinen Ausbildungsweg nach meinen Wünschen einschlagen zu können, und für ihre Unterstützung und Zuversicht bei der Bewältigung des eingeschlagenen Wegs.

Ihnen sei daher diese Arbeit gewidmet.

Christian Kaspar 


\section{Inhaltsüberblick}

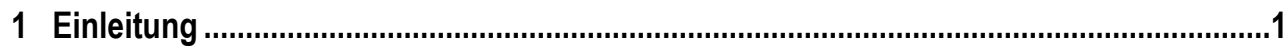

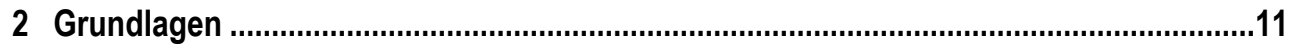

3 Strategische Herausforderungen in der Medienbranche .........................................65

4 Individualisierung von Mediengütern ..................................................................117

5 Inhalteverwertung im mobilen Internet ……......................................................179

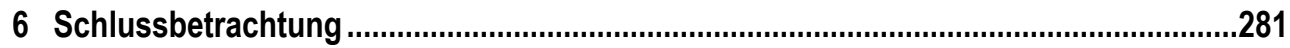

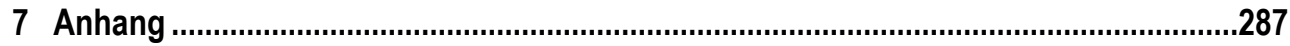

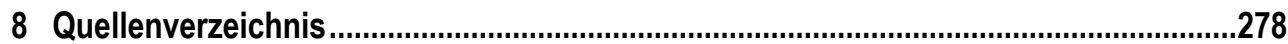





\section{Inhaltsverzeichnis}

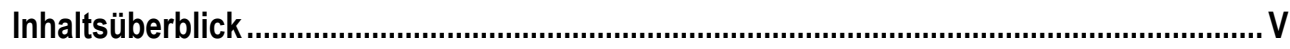

Abbildungsverzeichnis …............................................................................... XIII

Abkürzungsverzeichnis ................................................................................. XVII

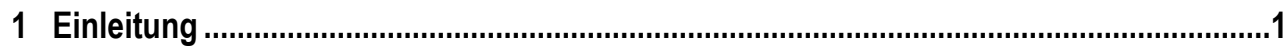

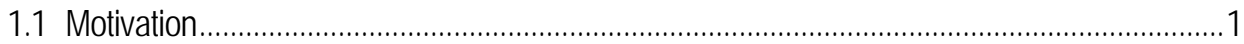

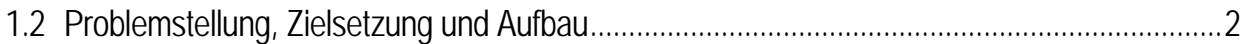

1.3 Inhaltliche und methodische Abgrenzung der Untersuchung .........................................

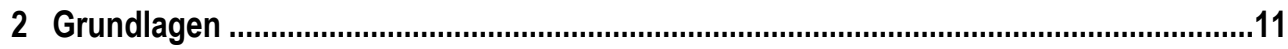

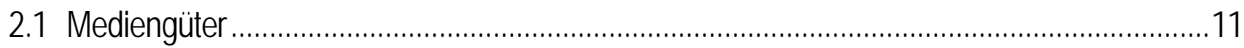

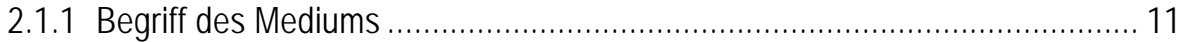

2.1.2 Eigenschaften von Mediengütern .......................................................... 13

2.1.2.1 Produktionsorientierte Perspektive .....................................................14

2.1.2.2 Verwendungsorientierte Perspektive ...............................................17

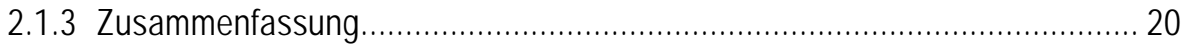

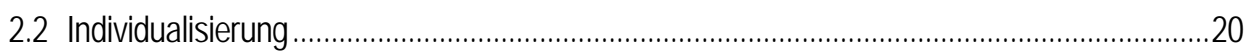

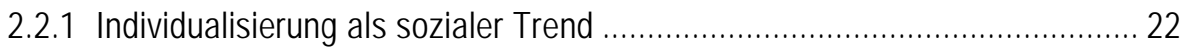

2.2.1.1 Individualisierung als gesellschaftlicher Wertewandel ............................22

2.2.1.2 Individualisierung als Segmentierung des Marktangebots.......................24

2.2.1.3 Individualisierung als Nachfragerationalisierung..................................27

2.2.1.4 Zusammenfassung ....................................................................30

2.2.2 Individualisierung als strategische Option der Marktbearbeitung ................. 31

2.2.2.1 Individuelles Kundenmarketing .........................................................32

2.2.2.2 Mass Customization im Industriebetrieb ...............................................35

2.2.2.3 Empfehlungssysteme für Dienstleistungen und Handel ............................38

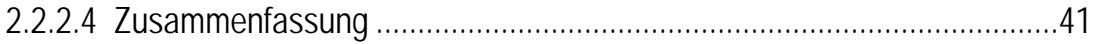

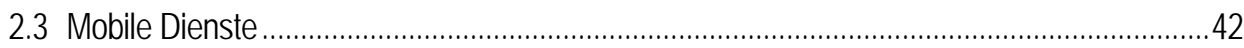

2.3.1 Mobilität im Rahmen von Anwendungssystemen ..................................... 43

2.3.2 Digitale Funknetztechnologien ........................................................... 45

2.3.2.1 Allgemeine Grundlagen der Mobilfunktechnik ....................................45

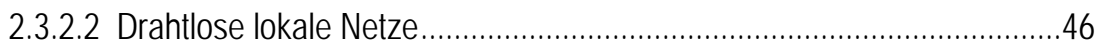

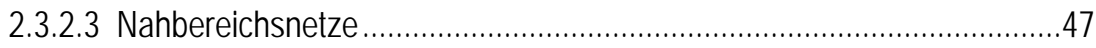

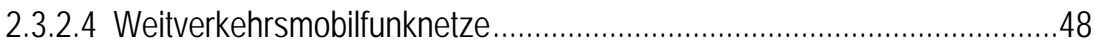


2.3.3 Technologien zur Bereitstellung mobiler Informationsdienste 51

2.3.3.1 Wireless Application Protocol (WAP). .52

2.3.3.2 Short Message Service (SMS) und Multimedia Messaging Service (MMS) .53

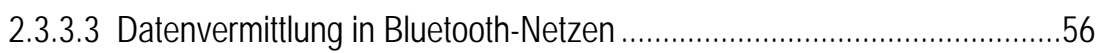

2.3.4 Anwendungsfelder für mobile Dienste ....................................................... 57

2.3.4.1 Einsatzvarianten mobiler Dienste .......................................................57

2.3.4.2 Betriebliche Anwendungsfelder mobiler Dienste ....................................58

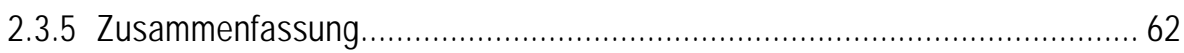

2.4 Zusammenfassung der grundlegenden Begriffe ..............................................................63

3 Strategische Herausforderungen in der Medienbranche ...........................................65

3.1 Konvergenzeffekte als Ausprägung der Umfelddynamik des Medienwettbewerbs...............65

3.1.1 Auslöser von Konvergenzeffekten im Medienumfeld.................................. 66

3.1.1.1 Veränderungen der technologischen Rahmenbedingungen....................67

3.1.1.2 Veränderungen der gesellschaftlichen Rahmenbedingungen .................69

3.1.1.3 Veränderungen der ökonomischen Rahmenbedingungen ......................70

3.1.2 Konvergenzdimensionen...................................................................... 70

3.1.2.1 Technologische Konvergenz...........................................................70

3.1.2.2 Branchenkonvergenz im Umfeld der Medienbranche.............................72

3.1.3 Bewertung der Konvergenzeffekte aus Sicht der Medienbranche ................ 73

3.2 Strukturanalyse des Medienwettbewerbs .....................................................................74

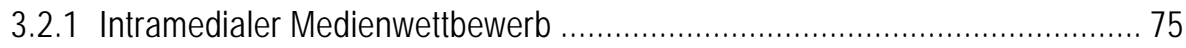

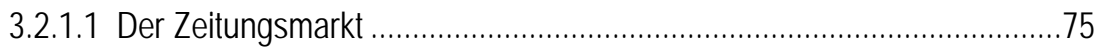

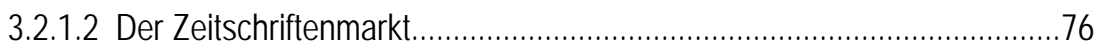

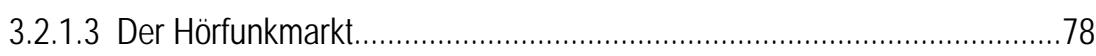

3.2.1.4 Der Fernsehmarkt..........................................................................

3.2.1.5 Das Internet als Segment des Medienmarkts........................................80

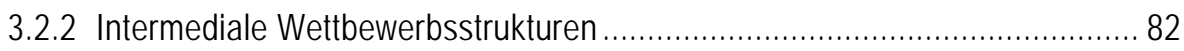

3.2.2.1 Entwicklung des Mediennutzungsverhaltens .......................................83

3.2.2.2 Umsatzentwicklung der Medienbranche auf dem Werbemarkt ...............85

3.2.3 Bewertung der Wettbewerbsstrukturen in der Medienbranche ...................... 86

3.3 Strategische Handlungsmöglichkeiten in der Medienbranche ...............................................8

3.3.1 Diversifikationsstrategien in der Medienbranche .................................... 89

3.3.1.1 Überblick über Diversifikationsmöglichkeiten eines Medienunternehmens .89

3.3.1.2 Diversifikation von Medienunternehmen durch das Angebot von

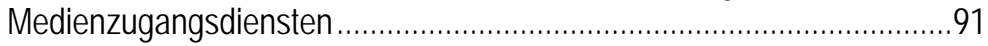

3.3.1.2.1 Das Angebot von Zugangsdiensten für Onlineinhalte .............92

3.3.1.2.2 Das Angebot von Rezeptionskontexten für Onlineinhalte ........94 
3.3.1.3 Diversifikation von Medienunternehmen durch Mehrfachverwertung von Inhalten .95

3.3.1.3.1 Technische Aspekte der Mehrfachverwertung. .96

3.3.1.3.2 Ausprägungsformen der digitalen Mehrfachverwertung..........97 3.3.1.3.2.1 Content Syndication........................................ 98

3.3.1.3.2.2 Cross-Media Management ................................. 99

3.3.2 Mehrwertstrategien für Medienunternehmen ............................................ 100

3.3.2.1 Ökonomische Grundlagen der Differenzierung....................................102

3.3.2.1.1 Produktdifferenzierung ........................................................103

3.3.2.1.2 Preisdifferenzierung .......................................................105

3.3.2.2 Differenzierung von Mediengütern.................................................106

3.3.2.2.1 Wertbildende Eigenschaften von Mediengütern...................107

3.3.2.2.2 Versioning und Bundling ...................................................108

3.3.2.2.3 Mobile und interaktive Mehrwertformate für Mediengüter .....110

3.3.2.2.4 Mehrfachverwendung von Inhalten und Individualisierung von Mediengütern.

3.3.3 Bewertung der strategischen Handlungsmöglichkeiten in der Medienbranche

3.4 Zusammenfassung der strategischen Herausforderungen von

Medienunternehmen.

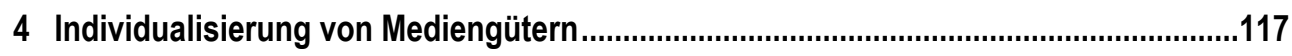

4.1 Individuelle Identifikation von Onlinerezipienten ...........................................................118

4.1.1 Nutzerprofile auf Grundlage von Befragung........................................ 118

4.1.1.1 Erhebung von Präferenzen durch Befragung ...................................118

4.1.1.2 Identifikation von Rezipienten anhand von Profilen ..............................121

4.1.1.3 Bewertung freiwilliger Profilangaben.....................................................123

4.1.2 Nutzerprofile auf der Grundlage von Verhaltensbeobachtungen ................. 124

4.1.2.1 Protokolldateien als Grundlage einer Beobachtung des

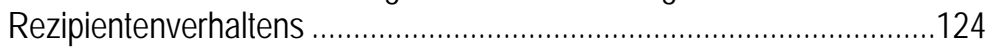

4.1.2.2 Verhaltensanalyse im Zugriffsprotokoll durch Data Mining..................125

4.1.2.2.1 Datenanalyse durch Data Mining .......................................126

4.1.2.2.2 Datenvorbereitung und -transformation von Webserver-

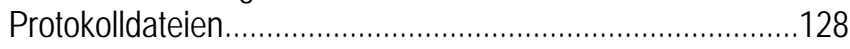

4.1.2.2.3 Mustererkennung in Webserver Protokolldateien .................129

4.1.2.3 Bewertung des Web Usage Mining zur Bestimmung von

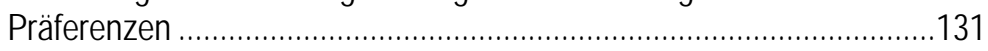

4.1.3 Rechtliche Aspekte der Identifikation von Onlinerezipienten ................................. 132

4.1.4 Bewertung der Möglichkeiten zur Identifikation von Onlinerezipienten........ 134

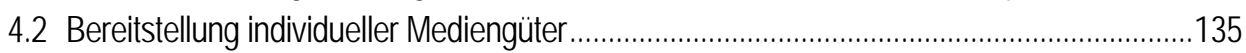


4.2.1 Eigenschaften von Systemen zur Individualisierung von Inhalteangeboten. 135

4.2.1.1 Formen der Individualisierung im Angebot von Mediengütern. 135

4.2.1.2 Aufbau von Individualisierungssystemen für Mediengüter. .138

4.2.2 Individualisierung auf Grundlage von Empfehlungssystemen 141

4.2.2.1 Individualisierung durch Selbstselektion. .141

4.2.2.2 Individualisierung durch eigenschaftsbasiertes Filtern 143

4.2.2.3 Kombination von Selbstselektion und eigenschaftsbasiertem Filtern. 145

4.2.3 Individualisierung auf Grundlage von Empfehlersystemen 146

4.2.3.1 Individualisierung durch Collaborative Filtering 147

4.2.3.1.1 Grundform von Collaborative Filtering-Algorithmen .147

4.2.3.1.2 Inhaltebezogenes Collaborative Filtering 149

4.2.3.2 Individualisierung durch beobachtungsbasiertes Filtern 151

4.2.3.2.1 Profilaggregation im Rahmen eines beobachtungsbasierten Empfehlungssystems .152

4.2.3.2.2 Ermittlung einer individuellen Empfehlung im Rahmen eines beobachtungsbasierten Empfehlungssystems .154

4.2.4 Zusammenfassende Bewertung der dargestellten Verfahren 155

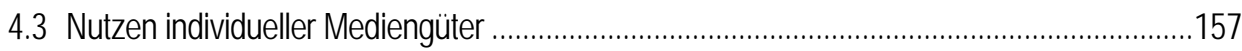

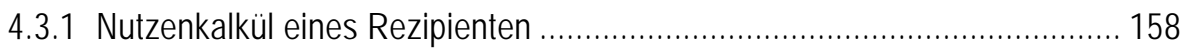

4.3.2 Konsumentscheidung eines Rezipienten ............................................... 160

4.3.3 Einfluss von Individualisierung auf die Konsumentscheidung eines Rezipienten 161

4.3.4 Bedingungen der Vorteilhaftigkeit individueller Mediengüter....................... 164

4.4 Wirkungsvergleich prototypischer Individualisierungssysteme ..........................................167

4.4.1 Der Internetauftritt der Universität Göttingen als Mediengut...................... 167

4.4.2 Implementierungsform prototypischer Individualisierungssysteme.............. 168

4.4.3 Ergebnisse einer experimentellen Anwendung der implementierten Verfahren.

4.4.3.1 Aufbau und Stichprobe der Erhebung................................................173

4.4.3.2 Ergebnisse der Teilnehmerbefragung................................................174

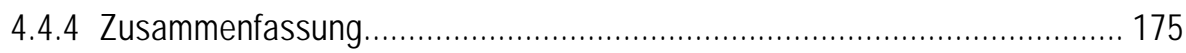

4.5 Zusammenfassung der Möglichkeiten einer Individualisierung von Mediengütern ..............176

5 Inhalteverwertung im mobilen Internet ..................................................................179

5.1 Stand der Forschung zum Geschäft mit mobilen Diensten .......................................179

5.2 Geschäftsmodelle für das Angebot von Mediengütern im mobilen Internet.........................182

5.2.1 Das Konzept des Geschäftsmodells als Analyseeinheit............................ 182

5.2.2 Rahmenbedingungen für Geschäftsmodelle inhalteorientierter Angebote im mobilen Internet. 
5.2.2.1 Rahmenbedingungen des Produkt- und Nutzenmodells inhalteorientierter mobiler Dienste.

5.2.2.2 Rahmenbedingungen des Wertschöpfungsmodells mobiler Dienste ....190

5.2.2.3 Rahmenbedingungen des Erlösmodells inhalteorientierter mobiler Dienste. .198

5.2.2.4 Zusammenfassung 201

5.2.3 Ausprägungsformen von Geschäftsmodellen für inhalteorientierte mobile Dienste 202

5.2.3.1 Fallstudie 1: Playboy Mobile. .204

5.2.3.1.1 Produkt- und Nutzenmodell von Playboy Mobile. .204

5.2.3.1.2 Wertschöpfungsmodell von Playboy Mobile. .207

5.2.3.1.3 Erlösmodell von Playboy Mobile .209

5.2.3.1.4 Ausblick. .210

5.2.3.2 Fallbeispiel 2: t-info SMS 210

5.2.3.2.1 Nutzen- und Produktmodell von t-info SMS .211

5.2.3.2.2 Wertschöpfungsmodell von t-info SMS .213

5.2.3.2.3 Erlösmodell von t-info SMS .214

5.2.3.2.4 Ausblick .215

5.2.3.3 Fallbeispiel 3: V-Card (12snap) 215

5.2.3.3.1 Produkt- und Nutzenmodell von V-Card .215

5.2.3.3.2 Wertschöpfungsmodell der V-Card .217

5.2.3.3.3 Erlösmodell der V-Card 217

5.2.3.3.4 Ausblick. .218

5.2.4 Zusammenfassung der Geschäftsmodellanalyse..................................... 218

5.3 Akzeptanz inhalteorientierter mobiler Dienste... 220

5.3.1 Stand der Forschung zur Akzeptanz mobiler Dienste 221

5.3.1.1 Ausprägungen der Akzeptanz als Forschungsgegenstand ..................221

5.3.1.2 Beiträge zur Akzeptanz mobiler Dienste.........................................223

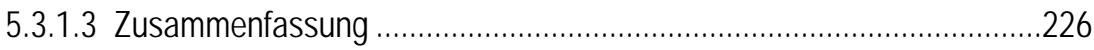

5.3.2 Analyse der Akzeptanz inhalteorientierter mobiler Dienste ......................... 226

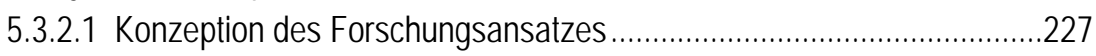

5.3.2.1.1 Forschungsziel und Forschungsleitfragen...........................227

5.3.2.1.2 Methodisches Vorgehen...................................................229

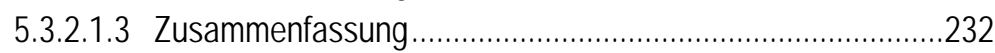

5.3.2.2 Evaluation einer inhaltebezogenen Segmentierung mobiler Nutzer ......232

5.3.2.2.1 Methodik und Aufbau der Untersuchung ..............................233

5.3.2.2.2 Ergebnisse der Untersuchung............................................234

5.3.2.2.3 Zusammenfassung der Befunde .......................................236

5.3.2.3 Akzeptanz mobiler Inhalteformate bei den Lesern von Printtiteln..........237 
5.3.2.3.1 Ziel, Stichprobe und Aufbau der Untersuchung ....................237

5.3.2.3.2 Ergebnisse der Leserbefragung ........................................238

5.3.2.3.2.1 Nutzungshäufigkeiten je Lesersegment........... 238

5.3.2.3.2.2 Nutzungshäufigkeiten je Dienstkategorie ......... 240

5.3.2.3.2.3 Motive der (Nicht-)Nutzung.............................. 243

5.3.2.3.3 Befunde der Untersuchung ..................................................244

5.3.2.3.4 Zusammenfassung der Leserbefragung .............................248

5.3.3 Zusammenfassung der Akzeptanzanalyse ............................................. 250

5.4 Mobilitätsgerechte Bereitstellung von Mediengütern .................................................250

5.4.1 Kontextadaptivität als Anforderung einer mobilitätsgerechten

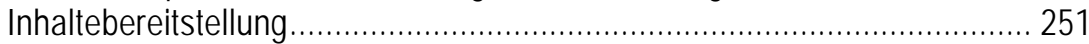

5.4.1.1 Maschinelle Situationswahrnehmung .............................................22

5.4.1.2 Kontextbezogene Systemadaption ..............................................254

5.4.1.3 Beiträge zu adaptiven mobilen Diensten ...........................................256

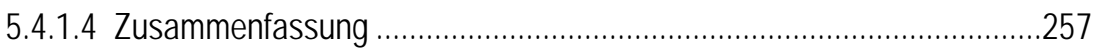

5.4.2 Mobilitätsgerechte Modellierung von Kontextdaten................................... 259

5.4.2.1 Anforderungen der Modellierung von Inhaltepräferenzen für mobile

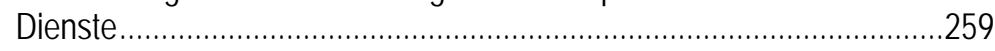

5.4.2.2 Semantische Repräsentation von Nutzermodellen auf Grundlage

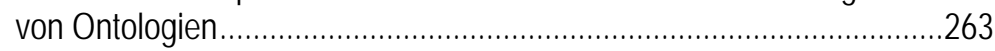

5.4.2.3 Datenintegration eines verteilten Kontextmodells................................267

5.4.2.4 Zusammenfassung ......................................................................270

5.4.3 Zugriffskontrolle für ein aggregiertes Kontextmodell ................................. 270

5.4.3.1 Forschungsbeiträge im Bereich der Datenkontrolle.............................271

5.4.3.2 Konzeption eines pseudonymen Nutzermodellierungssystems.............273

5.4.3.3 Zugriffskontrolle für Personendaten im Rahmen einer pseudonymen Nutzermodellierung ....................................................275

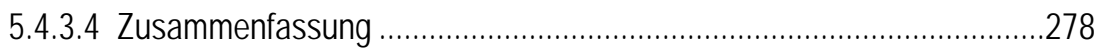

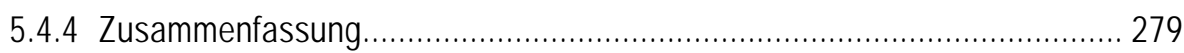

5.5 Zusammenfassung der Möglichkeiten der Inhalteverwertung im mobilen Internet..............280

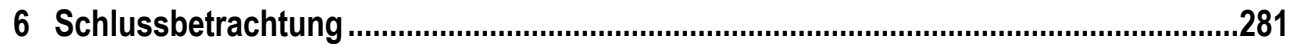

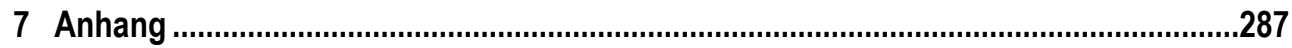

7.1 Ergebnisse der Erhebung zur Akzeptanz prototypischer

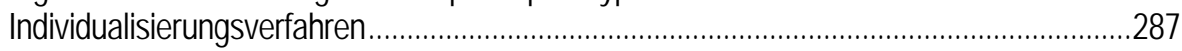

7.2 Ergebnisse der Befragung zur Mediennutzung ……...................................................288

7.3 Ergebnisse der Erhebung zur Akzeptanz mobiler Dienste.................................................289

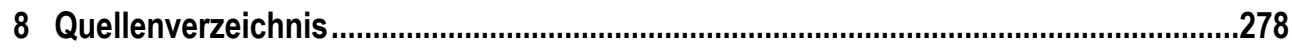




\section{Abbildungsverzeichnis}

Abbildung 1-1: Zentrale Forschungsfragen .........................................................................

Abbildung 1-2: Gang der Untersuchung und Aufbau der Arbeit..............................................

Abbildung 1-3: Forschungsansätze der ökonomischen Medienwirtschaft....................................6

Abbildung 2-1: Schichten eines Informationsguts ............................................................13

Abbildung 2-2: Wertschöpfungsstrukturen in der Medienbranche.............................................14

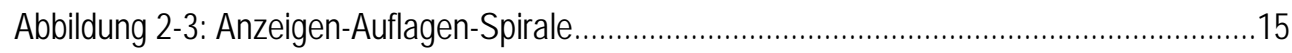

Abbildung 2-4: Typische Kostenstruktur in der Medienbranche ..........................................16

Abbildung 2-5: Betrachtungsdimensionen zur Individualisierung .........................................22

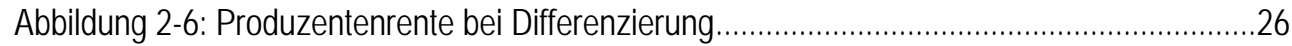

Abbildung 2-7: Zusammenfassung exogener Individualisierungstrends ..................................31

Abbildung 2-8: Einzelkundenorientierte Marketingkonzepte …………………........................34

Abbildung 2-9: Vorteilhaftigkeit der Mass Customization ......................................................38

Abbildung 2-10: Unterscheidungskriterien von Empfehlungssystemen ..................................39

Abbildung 2-11: Aufbau eines Bluetooth Controllers...........................................................48

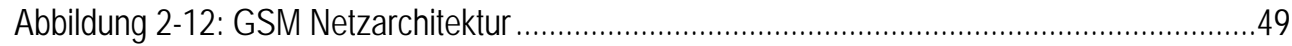

Abbildung 2-13: UMTS-Netzarchitektur.........................................................................51

Abbildung 2-14: Das WAP-Interaktionsmodell ...............................................................52

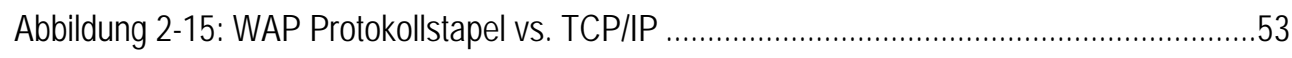

Abbildung 2-16: Ablauf einer SMS-gestützten Inhalteabfrage ................................................54

Abbildung 2-17: Anwendungsmöglichkeiten mobiler Dienste aus betriebswirtschaftlicher

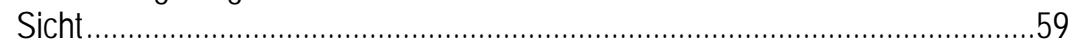

Abbildung 2-18: Mobile Mehrwertdienste .............................................................................61

Abbildung 2-19: Definitionen zum Begriff „Mobile Business“................................................62

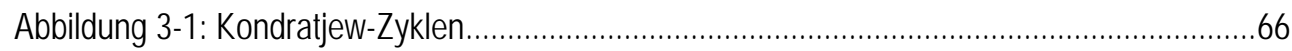

Abbildung 3-2: Entstehung von Multimediamärkten ...........................................................73

Abbildung 3-3: Entwicklung der Mediennutzung in Deutschland............................................84

Abbildung 3-4: Entwicklung der Auflagen von Zeitungen und Zeitschriften ...............................85

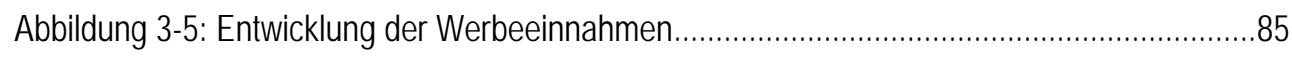

Abbildung 3-6: Veränderungsraten des Werbemarktes ...........................................................86

Abbildung 3-7: Strategienspektrum aus Sicht der Medienbranche .........................................89 
Abbildung 3-8: Konvergenzbedingte Diversifikationsmöglichkeiten aus Sicht der

Medienbranche . .90

Abbildung 3-9: Annäherung von Medien- und Telekommunikationsanbieter .............................91

Abbildung 3-10: Prinzip der Mehrfachverwertung ..................................................................95

Abbildung 3-11: Mehrfachverwertung auf der Grundlage von XML .......................................97

Abbildung 3-12: Organisationales Arrangement bei der Inhaltesyndizierung............................98

Abbildung 3-13: Beitragspotenziale des Kunden am Unternehmenswert ...............................102

Abbildung 3-14: Horizontale und vertikale Differenzierung .................................................104

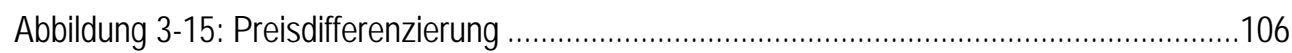

Abbildung 3-16: Wertbildende Eigenschaften eines Medienguts .........................................108

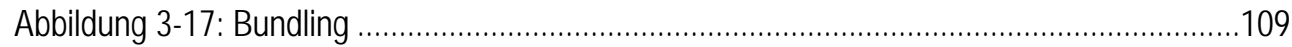

Abbildung 3-18: Mehrfachverwendung von Inhalten .......................................................113

Abbildung 4-1: Qualität von Benutzerprofilen ...............................................................119

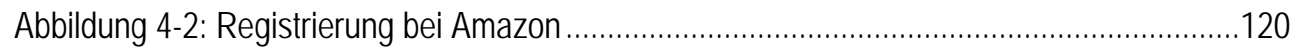

Abbildung 4-3: Inhalt eines Protokolleintrags ....................................................................... 125

Abbildung 4-4: Anwendungsbereiche von Data Mining im Internet.........................................126

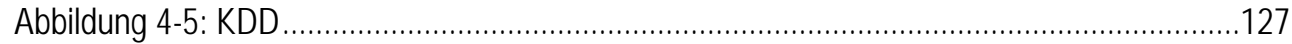

Abbildung 4-6: Datenbereinigung im Zugriffsprotokoll.................................................128

Abbildung 4-7: Binärcodierung zur Ähnlichkeitsbestimmung .................................................131

Abbildung 4-8: Zusammenfassung der vorgestellten Identifikationsverfahren .........................134

Abbildung 4-9: Individualisierungsformen im Angebot von Mediengütern................................136

Abbildung 4-10: Grundkonzept eines individuellen Medienguts.........................................137

Abbildung 4-11: Aufbau eines Individualisierungssystems................................................138

Abbildung 4-12: Varianten von Individualisierungssystemen ...............................................140

Abbildung 4-13: Individualisierung durch Selbstselektion im Onlineportal Yahoo!...................142

Abbildung 4-14: Ähnlichkeitsbestimmung durch eigenschaftsbasiertes Filtern........................144

Abbildung 4-15: Kombination aus Selbstselektion und eigenschaftsbasiertem Filtern .............146

Abbildung 4-16: Ratingmatrix beim Collaborative Filtering..................................................148

Abbildung 4-17: Content-boosted Collaborative Filtering .................................................150

Abbildung 4-18: Aufbau eines beobachtungsbasierten Filtersystems.....................................152

Abbildung 4-19: Profilaggregation durch Profil-Clusterung ..................................................153

Abbildung 4-20: Profilaggregation durch Pageviewclusterung .............................................154

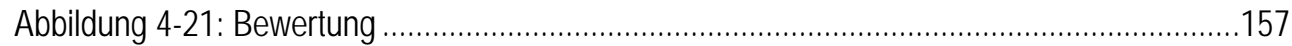

Abbildung 4-22: Notwendige Bedingung des Medienkonsums .........................................158 
Abbildung 4-23: Bruttonutzenfunktionen für ein individualisiertes und ein nichtindividualisiertes Bündel. .164

Abbildung 4-24: Prüfkriterien für die Individualisierungsqualität...........................................166

Abbildung 4-25: Auswahlseite des Instruments MyUni ..........................................................169

Abbildung 4-26:Kodierung des Nutzerprofils per Cookie......................................................170

Abbildung 4-27: Ausgabeseite von MyUni.......................................................................170

Abbildung 4-28: Auszug des Sitzungsprotokolls von MyBestBets ...........................................171

Abbildung 4-29: Datenselektion des MyBestBets-Filters......................................................171

Abbildung 4-30: Ausgabeformat des MyBestBets-Filters .....................................................172

Abbildung 5-1: Literaturbeispiele für Analysekriterien von Geschäftsmodellen.........................183

Abbildung 5-2: Teilnehmerlokalisierung im GSM-Netz ........................................................187

Abbildung 5-3: Orts- und Zeitspezifität von Bedürfnissen ..................................................188

Abbildung 5-4: Veränderungen der Wertschöpfungskette von Mobifunkbetreibern .................193

Abbildung 5-5: Beispiele für Modelle des Wertschöpfungssystems im mobilen Internet ..........195

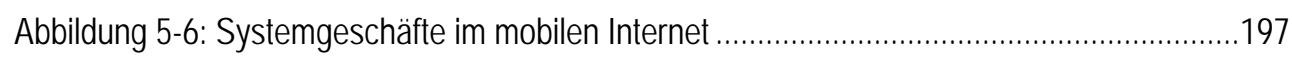

Abbildung 5-7: Varianten des Erlösmodells digitaler Medienprodukte .....................................198

Abbildung 5-8: Abrechnungsmodelle für mobile Dienste aus Sicht von Inhalteanbietern .........200

Abbildung 5-9: Klassifikationsraster inhalteorientierter mobiler Dienste..................................203

Abbildung 5-10: Technische Bereitstellung mobiler Dienste durch TFNM ...............................206

Abbildung 5-11: Transaktionsmodell von t-info SMS.........................................................212

Abbildung 5-12: Architekturmodell des Anwendungsystems von t-info.................................213

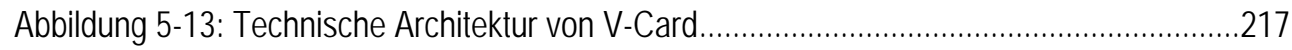

Abbildung 5-14: Zusammenfassender Überblick über die vorgestellten Geschäftsmodelle .....219

Abbildung 5-15: Mehrdimensionale Akzeptanzkonzeption....................................................223

Abbildung 5-16: Nutzungsgrade von Mobilfunktechnik in Abhängigkeit von Geschlecht

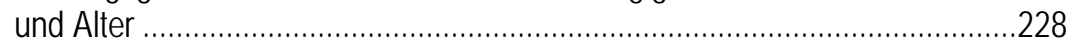

Abbildung 5-17: Forschungsleitfragen der Akzeptanzanalyse .............................................229

Abbildung 5-18: Formale Abgrenzung der durchgeführten Akzeptanzanalyse ........................232

Abbildung 5-19: Mittlere Nutzungshäufigkeit der Funktionen des Mobiltelefons .......................235

Abbildung 5-20: Nutzerinteresse an mobilen Dienstformaten für etablierte Inhalte ..................235

Abbildung 5-21: Mittelwertvergleich und Kreuzhäufigkeiten................................................236

Abbildung 5-22: Alters- und Einkommensstruktur der Befragungsteilnehmer..........................237

Abbildung 5-23: Anteile der Lesergruppen an der Stichprobe..............................................238

Abbildung 5-24: Endgerätehäufigkeiten anteilig je Lesersegment ........................................239 
Abbildung 5-25: Segmentübergreifende Nutzung kostenpflichtiger Dienste im mobilen Internet. .239

Abbildung 5-26: Nutzungsintensitäten je Dienstformat und Lesersegment.............................240

Abbildung 5-27: Nutzungsintensität von SMS-Diensten in Anteilen Lesergruppe......................241

Abbildung 5-28: Nutzungsintensität von MMS-Diensten in Anteilen der Lesergruppe ..............242

Abbildung 5-29: Nutzung von WAP-Diensten in Anteilen der Lesergruppe ............................242

Abbildung 5-30: Nutzung ortsbezogener Dienste in Anteilen der Lesergruppe.........................243

Abbildung 5-31: Bedingungen der zukünftigen Nutzung mobiler Inhalte..................................243

Abbildung 5-32: Ausprägungsgrade zweckneutraler Nutzungsmotive ...................................244

Abbildung 5-33: Inhaltebezogene Gratifikationen mobiler Dienste..........................................244

Abbildung 5-34: Vergleich der mittleren Nutzungsanteile von Mobiltelefon und Smartphone

Abbildung 5-35: Abhängigkeiten zwischen Leseverhalten und Nutzung des mobilen Internet. .246

Abbildung 5-36: Zusammenhangsmaße für Leserverhalten und Nutzung mobiler Dienste ......247

Abbildung 5-37: Zusammenhangsmaße für Leserverhalten und Nutzung mobiler Inhalteformate. .247

Abbildung 5-38: Verhältnis von Informations- und Unterhaltungsmotiv..................................248

Abbildung 5-39: Verhältnis von Neugier und Nutzungsgewohnheit ........................................248

Abbildung 5-40: Zusammenfassung der Erhebungsergebnisse............................................249

Abbildung 5-41: Aufbau eines Agentensystems ................................................................254

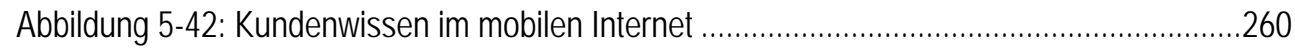

Abbildung 5-43: Klassenmodell einer inhaltebezogenen Nutzermodellierungssemantik ..........264

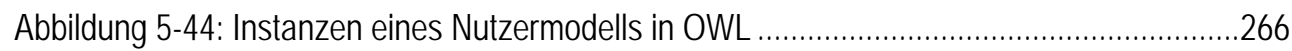

Abbildung 5-45: Codefragment eines Nutzermodells in OWL .............................................267

Abbildung 5-46: Austausch von Kontextdaten auf der Grundlage von Web ServiceTechnologien .269

Abbildung 5-47: Kommunikationsmodell einer pseudonymen Aggregation von Nutzermodellen.

Abbildung 5-48: Intermediation mit Aggregator und Bedürfnismanager. 


\section{Abkürzungsverzeichnis}

AG Aktiengesellschaft

Anm. d. Verf. Anmerkung des Verfassers

BDSG Bundes Datenschutzgesetz

BNEP Bluetooth Encapsulating Protocol

BSC Base Station Controller

bspw. beispielsweise

BSS Base Station Subsystem

BTS Base Transceiver Station

bzgl. bezüglich

CAE Computer-Aided Engineering

CAM Computer-Aided Manufacturing

CAP Computer-Aided Production

CAQ Computer-Aided Quality

CAS Computer-Aided Selling

CBCF content-boosted Collaborative Filtering

CDMA Code Division Multiple Access

CEPT European Conference of Postal and Telecommunications Administrations

CERN Centre Européen pour la Recherche Nucléaire

CF Collaborative Filtering

CIM Computer Integrated Manufacturing

CN Core Network

conf Konfidenzfaktor

COO Cell of Origin

CSMA/CA Carrier Sense with Collision Avoidance

CSS Cascading Stylesheet

DAML/OIL Defense (Advanced Research Projects Agency) Agent Markup Language/Ontology Inference Layer

DB Datenbank

DCF Distributed Coordination Function

DFWMAC Distributed Foundation Wireless MAC 
DNS Domain Name System

DSL Digital Subscriber Line

DSSS Direct Sequence Spread Spectrum

DTD Document Type Definition

DV Datenverarbeitung

DVD Digital Versatile Disc

EDV Elektronische Datenverarbeitung

ESS Extended Service Set

FDMA Frequency Division Multiple Access

FHSS Frequency Hopping Spread Spectrum

FTD Financial Times Deutschland

GAP Generic Access Profile

GK Grenzkosten

GMSC Gateway Mobile Switching Center

GPRS General Packet Radio Service

GSM Global System for Mobile Communications

GSM Global System for Mobile Communications

HLR Home Location Register

HSCSD High Speed Circuit Switched Data

HTML Hypertext Markup Language

HTTP Hypertext Transfer Protocol

i.e.S. / i.w.S. im engeren Sinne / im weiteren Sinne

ICE Information and Content Exchange Protocol

IFS Inter Frame Spacing

IMDb Internet Movie Database

IP Internet Protocol

IPTC International Press Telecommunications Council

IT Informationstechnologie

ITU International Telecommunication Union

luK Information und Kommunikation

KB Kilobyte

Kbit/s Kilobit pro Sekunde

KDD Knowledge Discovery in Databases 


$\begin{array}{ll}\text { KNN } & \text { Künstliches neuronales Netz } \\ \text { L2CAP } & \text { (Bluetooth) Logical Link Control and Adaptation Protocol } \\ \text { LBS } & \text { Location Based Service } \\ \text { MAC } & \text { Medium Access Control } \\ \text { Mbit/s } & \text { Megabit pro Sekunde } \\ \text { MBV } & \text { Market Based View } \\ \text { Mio. } & \text { Million } \\ \text { MMS } & \text { Multimedia Messaging Service } \\ \text { MMSC } & \text { Multimedia Messaging Service Center } \\ \text { Mrd. } & \text { Milliarde } \\ \text { MS } & \text { Mobile Station } \\ \text { MSC } & \text { Mobile Switching Center } \\ \text { NewsML } & \text { News Markup Language } \\ \text { NITF } & \text { News Industry Text Format } \\ \text { OBEX } & \text { (Bluetooth) Object Exchange Protocol } \\ \text { OeBF } & \text { Open eBook Forum } \\ \text { OFDM } & \text { Orthogonal Frequency Division Multiplexing } \\ \text { OMC } & \text { Operation and Maintenance Center } \\ \text { OWL } & \text { Web Ontology Language } \\ \text { P3P } & \text { Platform for Privacy Preferences } \\ \text { PCF } & \text { Point Coordination Function } \\ \text { PHY } & \text { Physical Layer } \\ \text { PLC } & \text { Physical Layer Convergence } \\ \text { PMD } & \text { Physical Medium Dependent } \\ \text { PPS } & \text { Produktionsplanung- und Steuerung } \\ \text { RFC } & \text { Request for Comment } \\ \text { RFID } & \text { Radio Frequency Identity } \\ \text { RNS } & \text { Radio Network Subsystem } \\ \text { RSS } & \text { Radio Subsystem } \\ \text { RSS } & \text { Rich Site Summary } \\ \text { SCP } & \text { Structure Conduct Performance } \\ \text { SDAP } & \text { Service Discovery Application Profile } \\ \text { SDP } & \text { Service Discovery Protocol } \\ & \end{array}$


SIG Special Interest Group

SIM Subscriber Identification Module

SIM Subscriber Identification Module

SMS Short Message Service

SMSC Short Messaging Service Center

sog. So genannte

SPP Serial Port Profile

STA Station

sup Supportfaktor

TCP Transmission Control Protocol

TCP/IP Transmission Control Protocol / Internet Protocol

TDDSG Teledienste Datenschutzgesetz

TDG Teledienstegesetz

TDMA Time Division Multiple Access

tf-idf term frequency- inverse document frequency

TFNM Tomorrow-Focus next media

TKG Telekommunikationsgesetz

UMTS Universal Mobile Telecommunications System

UMTS Universal Mobile Telecommunications System

UTRA UMTS Terrestrial Radio Access

VDZ Verband Deutscher Zeitschriftenverleger

VLR Visitor Location Register

W3C World Wide Web Consortium

WAP Wireless Application Protocol

WAP Wireless Application Protocol

WASP Wireless Application Service Provider

WLAN Wireless Local Area Network

WML Wireless Markup Language

WPAN Wireless Personal Area Network

XML Extensible Markup Language

XSL Extensible Stylesheet Language

XSLT XSL Transformations

z.T. $\quad$ zum Teil 


\section{Einleitung}

Das folgende Kapitel gibt eine Einführung in die vorliegende Arbeit. Die Motivation der Arbeit wird in Abschnitt 1.1 vorgestellt. In Abschnitt 1.2 werden die Problemstellung abgeleitet und der Aufbau der Arbeit erläutert. In Abschnitt 1.3 beschreibt die Forschungsmethodik der Arbeit und fasst das inhaltliche und methodische Vorgehen zusammen.

\subsection{Motivation}

Die Medienbranche war in den vergangenen Jahren mit einem radikalen Wandel der wirtschaftlichen Rahmenbedingungen konfrontiert. Nach einem stürmischen Wachstum zum Ende der 1990er Jahre zeichnete sich zuletzt anhand von zum Teil bedenklich sinkenden Umsatz- und Ertragszahlen eine strategische Bedrohung des Kerngeschäfts, wenn nicht sogar eine dauerhafte Existenzgefährdung $a b .{ }^{1}$ Die derzeitige wirtschaftliche Krise der Medienbranche kann allerdings nicht auf die mangelnde Nachfrage nach ihren Erzeugnissen zurückgeführt werden. Im Gegenteil war aktuellen Medienstatistiken zu Folge die durchschnittliche Medienkonsumdauer noch nie so hoch wie heute. ${ }^{2}$ Stattdessen ist festzustellen, dass Konsumenten von Mediengütern durch neue Möglichkeiten der digitalen Datenkommunikation ihre Informations- und Unterhaltungsbedürfnisse nicht mehr ausschließlich durch konventionell gebündelte Inhalte am Massenmarkt befriedigen, sondern gewünschte Inhalte und deren jeweiliges Format in Abhängigkeit von Tageszeit und Aufenthaltsort aus verschiedenen Quellen individuell selektieren und kombinieren. Die Möglichkeiten der digitalen Datenkommunikation stellen daher zwar nicht einzelne Produktgattungen der Medienbranche in Frage, aber deren standardisiertes, für den Massenmarkt gebündeltes Angebotsformat und damit auf grundsätzliche Weise die Idee der medialen Massenkommunikation.

Der Grund, warum die Medienbranche bisher kaum auf derartige Veränderungen der Nachfrage reagiert hat, besteht darin, dass Massenmedien aus ökonomischer Perspektive in erster Linie Werbeträger sind, deren Umsatz primär auf dem Werbemarkt erzielt wird. ${ }^{3}$ Kommerzielle Medienangebote nutzen dabei üblicherweise ihre Markenbekanntheit, um Kommunikationsreichweite zu erzeugen und diese an Werbetreibende zu vermarkten. ${ }^{4}$ Der Bedeutungsverlust

1 Vgl. Mings/White (2000), S. 63; Gerpott (2003), S. 90; Spiegel-Online (2003); Spiesecke (2004); Spiegel-Online (2004).

2 Vgl. Media Perspektive (2004), S. 64.

3 Vgl. Sennewald (1998), S. 33 und Sjurts (2002a), S. 13.

4 Vgl. Schumann/Hess (2001), S. 41. 
der Massenkommunikation und der dadurch bedingte Rückgang der durchschnittlich erzeugbaren Reichweite hat vor diesem Hintergrund zwei Auswirkungen: Auf der einen Seite sinkt mit dem reduzierten Reichweiteversprechen die wirtschaftliche Bedeutung etablierter Medienmarken auf dem Werbemarkt. Auf der anderen Seite bilden reichweitenbedingte Kostendegressionseffekte im Rahmen der Erzeugung von Mediengütern traditionell die wichtigste strategische Markteintrittsbarriere im Medienwettbewerb. ${ }^{5}$ Der Rückgang der durchschnittlichen Reichweiten verringert diese Barriere und eröffnet neuen Konkurrenten Markteintrittschancen. Als Folge dieser Entwicklung konkurrieren mittlerweile selbst in kleineren Nischen des Medienmarkts oft zwei bis drei Angebote mit sehr ähnlichem Profil in einem zwangsläufigen Verdrängungswettbewerb. Diese Wettbewerbsveränderungen in der Medienbranche führen zusammengefasst dazu, dass „Medienunternehmen (...) bei geringerer Verbreitung [ihrer Erzeugnisse, Anm. d. Verf.] innerhalb kürzerer Zeit und unter schärferem Wettbewerb Geld verdienen [müssen, Anm. d. Verf.]." ${ }^{6}$

Diese Wettbewerbsverschärfung und der daraus entstehende Handlungsdruck für Medienunternehmen bilden die zentrale Motivation dieser Arbeit. Ziel der Arbeit ist es, strategische Handlungsmöglichkeiten für die Medienbranche zu ermitteln, um den dargestellten Veränderungen im Wettbewerb zu begegnen, und diese Handlungsmöglichkeiten auf ihre Erfolgsaussichten zu bewerten.

\subsection{Problemstellung, Zielsetzung und Aufbau}

Entsprechend der etablierten Dialektik der Theorie des strategischen Managements können strategische Handlungsmöglichkeiten, mit denen Unternehmen auf die beschriebenen Veränderungen reagieren, grundsätzlich sowohl aus ressourcenorientierter Perspektive (1) als auch aus marktorientierter Perspektive (2) untersucht werden: ${ }^{7}$

(1) Die ressourcenorientierte Perspektive der strategischen Managementtheorie wurzelt in der Annahme einer heterogenen Verteilung von wettbewerbskritischen Ressourcen zwischen den Unternehmen einer Branche. ${ }^{8}$ Die zentrale Herausforderung eines strategischen Managements bildet daher die Identifikation kritischer Ressourcen (auch: „Kernressourcen“), wenn möglich ihr Erwerb, ihre Potenzialerhaltung und Weiterentwicklung sowie die Förderung ihrer Nutzung im Unternehmen. Kernressourcenpotenzial mit hoher Unternehmensspezifität

5 Vgl. Sjurts (2002a), S. 17.

6 Vgl. Bird/Künster/Vogelsang (2003), S. 72.

7 Vgl. u.a. Ansoff (1979); Waterman (1982); Gälweiler (1987); Hinterhuber (1992); Mintzberg (1994); Bea/Haas (1995); Eschenbach/Kunesch (1996); Kirsch (1997b); Porter (1998); Mintzberg (1999); Keuper (2001); Bryan (2002).

8 Ursachen dieser Heterogenität von Unternehmen bilden die langfristige, gewachsene Einbindung von Ressourcen in den spezifischen organisatorischen Kontext eines Unternehmens, die Unvollkommenheit der Faktormärkte und die befristete Immobilität von Ressourcen aufgrund (mitunter befristeter) vertraglicher Verfügungsrechte. Vgl. Barney (2002), S. 155 und Habann (1999), S. 3 ff. 
im Medienbetrieb besitzen insbesondere inhaltebezogene Ressourcen, bspw. die Verfügbarkeit von Inhalten als Inputfaktor von Mediengütern, etwa als Ergebnis des journalistischen Redaktionsprozesses oder als Folge des Einkaufs am Rechtemarkt, und die (langfristige) Fähigkeit zum Erwerb oder zur Entwicklung solcher wertvollen Inhalte. ${ }^{9}$ Das aus ressourcenorientierter Perspektive zentrale Erfolgspotenzial im Umgang mit den angesprochenen Veränderungen der Wettbewerbsbedingungen bildet vor diesem Hintergrund die Erhöhung der Ressourceneffizienz im Medienbetrieb. Von besonderer Bedeutung hierfür ist die Ausschöpfung von Synergiepotenzialen im Rahmen der Erzeugung von Mediengütern auf Grundlage einer Mehrfachnutzung von Inhalten. ${ }^{10}$

(2) Aus wettbewerbstrategischer Perspektive ist der Erfolg eines Unternehmens das Ergebnis seiner Positionierung im Rahmen der spezifischen Wettbewerbsstruktur der jeweiligen Branche. Ein Unternehmen kann seine Wettbewerbsposition innerhalb der Branche durch zwei strategische Maßnahmen beeinflussen: ${ }^{11}$ durch Differenzierung seines Leistungsangebots gegenüber verfügbaren Konkurrenzprodukten und durch Schaffung relativer Kostenvorteile in der Wertschöpfung gegenüber Konkurrenten. Ausschließlich kostenbezogene Strategien erscheinen in der Medienbranche sowohl vor dem Hintergrund einer geringen Preiselastizität der Nachfrage als auch aufgrund insgesamt niedriger Reproduktionskosten von Mediengütern als wenig vorteilhaft. Die zentrale Handlungsmöglichkeit zur Beeinflussung der Wettbewerbsposition bildet daher das Schaffen von Differenzierungsvorteilen gegenüber Konkurrenzprodukten.

Weder die Vorteilhaftigkeit einer inhaltlichen Differenzierung noch die Bedeutung der Mehrfachnutzung sind grundsätzlich neue Erkenntnisse für die Medienbranche. Beispiele für bekannte Differenzierungs- und Mehrfachnutzungsformen in der Medienbranche bilden etwa das variantenreiche Angebot im Marktsegment für Publikumszeitschriften oder das Mantelangebot von überregionalen Zeitungsverlagen für regionale Zeitungsausgaben. Durch die Digitalisierung im Rahmen der Erzeugung und Distribution von Mediengütern entstehen jedoch neue Möglichkeiten, um diese besser auf die jeweiligen Kundenbedürfnisse und den jeweiligen Rezeptionskontext abzustimmen und dennoch gleichzeitig Synergievorteile durch mehrfache Nutzung vorhandener Inhalte zu wahren. Die verwendungsneutrale, modularisierte Erzeugung von Inhalten und die verbesserte individuelle Identifikation der Rezipienten von Onlineinhalten eröffnen auf der einen Seite die Möglichkeit, Produktvarianten anzubieten, die optimal auf die individuellen Bedürfnisse eines Nutzers zugeschnitten sind und dennoch dem Kostenniveau einer Massenfertigung entsprechen. Dieser Vorgang wird auch als "Individualisierung“ bezeichnet. ${ }^{12}$

9 Vgl. Habann (1999); Brack (2003).

10 Vgl. Brack (2003), S. 140 und Hess/Schulze (2003), S. 1381.

11 Vgl. Porter (1998).

12 Das Thema Individualisierung findet im Zusammenhang mit der Medienbranche u.a. Erwähnung in Shapiro/Varian (1999), Brandtweiner (2000), Hass (2002), Rawolle (2002), Schumann/Hess (2002), Bird/ Künstner/Vogelsang (2003), Brack (2003), Hess/Schulze (2003), Hess (2004) und Sommerwerck (2004). 
Auf der anderen Seite etablieren sich auf der Grundlage des digitalen Mobilfunks neue Möglichkeiten der Inhaltedistribution, die es erlauben, Inhalte potenziellen Rezipienten besser und schneller zur Verfügung zu stellen als mit bislang existierenden Distributionsmedien. Im Zusammenhang mit dem Inhaltemarkt auf der Grundlage des Mobilfunks ist auch vom „mobilen Internet“ die Rede. ${ }^{13}$

An dieser Stelle setzt die Problemstellung der vorliegenden Arbeit an: Sie will aufzeigen, welche Möglichkeiten bestehen, Rezipienten massenmedialer Inhalte einen differenzierungswirksamen Mehrwert zu bieten. Als Grundlage eines solchen Mehrwerts werden in dieser Arbeit zwei Möglichkeiten untersucht: einerseits die Annäherung des Inhalteangebots an die individuelle Idealvorstellung eines Rezipienten durch das Maßschneidern individueller Bündel von Inhalten; andererseits der schnelle und ortsungebundene Zugriff auf Inhalte durch das mobile Internet. Die Arbeit verfolgt dabei eine zweifache Zielsetzung: Zum einen werden die Strategieoptionen Individualisierung und mobile Distribution von Inhalten jeweils auf den durch sie gestifteten Nutzen aus Rezipientensicht hin untersucht (Erklärungsziel). Zum anderen werden im Rahmen beider Strategieoptionen konzeptionelle Fragestellungen im Rahmen der Realisierung individueller und mobiler Mediengüter aufgeworfen und thematisiert (Gestaltungszie/). Die Arbeit orientiert sich vor dem Hintergrund dieser zweifachen Zielsetzung an vier zentralen Forschungsfragen (vgl. Abbildung 1-1):

1. Welche Handlungsmöglichkeiten bieten sich Medienunternehmen, um die derzeitige Krise der Branche zu bewältigen (Erklärungsziel)?

2. Wie können Mediengüter für einen individuellen Kunden maßgeschneidert werden (Gestaltungsziel)?

3. Welchen Nutzen stiften maßgeschneiderte Mediengüter aus Kundensicht (Erklärungsziel)?

4. Wie können Medienunternehmen das mobile Internet als Absatzmarkt für Mediengüter erschließen (Erklärungs- und Gestaltungsziel)?

Abbildung 1-1: Zentrale Forschungsfragen

Die Argumentation der Arbeit folgt dabei dem in Abbildung 1-2 dargestellten Aufbau: Zunächst werden in Kapitel 2 die im Mittelpunkt der Arbeit stehenden Begrifflichkeiten definiert und erläutert. Um sprachliche Ungenauigkeiten in Bezug auf den Gütercharakter der Erzeugnisse von Medienunternehmen im Onlinevertrieb und die Rolle des Kunden im Rahmen der Distribution vorzubeugen, wird in Abschnitt 2.1 der Begriff des "Medienguts“ eingeführt. Die zurzeit mit einer gewissen begrifflichen Unschärfe behaftete Thematik der „Individualisierung" wird in Abschnitt 2.2 im Hinblick auf verwandte Fragestellungen in Soziologie, Volkswirtschaftslehre, Industriebetriebslehre, Marketing und Informatik

13 Der Inhaltevertrieb im mobilen Internet wird u.a. in Bartussek (2001), Dettki/Ferrari/Resch (2001), Gregg (2001), Wirtz (2001a), Feldmann (2002) und Keuper (2002) behandelt. 
eingegrenzt. Die Begriffe des „mobilen Dienstes“ und des „mobilen Internet“ werden in Abschnitt 2.3 eingeführt und es werden die technischen Grundlagen mobiler Dienste in Bezug auf die Möglichkeiten des Inhaltevertriebs erläutert.

In Kapitel 3 wird die strategische Option einer Orientierung des Medienmanagements am individuellen Kundennutzen abgeleitet. Zunächst wird in Abschnitt 3.1 die Rahmendynamik des Wettbewerbs in der Medienbranche am technologieinduzierten Phänomen der Marktkonvergenz von Telekommunikations-, Medien und Informationstechnologie-(IT-)Branche erläutert. Abschnitt 3.2 analysiert die Wettbewerbsituation in der Medienbranche am Beispiel aktuell informierender Marktsegmente wie dem Zeitungs-, dem Zeitschriften-, dem Rundfunk- und dem Onlinemarkt. In Abschnitt 3.3 werden strategische Handlungsmöglichkeiten für die Medienbranche aufgezeigt, um den Kundennutzen im Rahmen der Bereitstellung von Inhalten zu erhöhen und dadurch den Kundenmehrwert zu steigern.

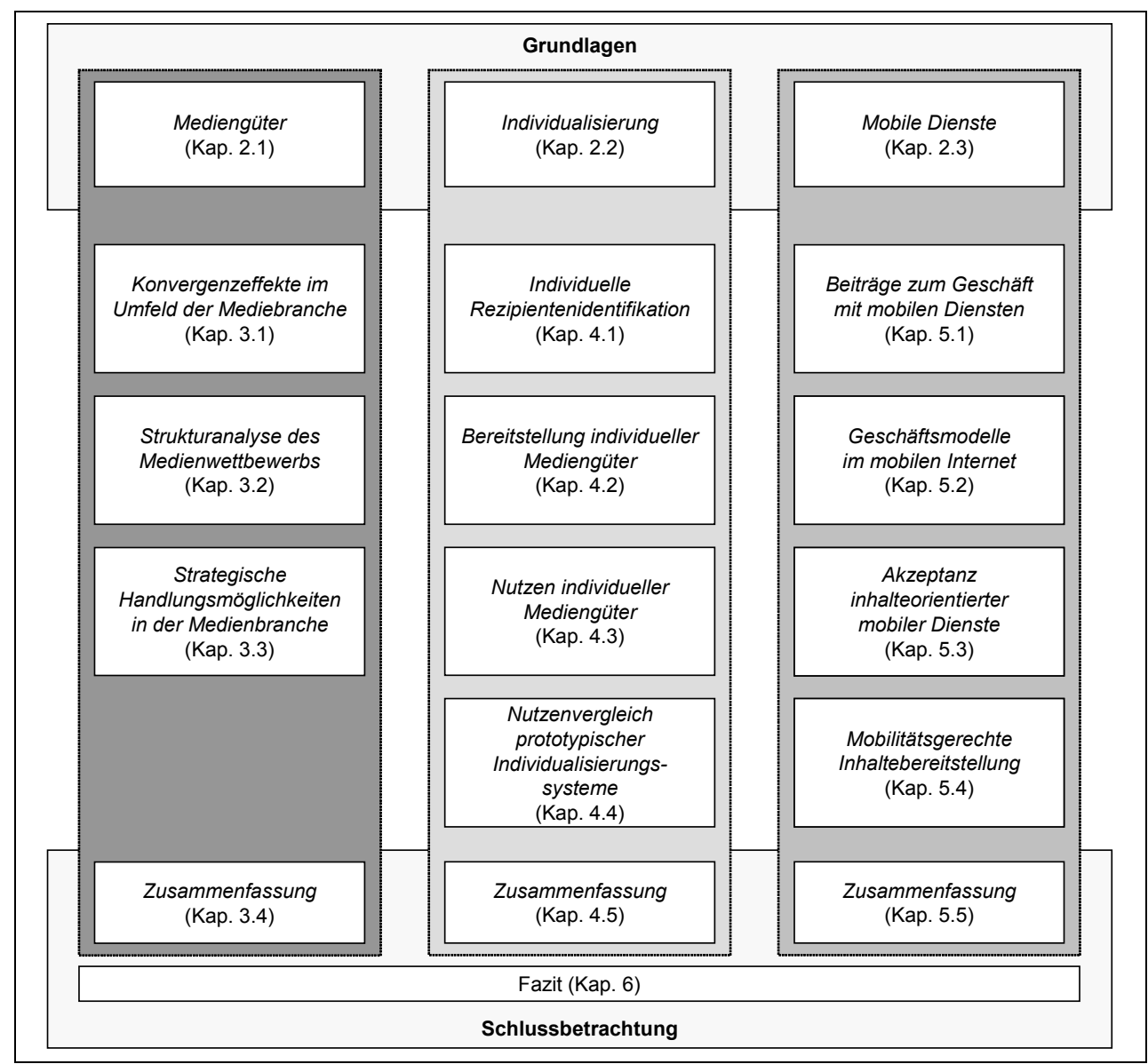

Abbildung 1-2: Gang der Untersuchung und Aufbau der Arbeit

Die Strategieoption der Individualisierung wird in Kapitel 4 im Detail untersucht. In Abschnitt 4.1 werden Möglichkeiten einer individuellen Identifikation der Konsumenten von digitalen Onlinemedien erläutert. Abschnitt 4.2 stellt Verfahren 
vor, mit denen digitale Mediengüter automatisch entsprechend individueller Kundenpräferenzen maßgeschneidert werden können. In Abschnitt 4.3 werden Kriterien für die relative Vorteilhaftigkeit maßgeschneiderter Mediengüter gegenüber dem standardisierten Inhaltevertrieb ermittelt. Diese Kriterien werden in Abschnitt 4.4 in einem Nutzerexperiment anhand prototypischer Realisierungen von Individualisierungssystemen für Mediengüter verifiziert.

In Kapitel 5 werden Gestaltungsmöglichkeiten für inhalteorientierte Angebote, die über Mobilfunknetze vertrieben werden, auf ihren Nutzen für die Rezipienten hin untersucht. Zunächst werden in Abschnitt 5.1 Rahmenbedingungen und Ausprägungsbeispiele von Geschäftsmodellen für die Verwertung von Inhalten im mobilen Internet dargestellt. In Abschnitt 5.2 werden Kriterien für die Akzeptanz des Inhaltevertriebs im mobilen Internet anhand der Ergebnisse empirischer Erhebungen, die im Rahmen dieser Arbeit durchgeführt wurden, ermittelt. Abschnitt 5.3 thematisiert offene konzeptionelle technische Fragen für die Umsetzung einer mobilitätsgerechten Inhaltebereitstellung für das mobile Internet. Die Arbeit schließt in Kapitel 6 mit einer Schlussbetrachtung.

\subsection{Inhaltliche und methodische Abgrenzung der Unter- suchung}

Das Untersuchungsobjekt der vorliegenden Arbeit bildet die Medienbranche mit ihren Erzeugnissen. Aus akademischer Sicht hat die Medienbranche durch das Forschungsfeld der ökonomischen Medienwirtschaft in den vergangenen zehn Jahren - nach zuvor langjährigem Nischendasein - eine breite Aufmerksamkeit erlangt. Dieser Wandel ist abzulesen an der großen Anzahl der jüngst in diesem Bereich veröffentlichten Forschungsbeiträge. In Hass (2002) findet sich ein Überblick über die unterschiedlichen Literaturstränge in der ökonomischen Medienwirtschaft, grafisch dargestellt in Abbildung 1-3.

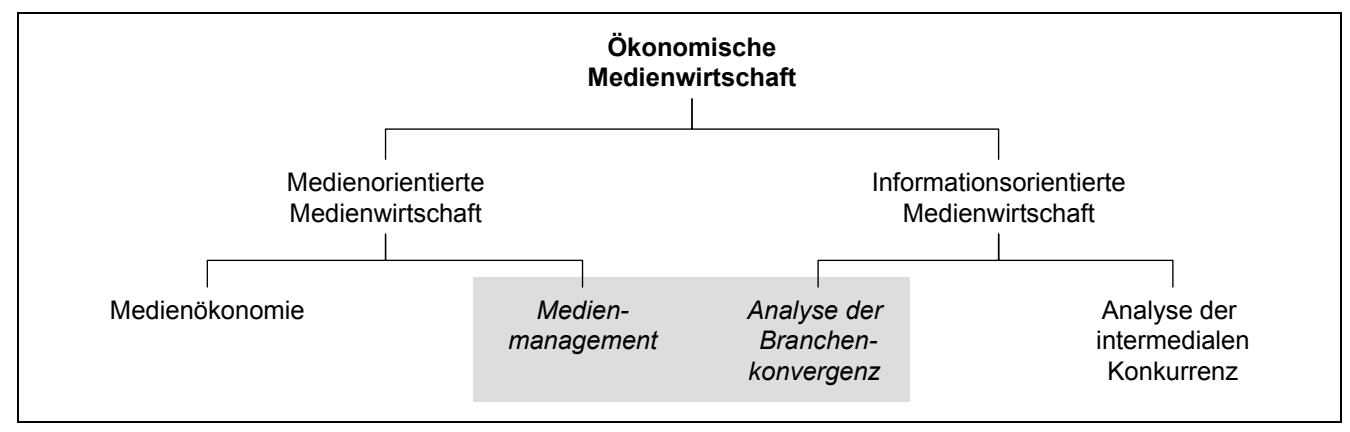

Abbildung 1-3: Forschungsansätze der ökonomischen Medienwirtschaft

Grundsätzlich kann zwischen traditionellen Ansätzen medienorientierter Medienwirtschaft und neueren struktur- bzw. informationsorientierten Ansätzen unterschieden werden. ${ }^{14}$ Medienorientierte Ansätze untersuchen ihr Erklärungs-

14 Vgl. Hass (2002), S. $7 \mathrm{ff}$. 
feld zumeist sektoral, d.h. in Segmenten, die anhand der unterschiedlichen Trägermedien gebildet werden. Im Bereich medienorientierter Ansätze kann weiter eine managementorientierte und eine ökonomische Perspektive unterschieden werden. Betrachtungsgegenstand der Medienökonomie bilden ökonomische Aggregate, also Unternehmen, Märkte oder Branchen. Ansätze der Kommunikations- bzw. der Finanzwissenschaft untersuchen dabei im Wesentlichen die Rolle der Medien innerhalb gesellschaftlicher Strukturen, insbesondere vor dem Hintergrund von Konzentrationstendenzen in den Medienmärkten. Industrieökonomische Ansätze fokussieren auf Wettbewerbsstrukturen innerhalb der unterschiedenen Mediensegmente und leiten daraus betriebwirtschaftliche Handlungsstrategien in Bezug auf betriebliche Ressourcen oder marktbezogenes Wettbewerbsverhalten ab. ${ }^{15}$ Die Herangehensweise im Medienmanagement ist demgegenüber funktionsorientiert, untersucht werden betriebliche Teilbereiche wie Produktion, Vermarktung und Finanzierung innerhalb von Medienunternehmen. ${ }^{16}$

Neuere Beiträge in der Medienwirtschaft thematisieren Mechanismen der Inhaltsvermarktung unter der Bedingung digitaler Erzeugung und Distribution. ${ }^{17}$ Dabei wird bewusst die Sichtweise der traditionellen Medienbranche verlassen und eine breitere informationsorientierte Sichtweise, die mitunter auch als „Internetökonomie" bezeichnet wird. ${ }^{18}$ Hass benennt zwei zentrale Anwendungsfelder der informationsorientierten Sichtweise in der Forschung der Medienwirtschaft: die Analyse der intermedialen Konkurrenz, insbesondere in Bezug auf die Beziehung zwischen Internet und ausgewählten traditionellen Vermarktungsformen, und die Untersuchung der Auswirkungen der Konvergenzprozesse der im Rahmen der Internetökonomie interagierenden Branchen auf die Rolle von Medienunternehmen als Inhaltsproduzenten und -distributoren. ${ }^{19}$

Den Untersuchungsgegenstand der vorliegenden Arbeit bilden neue technische Möglichkeiten zur Bündelung und Distribution von Inhalten in der Medienbranche. Aus Sicht der ökonomischen Medienwirtschaft adressiert diese Untersuchung sowohl funktionale als auch informationsorientierte Aspekte und ist daher in einem Überschneidungsbereich zwischen Medienmanagement und

15 Zu den deutschsprachigen Beiträgen zur industrieökonomisch ausgerichteten Medienökonomie gehören u.a. Sjurts (1996) und Sjurts (2002a), Heinrich (1999a), Heinrich (1999b) und Sennewald (1998); markt- bzw. ressourcenorientierte Betrachtungen finden sich darüber hinaus u.a. in Habann (1999), Wirtz (2001b).

16 Deutschsprachige Beiträge zum Medienmanagement bilden u.a. Karmasin/Winter (2000), Wirtz (2001a) und Schumann/Hess (2002).

17 Zu den informationsökonomischen Standardwerken zählen Kuhlen (1995), Shapiro/Varian (1999), Evans/Wurster (2000), Zerdick et al. (2001).

18 Der Begriff der Internetökonomie findet explizit Verwendung bei Zerdick et a. (2001), synonym werden in anderen Beiträgen die Begriff "Network Economy" u.a. in Shapiro/Varian (2001) oder "digital Economy" u.a. in Haertsch (2001) verwendet.

19 Fragen intermedialer Konkurrenz werden u.a. thematisiert in Sennewald (1998), Sjurts (2002a) und Sjurts (2002b); Konvergenzprozesse werden u.a. untersucht in Thielmann (2000), Zerdick et al. (2001), Scholz et al. (2001), Keuper (2002); Die Rolle der Medienbranche im Zuge der Konvergenz wird u.a. untersucht in Brandtweiner (2000), Vizjak/Ringlstetter (2001). 
informationsorientierter Medienwirtschaft angesiedelt (in Abbildung 1-3 grau hinterlegt). Als Forschungsmethodik wird vorwiegend der betriebswirtschaftliche Methodenkanon ${ }^{20}$ angewendet, im Detail finden jedoch darüber hinaus kommunikationswissenschaftliche und industrieökonomische Ansätze Anwendung:

- Die Entwicklung der Leithypothese des durchschnittlichen Rückgangs der Reichweite von Massenmedien erfolgt auf induktivem Wege. Grundlage der Schlussfolgerung bilden öffentlich zugängliche Branchendaten zum Medienwettbewerb. Zur Darstellung der Wettbewerbsituation wird auf industrieökonomische Ansätze zurückgegriffen. Bei der Beschreibung der Wettbewerbsituation werden aktuell produzierende Segmente wie der Zeitschriften-, der Zeitungs- und der Rundfunkmarkt fokussiert, unterhaltungsorientierte Segmente wie Musik- und Filmindustrie werden dagegen vernachlässigt.

- Die Definition des Rezeptionsnutzens von Inhalten in der Medienbranche erfolgt in Anlehnung an das voluntaristische Paradigma des Nutzen- und Belohnungsansatzes aus der kommunikationswissenschaftlichen Rezipientenforschung. Mediennutzung repräsentiert darin ein individuell bestimmtes, rational-zielorientiertes Wahlverhalten. Die Diskussion von Strategien zur Steigerung des Kundennutzens erfolgt durch theoriegeleitete Deduktion auf Grundlage der mikroökonomischen Markt- und Preistheorie, wodurch weitgehend von individualpsychologischen Fragestellungen des Produktnutzens abstrahiert wird.

- Die konzeptionelle Darstellung der Individualisierungsmöglichkeiten in Kapitel 4 und die Erläuterung einer mobilitätsgerechten Inhaltedistribution in Kapitel 5 erfolgen auf deduktivem Weg, durch die Benennung von Anforderungen und die Bestimmung einer Lösung aus fachlicher und datenverarbeitungs-(dv)technischer Sicht. Die Auswahl von Lösungsschritten erfolgt vor dem Hintergrund von Studien der jeweiligen Literatur sowie auf Grundlage eigener Forschungsbeiträge. In der Natur des zugrunde liegenden Gegenstandsbereichs der Individualisierung liegt es, dass Lösungsschritte dabei teilweise existierende oder im Rahmen der Arbeit fortentwickelte Algorithmen beinhalten.

- Die Bewertung des ökonomischen Nutzens einer Individualisierung von Mediengütern erfolgt auf der Grundlage einer ökonomischen Modellierung der Kaufentscheidung eines Rezipienten. Dabei wird ein Optimierungskalkül entwickelt, das auf bestehenden Modellen der mikroökonomischen Bündelungs- und Preistheorie aufsetzt.

- Die Modellierungsergebnisse werden auf empirisch-induktivem Wege verifiziert. Die Verifizierung erfolgt durch eine Kombination aus schriftlicher und mündlicher Befragung am Gegenstand zweier prototypisch implementierter Individualisierungsalgorithmen. Ebenfalls auf dem empirischinduktiven Weg erfolgt die Bewertung der Akzeptanz des Inhaltevertriebs

20 Zu den betriebswirtschaftlichen Forschungsmethoden gehören nach Bea/Dicht//Schweitzer (2000) im Wesentlichen Klassifizierung und Typologie, Induktion, Deduktion, Modellierung und Algorithmik. 
im mobilen Internet. Dazu wurden Onlinebefragungen durchgeführt, deren Ergebnisse statistisch und ökonometrisch ausgewertet wurden.

Zusammenfassend kann die Untersuchung wie folgt abgegrenzt werden: Das Untersuchungsobjekt ist die Medienbranche. Der konkrete Untersuchungsgegenstand ist die Bündelung und Distribution von Inhalten der Medienbranche. Untersuchungsmotivation bilden Veränderungen in den Wettbewerbsbedingungen der Medienbranche, die durch die Reduzierung durchschnittlicher Reichweiten massenmedialer Inhalte zu einer Wettbewerbsverschärfung führen. Die wichtigsten Einflussfaktoren der Untersuchung bilden neue Möglichkeiten zur individuellen Bündelung und Distribution von Inhalten im leitungsgebundenen und mobilen Onlinegeschäft. Untersuchungsziel ist es, zu zeigen, inwiefern die neuen Möglichkeiten im Rahmen der Bündelung und Distribution einen Beitrag leisten, einen Ausweg aus dem strategischen Reichweitendilemma in der Medienbranche zu finden. Im Mittelpunkt stehen sowohl technische Gestaltungsempfehlungen als auch die Erklärung des Nutzenbeitrags der Strategieoptionen der Individualisierung und der Inhaltedistribution im mobilen Internet aus Sicht des Kunden. 



\section{Grundlagen}

Im folgenden Kapitel wird ein Überblick über die zentralen Begriffe und die relevante Grundlagenliteratur der Arbeit gegeben. In Abschnitt 2.1 wird der Begriff des Medienguts in seiner hier zugrunde gelegten Verwendung eingeordnet und erläutert. In Abschnitt 2.2 wird die vielfältige Begriffs- und Theoriewelt in Verbindung mit dem Thema "Individualisierung" dargestellt. Abschnitt 2.3 definiert den in dieser Arbeit zugrunde gelegten Begriff des „mobilen Dienstes“. Zusätzlich wird ein Überblick über die Technik und die Anwendungsmöglichkeiten des Mobilfunks gegeben.

\subsection{Mediengüter}

Der Begriff der "Medien“ wurde in den vergangenen Jahren stark strapaziert, ob nun bspw. im Zusammenhang mit der volkswirtschaftlichen Diskussion um die Bedeutung des Unterhaltungs- und Informationssektors oder als Modewort der „Neuen“ Medien, synonym für sowohl Übertragungstechnologien als auch Endgeräte im Internetzeitalter. ${ }^{21}$ Dabei bleibt zunächst festzuhalten, dass der Begriff des Mediums, der bis zu Mitte des letzten Jahrhunderts „eher mit Elementen wie Wasser, Feuer und Luft oder mit spirituellen Medien verknüpft“22 wurde, in der heutigen Zeit grundsätzlich zwar neu, jedoch, wie die einleitenden Beispiele zeigen, sehr uneinheitlich belegt ist. Der folgende Abschnitt soll dazu dienen, Licht in die zum Teil populärwissenschaftliche Vermischung der Begriffe in Bezug auf Medien, Informations- und digitale Güter zu bringen (vgl. Abschnitt 2.1.1) und einen kurzen Überblick über die ökonomischen Besonderheiten der Medienbranche zu geben (vgl. Abschnitt 2.1.2). Abschnitt 2.1.3 fasst die Ergebnisse des Kapitels zusammen.

\subsubsection{Begriff des Mediums}

Im alltäglichen Sprachgebrauch lassen sich drei Facetten des Medienbegriffs, eine instrumentelle, eine organisatorische und eine gesellschaftliche, unterscheiden: ${ }^{23}$ Aus instrumenteller Perspektive repräsentiert ein Medium ein „In-

21 Vgl. Sennewald (1998), S. 9: „Als neu werden die in den letzten zehn Jahren entwickelten Medien bezeichnet. (...) Die neuen Medien sind ein Komplex von Informations- und Kommunikationstechniken, die als Leistungsbündel aus Produkten und Dienstleistungen verstanden werden können." Demgegenüber werden nach Sennewald Medien als traditionell bezeichnet, „wenn sich das technische Verfahren zur Herstellung oder zur Distribution in den letzten zehn Jahren nicht grundlegend geändert hat."

22 Hörisch (1998); vgl. außerdem Kiefer (2001), S. 14.

$23 \mathrm{Vgl}$. Beck (2002), S. 1. 
strument zur Verarbeitung von Informationen“24 oder technologischer, ein „Zeichentransportsystem“25 im Sinne einer „vermittelnden Instanz" zwischen kommunizierenden Parteien. Unter organisatorischem Blickwinkel lassen sich Medien als organisierte Sozialsysteme zur Erfüllung einer Informations- und Kommunikationsaufgabe beschreiben. Schließlich werden Medien auch als gesellschaftliche Institutionen verstanden, „zur Erfüllung von Leistungsbeiträgen an gesellschaftlichen Strukturen und Prozessen"26. Im Weiteren soll in Anlehnung an Saxer (1996) und Kiefer (2001) ein integrierender, tendenziell auf den Aspekt der Institution fokussierender Begriff der Medien verwendet werden. Medien sind demnach „komplexe institutionalisierte Systeme um organisierte Kommunikationskanäle von spezifischem Leistungsvermögen“27.

Um begrifflichen Verallgemeinerungen und Verwechslungen vorzubeugen, soll im Weiteren zwischen Medienunternehmen und Mediengütern unterschieden werden: Medienunternehmen werden häufig über ihren Leistungsprozess, durch das „Erzeugen, Bündeln und Distribuieren von Informationen und Unterhaltung" ${ }^{28}$ charakterisiert. Aus der hier zugrunde gelegten Sicht der Medienwirtschaft repräsentieren Mediengüter die Ergebnisse bzw. die Ausbringungsmenge der Leistungsprozesse eines Medienunternehmens ${ }^{29}$. „Aus Sicht der Mediennutzer produzieren Medienunternehmen Bündel von Texten, Bildern, verschiedenen Varianten von Audio- und Videoangeboten sowie in wachsendem Maße auch interaktiven Angeboten zur Befriedigung des Bedürfnisses nach Unterhaltung und Information." ${ }^{30}$

Aus einer technischeren Perspektive repräsentieren Mediengüter Informationsgüter, die an ein materielles bzw. digitales Trägermedium gebunden sind. ${ }^{31}$ Damit kann der Begriff des Medienguts solchen Definitionen des Informationsbegriffs gleichgesetzt werden, die Informationen über ihren „unvermeidbaren Dualismus zwischen materieller und immaterieller Sphäre"32 "charakterisieren. Vor dem Hintergrund dieses Dualismus können aus einer kommunikationstechnischen Sicht insgesamt fünf Schichten eines Informationsguts unterschieden werden (vgl. Abbildung 2-1): ${ }^{33}$

\footnotetext{
24 Vgl. Beck (2002).

25 Kiefer (2001), S. 15.

26 Kiefer (2001), S. 15.

27 Saxer (1996), S. 20; Kiefer (2001), S. 15.

28 Schumann/Hess (2002), S. 1.

29 Rawolle/Hess (2001), S. 2.

30 Rawolle (2002), S. 6.

31 Vgl. Hass (2002), S. 18

32 Bode (1997).

33 Vgl. Mowshowitz (1992), S. 234 ff.
} 


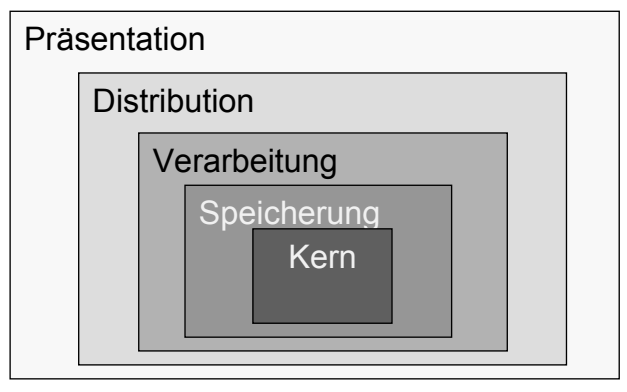

Abbildung 2-1: Schichten eines Informationsguts

Den Kern eines Informationsgutes bildet die transportierte Nachricht. Voraussetzung dafür, dass Nachrichten transportiert werden können, ist deren Speicherung auf einem Medium. Gespeicherte Nachrichten können distributionsgerecht verarbeitet werden, indem sie reorganisiert, aktualisiert, neu dargestellt oder durchsucht werden. Verarbeitete Nachrichten können über entsprechende Transportkanäle distribuiert werden. Dabei kann der jeweils gewählte Transportkanal bspw. durch seinen Aktualitätsgrad den Wert der transportierten Nachricht beeinflussen. Damit die transportierte Nachricht an einen Rezipienten übermittelt werden kann, muss die transportierte Nachricht bspw. durch das Transportmedium selbst oder durch ein spezielles Präsentationsmedium in verständlicher Form präsentiert werden.

Abhängig vom Interaktionsgrad zwischen Inhalteanbieter und -nachfrager unterscheidet Bode in Bezug auf Informationsgüter weiter zwischen Informations-(Sach-)Gütern und Informationsdienstleistungen. ${ }^{34}$ Vor dem Hintergrund der derzeit stattfindenden Entwicklungen im Rahmen der Informations- und Kommunikationstechnologien und der dadurch bedingten Veränderungen der Interaktionsprozesse ${ }^{35}$ scheint jedoch eine strikte Trennung zwischen Dienstleistungs- und Sachgüteraspekt eines Medienguts nicht mehr zeitgemäß. Die zunehmende Möglichkeit der Integration des Kunden und seiner Präferenzen in die Prozesse der Erstellung, Bündelung und Distribution medialer Inhalte ist ein Gegenstand dieser Arbeit und wird im Weiteren noch dezidiert betrachtet. Daher ist der Begriff des "Medienprodukts" für die weitere Untersuchung zu eng gefasst. Stattdessen wird im Weiteren der allgemeinere Begriff des „inhalteorientierten Dienstes“ bzw. - noch allgemeiner - des „Medienguts“ verwendet.

\subsubsection{Eigenschaften von Mediengütern}

Nach Rawolle (2002) kann für die Betrachtung der Merkmale von Mediengütern zwischen einer produktionsorientierten und einer verwendungsorientierten Per-

34 Vgl. Bode (1997) und Brandtweiner (2000), S. 33. Im Falle von Informationsdienstleistungen repräsentiert der Nachfrager einen „externen Faktor" der Leistungserbringung, ohne den eine Konkretisierung und damit Fertigstellung der Leistungserbringung unmöglich wäre.

35 Vgl. u.a. Piller (2001). 
spektive unterschieden werden. ${ }^{36}$ In produktionsorientierter Hinsicht sind drei Aspekte von Bedeutung: Die Struktur der Wertschöpfung, davon abhängig medienspezifische Aspekte der Kosten- und Erlösstruktur sowie die Möglichkeiten und Konsequenzen resultierend aus der zunehmenden Digitalisierung (vgl. Abschnitt 2.1.2.1). In verwendungsorienterter Hinsicht sind dagegen solche, in den Eigenschaften immaterieller Informationsgüter wurzelnde Wesensmerkmale darunter Netzeffekte und die Bewertungsproblematik von Mediengütern im Rahmen der Konsumentscheidung - zu erörtern (vgl. Abschnitt 2.1.2.2).

\subsubsection{Produktionsorientierte Perspektive}

Wie bereits angesprochen, lässt sich der Wertschöpfungsprozess in der Medienbranche grob zunächst in drei Schritte untergliedern: die Erzeugung, die Bündelung und die Distribution von Mediengütern. Abhängig vom jeweiligen Marktsegment kann diese Überblicksbetrachtung der Wertschöpfung in den verschiedenen Teilmärkten der Medienbranche durchaus unterschiedliche Formen annehmen. Klassischerweise erfolgt die Abgrenzung von Teilmärkten der Medienbranche auf der Grundlage der jeweils erzeugten Produkte bzw. ihren materiellen Trägern, bspw. in die Sektoren Zeitungen, Zeitschriften, Hörfunk, Fernsehen und Online. ${ }^{37}$ Abbildung 2-2 zeigt an den Beispielen des Zeitungs- und des Fernsehsegments einen kleinen Ausschnitt der unterschiedlichen Wertschöpfungsstrukturen in der Medienbranche.

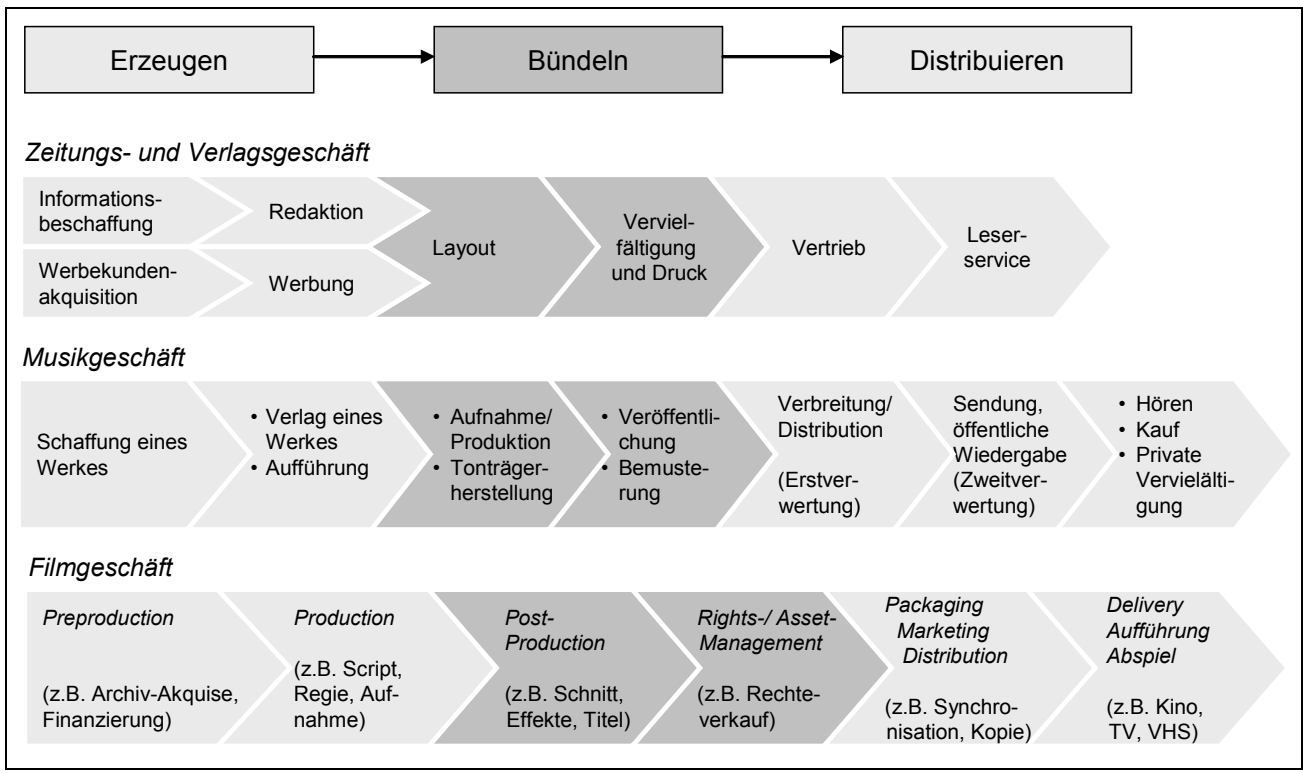

Abbildung 2-2: Wertschöpfungsstrukturen in der Medienbranche ${ }^{38}$

36 Vgl. Rawolle (2002), S. 6 f.

${ }^{37} \mathrm{Vgl}$. Boening-Spohr/Hess (1999), S. 3 f.

${ }^{38}$ Vgl. Zerdick et al. (2001), S. 62, S. 64 und S. 65. 
Mediengüter sind in Abhängigkeit von der Produktgattung durch unterschiedliche Erlösformen und -strukturen gekennzeichnet. Abbildung 2-2 impliziert bereits eine Reihe verschiedener Erlösformen in der Medienbranche: Abonnements, bspw. für Zeitungen oder Bezahlfernsehen; nutzungsunabhängige Gebühren, bspw. im öffentlichen Rundfunkbereich; staatliche Subventionen; Werbung sowie Erlöse aus nutzungsbezogenen Verkäufen und Einzeltransaktionen. Dabei weisen die verschiedenen Erlösformen von Mediengütern zum Teil komplexe Abhängigkeiten auf, wie kurz am Beispiel der Mechanismen der Werbefinanzierung erläutert werden soll:

Medienunternehmen stehen in einem vieldimensionalen Wettbewerb, unter anderem um das finanzielle Budget der Konsumenten, um den Zugang zu Inhalten auf der Ebene der Rechtevermarktung, im publizistischen Wettbewerb mit Konkurrenten und nicht zuletzt im Zeitwettbewerb mit anderen Formen der Information und Unterhaltung um die Aufmerksamkeit potenzieller Nutzer. ${ }^{39}$ Zwischen den genannten Wettbewerbsstrukturen existieren vielschichtige Abhängigkeiten, häufig zusammengefasst unter dem Stichwort der so genannten „Anzeigen-/Auflagen-Spirale": Attraktive publizistische Inhalte schaffen Aufmerksamkeit im Rezipientenmarkt. Mit steigenden Auflagen wächst auch das Interesse nach Werberaum auf dem Werbemarkt. Steigende Erlöse durch Abverkäufe und Werbeeinnahmen stärken die Marktposition in der Inhalteakquise, was wiederum die Qualität der publizistischen Inhalte erhöht. Abbildung 2-3 gibt die Interdependenzstruktur der Medienmärkte wieder:

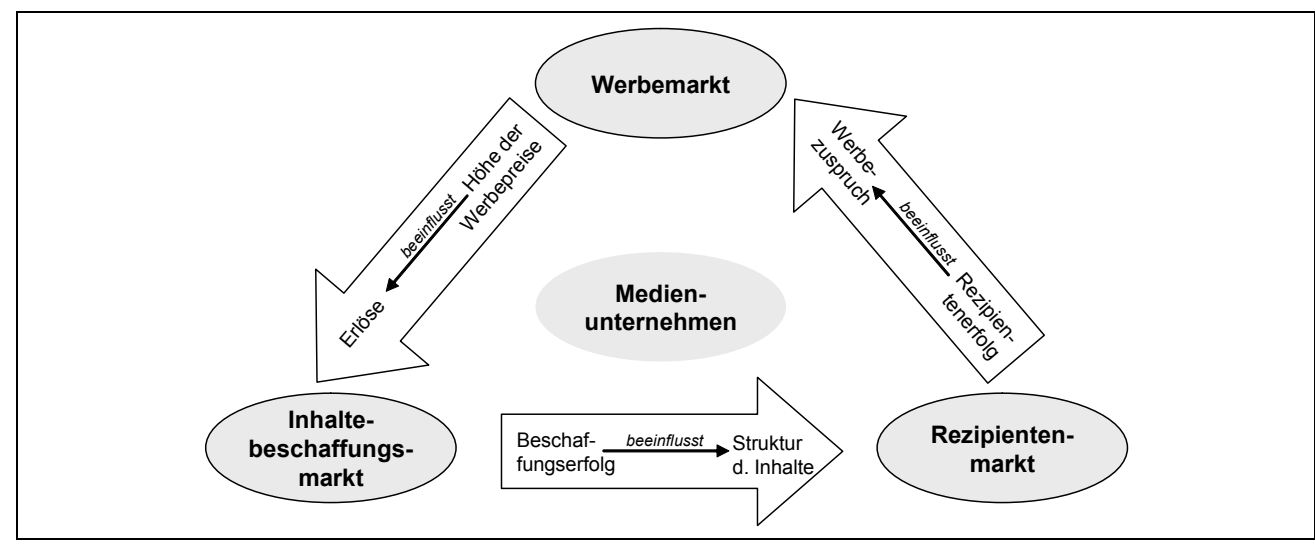

Abbildung 2-3: Anzeigen-Auflagen-Spirale ${ }^{40}$

Mediengüter werden häufig als Kuppelprodukte beschrieben, immer dann, „wenn ein Erwerbsunternehmen Annoncenraum als Ware erzeugt, die nur durch

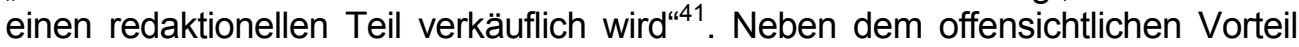
der Erschließung zusätzlicher Finanzierungsquellen am Werbemarkt liegen in der Beziehung zwischen redaktionellen Inhalten und Annoncenraum für Medienunter-

\footnotetext{
39 Vgl. Wirtz (2001a), S. 23 f.

40 Vgl. Wirtz (2001a), S. 22.

41 Bücher (1926).
} 
nehmen eine Reihe von Verbundvorteilen, bspw. durch die Reduktion der Produktionskosten und der gemeinsamen Verteilkosten im Vertrieb oder durch die „symbiotische Kopplung" gegenüber der Aufmerksamkeit und Wahrnehmung von Rezipienten. ${ }^{42}$

Ein weiteres, für viele Teilmärkte der Medienbranche gültiges Merkmal von Medienunternehmen sind Produktionsstrukturen mit sinkenden Durchschnittskosten. Sinkende Durchschnittskosten sind Ergebnis eines in der Regel hohen Fixkostenanteils - bspw. aufgrund hoher Aufwendungen für Druckanlagen, Fernsehstudios oder Redaktionsmitarbeiter - gegenüber im Verhältnis nur moderat wachsenden variablen Kosten bei steigender Ausbringungsmenge. Ergebnis dieser Kostenstrukturen ist ein natürlicher Konzentrationsprozess in den betroffenen Medienmärkten, resultierend aus der Möglichkeit zur Ausschöpfung von kostenreduzierenden Größenvorteilen. ${ }^{43}$ Abbildung 2-4 stellt einen typischen Kostenverlauf grafisch dar.

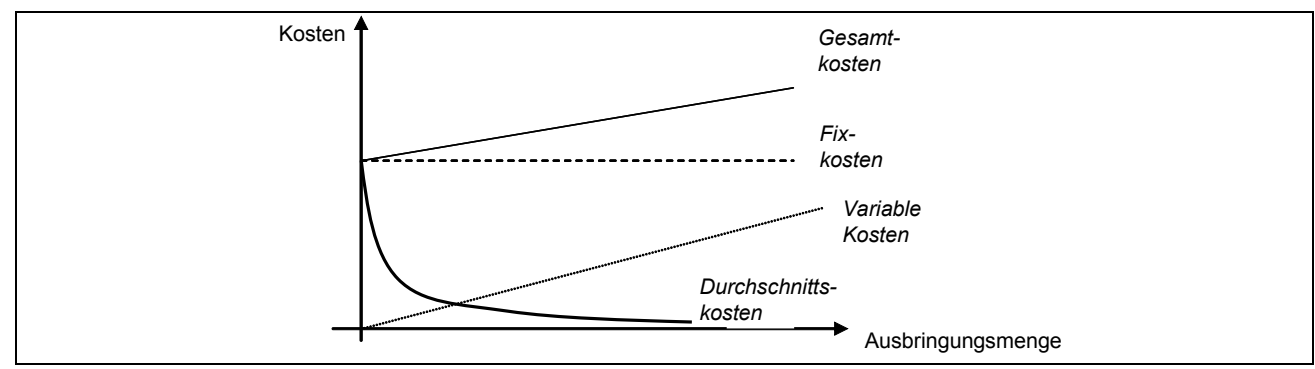

Abbildung 2-4: Typische Kostenstruktur in der Medienbranche

Wie bereits angesprochen, repräsentieren Mediengüter grundsätzlich (an materielle Trägermedien gebundene) Informationsgüter. Aus produktionsorientierter Sicht kann daher als Informationsgut jedes Gut verstanden werden, das als Folge von Bits codierbar und damit digitalisierbar ist. ${ }^{44}$ In diesem Zusammenhang muss allerdings zwischen digitalen Gütern und Informationsgütern differenziert werden: ${ }^{45}$

- Ein digitales Gut ist ein Gut, das in elektronischer Form, also vercodiert als Menge von Bits und Bytes vorliegt und somit über eine Netzwerkinfrastruktur geliefert werden kann, z.B. Computerprogramme.

- Ein Informationsgut ist ein Gut, das wegen seines Inhalts gekauft wird. Der wertbildende Bestandteil des Guts ist die Nachricht, sprich die Information oder die Unterhaltung, die es transportiert. ${ }^{46}$ Die transportierte Nachricht muss dabei für den Empfänger (subjektive) Signifikanz und Wirkung haben. ${ }^{47}$ Beispiele für Informationsgüter sind Bücher, Lexika, Tageszeitungen, Sportnachrichten oder Bedienungsanleitungen.

\footnotetext{
42 Kiefer (2001), S. 154.

$43 \mathrm{Vgl}$. Beck (2002), S. $28 \mathrm{f}$.

44 Vgl. Shapiro/Varian (1999), S. 3.

45 Vgl. Brandtweiner (2000), S.37.

46 Vgl. Abschnitt 2.1.1.

47 Vgl. Kotkamp (2001), S. 42 ff.
} 
- Ein digitales Informationsgut ist ein Gut, das beide Eigenschaften in sich vereint.

Aus der grundsätzlichen Digitalisierbarkeit von Mediengütern zum einen und der kontinuierlichen Weiterentwicklung digitaler Technologien zur Produktion und Distribution von Informationen zum anderen ergeben sich eine Reihe von Besonderheiten für die Bereitstellung und Distribution digitaler Inhalte, wie eine vereinfachte Erzeugung und Bearbeitung von Inhalten, deren vereinfachte Reproduzierbarkeit, erhöhte Speicher- und Übertragungskapazitäten für Inhalte, die Möglichkeit einer Kombination unter-schiedlicher Medientypen, die Möglichkeit interaktiver Komponenten in der Medienproduktion oder die vereinfachte Ent- und Neubündelung von Inhalten. ${ }^{48}$ Der vermehrte Einsatz digitaler Technologien als Träger von Mediengütern führt dabei nach Hass zu einer langfristigen "Desintegration“ von Träger-medium und Informationsgut. ${ }^{49}$ Dadurch entsteht zum einen für die - wie angesprochen - traditionell anhand verschiedener Trägermedien gezogenen Marktgrenzen innerhalb der Medienbranche ein Abgrenzungsproblem relevanter Wettbewerbsmärkte. Dieses Abgrenzungsproblem wird eingehend in Kapitel 3 thematisiert. Zum anderen schaffen die Möglichkeiten der interaktiven Produktgestaltung eine frühzeitige Einbindung von Kundeninformationen und -präferenzen in die Distribution und Produktion der Inhalte. Demzufolge lässt sich gegenüber dem dargestellten, dreistufigen Konzept ein um die vierte Stufe der „Konsumtion“ erweitertes Wertschöpfungsmodell vorstellen, in dem der Konsument statt seiner Rolle als passiver Rezipient eine aktive Rolle als „Prosument“ in der Wertschöpfung einnimmt. ${ }^{50}$

\subsubsection{Verwendungsorientierte Perspektive}

Mediengüter - dies wurde oben implizit vorausgesetzt - besitzen den Charakter ökonomischer Güter: sie sind knapp, dienen der Befriedigung menschlicher Bedürfnisse und sind auf Märkten handelbar. Versucht man eine Einordnung von Mediengütern in die verschiedenen Klassifikationsmöglichkeiten von Gütern in der Ökonomie, zeigen sich - neben der bereits angesprochenen Ambiguität in Bezug auf Sachgüter- und Dienstleistungscharakter von Mediengütern - eine Reihe von spezifischen Merkmalen, die im Folgenden näher untersucht werden sollen.

Vor dem Hintergrund der einleitend dargelegten institutionellen Betrachtungsperspektive des Medienbegriffs erfüllen Mediengüter eine gesellschaftliche und öffentliche Informations- und Unterhaltungsfunktion. Über den Grad der Verfügbarkeit von Mediengütern existiert eine kontroverse Diskussion in der Literatur: Mediengüter, die über den öffentlichen Rundfunk vertrieben werden, zeichnen sich bspw. durch eine (teilweise) Nicht-Ausschließbarkeit bestimmter Rezipienten vom möglichen Konsumentenkreis sowie durch eine Nicht-Rivalität

\footnotetext{
$48 \mathrm{Vgl}$. Hess/Rawolle (2001).

49 Vgl. Hass (2002), S. 11.

50 Vgl. Hass (2002), S. 19 f.; Zum Begriff des Prosumenten vgl. Toffler (1980); Meffert/Bruhn (1997).
} 
ihres Konsums aus. ${ }^{51}$ Dies lässt vermuten, dass Mediengüter Eigenschaften öffentlicher Güter auf weisen. ${ }^{52}$ Betrachtet man allerdings als Gegenbeispiele Konsumrivalität um vergriffene Kinokarten bzw. die Zugriffsrestriktionen im gebührenpflichtigen Kabelfernsehen oder durch limitierte Buchauflagen, zeigen sich Merkmale privater Güter. Diese Kontroverse ist zu lösen durch die bereits in Abbildung 2-1 angedeutete analytische Trennung zwischen medialem Inhalt und Träger: „Konsumrivalität besteht nur bei den Kopien auf materiellem Träger [also der im Rahmen von Bündelung und Distribution erbrachten Dienstleistung von Medienunternehmen; Anm. d. Verf.], nicht beim Inhalt. “53

Ein potenzieller Rezipient trifft seine Konsumentscheidung für ein Informationsgut in Abwägung zwischen seinem Freizeit- und Finanzbudget und seinem erwarteten Nutzen aus dem Konsum. ${ }^{54}$ Gegenüber der Konsumentscheidung für materielle Güter wird die Nutzenbewertung von Informationsgütern durch eine Reihe von Effekten beeinflusst:

(1) Ein Informationsgut zeichnet sich unter anderem dadurch aus, dass es „auch bei mehrfacher Nutzung nicht verbraucht wird. " ${ }^{55}$ Dies entspricht dem bereits angesprochenen Prinzip der Nichtrivalität. Der Wert eines Informationsguts bleibt jedoch nicht generell konstant, sondern kann entweder schnell nach der Erzeugung des Gutes an Wert verlieren (bspw. aktuelle Zeitungsmeldungen) oder sogar tendenziell mit dessen zunehmender Verbreitung ansteigen. ${ }^{56}$ Die Ursache letztgenannter positiver Rückkopplungen zwischen Verbreitung und Wert eines Informationsguts liegt in so genannten Netzwerkexternalitäten im Rahmen einer sozial oder insbesondere technisch vernetzten Mediennutzung. Netzwerkexternalitäten beschreiben grundsätzlich den Anstieg des Nachfragewerts eines Produkts als Nebenwirkung von dessen Nutzung. ${ }^{57}$ Dabei kann zwischen direkten und indirekten Rückkopplungseffekten in Bezug auf den Wert eines Guts in Abhängigkeit von seiner Nutzung und -verbreitung unterschieden werden: ${ }^{58}$

- Direkte Netzeffekte finden sich üblicherweise im Rahmen kommunikations- und interaktionsorientierter Dienste wie dem Telefon, E-Mail oder Filesharing-Netzwerken. Jeder zusätzliche Nutzer und jedes weitere im Netzwerk verfügbare Gut steigert dabei den Wert des Netzwerks. Im Zu-

51 Vgl. u.a. Beck (2002), S. 8.

52 Vgl. u.a. Wirtz (2001), S. 30; Heinrich (2001a), S. 71 und 94; Beck (2002), S. 14.

53 Kiefer (2001), S. 146.

54 Vgl. Schumann/Hess (2002), S. 39.

55 Picot/Franck (1988).

56 Vgl. Kiefer (2001), S. 147 und Zerdick et al. (2001), S. 156. Beispiele für Informationsgüter, die mit steigender Verbreitung an Wert gewinnen können, bilden etwa Titel der Unterhaltungsmusik oder wissenschaftliche Artikel und Studien.

57 Vgl. Hess (2000) und Schumann (1992); Netzeffekte beziehen also eine Gegenposition zu den Annahmen der neoklassischen Ökonomielehre, die von einem sinkenden, in dessen Knappheit begründeten Werts eines Gutes in Folge von dessen zunehmender Verbreitung ausgeht.

58 Vgl. Hess (2000). 
sammenhang mit der Verbreitung von Informationsgütern kann insbesondere bei werbefinanzierten Inhalten (aufgrund der bereits angesprochenen Anzeigen-Auflagen-Spirale) durch zunehmende Verbreitung des Gutes dessen Werbewert und dadurch auch sein inhaltlicher Wert für den einzelnen Konsumenten ansteigen.

- Indirekte Netzeffekte entstehen, wenn der Nutzen, den ein Gut stiftet, von der Verbreitung anderer Güter abhängt. Dies tritt vor allem dann auf, wenn Güter in Form von Basiselementen und komplementären Anwendungskomponenten angeboten werden. Beispiele für indirekte Netzeffekte bilden die Verbreitung von CD- oder VHS-Videoabspielgeräten, die sich vor allem aufgrund einer umfangreichen Anzahl abspielbarer Titel im jeweiligen Format gegenüber konkurrierenden Mediensystemen durchsetzen konnten.

- Ergänzend zu diesen beiden Formen kann der Konsum von Informationsgütern von einem sozialen Mitläufereffekt beeinflusst sein, verursacht durch Reproduktion medialer Inhalte in der Kommunikation im öffentlichen und privaten Lebensumfeld von Rezipienten. Bekanntes Beispiel ist das so genannte „Must-See TV“-Phänomen im amerikanischen Fernsehen. ${ }^{59}$

(2) Ein Informationsgut besitzt mindestens drei wertbildende Bestandteile: die zugrunde liegende Transportinfrastruktur, das jeweilige Speichermedium sowie - im Mittelpunkt der Konsumentscheidung stehend - die transportierte Nachricht. ${ }^{60}$ Die Bewertung der Nachricht bemisst sich dabei subjektiv nach qualitativen Kriterien, wie Aktualität, Exklusivität oder Spezifität. ${ }^{61}$ Eine solche Bewertung setzt jedoch eine Kenntnis des Inhalts und seiner Qualität voraus, die erst mit dem Konsum des Informationsguts erworben werden kann. ${ }^{62}$ Das resultierende Dilemma der Bewertung des Nachrichtenwerts eines Informationsguts durch einen Konsumenten wird auch als "Informationsparadoxon" bezeichnet. ${ }^{63}$ Zur Minderung dieses Entscheidungsdilemmas bieten sich verschiedene Möglichkeiten: Entsprechend der jeweiligen Mediengattung erstens die Veröffentlichung kostenfreier Auszüge aus dem Inhalt eines Informationsguts, bspw. Vorschauen von Kinofilmen im Fernsehen, Auszüge aus dem Inhalt von Büchern in

59 Als Must-See Ereignisse der amerikanischen Fernsehkultur gelten vor allem Fernsehserien wie die Cosby Show, Cheers, Seinfeld, Frasier oder Friends, die üblicherweise am Donnerstagabend zwischen 20 und 22 Uhr ausgestrahlt werden.

60 Vgl. Brandtweiner (2000), S. 41; Sjurts (2000), S. 8. Im Sinne der fünfschichtigen Sichtweise eines Informationsguts in Abschnitt 2.1.1 können neben Nachricht, Speichermedium und Distributionsinfrastruktur die jeweilige Verarbeitungs- und Präsentationsform der Nachricht einen spezifischen Wert generieren. Die Werterzeugung im Zusammenhang mit dem Verarbeitungs- und Präsentationsformat eines Medienguts wird in Abschnitt 3.3.2.2 vertieft.

61 Vgl. Beck (2002), S. 18.

62 Vgl. Heinrich (2001a), S. 99.

63 Erstmalig erwähnt bei Arrow (1962), S. 147: „Here is a fundamental paradox in the determination of demand for information; its value for the purchaser is not known until he has the information, but then he has in effect acquired it without cost." 
Zeitschriften oder auf der Buchrückseite oder die Titelseite von Zeitungen. ${ }^{64}$ Neben den eigentlichen Inhalten von Büchern, Filmen und Musikträgern werden auf Medienmärkten zweitens Bewertungsinformationen über erstere Inhalte als eigene Informationsgüter gehandelt, bspw. in Form von Theater-, Film und Fernsehkritiken im Feuilleton von Zeitungen bzw. in eigenen Fachsendungen und -zeitschriften. Als dritte Möglichkeit wird zunehmend die Etablierung von Marken als Bewertungssubstitut für die jeweiligen Inhalte im Rahmen der Konsumentscheidung diskutiert. Nach Hass besitzen Marken eine zweifache Signalfunktion für den Wertgehalt eines Informationsguts: Sie repräsentieren erstens eine Metainformation über einzelne Güter oder Güterbündel gleichartiger Formate im Sinne eines zusätzlichen, insbesondere im Rahmen von Wiederholungskäufen (neben Merkmalen wie Titel oder Autor) wirksam werdenden Kriteriums der Konsumentscheidung. Zweitens fungieren Marken als Reputationsmechanismus über Glaubwürdigkeit und Güte der langfristigen Leistungsqualität eines Unternehmens. ${ }^{65}$ Dabei ist von einem tendenziell volatilen Charakters positiver Reputation und einem vergleichsweise hohen Schadenspotenzial negativer Einzelbeurteilungen auszugehen. Als Reputationskapital fungieren dabei nicht nur direkt inhaltsbezogene Aspekte, sondern auch Kriterien der Bündelung und Distribution, bspw. die Prominenz eines Zeitungsherausgebers oder Beliebtheit eines Nachrichtensprechers. ${ }^{66}$

\subsubsection{Zusammenfassung}

Mediengüter werden in besonderer Weise durch die Entwicklungen im Bereich digitaler Informations- und Kommunikationstechnik beeinflusst, da ihr zentraler wertbildender Bestandteil informationeller Natur und damit vollständig digitalisierbar ist. Daher haben digitale Informations- und Kommunikationstechniken zum einen Auswirkungen auf die Rahmenbedingungen der Erzeugung und Bündelung von Inhalten. Zum anderen können Mediengüter vollständig digital und ohne physisches Trägermedium bspw. über Onlinenetze zum Endkunden transportiert werden. In Kapitel 3 werden daher die Herausforderungen für Medienunternehmen durch die Digitalisierbarkeit von Inhalten im Detail untersucht.

\subsection{Individualisierung}

Der folgende Abschnitt gibt einen einführenden Überblick über die Thematik der Individualisierung. Der Begriff der Individualisierung besitzt eine enge etymologische Verwandtschaft mit jenen des "Individuums“ und der "Individualität". Als Individuum ist jedes System bzw. jeder Organismus zu verstehen, der sich durch solche Eigenschaften und Merkmale, die kein anderes System besitzt, mit Einzigartigkeit auszeichnet. ${ }^{67}$ Der Grad der Einzigartigkeit eines individuel-

\footnotetext{
64 Vgl. Schumann/Hess (2002), S. 62 f.

65 Vgl. Heinrich (2001a), S. 162.

66 Vgl. Hass (2002), S. 55 f.

67 Vgl. Suarez (1976); Sonntag (1999), S. 8.
} 
len Systems wird als Individualität bezeichnet. Das Ausmaß an Individualität bemisst sich relativ in Bezug auf die Umwelt des individuellen Systems, durch seine Originalität und seine Soziabilität gegenüber anderen Systemen. ${ }^{68}$ Individualisierung kann demnach in einem ersten begriffstheoretischen Zugriff als eine Hervorhebung der einzigartigen Merkmale eines Gegenstands oder einer Person charakterisiert werden. ${ }^{69}$

Der Begriff der Individualisierung wurde ursprünglich in der soziologischen Fachliteratur im Zusammenhang mit der Diskussion des Wertewandels in der modernen westlichen Gesellschaft geprägt. ${ }^{70}$ Diese Diskussion der Veränderungen der gesellschaftlichen Lebensbedingungen wurde jedoch in den letzten Jahren auch in der Ökonomie und der Psychologie aufgegriffen. Neben der Beschreibung exogener Veränderungen in den Rahmenbedingungen sozialen Handelns wurden insbesondere in der Literatur zur Betriebswirtschaftslehre und zur Wirtschaftsinformatik Handlungsempfehlungen entwickelt, um diesen Veränderungen zu begegnen bzw. um sich diese Veränderungen zu Nutze zu machen. Von besonderer Bedeutung sind dabei die marketingtheoretische Überlegungen zum Aufbau individueller Kundenbeziehungen im Zusammenhang mit dem sog. „Beziehungsmarketing“ oder „Relationship Marketing“71, die industriebetrieblich geprägten Überlegungen zur „kundenindividuellen (Massen-)Fertigung" oder "Mass Customization"72 und die Beiträge aus den Bereichen Informatik und Wirtschaftsinformatik zur individuellen Anpassung von elektronischen Handelssystemen durch sog. „Empfehlungs- und Beratungssysteme“73.

Durch den vielschichtigen wissenschaftlichen Diskurs von Veränderungen und Handlungsempfehlungen sind im Zusammenhang mit dem Thema der Individualisierung sowohl eine begriffliche Vielfalt als auch vielfältige Argumentationen zur Erklärung von Formen der Individualisierung und deren Konsequenzen entstanden. Im Folgenden wird ein Literaturüberblick über die wichtigsten Beiträge im Rahmen der Diskussion zum Thema Individualisierung gegeben. Anhand der in Abbildung 2-5 dargestellten Systematik werden diese Beiträge entsprechend ihrer Implikationen für eine Einzelunternehmung in Betrachtungen exogener Entwicklungen und endogener Handlungsempfehlungen klassifiziert. In Abschnitt 0 werden zunächst die in den verschiedenen Forschungsbereichen als Trendaussagen formulierten Thesen zur Thematik der Individualisierung skizziert. In Abschnitt 2.2.2 werden Handlungsempfehlungen vorgestellt, die in der Literatur im Bereich der Betriebswirtschaftslehre und der

68 Vgl. Sonntag (1999), S. $10 \mathrm{f}$.

69 Vgl. Kron (2000), S. 7; Schackmann (2003), S. 9.

70 Vgl. u.a. Simmel (1900); Beck (1983); Beck (1986); Beck/Beck-Gernsheim (1990); Schweikart (1994); Beck (1995); Berger (1997); Wohlrab-Sahr (1997); Sonntag (1999); Meier (2003).

71 Vgl. u.a. Berry (1983); Kleinaltenkamp (1995); Diller (1995a); Peppers/Rogers (1996); Wehrli/Wirtz (1996); Burghard/Kleinaltenkamp (1996); Hildebrand (1997); Seller/Gray (1999).

72 Vgl. u.a. Pine (1993); Mertens (1995); Anderson/Pine (1997); Piller (2001).

73 Vgl. u.a. Mertens (1997); Resnick/Varian (1997); Konstan et al. (1997); Mertens et al. (2005). 
Wirtschaftsinformatik diskutiert werden, um dem Individualisierungstrend in Gesellschaft und Märkten zu begegnen. ${ }^{74}$

\begin{tabular}{|c|c|c|c|}
\hline $\begin{array}{l}\text { Soziologie: Gesellschaftlicher } \\
\text { Wertewandel (Kap. 2.2.1.1) }\end{array}$ & $\begin{array}{c}\text { Ökonomie: Segmentierung } \\
\text { des Angebots (Kap. 2.2.1.2) } \\
\downarrow\end{array}$ & $\begin{array}{l}\text { Psychologie: Rationalisierung } \\
\text { der Nachfrage (Kap. 2.2.1.3) }\end{array}$ & ळे \\
\hline & matve & & ภీ \\
\hline $\begin{array}{l}\text { Individuelles Marketing } \\
\text { (Kap. 2.2.2.1) }\end{array}$ & $\begin{array}{l}\text { Individuelle Fertigung } \\
\text { (Kap. 2.2.2.2) }\end{array}$ & $\begin{array}{l}\text { Beratungs- und Empfehlungs- } \\
\text { systeme (Kap. 2.2.2.3) }\end{array}$ & 站 \\
\hline
\end{tabular}

Abbildung 2-5: Betrachtungsdimensionen zur Individualisierung

\subsubsection{Individualisierung als sozialer Trend}

Der folgende Abschnitt skizziert Beiträge, die Individualisierung als einen sozialen Trend in der modernen abendländischen Gesellschaft beschreiben. Dieser Trend wird anhand von drei Dimensionen charakterisiert: als Ausprägungsform des allgemeinen gesellschaftlichen Wertewandels, beschrieben anhand von soziologischen Forschungsbeiträgen (vgl. Abschnitt 2.2.1.1); als ökonomischer Trend der voranschreitenden Segmentierung des Marktangebots (vgl. Abschnitt 2.2.1.2) und als sozialpsychologischer Trend der Rationalisierung des Verhaltens von Marktnachfragern (vgl. Abschnitt 2.2.1.3). Diese drei Aspekte sind nicht als unabhängige Entwicklungen zu verstehen. Vielmehr existieren vielschichtige Verflechtungen, die Gegenstand aktueller Wissenschaftsdebatten sind. Die Diskussion dieser Verflechtungen wird umfangsbedingt verkürzt in einer abschließenden Zusammenfassung wiedergegeben (vgl. Abschnitt 2.2.1.4).

\subsubsection{Individualisierung als gesellschaftlicher Wertewandel}

Die Thematik der Individualisierung beschäftigt die soziologische Forschung bereits seit Beginn des 20. Jahrhunderts, in erster Linie als Forschungsgegenstand im Zuge der Untersuchung sozialer Ungleichheit im Rahmen gesellschaftlicher Klassenstrukturen. ${ }^{75}$ Erstmalig formulierte Ulrich Beck in den 1980er Jahren die vergleichsweise radikale These eines Trends der gesellschaftsweiten Individualisierung im Sinne der Auflösung etablierter gesellschaftlicher Strukturmuster. ${ }^{76}$ Unter Individualisierung versteht Beck den gesellschaftlichen Prozess des Herauslösens des Individuums einerseits aus Schicht- und Klassen-

74 Es ist anzumerken, dass die hier vorgenommene Klassifikation bestehender Vorarbeiten in (aus Sicht einer Einzelunternehmung) exogene und endogene Individualisierungsformen nicht vollkommen überschneidungsfrei ist. So ist bspw. zum einen davon auszugehen, dass (exogene) Entwicklungen der Produktionstechnik, wie flexiblere und dennoch stärker automatisierte Fertigungsanlagen, erst (hier als endogen eingeordnete) Handlungsmöglichkeiten wie eine kundenindividuelle Güterfertigung ermöglichen. Zum anderen kommt die (hier als exogen beschriebene) Fragmentierung der Märkte z.T. erst durch eine (endogene) Angebotsdifferenzierung von Unternehmen zustande.

75 Vgl. bspw. Simmel (1900). Einen Überblick bieten Kron (2000), S. 7 f. und Kippele (1998).

76 Vgl. Beck (1983); Beck (1986); Beck (1990). 
milieus und andererseits aus traditionell-industriegesellschaftlichen Formen der Vergesellschaftung und Vergemeinschaftung. ${ }^{77}$ Dieser Wandel der Rolle des Individuums in der abendländischen Gesellschaft ist geprägt durch die Relativierung individueller Lebensläufe von sozialgeschichtlich geprägten Lebensstilen bzw. sozialstruktureller Milieus. ${ }^{78}$

Das heutige Verständnis von Individuum und Individualität ist in besonderem Maße mit der Herausbildung der modernen Marktwirtschaft im ökonomischen System verwoben. Eine von staatlichem Einfluss liberalisierte Ökonomie, wie sie erstmalig im 18. Jahrhundert von Adam Smith postuliert wurde, unterstreicht einerseits die ökonomische Dominanz des individuellen Gewinnstrebens zur Maximierung des Volkseinkommens gegenüber staatlicher Planlenkung, erforderte andererseits jedoch sowohl stabile Rahmenbedingungen für den Leistungsaustausch als auch die Sicherung des individuellen Wohlstandes gegenüber negativen Schwankungen im ungelenkten Wirtschaften. ${ }^{79}$ Der Prozess der Individualisierung repräsentiert demnach aus soziologischem Betrachtungsfokus in erster Linie eine Form der Soziabilität des Mitglieds einer Gesellschaft im verhaltensbeschränkenden Gemeinwesen. ${ }^{80}$ Vor dem Hintergrund der derzeit stabilen Gesellschaftsverhältnisse aufgrund von gesicherter nationaler Wohlfahrt und individueller Rechtssicherheit reduzieren sich die Eingriffe gemeinschaftlicher Reglementierungen in die individuellen Freiheiten insofern auf ein (bspw. im Vergleich zum mittelalterlichen Feudalwesen) minimiertes Mindestmaß. Dadurch entsteht die Reproduktion einer individuellen Differenzierung von Konsumansprüchen als eine gesellschaftliche Massenkultur. ${ }^{81}$ Derartige Prozesse der individuellen Reproduktion können im Zuge der gesellschaftlichen Liberalisierung in zweierlei Hinsicht beobachtet werden: ${ }^{82}$ in normativer Hinsicht als Wertewandel auf der Ebene kultureller Codes (1) und auf sozialstruktureller Ebene als empirisch belegbare Veränderungen von Mentalitäten, Verhaltensweisen und Einstellungen (2).

(1) Auf der Ebene kultureller Codes ist eine voranschreitende Enttraditionalisierung des Gemeinschaftswesens zu beobachten. ${ }^{83}$ Konkrete Indikatoren dieser Entwicklung bilden bspw. der Verfall des „puritanischen Ethos“ zugunsten einer intrinsischen Motivation als Hauptträger der Arbeitsmoral, ${ }^{84}$ der Abbau von Schuldgefühlen im Rahmen einer hedonistischen Genussmoralität des Kon-

77 Vgl. Hradil (1999); Meier (2003).

78 Vgl. Wiswede (1991), S. 23 f. Als Ursachen der gesellschaftlichen Individualisierung werden u.a. die gesellschaftliche Liberalisierung und die christliche Glaubenslehre mit der Sündenverantwortung der Einzelperson genannt.

79 Vgl. von Hayek (1952).

80 Vgl. Sonntag (1999), S. 10.

81 Vgl. Schweikart (1994), S. 4.

82 Vgl. Berger (1997), S. 81.

83 Vgl. Aretz (2000), S. 91.; Wiswede (1991), S. 21 ff.; Klages (1984); Beck (1986), S. 204.

$84 \mathrm{Vgl}$. von Rosenstiel (1993); Ein Beispiel eines solchen puritanischen Ethos bildet das Erstreben eines Gott gefälligen Werks in der eigenen Arbeit. 
sums ${ }^{85}$ oder die Abnutzung des sog. „demonstrativen Verbrauchs“ als prestigevermittelnde Quelle des Konsums ${ }^{86}$. Als Ursachen dieser Enttraditionalisierung werden u.a. üppigere Wahlmöglichkeiten im Bereich von Konsumgütern und Massenmedien und das Schwinden verbindlicher Modelle der Lebensführung angeführt. ${ }^{87}$ Individualisierung bedeutet insofern die Ablösung verbindlicher, gesellschaftsweiter Moralvorstellungen der Lebensführung von einem kritisch durch das Individuum reflektierten Wertepluralismus.

(2) Auf sozialstruktureller Ebene wird der Trend der Individualisierung anhand von gesellschaftlichen Einzeleffekten begründet. Konkrete Ausprägungen solcher Effekte bilden einerseits Nivellierungstendenzen des Lebensstandards in Bezug auf Einkommen, Ausgabeverhalten und Besitztatbestände ${ }^{88}$ andererseits die Differenzierung der Lebensstile, bspw. in Bezug auf die Nutzung von Konsumgütern oder die Kultivierung eines unterschiedlichen Geschmacks ${ }^{89}$. Als Ursachen dieser Effekte werden u.a. Gewinn an individueller Freiheit durch die Ausweitung politischer und sozialer Rechte, der Steigerung des materiellen Wohlstands sowie die Differenzierung und Pluralisierung von Wertorientierungen und Lebensstilen im Zuge der Gesellschaftsentwicklung genannt. ${ }^{90}$ Individualisierung bedeutet demnach im Gegensatz zu herkömmlichen Lebensstilen das Auftreten besonderer Spezialisierungs- und Anreicherungserscheinungen, die einen Pluralismus von Lebensstilen zur Folge haben und mit einer zunehmenden Heterogenität des Konsumverhaltens verbunden sind. ${ }^{91}$

\subsubsection{Individualisierung als Segmentierung des Marktangebots}

Die neoklassische Mikroökonomie unterstellt eine eingeschränkt individualistische Gesellschaftskonzeption, in der Konsumenten ihren individuellen Nutzen im Rahmen der Befriedigung ihrer Bedürfnisse durch den Konsum von Gütern maximieren. ${ }^{92}$ Das Marktangebot entsteht als gewinnoptimale Mengenanpassung des Produktionsvolumens von Güterproduzenten an einen exogen durch den Wettbewerb vorgegebenen Marktpreis. Im Wettbewerbsmarkt bildet sich immer ein langfristiges Marktgleichgewicht, bei dem der Preis der am Markt angebotenen Güter den Grenzkosten ihrer Produktion entspricht und dadurch von keinem Anbieter Gewinne realisiert werden können.

Neben den modellimmanenten Vereinfachungen wie vollkommene Preistransparenz und atomistische Angebots- und Nachfragestrukturen wird im neoklassischen Grundmodell des Marktes insbesondere die Granularität individueller

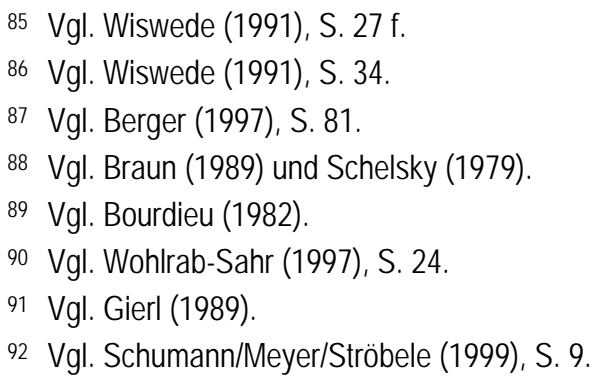


Konsumentenpräferenzen verkürzt dargestellt, indem von einer weitgehenden Homogenität von Güterangebot und -nachfrage ausgegangen wird. Individuelle Präferenzordnungen der Modellwelt unterscheiden lediglich zwischen Güterarten zur Befriedigung desselben Mangels, nicht aber bspw. zwischen verschiedenen Gütern derselben Güterart von unterschiedlichen Anbietern. ${ }^{93}$ Die zunehmende Diversifikation der Konsumentenbedarfe, der daraus resultierende Anstieg der Kapitalintensität langfristiger Investitionen und die aufkeimende Internationalisierung der Wettbewerbsstrukturen machte jedoch bereits ab den 1930 er Jahren grundlegende Mängel im neoklassischen Gleichgewichtsmodell des Marktes offenkundig. Globalwirtschaftliche Diskontinuitäten - prägende Ereignisse bildeten die Weltwirtschaftskrise Anfang der 1930er Jahre oder die globalen Ölkrisen in den 1970er Jahren - und die zunehmende Machtverlagerung auf Märkten in Richtung der Käuferschichten schufen die Notwendigkeit für einen grundlegenden Perspektivenwandel sowohl in der betrieblichen Planung als auch in den zugrunde liegenden ökonomischen Theorien.

Aus dieser Notwendigkeit heraus entstand in den 1930er Jahren die ökonomische Forschungsrichtung der sog. „Industrieökonomik“. Die Industrieökonomik erkennt in Abgrenzung zum neoklassischen Gleichgewichtsmodell des Marktes an, dass der Wettbewerb in vielen Märkten unvollkommener Natur ist. ${ }^{94}$ Ursachen solcher Unvollkommenheiten bilden qualitative Unterschiede im Marktangebot verschiedener Hersteller, bspw. aufgrund eines Innovationsvorsprungs durch betriebliche Forschung und Entwicklung, ${ }^{95}$ aufgrund der Heterogenität der Nachfragerbedürfnisse, aufgrund unvollkommener Information von sowohl Anbietern als auch Nachfragern über das Marktangebot ${ }^{96}$ oder aufgrund natürlicher Strukturphänomene, bspw. natürliche Oligopole oder Monopole, in bestimmten Märkten. ${ }^{97}$ Die Industrieökonomik untersucht, welche Zusammenhänge zwischen einer bestimmten Marktstruktur, dem Verhalten der Marktteilnehmer und dem durch sie in diesem Markt realisierbaren Ergebnis bestehen. ${ }^{98}$

Es gehört zu den zentralen Erkenntnissen der Industrieökonomik, dass es einem Unternehmen entgegen der neoklassischen Gleichgewichtstheorie ge-

93 Solche Marktbedingungen lagen insbesondere in den weitgehend undifferenzierten Angebotsmärkten in der Zeit vor und z. T. noch nach dem Zweiten Weltkrieg vor. Die zentrale Aufgabe des Managements lag daher in der Budgetierung der industriellen Produktion und der Kontrolle der Produktivität einer nach tayloristischen Prinzipien weitgehend variantenfreier Massenproduktion. Vgl. Haertsch (2000), S. 49; Bea/Haas (1995), S. 11; Hinterhuber (1992), S. 23

94 Vgl. Wied-Nebbeling (2004), S. 1; Bühler/Jäger (2002), S. 2.

95 Vgl. bspw. Schumpeter (1964), S. 99 ff. und Jacobson (1992), S. 782.

96 Vgl. bspw. Stigler (1961).

97 Bspw. existiert in Medienmärkten aufgrund von sinkenden Durchschnittskosten bei steigender Ausbringungsmenge die Tendenz einer natürlichen Monopolisierung. Vgl. Abschnitt 2.3.

98 Vgl. Tirole (1999), S. 2 f.; Bühler/Jäger (2002), S. 4 f.; Mason (1939); Bain (1986). Als Rahmenmethodik der industrieökonomischen Denkweise wurde das sog. „Structure-Conduct-Performance“ (SCP) Paradigma geschaffen, das den Erfolg (Performance) in einer Branche als Ergebnis der relativen Positionierung eines Unternehmens (Conduct) im Rahmen der spezifischen Wettbewerbsstruktur (Structure) der Branche. Vgl. u.a. Hümmer (2001), S. 30; Kirsch (1997a) S. 168; Wied-Nebbeling (2004), S. 1. 
lingen kann, positive Gewinne zu realisieren, wenn sich seine Produkte von denen der Konkurrenz unterscheiden. Diese Erfolgsbedingung wird auch als „Differenzierung" bezeichnet und beruht auf der Annahme, dass Kunden heterogene Präferenzen hinsichtlich bestimmter Merkmale von Güterangeboten besitzen. Die Präferenzen der Kunden können sich in sachlicher, persönlicher, räumlicher oder zeitlicher Hinsicht unterscheiden. ${ }^{99}$ Ein Unternehmen kann diese Präferenzheterogenität zu seinem Vorteil nutzen, indem es die Merkmale seines Güterangebots in Bezug auf unterschiedliche Präferenzdimensionen variiert. Dadurch kann das Unternehmen künstlich Marktsegmente erzeugen, innerhalb derer es einen gewissen monopolistischen Spielraum zu Preisgestaltung (bspw. durch Setzung der Preise $\mathrm{P}^{1}$ und $\mathrm{P}^{2}$ ) besitzt. Ein positiver Gewinn entsteht deshalb, weil ein Unternehmen mit differenziertem Angebot nicht, wie im neoklassischen Modellfall, als Preisnehmer auftreten muss, sondern gewinnoptimale Preise setzen kann (vgl. Abbildung 2-6).

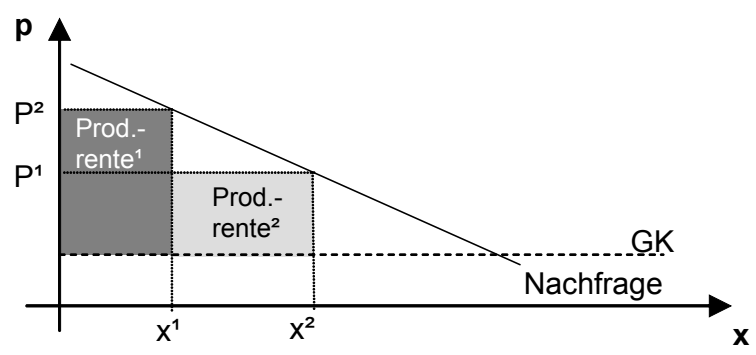

Abbildung 2-6: Produzentenrente bei Differenzierung

Diese industrieökonomischen Erkenntnisse wurden im Bereich der Managementlehre in Form des sog. "Market-Based-View“ (MBV) aufgegriffen. ${ }^{100} \mathrm{Ge}-$ mäß dem MBV muss ein Unternehmen für seine langfristige Strategiewahl zum einen die Struktur- und Wettbewerbsbedingungen der Branche berücksichtigen, in die es eintreten will, zum anderen kann das Unternehmen die Wettbewerbsstruktur der Branche durch gezielte strategische Manöver beeinflussen. Der prominenteste Vertreter des MBV, Michael Porter, identifizierte zwei grundlegende Wettbewerbsstrategien, mittels derer ein Unternehmen Vorteile gegenüber Konkurrenten im Branchenwettbewerb erzielen kann: ${ }^{101}$ die Optimierung der betrieblichen Kostenstrukturen zur Erzielung eines Kosten- und Preisvorteils gegenüber Konkurrenten und die bedürfnisgerechte Bedienung von Kundengruppen durch ein differenziertes Produktangebot. Dabei muss nach Porter zwischen den beiden Strategiealternativen eine strenge Auswahlentscheidung getroffen werden. Hintergrund bilden der zwangsläufige Anstieg der Komplexität in der Produktion bei Erhöhung der Variantenvielfalt und dadurch der Anstieg der direkten Kosten der Produktion, der Anstieg der Transaktionskosten

\footnotetext{
99 Vgl. Wied-Nebbeling (2004), S. 5.

100 Vgl. u.a. Mintzberg (1999), S. 101 ff.; Bea/Hass (1995), S. 24 f.

101 Vgl. Porter (1980).
} 
aufgrund von Variantenplanung und Abstimmung sowie die Reduktion von Verbund- und Skaleneffekten.

Da die Mittel der Wettbewerbsbeeinflussung durch Kostensenkung und Differenzierung theoretisch jedem Marktteilnehmer offen stehen, besteht nach Meinung einiger Autoren die logische Konsequenz des zunehmenden Überangebots an Gütern in vielen Märkten in einer zwangsläufigen Eskalation des Wettbewerbs, einem sog. „Hyperwettbewerb“. ${ }^{102}$ Ein solcher Hyperwettbewerb ist gekennzeichnet durch schnelle und dynamische Veränderungen der Wettbewerbsstrategien, wodurch sich die Wettbewerber eines Marktes nur kurzfristige Wettbewerbserfolge sichern können. Mit Verweisen auf Schumpeters Theorie zur disruptiven Natur von Innovationen aus den 1950er Jahren kann nach D'Aveni ein Unternehmen nur dann in einem solchen Hyperwettbewerb bestehen, wenn es durch gezielte Störmanöver (sog. „Disruptions“) stets einen Schritt voraus ist. ${ }^{103}$ Das Unternehmen eilt daher von einem Störmanöver zum nächsten. Im Umkehrschluss kann ein Unternehmen aus Sicht des MBV unter solchen Wettbewerbsbedingungen nur dann wiederholt Wettbewerbsvorteile erringen, wenn es konsequent die Vielfalt der von inm angebotenen Produktvarianten und dadurch die Zahl der unterschiedlichen von ihm bedienten Marktsegmente erhöht. Ein logischer Endpunkt einer solchen Entwicklung ist dann erreicht, wenn ein einzelnes bedientes Marktsegment gerade nur noch einen einzigen Käufer enthält, für den das Angebot individuell maßgeschneidert wird. Insofern repräsentiert Individualisierung aus ökonomischer Sicht eine wettbewerbsbedingte Eskalation der Segmentierung des Marktes zur Erringung positiver Gewinne.

\subsubsection{Individualisierung als Nachfragerationalisierung}

Eine Eskalation des Wettbewerbs, wie in Abschnitt 2.2.1.2 beschrieben, erhöht zwangsläufig die Angebotsvielfalt in den betroffenen Märkten. Dadurch entstehen zum einen zusätzliche Produktvarianten, die tendenziell besser auf individuelle Kundenwünsche zugeschnitten sind. Zum anderen hat dies jedoch zur Folge, dass für den einzelnen Kunden das Problem zunimmt, das jeweils für inn in Bezug auf Merkmalsausstattung und Preis optimale Gut zu finden. Es ist insofern von einem steigenden Informationsungleichgewicht zugunsten der Anbieter auszugehen. ${ }^{104}$ Das Problem, mit diesen nachfrageseitigen Veränderungen umzugehen, ist insofern sozial- und konsumpsychologischer Natur. Im Bereich der Sozial- und Konsumpsychologie wird zwar der Begriff der Individualisierung nicht neu belegt, es werden jedoch im Zusammenhang mit der Untersuchung von Konsumrationalität einige Effekte beschrieben, die in engem

102 Der Begriff des Hyperwettbewerbs wurde durch D'Aveni (1994) geprägt: „Hypercompetition is an envorinment characterized by intense and rapid moves, in which competitors must move quickly to build advantages and erode the advantages of their rivals. This speeds up the dynamic strategic interactions among competitors." (S. 7) Bereits 1984 wurde allerdings von McCann/Selsky (1984, S. 460) der Begriff „hyperturbulence“ eingeführt. Eine solche Hyperturbulenz repräsentiert eine vom Unternehmen nicht zu beherrschende oder zu beeinflussende Umweltturbulenz.

103 Vgl. D’Aveni (1994), S. 12.

104 Vgl. Fritz (2000), S. 68 und Zerdick et al. (1999), S. 151. 
Zusammenhang mit den bereits genannten Individualisierungstrends stehen und daher im Folgenden kurz erläutert werden.

1. Die Schaffung eines stabilen Marktgleichgewichts nach neoklassischem Ökonomieverständnis erfordert vollständige Information von sowohl Anbietern als auch Nachfragern über das Marktangebot und insbesondere über die am Markt gesetzten Preise. ${ }^{105}$ Vor dem Hintergrund der diversifizierten und differenzierten Angebotsstrukturen eines globalisierten „Marktraums“106 stellt eine solche umfassende Zweckrationalität jedoch eine hohe Anforderung an das Denkvermögen der involvierten Akteure. Bereits in den 50er Jahren des letzten Jahrhunderts stellte Simon fest, dass die für eine vollkommene Rationalität erforderliche Kenntnis und Voraussicht aller möglichen Konsequenzen bei Wahlentscheidungen in der Realität stets lediglich fragmentarisch bleibt. ${ }^{107}$ Die Annahme vollkommener Rationalität stellt deshalb nach Lindblom lediglich ein „synoptisches Ideal“108 dar, das nicht an bspw. die begrenzten intellektuellen Fähigkeiten des Menschen, sein generell begrenztes Wissen und die Aufwendigkeit vollständiger Situationsanalysen angepasst ist. ${ }^{109}$

2. Im Geiste des radikalen Konstruktivismus ${ }^{110}$ lässt sich das menschliche Problemlösen als ein Prozess der Informationsverarbeitung beschreiben, in dem der Entscheider durch seine Persönlichkeit in Form seiner im Langzeitgedächtnis gespeicherten Erfahrungen als Wahrnehmungsfilter gegenüber Reizsignalen im kapazitär vergleichsweise beschränkten Kurzzeitgedächtnis fungiert. ${ }^{111}$ Dadurch beschränkt sich das Such- und Auswahlverhalten zwischen Entscheidungsvariablen einer Problemstellung tendenziell in dem Rahmen erfahrungsgeprägter Muster, innerhalb derer lediglich kleine „inkrementale“ Änderungen, jedoch keine mit erhöhter Unsicherheit behafteten Änderungen im Hin-

$105 \mathrm{Vgl}$. Kirsch (1997a), S. 619 f.

106 Durch die Möglichkeiten medial vermittelter Kommunikation und dabei insbesondere durch das Internet werden nach Ansicht einiger Autoren die lokal gebundenen Marktplätze für physische Güter durch einen sich global ausdehnenden virtuellen Marktraum für Informationen und Güter aller Art abgelöst. Vgl. bspw. Weiber/Kollmann (2000), S. 48.

107 Vgl. Simon (1957), S. 81.

108 Vgl. Kirsch (1998), S. 181: Mit dem Attribut „synoptisch" wird unterstellt, dass der Entscheider über einen uneingeschränkten Überblick über alle Alternativen, alle möglichen Umweltsituationen und alle möglichen Ergebnisse der Alternativen verfügt.

109 Vgl. Braybrooke/Lindblom (1963), S. 113.

110 Der radikale Konstruktivismus beruht auf der neurobiologischen Erkenntnis, dass jede Form von Verstehen nicht ein getreues Abbild der Wirklichkeit, sondern durch die kognitiven Prozesse des Nervensystems lediglich ein internes Konstrukt der Außenwirklichkeit darstellt, das kognitive System des Gehirns demnach also eine operative Geschlossenheit aufweist. Vgl. Maturana/Valera (1987); Picot/Reichwald/Wiegand (1998), S. $83 \mathrm{f}$.

111 Vgl. Kirsch (1998), S. 183 f. 
blick auf vergangene, problemverwandte Entscheidungen vorgenommen werden. ${ }^{112}$

3. Wie Miller in den 50er Jahren anhand experimenteller Untersuchungen nachwies, beschränkt sich die spontane Kapazität menschlicher Informationsverarbeitung im Kurzzeitgedächtnis auf sieben (plus/minus zwei) Einzelkategorien. ${ }^{113}$ Eine Konfrontation mit einer über diese „magische" Grenze hinausgehende Anzahl an informatorischen Entscheidungsvariablen erzeugt durch eine Überlastung des Wahrnehmungsvermögens eine Form kognitiven Stresses beim Entscheider. Dieser Wahrnehmungsüberlastung wird auch als „information overload“ bezeichnet.

Wirtschaftssubjekte haben demnach zwar den Willen rational zu handeln, sie besitzen jedoch nicht ausreichend Informationen bzw. nicht ausreichend rationales Denkvermögen zu vollständiger Informationsverarbeitung. Die Entwicklungen im Bereich der mediengestützten Kommunikation, insbesondere durch die digitale Informations- und Kommunikationstechnik der letzten Jahre, haben jedoch zur Folge, dass Märkte zunehmend mediengestützt (bspw. über das Internet) abgewickelt werden und dadurch Eigenschaften vollkommener Märkte entwickeln: ${ }^{114}$ Das Internet ist global verfügbar, es besitzt das Potenzial zu uneingeschränkter Markttransparenz, es verursacht nur marginale Transaktionskosten und erlaubt eine schnelle Interaktion der Marktteilnehmer auf einem elektronischen Markt. Dies hat drei Auswirkungen auf die Bedingungen der Informationsverarbeitung durch Konsumenten:

1. Informations- und Kommunikationsvorgänge können mit größerer Reichhaltigkeit und Reichweite durchgeführt werden. ${ }^{115}$ Dadurch verbessern sich die Möglichkeiten für den Konsumenten, Angebotsinformationen über seinen Produktwunsch zu verarbeiten.

2. In Verbindung mit dem Internet sind neue Möglichkeiten entstanden, Transaktionsprozesse vollständig mediengestützt zu verarbeiten. Als Folge dessen kommt es in einigen Branchen zur Ausschaltung von Intermediären zwischen Produzenten und Endkonsumenten, bspw. im Musikgeschäft oder im Bereich der Sportartikelherstellung durch Direktvermarktung der Erzeugnisse vom Hersteller ohne Zwischenhändler an den Konsumenten. ${ }^{116}$ Insofern dadurch die Vorselektion durch den Handel entfällt, erhöht sich jedoch die Menge an Informationen, die ein Konsument bei der Suche nach geeigneten Angeboten bzgl. seines Produktwunsches verarbeiten muss.

3. Durch die mediengestützte Transaktionsabwicklung im Internet wird die Angebots- und Transaktionstransparenz des Markts erhöht. Ausprägungsbeispiele für eine solche Transparenzerhöhung bilden Spei-

\footnotetext{
112 Vgl. Kirsch (1998), S. 185.

$113 \mathrm{Vgl}$. Miller (1956).

114 Vgl. Meffert (2001), S. 166.

$115 \mathrm{Vgl}$. Evans/Wursterer (2000), S. 37.

116 Vgl. Becker (2001), S. 101.
} 
cherfunktionen für abgeschlossene Transaktionen oder Suchfunktionen für Onlineangebote. Dadurch erhöht sich tendenziell die Chance eines Konsumenten, mit geringeren Transaktionskosten zu seinem Produktwunsch den Anbieter mit dem günstigsten Preis zu finden. ${ }^{117}$ Diese Transparenz wird zusätzlich erhöht durch das Informationsangebot neuer spezialisierter Informationsintermediäre wie bspw. virtuelle Communities, Auktionshäuser, Informationsbroker, Preisagenturen, Portale oder Onlinemagazine. ${ }^{118}$

Zusammenfassend ist einerseits davon auszugehen, dass die Informationsmenge, die einem Konsument im Rahmen der Suche nach einem wunschgemäßen Produkt zur Verfügung steht, durch die allgemeine Wettbewerbsintensivierung und speziell durch die Virtualisierung von Markttransaktionen im Internet zugenommen hat. Zum anderen bieten sich dem Konsumenten durch Suchfunktionen oder Preisagenten zusätzliche Instrumente, die die Möglichkeiten der Informationsverarbeitung im Rahmen seiner Suche verbessern. Es wird in diesem Zusammenhang auch von einer steigenden "Informatisierung" der Gesellschaft gesprochen. ${ }^{119} \mathrm{Um}$ dieses Informationspotenzial ausschöpfen zu können, muss der Konsument jedoch zusätzliches Wissen entwickeln, um mit den entsprechenden Instrumenten wie bspw. einer Onlinesuche umgehen zu können. Entsprechend ist eine Folge der steigenden Wettbewerbsintensität und der Informatisierung die Notwendigkeit der Wissensintensivierung und dadurch der Rationalisierung des Nachfrageverhaltens. ${ }^{120}$

\subsubsection{Zusammenfassung}

Zusammenfassend können vier interdependente Argumentationen ((1)-(4)) identifiziert werden, die die Individualisierung von Angebot und Nachfrage im Sinne exogener gesellschaftlicher Trends zum Gegenstand haben (vgl. Abbildung 2-7): Individuelle Rechtssicherheit und die allgemeine Stabilisierung der gesellschaftlichen Wohlfahrt bilden die historische Grundlage der Entwicklung des Wirtschaftsprinzips des Eigennutzens (1). Der zunehmende Eigennutzen spiegelt sich zum einen auf gesellschaftlicher Ebene in einem wachsenden Wertepluralismus wider, der sich auf der Ebene privater Haushalte u.a. in einem Wandel in der Arbeitsauffassung und im Konsumverhalten niederschlägt (2). Zum anderen ist die Zunahme des Güterangebots aufgrund allgemein verbesserter Produktionsverfahren sowie liberalisierter und globalisierter Marktstrukturen festzustellen. Dem daraus resultierenden Überangebot an Gütern (3) und Marktinformationen steht eine Rationalisierung der gesellschaftsweiten Informationsverarbeitungsfähigkeiten gegenüber (4). Diese informationsbezogene Rationalisierung wird unterstützt durch reichhaltigere Informationsund Kommunikationsmöglichkeiten, eine gestiegene Markttransparenz und

117 Vgl. Bliemel/Fassott (2000), S. 17; Zerdick et al. (1999), S. 152.

118 Vgl. Meffert (2001), S. 164.

$119 \mathrm{Vgl}$. Kuhlen (1995), S. 49.

120 Vgl. Kuhlen (1995), S. 8. 
eine zunehmende Intermediation im Bereich spezialisierter Informationsdienstleister.

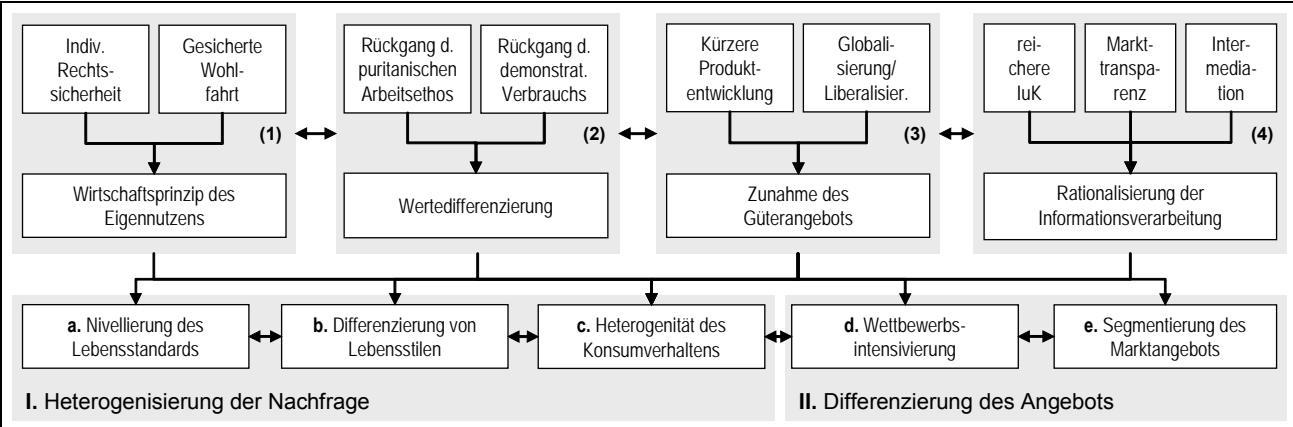

Abbildung 2-7: Zusammenfassung exogener Individualisierungstrends

Im Voranstehenden wurden ausgehend von diesen vier Rahmenentwicklungen fünf Trends $(a-e)$ identifiziert, die mit einer gesellschaftsweiten Individualisierung einhergehen: Auf der Ebene der Haushalte wurden die Trends der Nivellierung von Haushaltseinkommen und Lebensstandards (a) mit der Folge zunehmend hedonistisch geprägter Lebensstile (b) und einer Heterogenität des Konsumverhaltens (c) festgestellt. Es ist insofern auf der Nachfrageseite abendländischer Märkte von einer Heterogenisierung der Güternachfrage auszugehen (I). Auf der Ebene des Güterangebots wurde eine Intensivierung des Wettbewerbs (d) in Verbindung mit einer Segmentierung des Marktangebots (e) beschrieben. Es ist daher auf der Angebotsseite zusammenfassend von einer zunehmenden Differenzierung des Marktangebots (II) auszugehen.

\subsubsection{Individualisierung als strategische Option der Marktbear- beitung}

In Abschnitt 2.2.1 wurde Individualisierung als ein exogener Umwelttrend beschrieben, der zu einer Heterogenisierung der Güternachfrage und zu einer Differenzierung des Warenangebots geführt hat. Bislang wurde das Verhalten von Unternehmen in Verbindung mit diesen Rahmenbedingungen lediglich als strukturdeterministische Reaktion und dadurch trendverstärkende Variable berücksichtigt. Demgegenüber werden im Folgenden die Forschungsergebnisse im Bereich solcher Gestaltungsempfehlungen zusammengefasst, die Reaktion auf die genannten Veränderungen der Rahmenbedingungen in Form eines individuell auf den Kunden maßgeschneiderten Angebots zum Gegenstand haben. Individualisierung wird dabei nicht als soziale Rahmenbedingung, sondern als eine Option der Marktbearbeitung betrachtet. ${ }^{121}$ In diesem Zusammenhang sind drei wesentliche Denkrichtungen zu berücksichtigen, die in der betriebswirtschaftlichen Literatur eine besondere Beachtung finden: Die Untersuchungen zum kundenindividuellen Marketing (vgl. Abschnitt 2.2.2.1), die Untersu-

121 Vgl. bspw. Mayer (1993), S. 35. 
chungen zu den Einsatzmöglichkeiten und Folgen von Informationsverarbeitungstechniken im Bereich des Industriebetriebs (vgl. Abschnitt 2.2.2.2) und im Bereich des Handels (vgl. Abschnitt 2.2.2.3).

\subsubsection{Individuelles Kundenmarketing}

Das Wesen der modernen Massenproduktion begründet ein strategisches Wachstumsdilemma: ${ }^{122}$ Die zentralen Vorteile eines Massenanbieters bilden Größen- und Verbundeffekte im Rahmen von Produktion und Vermarktung. Je größer ein Konkurrent ist, desto größer der resultierende Wettbewerbsvorteil. Am Markt entsteht ein Wettbewerb um die Größe des Marktanteils, mit dem Effekt steigender Rabatte und sinkender Preise. Aufgrund der dadurch sinkenden Durchschnittsgewinne der einzelnen Wettbewerber steigt wiederum die Bedeutung der Unternehmensgröße.

In Abschnitt 2.2.1.2 wurde gezeigt, dass in vielen Märkten die Intensivierung des Wettbewerbs zu einer wachsenden Segmentierung der Marktbearbeitung durch konkurrierende Anbieter geführt hat. Aus Sicht eines einzelnen Wettbewerbers hat diese voranschreitende Marktsegmentierung zwei Konsequenzen:

- Trotz der steigenden Segmentierung sind nur noch selten Zugewinne zusätzlicher Marktanteile möglich. Weil selbst in kleineren Marktsegmenten in der Regel mehr als ein Anbieter vertreten ist, findet ein Verdrängungswettbewerb zwischen diesen Anbietern statt. Zudem sinkt im Zuge der insgesamt gestiegenen Werbepenetration in den Märkten die Aufmerksamkeit potenzieller Kunden. ${ }^{123}$

- Durch die in Abschnitt 2.2.1.3 angesprochene Erhöhung der Transparenz mediengestützt abgewickelter Märkte und Transaktionen besteht die Möglichkeit, dass Kunden selbst marginale Unterschiede in den Merkmalen oder Preisen der Güter verschiedener Anbieter zu ihrem Vorteil ausnutzen. Insofern ist von einer gesunkenen durchschnittlichen Bindung von Kunden an einzelne Anbieter auszugehen. ${ }^{124}$

Bereits Anfang der 1990er Jahre wurde daher in der Marketing-Literatur ein Umdenken gefordert, weg vom Marketing zur Erringung von Marktanteilen am (ggf. segmentierten, aber dennoch) anonymen Massenmarkt hin zu einem Marketing zur Steigerung der Kundenbindung und dadurch der Häufigkeit von Wiederholungskäufen eines individuellen Kunden (durch sog. „KundenanteilsMarketing"). Diese Forderung wird im Wesentlichen auf die Annahme gestützt, dass der Großteil der Geschäfte eines Unternehmens von lediglich einem kleinen Teil seiner Kunden getätigt wird. ${ }^{125}$ Vor dem Hintergrund dieser Annahme kann geschlussfolgert werden, dass eine Konzentration der Marketingaktivitäten eines Unternehmens auf lediglich diesen Teil ihres potenziellen Kundenkreises sowohl mit kosten- als auch effektivitätsspezifischen Vorteilen verbun-

\footnotetext{
122 Vgl. Mayer (1993), S. $120 \mathrm{f}$.

123 Vgl. Hildebrand (1997), S. 16.

124 Vgl. Mayer (1993), S. 124.

125 Vgl. Peppers/Rogers (1996), S. 32.
} 
den ist. Eine solche Konzentration der Marketingaktivitäten setzt dreierlei voraus: Im ersten Schritt muss der potenzielle Gesamtmarkt so segmentiert werden, dass „wertvolle“ Kunden identifiziert werden können, die im Verhältnis zum Gesamtaufwand einer Marketingstrategie signifikant höhere Rückflüsse erzeugen. Im zweiten Schritt muss eine dialogorientierte Kundenkommunikation zur Ermittlung der Bedürfnisse dieser Kunden aufgebaut werden. Auf der Grundlage einer solchen dialogorientierten Kommunikation kann dann im dritten Schritt das Angebot individuell angepasst werden.

Die Auseinandersetzung mit dem Problem der Steigerung von Kundenanteil und Kundenbindung durch eine Einzelkundenorientierung hat in der Marketingliteratur zu einer beinahe inflationär anmutenden Begriffsvielfalt geführt: Der Begriff des sog. „Database Marketing“ unterstreicht in erster Linie den instrumentellen Aspekt von Informationssystemen zur Umsetzung der notwendigen Filterung und Speicherung von Kundeninformationen im Rahmen der Marktsegmentierung und Kundenansprache. ${ }^{126} \mathrm{Im}$ Zusammenhang mit den Begriffen des sog. „One-to-One Marketing“127, „Segment-of-One-Marketing“"128 oder „Target Marketing“ ${ }^{129}$ wird dagegen stärker die Orientierung am Einzelkunden als strategische Option der Marktbearbeitung in Abgrenzung zur traditioneller Strategie der standardisierten Marktbearbeitung hervorgehoben. Schließlich wird durch Überlegungen zum sog. „Dialogmarketing“"130, „Relationship Marketing“ oder „Beziehungs-Marketing“" ${ }^{131}$ die dialogorientierte Natur einer langfristig aufzubauenden Kundenbeziehung betont.

Eine vergleichsweise populäre Systematisierung im Zusammenhang mit den sehr heterogenen Ansätzen der Marketingliteratur zum Thema Einzelkundenorientierung stammt von Link und Hilderand aus dem Jahr $1995 .{ }^{132}$ Darin werden die denkbaren Ansätze einer Einzelkundenorientierung des Marketing nach zwei Dimensionen klassifiziert: Zum einen wird nach dem Individualisierungsgrad der angebotenen Leistung und der dafür notwendigen Integration von Kundendaten in die Erzeugung zwischen maßgeschneiderten Angeboten mit hohem Integrationsgrad von Kundendaten und standardisierten Angeboten mit geringem Kundenintegrationsgrad unterschieden. Zum anderen wird, anhand des beabsichtigten wiederkehrenden Interaktionsgrads von Anbieter und Nachfrager, zwischen solchen Ansätzen, die eine geringe Interaktion mit dem Kunden erfordern und damit zukünftige Transaktionen unberücksichtigt lassen und solchen, die zukünftige Transaktionen mit einbeziehen und dafür eine hohe Interaktion mit dem Kunden erfordern, differenziert. Anhand dieser zwei Dimensi-

126 Vgl. u.a. Shaw/Stone (1988); Schüring (1991); Roberts (1992); Kreutzer (1992); Wilde (1992); Huldi (1992); Nash (1993); Link (1993); Petrison/Blattberg/Wang (1993). Einen Überblick bietet: Seller/Gray (1999).

127 Vgl. Peppers/Rogers (1996).

$128 \mathrm{Vgl}$. Winger/Edelman (1989); McQuaid (1990).

129 Vgl. Köhler (1994).

130 Vgl. Schleuning (1994); Schäfer (1995).

131 Vgl. Berry (1983); Beinlich (1995); Kern (1990); Homburg (1995); Wehrli/Wirtz (1996); Rieker (1995).

132 Vgl. Link/Hildebrand (1995); Hildebrand (1997), S. 41 f. 
onen lassen sich vier Grundtypen der Marktbearbeitung unterscheiden (vgl. Abbildung 2-8):

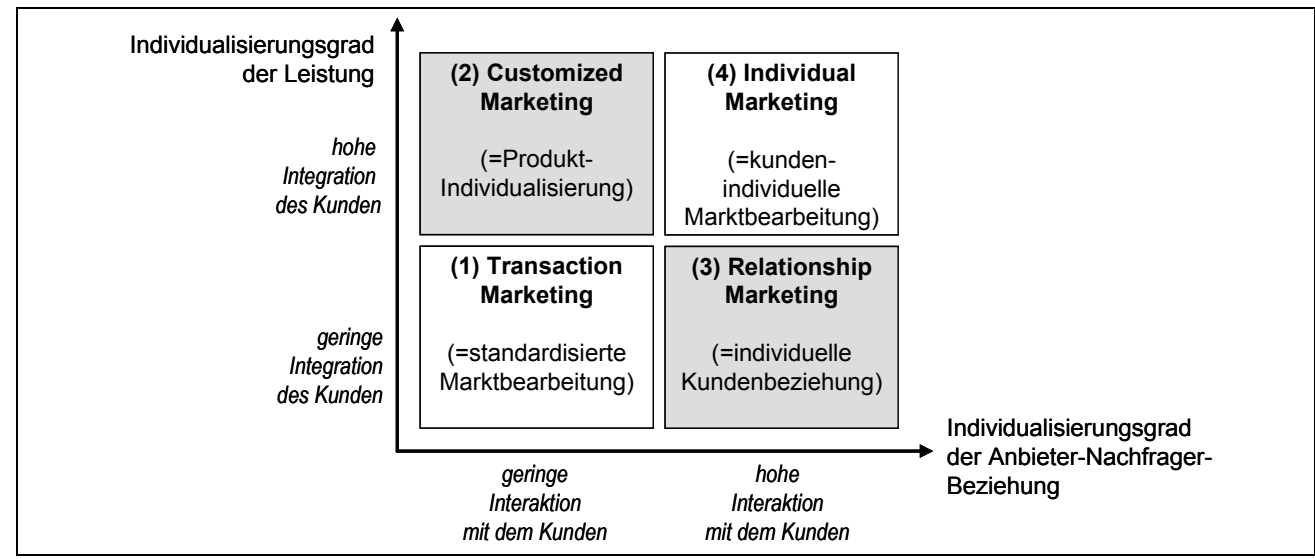

Abbildung 2-8: Einzelkundenorientierte Marketingkonzepte ${ }^{133}$

(1) Die konventionelle Form der Marketingkonzeption mit standardisierten Leistungen und geringer Kundeninteraktion kann als „Transaction Marketing" oder Transaktionsmarketing bezeichnet werden. Es findet keine Form der Individualisierung statt.

(2) Die Marketingkonzeption des „Customized Marketing“ benutzt Bedürfnisse eines individuellen Kunden dafür, ein Angebot nach dessen Wünschen maßzuschneidern. Individualisierung bedeutet hier, eine den individuellen Bedürfnissen entsprechende Produktvariante zu erzeugen. ${ }^{134}$ Es wird jedoch kein Dialog mit dem Kunden für eine langfristige Kundenbindung aufgebaut. Angebote nach dem Prinzip des Customized Marketing haben daher üblicherweise Kontraktgutcharakter. ${ }^{135}$ Das Customized Marketing rechtfertigt seine Vorteilhaftigkeit zum einen über einen höheren Kaufanreiz maßgeschneiderter Produkte auf Kundenseite und zum anderen über erzeugungsorientierte Effizienzpotenziale maßgeschneiderter Güter aufgrund eines wachsenden Automatisierungsgrads der industriellen Fertigung auf Angebotsseite. Damit steht das Customized Marketing in enger Beziehung zu dem im Nachstehenden noch eingehender behandelten Konzept der "Mass Customization“ (vgl. Abschnitt 2.2.2.2).

(3) Die Beziehung zwischen Kunden und Anbietern wurde bereits Ende der 1920er Jahre ökonomisch untersucht. ${ }^{136}$ Der Begriff des „Relationship

$133 \mathrm{Vgl}$. Hildebrand (1997), S. 41.

134 Vgl. Mayer (1992), S 36 f.; Schneider (1997), S. 13 f.

135 Vgl. Jacob (1995), S. 44 f. Ein Kontraktgut ist zum Kaufzeitpunkt noch kein fertiges Produkt, sondern repräsentiert zunächst ein Leistungsversprechen, das erst in Verbindung mit einem konkreten Variantenwunsch bzw. der konkreten Bestellung eines Kunden erfüllt wird. Vgl. auch Kass (1992) und (1995).

136 Vgl. Hildebrand (1997), S. 44; Domizlaff (1929). 
Marketing“ oder „Beziehungsmarketing“ wurde allerdings erst 1983 durch Berry geprägt. ${ }^{137}$ Als Beziehungsmarketing sind alle Marketingaktivitäten zu verstehen, die den Aufbau, die Entwicklung und die Pflege von Austauschbeziehungen zum Gegenstand haben. ${ }^{138} \mathrm{Im}$ Vergleich zum Customized Marketing besitzt die Literatur zum Beziehungsmarketing einen ungleich vielfältigeren Umfang. ${ }^{139}$ Dabei werden zum Teil unterschiedliche Aspekte des Beziehungsmarketing thematisiert: ${ }^{140}$ Anstelle der individuellen Leistungsgestaltung des Customized Marketing bedeutet Individualisierung aus Sicht des Beziehungsmarketing, eine individuelle Kommunikation zum Kunden aufzubauen. ${ }^{141}$ Diese Kommunikation dient dazu, eine individuelle Beziehung mit dem Kunden zu entwickeln und somit einen Wettbewerbsvorteil zu schaffen, der von Konkurrenten nicht imitiert werden kann. ${ }^{142}$ Die Kommunikation zwischen Anbieter und Kunden besitzt den Charakter einer dialogartigen Interaktion. Diese Interaktion ersetzt das „Beeinflussungsmanagement" des konventionellen Transaktionsmarketing, wobei der Kunde und seine Bedürfnisse konsequent und transaktionsübergreifend in die Wertschöpfungsprozesse des Anbieters integriert werden. Der Erfolg des Beziehungsmarketing ist daher tendenziell langfristiger Natur und kann sich sowohl in qualitativen Vorteilen wie der Erhöhung der Kundenbindung und einem Imagegewinn als auch quantitativ in Form von Erlössteigerungen oder Kostensenkungen niederschlagen.

(4) Das „Individual Marketing“ repräsentiert den Extremfall einer hohen Kundenintegration und einer ausgeprägten Interaktion zwischen Kunden und Anbietern. Unter Individual Marketing ist die Anfertigung maßgeschneiderter Produkte auf eine individuelle Bestellung hin zu verstehen. Dies entspricht der typischen Arbeitsweise in Handwerksberufen wie dem Schneiderei- oder Schustereiwesen. Jedes Produkt ist eine individuelle Auftragsanfertigung, es werden keine (bzw. nur in geringem Maße) lagerfähigen Standardwaren erzeugt. Neben handwerklicher Arbeit bilden weitere Beispiele für ein Individual Marketing die Individualfertigung von Industrieanlagen oder das Programmieren von Individualsoftware.

\subsubsection{Mass Customization im Industriebetrieb}

In Abschnitt 2.1.2 wurde erläutert, dass die strategische Managementlehre fordert, dass sich ein Unternehmen im Rahmen seiner Wettbewerbsstrategie entweder für einen hohen Differenzierungsgrad oder für ein niedriges Kostenniveau durch eine hochgradig standardisierte Leistungserbringung entscheiden

137 Vgl. Berry (1983), S. 25.

$138 \mathrm{Vgl}$. Morgan/Hunt (1994), S. 22.

139 Einen Überblick bietet Hildebrand (1997), S. 44 ff.

140 Vgl. Diller (1995), S. 55.

141 Vgl. Berry (1995), S. 240; Shani/Chalasani (1993).

142 Vgl. Jüttner/Wehrli (1994), S. 54. 
muss. Diese Alternativenauswahl ist nach Porter strikt dichotom, eine gemeinsame Verfolgung beider Optionen im Sinne einer hybriden Strategie ist nur im Ausnahmefall einer geschützten Marktnische erfolgreich. ${ }^{143}$ Als Begründung dieser strikten Dichotomie gilt, dass ein hoher Differenzierungsgrad mit einer hohen Varietät der Erzeugnisse verbunden ist. Diese Varietät hat zwangsläufig einen Anstieg der Kosten zur Folge. Nach Pine/Anderson besitzt eine Differenzierungsstrategie im Zeitalter nachfrageorientierter Märkte ein zweifaches Varietätsproblem: ${ }^{144}$ Erstens, die externe Varietät aus Kundensicht, die es im Sinne kundenindividueller Bedürfnisbefriedigung zu maximieren gilt. Zweitens, die interne Varietät von Produktions- und Vertriebsprozessen, die es umgekehrt durch Standardisierung von Fertigungsschritten und Reduktion der organisatorischen Komplexität zu minimieren gilt.

Nach Piller vernachlässigt jedoch eine solche Begründung die Auswirkungen einerseits der informations- und kommunikationstechnischen Entwicklungen, andererseits moderner Prinzipien flexibler, modularer Variantenfertigung sowie der sich daraus ergebenden Möglichkeiten zur Realisierung gleichzeitiger Differenzierungs- und Kostenvorteile in der Produktion. ${ }^{145}$ Moderne luK-Technologien ermöglichen eine Handhabung der aus einer hohen Varietät der Leistungsproduktion resultierenden Komplexität in zweifacher Weise: Sie bewirken einerseits eine Reduktion der Planungsunsicherheit durch eine Erhöhung der Interaktivität im direkten Kundenkontakt, bspw. bei der Ermittlung von Kundenwünschen und Produktbewertungen. ${ }^{146}$ Andererseits verbessern integrierte inner- und zwischenbetriebliche Informations- und Planungssysteme in Verbindung mit einer leistungsfähigen Datenvernetzung die Möglichkeiten organisatorischer Kommunikation und dadurch der Koordination betrieblicher Leistungsprozesse. ${ }^{147}$ Eine solche integrierte Unterstützung der betrieblichen Planungs- und Erzeugungsprozesse wird im Industriebetrieb auch als „Computer Integrated Manufacturing" (CIM) bezeichnet. ${ }^{148}$

Das Ziel der Einführung eines CIM-Konzepts besteht darin, die industriellen Herstellungs-, Planungs- und Steuerungsprozesse flexibler und modularer zu

\footnotetext{
143 Vgl. Porter (1999).

$144 \mathrm{Vgl}$. Anderson/Pine (1997), S. $45 \mathrm{f}$.

145 Vgl. Piller (2001), S. 110.

146 Vgl. Piller (2001), S. 226 f.

147 Vgl. bspw. Picot/Reichwald/Wigand (1998), S. 91.

148 Das CIM-Konzept beruht auf einer Integration der Informationsverarbeitung einerseits innerhalb und andererseits zwischen den betriebswirtschaftlichen und technischen Aufgaben eines Industriebetriebs. Zur Unterstützung von betriebswirtschaftlichen Aufgaben werden sog. Produktionsplanungsund Steuerungs- (PPS-)systeme eingesetzt, auf technischer Seite kommt ein Bündel sog. Computer Aided (CA) Systeme wie bspw. Systeme für die computergestützte Entwicklung (Computer Aided Engineering, CAE) oder computergestützte Herstellung (Computer Aided Manufacturing, CAM) zum Einsatz. Eine Integration zwischen betriebswirtschaftlicher und technischer Datenverarbeitung kann dadurch gewährleistet werden, dass sowohl kaufmännische Auftragsdaten als auch technische Fertigungsdaten für ein Produkt in einer gemeinsamen Datenbank gespeichert werden, um bspw. Dateninkonsistenzen zu verringern. Vgl. Scheer (1990), S. 2 und 12; Mertens et al. (2005), S. 142.
} 
gestalten, ohne zusätzliche Kosten zu verursachen. ${ }^{149}$ Eine solche Flexibilisierung und Modularisierung kann bspw. dadurch erreicht werden, dass der Vorfertigungsgrad der Produktbestandteile durch flexibel kombinierbare Produktmodule erhöht wird. Solche Fertigungsstrukturen erlauben eine Erhöhung der externen Produktvarietät in Form einer flexiblen Modulkonfiguration zwischen verschiedenen Produktionslinien mit einer gleichzeitigen Wahrung der Skalenund Verbundeffekte der Massenproduktion. ${ }^{150}$ Dies kann bei konsequenter Verfolgung des CIM-Gedankens langfristig dazu führen, dass „als Folge der Informationswirtschaft (...) die klassische Massenproduktion durch die Fertigung kundenindividueller Güter zu einem Kostenniveau vergleichbarer Massengüter abgelöst [wird]. “151 Diese Fertigungsform wird auch als „Mass Customization“152 bzw. „Massen-Maßfertigung“"153 oder „kundenindividuelle Massenproduktion“"154 bezeichnet.

Mass Customization repräsentiert aus Fertigungssicht das Äquivalent zu der im Voranstehenden erläuterten Sichtweise des Customized Marketing. Aus Wettbewerbssicht bildet Mass Customization eine hybride Wettbewerbsstrategie aus Massenproduktion („Mass Production“) und Einzelfertigung („Customization“). Eine Mass Customization Strategie kann neben den Möglichkeiten der computergestützten Fertigung durch eine Reihe weiterer IV-Systeme unterstützt werden, wie bspw. elektronische Produktkataloge, Konfigurationssysteme oder ein Supply Chain Management. ${ }^{155}$ Die Vorteilhaftigkeit der Mass Customization kann sowohl aus Erlössicht als auch aus Kostensicht begründet werden: Aus Erlössicht kann ein individuelleres Leistungsangebot bspw. dazu führen, dass das Angebot für den Kunden attraktiver erscheint und dadurch die Absatzmenge gesteigert wird oder sich für den Anbieter Preisspielräume eröffnen. Aus Kostensicht kann einerseits der Aufbau einer langfristigen interaktiven Beziehung zum Kunden zu zusätzlichen Lern- und Verbundeffekten führen. Andererseits können durch integrierte Planung und Fertigung die Fertigungs- und Lagerkosten reduziert werden (vgl. Abbildung 2-9).

149 Beispiele für Unternehmen, die eine große Reihe von Produktvarianten traditionell auf der Grundlage von vorgefertigten Modulen anbieten bilden im Fotokopierbereich Canon und Xerox, im Werkzeugebereich Black\&Decker oder im Bereich der Unterhaltungselektronik Sony. Vgl. Piller (2001), S. 228 f.

150 Vgl. Picot/Reichwald/Wigand (1998), S. 91.

151 Piller (2001), S. 200. Neben dem von Piller betonten „Folgen der Informationswirtschaft" als Grundlage der Mass Customization kann darüber hinaus die Entwicklung intelligenter IT- und Produktionskonzepte genannt werden.

152 Vgl. Davis (1987).

153 Vgl. Mertens (1995), S. 503.

154 Vgl. Piller (2001), S. 205.

$155 \mathrm{Vgl}$. Mertens (1995), S. 304. 


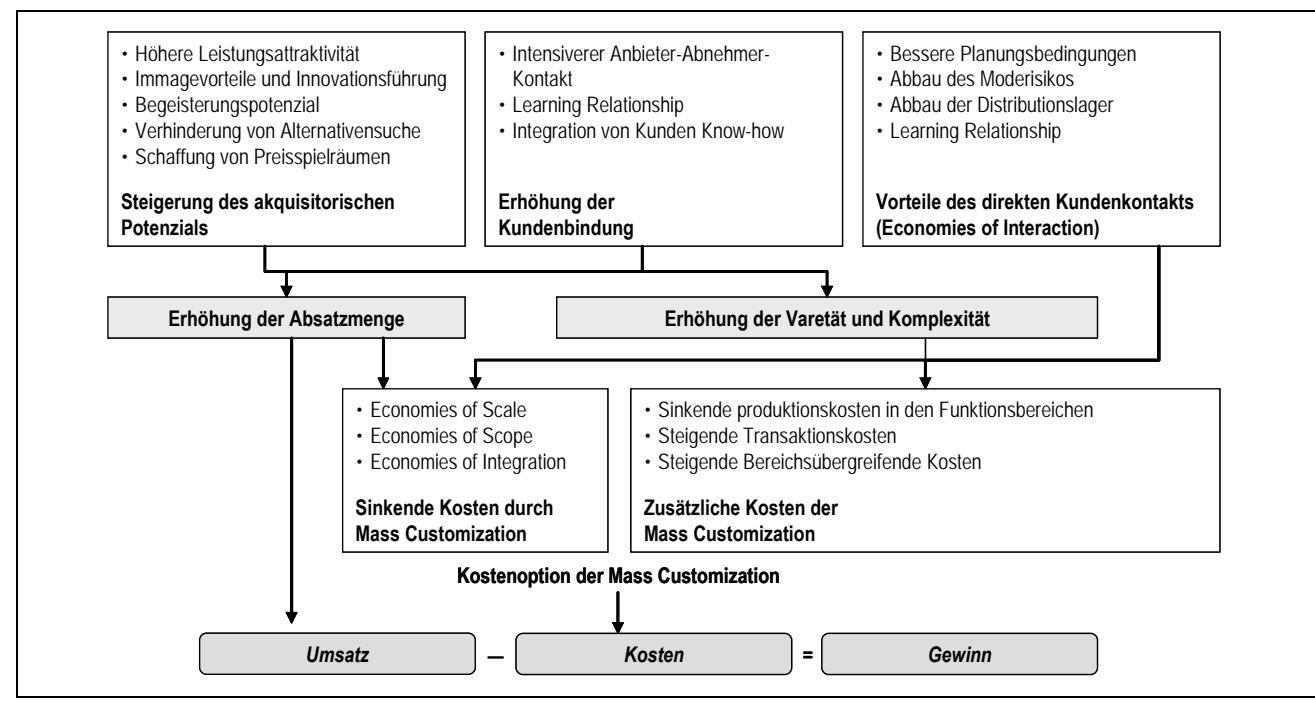

Abbildung 2-9: Vorteilhaftigkeit der Mass Customization

Eines der bekanntesten Beispiele für Mass Customization in der industriellen Fertigung bildet der Dienst „Nike-ID“ des amerikanischen Sportartikelherstellers Nike, durch den der Nutzer einen individuellen Turnschuh gestalten kann. ${ }^{156}$ Eine Auswahl von über 20 Grundtypen der Nike-Schuhmodellreihe kann in Bezug auf die Farbgebung der einzelnen Aufbauelemente, wie Sohle, Zunge oder Obermaterial des Überschuhs konfiguriert, mit einem persönlichen Logo individuell signiert und natürlich in der richtigen Größe bestellt werden. Einige Modelle bieten zudem die Möglichkeit, die Weite und die Dämpfungsstärke der Sohle zu bestimmen.

\subsubsection{Empfehlungssysteme für Dienstleistungen und Handel}

Trotz der im voranstehenden Abschnitt beschriebenen Möglichkeiten, Güter individuell in industriebetrieblicher Massenfertigung herzustellen, existieren zum einen Produkte wie bspw. in Buchform gedruckte Romane oder auf DVD gepresste Kinofilme, die kaum oder gar nicht individuell maßgeschneidert werden können. Zum anderen ist der Hauptbestandteil des Outputs eines Dienstleistungsunternehmens grundsätzlich immateriell, die Leistungserbringung erfolgt immer in gewisser Weise für den Kunden maßgeschneidert. Dennoch besteht gleichwohl auch für den Dienstleistungsbereich und den Bereich der nur geringfügig maßschneiderbaren Produkte aufgrund des Überangebots in den meisten Märkten für die Kunden ein zunehmendes Auswahlproblem. Um diesem Auswahlproblem begegnen zu können, ist es möglich, die Prinzipien der Mass Customization so anzuwenden, dass nicht das Produkt selbst, sondern lediglich seine Präsentation im Rahmen eines Auswahlkatalogs in Form einer individuellen Empfehlung maßgeschneidert wird. Systeme, die eine derartige Empfeh-

156 Vgl. www.nikeid.com. 
lung automatisch für einen individuellen Kunden abgeben, werden auch als "Beratungssysteme“ oder „Empfehlungssysteme“ (i.w.S.) bezeichnet. ${ }^{157}$

Empfehlungssysteme können nach drei Kriterien unterschieden werden (vgl. Abbildung 2-10): nach dem verwendeten Dateninput des Systems (1), nach der eingesetzten Empfehlungsmethode (2) und nach den Gestaltungsoptionen des vom System erzeugten Output (3).

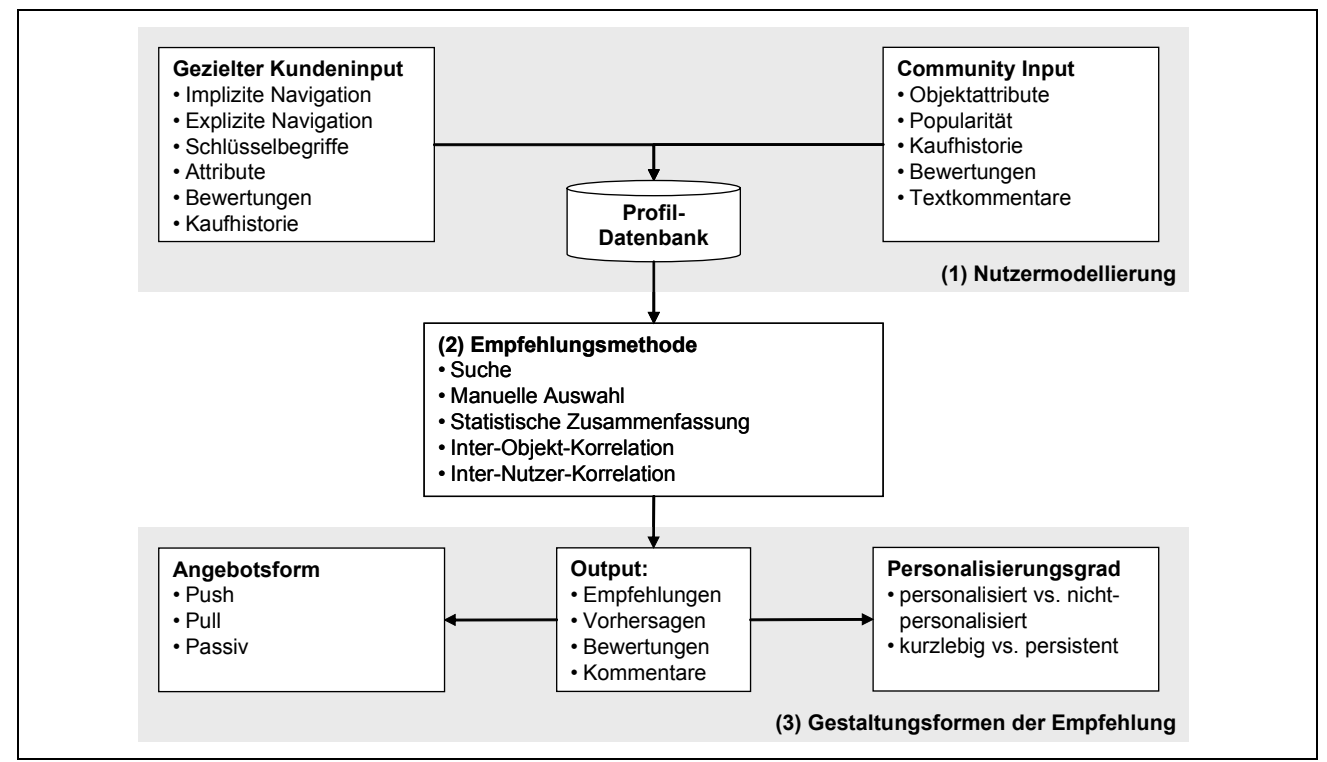

Abbildung 2-10: Unterscheidungskriterien von Empfehlungssystemen

(1) Um plausible Empfehlungen abgeben zu können, benötigt ein Empfehlungssystem zunächst Angaben über die Kaufpräferenzen der jeweiligen Kunden. Die Ermittlung von kaufbezogenen Kundenmerkmalen erfolgt üblicherweise durch eine Nutzermodellierungskomponente. ${ }^{158}$ Die Nutzermodellierungskomponente kann Kaufpräferenzen entweder auf der Grundlage von Einzelkundenangaben oder anhand von gruppenbezogenen Angaben ermitteln. Als Quelle von Einzelkundenangaben können Beobachtungen des Navigationsverhaltens (implizite Navigation) sowie explizite Befragungen des Kunden nach seinem Navigationsweg (explizite Navigation), nach Schlüsselbegriffen oder anderen präferierten Referenzangaben verwendet werden. Gruppenbezogene Angaben können bspw. von bestimmten Kundentypen gebräuchliche Klassifikationen (Objektattribute), statistische Popularitätsangaben oder gruppentypische Bewertungen verwendet werden. Die jeweils vom Nutzermodellierungssystem erhobenen Einzelangaben werden in individuellen oder gruppenbezogenen Profilen gespeichert.

157 Vgl. Runte (2000); Mertens (1997), S. 401

$158 \mathrm{Vgl}$. Mertens (1995), S. 505. 
(2) Grundsätzlich existieren fünf verschiedene Verfahren, um durch ein intelligentes System Kaufempfehlungen zu erzeugen: Unter Verwendung von Funktionen aus dem sog. "Information Retrieval“" ${ }^{159}$ können Konsumenten Suchanfragen über eine indizierte Datenbank von Produktangaben abschicken und erhalten als Ergebnis eine Liste aller mit dem Suchbegriff übereinstimmenden Produkte. Umgekehrt kann ein Konsument manuell aus vorgeordneten Produktlisten das für inn relevante Angebot selbst auswählen. Zusätzlich können automatisch oder händisch generierte statistische Kurzzusammenfassungen verwendet werden, um zusätzliche Selektionskriterien für eine Suchfunktion oder manuelle Auswahllisten zu schaffen. Komplexere Verfahren verwenden statistische Korrelationen und Inferenzen, um Ähnlichkeiten entweder zwischen den Profilen verschiedener Konsumenten (Inter-Nutzer-Relationen) oder verschiedenen Produkten (Inter-Objekt-Relationen) zu bestimmen. Gebräuchlich ist in diesem Zusammenhang die Unterscheidung zwischen „Empfehlungssystemen“ (i.e.S.) bzw. „Recommendation Systems“ auf der einen Seite und „Empfehlersystemen“ bzw. „Recommender Systems“ auf der anderen Seite. ${ }^{160}$ Die erstere Gruppe verwendet Inferenzalgorithmen, die auch als „eigenschaftsbasierte Filterverfahren" oder "feature-based filtering" bezeichnet werden. Eigenschaftsbasierte Filter ziehen einen direkten Vergleich zwischen den Profilmerkmalen eines Nutzers und den Eigenschaften verfügbarer Produktbeschreibungen und treffen eine Auswahl der Produkte, deren Produktbeschreibung mit den Nutzerbedürfnissen am besten übereinstimmen. Dabei kommen in der Regel statistische Klassifikationsalgorithmen zum Einsatz. ${ }^{161}$ Die Gruppe der Empfehlersysteme verwendet dagegen Inferenzalgorithmen, die auch als kollaborative Filter oder "Collaborative Filtering" bezeichnet werden. Collaborative FilteringVerfahren leiten individuelle Angebote indirekt, anhand struktureller Gemeinsamkeiten in den Präferenzen verschiedener Nutzer oder Nutzergruppen ab. ${ }^{162}$ Anstelle einer direkten Ähnlichkeitsüberprüfung zwischen Inhalteeigenschaften und Nutzerpräferenzen werden zunächst signifikante Präferenzähnlichkeiten zwischen verschiedenen Nutzern bestimmt. Auf der Grundlage solcher Präferenzähnlichkeiten treffen Collaborative Filtering-Verfahren für einen Konsumenten eine Auswahl, die aus solchen Produkten besteht, die ein Nutzer mit ähnlichen Präferenzen nachgefragt hat.

(3) Ein Empfehlungssystem kann verschiedene Ergebnisse liefern, um die Auswahl für einen Konsumenten zu erleichtern. Beispiele für die Möglichkeiten der Auswahlunterstützung bilden die Auflistung von Kaufempfehlungen, die Vorhersage nächster Navigationslinks sowie Bewertungen und Kommentare zu

159 Vgl. bspw. Ferber (2003).

160 Vgl. Runte (2000), S. 10.

161 Im Rahmen solcher Klassifikationsalgorithmen können verschiedene Verfahren zum Einsatz kommen wie bspw. Vektorraummodelle (vgl. Salton/Wong/Yang (1975)), Verfahren auf Grundlage kombinierter Wahrscheinlichkeiten (vgl. Pazzani/Billsus (1997)), Entscheidungsbäume oder neuronale Netze (vgl. Yang/Liu (1999)).

162 Vgl. Herlocker/Konstan/Borchers/Riedl (1999); Melville/Mooney/Nagarajan (2002); Schafer/Konstan/Riedl (2001). 
betrachteten Produktbeschreibungen. Dabei kann das Empfehlungssystem konkret eine eigene Kommunikationsschnittstelle zum Kunden besitzen, über die das Systemergebnis entweder in Push- oder Pull-Form distribuiert wird, oder das Systemergebnis wird lediglich (passiv) in die Produktbeschreibung integriert. Insofern ist zwischen solchen Systemergebnissen zu unterscheiden, die einen Bezug zu den Kaufpräferenzen eines individuellen Nutzers bzw. einer spezifischen Gruppe aufweisen und somit kurzlebigen, dynamischen Charakter besitzen, und solchen, die für alle Nutzer gleich und damit persistent sind.

\subsubsection{Zusammenfassung}

Die Veränderungen, die zu einer gesellschaftlichen Individualisierung geführt haben (vgl. Abschnitt 2.2.1) sind ebenso wie die Entwicklungen neuer Gestaltungsoptionen einer individuelleren Marktbearbeitung (vgl. Abschnitt 2.2.2) eng geknüpft an die Entwicklung der digitalen Informations- und Kommunikationstechnik. Dennoch beschieden Zerdick et al. noch vor vier Jahren den Möglichkeiten der Personalisierung, mehr Versprechen denn Realität der Internetökonomie zu sein. ${ }^{163}$ Diese Einschätzung trifft heute nicht mehr zu: Neben den bereits genannten Beispielen kundenindividueller Massenfertigung bilden die Onlinedienste des Buchhandels Amazon und des Auktionshauses Ebay derzeit die bekanntesten Beispiele für die Verwendung von Empfehlungssystemen. Bei Amazon kommen alle fünf beschriebenen Varianten von Empfehlungsmethoden zum Einsatz: Manuelle Auswahl (bspw. in der Rubrik „Stöbern“), Suchfunktionen, Kurzbeschreibungen, Kundenbewertungen und profilgestützte individuelle Produktempfehlungen. Dennoch repräsentieren Individualisierungsformen insbesondere im Bereich der Wirtschaftsinformatik einen vergleichsweise jungen Forschungsgegenstand, der teilweise durch begriffliche Unschärfe gekennzeichnet ist. Vor dem Hintergrund der ausführlichen Herleitung der begrifflichen und theoretischen Individualisierungsdimensionen sei Individualisierung im Weiteren weniger als gesellschaftliche Entwicklung als vielmehr als eine strategische Handlungsoptionen aus einzelbetrieblicher Sicht verstanden. Der Fokus der Arbeit liegt dabei auf der Umsetzung und den Konsequenzen des Angebots maßgeschneiderter Mediengüter. Dies wird schwerpunktmäßig in Kapitel 4 untersucht. Insofern der wertbildende Bestandteil eines Medienguts immaterieller Natur ist, werden insbesondere Möglichkeiten geprüft, Empfehlungssysteme zu verwenden, um individuelle Mediengüter automatisch zu erzeugen. Marketingbezogene Aspekte des Aufbaus einer nachhaltigen Kundenbeziehung werden dagegen weitgehend vernachlässigt. Ebenso wird im weiteren Verlauf der Arbeit der, im Vergleich zum Begriff der Individualisierung tendenziell unscharfe Begriff der „Personalisierung“ vermieden, der sich insbesondere in der Managementliteratur teils als Synonym für ein einzelkundenorientiertes Marketing, teils als Synonym für Mass Customization und teils sogar als Überbegriff für alle Formen einer einzelkundenorientierten Marktbearbeitung eingebürgert hat. ${ }^{164}$

$163 \mathrm{Vgl}$. Zerdick et al. (2001), S. 113.

164 Vgl. bspw. Runte (2000); Gora/Röttger-Gerigk (2002); Welsch-Lehmann (2001). 


\subsection{Mobile Dienste}

Mobile Dienste repräsentieren sowohl aus technischer als auch aus ökonomischer Sicht ein vergleichsweise junges Forschungsgebiet. Dabei war insbesondere die ökonomische Bewertung der Marktpotenziale mobiler Dienste in den vergangenen Jahren durch eine Reihe von Fehlerwartungen geprägt: Eine anfängliche Euphorie begründete sich im Wesentlichen durch Wachstumserwartungen im Bereich nicht-sprachorientierter Dienste, ${ }^{165}$ die den Fokus der hier zugrunde liegenden Untersuchung bilden. Optimistische Analysen der Marktentwicklung prognostizierten ein durchschnittliches Jahreswachstum von 100 bis über $200 \%$ mit einem erwarteten Gesamtvolumen des europäischen Umsatzes von über $23 \mathrm{Mrd}$. EUR bereits für das Jahr 2003. ${ }^{166}$ Diese Erwartungen scheinen sich jedoch bei weitem nicht zu erfüllen. Das Ministerium für Wirtschaft und Arbeit der Bundesrepublik Deutschland weist 2003 für Europa lediglich ein Umsatzvolumen von 71 Mio. US-\$ aus. Auf dieser Basis wird ein Wachstum auf insgesamt 119 Mio. US-\$ bis 2007 prognostiziert. ${ }^{167}$

Fehlende erfolgreiche Geschäfts- und Produktkonzepte führten aufgrund der Diskrepanz zwischen Erwartung und Realität zu einer Unsicherheit in Bezug auf die Marktchancen mobiler Dienste. Eine Hauptursache hierfür ist die anhaltend hohe Komplexität und Dynamik im Bereich mobiler Technologien. Drei Determinanten sind dafür wesensgebend: ${ }^{168}$ erstens, die Unsicherheit in Bezug auf die Entwicklung und Etablierung dominierender Technologie- und Branchenstandards; zweitens, die Komplexität der Realisierung mobiler Dienste; drittens, die Vielfalt involvierter Akteure im Rahmen der Bereitstellung mobiler Dienste für Endnutzer.

Im Folgenden werden zentrale Aspekte mobiler Technologien als Grundlage Erfolg versprechender mobiler Dienste dargestellt. Vor dem Hintergrund der angesprochenen dreifachen Unsicherheitsproblematik werden Anwendungsformen auf drei Ebenen erfasst: erstens, auf der Netzwerkebene unter Berücksichtigung der derzeitig verfügbaren Technologiealternativen zum Aufbau von digitalen Funknetzen (vgl. 2.3.2); zweitens, auf der Dienstebene unter Berücksichtigung verschiedener Übertragungsstandards zur Entwicklung mobiler Datendienste (vgl. Abschnitt 2.3.3); drittens, auf der Geschäftsebene zur Identifikation Nutzen stiftender Anwendungsszenarien aus Anwendersicht (vgl. Abschnitt 2.3.4). Zuvor wird in Abschnitt 2.3.1 ein einleitender Überblick über die grundlegenden Forschungsaspekte in Bezug auf mobile Dienste aus technischer Sicht gegeben.

\footnotetext{
165 Vgl. Buse (2002), S. 97.

166 Vgl. Müller-Verse (1999).

167 Vgl. Graumann/Köhne (2003).

$168 \mathrm{Vgl}$. Lehmann/Lehner (2003), S. 8.
} 


\subsubsection{Mobilität im Rahmen von Anwendungssystemen}

Traditionell spielt Mobilität im Sinne räumlicher Entfernungen im Zusammenhang mit der technischen Realisierung von Anwendungssystemen lediglich eine untergeordnete Rolle. ${ }^{169}$ Stattdessen wird in der Informatik üblicherweise von räumlichen Gegebenheiten abstrahiert, wodurch gemäß dem Prinzip der so genannten Zugriffs- oder Verteilungstransparenz die logische Unabhängigkeit von Informationen und ihrem physischen Speicherort gewährleistet wird. ${ }^{170}$ Erst durch die Möglichkeiten einer drahtlosen Datenkommunikation rückt auch die physische Mobilität von Endgeräten und Nutzern in den Mittelpunkt des Interesses. Um eine solche Mobilität von Nutzern und Endgeräten gewährleisten zu können, müssen Kommunikationssysteme allerdings Basisfunktionalitäten zur Verfügung stellen, um die Mobilität eines Nutzers oder Endgeräts transparent zu unterstützen. Aus technischer Sicht kann dabei zwischen primären und sekundären Mobilitätsformen unterschieden werden. ${ }^{171}$

Zu den primären Mobilitätsformen zählen (1) Endgerätemobilität, (2) Personenmobilität, (3) Dienstmobilität und (4) Sitzungsmobilität. Sekundäre Mobilitätsformen decken dagegen systemtechnische und administrative Aspekte der primären Mobilitätsformen ab und gliedern sich in Intrasystem- und Intersystemmobilität sowie intraorganisationale und interorganisationale Mobilität.

(1) Unter Endgerätemobilität wird die räumliche Beweglichkeit von portablen Geräten wie z.B. Mobiltelefonen, Notebooks, etc. verstanden. Endgerätemobilität wird durch Kommunikationsnetze entweder in Form kontinuierlicher (bei drahtloser Verbindung) oder diskreter Endgerätemobilität unterstützt. Bei der diskreten (üblicherweise drahtgebundenen) Endgerätemobilität ist das Gerät transparent für die genutzten Dienste an verschiedenen Zugangspunkten mit dem Kommunikationsnetz verbunden (z. B. Telefondosen in verschiedenen Räumen). Demgegenüber kann ein Endgerät auf Grundlage des schnurlosen DECT-Standard (Digital Enhanced Cordless Telecommunications) kontinuierlich in verschiedenen Räumen verwendet werden, solange es über Funk mit seiner Basisstation verbunden bleibt. Eine kontinuierliche Dienstnutzung eines Teilnehmers über den Wirkungsbereich einer Sendestation hinaus erfordert zusätzlich das Weiterreichen der Funkverbindung durch so genanntes „Handover" zwischen verschiedenen Sendestationen (Mikromobilität) und das Weiterreichen der Verbindung durch so genanntes „Roaming“ zwischen Mobilfunknetzen verschiedener Betreiber (Makromobilität).

(2) Personenmobilität ermöglicht es einem Nutzer, unabhängig von dem aktuellen Netzzugangspunkt und dem gerade verwendeten Endgerät, seine Identität gegenüber dem Netz aufrecht zu erhalten. Notwendig hierfür ist die Verfügbarkeit der Benutzerprofile über Endgerät-, Betreiber- und Netzgrenzen hinweg. Die Voraussetzung für die Inanspruchnahme der Dienste stellt eine Registrierung des Nutzers im Netz mit seinen Identifikationsmerkmalen (z.B. durch eine

\footnotetext{
$169 \mathrm{Vgl}$. Hess et al. (2005).

170 Vgl. Coulouris/Dollimore/Kindberg (2002), S. 42 f.

171 Vgl. Küpper/Reiser/Schiffer (2004).
} 
Nutzerkennung und ein Passwort) dar. Personen- und Endgerätemobilität hängen daher in Funknetzen häufig eng zusammen. So werden bspw. in GSM- und UMTS-Netzen sowohl die Personen- als auch die Endgerätemobilität durch die Authentifizierung des Nutzers mittels des sog. Subscriber Identification Module (SIM) beim Mobilfunknetz erreicht.

(3) Dienstmobilität erlaubt es dem Nutzer, nach dem „Anytime-Anywhere“Paradigma mit einem beliebigen Endgerät an einer beliebigen Stelle eines Kommunikationsnetzes Dienste in der von ihm bevorzugten Form zu nutzen. Eine nutzerspezifische Anpassung des Dienstes ist dabei im Wesentlichen davon abhängig, in welchem Rahmen das genutzte Endgerät und nutzerspezifische Diensteinstellungen im aktuellen Netz unterstützt werden. Die Speicherung der Diensteinstellungen kann u.a. in einer Smart Card, einer Datenbank des Netzbetreibers oder auf einem Server des Teilnehmers erfolgen. Eine weitere Herausforderung ist die Anpassung des jeweiligen Dienstes an eine veränderte Umgebung, z.B. an neue Endgeräte oder Netzinfrastrukturen.

(4) Die Sitzungsmobilität beruht auf dem so genannten Sitzungskonzept. Eine Sitzung wird definiert als eine temporäre Beziehung zwischen verteilten Dienstkomponenten, welche die zur Erbringung einer bestimmten Aufgabe benötigten Ressourcen zusammenfasst. Eine Sitzung kann eingerichtet, terminiert, unterbrochen und reaktiviert werden. Um zu ermöglichen, dass eine Sitzung temporär unterbrochen und ggf. auf ein anderes Endgerät oder in ein anderes Netz verlagert wird, muss der Zustand und Verlauf der Sitzung gespeichert werden. Bei der diskreten Sitzungsmobilität kann der Dienst nur zu bestimmten Synchronisationspunkten verlagert werden, bei der kontinuierlichen Sitzungsmobilität kann der Dienst zu jedem Zeitpunkt unterbrochen werden.

Endgeräte- und Personenmobilität werden derzeit bereits von den meisten verfügbaren Funktechnologien unterstützt. Die Funknetzstandards für die Weitverkehrskommunikation wie GSM (Global System for Mobile Communications) und UMTS (Universal Mobile Telecommunications System) erlauben bspw. sowohl eine kontinuierliche Dienstnutzung innerhalb des Netzes als auch die Erreichbarkeit eines Nutzers unter seiner Telefonnummer in fremden Mobilfunknetzen. ${ }^{172}$ Des Weiteren sollen zukünftig auch eine netzübergreifende Authentifizierung und eine Abrechnung der Nutzungsgebühren in WLAN-basierten Umgebungen möglich sein. Demgegenüber werfen Dienst- und Sitzungsmobilität bislang noch technische und insbesondere sicherheitstechnische Probleme auf. Daher werden Dienst- und Sitzungsmobilität bislang weder in GSM-Netzen noch in WLAN-Umgebungen umgesetzt. Insofern ist für ein Verständnis der technischen Rahmenbedingungen mobiler Dienste in erster Linie die Kenntnis der derzeit existierenden Mobilfunktechnologien erforderlich. Im Folgenden wird daher hierüber ein Überblick gegeben.

172 Vgl. Eberspächer/Vögel/Bettstetter (2001); Kaaranen/Ahtiainen/Laitinen/Naghian/Niemi (2001). 


\subsubsection{Digitale Funknetztechnologien}

Eine Untersuchung von mobilen Netztechnologien und Anwendungen beschränkte sich in der Vergangenheit häufig auf die etablierten Technologiestandards bzw. deren Fortentwicklungen im Rahmen kommerzieller Mobilfunkkommunikationsnetze. Mittlerweile zeichnet sich jedoch ab, dass auch Technologien, die für den Netzwerkaufbau drahtloser, lokal begrenzter Infrastrukturen entwickelt wurden, echte und im Vergleich deutlich kostengünstigere Alternativen für Daten-, mittelfristig auch für Kommunikationsnetze darstellen. ${ }^{173}$ Daher sollen im Folgenden beide Architektur- und Technologievarianten vorgestellt werden. In Abschnitt 2.3.2.1 werden zunächst grundlegende Aspekte der Mobilfunktechnik erläutert. Im Weiteren werden die zentralen Standards für drahtlose lokale Netze (vgl. Abschnitt 2.3.2.2), Nahbereichsnetze (vgl. Abschnitt 2.3.2.3) und Weitverkehrsmobilfunknetze (vgl. Abschnitt 2.3.2.4) beschrieben.

\subsubsection{Allgemeine Grundlagen der Mobilfunktechnik}

Generell können in mobilen Funknetzen Kommunikationsverbindungen zwischen beweglichen und ortsfesten Stationen (Infrastrukturnetzwerke) oder ausschließlich zwischen beweglichen Stationen (Ad-hoc-Netzwerke) aufgebaut werden. ${ }^{174}$ Damit unterscheiden sich Mobilfunknetze von Festnetzen, indem sie neben der (auch von Festnetzen unterstützten) Nutzermobilität zusätzlich die Mobilität von Endgeräten gewährleisten. An die Stelle einer festen Übertragungsleitung tritt im Mobilfunk ein ungerichteter Funkkanal. Anders als in analogen Funknetzen, in denen das Kommunikationssignal unmittelbar als kontinuierliche Signalwelle übertragen wird, wird beim digitalen Mobilfunk das ursprüngliche Signal vom Endgerät in Folgen von Bits und Bytes kodiert, als diskretes Signal übertragen und vom Empfängergerät dekodiert. Dabei kommen im Rahmen der Signalübertragung zwei Verfahren zur Anwendung: ${ }^{175}$ Verfahren, die den mehrfachen Zugriff auf das Funkträgermedium erleichtern, sog. "Multiplex“ Verfahren (1) und sog. „Bandspreizverfahren“ zur Verringerung von Übertragungsstörungen (2).

(1) Das ökonomisch nutzbare Frequenzspektrum ist durch die Art der Nutzung und den Stand der Technik eine nur begrenzt verfügbare Ressource und stellt einen Engpass bei der mobilen Funkübertragung dar. ${ }^{176}$ Das Frequenzband der deutschen D-Netze umfasst bspw. zusammen eine Bandbreite von lediglich rund $25 \mathrm{MHz}$ im Frequenzbereich von $900 \mathrm{MHz}$ und ist in 124 Kanäle unterteilt. Um einen gleichzeitigen Zugriff auf das Funkübertragungsmedium von mehr als 124 gleichzeitigen Kommunikationsvorgängen zu ermöglichen, bedarf es also Verfahren zur Gewährleistung von kollisionsfreien Mehrfachzugriffen auf dieselben Übertragungskanäle. Durch sog. „Multiplexen“ kann ein Medium durch

\footnotetext{
173 Vgl. Webb (2001); Sikora (2001).

$174 \mathrm{Vgl}$. Müller/Eymann/Kreutzer (2003), S. 150.

$175 \mathrm{Vgl}$. Kaspar/Hagenhoff (2005).

176 Vgl. Heutmann/Ewers (2003).
} 
Unterteilung nach Zugriffsraum, Zeit, Frequenz oder Code verschiedenen Nutzern störungsfrei zur Verfügung gestellt werden. Im digitalen Mobilfunk kommen dabei drei unterschiedliche Verfahren z. T. gemeinsam zur Anwendung: ${ }^{177}$ Beim Time Division Multiple Access (TDMA) werden die verschiedenen Kommunikationsvorgänge zeitlich gestaffelt auf die Übertragungskanäle exklusiv verteilt. Beim Frequency Division Multiple Access wird jedem Kommunikationsteilnehmer eine exklusive Frequenz zugewiesen. Beim aufwändigeren Code Division Multiple Access (CDMA) senden zwar alle Kommunikationsteilnehmer gleichzeitig auf derselben Frequenz, die einzelnen Kommunikationsvorgänge werden jedoch mit einem eindeutigen Code verschlüsselt und können dadurch unabhängig identifiziert werden.

(2) Anders als bei leitungsgebundenen Netzen erfolgt die Signalausbreitung in Funknetzen stets geradlinig, vergleichbar mit Lichtwellen. Objekte im Verlaufsweg der Senderichtung können die Signalausbreitung behindern. Dabei sind Phänomene zu beobachten wie Signalabschattungen durch Hindernisse, Signalreflexionen, bspw. durch große Flächen, Signalstreuungen durch kleine Objekte oder Signalbeugungen an scharfen Kanten. ${ }^{178}$ Weitere, z. T. aus den erstgenannten resultierende Phänomene der Funksignalausbreitung sind: Freiraumdämpfung, d.h. die Abnahme der Empfangsleistung eines Signals mit zunehmender Entfernung; Mehrwegeausbreitungen von Signalen in Form zeitlich versetzter Empfangsimpulse (bspw. aufgrund von reflektierten Signalen); Signalfading, d. h. Schwankungen in der Signalleistung bspw. im Zuge von Bewegungen der Kommunikationsteilnehmer. Aufgrund dieser Störphänomene im Rahmen der Signalübertragung besteht die Gefahr einer Signalauslöschung während des Kommunikationsvorgangs. Um die Auswirkungen solcher Signalstörungen zu reduzieren, wird im Rahmen sog. Bandspreizverfahren die ursprüngliche Übertragungsbandbreite eines Signals auf eine größere Bandbreite verteilt. ${ }^{179}$ Die daraus resultierende Einschränkung an verfügbarem Frequenzraum kann durch die Kombination von Bandspreizverfahren in Verbindung mit Verfahren des Mehrfachzugriffs minimiert werden. Beispiele solcher Kombinationsformen bilden das Frequency Hopping Spread Spectrum (FHSS), bei dem jeder Sender in einer festgelegten Abfolge die Frequenz wechselt und das Direct Sequence Spread Spectrum (DSSS), bei dem das gespreizte Originalsignal mit einer vorgegebenen Pseudozufallszahl codiert wird.

\subsubsection{Drahtlose lokale Netze}

Drahtlose lokale Netze sind gemäß der Spezifikationsnummer 802.11 durch den amerikanischen Ingenieursverband IEEE standardisiert. Die wesentlichen Entwurfsziele des 802.11-Standards bilden Anwendungs- und Protokolltransparenz, nahtlose Festnetzintegration und die weltweite Betriebsmöglichkeit im lizenzfreien Funkfrequenzband (dem sog. „Industrial, Scientific and Medical“

\footnotetext{
177 Vgl. Schiller (2002), S. 347 f. und Müller/Eymann/Kreutzer (2003), S. 53 f.

178 Vgl. Schiller (2002), S. 350 f.

179 Vgl. Schiller (2002), S. 352 f.
} 
[ISM] Band). ${ }^{180}$ Der ursprüngliche 802.11-Standard von 1997 beschreibt drei Sendevarianten: eine Infrarotvariante im Lichtwellenbereich von 850-950 nm und - ökonomisch von größerer Bedeutung - zwei funkbasierte Varianten im Frequenzband von $2,4 \mathrm{GHz} .{ }^{181}$ Bei einem vorgesehenen Spektrum der Sendeleistung zwischen mindestens $1 \mathrm{~mW}$ und in Europa maximal $100 \mathrm{~mW}$ kann in den Funkvarianten eine Kanalkapazität von 1-2 Mbit/s erreicht werden. Zur Reduktion der Kanalknappheit wird das FHSS-Verfahren mit 2,5 Frequenzwechseln pro Minute eingesetzt. ${ }^{182}$ Typischerweise werden WLANs aufgrund einer größeren Sendereichweite von ca. $300 \mathrm{~m}$ im Infrastrukturmodus betrieben, bei dem die gesamte Kommunikation eines Clients über einen Zugangspunkt (Access Point) erfolgt. Der Zugangspunkt dient dabei der Versorgung sämtlicher Clients in seinem Empfangsbereich oder als Funkbrücke zu benachbarten Zugangspunkten oder leitungsgebundenen Netzen.

Weiterentwicklungen des ursprünglichen 802.11-Standards setzen vorwiegend im Bereich der physischen Signalübertragung an: ${ }^{183}$ Beim 802.11aStandard wird anstelle des ursprünglichen $2,4 \mathrm{GHz}$-Bandes auf das $5 \mathrm{GHz}$ Band ausgewichen. Kombiniert mit einem neuen Modulationsverfahren, dem sog. Orthogonal Frequency Division Multiplexing (OFDM), soll dadurch eine Kapazität von bis zu $54 \mathrm{Mbit} / \mathrm{s}$ erreicht werden. Demgegenüber weiterhin im 2.4 $\mathrm{GHz}$-Band operierend, allerdings u.a. erweitert um das kodierte Bandspreizverfahren DSSS und einen verbesserten Signal-Rausch-Abstand, erreicht der derzeit am weitesten verbreitete Standard 802.11b eine Kapazität von bis zu $11 \mathrm{Mbit} / \mathrm{s}$.

\subsubsection{Nahbereichsnetze}

Unter dem Beinamen des dänischen Königs Harald „Blauzahn“, der im 10. Jahrhundert Norwegen und Dänemark vereinte, gründeten die Firmen Ericsson, Nokia, IBM, Toshiba und Intel 1998 eine „Special Interest Group“ für Funknetzwerke für den Nahbereich. ${ }^{184}$ Bluetooth repräsentiert eine Realisierungsform des IEEE Standards 802.15 für Nahbereichsnetze. Ebenso wie WLAN-Netze senden Bluetooth-Geräte im lizenzfreien 2,4 GHz-Band, weshalb es zwischen beiden Netztechnologien zu Interferenzen kommen kann. In Bluetooth-Netzen stehen in der Regel 79 Kanäle zur Verfügung, als Bandspreizverfahren wird FHSS mit 100 Sprüngen pro Sekunde eingesetzt. Alle Geräte mit derselben Sprungsequenz bilden ein gemeinsames sog. „Piconetz“. ${ }^{185}$ Darin sind zwei Dienstklassen spezifiziert, ein synchron-verbindungsorientiertes und ein asynchron-verbindungsloses Verfahren. Bei einer maximalen Sendeleistung von $10 \mathrm{~mW}$ errei-

\footnotetext{
180 Vgl. Schiller (2003).

181 Vgl. IEEE (2001).

182 Vgl. IEEE (2001).

$183 \mathrm{Vgl}$. Schiller (2002).

184 Vgl. SIG (2004); Müller/Eymann/Kreutzer (2003)

$185 \mathrm{Vgl}$. Müller/Eymann/Kreutzer (2003).
} 
chen einzelne Bluetooth-Geräte dabei einen Senderadius von $10 \mathrm{~m}$ bis (theoretisch) maximal $100 \mathrm{~m}$ und eine Datenkapazität bis zu $723 \mathrm{Kbit} / \mathrm{s}{ }^{186}$

Zentrale Einsatzgebiete für Bluetooth-Technologien sind die Verbindung von Peripheriegeräten wie Maus, Kopfhörer, Kfz-Elektronik, Küchengeräte oder die Brückenfunktion zwischen verschiedenen Netztypen wie die Vernetzung zwischen leitungsgebundenen Computernetzwerken und mobilen Funknetzzugangsgeräten. ${ }^{187}$ Bluetooth-Netze werden dazu in der Regel als ad-hoc Netze zusammengeschlossen. Ad-hoc Netze benötigen keine dezidierten Zugangspunkte, die mobilen Endgeräte kommunizieren gleichberechtigt und direkt mit anderen Endgeräten in Empfangsreichweite. Es dient immer genau ein Gerät in einem Netz aus insgesamt maximal acht Geräten als Leitstation zur Vorgabe und Synchronisation der Sprungfrequenz. ${ }^{188}$ Ein Bluetooth-Endgerät kann gleichzeitig an mehreren verschiedenen Piconetzen teilnehmen, jedoch zu einem bestimmten Zeitpunkt aktiv immer nur in einem dieser Netze kommunizieren. Solche überlappenden Netzstrukturen werden als Scatter-Netze bezeichnet.

Ein Bluetooth-Controller, die Sende-Empfangseinheit eines BluetoothEndgeräts, besteht aus drei Bausteinen: Einem Funkempfänger, einer Einheit zur Abwicklung und Überwachung der physischen Funkverbindung und einer Einheit für das Management des Linkaufbaus und der Konfiguration verbundener Geräte. Über eine standardisierte Schnittstelle, dem sog. Host Controller Interface $(\mathrm{HCl})$, erhält ein Host den Steuerungszugriff auf eine in der Regel integrierte Einheit (vgl. Abbildung 2-11). Ein Bluetooth Host ist dabei gewöhnlich eine Softwarekomponente als Bestandteil des Betriebssystems auf dem mobilen Nutzerendgerät. ${ }^{189}$

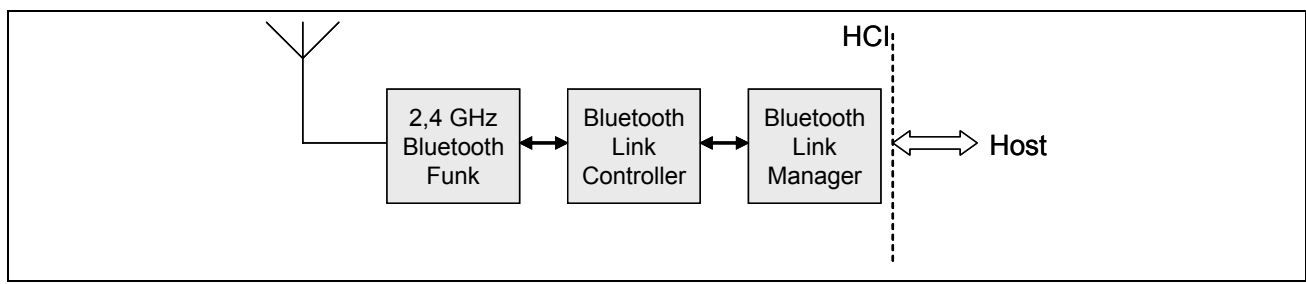

Abbildung 2-11: Aufbau eines Bluetooth Controllers

\subsubsection{Weitverkehrsmobilfunknetze}

Unter dem Namen „Group Spécial Mobile“ (GSM) gründete die Europäische Konferenz der Post- und Fernmeldeverwaltungen (CEPT) ein Konsortium zur Koordinierung und Standardisierung eines künftigen paneuropäischen Mobiltelefonnetzes, später unter demselben Akronym umbenannt in „Global System for

186 Vgl. Bakker/McMichael (2002).

$187 \mathrm{Vgl}$. Diederich/Lerner/Lindemann/Vehlen (2001).

$188 \mathrm{Vgl}$. Schiller (2002); Haartsen (2003); Nokia (2003).

$189 \mathrm{Vgl}$. Kumar/Kline/Thomposen (2004). 
Mobile Communications". ${ }^{190}$ GSM-basierte Mobilfunknetze existieren derzeit in drei Varianten weltweit mit 900, 1.800 und $1.900 \mathrm{MHz}$ und vernetzen derzeit rund 800 Mio. Teilnehmer in 190 Ländern. ${ }^{191}$ Der Medienzugriff mobiler Geräte auf das Funknetz erfolgt in Europa per Zeit- und Frequenzmultiplexverfahren über eine Luftschnittstelle, genannt $U_{\mathrm{M}}$, mit 124 Sendekanälen mit je $200 \mathrm{kHz}$ im Frequenzband zwischen 890-915 MHz (uplink) bzw. 935-960 MHz (downlink). ${ }^{192}$ Im GSM sind drei Dienstklassen vorgesehen:

- Trägerdienste zur Datenübertragung zwischen Netzzugangspunkten; dabei sind sowohl leitungsvermittelnde Verfahren als auch paketvermittelnde Verfahren mit 2.400, 4.800 und 9.600 Bit/s synchron oder 300-1.200 Bit/s asynchron spezifiziert.

- Teledienste zur Sprachübertragung bei ursprünglich $3,1 \mathrm{KHz}$ und für weitere nichtsprachliche Anwendungen wie Fax-, Sprachspeicher- und Mitteilungsdienste (SMS);

- Zusatzdienste wie Rufnummernweiterleitung, -unterdrückung, Anklopfen etc.

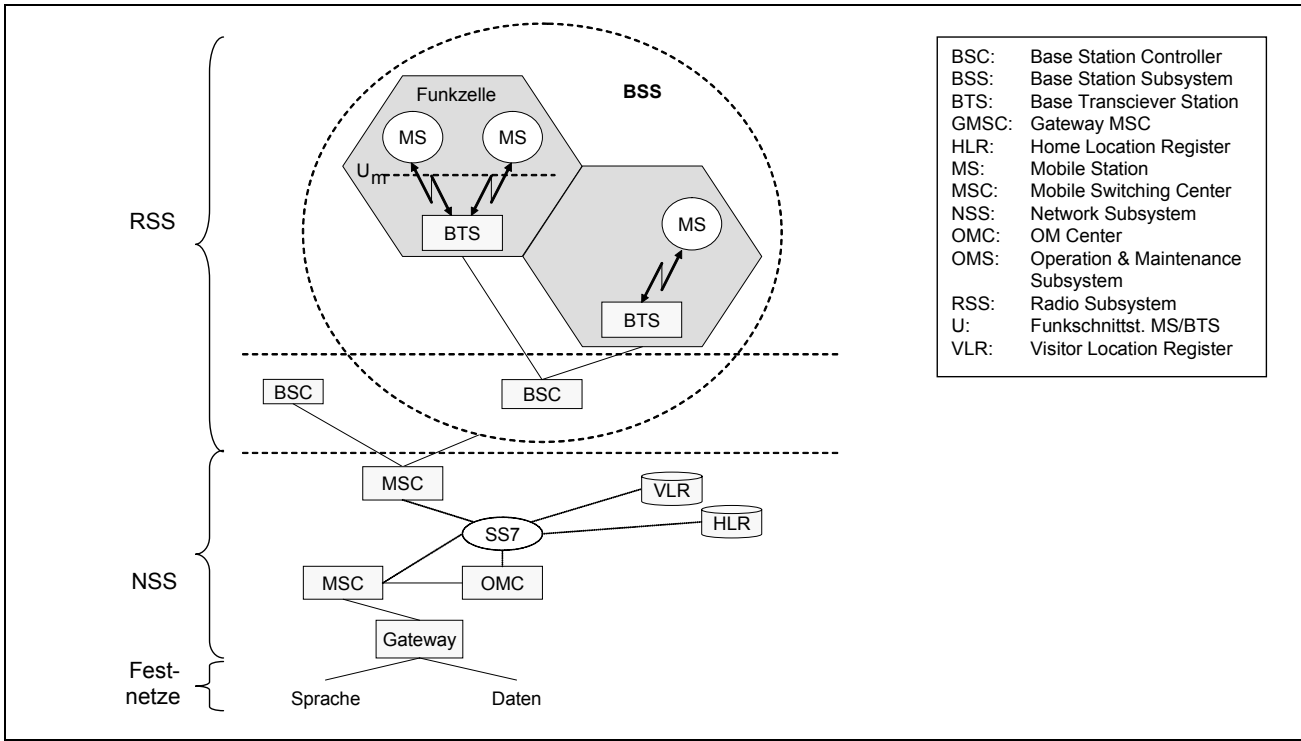

Abbildung 2-12: GSM Netzarchitektur193

Die Architektur eines flächendeckenden GSM-Netzes ist im Vergleich zu lokalen Funknetzvarianten deutlich komplexer (vgl. Abbildung 2-12). Die Netzarchitektur besteht aus drei Subsystemen: dem Funksubsystem (1), dem Vermittlungssubsystem (2) und dem Betriebs- und Wartungssubsystem (3). ${ }^{194}$

\footnotetext{
190 Vgl. Schiller (2003).

$191 \mathrm{Vgl.} \mathrm{GSM} \mathrm{Association} \mathrm{(2003).}$

192 Vgl. Schiller (2002).

193 Vgl. Schiller (2002), S. 377.

194 Vgl. Müller/Eymann/Kreutzer (2003); Schiller (2002).
} 
(1) Das Funksubsystem (Radio Subsystem, RSS), ein flächendeckendes zellulares Funknetz, besteht aus mehreren Basisstation-Subsystemen (Base Station Subsystems, BSS). Ein BSS besitzt mindestens einen Base Station Controller (BSC) der wiederum mehrere Base Transceiver Stations (BTS), vergleichbar mit den Zugangspunkten in WLAN-Netzen, steuert. Ein BTS versorgt in der Regel eine Funkzelle mit einem Zellradius von $100 \mathrm{~m}$ bis maximal $35 \mathrm{~km}$.

(2) Den Hauptbestandteil eines GSM-Netzes bildet das VermittlungsSubsystem (Network Subsystem, NSS), dem sämtliche verwaltungstechnischen Aufgaben obliegen. Kernelemente sind Schaltzentralen (Mobile Switching Center, MSC), die ein Signal im Netz einem authentifizierten Teilnehmer zuordnen. Die Authentifizierung erfolgt auf der Grundlage zweier Datenbanken: im Heimatregister (Home Location Register, HLR) sind alle vertragsspezifischen Daten eines Nutzers sowie sein Aufenthaltsort gespeichert; im Aufenthaltsregister (Visitor Location Register, VLR), üblicherweise einem MSC zugeordnet, sind alle aktuell im Zuständigkeitsbereich des MSC befindlichen Teilnehmer gespeichert.

(3) Die Überwachung und Steuerung von Netzwerk- und Funksubsystem erfolgt durch ein Betriebs- und Wartungssubsystem (Operation and Maintenance System, OMC). Das OMC führt die Registrierung von Mobilstationen und Nutzerberechtigungen durch und erzeugt teilnehmerspezifische Berechtigungsparameter.

Den wesentlichen Kritikpunkt am GSM-Netz bildet die geringe Kanalkapazität bei der Signalübertragung. Mit einer Reihe von Weiterentwicklungen wird versucht, diese Einschränkung zu verringern: ${ }^{195}$ Beim High-Speed Circuit Switched Data (HSCSD)-Verfahren werden mehrere Zeitschlitze im Rahmen des Zeitmultiplex für ein leitungsvermittelndes Signal zusammengefasst. Der General Paket Radio Service (GPRS) ist ein paketvermittelndes Verfahren, das ebenso wie das HSCSD-Verfahren mehrere Zeitschlitze zusammenfasst, Kanalkapazitäten jedoch nur im Falle tatsächlicher Datenübertragung belegt. GPRS erfordert zusätzliche Systemkomponenten im Rahmen des Vermittlungssubsystems und erlaubt eine theoretische Übertragungskapazität von 171,2 KBit/s.

Der Universal Mobile Telecommunication Service (UMTS) repräsentiert im Grunde auch eine evolutionäre Weiterentwicklung von GSM (vgl. Abbildung 2-13). Entwicklungsziel ist eine höhere Übertragungskapazität für Datendienste mit einer minimalen Datenrate von 144 kBit/s in ländlichen Regionen und einer maximalen Datenrate von bis zu $2 \mathrm{Mbit} / \mathrm{s}$ in Ballungszentren. Kern der Fortentwicklung bildet eine erweiterte Luftschnittstelle „Universal Terrestrial Radio Access" (UTRA) zwischen den Funksubsystemen (Radio Network Subsystem, RNS) und dem UMTS-Kernnetz (Core Network, CN). Diese nutzt eine Trägerfrequenz der Bandbreite im Bereich von 1,9-2,1 GHz und setzt eine Breitband-CDMA-Technik mit DSSS als Bandspreizverfahren ein.

195 Vgl. Schiller (2002). 


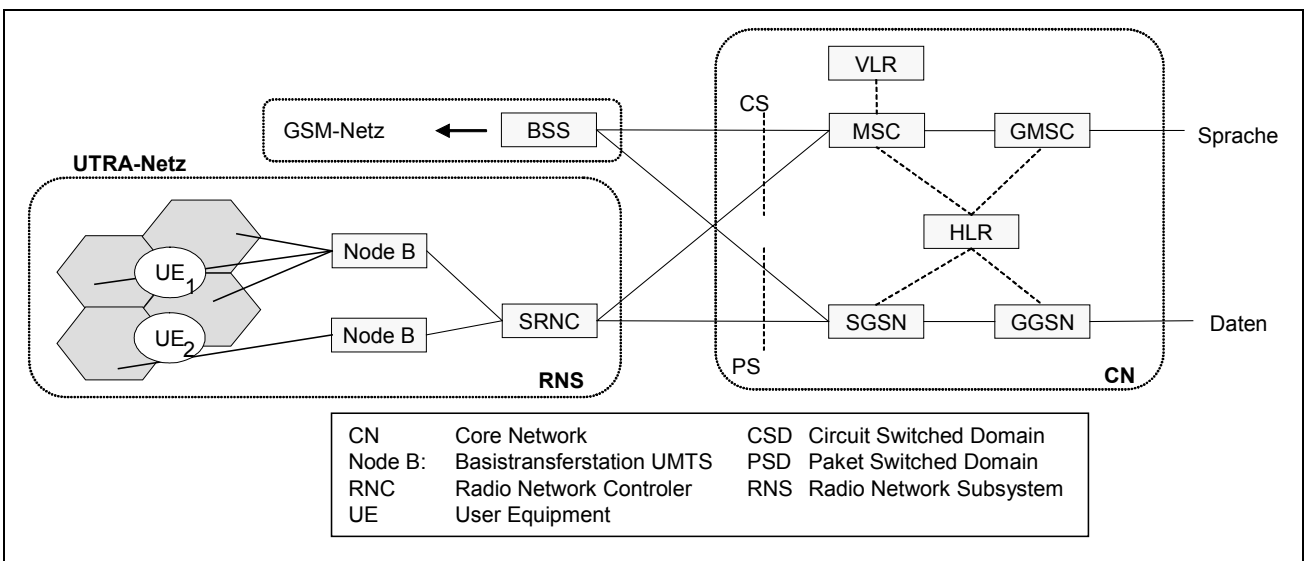

Abbildung 2-13: UMTS-Netzarchitektur

Für die Signalübertragung zwischen Endgeräten (User Equipment UE) und Netzwerk (Radio Network System, RNS) sind bei UMTS zwei Konzepte spezifiziert, die nebeneinander auf einem Zugangsknoten, dem sog. Node-B, möglich sind: ${ }^{196}$ Der UTRA-FDD-Modus für symmetrische Übertragungen in Ballungszentren nutzt eine Kombination aus CDMA zur Teilnehmersignaltrennung und FDMA zur Signalrichtungstrennung zwischen Uplink und Downlink. Der UTRATDD-Modus für kleinere Mobilfunkzellen trennt Uplink und Downlink in einem ungepaarten Spektrum durch Zeitschlitzkombinationen.

Im Kernnetz kann zwischen einem leitungsvermittelnden (Circuit Switched, CS) und einem paketvermittelnden (Paket Switched, PS) Bereich unterschieden werden. Der leitungsvermittelnde Bereich stellt im Wesentlichen auf Grundlage der bereits aus dem GSM-Netz bekannten Komponenten (MSC, VLR, GMSC) eine Verbindung zu klassischen Telefonnetzen her. Im paketvermittelnden Bereich kommen dagegen Komponenten der GPRS-Technik wie Serving GPRS Support Node und Gateway GPRS Support Node (SGSN, GGSN) zum Einsatz.

\subsubsection{Technologien zur Bereitstellung mobiler Informations- dienste}

Die vorgestellten Netztechnologien verkörpern zunächst lediglich Trägerschichten und ermöglichen alleine keinen Informationsaustausch auf Anwendungsebene. Im Folgenden werden daher, darauf aufbauend, Protokollstandards zur Datenübertragung für die Entwicklung von Informationsdiensten vorgestellt. Derzeit existieren drei unterschiedliche Verfahren, die einen Austausch von Inhalten auf Grundlage von Mobilfunknetzen ermöglichen: Dienstformen auf der Grundlage des Wireless Application Protocol (WAP, vgl. Abschnitt 2.3.3.1), die Datenübertragung auf der Grundlage von Kurz- und Multimedianachrichten

196 Vgl. Lehner (2003). 
(SMS und MMS, vgl. Abschnitt 2.3.3.2) und die Datenübertragung durch spezielle Verfahren der Bluetooth-Spezifikation (vgl. Abschnitt 2.3.3.3).

\subsubsection{Wireless Application Protocol (WAP)}

Ebenso wie im leitungsgebundenen Internet kann auch in Mobilfunknetzen die Datenübertragung grundsätzlich auf der Grundlage von HTML und TCP/IP erfolgen. Daher wird die mobile Datenübertragung häufig auch als „mobiles Internet" bezeichnet. Neben dem Problem der geringen Darstellbarkeit von Inhalten aufgrund der kleinen Displays von Mobilfunk-Endgeräten kann jedoch insbesondere der Einsatz von TCP in Mobilfunknetzen mit Problemen verbunden sein und zu unerwünschten Leistungseinbußen führen. ${ }^{197}$ Vor diesem Hintergrund entwickelte ein Herstellerkonsortium von Mobilfunkendgeräten 1997 das Wireless Application Protocol (WAP), mit dem Ziel, die Übertragung von Internetinhalten und Datendiensten für mobile Endgeräte zu verbessern. WAP repräsentiert einen De-facto-Standard, der durch das von Ericsson, Motorola und Nokia ins Leben gerufene WAP-Forum überwacht wird.

In dem vom WAP-Forum definierten Interaktionsmodell für die Entwicklung WAP-gestützter Dienste für mobile Endgeräte fungiert WAP als Kommunikationsplattform zwischen dem mobilen Endgerät und einem WAP-Gateway. Das Gateway, ein spezieller Server ähnlich einem Proxy-Server, übersetzt WAPAnfragen in HTTP-Nachrichten und leitet diese an Internetserver weiter, die die vom Nutzer gewünschten Inhalte vorhalten (vgl. Abbildung 2-14). ${ }^{198}$

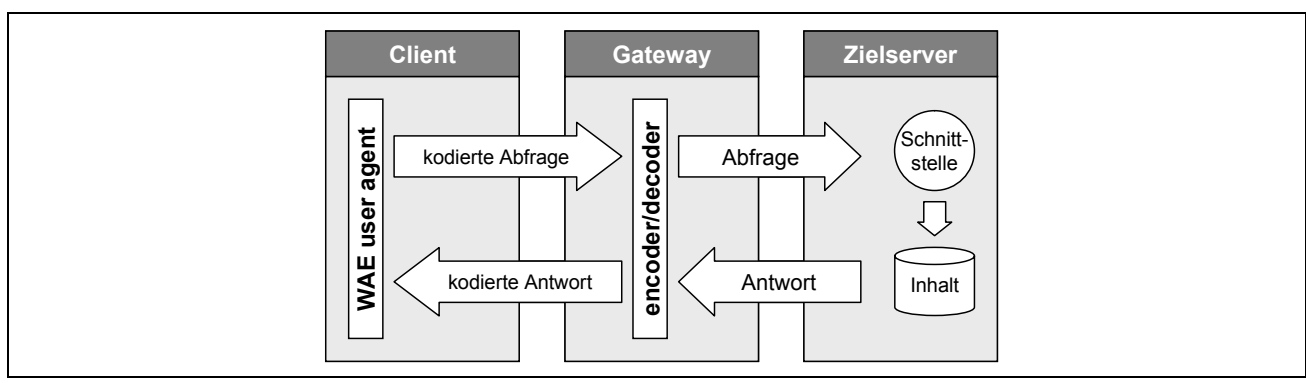

Abbildung 2-14: Das WAP-Interaktionsmodell

Unter dem Begriff WAP wird eine Reihe verschiedener Protokolle zusammengefasst, die entlang der Schichten im ISO/OSI Modell verschiedene Aufgaben für die Datenübertragung von oder zu mobilen Endgeräten unterstützen. Dazu gehören sowohl die Protokolle zur Datenübertragung zwischen WAP-Gateway und Endgerät als auch bspw. die Auszeichnungssprache WML (vgl. Abbildung 2-15): ${ }^{199}$ Das Wireless Datagramm Protocol (WDP) sorgt für eine transparente Übertragung von Datenbits unabhängig vom verwendeten darunter liegenden Trägerdienst. Das Wireless Transaction Protocol (WTP) ermöglicht interaktive

\footnotetext{
197 Vgl. Lehner (2003).

$198 \mathrm{Vgl}$. Deitel/Deitel/Nieto/Steinbuhler (2002).

199 Vgl. Lehner (2003).
} 
Browsing-Sessions durch Gewährleistung zuverlässiger Transaktionen zwischen Client und Server. Das Wireless Session Protocol (WSP) ermöglicht zwei Sitzungsdienste, einen verbindungsorientierten und einen verbindungslosen. Zusätzlich stellt WSP eine kompakte Semantik für HTTP 1.1 bereit. Die Wireless Application Environment (WAE) kombiniert Mobilfunk- und WebTechnologien. Konkret wird zwischen Nutzer Agenten (User Agents), wie dem Browser, zur Interpretation übermittelter Inhalte oder der Software zur Steuerung von Telefonbuchfunktionen mit WAP, und Diensten bspw. auf Grundlage von WML oder WMLScript unterschieden. In Abbildung 2-15 werden die Schichten von WAP anhand des ISO/OSI-Modells dem TCP/IP-Modell gegenüber gestellt.

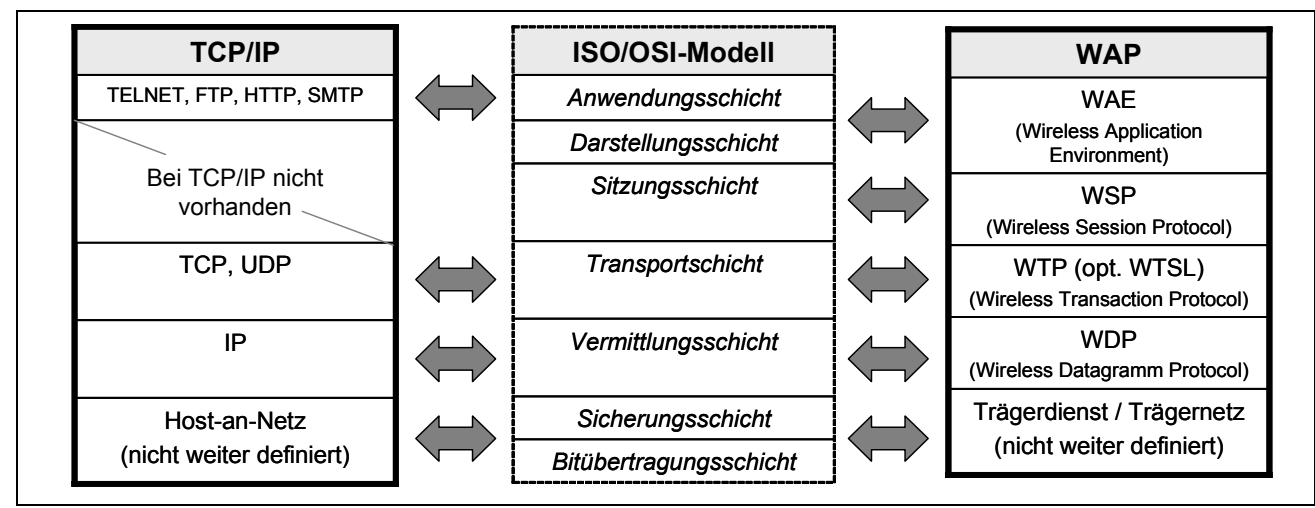

Abbildung 2-15: WAP Protokollstapel vs. TCP/IP

\subsubsection{Short Message Service (SMS) und Multimedia Messaging Service (MMS)}

Der Short Message Service (SMS) ist ein üblicherweise als Basisdienst von GSM-Netzen implementiertes System, das den Austausch von kurzen Textnachrichten von bis zu 160 Zeichen zwischen Mobilfunknutzern erlaubt. Seitdem erstmalig im Jahr 1992 eine Kurznachricht in europäischen GSM-Netzen übertragen wurde, verzeichnen SMS-gestützte Dienste ein kontinuierliches Nachfragewachstum. Während bereits 2001 weltweit 102,9 Mrd. Nachrichten versandt wurden, stieg die Zahl von SMS-Nachrichten auf $168 \mathrm{Mrd}$. im Jahr $2003{ }^{200}$ Die Spezifikation des SMS wurde ursprünglich durch das European Telecommunications Standards Institute (ETSI) entwickelt, später vom Standardisierungsgremium für Mobilfunktechnologien der dritten Generation, dem „3rd Generation Partnership Project“ (3GPP), weitergeführt. ${ }^{201}$

Der SMS wurde ursprünglich für den Bereich der Mensch-zu-MenschKommunikation entwickelt, um Kurznachrichten zwischen zwei Mobilfunkteilnehmern auszutauschen. Dabei kann eine Nachricht immer nur genau von ei-

200 Vgl. Le Bodic (2003), S. 36.

$201 \mathrm{Vgl}$. Le Bodic (2003), S. 35. 
nem Sender an genau einen Empfänger übertragen werden. Neben einer solchen textgestützten Kommunikation zwischen Mobilfunkteilnehmern kann der SMS auch zur Inhaltevermittlung im Rahmen von Maschine-zu-MenschKommunikation eingesetzt werden. ${ }^{202}$ Dabei lassen sich zwei Anwendungsformen unterscheiden: SMS-gestützte Nachrichten können dazu verwendet werden, einem Mobilfunkteilnehmer anzuzeigen, dass eine Sprach-, Fax- oder Emailmitteilung auf inn wartet. Die am weitesten verbreitete Anwendung von SMS bildet die Übertragung von kommerziellen Kurzinhalten, wie bspw. Nachrichten oder Werbung. Üblicherweise muss ein Rezipient einen solchen Dienst zuvor anfordern. Vor dem Hintergrund der beschränkten Übertragungskapazitäten einer SMS-Nachricht kann eine solche Nachricht auch dazu verwendet werden, einen Inhalt nicht direkt per SMS zu übertragen, sondern mit der Nachricht lediglich eine Adresse zu übermitteln, von der aus Inhalte entweder per WAP betrachtet oder auf das Endgerät herunter geladen werden können.

Neben den beschriebenen Komponenten einer GSM-Netzarchitektur erfordert der Betrieb eines SMS-Systems drei zusätzliche Komponenten: ${ }^{203}$

- Die Short Message Entity (SME) ist üblicherweise eine Softwarekomponente im Betriebssystem des mobilen Endgeräts, das Kurznachrichten versendet, empfängt und anzeigt. Im Falle von Informationsdiensten kann ein SME auch ein Server sein, der mit dem SMSC in Verbindung steht. Ein solcher Server wird auch als Externe SME (ESME) bezeichnet.

- Das Short Message Centre (SMSC) ist für das Speichern und Weiterversenden von Kurznachrichten im GSM-Netz verantwortlich. Kann eine Nachricht vorübergehend nicht an einen Empfänger versendet werden, verzögert das SMSC die Auslieferung. Theoretisch kann ein einziges SMSC den Kurznachrichtenversand mehrerer Mobilfunknetze organisieren.

- Gateways, bspw. zu Email- oder Faxsystemen, verbinden das SMSC mit alternativen Kommunikationssystemen wie dem Internet.

SMS-gestützte Kurznachrichten werden üblicherweise von mobilen Endgeräten an das SMSC versendet (vgl. Abbildung 2-16).

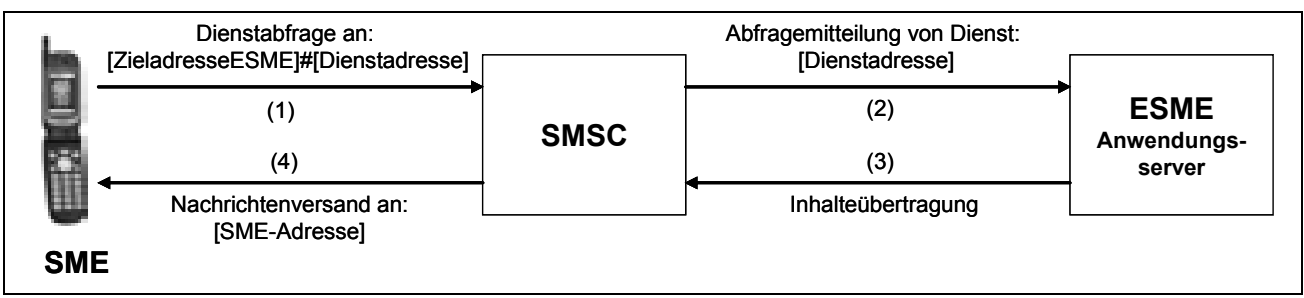

Abbildung 2-16: Ablauf einer SMS-gestützten Inhalteabfrage

Die Adressierung des Empfängers erfolgt über die Teilnehmerrufnummer des Empfängers. Im Falle der Anforderung eines SMS-gestützten Informationsdienstes kann die Empfängeradressierung mit Sub-Adressinformationen zur Bestimmung des abzurufenden Inhalts versehen werden (1). Das SMSC über-

202 Vgl. Schiller (2003), S. $132 \mathrm{f}$.

203 Vgl. Le Bodic (2003), S. 40 f. 
trägt die Nachrichtenanfrage an den ESME-Server (2). Der ESME-Server liefert den angeforderten Inhalt an das SMSC zurück (3), das diesen an den Absender der Abfrage versendet (4).

Vor dem Hintergrund der deutlichen Formatbeschränkung von SMSgestützten Kurznachrichten für die kommerzielle Inhalteverbreitung, der Verfügbarkeit größerer Übertragungsbandbreiten in den Mobilfunknetzen der Generationen 2.5 und 3 sowie einer erwarteten Nachfrage im Bereich der drahtlosen Übertragung von multimedialen Inhalten spezifizierten 3GPP und das WAP-Forum gemeinsam die technischen Grundlagen eines Multimedia Messaging Service (MMS). ${ }^{204}$ Der MMS repräsentiert eine evolutionäre Weiterentwicklung von SMS und WAP zur nachrichtengestützten Übertragung multimedialer Inhalte wie Texte, Bilder, Musik und Videos. Die Anwendungsszenarien von MMS gleichen jenen von SMS, mit dem Unterschied des breiteren Formatspektrums übertragbarer Inhalte. ${ }^{205}$ Die kommerzielle Einführung von MMSgestützten Diensten in Deutschland erfolgte im April 2002. ${ }^{206}$

Gegenüber bestehenden GSM/SMS-Systemarchitekturen erfordert die Ausführung von MMS-gestützten Diensten drei weitere Systemkomponenten: ${ }^{207}$ Der MMS User-Agent ist eine Software, die auf dem mobilen Endgerät ausgeführt wird und für den Versand, das Empfangen und die Interpretation von MMS-Nachrichten verantwortlich ist. Das MMS Relay sorgt für die Übertragung von MMS-Nachrichten innerhalb des jeweiligen Trägernetzes. Der MMS Server speichert auszuliefernde Nachrichten. MMS Relay und Server werden vor dem Hintergrund des Vokabulars von der SMS-Systemarchitektur auch gemeinsam als MMS Center (MMSC) bezeichnet. Das MMSC unterstützt die Übertragung von MMS-Nachrichten durch zwei Verfahren: Auf der Grundlage des sog. "Batch Mode" werden alle zur Nachricht gehörenden Inhalte vollständig an das mobile Endgerät des Nachrichtenempfängers übertragen. Der sog. „Streaming Mode" überträgt dagegen nur Nachrichtenteile, die vom Empfänger editiert und dann weiter versendet werden können.

Eine MMS-Architektur erlaubt die Übertragung von MMS-Nachrichten auf zwei unterschiedlichen protokollarischen Wegen: ${ }^{208}$ Bei der Übertragung von MMS-Nachrichten auf der Grundlage der WAP MMS 1.0 Spezifikation wird eine MMS-Nachricht zwischen dem mobilen Endgerät und dem MMSC zunächst per WAP an ein WAP-Gateway versandt, das die empfangenen Inhalte als HTTPNachricht umkodiert und an das MMSC weiterreicht. Die Spezifikation WAP MMS 1.1 erlaubt den Nachrichtenversand zwischen dem mobilen Endgerät und dem MMSC dagegen direkt auf der Grundlage von HTTP.

\footnotetext{
204 Vgl. Le Bodic (2003), S. $197 \mathrm{f}$.

$205 \mathrm{Vgl}$. Guthery/Cronin (2003), S. 7-13.

206 Vgl. Le Bodic (2003), S. 199.

207 Vgl. Le Bodic (2003), S. 200 f. und Guthery/Cronin (2003), 20 f.

208 Vgl. Le Bodic (2003), S. 204 und 206.
} 


\subsubsection{Datenvermittlung in Bluetooth-Netzen}

Typischerweise werden die lizenzierten Bereiche des Funkfrequenzspektrums zur Weitstreckenkommunikation genutzt. Zur Nahverkehrskommunikation wird dagegen entweder auf Infrarot (IrDA) oder das lizenzfreie Funkfrequenzspektrum zurückgegriffen. ${ }^{209}$ Weitverkehrsnetze wie GSM oder UMTS zeichnen sich dabei zwar sowohl für Sprach- als auch Datenübertragung durch eine hohe Dienstqualität, gleichzeitig jedoch aufgrund hoher Investitionskosten für Infrastruktur und Lizenzerwerb durch hohe Übertragungspreise aus. Technologien für Nahverkehrsnetze besitzen dagegen entweder eine sehr niedrige Leistungskapazität (IrDA) oder ein auf den Bereich des Zugriffs auf Datennetze (WLAN) eingeschränktes Anwendungsspektrum.

Zielsetzung der Entwicklergruppe von Bluetooth war vor dem Hintergrund dieser Einschränkungen die Gewährleistung einer kostengünstigen, universellen Verbindung zwischen portablen Endgeräten sowohl mit Kommunikationsfunktionen als auch mit Rechenkapazitäten. ${ }^{210}$ Anders als bspw. WLAN oder UMTS definiert die Bluetooth-Spezifikation ein vollständiges System von der Funkübertragungs- bis zur Anwendungsschicht. In der Spezifikation werden zwei Ebenen unterschieden: die technische Kernspezifikation mit der Beschreibung des Protokollstapels (1); die Anwendungsebene mit autorisierten Profilen für spezifizierte Anwendungsfälle (2).

(1) In der Architektur des Bluetooth-Protokollstapels werden zwei Komponenten unterschieden: Der Bluetooth-Host und der bereits im voranstehenden Kapitel vorgestellte Bluetooth-Controller. Dem Bluetooth-Host, üblicherweise eine Softwarekomponente im Betriebssystem eines Nutzerendgeräts, stehen im Kern fünf Protokolle zur Verfügung, die eine Integration von Bluetooth Verbindungen mit anderen Spezifikationen ermöglichen. Das Logical Link Control and Adaption Protocol (L2CAP) ermöglicht einen Mehrfachzugriff verschiedener logischer Verbindungen der darüber liegenden Schichten auf das Funkfrequenzspektrum. Die Identifikation von verfügbaren Bluetooth Diensten erfolgt durch das Service Discovery Protocol (SDP). Bestehende Datenverbindungen wie Punkt-zu-Punkt Verbindungen oder WAP-Dienste werden entweder über RFCOMM oder das Bluetooth Encapsulating Protocol (BNEP) übertragen. RFCOMM ist ein einfaches Transportprotokoll, das die Funktionsweise eines seriellen Ports emuliert. BNEP kapselt Pakete aus bestehenden Datenverbindungen und versendet diese direkt über das L2CAP. Das Object Exchange Protocol (OBEX) wurde aus der Infrarot-Technologie zur Übertragung von Dokumenten wie Visitenkarten (vCard) adaptiert.

(2) Bluetooth Profile repräsentieren Nutzungsmodelle für BluetoothTechnologien mit spezifizierten Interoperabilitäten für vordefinierte Funktionen. Bluetooth Profile unterliegen einem strengen Zertifizierungsverfahren (Qualification) durch die SIG. Es wird zwischen allgemeinen Transportprofilen und nutzungsmodellorientierten Anwendungsprofilen unterschieden. Zu den allgemeinen

$209 \mathrm{Vgl}$. Kumar/Kline/Thomposen (2004).

$210 \mathrm{Vgl}$. Haartsen/Allen/Inouye/Joeressen/Naghshineh (1998). 
Profilen gehören bspw. das Generic Access Profile (GAP) zur Unterstützung von Geräteidentifikation, Linkmanagement und Sicherheit, das Serial Port Profile (SPP) zur Emulierung von seriellen Kabelverbindungen auf der Basis von RFCOMM oder das Generic Object Exchange Profile für Austauschfunktionen wie Synchronisation oder Dateitransfer. Nutzungsmodellorientierte Profile umfassen zum einen typische Szenarien für einen Kabelersatz in der Gerätekommunikation im Kurzstreckenbereich wie die Verwendung des Mobiltelefons als Modem, als Fax, die Verbindung zu einem Headset oder als Netzwerkverbindung in ein LAN. Zum anderen werden Profile für den Austausch von Dokumenten, für Push-Dienste und für die Synchronisation bspw. mit Anwendungen auf Computerterminals angeboten. Zusätzlich erlauben Bluetooth Profile die Verwendung des Bluetooth-fähigen Mobiltelefons, neben dessen typischem Einsatz im Mobilfunknetz, als Funkgerät innerhalb bspw. eines betrieblichen Funknetzes oder als schnurloses Telefon in Verbindung mit einem Festnetztelefonanschluss.

\subsubsection{Anwendungsfelder für mobile Dienste}

Das zentrale Bewertungsproblem im Rahmen der kommerziellen Vermarktung der Mobilfunktechnik entspringt der Fragestellung, ob mobile Dienste lediglich eine evolutionäre Entwicklung des Electronic Business darstellen oder revolutionäres Innovationspotenzial bspw. für die innerbetriebliche Organisation oder zur Entwicklung eines neuen Wirtschaftszweigs besitzen. ${ }^{211}$ Vor dem Hintergrund dieser Bewertungsproblematik werden in Abschnitt 2.3.4.1 die Einsatzvarianten der beschriebenen Mobilfunktechniken im Überblick zusammengefasst. Darauf aufbauend werden in Abschnitt 2.3.4.2 plausible Einsatzmöglichkeiten mobiler Dienste aus betriebswirtschaftlicher Sicht benannt.

\subsubsection{Einsatzvarianten mobiler Dienste}

Zu den voranstehenden Erläuterungen der Mobilfunktechnik können zusammenfassend drei generelle Einsatzvarianten mobiler Dienste unterschieden werden:

(1) Die WLAN-Technik ermöglicht in Erweiterung zum leitungsgebundenen Ethernet einen portablen Zugang zu Datennetzen auf der Grundlage von TCP/IP über eine Luftschnittstelle. Neben dem Vorteil der räumlichen Flexibilität des Datenzugriffs können jedoch keinerlei zusätzliche Nutzungsvorteile wie PushFunktionen oder Geräteverbindungen und dadurch keine grundsätzlich neuen Dienstformen realisiert werden.

(2) Die Bluetooth-Technologie wurde - wie angesprochen - ursprünglich als Kabelersatz für die Verbindung räumlich naher Geräte entwickelt. Die geringe Größe und der geringe Stromverbrauch von Bluetooth-Systemen machen den Einsatz vor allem in alltäglichen Situationen als ubiquitäre Vernetzungstechnik plausibel. Dabei sind wiederum zwei Nutzungsformen vorstellbar: zum einen die Vernetzung zwischen mobilen Nutzerendgeräten als Alternative zu beste-

211 Vgl. Zobel (2001). 
henden Infrastrukturnetzen sowohl für Daten- als auch Sprachkommunikation; zum anderen als Grundlage einer einfachen und schnellen Verbindung zwischen Nutzerendgeräten und stationären Systemen bspw. im Rahmen von Umgebungsinformations- oder Warenabrechnungssystemen.

(3) Bereits die Standards der Generation 2.5 (bspw. GSM/GRPS) im lizenzpflichtigen Mobilfunk ermöglichen eine zuverlässige Sprachkommunikation und zumindest akzeptable Kapazitäten für den Datenverkehr. Problematisch sind jedoch die in der Regel hohen Verbindungskosten, weshalb diese Mobilfunktechnik im Bereich des Datenverkehrs zumindest mittelfristig keine echte Alternative zu leitungsgebundenen Netzen darstellt. Anstelle eines portablen Datennetzzugangs erscheint hier das Szenario spezieller Informationsdienste für die Bedürfnisse mobiler Nutzer plausibel, die gegenüber der stationären Netzwerknutzung einen zusätzlichen Mehrwert produzieren. Als Mehrwertfaktoren werden unter anderem die einfache, in GSM bspw. über die Aufenthaltsregister (HLR/VLR) mögliche Teilnehmerlokalisierung oder die über die SIM-Karte der Mobilfunkgeräte jederzeit eindeutige Personenidentifikation eines Teilnehmers diskutiert. In Verbindung mit der in der WAP-Spezifikation verankerten Möglichkeit von Push-Diensten kann dadurch solche Dienste realisiert werden, die die aktuelle Aufenthaltssituation eines Teilnehmers erkennen und automatisch ein kontextadaptives Angebot für den Nutzer bereitstellen. ${ }^{212}$

Die Zusammenfassung zeigt, dass die jeweiligen Formen der Mobilfunktechnik spezifische Anwendungsfelder für Datendienste plausibel erscheinen lassen. Insofern kann datengestützten mobilen Diensten zweifelsohne ein signifikantes Marktpotenzial beschieden werden. Dennoch scheint eine Wiederholung der revolutionären Veränderungen durch die Internettechnologien aufgrund von Mobilfunktechnologien eher unwahrscheinlich. Mobilfunktechniken können einen zusätzlichen Nutzen gegenüber leitungsgebundenen Netzen stiften, und es können neue Dienstformen unter Ausnutzung bestehender Mehrwertfaktoren der Mobilfunktechnik entstehen. Diese Vorteile sind jedoch weitgehend effizienzbezogener Natur, etwa durch die bessere Integrationsfähigkeit verteilter Geräte und System oder einen bequemeren Datenzugriff. Um plausible und ökonomisch Erfolg versprechende Anwendungsfelder mobiler Dienste identifizieren zu können, ist zu prüfen, in welcher Form solche Effizienzpotenziale im Rahmen der betrieblichen Wertschöpfung ausgeschöpft werden können. Dies ist Gegenstand des folgenden Abschnitts.

\subsubsection{Betriebliche Anwendungsfelder mobiler Dienste}

Die Betriebswirtschaftslehre befasst sich mit Mobilität traditionell im Rahmen betrieblicher Leistungserstellungs- und Absatzprozesse, in denen eine räumliche Unabhängigkeit von Aufgabenträgern oder Abnehmern gefordert ist. Ein sinnvolles Anwendungsgebiet aus betrieblicher Sicht existiert daher überall dort, wo eine räumliche Unabhängigkeit unterstützt werden muss. Aus ökonomischer Sicht lassen sich grundsätzlich drei Ansatzpunkte für eine Untersu-

212 Vgl. Abschnitt 5.4.1. 
chung von Potenzialen räumlicher Unabhängigkeit identifizieren (vgl. Abbildung 2-17): ${ }^{213}$ Mobilität zur Unterstützung und Optimierung der betrieblichen Wertschöpfung und Leistungserstellung (1); Mobilität im Rahmen der Koordination und Abwicklung des Leistungstransfers einer Markttransaktion zwischen Anbieter und Nachfragern (2) und Informationsprodukte bzw. Informationsdienstleistung im Sinne ökonomischer Güter, die speziell für mobile Nutzer bereitgestellt werden (3).

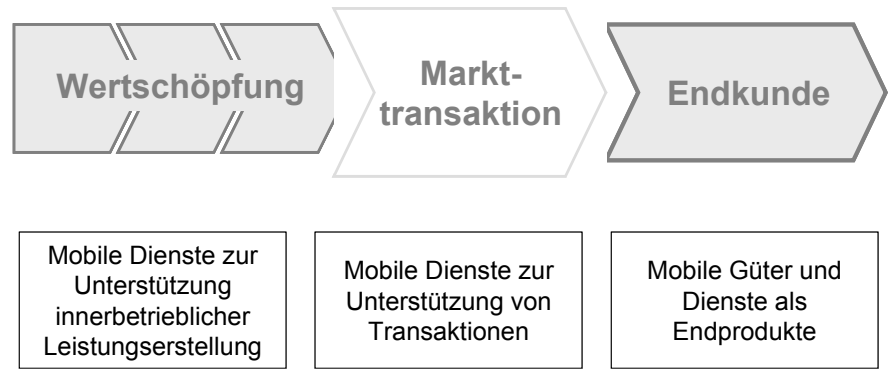

Abbildung 2-17: Anwendungsmöglichkeiten mobiler Dienste aus betriebswirtschaftlicher Sicht

(1) Im Rahmen der Leistungserstellung kann Mobilität zur Unterstützung und Optimierung der innerbetrieblichen und unternehmensübergreifenden Wertschöpfung dienen. Dabei sollen vor allem Effizienz und Effektivität von Prozessen gesteigert werden. Dieses Ziel wird durch eine engere Verzahnung mobiler, wertschöpfungsbezogener Aufgabenträger mit der IT-Infrastruktur während der jeweiligen mobilen Aufgabendurchführung gewährleistet. Aufgabenträger sind entweder Mitarbeiter des Unternehmens oder Maschinen. Eine mobile Unterstützung personeller Aufgabenträger wird üblicherweise dort realisiert, wo wertschöpfungsrelevante Aufgaben in einem durch Mobilität geprägten Umfeld zu erbringen sind. Beispiele bilden etwa die Durchführung von Wartungsprozessen durch Servicetechniker oder die Vertriebskoordination von Außendienstmitarbeitern. Zusätzlich zur direkten Unterstützung personeller Aufgabenträger bestehen Möglichkeiten zu Effizienz- und Effektivitätssteigerungen durch die Vernetzung physischer Objekte der realen Welt und betrieblicher Informationssysteme. Im Idealfall kann der Mensch als bisheriger Mediator an der Schnittstelle (Tastatureingabe, Barcodescanner o. Ä.) entfallen und durch die direkte Maschine-Maschine-Kommunikation ersetzt werden. ${ }^{214}$ Ausgehend von der weiter zunehmenden Miniaturisierung der Mobilfunktechnik entsteht ein Vernetzungspotenzial, mit dem dann bislang ausschließlich passive Produktionsressourcen zu aktiven maschinellen und ggf. mobilen Aufgabenträgern in physischen Wertschöpfungsprozessen werden. Dadurch können bspw. im Rahmen der Warenlogistikkette leistungsfähige Mini-Chip-Technologien mit der so genannten Radio Frequency Identification (RFID) kombiniert werden, wodurch damit ausgestattete Materialflusskomponenten zu „intelligenten“ mobilen und

\footnotetext{
$213 \mathrm{Vgl}$. Hess et al. (2005).

214 Vgl. Fleisch (2001), S. $273 \mathrm{ff}$.
} 
dadurch teilautonom agierenden Objekten (so genannten "Smart Devices“) werden.

(2) Der zweite Einsatzbereich mobiler Dienste im betriebswirtschaftlichen Kontext umfasst die Unterstützung von Markttransaktionen, d.h. die Koordination und ggf. die Durchführung des Leistungsaustausches eines Sachgutes oder einer Dienstleistung. Transaktionsprozesse lassen sich allgemein in die Phasen Anbahnung, Vereinbarung und Abwicklung sowie die nachgelagerte AfterSales-Phase unterteilen. Zu den Bestandteilen einer Markttransaktion zählen vor diesem Hintergrund alle hierfür relevanten informatorischen sowie koordinativen Aktivitäten. Mobile Dienste eröffnen hierbei das Potenzial der Senkung von Transaktionskosten, die bei der Durchführung des Leistungsaustauschs hinsichtlich der Anbahnungs-, Vereinbarungs-, Abwicklungs-, Kontroll- und Anpassungsprozesse anfallen: ${ }^{215} \mathrm{Im}$ Rahmen der Vertragsanbahnung können z. B. mobile Preisvergleichsdienste oder ortsabhängige Anbieterverzeichnisse zum Einsatz kommen. In der Vereinbarungsphase und der Abwicklungsphase können bspw. mobile Zahlsysteme verwendet werden, die im Falle digitaler Produkte eine Vereinbarung und Abwicklung über das mobile Endgerät ohne Medienbruch erlauben. In der After-Sales-Phase können mobile Dienste genutzt werden, um durch Feedbackmechanismen eine gezieltere Kundenansprache zu ermöglichen und Aktivierungsanreize für weiterführende Transaktionen gezielt einzusteuern.

(3) Der dritte Anwendungsbereich im betriebswirtschaftlichen Kontext umfasst mobile Anwendungen als ökonomische Güter. Eine Differenzierung der Anwendungsfelder mobiler Dienste in diesem Anwendungsbereich wird häufig aus drei Perspektiven vorgenommen:

- Aus produktorientierter Sicht kann zwischen vier Anwendungsklassen unterschieden werden: informationsorientierte Dienste wie Fahrpläne, Börseninformationen oder Sportnachrichten; applikationsorientierte Dienste wie Computerspiele, Übersetzungsdienste oder Terminplaner; transaktionsorientierte Dienste wie Reservierungssysteme, Auktionen oder Tauschbörsen; kommunikationsorientierte Dienste wie Email, Diskussionsforen oder Chats. ${ }^{216}$

- Aus Sicht einer Anwendertypologie kann bspw. zwischen Geschäftsinteressen (Business), privaten Endkunden (Consumer), Betriebsmitarbeitern (Employee) und der Unternehmensleitung (Administration) unterschieden werden. ${ }^{217}$ Durch wechselseitige Kombination von Kommunikationsarrangements zwischen den verschiedenen Anwendertypen lassen sich 16 Anwendungscluster abgrenzen (bspw. B2B, B2C, etc.).

215 Vgl. Picot/Reichwald/Wigand (2001), S. 50.

${ }^{216}$ Vgl. Schumann (2002) S. 7 und Hess/Rawolle (2001). Informationsgüter, entsprechend der in Abschnitt 2.1 vorgenommenen Begriffsdefinition, bilden im Rahmen dieser Klassifikation in erster Linie informationsorientierte mobile Dienste.

217 Vgl. Diederich/Lerner/Lindemann/Vehlen (2001), S. 232. 
- Aus der Sicht technologischer Spezifika des Mobilfunks wird häufig eine Reihe von Anwendungsfeldern, so genannte Technologiewertbeiträge, ${ }^{218}$ abgeleitet. Im Mittelpunkt dieser Spezifika stehen in der Regel zwei Aspekte: die allgegenwärtige und vor allem ortsunabhängige Verfügbarkeit mobiler Endgeräte und die eindeutige Identifizierbarkeit mobiler Anwender. Während die Ortsunabhängigkeit ein definitionsgemäßes Kriterium von Anwendungen und Diensten jeglicher Form auf der Grundlage von Mobilfunknetzen darstellt, bildet die Identifizierbarkeit ein spezielles Kriterium für Anwendungen in flächendeckenden Kommunikationsnetzen des kommerziellen Mobilfunks wie GSM oder UMTS. Petersmann und Nicolai identifizieren im B2C-Bereich aus den ortsunabhängigen bzw. lokalisierbaren Eigenschaften mobiler Technologien jeweils 2 Anwendungsfelder - ortsbezogene und zeitkritische Dienste - im Spektrum der vier Produktklassen Information, Kommunikation, Selektion und Transaktion des Mobile Business (vgl. Abbildung 2-18). ${ }^{219}$ Innerhalb der einzelnen Matrixfelder kann wiederum zwischen klassischen Pull- (also Selbstbedienungs-)Angeboten des Nutzers und Push-Angeboten unterschieden werden. In der entstehenden 2x8 Feldertafel bestimmen die Autoren dann derzeit bereits verfügbare (1)-3) und Erfolg versprechende, jedoch noch nicht realisierte Anwendungsformen (4)-8).

\begin{tabular}{|c|c|c|c|c|c|}
\hline & Information & Kommunikation & Selektion & Transaktion & (1) w ftd com/wireless \\
\hline 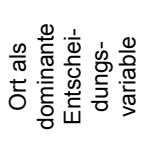 & $\begin{array}{r}\text { (4) } \\
\text { PULL }\end{array}$ & $\begin{array}{l}\text { PUSH } \\
\text { (2) } \\
\\
\text { PULL }\end{array}$ & PUSH & $\begin{array}{ll}\text { PUSH } & \\
& \\
& \text { (6) } \\
& \text { PULL }\end{array}$ & $\begin{array}{l}\text { (2) www.wapaparty.de } \\
\text { (3) www.jamba.de } \\
\text { (4) EC-Automatensuche } \\
\text { (5) Mobiles Finanzportal } \\
\text { (6) Zahlungsmittelfunktion }\end{array}$ \\
\hline 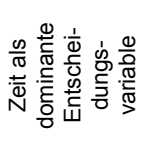 & $\begin{array}{ll}\text { PUSH } & \\
& \\
& \text { (1) } \\
& \text { PULL }\end{array}$ & PUSH & $\begin{array}{l}\text { PUSH } \\
\text { (3) } \\
\\
\\
\text { (5) } \\
\\
\end{array}$ & $\begin{array}{ll}\text { PUSH } & \\
\text { (7) } & \\
& \\
& 8 \\
& \text { PULL }\end{array}$ & (8) Wertpapier-Order \\
\hline
\end{tabular}

Abbildung 2-18: Mobile Mehrwertdienste

Die betrieblichen Anwendungsfelder mobiler Dienste werden übergreifend häufig unter dem Begriff des "Mobile Business" zusammengefasst. Hinter diesem Begriff verbirgt sich allerdings keine einheitlich verwendete Definition. Im Gegenteil ist eine breite Vielfalt an Definitionen - augenmerklich um das Jahr 2001, in der Hochzeit der Mobilfunkeuphorie im Zusammenhang mit den Lizenzversteigerungen für die geplanten UMTS-Netze in Europa - entstanden. Abbildung 2-19 zeigt einige Beispiele der in dieser Zeit veröffentlichten Begriffsdefinitionen.

$218 \mathrm{Vgl}$. Zobel (2001), S. 63.

219 Vgl. Nicolai/Petersmann (2001), S. 17. 


\begin{tabular}{|ll|}
\hline Hartmann/Dirksen (2001), S. 16: & $\begin{array}{l}\text { Unter Mobile Business versteht man Geschäftsmodelle, bei denen Geschäftsprozesse wie z.B. Daten- und } \\
\text { Informationsaustausch durch die Integration von mobilen Endgeräten wie Handys oder PDAs unabhängig } \\
\text { von Ort und Zeit abgefragt, beeinflusst oder sogar gesteuert werden können. }\end{array}$ \\
Reichwald et al. (2002), S. 8: & $\begin{array}{l}\text { Mobile Business die Gesamtheit der über ortsflexible datenbasierte und interaktive Informations- und } \\
\text { Kommunikationstechnologien (z.B. Mobiltelefone, PDAs) abgewickelten Geschäftsprozesse. }\end{array}$ \\
Scheer et al. (2001), S. 30: & $\begin{array}{l}\text { Unter Mobile Business wird (...) die kommerzielle Nutzung von nicht drahtgebundenen Diensten zur } \\
\text { Unterstützung von Geschäftsprozessen in Unternehmen, zwischen Unternehmen und deren Lieferanten } \\
\text { sowie an der Schnittstelle zwischen Unternehmen und Kunden verstanden. }\end{array}$ \\
Steimer (2001), S. 137: & $\begin{array}{l}\text { Mobile Business umfasst die ortsgebundene (mobile) Beschaffung, Verarbeitung und Bereitstellung von } \\
\text { Informationen aller Art, zur Abwicklung von Geschäfts- und Kommunikationsvorgängen unter Einsatz } \\
\text { mobiler Endgeräte und Nutzung geeigneter Dienste und Infrastrukturen. }\end{array}$ \\
Wirtz (2001), S. 45: & $\begin{array}{l}\text { Unter dem Begriff Mobile Business wird die Anbahnung sowie die teilweise respektive vollständige } \\
\text { Unterstützung, Abwicklung und Aufrechterhaltung von Leistungsaustauschprozessen mittels elektronischer } \\
\text { Netze und mobiler Zugangsgeräte verstanden. }\end{array}$ \\
Znter Mobile Business verstehe ich alle auf mobilen Geräten ("Devices") ausgetauschten Dienstleistungen, \\
Waren sowie Transaktionen.
\end{tabular}

Abbildung 2-19: Definitionen zum Begriff „Mobile Business“

Wie Wirtz feststellt, liegt die Übereinstimmung in den existierenden Definitionen darin, dass die Benutzung mobiler Endgeräte im Zusammenhang mit wirtschaftlichen Akteuren als zentrales Definitionskriterium genannt wird. ${ }^{220}$ Der Unterschied zwischen den jeweiligen Definitionen liegt in der Hervorhebung unterschiedlicher Aspekte des Einsatzes mobiler Endgeräte im wirtschaftlichen Handeln: der Informationsaustausch zwischen Akteuren (bspw. Steimer, Hartmann/Dirksen), betriebliche oder absatzbezogene Geschäftsprozesse (Scheer/Feld/Göbl, Reichwald/Meier/Frehmut) oder der Austausch von Dienstleistungen und Gütern (Zobel, Wirtz). In Anlehnung an die Begriffseinteilungen im Rahmen der (Fest-)Netz- und speziell internetbasierten Unterstützung betrieblicher Prozesse in E-Commerce und E-Business wird im Rahmen mobiler Anwendungen häufig eine Abgrenzung zwischen Mobile Business und Mobile Commerce vorgenommen. Mobile Commerce umfasst dabei diejenige Teilmenge des Mobile Business, „welche die reine Durchführung von mit monetären Werten verbundenen Transaktionen beschreibt. “221

\subsubsection{Zusammenfassung}

Der Überblick über die verschiedenen drahtlosen Netztechniken macht deutlich, dass der Mobilfunk das Potenzial einer konkurrenzfähigen Technik zu leitungsgebundenen Netzen nicht nur im Bereich der Sprachübertragung besitzt. Dennoch, so zeigt Abschnitt 2.3.4, existiert derzeit noch keine allgemeine in der $\mathrm{Li}$ teratur geteilte Meinung in Bezug auf die Strukturierung ökonomisch sinnvoller Einsatz- und Anwendungsmöglichkeiten mobiler Dienste.

Vor dem Hintergrund der dreifachen Anwendungsmöglichkeiten mobiler Dienste im betriebswirtschaftlichen Kontext (zur Unterstützung der betrieblichen Leistungserbringung, zur Unterstützung der Abwicklung von Markttransaktionen und als Endprodukte; vgl. Abschnitt 2.3.4.2) liegt der Fokus dieser Arbeit auf der Untersuchung möglicher Anwendungsformen mobiler Dienste als Endprodukte für Privatkunden. Dabei werden insbesondere Möglichkeiten geprüft, Mobilfunknetze

220 Vgl. Wirtz (2001b), S. 44 f.

221 Buse (2002), S. 92. 
als Vertriebskanäle für digitale Mediengüter zu nutzen. Diese Prüfung wird schwerpunktmäßig in Kapitel 5 vorgenommen. Im Weiteren wird der Begriff „mobiles Internet“" synonym zum voran stehend erläuterten (allgemeineren) Begriff des mobilen (Daten-)Dienstes verwendet. Es wird dagegen eine Verwendung der - wie oben dargestellt - vergleichsweise unscharfen Begriffe "Mobile Business" oder „Mobile Commerce“ vermieden.

\subsection{Zusammenfassung der grundlegenden Begriffe}

In Kapitel 2.1 wurde der Begriff des Medienguts eingeordnet und Besonderheiten in der Güternatur der Erzeugnisse der Medienbranche dargestellt. Als Mediengut ist ein immaterieller Inhalt zu verstehen, der gebunden an ein Übertragungsmedium kommerziell zum Zwecke der Information oder Unterhaltung für ein Publikum bereitgestellt wird. Der Begriff der Individualisierung wurde in Kapitel 2.2 multiparadigmatisch untersucht, und es wurden Handlungsmöglichkeiten für eine betriebliche Einzelkundenorientierung vorgestellt. Unter Individualisierung ist im Weiteren die einzelbetriebliche Handlungsoption der Bereitstellung maßgeschneiderter Produkte und Dienstleistungen zu verstehen. In Kapitel 2.3 wurden die technischen Grundlagen des Mobilfunks erläutert und Anwendungsmöglichkeiten im betrieblichen Umfeld dargestellt. Unter mobilen Diensten sind Datendienste zu verstehen, die Endkunden zu kommerziellen Zwecken auf der Grundlage von Mobilfunktechniken bereitgestellt werden. 



\section{Strategische Herausforderungen in der Medienbranche}

Das folgende Kapitel befasst sich mit der Begründung eines am individuellen Kundennutzen ausgerichteten strategischen Managements in der Medienbranche. In Abschnitt 3.1 werden die zentralen Veränderungen der Rahmenbedingungen des Wettbewerbs im Zusammenhang mit sog. „Konvergenzeffekten“ im Marktumfeld der Medienbranche untersucht. Anhand ausgewählter Branchendaten wird in Abschnitt 3.2 im Rahmen einer Situationsanalyse des Medienwettbewerbs eine Entwicklung in Richtung eines allgemeinen Reichweitenverlusts traditioneller Massenmedien und einer dadurch bedingten Wettbewerbsverschärfung aufgezeigt. In Abschnitt 3.3 werden aus Sicht der Medienbranche Handlungsmöglichkeiten benannt, um dieser Wettbewerbsverschärfung zu begegnen. Die Ergebnisse des Kapitels werden in Abschnitt 3.4 zusammengefasst.

\subsection{Konvergenzeffekte als Ausprägung der Umfelddynamik des Medienwettbewerbs}

Wie bereits einleitend angedeutet wurde, sieht sich die Medienbranche derzeit mit tief greifenden Veränderungen konfrontiert. Im Folgenden werden die Ausprägungen und Ursachen dieser Veränderungen näher untersucht. Ausgangspunkt der Untersuchung der Wettbewerbsveränderungen bildet eine allgemeine Erörterung der Rahmendynamik im Wettbewerbsumfeld der Medienbranche. Diese Rahmendynamik wird vor allem in der ökonomisch geprägten Literatur unter dem Begriff der „Konvergenz“ subsumiert.

Konvergenz kann allgemein verstanden werden als ein „Prozeß der Interaktion zwischen Unternehmensumwelt bzw. Wettbewerbstrukturen und Unternehmensstrategie, der zur strukturellen Verbindung bislang getrennter Märkte führt. “222 Der Begriff Konvergenz ist allerdings in der Literatur zur Medienwirtschaft nicht einheitlich belegt: Funktionale Überlappungen von Fernsehen und Computer ${ }^{223}$ oder die Marktdurchdringung multimedialer Angebote werden ebenso genannt wie die Virtualisierung betrieblicher Strukturen ${ }^{224}$ und Verschmelzung ganzer Marktbereiche ${ }^{225}$. Zu einer genaueren Bestimmung von Konvergenzeffekten im Umfeld der Medienbranche werden daher im Folgenden

\footnotetext{
222 Thielmann (2000), S. 9.

223 Vgl. Stipp (1999).

$224 \mathrm{Vgl}$. Wirtz (2001a).

225 Vgl. Thielmann (2000), S. 9.
} 
zunächst die Auslöser konvergierender Effekte für die Medienbranche beschrieben (vgl. Abschnitt 3.1.1), bevor im Weiteren Konvergenzdimensionen (vgl. Abschnitt 3.1.2) dargestellt werden. In Abschnitt 3.1.3 werden die aufgezeigten Konvergenzeffekte zusammenfassend aus Sicht der Medienbranche bewertet.

\subsubsection{Auslöser von Konvergenzeffekten im Medienumfeld}

Beim Thema der Konvergenz "geht es nicht nur um Technologie, sondern um Dienste und neue Wege wirtschaftlicher und gesellschaftlicher Interaktionen", urteilt die Europäische Kommission in ihrem Grünbuch zur Konvergenz der Branchen Telekommunikation, Medien und Informationstechnologie. ${ }^{226}$ Das Zusammenwirken der Dynamik von gesellschaftlichen Trends, technologischem Fortschritt und ökonomischen Prinzipien wird seit Jahrzehnten in zahlreichen Diskussionen thematisiert. Das bekannteste Beispiel zur Erklärung der Bedeutung technologischer Innovationen für Gesellschaft und Ökonomie bildet die auf Kondratjew (1984) zurückgehende Theorie der langen Wellen. Demnach beeinflussen technologische Innovationen, bspw. die Erfindung der Dampfmaschine im 19. Jahrhundert oder die Entdeckung der Elektrizität zu Beginn des 20. Jahrhunderts, in zyklischen Abständen massiv sowohl den gesellschaftlichen Alltag als auch die gesamtwirtschaftliche Entwicklung. ${ }^{227}$ Seit Beginn des 18. Jahrhunderts können dabei insgesamt fünf derartige Zyklen, die als "lange Wellen" oder auch als "Kondratjew-Zyklen" bezeichnet werden, bestimmt werden (vgl. Abbildung 3-1). ${ }^{228}$

\begin{tabular}{|c|c|c|c|c|}
\hline \multicolumn{4}{|c|}{$\begin{array}{l}\text { Industrie- } \\
\text { gesellschaft }\end{array}$} & $\begin{array}{l}\text { Informations- } \\
\text { gesellschaft }\end{array}$ \\
\hline $\begin{array}{l}\text { Basisinnovation (BI): } \\
\text { - Dampfmaschine }\end{array}$ & $\begin{array}{l}\text { BI: } \\
\text { - Eisenbahn } \\
\text { - Stahlindustrie }\end{array}$ & $\begin{array}{l}\text { BI: } \\
\text { • Elektrizität } \\
\text { - Chemische Industrie }\end{array}$ & $\begin{array}{l}\text { BI: } \\
\text { - Molekularbiologie } \\
\text { - Automobilindustrie }\end{array}$ & $\begin{array}{l}\text { BI: } \\
\text { - Informations- } \\
\text { technologie } \\
\text { - Internet }\end{array}$ \\
\hline 1. Kondratjew & 2. Kondratjew & 3. Kondratjew & 4. Kondratjew & 5. Kondratjew \\
\hline 1800 & 1850 & 1900 & 1950 & 1990 \\
\hline
\end{tabular}

Abbildung 3-1: Kondratjew-Zyklen

226 EU (1999).

227 Vgl. Kondratjew (1984), S. $60 \mathrm{ff}$.

${ }^{228}$ Kondratjew selbst stellte 1926 zweieinhalb dieser langen Wellen fest, wobei die Bezeichnung des „Kondratjew-Zyklus“ für diese Wellen auf Schumpeter (1964) zurückgeht. Nefiodow (2001) identifizierte retrospektiv insgesamt fünf Kondratjew-Zyklen, denen die jeweils in Abbildung 3-1 angegebenen Basisinnovationen beigeordnet werden können. Als aussichtsreiche Kandidaten für die Basisinnovationen des bereits begonnenen sechsten Zyklus werden derzeit vor allem biotechnologische Entwicklungen eingeschätzt. 
Der Theorie der langen Wellen liegt die Vermutung zugrunde, dass technologische Innovationen und gesellschaftliche sowie ökonomische Entwicklungen sich gegenseitig beeinflussen und dadurch die Rahmenbedingungen des Wettbewerbs einer Branche nachhaltig verändern. Diese drei Aspekte - technologische, gesellschaftliche und ökonomische Veränderungen - werden daher im Folgenden als Rahmenbedingungen möglicher Wettbewerbsveränderungen in der Medienbranche näher untersucht.

\subsubsection{Veränderungen der technologischen Rahmenbedingungen}

Den zentralen Motor des Wandels von der Industrie- zur Informationsgesellschaft bilden die Entwicklungen im Rahmen der Informations- und Kommunikationstechnik (luK-Technik). Der technologische Fortschritt kann an drei Eckpunkten festgemacht werden: am Kapazitätenzuwachs der elektronischen Daten- und Informationsverarbeitung (1); an der Ausweitung der elektronischen Vernetzung (2); am Einzug der Digitalisierung in den Bereich der Signalübertragung (3). ${ }^{229}$

(1) Gordon Moore von der Firma Intel veröffentlichte 1965 seine Beobachtung, dass die Integrationsdichte von Halbleitertransistoren eines Mikroprozessors exponentiell wächst. Seine Vorausschau, dass sich die Zahl der Transistoren eines Mikroprozessors bei gleich bleibenden Produktionskosten alle 18 Monate verdoppelt, wurde als "Gesetz von Moore" bekannt. Dahingestellt, ob es sich bei diesem Gesetz um die Weitsicht eines Visionärs oder - wie auch behauptet - um eine "self-fulfilling prophecy“ der Branche handelt, ${ }^{230}$ wird das Gesetz von Moore bis heute weitgehend als gültig anerkannt. ${ }^{231}$ Gleichzeitig ist neben der Steigerung der Rechenleistung eine Miniaturisierung der Gerätetechnik zu verfolgen. Beispiele einer solchen Miniaturisierung finden sich zum einen im Bereich von Speichermedien, wo in den vergangenen 50 Jahren eine Entwicklung von Lochkarten über Magnetbänder hin zu münzgroßen Mikrotransistoren vollzogen wurde. Zum anderen ist in der elektronischen Endgerätetechnik ein Trend zu Miniaturisierung und Arbeitsteilung zu beobachten, der häufig unter dem Begriff des „Ubiquitous Computing“ subsumiert wird. Das Ubiquitous Computing kennzeichnet eine Evolution der Computertechnologie von Mainframe-Systemen über PC-Systeme hin zu verteilten, arbeitsteiligen Minicomputern. Die Vision des Ubiquitous Computing besteht darin, dass Computer allgegenwärtig, also „ubiquitär“, sein werden. Trotz ihrer Allgegenwärtigkeit erscheinen diese Computer den Anwender nicht mehr als ein einzelner, identifizierbarer Gegenstand, sondern verschmelzen mit Objekten der physischen Realität, wodurch sie praktisch unsichtbar werden. ${ }^{232}$ Für die Medienbranche können sich durch solche allgegenwärtigen Mini-Computer zukünftig neue Möglichkeiten der Inhaltedistribution eröffnen. Bspw. stellte jüngst der finnische Mobiltelefonhersteller

$229 \mathrm{Vgl}$. Zerdick et al. (2001), S. 150.

$230 \mathrm{Vgl}$. Scholz/Stein/Eisenbeis (2001), S. 73.

$231 \mathrm{Vgl}$. Intel (2003); Zerdick et al. (2001), S. 118; FAZ (2001), S. 34.

$232 \mathrm{Vgl}$. Leimeister/Krcmar (2002). 
Nokia ein Telefon mit integriertem Funkscanner für Warenetiketten vor, mit dem, nach einem Werbeszenario, Musikstücke aus Datensendern, die in Werbeplakaten versteckt sind, herunter geladen werden können. ${ }^{233}$

(2) Im Mai 2004 erfasste eine Studie zur Onlinenutzung in Deutschland insgesamt knapp 36 Millionen Internetteilnehmer. ${ }^{234}$ Der derzeitige Mobilfunkstandard GSM ist heute in 180 Ländern vertreten und vernetzt weltweit rund 900 Mio. Teilnehmer. Die wachsende Netzdichte kann erklärt anhand von positiven Netzwerkexternalitäten: Nach dem auf den Erfinder des Ethernet, Robert Metcalfe, zurückgehenden Gesetz der Netzwerke steigt der Wert eines Netzes im Quadrat zur Zahl seiner Teilnehmer. Dabei unterliegt der Netzwert zwei Bedingungen: der Standardisierung der Netzwerkkommunikation und der Attraktivität der verfügbaren Inhalte. Die aus einem militärischen Forschungsprojekt der späten 60er Jahre hervor gegangenen Protokolle „Transmission Control Protocol“ (TCP) und das "Internet Protocol“ (IP) wurden im April 1993 vom Europäischen Zentrum für Kernforschung (CERN) zur kostenlosen Nutzung freigegeben. Diese beiden Protokolle bilden derzeit den weltweit akzeptierten De-factoStandard der Datenkommunikation sowohl im leitungsgebundenen als auch im mobilen Internet. Die inhaltliche Dichte der Netzstruktur kann demgegenüber anhand der Anzahl der registrierten Zugangsadressen bestimmt werden. Nach einer Erhebung der Bundesregierung existierten im zweiten Quartal 2004 weltweit über 233 Mio. Domain-Namen mit eigener IP-Adresse (Hosts) ${ }^{235}$ Aus Sicht der Medienbranche repräsentieren Datennetze daher Übertragungsmedien, die nicht (mehr) nur eine speziell geschulte Teilnehmernische bedienen, sondern zunehmend die Reichweite traditioneller Massenmedien besitzen.

(3) Die bis vor einigen Jahren vorherrschenden analogen Übertragungsverfahren folgten dem Prinzip von Radiowellen: ${ }^{236}$ Die Bandbreite des Übertragungsmediums wurde in beliebig viele Frequenzbänder unterteilt, denen dann bestimmte Sende- oder Empfangsaufgaben zugeordnet wurden. Um digitale Daten, z.B. aus der elektronischen Datenverarbeitung zu versenden, mussten diese zunächst in analoge Form übersetzt, übertragen und beim Empfänger redigitalisiert werden. ${ }^{237}$ Digitale Übertragungsverfahren ermöglichen demgegenüber Übertragungswege für Datenpakete, unabhängig ob im Sprach-, Text- oder Bildformat, die ohne Qualitätsverluste beim Empfänger unmittelbar weiterverarbeitet werden können. Dabei ist zwischen leitungsvermittelnden und paketvermittelnden Übertragungsverfahren zu unterscheiden: Leitungsvermittelnde Netze etablieren eine permanente physische Verbindung zwischen kommunizierenden Einheiten. Für die Dauer des Kommunikationsvorgangs bleibt der Verbindungskanal exklusiv für die kommunizierenden Einheiten reserviert. Paketvermittelnde Netze spalten Nachrichten vor deren Versendung

\footnotetext{
$233 \mathrm{Vgl}$. Nokia (2004).

234 Vgl. Zerdick et al. (2001), S. 152 und Oebbeke (2003).

$235 \mathrm{Vgl}$. ISC (2003).

$236 \mathrm{Vgl}$. Scholz/Stein/Eisenbeis (2001), S. 55.

237 Vgl. Zerdick et al. (2001), S. 140.
} 
zunächst in verschiedene Datenpakete auf. Jedes Paket wird individuell übertragen und kann auf unterschiedlichen Übertragungswegen zum Bestimmungsort gelangen. Erst beim Zieladressaten werden die Pakete wieder zur Originalnachricht kompiliert. Der Vorteil paketorientierter Übertragungsverfahren, wie bspw. das GPRS-Verfahren im Mobilfunk, besteht in einer effizienteren Nutzung der verfügbaren Übertragungsbandbreiten und dadurch in einer höheren durchschnittlichen Übertragungskapazität, wodurch sich derartige Netze aus Sicht der Medienbranche verstärkt auch als Trägermedien für multimediale Inhalte eignen.

\subsubsection{Veränderungen der gesellschaftlichen Rahmenbedingungen}

Die technologischen Veränderungen erzeugten in vielen Ländern - darunter insbesondere den USA - einen gesamtwirtschaftlichen Wachstumsschub. Ausgelöst durch Wachstumserwartungen strategischer Investoren schien das Branchenwachstum der Biotechnologie, Automatisierungstechnik, Medien, Telekommunikation und Informationstechnik die zyklischen Abhängigkeiten von Beschäftigungsquote, Inflationsrate, Zahlungsbilanzgleichgewicht und Wirtschaftswachstum aufgehoben zu haben. ${ }^{238}$ Dieses Branchenwachstum schuf den Mythos neuer ökonomischer Gesetzmäßigkeiten. Die so genannte „New Economy" wurde zu einem Generator aber auch Magneten im Arbeitsmarkt, mit knapp 2 Mio. Beschäftigten in informations- und technologieaffinen Berufen im Jahr 2001. ${ }^{239}$ Diese Verschiebung am Arbeitsmarkt erzeugte zudem neue Arbeitszeitmodelle, Arbeitsformen und Qualifikationsvoraussetzungen. Ein früher Eintritt ins Berufsleben, insgesamt längere Lebensarbeitszeiten und eine zunehmende Zahl von Heimarbeitsplätzen sind nur einige Beispiele dieser Veränderungen.

Mit den wachsenden Ansprüchen an technologischen Grundfertigkeiten, die Voraussetzung kreativen und komplexen Denkens für die Bewältigung der Dynamik in Wettbewerb und Branchenumfeld verliert die menschliche Arbeitskraft den Charakter einer "Commodity“. ${ }^{40}$ Indikatoren dieser Veränderungen bilden zunehmend forschungsintensive Produkte und ein allgemeiner Komplexitätsanstieg der Arbeitsorganisation. ${ }^{241}$ Das Ergebnis dieser Veränderungen in Bezug auf die Arbeitsqualifikation bildet ein Bedeutungszuwachs expliziten, theoretischen Wissens. Die gesamtgesellschaftliche Prägung dieses Bedeutungszuwachses ist aus Sicht der Massenmedien u.a. in einem Wandel der inhaltlichen Nachfrage abzulesen. Bspw. gewinnt gegenüber rein unterhaltungsorientierten Angeboten die Nachfrage nach informationsorientierten Angeboten eine wachsende Bedeutung.

\footnotetext{
238 Vgl. Krugman (1997) und Shepard (1997).

239 Vgl. Wirtz (2001b), S. 9.

$240 \mathrm{Vgl}$. Haertsch (2000), S. 14.

241 Vgl. Drucker (1994).
} 


\section{1. 1.3 Veränderungen der ökonomischen Rahmenbedingungen}

Die makroökonomische Entwicklung der vergangenen Jahrzehnte war geprägt durch eine Globalisierung von Märkten und wirtschaftlichen Verflechtungen. Globalisierung erfordert den Abbau von nationalen Regulierungen und Handelsschranken. Beispiele für Wettbewerbsveränderungen im Medienumfeld durch Liberalisierung der Märkte bilden zum einen die schrittweise Deregulierung des deutschen Rundfunkwesen, mit der Einführung des privaten Rundfunks 1984 als Grundstein des heutigen dualen Rundfunksystems, oder die Öffnung des europäischen Telekommunikationssektors durch die Abschaffung der staatlichen Monopole in Netzbetrieb und Sprachtelephonie auf der Grundlage des Telekommunikationsgesetzes (TKG) vom 25.07.1996. ${ }^{242}$ Dieser Wegfall der nationalen Zutrittsschranken öffnete zum einen den Weg für neue Konkurrenz im Wettbewerb. Die Deregulierung des Marktes erlaubte zum anderen differenzierte Geschäftsmodelle entlang der Wertschöpfungsstrukturen. ${ }^{243}$

Der Aufbruch der nationalen Monopole wurde durch einen weiteren Faktor begünstigt. Das Engagement in multinationale Unternehmensverbindungen, bspw. zum Ausbau von Ressourcensynergien und Skaleneffekten, setzt insbesondere im Bereich von Medien- und Telekommunikationsmärkten, eine breite Kenntnis der nationalen Kundenbedürfnisse und Marktdeterminanten voraus. Die zunehmende gesellschaftliche Informations- und Wissensdurchdringung trug jedoch gleichzeitig zu einer Abminderung der kulturellen Gegensätze und damit nationalen Unterschieden solcher Märkte bei. Ein Beispiel für eine globale Verwertungsstrategie von Inhalten in der Medienbranche, die von diesen Veränderungen profitiert, bildet die amerikanische Filmindustrie, deren Produkte in allen Ländern des westlichen Kulturkreises vermarktet werden.

\subsubsection{Konvergenzdimensionen}

Nachdem die Triebkräfte der Veränderungen von Unternehmensumfeld und Wettbewerbsbedingungen identifiziert sind, bleibt zu klären, in welchen Formen konkrete Ausprägungen konvergenzbedingter Transformationsprozesse in Märkten und Wettbewerbsstrukturen zu beobachten sind. Für die Analyse der strategischen Wettbewerbsbedingungen sind dabei zwei Dimensionen von Relevanz: Konvergierende Trends auf technologischer Ebene (vgl. Abschnitt 3.1.2.1) und Nivellierungs- und Verschmelzungsformen in den Wertschöpfungsstrukturen bestehender Branchen (vgl. Abschnitt 3.1.2.2).

\subsubsection{Technologische Konvergenz}

Die in Kapitel 3.1.1 dargestellten Veränderungen sind in besonderer Weise durch die Evolution der Informations- und Kommunikationstechnik geprägt. Ein Effekt des Innovationsdreiecks aus Digitalisierung, Vernetzung und verbesserten Verarbeitungs- und Speichertechnologien ist die Ausprägung neuer Funktionen

\footnotetext{
242 Vgl. Heinrich (1999b), S. 103.

243 Vgl. Zerdick et al. (2001), S. 109.
} 
durch die vielschichtige Integration, Anreicherung und Ausdifferenzierung der Technologien in verschiedenen Anwendungsbereichen. Drei wesentliche Entwicklungen bilden das Ergebnis dieser Effekte:

Erstens die Entwicklung multimedialer Eigenschaften von stationären Geräten, Kommunikationsdiensten und Übertragungsnetzwerken. Das Internet bildet eine wichtige Ausprägung für ein solches Zusammenwachsen von informationsverarbeitender Hardware, integrierten Anwendungen zum Abrufen von Datenformaten in Text, Bild und Ton und sowohl schmal- als auch breitbandigen Übertragungsverfahren. Insbesondere in Bezug auf die Übertragungsnetzwerke vereinigen sich derzeit eine Reihe unterschiedlicher Trends. Wie eine Studie im Auftrag des Bundesministeriums für Wirtschaft und Technologie prognostiziert, wird die Trennung zwischen Sprach- und Datennetzen in Zukunft vollständig verschwinden. ${ }^{244}$

Zweitens der zunehmende Wegfall von Kapazitätsschranken der Übertragungstechnik. Trotz alternativer und konkurrierender Zugangstechnologien wie bspw. Glasfasernetze, Rundfunkkabelnetze oder Stromleitungen lässt sich dabei zum einen die Entwicklung in Richtung durchgängig breitbandiger Übertragungsverfahren beobachten. Gleichzeitig zeichnet sich eine Entwicklung konvergierender Netzeigenschaften und Standards im Bereich einerseits stationärer, andererseits mobiler Dienste ab. Wenn auch gegenüber leitungsgebundenen Verfahren mit einer zeitlichen Entwicklungsverzögerung von rund drei bis fünf Jahren, beschreiten auch mobile Technologien den Weg in Richtung breitbandiger Übertragungskapazitäten. Damit treten mobile Übertragungsverfahren nicht nur vermehrt in ein Konkurrenzverhältnis zu stationären Diensten. Zunehmend entstehen auch Kombinations- und Integrationsformen. Ausprägungen stellen bspw. die bereits in Kapitel 2 dargestellten drahtlosen Netzsysteme dar, die im Wesentlichen auf dem Ethernet-Standard für drahtgebundene Datennetze basieren, jedoch eine Luftschnittstelle im lizenzfreien Funkfrequenzbereich bieten. Ein anderes Beispiel bildet die Entwicklung konvergierender Netzeigenschaften und -standards zwischen leitungsgebundenen und mobilen Datendiensten. Der in Kapitel 2 erläuterte Begriff des „mobilen Internet“ bildet selbst ein Ausprägungsbeispiel solcher Konvergenzeffekte. ${ }^{245}$

Drittens wird die digitale Datenkommunikation insbesondere bei Inhalteanbietern und -nachfragern durch weitere technische Entwicklungen begleitet. Solche Entwicklungen umfassen bspw. Content-Management-Systeme zur arbeitsteiligen Planung, Erzeugung, Gestaltung, Verwaltung und Publikation digitaler Inhalte, Rechtemanagementsysteme zur Unterstützung der Durchsetzung von Verwertungsrechten, elektronische Zahlungssysteme zur Unterstützung und Abrechnung von Geschäften im Internet, digitale Wasserzeichen zur urheberrechtlichen Zuordnung von Inhalten und Austauschstandards für einen automatisierten Datenaustausch, unabhängig von anwendungsspezifischen Auszeichnungssemantiken. ${ }^{246}$

\footnotetext{
244 Vgl. Büllingen/Stamm (2001), S. 17.

245 Vgl. u.a. Lehner (2002) und Best (2002).

$246 \mathrm{Vgl}$. Hess (2002), S. 574.
} 


\section{1.2.2 Branchenkonvergenz im Umfeld der Medienbranche}

Durch die Möglichkeiten der digitalen Datenkommunikation werden digitale Kommunikationsnetze wie das Internet oder der digitale Mobilfunk potenziell zu Trägermedien publizistischer Massenkommunikation. Der Einsatz digitaler Kommunikationstechnologien als Träger der Massenkommunikation führt dabei nach Hass zu einer langfristigen „Desintegration“ von Trägermedium und transportierter Nachricht. ${ }^{247}$ Dadurch entsteht zum einen für die traditionell anhand von Trägermedien unterschiedenen Segmente der Medienbranche ein Abgrenzungsproblem relevanter Wettbewerbsmärkte. Zum anderen erzeugen die Möglichkeiten der Datenkommunikation „strukturelle Kopplungen“ ursprünglich getrennter Wertschöpfungsaktivitäten zwischen Telekommunikations-, IT- und Medienbranche. ${ }^{248}$ Diese Kopplung folgt laut Zerdick et al. einem zweistufigen Muster: Digitalisierung und Datenvernetzung sorgen zum einen für eine Verschmelzung der Leistungsketten von Telekommunikations- und Informationstechnik. Zum anderen bedingt das Zusammenwachsen von Individual- und Massenkommunikation eine Annäherung von Telekommunikation und Informationstechnologie an die Medienbranche.

Nach Müller und Heitmann können im Rahmen solcher strukturellen Kopplungseffekte drei Formen der angebotsseitigen Konvergenz von Branchen unterschieden werden: Konvergenz auf der Ebene der Wertschöpfungsstufen durch neu entstehende Zulieferer-Abnehmer-Beziehungen, Konvergenz durch Komplementarität sich gegenseitig erlöswirksam beeinflussender Leistungen und Konvergenz auf Wettbewerbsebene durch Substitutionsangebote. ${ }^{249} \mathrm{Er}$ gebnis dieses Annäherungsprozesses der drei Branchen ist der ständige Bedeutungszuwachs der Überschneidungsbereiche. Beispiele für derartige neue Überschneidungsbereiche zwischen den Wertschöpfungen der drei Branchen bilden die Übertragung von Daten und die dafür notwendige Bereitstellung und Paketierung von Netzen und Diensten, das Angebot von Diensten für Suche und Navigation in digital vernetzenden Inhalten, das Angebot von Mehrwertdiensten bspw. zur Gewährleistung von Interaktivität im Rahmen der Rezeption massenmedialer Inhalte sowie das Angebot von Endgeräten, das eine interaktive und multimediale Nutzung von digitale vernetzten Inhalten ermöglicht. Abbildung 3-2 zeigt das denkbare Ergebnis eines solchen branchenbezogenen Konvergenzprozesses.

247 Hass (2002), S. 11: „Als Folge des Übergangs „von physischer und medienspezifischer zu nichtphysischer und medienunabhängiger Produktion, Redaktion und Distribution von Informationen (...) lassen sich nunmehr ein und dieselben Inhalte über verschiedene Medienkonfigurationen verbreiten."

248 Zerdick et al. (2001), S. 141.

249 Vgl. Müller/Heitmann (2002). 


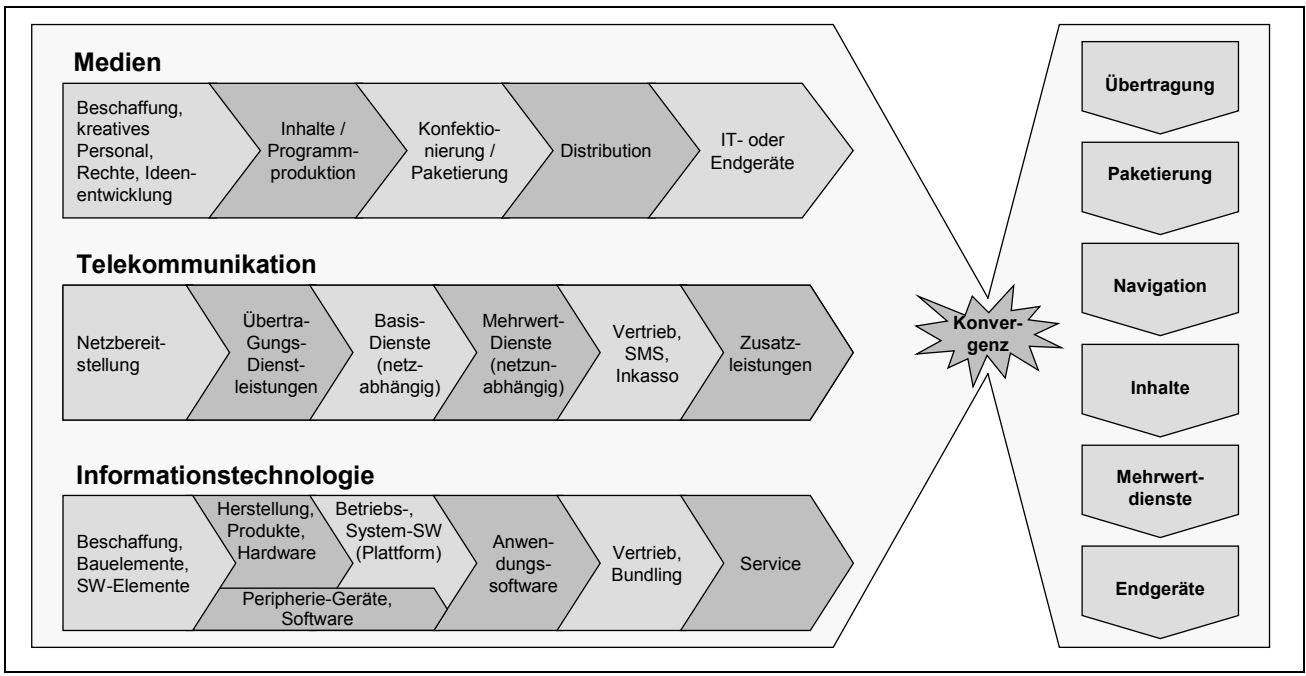

Abbildung 3-2: Entstehung von Multimediamärkten250

Für die aus der Konvergenz von Telekommunikations- IT- und Medienbranche entstehenden Multimediamärkte prognostiziert Keuper drei komplementäre Erlebniswelten: Das M(obile)-Business im mobilen Internet, das $\mathrm{E}$ (lectronic)Business im stationären Internet und das T(elevision)-Business im interaktiven digitalen Fernsehen. ${ }^{251}$ Mag man auch im Zweifel über die jeweilige Eintrittswahrscheinlichkeit der drei genannten Erlebniswelten sein - betrachtet man die Rolle der Medien, ist die vollkommene Neudefinition der Rolle der Medien bzw. eines Medienunternehmens im Rahmen konvergenter Märkte evident: War die traditionelle Rolle eines Medienunternehmens festgelegt auf die eines vollintegrierten Unternehmens zur Distribution von Inhalten in einem bestimmten Medienkanal, ist ihre Rolle im konvergenten Märkten deutlich enger gefasst, als Anbieter von Inhalten, die über fremde Übertragungskanäle wie bspw. Telekommunikationsnetze verwertet werden. Dadurch löst sich die Branchenabgrenzung der Medienindustrie zwangsläufig von ihrem traditionell engen Bezug zu jeweils einem Distributionskanal von spezifischem Leistungsvermögen.

\subsubsection{Bewertung der Konvergenzeffekte aus Sicht der Medien- branche}

Zusammenfassend lassen sich im Rahmen der aufgezeigten Konvergenzeffekte aus Sicht der Medienbranche drei Veränderungstendenzen ableiten: Durch die Möglichkeiten der Digitalisierung können erstens Inhalte medienneutral erzeugt werden, wodurch sie potenziell in unterschiedlichen Trägermedien verwendet werden können. Zweitens entstehen durch die digitale Datenkommunkation neue Distributionskanäle in Form von digitalen Kommunikationsnetzen

250 In Anlehnung an Zerdick et al. (2001), S. 144.

251 Vgl. Keuper (2002), S. 653. 
für die durch Medienunternehmen erzeugten Inhalte. Dies hat drittens Auswirkungen auf das Branchenverständnis von Medienunternehmen, weg von Anbietern vollständiger Produkte zur Massenunterhaltung und hin zu Inhaltespezialisten im Rahmen einer vernetzten Wertschöpfung für multimediale netzbasierte Angebote.

Aus Sicht der Medienbranche ist die Frage zu klären, inwieweit die aufgezeigten Veränderungspotenziale ein neues Branchenverständnis determinieren, also revolutionäre Implikationen für die Medienbranche besitzen, oder lediglich neue (alternative) Handlungsmöglichkeiten mit evolutionärem Charakter entstehen. Vor dem Hintergrund der bislang primär aus angebotsseitiger Perspektive aufgezeigten Entwicklungen ist diese Frage eng geknüpft an die Entwicklung der Nachfrage nach den Produkten und Inhalten der Medienbranche. Die Nachfrageentwicklung birgt das zentrale Unsicherheitsmoment für die Ermittlung einer strategischen Handlungsempfehlung in der Medienbranche. Die Unsicherheit besteht dabei in dreifacher Hinsicht: „Es ist unklar, ob überhaupt eine Nachfrage besteht, wenn ja, wie groß das Marktvolumen für spezifische Produkte sein wird. Weiterhin ist unklar, welche Bestandteile eines Dienstes aus Konsumentensicht welche Differenzierungswirkung erzielen.“252

Nach Keuper kann hinsichtlich der durch Konvergenzeffekte verursachten Nachfrageveränderungen ein komplementäres und eines kompetitives Konvergenzparadigma unterschieden werden: ${ }^{253}$ Unter dem kompetitiven Paradigma versteht Keuper die Verschmelzung zweier Branchen aufgrund der konvergenzbedingten, funktional-äquivalenten Beurteilung ihrer Produkte durch Kunden. Demgegenüber beschreibt das komplementäre Paradigma die Bildung eines zusätzlichen, neuen Marktes in Folge der Leistungsbündelung und -integration der Leistungen zweier Branchen. Um die Auswirkungen der Konvergenz auf die Nachfrage in der Medienbranche zu bestimmen ist daher der Substitutionsgrad neuer und bestehender Angebote zu untersuchen.

Vor diesem Hintergrund werden im Folgenden zunächst die Wettbewerbsstrukturen sowohl in den bestehenden als auch neuen Angebotsbereichen von Inhalten ermittelt. Anhand dessen wird der Substitutionsgrad zwischen alten und neuen Angebotsformen bewertet.

\subsection{Strukturanalyse des Medienwettbewerbs}

Die folgende Untersuchung der strukturellen Wettbewerbskräfte im Medienbereich fokussiert zwei Aspekte: erstens, die den jeweiligen Einzelsegmenten der Medienbranche innewohnenden (intramedialen) Wettbewerbsstrukturen und die daraus abzuleitenden Strategieoptionen zur Bewertung des existierenden Handlungsdrucks im Rahmen der traditionellen Marktbereiche (vgl. Abschnitt 3.2.1); zweitens, die Veränderungen und Auswirkungen neuer, digitaler Inhalteangebote auf den (intermedialen) Wettbewerb in den etablierten Marktsegmenten der Medienbranche (vgl. Abschnitt 3.2.2).

\footnotetext{
252 Müller/Heitmann (2002), S. 7.

253 Vgl. Keuper (2002), S. 624 und Thielmann (2000), S. 14.
} 


\subsubsection{Intramedialer Medienwettbewerb}

Im Folgenden werden die strukturimmanenten Wettbewerbsbedingungen in den Medienmärkten untersucht. Nach dem Bedarfsmarktkonzept werden vier Teilmärkte unterschieden: der Zeitungsmarkt (vgl. Abschnitt 3.2.1.1), der Zeitschriftenmarkt (vgl. Abschnitt 3.2.1.2), der Rundfunkmarkt - aufzuspalten in Hörfunk (vgl. Abschnitt 3.2.1.3) und Fernsehen (vgl. Abschnitt 3.2.1.4) - und der Inhaltemarkt im Internet (vgl. Abschnitt 3.2.1.5).

\subsubsection{Der Zeitungsmarkt}

Zeitungen sind Druckschriften zu aktuellen Informationen mit regelmäßiger Erscheinungsfolge. Im Marktsegment für Zeitungen können Untersegmente entsprechend der Erscheinungshäufigkeit (bspw. Tages- und Wochenzeitungen), dem Verbreitungsgebiet (bspw. regionale und überregionale Zeitungen) und der Verbreitungsart (Straßenverkaufs- vs. Abonnementzeitungen) gebildet werden. ${ }^{254}$ Die folgende Untersuchung beschäftigt sich überblicksartig mit dem gesamten Segment.

Der Zeitungsmarkt repräsentiert mit Nettowerbeeinnahmen in Höhe von knapp 4,5 Mrd. EUR nach wie vor den größten Werbeträger im gesamten Mediensektor. ${ }^{255}$ Der Markt verzeichnet auf Anbieterseite in den letzten zehn Jahren leicht sinkende Teilnehmerzahlen. ${ }^{256}$ Nachdem im Jahr 2000 mit 10,79 Mrd. EUR gegenüber 18,64 Mrd. DM 1999 letztmalig ein leichtes Umsatzwachstum erzielt wurde, ${ }^{257}$ sank der Umsatz in den drei Folgejahren um durchschnittlich 6,2\% auf 8,9 Mrd. EUR im Jahr 2003. ${ }^{258}$ Die Hauptursache dieses Umsatzrückgangs bildet ein Einbruch im Anzeigengeschäft, das allein im Jahr 2001 einen Rückgang um 13,9\% verzeichnete. Die Umsatzverteilung zwischen den Anbietern liefert ein differenziertes Bild: Während der Marktanteil der fünf größten Abonnementzeitungen zwischen 1989 und 2002 von 24,9\% auf 28,8\% stieg, sank im selben Zeitraum der Anteil der reichweitenstärksten Zeitungen im vergleichsweise hochkonzentrierten Segment des Straßenverkaufs von 98,8\% auf $94,6 \%$. Segment-übergreifend blieb die Konzentration der größten fünf Zeitungsanbieter mit 42,8\% (1989) und 42,3\% (2002), ebenso der größten zehn Anbieter mit 54,8\% (1989) und 56,3\% (2002) nahezu konstant. ${ }^{259}$

Durch die starke inhaltliche Differenzierung auf der Grundlage des redaktionellen Profils der einzelnen Blätter ist die Flexibilitätsspielraum im redaktionellen Angebot gegenüber Präferenzänderungen der Nachfrage an den Grenzen des gewählten Profils relativ gering. In Verbindung mit einer gleichzeitig schwach ausgeprägten Nachfrageflexibilität in Bezug auf die alternative Rezep-

254 Vgl. Sjurts (2002a) und Sjurts (1996).

255 Vgl. Keller (2004), S. 51: 4,45 Mrd. EUR.

256 Vgl. Media Perspektive (2004), S. 41.

257 Vgl. Sjurts (1996), S. 11 und Media Perspektive (2002), S. 43.

258 Vgl. Keller (2004), S. 82.

259 Vgl. Sjurts (2002a), S. 28. 
tion verschiedener Zeitungen - aufgrund der über das redaktionelle Profil geprägten „Leser-Blatt-Bindung“ - sind die Preiselastizitäten in der Zeitungsnachfrage ebenfalls gering. Die Gefahr intramedialer Substitution ist daher schwach ausgeprägt. Demgegenüber weist der Anzeigenmarkt eine hohe Flexibilität, sowohl hinsichtlich der Angebotsformen für Werberaum seitens der Zeitungsverlage, als auch in Bezug auf hohe intramediale Preiselastizitäten der Werberaumnachfrage auf. Die Nachfrage im Anzeigenmarkt wird wesentlich durch den bereits beschriebenen Volumeneffekt der "Anzeigen-Auflagen-Spirale“ bestimmt. Dieser Volumeneffekt erzeugt in Verbindung mit den für den Zeitungsmarkt typischen Kostenstrukturen, die durch hohe Fixkostenanteile und sinkende Durchschnittskosten geprägt sind, strukturelle Barrieren gegen einen Marktzutritt potenzieller neuer Wettbewerber.

Die dominierende Wettbewerbsstrategie im deutschen Zeitungsmarkt bildet eine reaktive, auf die Durchdringung der traditionellen Märkte gerichtete Differenzierung des redaktionellen Angebots, bspw. durch die Einführung von Supplements in den 1980er Jahren, das Engagement der Zeitungsverlage im privaten Hörfunk in den 1990ern sowie durch die aktuelle Regionalisierungstendenz der Zeitungsinhalte. Vermehrt haben die Zeitungsverlage mit einem allgemeinen Rückgang des Leserinteresses speziell in jüngeren Lesergruppen zu kämpfen, was in der dargelegten Umsatzstagnation sowie in den leicht rückläufigen Auflagenzahlen (die Gesamtauflage der deutschen Tagespresse sank zwischen 1997 und 2003 von 29,4 auf 26,8 Mio. Exemplare) zum Ausdruck kommt. ${ }^{260}$

\subsubsection{Der Zeitschriftenmarkt}

Zeitschriften sind periodisch erscheinende Druckschriften mit kontinuierlicher Stoffdarbietung, die mindestens viermal jährlich herausgegeben werden. ${ }^{261}$ Untersegmente können entsprechend der Zielgruppe (bspw. Publikums- und Fachzeitschriften), der inhaltlichen Spezialisierung (bspw. Frauen-, Programmoder aktuelle Zeitschriften) oder der Erscheinungshäufigkeit (bspw. Wochenmagazin und Monatsmagazin) unterschieden werden. Die folgende Untersuchung fokussiert den kommerziell wichtigeren Markt für Publikumszeitschriften mit einem Schwerpunkt auf dem auflagenstärksten Segment der mindestens 14tägig erscheinenden Titel.

1997 wurden insgesamt 8.740 Zeitschriftentitel produziert, wovon 1.685 auf das umsatzstärkste Segment der Publikumszeitschriften entfielen. ${ }^{262}$ Die Zahl der Publikumstitel stieg bis zum Jahr 2000 auf 2.040 und bis 2003 auf 2.300 Titel $a^{263}$ Der Umsatz im Zeitschriftenmarkt verzeichnete in den 90er Jahren ein kontinuierliches Wachstum von jährlich rund $6 \%$ und stieg damit von 14,7 Mrd. DM (1991) auf 19,378 Mrd. DM (1998). ${ }^{264}$ Der Anteil der Anzeigenerlöse

\footnotetext{
260 Vgl. Media Perspektive (2003), S. 43.

261 Vgl. Sjurts (2002a), S. 64.

262 Vgl. Sjurts (1996), S. 68.

$263 \mathrm{Vgl}$. VDZ (2001).

264 Vgl. Heinrich (2001a), S. 36 und S. 334.
} 
rangiert gegenüber dem Zeitungsmarkt auf niedrigerem Niveau mit 1,79 Mrd. EUR (1997) bzw. 2,24 Mrd. EUR (2000). Ähnlich wie am Zeitschriftenmarkt sanken die Werbeumsätze der Zeitschriftenverlage seit 2001 jährlich um durchschnittlich 6,1\% auf 1,86 Mrd. EUR (2003). ${ }^{265}$

Gegenüber dem Zeitungssektor zeichnet sich im Markt für Zeitschriften eine deutlich stärkere oligopolistische Konzentrationstendenz ab. Bestimmend für den Zeitschriftenmarkt ist der Wettbewerb zwischen den vier umsatzstärksten Verlagshäusern - Bauer, Springer, Burda und Gruner+Jahr - die 58,6\% der Umsatzerlöse $e^{266}$ und $61,18 \%$ der Gesamtauflage ${ }^{267}$ des Markts auf sich vereinen. Dabei ist die Konzentrationstendenz bezogen auf den Gesamtmarkt insgesamt abnehmend. Sie sank zwischen 1980 und 2000 von $66,9 \%$ auf $58,6 \%$, im wiederum umsatzstärksten Segment der Publikumszeitschriften der mindestens 14tägig erscheinenden Titel jedoch leicht steigend, von $76,1 \% \mathrm{im}$ Jahr 1975 auf $77,2 \%$ im Jahr 2000.

Während die Gesamtauflage im Zeitschriftenmarkt zwischen 1980 und 2000 von 63,5 Mio. verkaufter Exemplare auf 92,5 Mio. ${ }^{268}$ ausgeweitet wurde, sank im selben Zeitraum die Durchschnittsauflage je Zeitschriftentitel drastisch von 741.000 auf 541.000 Exemplare. ${ }^{269}$ Die gesunkene Durchschnittsauflage lässt sich erklären durch die starke interessensspezifische Differenzierung des Zeitschriftenangebots. Alleine im Jahr 2000 entstanden 409 neue Zeitschriftentitel als Ausdruck einer allgemein im Vergleich zum Zeitungsmarkt höheren Angebotsflexibilität der Zeitschriftenverlage. ${ }^{270}$ Diese interessensspezifische Ausdifferenzierung des Titelangebots verringert dabei einerseits die Preiselastizitäten der Rezipientennachfrage, sie schafft andererseits eine zielgruppenspezifische Kanalisierung der Werberaumnachfrage in den einzelnen Zeitschriftentiteln mit der Folge einer geringeren Nachfrageflexibilität am Anzeigenmarkt. Die Bedingungen des Marktzutritts im Zeitschriftenmarkt entsprechen weitgehend denen des Zeitungsmarkts und werden daher nicht gesondert betrachtet.

Trotz der hohen Konzentration im Zeitschriftenmarkt existiert ein heftiger Wettbewerb zwischen den Marktteilnehmern, ausgelöst durch eine Phase der Wachstumsstagnation zu Beginn der 1980er Jahre, der in der Vergangenheit in mehreren Konfrontationsrunden insbesondere auf den drei Wettbewerbsfeldern Programmpresse, Frauenzeitschriften und aktuelle Magazine ausgetragen wurde. Als Folge dieser Konfrontationen präsentiert sich der Markt heute mit einer breiten, differenzierten Angebotspalette mit insgesamt niedrigem Preisniveau. Aufgrund der hohen Angebotsflexibilität konnte der Zeitschriftenmarkt vom Boom der New Economy zum Ende der 1990er durch Titelinnovationen im Bereich von Finanzdienstleistungen, Telekommunikation und Internet profitieren, steht jedoch im Zuge des derzeit auch in diesem Segment schwindenden Rezi-

265 Vgl. Media Perspektive (2002).

266 Vgl. Sjurts (2002a), S. 98.

267 Vgl. Schulz (2001).

268 2003: 86,81 Mio.

269 Vgl. Sjurts (2002a), S. 98 und S. 102.

270 Vgl. Sjurts (2002a), S. 99. 
pienteninteresses - EDV-Magazine verbuchten 2001 einen Nachfragerückgang von $20,4 \%$, Wirtschaftsblätter sogar von $29 \%{ }^{271}$ - vor der Herausforderung der Marktkonsolidierung und Risikominimierung. Dominierende Wettbewerbsstrategie der Akteure am Zeitschriftenmarkt bildet eine reaktive, intramedial ausgerichtete Differenzierung. Beispiele einer solchen intramedialen Differenzierung bilden Dachmarkenstrategien zur Schaffung von Käufervertrauen (so genannte "Line Extensions“, wie bspw. die Übertragung der Zeitschriftenmarke „Stern“ im Verlag Gruner+Jahr auf die Zeitschriftentitel „Biografie“, „Campus\&Karriere“, "Chronik“ und „Neon“), sowie kostenreduzierende Vertriebsformate (wie die in A5-ähnlichen Kompaktformaten erscheinenden Frauenzeitschriften „young“ im Burda Verlag oder "Glamour" im Condé Nast Verlag). ${ }^{272}$

\subsubsection{Der Hörfunkmarkt}

Als Hörfunk wird die auditive Sparte des Rundfunks verstanden. Untersegmente des Hörfunkmarkts können anhand der Eigentümerstrukturen (öffentlichrechtliche und private Sendeanstalten), der inhaltlichen Ausrichtung (bspw. Nachrichten- und Musiksender) und der Sendereichweite (bspw. landesweite und lokale Sender) unterschieden werden. Der Hörfunk repräsentiert mit einem Zeitaufwand von rund 155 Minuten pro Werktag das meistgenutzte Medium in Deutschland. ${ }^{273}$

Auf dem Hörfunkmarkt besteht derzeit auf Landesebene ein mäßig weites, auf Lokalebene weites Angebotsoligopol. Die Programmzahl der Radiostationen in Deutschland stieg nach der Liberalisierung der Mediengesetze 1986 von 63 Programmen, darunter 24 privat finanzierte, in den 1990ern bis auf insgesamt 240 Programme. ${ }^{274}$ Der Werbemarkt erwirtschaftete im Jahr 2000 einen Umsatz von 628 Mio. EUR und musste nach einem Jahrzehnt stabiler, jährlicher Wachstumsraten von konstant über $5 \%$ im Folgejahr erstmals Einbußen von 7,5\%, im Jahr 2002 um 12,2\%, verzeichnen. ${ }^{275}$ Während sich der Werbemarkt sowohl im Angebot als auch in der intramedialen Nachfrage durch eine hohe Flexibilität auszeichnet, ist der Programmvertrieb, nachfrageseitig aufgrund der nachweislichen, sowohl intra- als auch intermediären Programmtreue der Rezipienten, angebotsseitig aufgrund der wachsenden Programmdifferenzierung der Sendestationen, durch geringe Preiselastizitäten und somit durch eine geringe intramediale Substitutionsneigung gekennzeichnet. Wettbewerbshemmend wirken sich des Weiteren eine Reihe von Marktzutrittsschranken aus: strategische Barrieren, durch eine hohe, rückwärts gerichtete Wertschöpfungsintegration der einzelnen Sendestationen in die vergleichbar teure Programmproduktion; strukturelle Barrieren durch den analog bereits im Rahmen der Druckmedien als „Anzeigen-Auflagen-Spirale“ charakterisierten Volumeneffekt

\footnotetext{
$271 \mathrm{Vgl}$. Schulz (2001), S. 7.

272 Vgl. Rzesnitzek (2003), S. 238 f.

$273 \mathrm{Vgl}$. Heinrich (2001b), S. 72.

274 Vgl. Sjurts (2002a), S. 186.

275 Vgl. Sjurts (2002a), S. 196.
} 
zwischen Rezipienten- und Werberaumnachfrageinteresse; sowie die in den Landesmediengesetzen verankerte Limitierung des Marktzutritts als institutionelle Hürde.

Zusammenfassend lässt sich der Hörfunkmarkt nach einer Expansions- und Differenzierungsphase seit der Marktliberalisierung in der zweiten Hälfte der 1980er Jahre als weitgehend gesättigter, jedoch von intermediären Substitutionsneigungen der Rezipienten weitgehend unbelasteter Markt. Dominierende Strategie bildet eine, in Bezug auf das Programmangebot reaktive, im Werbemarkt häufig innovative Angebotsdifferenzierung. Offen sind derzeit die zukünftigen Auswirkungen der zunehmenden Alternativenvielfalt der Programmdistribution wie Internetradio und digital-terrestrischer Rundfunk.

\subsubsection{Der Fernsehmarkt}

Der Fernsehmarkt als audio-visuelle Sparte des Rundfunks kann nach denselben Kriterien wie der Hörfunk segmentiert werden. Trotz des europaweit größten Programmangebots von 15 öffentlich-rechtlichen und 22 privaten Kanälen präsentiert sich der Fernsehmarkt als hochkonzentriertes Mediensegment mit drei dominierenden Anbietern: ${ }^{276}$ Erstens die öffentlich-rechtlichen Sendestationen, zweitens die zum ehemaligen Kirch-Konzern gehörenden Kanäle und drittens die Stationen der Bertelsmann AG. Diese Dreiteilung spiegelt sich in den Zuschauermarktanteilen der einzelnen Programme wieder, wonach auf öffentlichrechtliche Stationen ein Anteil von 42,4\%, auf die Sender der ehemaligen KirchGruppe 23,2\% (Sat1, Pro7, Kabel1, DSF) und auf die Stationen der Bertelsmann AG (RTL, RTL2, VOX, SuperRTL) $26,4 \%$ entfallen. ${ }^{277}$ Der Werbemarkt erlebte in den 1990er Jahren ein kontinuierliches Wachstum mit stabilen Zuwachsraten von jährlich über $6 \%$ und erwirtschaftete im Jahr 2000 einen Gesamtumsatz von 4,7 Mrd. EUR. ${ }^{278}$ Erstmalig wurde im Jahr 2001 ein Negativwachstum von $-5,1 \%$ verzeichnet. ${ }^{279}$ Nach Heinrich kann auf dem Werbemarkt von einem marktbeherrschenden Oligopol dreier Anbieter, RTL mit 30,2\%, Sat. 1 mit 20,4\% und Pro7 mit 18,4\% Marktanteil, ausgegangen werden. ${ }^{280}$

Im Gegensatz zum Hörfunkmarkt sind Fernsehzuschauer in ihrer Programmauswahl sehr flexibel. Während sich einerseits der durchschnittliche Zeitaufwand für das Fernsehen in den vergangenen 30 Jahren von 70 Minuten auf 145 Minuten mehr als verdoppelte, kann andererseits ein Wandel von einer gezielten Programmrezeption zu einem unsystematischen Wahrnehmung des Fernsehens als Hintergrundsunterhaltung in Konkurrenz zum Hörfunk beobachtet werden. Das Programmangebot weist zunehmend Merkmale eines Hyper-

\footnotetext{
276 Vgl. Sjurts (2002a), S. 240.

277 Vgl. Sjurts (2002a), S. 243 und KEK (2003).

$278 \mathrm{Vgl}$. ZAW (2001).

279 Gefolgt von einem Rückgang um 11,5\% (2002) und 3,7\% (2003). Derzeit verzeichnet die Branche einen Werbeumsatz von 3.811,27 Mio. EUR. Vgl. ZAW (2004), S. 278.
}

280 Vgl. Heinrich (2001b), S. 464 und ZAW (2004), S. 278 (Zahlen von 2003). 
wettbewerbs $^{281}$ auf, mit abnehmenden Programmlebenszyklen und kurzen Innovationsintervallen neuer Programmformate. ${ }^{282}$ Der Werbemarkt besitzt sowohl auf Angebots- als auch Nachfrageseite ein hohes Maß an Flexibilität mit der Folge einer hohen Wettbewerbsintensität insbesondere zwischen den privaten Anbietern. Die Möglichkeiten des Marktzutritts werden eingeschränkt durch ähnliche Hemmnisse wie im Hörfunkmarkt und müssen hier nicht weiter betrachtet werden.

Ohne an dieser Stelle eine Typunterscheidung zwischen den einzelnen Programmen vorzunehmen - denkbar wäre bspw. die Abgrenzung zwischen Voll-, Regional-, Sparten und Abonnementprogrammen - verfolgen alle Marktteilnehmer eine, sowohl in Bezug auf den Rezipienten- als auch Werbemarkt, innovative, intramedial ausgerichtete Differenzierungsstrategie. Als in absehbarer Weise für die nahe Zukunft für den Fernsehmarkt wettbewerbsprägend lassen sich zwei Ereignisse in der jüngeren Vergangenheit bewerten: Erstens die Insolvenz der Kirch-Media-Holding und der dadurch ermöglichte Einstieg der P7S1 Holding ${ }^{283}$, einer Tochtergesellschaft der amerikanischen Saban Capital Group, in das deutsche Fernsehgeschäft. Zweitens die wachsende Bedeutung der Digitalisierung und die dadurch eröffneten Wege in die Multimedialität, sowohl im Hörfunk als auch im Fernsehmarkt. Heinrich benennt in diesem Zusammenhang drei Elemente eines sich abzeichnenden Wandels: die Entwicklung des Schnittfelds zwischen Rundfunk und Telekommunikation zur Schlüsselbranche mit hohem Wachstumspotenzial; zunehmende Vertriebskapazitäten bei gleichzeitig sinkenden Kosten; der Trend in Richtung von Differenzierung und, in Folge der möglichen Interaktivität, Individualisierung des Programmangebots.

\subsection{5 Das Internet als Segment des Medienmarkts}

Öffentliche, elektronische Datennetze wie das Internet weisen Wesensmerkmale von Massenmedien auf: sie ermöglichen den Transport von Inhalten - aktuelle Informationen, Werbung oder Unterhaltung - zum Zwecke der Massenkommunikation. Betrachtet man demnach das Internet im Lichte der traditionellen Abgrenzung von Marktsegmenten der Medienbranche anhand des verwendeten Trägermediums der Inhaltedistribution, kann auch das Internet als neues Marktsegment der Medienbranche charakterisiert werden. Anders als die etablierten Print- und Rundfunkmedien ist das Internet ein multifunktionales Medium. Es dient gleichermaßen der Kommunikation, als Informationsquelle, zur interaktiven Unterhaltung und als virtuelles Einkaufszentrum. Potenzielle Konkurrenz für ein redaktionelles Inhalteangebot im Internet bilden daher kommunikations- und transaktionsorientierte Angebote, Werbeinhalte bspw. aus dem Bereich der Konsumgüterindustrie sowie nicht-kommerzielle Inhalte privater Internetnutzer.

\footnotetext{
281 Zum Begriff des Hyperwettbewerbs vgl. D'Aveni (1994) und Hümmer (2001).

282 Vgl. Sjurts (2002a).

283 Vgl. P7S1 Holding (2004).
} 
Die Zahl der Internetnutzer in Deutschland stieg zwischen 1997 und 2000 jährlich um 60-70\%. Seit 2001 schwächt sich das Wachstum bei einem durchschnittlichen Zuwachs von 24\% spürbar ab, im Jahr 2004 wurde lediglich ein Nutzerwachstum von 4\% verzeichnet. ${ }^{284}$ Den derzeit rund 35,7 Mio. Internetnutzern in Deutschland steht weltweit ein Angebot von mehr als 17 Mio. Hosts, davon 2,5 Mio. aus Deutschland, zur Verfügung. ${ }^{285}$ Zur Bemessung der Reichweite einzelner Angebote werden sowohl nutzerbezogene Maße als auch nutzungsbezogene Maße verwendet. Zu den fünf hinsichtlich der Nutzerzahlen größten Anbietern aus Deutschland gehören derzeit T-Online mit 6,6 Mio. Besucher, Google mit 6,3 Mio., gefolgt von MSN.de mit 5,6 Mio., Web.de mit 5,2 Mio. und Freenet.de mit rund 5 Mio. Besuchern. ${ }^{286}$ Zur den am häufigsten genutzten Inhalten gehört das Angebot von Bild.t-online.de mit monatlich knapp 240 Mio. abgerufenen Seiten, RTL.de mit 234 Mio. Seiten, gefolgt von Spiegel Online mit 112 Mio. Seiten, Sat1 Online mit 103 Mio. und Focus Online mit 97,3 Mio. Seiten. ${ }^{287}$

Das Internet zeichnet sich aufgrund der geringen Kosten einer digitalen Remission und Distribution von Onlineinhalten als ein Segment des Medienmarkts mit geringen Marktzutrittsschranken aus. Aufgrund der geringen Distributionskosten können Anbieter zudem sehr flexibel auf Änderungen der Nachfragepräferenzen oder aktuelle Geschehen sowohl auf dem Rezipienten- als auch auf dem Werbemarkt bzw. reagieren. ${ }^{288}$ Ähnlich flexibilitäts- und dadurch wettbewerbsverstärkend wirken sich die geringen nachfrageseitigen Umstellungskosten von Rezipienten und Anzeigenkunden aus.

Gemessen an den derzeitigen Vertriebs- und Werbeumsätzen in den traditionellen Medienmärkten stellt das Internet nach wie vor ein kleines Marktsegment dar. Mit 257 Mio. EUR beliefen sich die Netto-Werbeeinnahmen im Onlinegeschäft im Jahr 2003 auf wenig mehr als 1\% des Gesamtwerbeertragsvolumens der Medienbranche. ${ }^{289}$ Direkte Erlöse auf dem Online-Rezipientenmarkt besitzen lediglich ein Umsatzvolumen von 86 Mio. EUR, wobei derzeit ein dreistelliges Umsatzwachstum zu verfolgen ist. ${ }^{290}$ Dennoch wird der Markt für direkte Umsätze mit Bezahlinhalten in Deutschland nach einer Schätzung des Verbands Deutscher Zeitschriftenverleger bis ins Jahr 2005 lediglich ein Gesamtvolumen von 127 Mio. EUR erreichen. ${ }^{291}$ Die geringe Zahlungsbereitschaft von Rezipienten für Onlineinhalte hat drei Ursachen: Erstens, die traditionelle Gratismentalität im Internet, das seinen Wandel von einem Medium des freien Informationsaustauschs zwischen akademischen oder staatlichen Forschern zu einem kommerziellen Kommunikationskanal noch nicht vollständig

284 Vgl. Media Perspektive (2003), S. 83; von Eimeren/Gerhard/Frees (2004), S. 351 f.

$285 \mathrm{Vgl}$. Sjurts (2002a), S. 311.

$286 \mathrm{Vgl}$. Werben und Verkaufen (2002).

287 Vgl. Sjurts (2002a), S. 315; Werben und Verkaufen (2002).

288 Vgl. Wirtz (2001a), S. 388.

289 Vgl. von Reibnitz (2004).

290 Vgl. von Reibnitz (2004).

$291 \mathrm{Vgl}$. Ecin (2002). 
vollzogen hat. Zweitens, die duale Gebührenstruktur von Onlineinhalten für Medienzugang und Nutzungsdauer im Internet, ${ }^{292}$ wodurch der Reservationspreis für die Onlinerezeption von Inhalten sinkt. Drittens, das gebührenfinanzierte und dadurch kostenfreie Onlineangebot von öffentlich-rechtlichen Rundfunkanstalten, das insbesondere im nachfragestärksten Inhaltesegment aktueller Nachrichten im Internet in Konkurrenz zu gebührenpflichtigen Angeboten steht.

Aufgrund der (zumindest noch) geringen Bedeutung von Bezahlinhalten repräsentiert das Internet aus kommerzieller Sicht im Wesentlichen einen Werbeträger. Das zentrale Entscheidungskriterium für die Werberaumnachfrage bildet die generierbare Rezipientenreichweite der an den Werberaum gekoppelten Inhalte sowie die vom Anbieter zugrunde gelegten, in der Regel reichweitenabhängigen Werberaumtarife. Der Zugang zu Aufmerksamkeit schaffenden, internetfähigen Inhalten ist daher eine zentrale Hürde für den Marktzutritt neuer Wettbewerber im Internet. ${ }^{293}$ Als weitere strukturelle Hürde für den Marktzutritt existieren auch im Internet Skaleneffekte in Form der WerbebannerReichweiten-Spirale, analog zu den beschriebenen Effekten von AnzeigenAuflagen-Spirale der Druckmedien. ${ }^{294}$

\subsubsection{Intermediale Wettbewerbsstrukturen}

Die Frage, ob und in welchem Ausmaß die Einführung einer neuen Mediengattung in Konkurrenz zu bestehenden Übertragungsformen tritt, wurde bereits im Zusammenhang mit der Einführung des Radios und des Fernsehens kontrovers diskutiert. Weitgehende Gültigkeit, auch für die Systeme moderner Massenmedien, wurde der 1913 von Wolfgang Riepl in Bezug auf die Nachrichtenmedien des Altertums formulierte Hypothese beschieden, wonach Medien „wenn sie nur einmal eingebürgert und für brauchbar befunden worden sind, auch von den vollkommensten und höchst entwickelten niemals wieder gänzlich und dauerhaft verdrängt und außer Kraft gesetzt werden (...), sondern sich neben diesen erhalten (...). “295

Im folgenden Abschnitt wird diese auch als „Riepls Gesetz“ in der kommunikationswissenschaftlichen Literatur bekannte Hypothese insbesondere in Bezug auf einen messbaren Einfluss des Internet auf die Mediennutzung untersucht. Dabei werden zwei Indikatoren für den Überprüfung des Einflusses analysiert: Die Entwicklung des Mediennutzungsverhaltens der Rezipienten (vgl. Abschnitt 3.2.2.1) und die Entwicklung der Umsätze auf dem Werbemarkt (vgl. Abschnitt 3.2.2.2).

$292 \mathrm{Vgl}$. Mings/White (2000), S. $64 \mathrm{f}$.

$293 \mathrm{Vgl}$. Klein (2001), S. 102 und Sjurts (2002a), S. 318.

294 Vgl. Wirtz (2001a), S. 390 und Sjurts (2002a), S. 318.

295 Hagen (2003). 


\subsubsection{Entwicklung des Mediennutzungsverhaltens}

Riepls Gesetz unterstellt eine komplementäre Nutzung verschiedener Mediengattungen. Argumente, die eine solche komplementäre Mediennutzung im Onlinezeitalter plausibel erscheinen lassen, bilden Unterschiede der Rezeptionsbedingungen in Bezug auf aktive („lean forward“) und passive („lean backward“) Konsumgewohnheiten ${ }^{296}$ oder die derzeit noch zum Teil eingeschränkten Darstellungsmöglichkeiten oder technologischen Schranken digitaler Medien hinsichtlich audio-visueller Aufbereitung. Demgegenüber lassen sich drei Argumente gegen eine dauerhafte, komplementäre Nutzung traditioneller und digitaler Medien anführen: ${ }^{297}$ eine aus Nachfragesicht zunehmende Substituierbarkeit verschiedener Medienformen (1); die Veränderung der Rezeptionsgewohnheiten vom habitualisierten Lesen in Richtung einer habitualisierten Nutzung elektronischer und vermehrt digitaler Medien (2); die Verlagerung der Rezeptionsgewohnheiten in Richtung elektronischer, audio-visuell darstellender Medien (3).

(1) In der kommunikationswissenschaftlichen Literatur wird üblicherweise davon ausgegangen, dass die Medienrezeption einer Nutzerpräferenzordnung unterliegt, nach der die Vermittlung kurzer, gebündelter Nachrichten durch elektronische Medien erfolgt, während inhaltlich detaillierte Hintergrundsinformationen tendenziell durch textbasierte Medien vermittelt werden. ${ }^{298}$ Allerdings mangelt es dieser These bislang an ausreichend empirischer Fundierung. Zudem vernachlässigt diese Präferenzthese nach Sennewald die wachsende Angebotsdifferenzierung und die daraus resultierende wachsende inhaltliche Tiefe der Angebote von Rundfunk- und Onlinemedien gegenüber textbasierten Medien. ${ }^{299}$ Insofern ist zum einen von einer grundsätzlichen Indifferenz von Rezipienten hinsichtlich des konsumierten Inhalteformats sowie von einer langfristigen wechselseitigen Substituierbarkeit zwischen den verschiedenen Mediengattungen auszugehen.

(2) Eine umfassende Studie von ARD und ZDF zum Onlinenutzungsverhalten aus dem Jahr 2002 zeigt eine prägnante Spaltung der Nutzerschaft in zwei, derzeit gleich große Gruppen: ${ }^{300}$ auf der einen Seite eine Gruppe von Nutzern, "die durch ein aktiv-dynamisches Verhalten gekennzeichnet sind, die relativ häufig und kontinuierlich online sind, deren Vielseitigkeit der Nutzanwendung und deren Aufgeschlossenheit für das Medium erkennen lässt, dass sie ein fortgeschrittenes Aneingnungsniveau des Mediums erreicht haben“; auf der anderen Seite eine Nutzergruppe, „die das Medium eher selektiv (...) auf wenige Funktionen beschränkt wahrnimmt und nutzt." Als wesentliches Zuordnungskriterium von Nutzern in die genannten Gruppen ermittelte diese Untersuchung

\footnotetext{
296 Vgl. Beck (1999).

297 Vgl. Sennewald (1998), S. $112 \mathrm{ff}$.

298 Vgl. Picard (1989), S. 22 f.; Stahmer (1995), S. 69.

$299 \mathrm{Vgl}$. Sennewald (1998).

300 Vgl. Oehmichen/Schröter (2002), S. 378.
} 
die grundsätzliche Netzerfahrung eines Nutzers im Medium Internet. Insofern lassen diese Ergebnisse die Schlussfolgerung zu, dass eine zunehmende gesellschaftliche Diffusion und Gewöhnung an die vergleichbar jungen Onlinemedien, deren Bedeutung für die Planung des Zeitbudgets in Konkurrenz zu alternativen Medienformaten durch die Nutzer und eine routinierte und aktive Nutzung in ähnlichem Maße gewinnen lässt.

(3) Eine Untersuchung der allgemeinen Nutzernachfrage zeigt, dass sich im Zeitraum zwischen 1970 und 2000 die durchschnittliche Medienrezeption pro Woche über alle Mediengattungen hinweg von 221 Minuten auf 444 Minuten verdoppelt hat. ${ }^{301}$ Während bei der Nutzung von Printerzeugnissen ein leichter Rückgang festzustellen ist - die durchschnittliche, wöchentliche Lesedauer von Zeitungen sank im selben Zeitraum von 35 auf 30 Minuten, im Zeitschriftenbereich zwischen 1980 und 2000 nur unwesentlich von 11 auf 10 Minuten - erfreuten sich besonders audiovisuelle Medien einer wachsenden Nachfrage. Wie Abbildung 3-3 verdeutlicht, hatte die Diffusion der Online-Medien seit Anfang der 90er Jahre eine positiv verstärkende Wirkung auf die bereits seit Mitte der 80er Jahre bestehende Entwicklungstendenz in Richtung einer insgesamt steigenden Rezeptionsdauer bei stagnierender Nachfrage nach Printmedien.

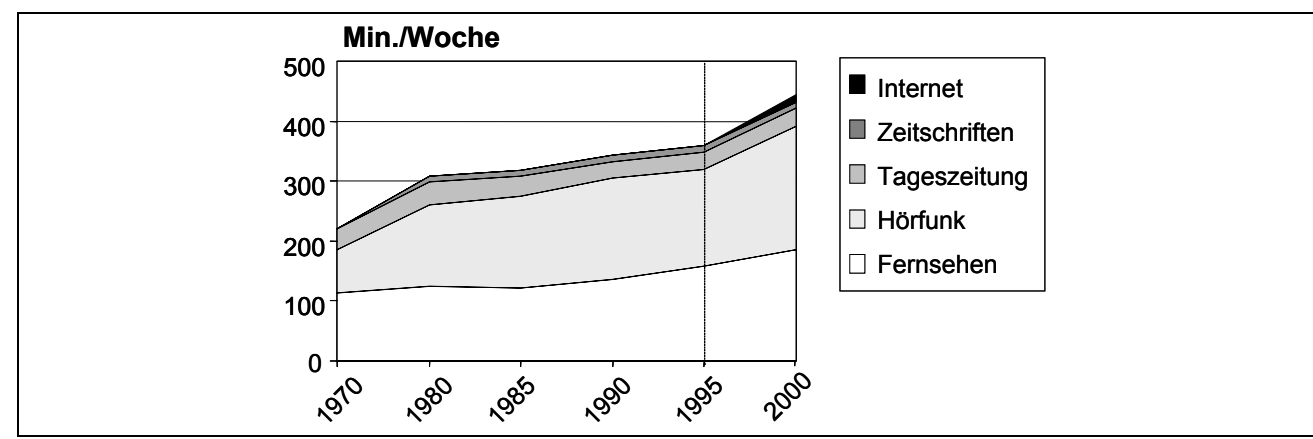

Abbildung 3-3: Entwicklung der Mediennutzung in Deutschland302

Die Rezeptionsverlagerung von Printerzeugnissen in Richtung elektronischer Medien spiegelt sich auch in der Reichweitenstatistik wieder (vgl. Abbildung 3-4): Trotz steigender Rundfunkgebühren wächst die Anzahl gemeldeter Hörfunk- und Fernsehgeräte seit den 70er Jahren nahezu unverändert um jährlich durchschnittlich rund 1,6\%. Deutlich geringer fällt mit 0,51\% das durchschnittliche Wachstum der verkauften Auflage der Zeitschriftenverlage in den vergangenen zehn Jahren aus. Im gleichen Zeitraum verzeichnete der Zeitungsmarkt sogar einen durchschnittlichen Rückgang der verkauften Auflage um jährlich 1,26\%.

\footnotetext{
301 Vgl. Media Perspektive (2002), S. 64.

302 Vgl. Media Perspektive (2004), S. 64.
} 


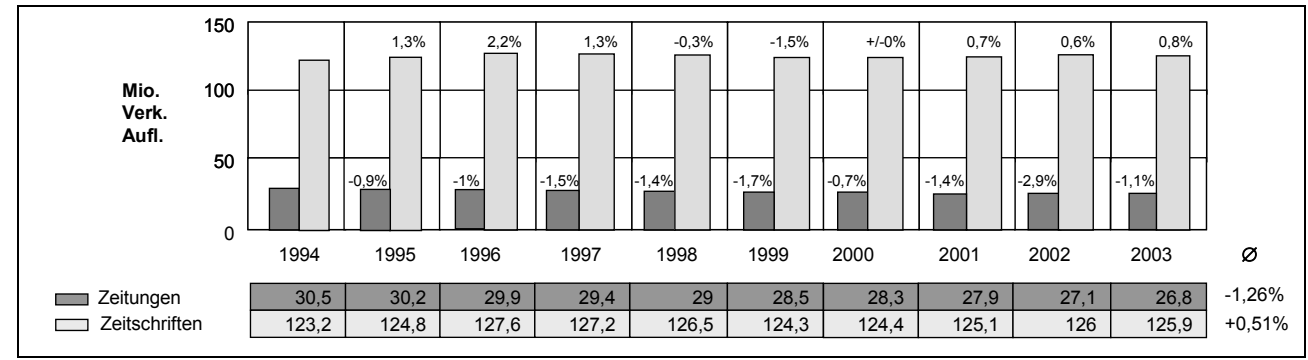

Abbildung 3-4: Entwicklung der Auflagen von Zeitungen und Zeitschriften

Zusammenfassend lassen sich keine deutlichen Substitutionseffekte etablier Mediengattungen durch das Internet feststellen. Dennoch deutet die nachweisliche Wachstumsstagnation im Printbereich, in Verbindung mit der zunehmenden Habitualisierung der Nutzung elektronischer und digitaler Vertriebsmedien insbesondere in jungen Käuferschichten, auf eine mittelfristige, demografisch bedingte Substitution hin.

\subsubsection{Umsatzentwicklung der Medienbranche auf dem Werbemarkt}

Ein im Vergleich zur Mediennutzung deutlich unterschiedliches Bild liefert die Entwicklung der Werbeumsätze in den verschiedenen Mediensegmenten. Auf dem Werbemarkt ereignete sich in den letzten Jahren eine der „schwersten Krisen der Nachkriegszeit" ${ }^{* 303}$. Diese Krise besitzt zwei Auslöser: zum einen die allgemeinen konjunkturell bedingten Sparmaßnahmen im Bereich der Werbung - als Schuldtragende der Werbemarktkrise gelten die durch Konsolidierungen stark getroffenen Unternehmen der Märkte Telekommunikation, Energieversorgung und Finanzen mit Werbeetatkürzungen im „deutlich zweistelligen Bereich“304; zum anderen, die wachsende Vielfalt und das damit einhergehende Überangebot an Mediengütern.

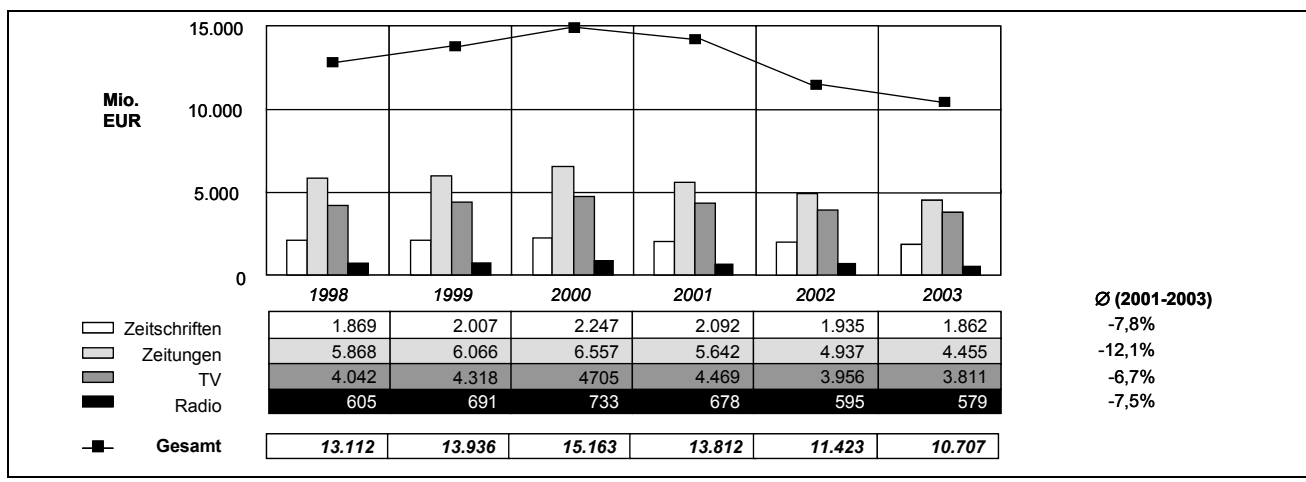

Abbildung 3-5: Entwicklung der Werbeeinnahmen305

303 Manager-Magazin (2002).

304 Horizont 04/2003, S. 34.

$305 \mathrm{Vgl}$. Heinrich (2001a) und ZAW (2004). 
Gegenüber dem Allzeithoch von über 15 Mrd. EUR im Jahr 2000 brach die Gesamtnachfrage nach Werberaum in der Medienbranche im Folgejahr um knapp $9 \%, 2002$ nochmals um 4,4\% ${ }^{306}$, ein. Ein vollkommen gegensätzliches Bild liefert die Entwicklung der Werbeumsätze im Onlinebereich, die 2002 im Vergleich zum ohnehin über $20 \%$-igen Wachstum im Vorjahr sogar noch zulegen konnte - wenngleich auch bei einem insgesamt niedrigen Umsatzvolumen von 185 Mio. EUR im Jahr 2001 bzw. 224 Mio. in 2002. Abbildung 3-6 verdeutlicht die Nachfrageentwicklungen für die einzelnen Branchensegmente.

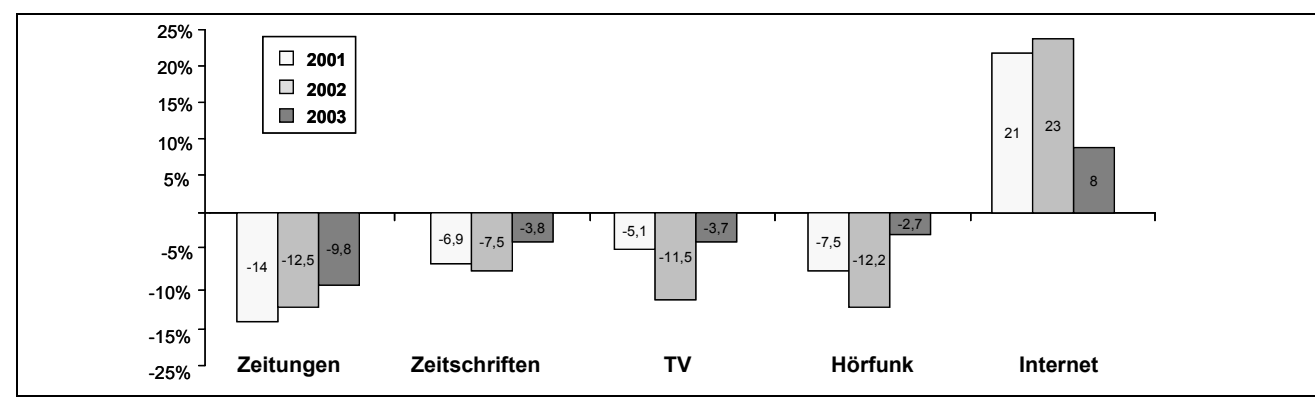

Abbildung 3-6: Veränderungsraten des Werbemarktes307

Die Ableitung einer eindeutigen Beziehung zwischen der Entwicklung des Werbemarkts zwischen traditionellen und Onlinemedien ist dennoch problematisch: Zum einen ist die langfristige Entwicklung der Zuwachsraten der Onlinewerbung strittig - Experten propagieren ein Erreichen der 2\%-Marke am Umsatzgesamtvolumen der Medienwerbung kontrovers im Zeitraum zwischen 2004 und $2006 .{ }^{308}$ Zum anderen zeichnet sich, ohne eine nachweisliche Existenz etwaiger gegenläufiger Veränderungen der Werbenachfrage im Onlinegeschäft, eine langsame Erholung der Nachfrage am Werbemarkt in den traditionellen Mediensegmenten ab. ${ }^{309}$ Eine dauerhafte Substitutionsbeziehung im derzeitigen Ausmaß der dargestellten Schere zwischen dem Rückgang der Werbeumsätze in den traditionellen Mediensegmenten und dem Wachstum der Onlinewerbung scheint daher langfristig unwahrscheinlich.

\subsubsection{Bewertung der Wettbewerbsstrukturen in der Medien- branche}

Ein segmentübergreifener Blick auf die Wettbewerbssituation in den Medienmärkten liefert das Bild weitgehend gesättigter Märkte mit hoher Wettbewerbsintensität, sowohl in den Rezipienten- als auch Werbemärkten. Der hohe Marktsättigungsgrad ist insbesondere im Bereich des Inhalteangebots in gedruckter Form zum einen abzulesen an einer Stagnation des absoluten Reich-

\footnotetext{
306 Vgl. Horizont 04/2003, S. 36.

307 Vgl. VDZ (2003) und Media Perspektive (2002), S. 65.

$308 \mathrm{Vgl}$. Meyer-Lucht (2002).

309 Vgl. Horizont 04/2003, S. 36.
} 
weitenwachstums. Zum anderen sind die dargestellten Marktsegmente gekennzeichnet durch ein vielfältiges Angebot mit steigendem Differenzierungsgrad. Die Folge aus absoluter Reichweitenstagnation und zunehmendem Differenzierungsgrad ist ein zwangsläufiger Reichweitenverlust einzelner Mediengüter. Dies bewirkt eine zunehmende Machtverlagerung zu Gunsten der jeweiligen Nachfragergruppen im Rezipienten- und Werbemarkt.

Auf dem Rezipientenmarkt lässt sich eine Verlagerung der Mediennutzung in Richtung audio-visueller Medien und speziell multimedialer Angebote nachzeichnen. Die zunehmende Habitualisierung der Nutzung elektronischer Medien, die sinkenden Kapazitätsschranken der digitalen Informations- und Kommunikationstechnik, die steigende Bedienungsfreundlichkeit digitaler Endgeräte und die grundsätzlichen Vorteile digitaler Informationsdistribution wie Zugriffsschnelligkeit oder die Möglichkeit zu interaktiver Vertriebsgestaltung konstituieren die wachsende Bedeutung digitaler Medien, gleichermaßen für den inhaltlichen Rezipienten-, als auch den Werbemarkt. Eine signifikante Substitutionsbeziehung zwischen traditionellen und digitalen Medienmärkten ist, wie dargestellt, zwar derzeit nicht nachzuweisen, jedoch aufgrund der geringen Akzeptanz von Inhalten in gedruckter Form bei jüngeren Nachfragergruppen demografisch langfristig absehbar.

Insgesamt wettbewerbsverstärkend wirkt sich die allgemeine konjunkturelle Schwäche auf die Medienmärkte aus. Eine wesentliche Rolle für den Grad der Betroffenheit von der allgemeinen Rezession spielt das Ausmaß der jeweiligen Erlösabhängigkeiten der Mediensegmente vom Werbemarkt. Die Rezession hat insbesondere den Markt für überregionale Tageszeitungen hart getroffen, in dem ein Werbe- und Anzeigenerlösanteil von durchschnittlich $60 \%$ üblich ist. ${ }^{310}$ Die Reaktion der Branche erfolgte durch Kosteneinsparungen und Personalabbau. ${ }^{311}$ Bspw. kündigte der Süddeutsche Verlag, Herausgeber der Süddeutschen Zeitung, aufgrund von Millionenverlusten im Geschäftsjahr 2001 trotz Auflagenwachstum Mitarbeiterentlassungen in Höhe von 20\% und eine Fremdbeteiligung durch die Südwestdeutsche Medienholding in Höhe von $18 \%$ an. ${ }^{312}$

Wie bereits in Kapitel 2 dargestellt wurde, repräsentieren Mediengüter in erster Linie Werbeträger, die Aufmerksamkeit durch das Interesse an dem inhaltlichen Produktbestandteil erzeugen und vermarkten. Einen wesentlichen Erfolgsfaktor für den Branchenwettbewerb in den Medienmärkten stellt der Zugang zu vermarktungsfähigen Inhalten dar. Die Schaffung und mediengerechte Produktion von Inhalten ist kostenspielig, zeitaufwändig und eingeschränkt durch die Verfügbarkeit dazu notwendigen kreativen Potenzials. Eine etablierte Wettbewerbsstrategie in der Medienbranche bildet daher, bspw. im Fernseh- und Hörfunkmarkt mit einer wachsenden Zahl an Eigen- bzw. Vertragsproduktionen, eine intramediale, vertikal rückwärts gerichtete Integrationsstrategie zur Eindämmung möglicher Lieferantenmacht. ${ }^{313}$ Das Problem des zunehmenden Ver-

310 Vgl. Wössner (2001), S. 22 f.

$311 \mathrm{Vgl}$. Hautsch (2002b).

$312 \mathrm{Vgl}$. Handelsblatt (2003).

313 Vgl. Sjurts (2002b), S. 14 f. 
lusts durchschnittlicher Produktreichweiten kann jedoch durch eine defensive Ressourcensicherung nicht behoben werden. Vielmehr weist die aktuelle Wettbewerbsituation auf ein grundsätzliches, durch das Überangebot bedingtes Problem des Aufmerksamkeitsverlusts der traditionellen Massendistribution von Inhalten und Werbebotschaften hin. Im folgenden Kapitel werden daher Handlungsmöglichkeiten dargestellt, um dem Problem des Aufmerksamkeitsverlusts zu begegnen.

\subsection{Strategische Handlungsmöglichkeiten in der Medien- branche}

In Abschnitt 3.1 wurde gezeigt, dass die Entwicklung der technischen, gesellschaftlichen und ökonomischen Rahmenbedingungen des Wettbewerbs in der Medienbranche zu Veränderungen in den Möglichkeiten der Erzeugung und der Distribution von Inhalten führen. Die Situationsanalyse des Medienwettbewerbs in Abschnitt 3.2 machte deutlich, dass die bestehenden Medienmärkte durch einen hohen Sättigungsgrad und hohe Wettbewerbsrivalität unter den konkurrierenden Anbietern gekennzeichnet sind. Die Folge dieser Wettbewerbssituation bilden einerseits sinkende durchschnittliche Produktreichweiten und andererseits schrumpfende Einnahmen sowohl auf dem Rezipienten- als auch auf dem Werbemarkt. Dennoch konnte nachgewiesen werden, dass die Nachfrage nach Inhalten der Medienbranche aufgrund eines wachsenden Informationsund Unterhaltungsbedarfs insgesamt ansteigt.

Im Folgenden wird gezeigt, dass sich aus Sicht eines Medienunternehmens, dessen traditionelles Kerngeschäft im Bereich der Inhaltebündelung angesiedelt ist, ${ }^{314}$ zwei Handlungsoptionen anbieten, um der Umsatzstagnation zu begegnen (vgl. Abbildung 3-7): die Erschließung neuer Einnahmequellen durch Diversifikation der Unternehmenswertschöpfung (Abschnitt 3.3.1) oder die Erhöhung der Einnahmen im bestehenden Geschäft durch eine Steigerung des relativen Wertbeitrags eines bestehenden Kunden (Abschnitt 3.3.2). Diversifikationsmöglichkeiten bilden einerseits vorwärts- und rückwärtsgerichtete vertikale Integrationsformen der Wertschöpfung in bestehenden Vertriebsmärkten sowie andererseits im Angebot von Leistungen außerhalb des bestehenden ProduktMarkt-Spektrums, entweder im Bereich des Medienzugangs oder durch mehrfache Verwertung der erzeugten Inhalte in verschiedenen Absatzkanälen. Bestehende Kunden eröffnen dagegen dann einen Mehrwert, wenn entweder die Erzeugung der Ihnen angebotenen Leistung durch Nutzung von Ressourcensynergien einen geringeren Ressourcenverbrauch aufweist, oder wenn diese Kunden für ein differenziertes Angebot einen höheren Betrag zu zahlen bereit sind. ${ }^{315}$

\footnotetext{
314 Vgl. Kapitel 2.
}

315 Die zur besseren Systematisierung strategischer Handlungsmöglichkeiten vorgenommene Trennung zwischen Mehrfachverwendung von Inhalten und den Möglichkeiten einer Umsatzerhöhung durch Differenzierung wird in der Praxis in dieser getrennten Form kaum anzutreffen sein. Vielmehr ermögli- 


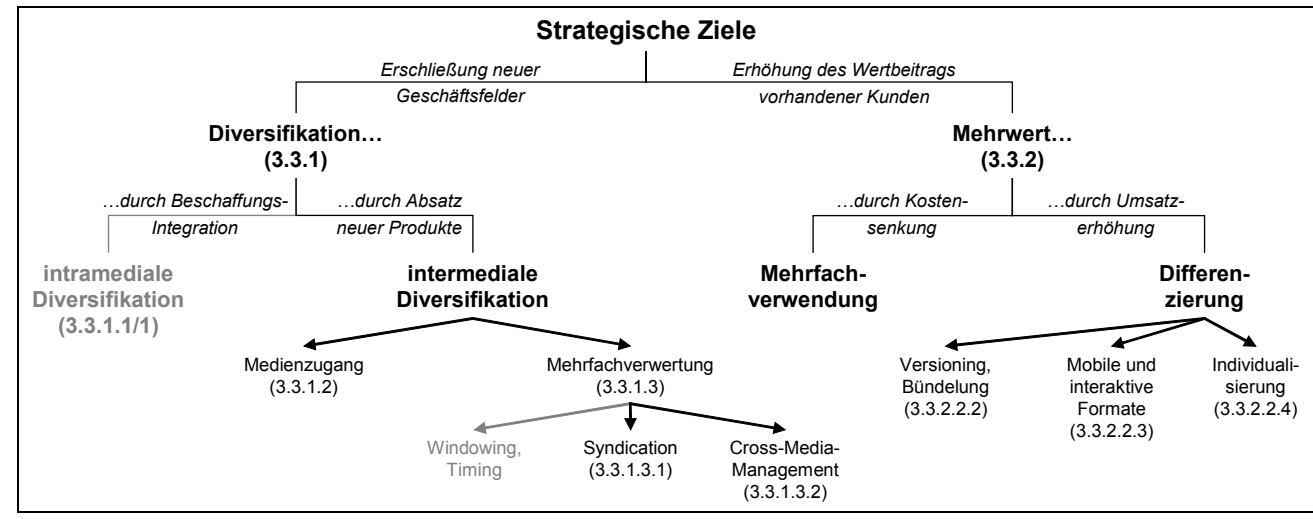

Abbildung 3-7: Strategienspektrum aus Sicht der Medienbranche

\subsubsection{Diversifikationsstrategien in der Medienbranche}

Als Diversifikationsstrategien sind Aktivitäten zu verstehen, die Wertschöpfung des Unternehmens in Produkt-Markt-Bereiche auszudehnen, die bisher vom Unternehmen nicht abgedeckt wurden. ${ }^{316}$ Diversifikationsstrategien können zum einen differenziert werden nach dem Verwandtschaftsgrad der für den neuen Wertschöpfungsbereich notwendigen Ressourcen im Verhältnis zu den bestehenden Unternehmensressourcen in verwandte bzw. unverwandte Diversifikation, zum anderen nach dem Verhältnis von Ausgangs- und Zielbranche in vertikale, auf dieselbe Branche gerichtete Entscheidungen, horizontale, auf andere Zielbranchen gerichtete Aktivitäten und diagonale, auf sowohl andere Zielbranchen als auch andere Wertschöpfungsstufen bezogene Vorhaben. ${ }^{317}$

Abschnitt 3.3.1.1 gibt einen Überblick über die grundsätzlichen Möglichkeiten eines Medienunternehmens zur Diversifikation seiner Wertschöpfung. Abschnitt 3.3.1.2 erläutert die Möglichkeit des Angebots von Dienstangeboten für den Zugang zu Onlinemedien durch ein Medienunternehmen. Abschnitt 3.3.1.3 stellt Ausprägungsformen einer Diversifikation durch mehrfache Verwertung von Inhalten vor.

\subsubsection{1 Überblick über Diversifikationsmöglichkeiten eines Medien- unternehmens}

Vor dem Hintergrund der in Kapitel 3.1 dargelegten Konvergenzeffekte im Umfeld des Medienwettbewerbs können Diversifikationsentscheidungen von Me-

chen gerade die Kostensenkungspotenziale einer Mehrfachverwendung von Inhalten eine verstärkte Differenzierung des Angebots. Vor dem Hintergrund der zugrunde liegenden Thematik dieser Arbeit wird daher die Option der Mehrfachverwendung von Inhalten konkret im Zusammenhang mit den Möglichkeiten einer Individualisierung von Mediengütern in Abschnitt 3.3.2.2.4 behandelt.

316 Vgl. u.a. Kieser/Kubicek (1992), S. 230 f. und Picot/Diet//Franck (1997), S. 256.

317 Vgl. Kieser/Kubicek (1992), S. 231 und Sjurts (2002a), S. 344 f. 
dienunternehmen aus drei Perspektiven begründet sein (vgl. Abbildung 3-8): eine vertikale, verwandte Diversifikation durch Rückwärtsintegration in zusätzliche Marktbereiche der Inhalteerzeugung (1); eine horizontale, unverwandte Diversifikation in den Marktbereich des digitalen, netzgestützten Medienzugangs (2); eine vertikale, unverwandte Diversifikation in den Bereich des digitalen Medienvertriebs durch Mehrfachverwertung gebündelter Inhalte (3).

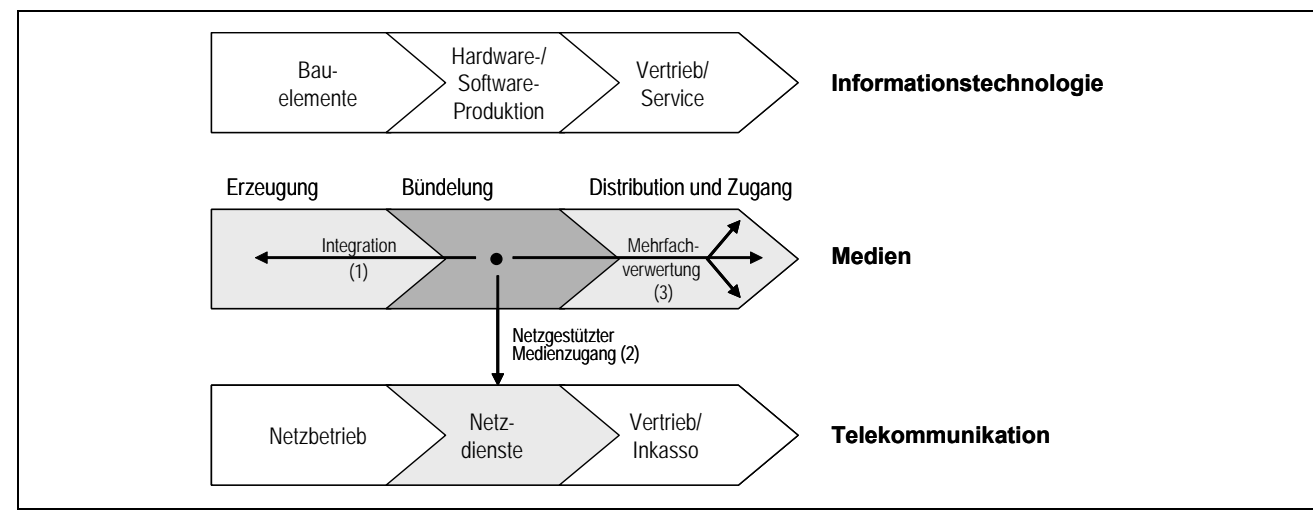

Abbildung 3-8: Konvergenzbedingte Diversifikationsmöglichkeiten aus Sicht der Medienbranche

(1) Wie bereits in Abschnitt 3.2.3 angesprochen, bildet der Zugang zu vermarktungsfähigen Inhalten einen zentralen Erfolgsfaktor im Wettbewerb um die Rezipientenaufmerksamkeit. Dass sich Medienunternehmen dieses Erfolgsfaktors bewusst sind, spiegelt die Formierung von Medienkonzernen mit hohem vertikalem Integrationsgrad in den letzten Jahren wieder. Mit der Marktkonsolidierung von sechs global agierenden Medienkonzernen, AOL Time Warner, Walt Disney Company, Vivendie Universal, Viacom, Bertelsmann und News Corporation hat diese Integrationswelle zurzeit ein vorläufiges Ende gefunden. Daher wird diese Diversifikationsform im Folgenden nicht gesondert betrachtet. ${ }^{318}$

(2) Digitalisierung und Vernetzung führen, wie in Abschnitt 3.1.2.2 gezeigt wurde, zu einer Annäherung von Medien- und Telekommunikationsbranche. Im Bereich des digitalen, netzbasierten Medienzugangs entstehen neue Wertschöpfungsmöglichkeiten, die außerhalb des ursprünglichen Produkt-Markt-Spektrums von sowohl Medien- als auch Telekommunikationsbranche liegen. Neben dem physischen und logischen Zugang zum Datennetz bietet sich die Möglichkeit der Vermarktung von Kontextinformationen im Rahmen der netzbasierten Inhaltenachfrage durch das Angebot von Portalen und Aggregationen. Die Möglichkeit einer derartigen, aus Mediensicht vertikalen, unverwandten Diversifikation wird in Abschnitt 3.3.1.2 untersucht.

(3) In Abschnitt 3.1.2.1 wurden neue Möglichkeiten der digitalen Erzeugung und Bündelung von Inhalten als Ergebnis konvergierender Technologieentwicklung aufgeführt. Von besonderer Bedeutung sind in diesem Zusammenhang neue DV-technische Inhalteformate, bspw. auf der Grundlage der Auszeichnungs-

$318 \mathrm{Vgl}$. Sjurts (2002a), S. $364 \mathrm{ff}$. 
sprache $\mathrm{XML}$, die es erlauben, ein einmalig erzeugtes Mediengut mehrfach in unterschiedlichen Distributionskanälen zu verwerten. Abschnitt 3.3.1.3 zeigt Möglichkeiten einer solchen Mehrfachverwertung im Sinne einer horizontal unverwandten Diversifikation auf.

\subsection{2 Diversifikation von Medienunternehmen durch das Angebot von Medienzugangsdiensten}

In dem Maße, in dem digitale Kommunikationsmedien die Rolle von Träger bzw. Übertragungsmedien von Inhalten im Rahmen der Massenkommunikation übernehmen, kommt es zur bereits angesprochenen Annäherung der traditionellen Wertschöpfungsbereiche von Telekommunikations- und Medienbranche im Bereich der Inhaltedistribution auf der Grundlage digitaler Kommunikationsnetze. Die Onlinedistribution von Inhalten schafft dabei zwei neue Leistungssegmente, die tendenziell außerhalb des typischen Produkt-Markt-Spektrums von Medien- und Telekommunikationsbranche stehen: Das Angebot von Netzzugangsdienstleistungen (1) und das Angebot von inhaltlichen Informationskontexten zur Unterstützung der Navigation und Rezeption von Onlineinhalten (2). ${ }^{319}$

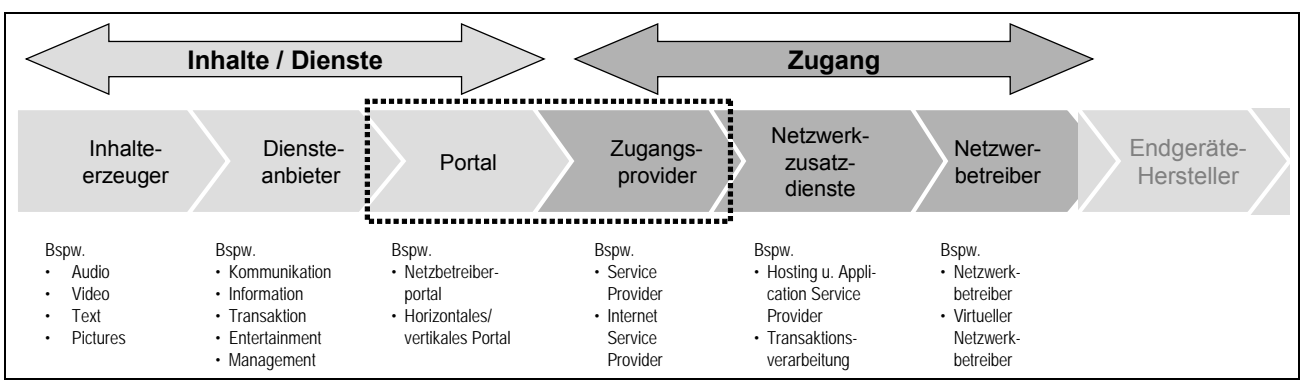

Abbildung 3-9: Annäherung von Medien- und Telekommunikationsanbieter 320

(1) Um digitale Kommunikationsnetze als Träger publizistischer Massenkommunikation nutzen zu können, muss die Leistungskette des typischerweise von Telekommunikationsunternehmen abgewickelten Netzbetriebs und -zugangs um solche Leistungen erweitert werden, die einen logischen Datennetzzugang von sowohl Inhalteanbietern als auch -Rezipienten gewährleisten. Abschnitt 3.3.1.2.1 untersucht das Angebot von Zugangsdienstleistungen für Onlineinhalte und bewertet Handlungsmöglichkeiten aus Sicht der Medienbranche.

319 Im Hinblick auf den Verwandtschaftsgrad des Angebots von Informationskontexten mit dem typischen Leistungsspektrum von Medien- und Telekommunikationsunternehmen ist anzumerken, dass das Angebot von Kontextinformationen, bspw. in Form von Branchenführern oder Telefonbüchern, auch zuvor schon durch Unternehmen dieser Branchen vereinzelt wahrgenommen wurde. Im Kern stellt jedoch das Angebot von Portalen oder Aggregaten für die meisten Unternehmen dieser Branchen eine notwendige Erweiterung ihres Leistungsspektrums dar, insofern entweder Telekommunikationsunternehmen ihr Verzeichnisangebot um weiterführende Inhalte erweitern müssen oder Medienunternehmen über das eigene Inhalteangebot hinausgehende Verzeichnisstrukturen aufbauen müssen.

320 In Anlehnung an: Gregg (2001), S. 38. 
(2) Die Inhaltedistribution in digitalen Kommunikationsnetzen erfolgt durch die logisch eindeutige Ressourcenadressierung der IP-Adresse, sowie durch relative Verknüpfungen zwischen einzelnen Inhalten nach dem Hypertextprinzip. Die Logik der Adressierung ist sowohl vom Speicherort, als auch von der inhaltlichen Bedeutung einer Inhaltsressource unabhängig. ${ }^{321}$ Daher kann ein Inhalt nur dann rezepiert werden, wenn entweder seine exakte Adresse bekannt ist, oder der Rezipient Hypertextverknüpfungen folgt, die in von ihm bekannten Inhalten hinterlegt sind. Dies eröffnet ein Marktpotenzial für strukturierte, bspw. nach inhaltlichen Kriterien vordefinierte Adresskataloge als Kontextinformationen der Rezeption von Onlineinhalten. In Abschnitt 3.3.1.2.2 werden Angebotsformen für Rezeptionskontexte zur Unterstützung von Navigation und Auffinden von digital vertriebenen Inhalten vorgestellt.

\subsection{Das Angebot von Zugangsdiensten für Onlineinhalte}

Im Zuge der veränderten Rolle digitaler Kommunikationsnetze als inhaltliche Trägermedien erweitert sich das Funktionsspektrum von Kommunikationsnetzen von der Gewährleistung von Individualkommunikation um Aspekte der Bereitstellung von Inhalten im Sinne der Massenkommunikation. Eine Distribution von Inhalten über digitale Kommunikationsnetze erfordert Dienstleistungen im Zusammenhang mit dem sowohl logischen als auch physischen Netzzugang. $\mathrm{Zu}$ diesen Leistungen gehören bspw. eine konfliktfreie Zuteilung eindeutiger Internet- (IP-) Adressen für zu distribuierende Inhalte oder die Bereitstellung garantierter Übertragungsbandbreiten. Derzeit können zwei Geschäftsformen im Bereich des inhaltlichen Medienzugangs unterschieden werden: erstens, das Angebot des technischen Netzwerkzugang durch sog. "Internet Service Provider; zweitens, die an einen technischen Zugang geknüpften inhaltlichen Zusatzleistungen wie Einstiegsportale und Kommunikationsdienste, die typischerweise angeboten durch sog. "Online Service Provider" angeboten werden. ${ }^{322} \mathrm{Im}$ Bereich der Zugangsdienstleistung existieren drei denkbare Verkaufsmodelle: ${ }^{323}$ der Vollanschluss über eine eigene Leitung mit monatlicher Grundgebühr zuzüglich zeit- oder volumenabhhängiger Nutzungsentgelte; eine rein zeitabhängige Abrechnung ohne vorherige Anmeldung sowie eine zeitabhängige oder pauschale Abrechnung nach vorheriger Dienstregistrierung.

Das branchentypische Ressourcenprofil im Telekommunikationsgeschäft zeichnet sich einerseits durch besitzbasierte Ressourcen im Bereich physischer Übertragungsnetze sowie einer kritischen Kundenmasse, andererseits durch Kompetenzen im Bereich von Netz- und Kundenmanagement aus. ${ }^{324}$ Wie Klein ausführt, führen der hohe Wettbewerb in der Telekommunikationsbranche aufgrund der zunehmenden Sättigung der Kommunikationsmärkte sowie der

\footnotetext{
321 Theoretische Fortentwicklungen einer semantischen Ressourcenadressierung werden unter dem Begriff „Semantic Web“ zusammengefasst, besitzen jedoch derzeit noch kaum praktische Bedeutung im Bereich der Massenkommunikation. Vgl. Daconta/Obrst/Smith (2003).

322 Vgl. Wirtz (2001b), S. 256 und Klein (2001), S. 99 f.

$323 \mathrm{Vgl}$. Klein (2001), S. 99.

324 Vgl. Thielmann (2000), S. 80.
} 
Preisverfall im Bereich des Internetzugangs zu einer Bedrohung rein verbindungsabhängiger Geschäftsmodelle von Dienstanbietern im Internet. ${ }^{325} \mathrm{Die}$ Erweiterung um inhaltebezogene Dienste ist für eine langfristige wettbewerbsstrategische Differenzierung zur Vermeidung eines Preiswettbewerbs notwendig. ${ }^{326}$ Aus Sicht der Telekommunikationsbranche repräsentieren Inhaltsangebote demnach Systemgüter, die einen Zusatznutzen als strategischen Erfolgsfaktor des Wettbewerbs im Geschäftsbereich des Netzzugangs ermöglichen.

Für Medienunternehmen in traditionellen Geschäftsbereichen, speziell in nicht-elektronischen Mediensegmenten wie Zeitschriften oder Zeitungsmärkten, ist die Produktion des Trägermediums lediglich von nachrangiger strategischer Bedeutung. Zur Realisierung von Verbundeffekten in der Produktion bzw. zur Reduktion von Zulieferabhängigkeiten ist ein hoher, vertikaler Integrationsgrad branchentypisch. ${ }^{327}$ Die Frage des Medienformats sowie der Zugang zum Vertriebsnetz nimmt im Rahmen der elektronischen, insbesondere der digitalen Verwertung in dreifacher Hinsicht eine zentrale Bedeutung ein:

Erstens bildet aus Sicht eines Medienunternehmens der Betrieb und Zugang zum digitalen Kommunikationsnetz eine notwendige Systemleistung für die Konsumierbarkeit online vertriebener Mediengüter. ${ }^{328}$ Zweitens spielt die Auswahl und Produktion des Medienformats, bspw. in Bezug auf den Detaillierungsgrad der Modularisierung im Rahmen der Mikrostruktur oder die Klassifizierbarkeit und Kombinierbarkeit, eine entscheidende Rolle für die Möglichkeiten einer erlöswirksamen Mehrfachverwertung wie Syndizierung oder Target-Advertising. ${ }^{329}$ Drittens bildet häufig erst die Abwicklung des materiellen Medienzugangs für Kunden, im Falle digitaler Onlinemedien eine Zugangsdienstleistung zu digitalen Kommunikationsnetzwerken, die Voraussetzung, sowohl eine breite potenzielle Rezipientenvielfalt in der Inhaltsvermarktung zu erreichen, als auch kundenspezifische Informationen wie Personalien oder Konsumpräferenzen zu erwerben.

Die Diversifikation von Medienunternehmen in den Bereich des Netzzugangs ist jedoch mit einem gewissen Risiko behaftet. Wie Picot/Diet//Frank feststellen, ist eine Unternehmensdiversifikation in Bereiche, die mit dem ursprünglichen Stammgeschäft unverbunden ist, in vielen Fällen ineffizient. ${ }^{330}$ Ursache solcher Ineffizienzen bilden in der Regel fehlende kritische Unternehmensressourcen für das diversifizierte Geschäftsfeld. Aus Sicht eines Medienunternehmens bilden Ressourcen, die für den Markt des Netzzugangs neu erworben werden müssen, bspw. Fähigkeiten im Bereich der Abrechnung von Netzleistungen sowie Kenntnisse im Bereich der Zuteilung und Steuerung von Übertragungskapazitäten.

\footnotetext{
325 Vgl. bspw. Ecin (2003).

326 Vgl. Klein (2001), S. 102.

327 Vgl. Sjurts (2002b), S. 13 und Sjurts (2002a), S. 348.

328 Zum Begriff des Systemguts, vgl. Taschner (2001).

$329 \mathrm{Vgl}$. Abschnitt 3.3.1.3.2 und Abschnitt 3.3.2.2.4.

$330 \mathrm{Vgl}$. Picot/Diet//Frank (1997), S. 256.
} 


\subsection{Das Angebot von Rezeptionskontexten für Onlineinhalte}

Die grundsätzliche Modularität digital erzeugter Inhalte ermöglicht, wie angesprochen, eine getrennte Vermarktung der Inhaltselemente von strukturbezogenen Informationen zu Klassifikation und Systematik, also den strukturellen Inhaltekontexten. ${ }^{331}$ Nach Böning-Spohr/Hess (2000) eröffnen sich für die Vermarktung solcher Informationskontexte im Rahmen einer Bündelung von Inhalten in der digitalen Verwertungskette zwei denkbare Geschäftsmodelle: ${ }^{332}$ Erstens, das Angebot von Portalen zur Navigationsunterstützung des Nutzers durch eine Bündelung von Inhaltsadressen. Zweitens, die materielle, inhaltliche Neukombination einzelner Inhaltselemente durch so genannte Aggregatoren.

Wesentlich im Falle sowohl von Portal als auch Aggregator ist eine strukturelle Veränderung von bereits in gebündelter Form vorliegenden Informationsgütern. Nach Brandtweiner lassen sich Informationsgüter generell als Produktbündel interpretieren. ${ }^{333}$ Diese Bündelinterpretation kann nach Rawolle bei digital erzeugten Formaten sowohl aus einer Makro- als auch aus einer Mikrostruktur bewertet werden. ${ }^{334}$ Während die Perspektive der Makrostruktur ein Informationsgut im Sinne geschlossener Medienobjekte, also in Form eines Textes, eines Bildes oder einer Audio-Sequenz als kleinste betrachtete Einheit versteht, repräsentiert die Mikrostruktur die Zerlegung solcher Objekte in einzelne Inhaltselemente. Die Perspektive der Mikrostruktur kann verdeutlicht werden anhand des Beispiels eines textbasierten Informationsguts, interpretierbar als ein Bündel aus einer Überschrift, einer Quellenangabe und einem Textkörper. Ein Portal in der Begriffsabgrenzung von Böning-Spohr/Hess zielt dabei nicht auf eine Trennung zwischen Inhalt und dem vom Autor zugrunde gelegten thematischen Kontext, also eine Neuformung der Mikrostruktur eines Medienguts, sondern bietet einen Metakontext im Sinne einer thematischen oder zielgruppenspezifischen Makrostruktur zur Kategorisierung der im Portal erfassten Inhalte. Demgegenüber bildet der Leistungsschwerpunkt im zweitgenannten Geschäftsmodell der Aggregatoren eine bewusste Re-Kontextualisierung von Inhalten. Verfügbare Inhalte werden vom Aggregator sowohl hinsichtlich ihrer inhaltlichen Mikro- als auch ihrer Makrostruktur in zielgruppenspezifische Themenkategorien zusammengeführt. Dabei kann in der Praxis zwischen Aggregatoren im engeren Sinne, die eine Bündelung für eine konkrete, abgrenzbare Zielgruppe vornehmen, und Aggregatoren im weiteren Sinne oder „Onlinediensten", die neben einem Zugang zum Daten- und Kommunikationsnetz lediglich eine grobe thematische Segmentierung in einer eigenen Startseite vornehmen, unterschieden werden. ${ }^{335}$

\footnotetext{
331 Vgl. Wirtz (2001b), S. 243.

$332 \mathrm{Vgl}$. Böning-Spohr/Hess (2000).

$333 \mathrm{Vgl}$. Brandtweiner (2000), S. 71

334 Vgl. Rawolle (2002), S. 16.

335 Vgl. Böning-Spohr/Hess (2000).
} 


\subsection{3 Diversifikation von Medienunternehmen durch Mehrfachverwer- tung von Inhalten}

Durch die Technologiekonvergenz von computergestützter Informationsverarbeitung, Netz- und Kommunikationstechnik sowie Medienerzeugung entstehen aus Sicht der Medienbranche, wie in Abschnitt 3.1.2.1 dargestellt, neue Möglichkeiten der digitalen Erzeugung und Bündelung von Inhalten. Die Digitalisierung im Rahmen der Leistungsprozesse der Medienbranche wird begünstigt durch allgemein computerunterstützte Arbeitsplätze, die Entwicklung von Datenbanken zur Speicherung und Archivierung multimedialer Inhalte sowie durch Content-Management-Systeme zur integrierten Erzeugung, Bündelung und Distribution von Inhalten.

Trotz Computer- und Systemunterstützung fallen dennoch bspw. im Bereich der Printmedien etwa 50\% der Herstellkosten eines Medienguts im Bereich der Inhalteerzeugung und Bündelung an. ${ }^{336}$ Digital erzeugte Inhalte sind jedoch anders als traditionell bzw. analog erzeugte Inhalte nicht an ein spezifisches Trägermedium gebunden, sondern können auf jedem digitalen Träger vertrieben werden. Dadurch können mit digitalisierten Inhalten zusätzliche Skaleneffekte der Distribution durch eine mehrfache Verwertung des digitalen Inhalts in verschiedenen Trägermedien, bspw. als digitale Druckvorlage einer Zeitschrift oder als Onlineausgabe der geruckten Zeitschrift, realisiert werden (vgl. Abbildung 3-10).

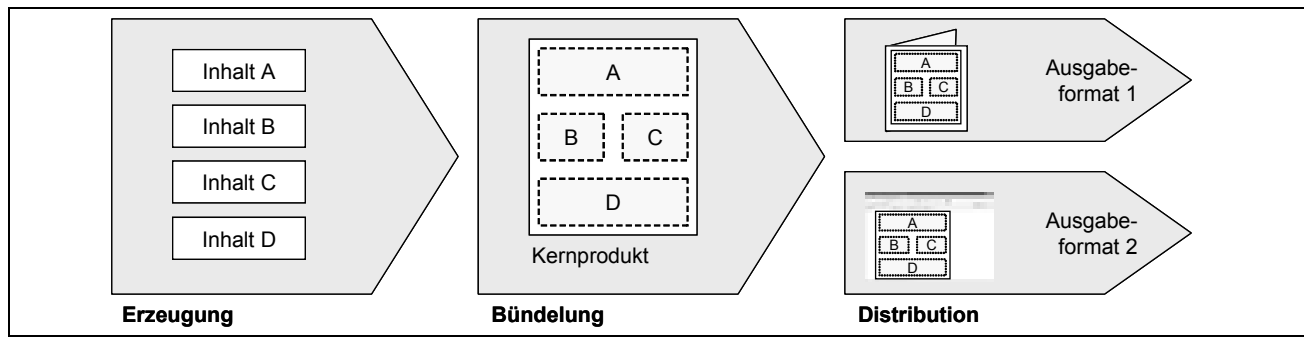

Abbildung 3-10: Prinzip der Mehrfachverwertung

Das Prinzip der Mehrfachverwertung ist in der Medienbranche, bspw. im Verlagswesen in Form einer mehrfachen Verwertung von Buchtiteln als gebundene und als Taschenbuchausgabe oder in der Filmindustrie in Form einer Verwertung von Filmen in Kino, Video, Bezahlfernsehen und Rundfunk, hinreichend bekannt und werden daher im Weiteren nicht gesondert betrachtet. Diese Spielart der Mehrfachverwertung wird auch als "Windowing“ oder "Timing" bezeichnet. ${ }^{337}$ Durch Digitalisierung von Inhalten entstehen jedoch zusätzliche Möglichkeiten der Mehrfachverwertung, die weitgehend ohne zusätzliche Erzeugungskosten realisiert werden können. In Abschnitt 3.3.1.3.1 werden zunächst technische Aspekte einer digitalen Mehrfachverwertung erläutert. In Ab-

336 Vgl. Hess (2004), S. 60.

337 Vgl. u.a. Schumann/Hess (2002); S. 74, Vizjak/Ringlstetter (2001); Schumann (2003). 
schnitt 0 werden Ausprägungsformen einer digitalen Fremd- und Eigenverwertung dargestellt.

\subsection{Technische Aspekte der Mehrfachverwertung}

In der Regel erzeugen und bündeln Medienunternehmen einzelne Inhalteelemente speziell für einen bestimmten Produkttyp, bspw. eine Zeitung, eine Zeitschrift oder eine Rundfunksendung. Dieses Vorgehen wird auch als „kernproduktorientiert" bezeichnet. ${ }^{338} \mathrm{Um}$ ein solches Kernprodukt in verschiedenen Distributionskanälen verwerten zu können, muss das gebündelte Produkt in einem medienneutralen Datenformat gespeichert werden.

Nicht jedes digitale Datenformat eignet sich gleichermaßen für den Einsatz in verschiedenen Zielmedien. Bspw. werden im Printbereich in der Regel Formate wie Quark Express, Adobe Framemaker oder Microsoft Word verwendet, während im netzbasierten Vertrieb Auszeichnungssprachen wie HTML oder WML zum Einsatz kommen. ${ }^{339}$ Als viel versprechend für eine verwendungsneutrale Datenformatierung gelten die im Umfeld der Auszeichnungssprache XML angesiedelten Spezifikationen. ${ }^{340}$ XML ist ebenso wie HTML eine strukturierte Auszeichnungssprache. ${ }^{341}$ Während jedoch die HTML-Syntax vordefinierte Datentypen zur Inhaltebeschreibung besitzt, können die Datentypen bei XML durch sog. Formattyp Definitionen (wie bspw. auf Grundlage der Document Type Definition, DTD) flexibel sowohl hinsichtlich Struktur als auch hinsichtlich inres Wertebereichs definiert werden. ${ }^{342}$ Dadurch kann die Verwendung des Inhalts eines Medienguts sowie sein Darstellungsformat unabhängig vom Inhalt durch Typ-gebundene Verwendungsanweisungen festgelegt werden.

Abbildung 3-11 zeigt schematisch die Möglichkeiten einer Mehrfachverwertung auf der Grundlage von XML am Beispiel einer textbasierten Nachricht. Zunächst werden die einzelnen Inhalte zu einer Nachricht gebündelt. Diese Nachrichtenbündelung wird im XML Format gespeichert und die Formattypdefinitionen festgelegt. Das Ausgabeformat kann bspw. durch Darstellungsanweisungen in einem Cascading Stylesheet $\left(\mathrm{CSS}^{343}\right)$ oder durch Anweisungen in der Extensible Stylesheet Language $\left(\mathrm{XSL}^{344}\right)$ festgelegt werden. Inhalte, die auf

338 Vgl. Rawolle (2002).

339 Vgl. Schumann (2003), S. 119.

340 Die Metasprache XML wurde im Februar 1998 als Standard in der Version 1.0 vom World Wide Web Consortium (W3C) verabschiedet, vgl. W3C (2004a).

$341 \mathrm{Vgl}$. Weitzel/Harder/Buxmann (2001), S. 18.

342 Vgl. Phillips (2000), S. 13; Rawolle (2002), S. 52; Hess (2004), S. 63 ff.

343 Das Cascading Style Sheet ist ein einfacher Mechanismus, um Layoutangaben und Inhalte einer Webseite zu trennen. Die Spezifikation der CSS Syntax wurde 1996 vom W3C verabschiedet, vgl. W3C (1999).

344 Die Extensible Stylesheet Language ist eine Sprachfamilie zur Definition von Layoutangaben auf der Grundlage von XML. XSL besteht aus drei Sprachbestandteilen: Die hier im Beispiel verwendete Objektformatierungssprache XSL Formatting Objects (XSL-FO), die Verweissprache XML Path Language (XPath) und die Transformationssprache XSL Transformations (XSLT). Die Spezifikation der XSLFO Syntax wurde 2001 vom W3C verabschiedet, vgl. W3C (2001). 
einem gültigen XML-Format beruhen, können automatisch in unterschiedliche Ausgabeformate transformiert werden. Dieser Vorgang wird auch als „Transcoding" bezeichnet. ${ }^{345}$ Eine solche Transformation erfolgt in der Regel im Zuge der Definition des Ausgabeformats und kann bspw. durch die zur XSL Spezifikation gehörenden Programmiersprache Extensible Stylesheet Language Transformation (XSLT) umgesetzt werden.

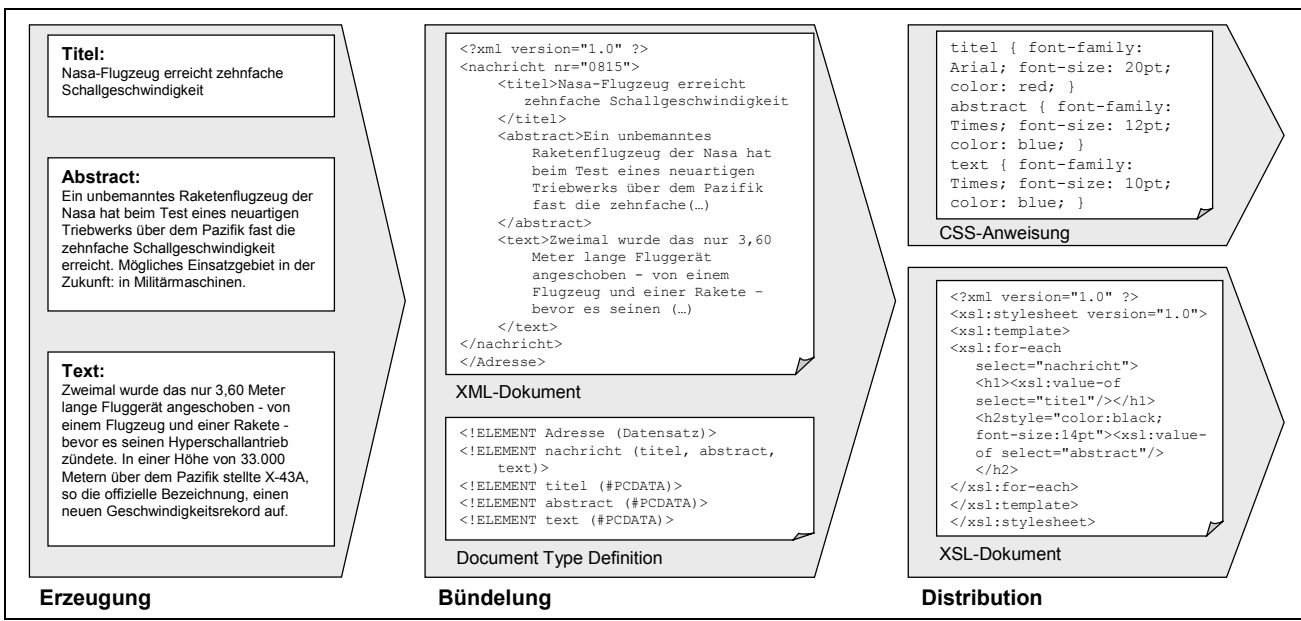

Abbildung 3-11: Mehrfachverwertung auf der Grundlage von XML

\subsection{Ausprägungsformen der digitalen Mehrfachverwertung}

Eine Mehrfachverwertung von Inhalten verursacht zusätzliche, vorwiegend fixe Kosten im Bereich der Erfassung, Gestaltung und Nachbearbeitung von Inhalten durch die notwendige Inhaltekonvertierung in ein medienneutrales Datenformat. Daher ist eine Inhaltekonvertierung nur dann sinnvoll, wenn die Konvertierungskosten durch zusätzliche Einnahmen kompensiert werden können.

Neben dem Vorteil der mehrfachen Verwertung der gebündelten Inhalte erlaubt XML zudem speziell bei textbasierten Inhalten eine modulare Inhalteverwertung durch die Definition unterschiedlicher Formattypdefinitionen. Dadurch sind medienneutrale Inhalteformate praktisch unbegrenzt innerhalb und außerhalb des Erzeugerunternehmens verwertbar. Bei den Ausprägungsformen einer digitalen Mehrfachverwertung wird daher üblicherweise zwischen Formen der Eigen- und der Fremdverwertung unterschieden. Formen der Fremdverwertung werden üblicherweise unter dem begriff „Content Syndication“ oder "Syndizierung von Inhalten" zusammengefasst (vgl. Abschnitt 3.3.1.3.2.1). Die mehrfache Eigenverwertung von Inhalten wird auch als "Cross Media Management" bzw. „Cross Media Publishing“ bezeichnet (vgl. Abschnitt 3.3.1.3.2.2).

$345 \mathrm{Vgl}$. Oesterdiekhoff (2004). 


\subsection{Content Syndication}

Content Syndication bezeichnet die Bündelung und den Vertrieb von Informationsgütern oder Verwertungsrechten an Geschäftskunden zum Zwecke einer kommerziellen Weiterverwertung. ${ }^{346}$ An die Stelle einer Einzelvermarktung von Inhalten an Endkunden tritt bei der Syndizierung der Vertrieb von Inhalten an einen Intermediär zur Weiter- oder Endvermarktung. ${ }^{347}$ Der Urheber berechnet diesem Intermediär dabei in der Regel höhere Preise als bei der Einzelvermarktung des Inhalts an Endkunden.

Syndizierung findet primär auf der Ebene der Bündelung von Mediengütern statt. Dabei wird das Modell der inhaltlichen Verwertung um die Rolle des sog. Syndikators als Handelsmittler für Inhalte erweitert. Das Erfolgspotenzial einer Syndizierung beruht auf zwei Annahmen: ${ }^{348}$ Extranets von Unternehmenskooperationen, E-Commerce Seiten im Internet oder Seiten mit Inhalten zu Spezialthemen können zum einen Mehrwerte für ihre Kunden durch Integration fremder Inhalte ermöglichen. Ein Beispiel für einen solchen Mehrwert bildet ein unabhängiger redaktioneller Produkttest auf einer E-Commerce Seite. Dadurch steigt die Nachfrage nach weiterverwertbaren Inhalten. Zum anderen sinken durch die Wiederverwendbarkeit digital und ggf. medienneutral erzeugter Inhalte die Transaktionskosten der Distribution und Integration fremder Inhalte. Diese Transaktionskostendifferenz kann durch Handelsmittler als Gewinnmarge in Form von Bündelungs- und Interaktionsdienstleistungen abgeschöpft werden.

Ergebnis ist eine theoretische Dreiecksbeziehung zwischen dem Urheber von Inhalten oder auch „Originator", dem „Syndikator" als Handelsmittler und dem Vertreiber der Inhalte oder "Distributor" im Endkundengeschäft (vgl. Abbildung 3-12). ${ }^{349}$

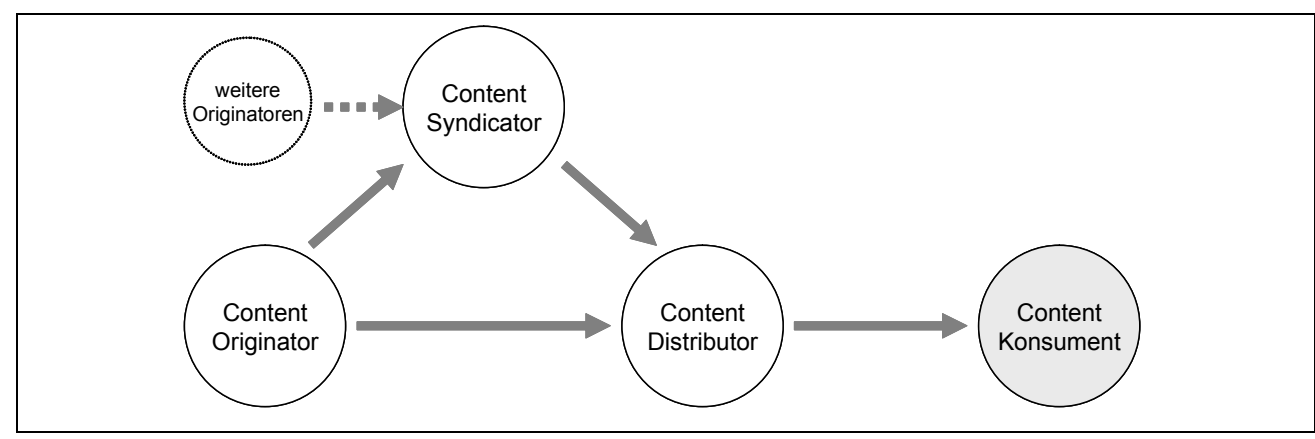

Abbildung 3-12: Organisationales Arrangement bei der Inhaltesyndizierung

$346 \mathrm{Vgl}$. Anding/Hess (2000), S. 3.

347 Die Idee der Syndizierung ähnelt dem in der Medienbranche bereits seit der zweiten Hälfte des 19. Jahrhunderts bekannten Geschäftsprinzip von Nachrichtenagenturen (vgl. Zöttl (2003)). Während Nachrichtenagenturen jedoch journalistisch weitgehend unbearbeitete aktuelle Einzelmeldungen an Medienunternehmen vertreiben, erfolgt im Rahmen der Syndizierung ein Vertrieb der Endprodukte der (ggf. journalistischen) Erzeugung und Bündelung von Inhalten durch ein Medienunternehmen an Dritte.

$348 \mathrm{Vgl}$. Hess (2004).

349 Vgl. Hess/Anding (2002), S. 167. 
In der Praxis nehmen nicht nur unabhängige Intermediäre, sondern zunehmend insbesondere Inhalteersteller und -vertreiber die Rolle eines Syndikators ein. ${ }^{350}$ Ein Beispiel für Syndizierungsangebote von deutschen Inhalteerstellern bildet das Angebot der Tomorrow-Focus AG als Syndikator der Verlagsgruppen Milchstraße und Hubert Burda. Nach einer Reihe von Unternehmenskonkursen unabhängiger Syndikatoren wie des amerikanischen Unternehmens iSyndicate oder der deutschen Unternehmen 4Content und Contonomy zeichnet sich derzeit eine zunehmende Rollenverschmelzung von Urhebern und Syndikatoren ab.

Aus technischer Sicht ist der Wert syndizierter Inhalte in erster Linie abhängig von ihrer Integrierbarkeit in fremde Angebote. Der Erfolg einer Fremdverwertung von Inhalten ist daher abhängig vom Standardisierungsgrad des Formats syndizierter Inhalte. Das Standardisierungsproblem besteht nicht nur auf syntaktischer Ebene, wofür sich entsprechend der in Abschnitt 3.3.1.3.1 angestellten Vorüberlegungen die Auszeichnungssprache XML etabliert hat, sondern insbesondere in Bezug auf die verwendete Formattypdefinition. Derzeit existieren eine Reihe unterschiedlicher Formattypdefinitionen für die Syndizierung von Inhalten auf der Grundlage von XML, wie bspw. das News Industry Text Format (NITF) ${ }^{351}$, NewsML ${ }^{352}$ oder Rich Site Summary (RSS) ${ }^{353}$.

\subsection{3.2.2Cross-Media Management}

Cross-Media Strategien umfassen alle Vermarktungskonzepte eines Unternehmens, die sich auf mindestens zwei Medienformen beziehen. ${ }^{354}$ Die Vorteilhaftigkeit von Cross-Media Strategien beruht auf zwei Annahmen: ${ }^{.35}$ Durch die

350 Vgl. Werbach (1999).

351 Vgl. IPTC (2004a): NITF ist ein Formatstandard für vor allem textbasierte Nachrichten, der bereits Anfang der 1990er Jahre von der Newspaper Association of America (APA) und dem International Press Telecommunications Council (IPTC), einem weltweiten Verband von Nachrichtenagenturen, entwickelt wurde. NTIF ist seit der Verabschiedung des XML Standards 1998 vollständig XMLkompatibel und besitzt unter anderem Datentypen zur Beschreibung des Textaufbaus sowie für Metainformationen bspw. zum thematischen Bezug oder der Versionshistorie einer Textnachricht. NITF ist der derzeit am meisten verwendete Standard für die Syndizierung von Textnachrichten.

352 Vgl. IPTC (2004b): NewsML wurde im Oktober 2000 ebenfalls vom IPTC verabschiedet. Im Unterschied zu NITF dient NewsML neben texbasierten Nachrichten auch zur Beschreibung von Inhalten verschiedener Medienformate. NewsML wird vor allem von den deutschen Nachrichtenagenturen unterstützt. Alle NTIF konformen Inhalte besitzen auch ein gültiges NewsML Format.

353 Vgl. Hammersley (2003): RSS, auch unter der Definition „Really Simple Syndication“ bekannt, wurde 1999 in der Version 0.9 von Netscape zur Synchronisation von Portalen für den Portaldienst „My Netscape Service" entwickelt. Nach einer z.T. parallelen Weiterentwicklung der Firmen Netscape und UseLand liegt RSS derzeit in der Version 2.0 vor. Die wachsende Popularität von RSS rührt vor allem von dessen einfachem Metadatenformat her, das sich besonders für kleine Endgeräte oder Email Newsletter eignet. RSS Formate können von speziellen RSS Readern, sog. „Aggregatoren“ gelesen und ausgegeben werden. Durch die Integration eines RSS Aggregators in der ersten offiziellen Version des Opensource Browsers Firefox in Form sog. "Life Bookmarks" ist darüber hinaus eine wachsende Bedeutung von RSS für die Endnutzerdistribution von Inhalten absehbar.

354 Vgl. Sjurts (2002a), S. 346; Sjurts (2002b), S. 4 f.; Müller-Kalthoff (2002), S. 20.; Hess/Schulze (2003). $355 \mathrm{Vgl}$. Hess (2004). 
zunehmende funktionale Konvergenz von speziell elektronischen Medienformen konsumieren Inhalterezipienten ähnliche Inhalte, bspw. im Verlauf eines Tages, in unterschiedlichen Medienformen. Um die Aufmerksamkeit des Rezipienten dauerhaft erregen zu können und die Gefahr einer Substitution durch den Rezipienten zu verringern, muss das inhaltliche Angebot eines Inhalteanbieters in jedem Vertriebskanal präsent sein. Eine Mehrfacherstellung und Bündelung von Inhalten spezifisch für jedes Vertriebsformat ist jedoch aufwändig und teuer.

Wie auch im Rahmen der Syndizierung bestehen bei Cross-Media Strategien aus technischer Sicht Synergiepotenziale einer mehrfachen Verwertung von Inhalten durch eine Standardisierung des Inhalteformats auf der Leistungsebene der Bündelung. Beispiele für standardisierte Datentypdefinitionen für eine Mehrfachverwertung von Inhalten im Verlagswesen bilden bspw. Open eBook $^{356}$ und DocBook ${ }^{357}$.

\subsubsection{Mehrwertstrategien für Medienunternehmen}

Eine Mehrfachverwertung von Inhalten ist in der Regel mit einem zusätzlichen Aufwand aufgrund der notwendigen Standardisierung und ggf. Konvertierung von Inhalten in ein verwertungsneutrales Format verbunden. Entsprechend ist eine digitale Verwertung von Inhalten immer nur dann erfolgreich, wenn durch eine ausreichende zusätzliche Aufmerksamkeit zusätzliche Einnahmen erzeugt werden können. Die schleppende Etablierung insbesondere von direkten Erlösen bei Onlineinhalten belegt jedoch, dass ein digitales Inhalteangebot nicht automatisch einen erlöswirksamen Wertbeitrag für Medienunternehmen erzeugt. Die Ursache dafür liegt vor allem in der mangelnden Kompensation des Lernaufwands der Nutzung von Online-

356 Vgl. OeBF (2002): Open eBook ist ein XML-basierter Standard, der 1999 durch das Open eBook Forum, einem Industriekonsortium unter Beteiligung von Microsoft, Adobe und Palm entwickelt wurde. Die Datentypdefinition des Open eBook Formats enthält Elementtypen zur Beschreibung eines gebündelten Inhalts hinsichtlich Metainformationen entsprechend dem Dublin-Core-Standard, den im Bündel enthaltenen inhaltlichen Elemente, ihrer Reihenfolge und optionalen strukturellen inhaltichen Ordnungen dieser Elemente. Das eBook Format wird zurzeit weniger als verwendungsneutrales Inhalteformat sondern insbesondere im Zusammenhang mit einer digitalen Endverwertung von Buchtiteln diskutiert. Für die Rezeption von Inhalten im eBook Format werden Software Reader wie bspw. von der Firma Adobe sowie, kommerziell jedoch weniger erfolgreich, spezielle Hardware Reader wie von der Firma Gemstar.

357 Vgl. Walsh/Muellner (2004): Das DocBook-Format wurde erstmalig 1992 von der Davenport Gruppe veröffentlicht, einem Konsortium von Herstellern technischer Dokumentationen unter Führung des amerikanischen Sachbuchverlags O'Reilly. DocBook repräsentiert einen de-facto-Standard für technische Dokumentationen, die mehrfach, bspw. als interaktive Hilfefunktionen oder gedruckte Manuale für Softwareprodukte, vertrieben werden. Das DocBook Format liefert umfangreiche Datentypdefinitionen zur Beschreibung eines Inhalts anhand von logischen und physischen Strukturinformationen (bspw. Reihe, Band oder Abschnitt), Metainformationen (bspw. Autor, Titel, Herausgeber) sowie zahlreiche, bspw. in der HTML-Syntax nicht vorgesehene Beschreibungselemente für insbesondere technische Inhalte wie Absätze, Fußnoten, Zitate oder Indizes. 
medien durch ihren Kundennutzen: ${ }^{358}$ Neben nominalen Kosten der Inhalterezeption wie dem Verkaufspreis einer Zeitung oder den Nutzungsgebühren eines Onlinemediums erfordert die Rezeption eines Inhalts aus Konsumentensicht einen Lern- und Suchaufwand in Bezug auf den Konsum des jeweiligen Distributionsformats. Vor dem Hintergrund des inhaltlichen Überangebots auf dem Medienmarkt muss ein zusätzlicher Distributionskanal daher diesen Lernaufwand durch einen spezifischen Mehrwert nicht nur ausgleichen, sondern um den jeweils erhobenen Preis überkompensieren. ${ }^{359}$

Wertorientierte Überlegungen im Zusammenhang mit einer Unternehmens- oder Kundenbewertung stehen in der Tradition der vor allem von Rappaport 1986 geforderten Orientierung des betrieblichen Führungshandelns am Unternehmenswert aus Anlegersicht, dem sog. „Shareholder Value“. ${ }^{360}$ Gegenstand solcher streng wertorientierten Betrachtungen bilden Zahlungsströme, sog. "Cash Flows“, in einem bestimmten Zeitraum. ${ }^{361}$ Der (Netto-)Beitrag eines Kunden zum Unternehmenswert kann vor diesem Hintergrund als die Summe der kundenbezogenen Ein- und Auszahlungen durch getätigte Transaktionen während der gesamten Dauer der Kundenbeziehung interpretiert werden.

Die Einzahlungen durch einen Kunden im Rahmen der von inm getätigten Transaktionen stehen in Zusammenhang mit dessen individueller Zahlungsbereitschaft für das jeweilige Produkt. Die Zahlungsbereitschaft wird durch zwei Faktoren bestimmt: dem von inm individuell wahrgenommenen Produktnutzen im Sinne eines "Gebrauchswerts" und dem objektiven Tauschwert, widergespiegelt durch den Ressourcenverzehr. ${ }^{362}$ Neben rein monetär erfassbaren Beiträgen können Kunden zusätzliche Wertbeiträge liefern, bspw. in Form von Produktbewertungen als Anregungen für Produktneuentwicklungen oder durch Empfehlungen gegenüber anderen Kunden. Entsprechend besitzen Kunden aus Anbietersicht neben einem reinen Erlöspotenzial

358 Vgl. Fink (2002). Eine zusätzliche Ursache mag darin bestehen, dass sich Onlineformate für einige Inhaltetypen schlicht als unpraktisch erwiesen haben.

359 Vgl. Brack (2003), S. 15.

360 Vgl. Rappaport (1994), S. 53 f.

361 Vgl. Meffert (1995); Wyner (1996); Krafft/Marzian (1997).

362 Vgl. Meier (2002), S. 19 f.; Eine der ersten differenzierten ökonomischen Untersuchung der wertbildenden Eigenschaften von Gütern getrennt nach Gebrauchs- und Tauschwert finden sich Bezug nehmend auf Überlegungen des englischen Staatsphilosophen John Locke von 1696 - bei Karl Marx. Nach Marx kann steht der Gebrauchswert eines Gutes für seine Nützlichkeit im Zuge seines Gebrauchs oder seiner Konsumtion durch ein Individuum (vgl. Marx (1872), S. 32). Der Tauschwert eines Gutes ist dagegen das quantitative Austauschverhältnis eines Gutes mit anderen Gütern. Die Höhe des Tauschwerts bemisst sich nach dem Umfang der zur Herstellung des Gutes aufgewendeten Ressourcen (wobei Marx das breite Spektrum denkbarer Aufwandsarten auf eine einzige Ressource, nämlich die aufgewendete menschliche Arbeit, reduziert; vgl. Marx (1872), S. 36). 
ein Ressourcenpotenzial, das dem auszahlungswirksamen Ressourcenverbrauch entgegensteht (vgl. Abbildung 3-13). ${ }^{363}$

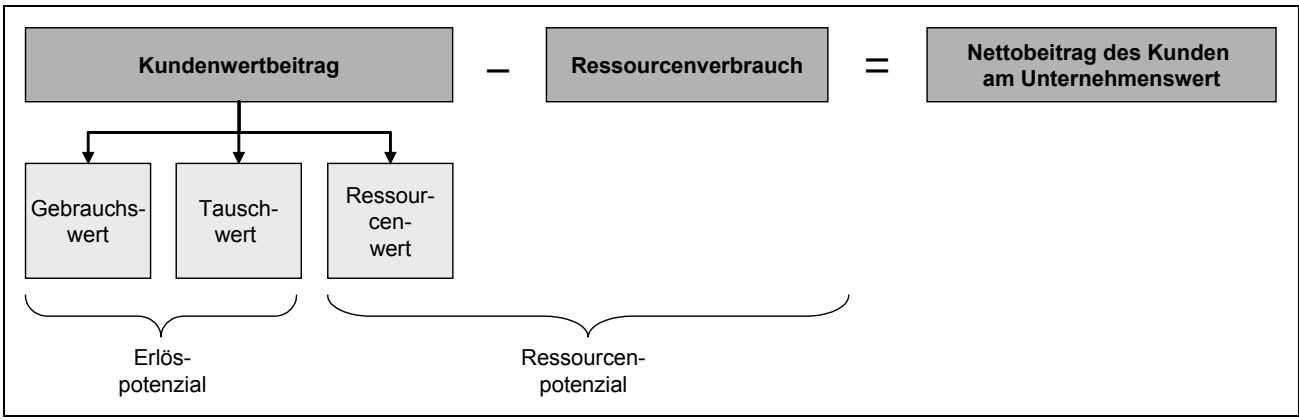

Abbildung 3-13: Beitragspotenziale des Kunden am Unternehmenswert

Ein Mediengut liefert immer dann einen relativen Beitrag für den Unternehmenswert eines Medienunternehmens, wenn dadurch entweder der erlösbezogene Kundenwertbeitrag oder das Ressourcenpotenzial des Unternehmens gesteigert wird. Zur Steigerung des erlöswirksamen Kundenwertbeitrags muss eine wettbewerbsbezogene Differenzierungswirkung gegenüber vergleichbaren Konkurrenzprodukten geschaffen werden. Die Zahlungsbereitschaft eines Kunden steigt dabei in dem Maße an, in dem das Angebot seinen Bedürfnissen entspricht. Zur Steigerung des Ressourcenpotenzials eines Medienunternehmens ist einerseits eine stärkere Integration des ressourcenwerten Kundenbeitrags und andererseits eine Reduktion des Ressourcenverbrauchs anzustreben. In Anlehnung an die im voranstehenden Abschnitt behandelten Ausprägungsformen einer mehrfachen Verwertung von Inhalten wird die Reduktion des Ressourcenverbrauchs mit dem Begriff der Mehrfachverwendung von Inhalten in Verbindung gebracht.

In Abschnitt 3.3.2.1 werden die Möglichkeiten eines differenzierten Angebots im Hinblick auf die Unterscheidung zwischen individuellem Gebrauchswert und objektivem Tauschwert anhand von mikroökonomischen Überlegungen operationalisiert. In Abschnitt 3.3.2.2 werden Möglichkeiten einer differenzierten Bereitstellung von digitalen Mediengütern zur Steigerung des Kundennutzens unter der Nebenbedingung einer mehrfachen und dadurch verbrauchsminimierenden Ressourcenverwendung erläutert.

\subsubsection{1 Ökonomische Grundlagen der Differenzierung}

Das Differenzierungsproblem lässt sich allgemein beschreiben als das „Bemühen einer Unternehmung (...), sich in der Wahrnehmung der Abnehmer von der Konkurrenz entscheidend abzuheben. ${ }^{364}$ Nach Chamberlin löst die Differenzierung das theoretische Spannungsfeld in der Erklärung von Marktstrukturen zwischen den in ihren Annahmen unrealistischen oder zumindest seltenen Phä-

\footnotetext{
363 Vgl. Meier (2002), S. 16 f.

364 Bohn (1993), S. 2. Vgl. außerdem Abschnitt 2.2.1.2.
} 
nomenen vollkommener Konkurrenz und Monopol. ${ }^{365}$ Zentrale Annahmen mikroökonomischer Untersuchungen der Differenzierungsproblematik bilden ein Wettbewerbsmarkt mit zumindest oligopolistischen Anbieterstrukturen und Nachfragestrukturen mit heterogenen Präferenzen hinsichtlich optimaler Leistungen. ${ }^{366}$ Eine Produktdifferenzierung existiert im Falle solcher Marktstrukturen immer dann, wenn die Nachfrage zwischen konkurrierenden Leistungsangeboten dieses Markts eine zumindest unvollkommene Substituierbarkeit, also Kreuzpreiselastizitäten ungleich Null aufweisen. ${ }^{367}$ Je größer der Grad der Differenzierung und damit je geringer die Kreuzpreiselastizitäten der Güter eines Markts, desto geringer die Wettbewerbsrivalität zwischen den Anbietern auf diesem Markt.

Während unter mikrökonomischer Perspektive die grundsätzliche Bedeutung und Wirkungsstruktur thematisiert wird, analysiert die wettbewerbstrategische Literatur Möglichkeiten zur Formung und Ausnutzung von Substitutionsgrenzen in der Marktnachfrage. Nach Schreyögg verfolgt eine Differenzierungsstrategie das Ziel, „bei bestimmten Produkten und deren Verwendungsmöglichkeiten Unterschiede zu schaffen; sie aus der Zone der Homogenität herauszuführen." ${ }^{368}$ Eine solche Differenzierung kann auf zwei Wegen erzielt werden: erstens, durch eine Heterogenisierung des Leistungsangebots auf der Grundlage einer Differenzierung der Merkmalskonfiguration (= Produktdifferenzierung, vgl. Abschnitt 3.3.2.1.1); zweitens, durch eine Segmentierung des Marktes entsprechend der Zahlungsbereitschaft für ansonsten homogene Güter (= Preisdifferenzierung, vgl. Abschnitt 3.3.2.1.2);

\subsection{Produktdifferenzierung}

Das Gestaltungsziel der Produktdifferenzierung bildet die Produktion und das Angebot eines Produkts, „das sich in Qualität und Service von den Konkurrenzprodukten deutlich abhebt." ${ }^{\text {"369 }}$ Eine solche Differenzierung kann unter zweifacher strategischer Zielsetzung verfolgt werden: zum einen zur Schaffung eines differenzierten Kundennutzens, zum anderen zur Verringerung des Preiswettbewerbs und zur Schaffung monopolistischer Preissetzungsspielräume. ${ }^{370}$

Das bekannteste ökonomische Modell der Produktdifferenzierung geht auf Lancaster (1979) zurück. Lancaster unterscheidet verschiedene Ausprägungsformen einer Produktdifferenzierung anhand von Unterschieden im Ressourcenverzehr der Herstellungsverfahren verschiedener Produktlinien. ${ }^{371}$ Der Ressourcenverzehr der Herstellung bestimmt dabei das objektive Nutzenniveau der im Rahmen eines Herstellungsverfahrens erzeugbaren Produktvarianten einer

\footnotetext{
365 Vgl. Chamberlin (1954), S. $76 \mathrm{ff}$.

366 Vgl. Bain (1968), S. 30.

367 Vgl. Bohn (1993), S. 14.

368 Schreyögg (1984), S. 26.

369 Bea/Haas (1995), S. 166.

370 Vgl. Brandtweiner (2000), S. 80.

371 Vgl. Lancaster (1979).
} 
Produktlinie. Eine Differenzierung gegenüber den individuellen Präferenzen auf der Nachfrageseite des Markts kann in zweierlei Form erfolgen: erstens, durch die (horizontale) Variation spezifischer Merkmale im Rahmen der Produktion qualitativ gleichwertiger Produktvarianten ( $\mathrm{X}$ und $\mathrm{Y}){ }^{372}$ zweitens, durch die Schaffung eines (vertikal) höheren objektiven Nutzenniveaus (IK') mit einem qualitativ höherwertigen Angebot ( $\left.\mathrm{X}^{\prime}\right)$ durch Veränderung von Produktionstechnologie oder Ressourceneinsatz (vgl. Abbildung 3-14). ${ }^{373}$

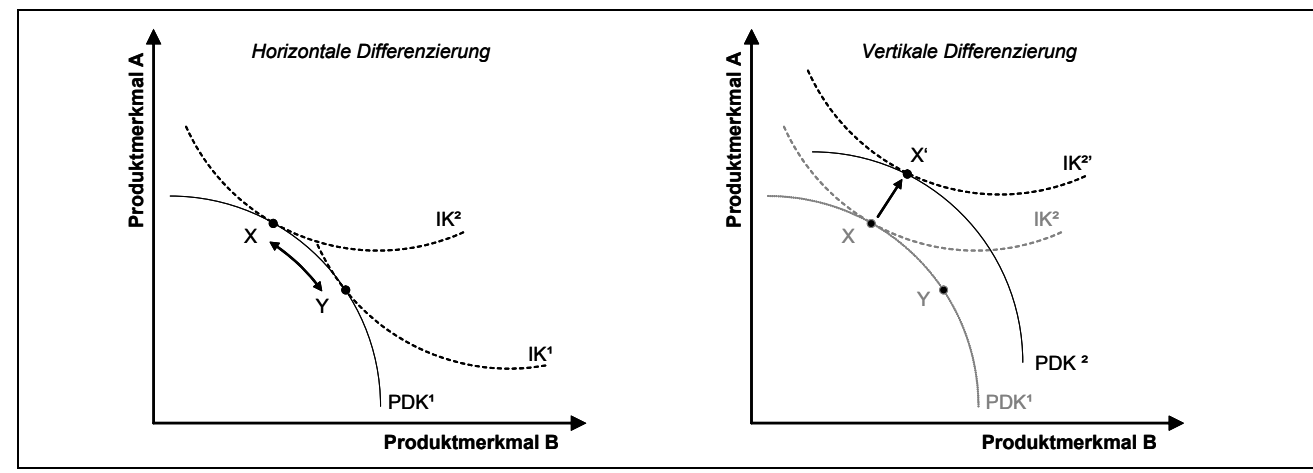

Abbildung 3-14: Horizontale und vertikale Differenzierung

Kirsch und Ringlstetter greifen das Konzept von Lancaster als „Differenzierung durch Qualität" auf, räumen jedoch ein, dass die Bewertung von Leistungen durch Kunden üblicherweise nicht eindeutig horizontal oder vertikal skalierten Präferenzmaßen unterliegt, sondern in „Merkmalsräumen“ von grundsätzlich ndimensionaler Natur erfolgt. ${ }^{374}$ Der Kunde trifft seine Kaufentscheidung aus der Vielfalt angebotener Leistungen als Nutzenmaximierer zwischen der von ihm präferierten Idealvorstellung und der empirischen Wirklichkeit des Marktangebots in Bezug auf seine Kaufkriterien. Die Nutzenbewertung erfolgt dabei durch einen zweifachen Hypothesenabgleich zwischen Wirklichkeit und Idealvorstellung: Zum einen werden über Hypothesenketten zwischen den erkennbaren Produktmerkmalen und den Idealkriterien logische Verknüpfungen erzeugt und der Funktionalnutzen bewertet. Zum anderen werden sämtliche in Verbindung mit dem Produktkonsum über die gesamte Produktlebensdauer antizipierbaren Kosten summiert und ein Wirtschaftlichkeitsnutzen bewertet.

372 Beispiel für eine horizontale Differenzierung von Gütern bildet die Herstellung zweier Zeitungsvarianten, wovon eine Zeitung $(X)$ einen Inhaltefokus auf Politik und Kulturellem und die zweite Zeitung $(Y)$ Sportnachrichten enthält. Ein Beispiel für eine vertikale Differenzierung bildet dagegen die Herstellung einer Zeitschriftenvariante in geklammertem Schwarzweiß-Druck $(X)$ sowie einer zweiten Variante mit geleimten Hochglanzfarbseiten ( $\left.X^{\prime}\right)$.

373 Die im Rahmen einer Produktionstechnologie erzeugbaren Produktvarianten werden bestimmt durch eine konvexe Produktdifferenzierungskurve (PDK), die individuellen Präferenzen der Konsumenten bzgl. der dargestellten Merkmale durch konkave Indifferenzkurven (IK). Die Punkte X und Y, bzw. X' und $Y^{\prime}$ repräsentieren jeweils optimale Konsumentscheidungen individueller Konsumenten.

374 Vgl. Kirsch (1997), S. 472 f. 
Das Optimierungskriterium für die angestrebte Verringerung der Distanz zwischen Real- und Idealleistung einer Produktlinie bildet die Varietät, im Sinne der „Mächtigkeit der Menge der angebotenen Leistungsvarianten“. Eine Erhöhung der Variantenzahl steigert aufgrund differenzierter Preissetzung den Stückgewinn. Die Zahl der Varianten erhöht sowohl zum einen die Komplexität und dadurch die Transaktionskosten der Produktion, als auch zum anderen die direkten Produktionskosten - allerdings durch Verbund- und Skaleneffekte bei eingeschränkter Variantenvielfalt zunächst nur in begrenztem Maße. Eine darüber hinausgehende Ausdehnung der Varietät führt schließlich nur noch zu geringeren Preissteigerungsmöglichkeiten, gleichzeitig jedoch zu exponentiell wachsenden Transaktionskosten mit dem Ergebnis von sinkenden oder sogar negativen Stückgewinnen. ${ }^{375}$

\subsection{Preisdifferenzierung}

Die Merkmalsdifferenzierung einer produzierten Leistung kann Spielraum für eine differenzierte Preissetzung eröffnen. Die unterschiedlichen Modelle zur Preisdifferenzierung setzen dies z.T. jedoch nur implizit voraus, bspw. als Rechtfertigung unterschiedlicher Preise für unterschiedliche Konsumentengruppen. Preisdifferenzierung beruht auf der Erkenntnis, dass Konsumenten aufgrund von Unterschieden in ihren Produktpräferenzen, aufgrund von Informationsasymmetrien oder räumlich isolierten Märkten eine unterschiedlich hohe Zahlungsbereitschaft für dasselbe Produkt besitzen. Ziel der Preisdifferenzierung ist die Optimierung der Preissetzung entsprechend der jeweiligen nutzerspezifischen Reservationspreise zur Abschöpfung der Konsumentenrente. ${ }^{376}$

Abbildung 3-15 stellt den Unterschied zwischen gewinnoptimaler Gesamtmarktstrategie und differenzierter Preissetzung im Monopolfall unter vereinfachender Annahme konstanter Grenzkosten dar. Die gewinnoptimale Absatzmenge $x^{*}$ wird im Fall der Gesamtmarktstrategie bestimmt durch den Schnittpunkt von Grenzerlös- und Grenzkostenkurve, dem so genannten "Cournot'schen Punkt" (C). Der entsprechende Monopolpreis $p^{*}$ ergibt sich anhand der gewinnoptimalen Absatzmenge über die Preis-Absatzfunktion. ${ }^{377}$ Existieren am Markt allerdings unterschiedliche Käufer mit heterogenen Nutz- und damit Preisvorstellungen, kann eine differenzierte Preissetzung von $\mathrm{p}^{1 *}$ oder $\mathrm{p}^{2 *}$ gegenüber einem Einheitspreis eine Gewinnsteigerungsmöglichkeit bieten.

\footnotetext{
375 Vgl. Bohn (1993), S. 82.
}

376 Als Reservationspreis wird die maximale Zahlungsbereitschaft, also der Preis, den ein Konsument für den Erwerb eines Gutes höchstens ausgeben würde, verstanden. Vgl. u.a. Gehrke/Burghardt/Schumann (2002), S. 3.

377 Vgl. für den Fall konstanter Grenzkosten u.a. Meffert (2000), S. 514 ff.; für den Fall nicht-linearer Kosten vgl. u.a. Schumann/Meyer/Ströbele (1999), S. 284 ff. 

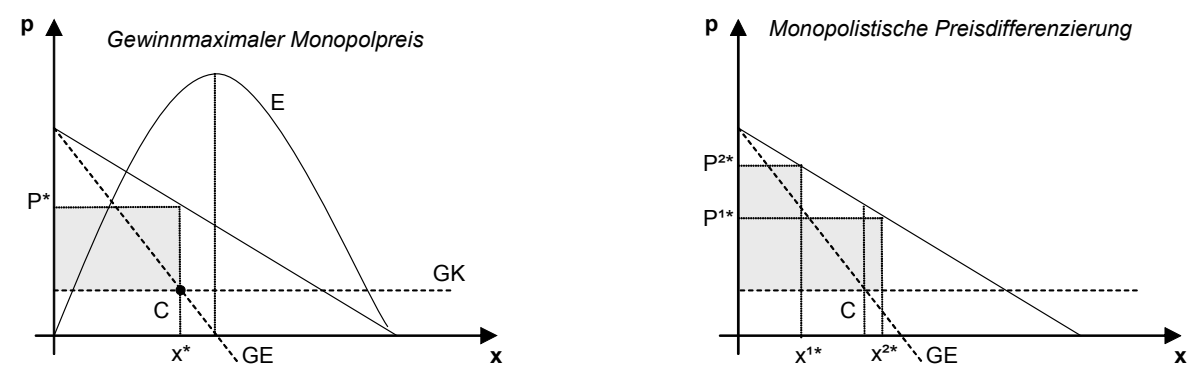

Abbildung 3-15: Preisdifferenzierung

Nach der Optimalitätsbedingung von Stackelberg kann ein Produzent durch Setzung unterschiedlicher Preise seinen Erlös gegenüber der ursprünglichen Rente bei Setzung eines Einheitspreises für den Gesamtmarkt steigern. ${ }^{378}$ Dies erfordert gem. der Preisdifferenzierung ersten Grades nach Pigou, dass jedem Konsumenten sein individueller Reservationspreis verrechnet wird. Dies setzt zum einen eine annähernd vollständige Information über die Reservationspreise der Produktnachfrage voraus, zum anderen die grundsätzliche Spaltbarkeit des Markts in verschiedene Segmente, bspw. durch eine segmentspezifische Merkmalsgestaltung unterschiedlicher Produktvarianten. Liegt diese nicht vor, bieten sich zwei Alternativen: ${ }^{379}$ erstens durch eine Segmentierung des Marktes in Gruppen mit möglichst homogenen Zahlungsbereitschaften; dabei wird ein Produkt in jedem Nachfragersegment jeweils mit einem unterschiedlichen Preis angeboten. ${ }^{380}$ Zweitens durch eine vollständige (vertikale) Spaltung des Gesamtmarkts auf solche Weise, dass sich ,in jedem Submarkt Käufer aller oder aber zumindest mehrerer Preisschichten befinden. “381

Aus den mikroökonomischen Vorüberlegungen zur Differenzierung können zwei Konsequenzen für die Vermarktung von Mediengütern abgeleitet werden: Zum einen kann eine Differenzierungswirkung einer angebotenen Leistung entweder über objektiv beurteilbare Qualitätsaspekte (Tauschwert) oder durch den individuellen Gebrauchswert im Rahmen qualitativ gleichwertiger Varianten erzeugt werden. Zweitens ist das Differenzierungspotenzial in einem Marktsegment aus Anbietersicht umso höher einzuschätzen, je individueller Nachfragebedürfnisse in diesem Segment identifiziert werden können.

\subsubsection{Differenzierung von Mediengütern}

Eine unmittelbare Anwendung der mikroökonomischen Differenzierungstheorie auf die Medienbranche ist aus zwei Gründen problematisch: Anders als bspw.

378 Vgl. Brandtweiner (2000), S. 83.

379 Vgl. Pigou (1950), S. 279 und Brandtweiner (2000), S. 89.

380 Beispiel einer solchen Form der Preisdifferenzierung in der Medienbranche bildet der reduzierte Abonnementpreis einer Tageszeitung für Schüler und Studenten.

381 Beispiel dieser Form der Preisdifferenzierung bildet ein regional unterschiedlicher Straßenverkaufspreis einer Tageszeitung. 
in Konsumgütermärkten, wie dem Bekleidungs- oder dem Automobilmarkt, ist die Qualitätsbeurteilung von Mediengütern durch deutlich heterogenere Präferenzstrukturen gekennzeichnet. Zudem unterliegen Mediengüter aufgrund ihrer Erfahrungsguteigenschaften aus Rezipientensicht einem Bewertungsproblem. Im Folgenden werden daher zunächst wertbildende Merkmale von Mediengütern als Ansatzpunkte einer Bestimmung von differenzierungswirksamen Mehrwertpotenzialen bestimmt (vgl. Abschnitt 3.3.2.2.1). Im Weiteren werden dann Differenzierungsvarianten für Mediengüter dargestellt.

\subsection{Wertbildende Eigenschaften von Mediengütern}

Wie bereits in Kapitel 2 erläutert wurde, bildet die transportierte Nachricht die primäre Nutzen stiftende und dadurch aus Nutzersicht kaufentscheidende Komponente eines Medienguts. Die Bewertung des Nutzens eines Medienguts kann jedoch bspw. in Abhängigkeit vom Bildungsgrad oder den Interessen erheblich zwischen verschiedenen Nutzern divergieren. Die Frage nach den Bestimmungsfaktoren der Mediennutzung wird in der kommunikationswissenschaftlichen Sozialforschung bereits seit den 1970er Jahren erörtert. ${ }^{382}$ Die Mediennutzenforschung (nach dem sog. „Uses and Gratifications Approach“) untersucht Bedürfnisse, die durch die Nachfrage nach Mediengütern gestillt werden. ${ }^{383}$ Unterstellt, dass der Mediennutzung ein rationales Wahlverhalten zugrunde liegt, können vier Bedürfniskategorien bestimmt werden: kognitive Unterhaltungs- und Informationsbedürfnisse, denen ein bestimmter Nutzungszweck zugrunde liegt; affektive Bedürfnisse, die durch besondere Bedingungen der Nutzungssituation ausgelöst werden; integrative Bedürfnisse zur Linderung sozialer Spannung oder zur Zerstreuung von Langeweile; interaktive Bedürfnisse zum Austausch mit anderen Mediennutzern. ${ }^{384}$ Auf medialer Ebene kommen drei Quellen einer Befriedigung dieser Bedürfnisse in Frage: die transportierte Nachricht, das Übertragungsformat dieser Nachricht und der Kontext der Mediennutzung (vgl. Abbildung 3-16). ${ }^{385}$

Kriterien für den Wert eines Medienguts auf inhaltlicher Ebene bilden die Breite und die Tiefe der kommunizierten Inhalte, ihre überprüfbare Validität und insbesondere bei informationsorientierten Inhalten, ihr Aktualitätsgrad. Der Aktualitätsgrad eines Inhalts, den ein Mediennutzer wahrnimmt, ist jedoch nicht allein eine Frage der Erzeugung, sondern vor allem durch die Periodizität des Distributionsformats bestimmt. Neben der Aktualität der Distribution bilden performative (Performance) und nutzungsökonomische Kriterien (Usability) des Distributionsformats weitere Nutzen stiftende Aspekte eines Medienguts. Dazu gehören bspw. die Verfügbarkeit des Mediums, die Geschwindigkeit der Möglichkeit eines Inhaltezugriffs und die Ökonomie der logischen Inhaltestruktur. Der Gebrauchswert des Medienformats kann dabei in Abhängigkeit der Nutzungssituation variieren. Bspw. kann eine Fernsehsendung ausreichende Zugriffsbequemlichkeit und inhaltliche Übersicht im Rahmen der Feierabendunterhaltung geben, jedoch für einen schnellen

\footnotetext{
382 Vgl. Palmgreen (1983) und Palmgreen (1984).

$383 \mathrm{Vgl}$. Leber (1988), S. 60.

384 Vgl. Leber (1988), S. 65 f.

$385 \mathrm{Vgl}$. Fink (2002).
} 
Informationszugriff im Arbeitsalltag ungenügend sein. Insofern transportiert der situative Kontext der Mediennutzung wertbildende Kriterien für den Gebrauchswert von sowohl der Nutzbarkeit des Formats als auch der Relevanz transportierter Inhalte.

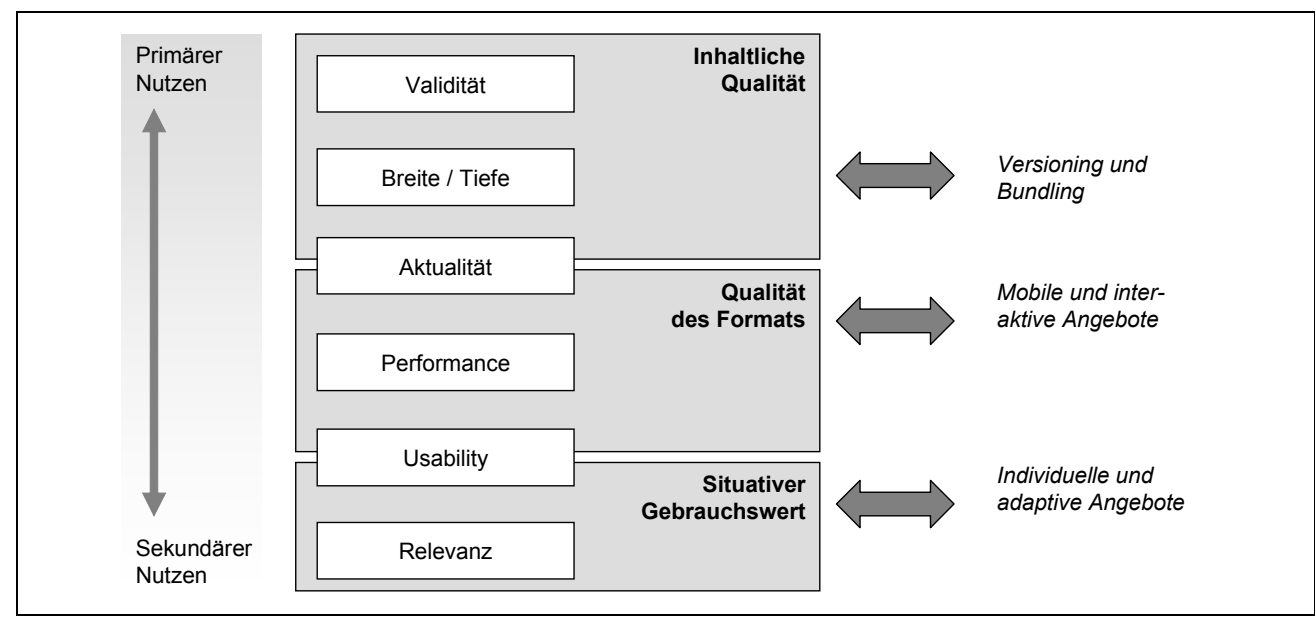

Abbildung 3-16: Wertbildende Eigenschaften eines Medienguts

Entsprechend diesen drei Ebenen der Bedürfnisbefriedigung können bei Mediengütern drei Differenzierungstypen unterschieden werden, die folgend näher erläutert werden: die Differenzierung durch Variation der inhaltlichen Qualität (Abschnitt 3.3.2.2.2); die Differenzierung durch Variation der Qualität des Distributionsformats, insbesondere durch mehrwertbildende Faktoren digitaler Distributionskanäle wie Mobilfunk oder interaktives Fernsehen (Abschnitt 3.3.2.2.3); die Differenzierung durch Individualisierung des Angebots zur Steigerung des situativen Gebrauchswerts (Abschnitt 3.3.2.2.4).

\subsection{Versioning und Bundling}

Eine zentrale Voraussetzung eines differenzierten Angebots mit zumindest gruppenspezifischer Preissetzung bildet die Kenntnis von Nachfragebedürfnissen und Preisrestriktionen. Sind diese nicht verfügbar oder nur mit hohem Aufwand zu ermitteln, bildet das Angebot einer begrenzten Anzahl von Produktvarianten für verschiedene Käufersegmente eine alternative Differenzierungsstrategie. Diese Form der Variantenstrategie wird im Bereich von Informationsgütermärkten auch als „Versioning“ oder „Versionierung“ bezeichnet. ${ }^{386}$

Eine Versionierung kann nach zweierlei Prinzipen erfolgen: ${ }^{387}$ erstens durch eine bewusste Adressierung ausgewählter Bedürfnisgruppen; zweitens durch eine Marktspaltung in Form einer Selbstselektion der Nutzer. Shapiro und Varian nennen eine Vielzahl von Beispielen denkbarer Dimensionen einer Produktversionierung, darunter die zeitliche Verzögerung von Veröffentlichung und Vertrieb, den Grad der funktionalen Ausgestaltung der angebotenen Dienste oder

386 Vgl. Schumann/Hess (2002), S. 75; Vizjak/Ringlstetter (2001), S. 12; Brandtweiner (2000), S. 113 ff. 387 Vgl. Shapiro/Varian (1997), S. 54. 
der inhaltliche Umfang des vertriebenen Informationsguts. ${ }^{388}$ Eine Variation der inhaltlichen Qualität kann dabei auf drei Ebenen erfolgen: auf der Ebene des inhaltlichen Kernprodukts, auf der Ebene flankierender Zusatzinhalte und durch Metainformationen zu den ersten beiden Ebenen.

Das Beispiel für eine Mehrwert stiftende zeitliche Variation des Kerninhalts bildet das Angebot der Zeitschrift „der Spiegel“, die Inhalte der wöchentlich am Montag erscheinenden Printausgabe bereits am Wochenende in kostenpflichtigen Onlineauszügen zu lesen. Flankierende Zusatzinhalte werden bspw. durch Kritiken, Kundenbewertungen oder Suchmaschinen erzeugt. Der Mehrwert solcher Inhalte kann einerseits in der Steigerung des Nutzungskomforts, andererseits in der Reduktion der Bewertungsunsicherheit für Rezipienten begründet sein. Einen ähnlichen Mehrwert adressieren publizierte Metainformationen bspw. in Form eine Auswertung von Nutzungshäufigkeiten publizierter Inhalte.

Als spezielle Form der Versionierung kann das Konzept der Güterbündelung, das sog. „Bundling“, interpretiert werden ${ }^{389}$ Die Bündelung von Gütern als Instrument der Preisdiskriminierung wurde erstmalig ausführlich untersucht von Adams und Yellen (1976), erweitert von Schmalensee (1984). ${ }^{390}$ Gegenüber einer reinen Einzelvermarktung (= „pure components“) von Gütern kann eine Vermarktung in gebündelter Form eine Einsparung von Produktions- Transaktions- und Informationskosten, die Förderung von Güterkomplementaritäten sowie eine Segmentierung der Nachfrage bewirken. Die Autoren unterscheiden neben der Einzelvermarktung zwei Formen der Bündelung (vgl. Abbildung 3-17): die reine Bündelstrategie (= „pure bundeling“) und die gemischte Bündelstrategie als gleichzeitiges Angebot von Einzel- und Bündelvermarktung (= „mixed bundeling“).

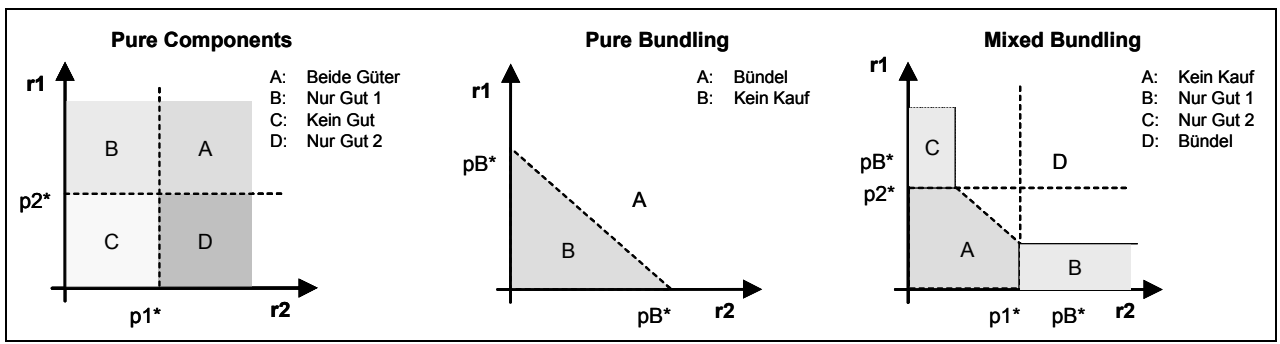

Abbildung 3-17: Bundling391

Die Vorteilhaftigkeit der Bündelung lässt sich nach Adams und Yellen nicht generell festlegen, sondern ist abhängig von der Streuung der Reservationspreise der einzelnen Bündelkomponenten und den jeweiligen Einzel- und Bündelkosten der Produktion. Unter Annahme bivariat normalverteilter Reservationspreise zweier "symmetrischer Produkte" - gemeint sind Produkte mit gleicher Nachfrage- und Kostenstruktur - beschreibt Schmalensee die Vorteilhaftigkeit einer Bündelstrategie in Abhängigkeit von der Korrelation der jeweiligen Zahlungsbe-

388 Vgl. Shapiro/Varian (1997), S. $56 \mathrm{ff.}$

389 Vgl. Brandtweiner (2000), S. 71.

$390 \mathrm{Vgl}$. Adams/Yellen (1976), Schmalensee (1984).

391 Dargestellt sind Formen der Bündelung zweier Güter r1 und r2 zum Preis $p$. 
reitschaft für jeweils eine Bündelkomponente und der durchschnittlichen Gesamthöhe der Zahlungsbereitschaft für ein Bündel. Bündelung erweist sich deshalb als vorteilhaft, weil die Konsumentenrente von einem Produkt auf ein anderes übertragen wird. ${ }^{392}$ Dies setzt zum einen voraus, dass die Zahlungsbereitschaft für beide Produkte negativ korreliert ist, also die Nachfrage nach den Komponenten heterogen ist. Zum anderen, so führen Olderog und Skiera anhand von Simulationsmodellen aus, ist Bündelung nur dann sinnvoll, wenn die Zahlungsbereitschaft die jeweiligen variablen Kosten der Reproduktion deutlich übersteigen. ${ }^{393}$ Eine solche Kostenstruktur mit geringen variablen Kosten, so führt Skiera weiter aus, zeichnet insbesondere die Produktion digitaler Informationsgüter aus. ${ }^{394}$ Daher wird der Bündelung eine hohe Relevanz für die Differenzierung von Informationsgütern beschieden. Ein Beispiel für eine mehrwertstiftende Bündelungsform von Onlineinhalten, die über das typische Bündeln von Inhalten bspw. in einer Zeitung oder Zeitschrift hinausgeht, bildet das kostenpflichtige Angebot von Artikelarchiven oder themenspezifischen Artikeldossiers.

\subsection{Mobile und interaktive Mehrwertformate für Mediengüter}

Aus informationswissenschaftlicher Sicht können digitale Inhalteformate gegenüber traditionellen Markt- und Distributionsformen von Inhalten sowohl aus Anbieter- als auch Nachfragersicht mehrwertbildende Eigenschaften besitzen. Solche von Kuhlen auch als „informationelle Mehrwerte“ bezeichnete Eigenschaften des Distributionsformats entstehen durch Formen anbieterseitiger Informationsarbeit im Rahmen der kanalspezifischen Formataufbereitung. ${ }^{395}$ Die mehrwertstiftende Eigenschaft der Informationsarbeit beruht auf einer Unterstützung der Konvertierung von Informationen in ein durch den Rezipienten anwendbares Wissen. Beispiele für eine solche mehrwertstiftende Informationsarbeit bildet das Referieren und Indizieren von Inhalten, die Konvertierung von Inhalten in logisch verknüpfte Hypertexte oder die Wissensrepräsentation durch eine intelligente Produktempfehlung. ${ }^{396}$ Informationsarbeit wie das Erstellen von Hypertexten mag einen formatbezogenen Mehrwert gegenüber traditionellen Distributionsformen bilden, die wettbewerbsbezogene Differenzierungswirkung von Onlineformaten ist jedoch - wie bereits in Kapitel 4 erläutert - aufgrund des Überangebots werbe- und rundfunkgebührenfinanzierter Onlineinhalte gering. Besondere Bedeutung wird derzeit daher neuen Distributionskanälen wie dem mobilen Internet oder dem interaktiven, digitalen Fernsehen beigemessen.

Als digitales interaktives Fernsehen werden Dienstformen verstanden, die digitalisierte Bewegtbilder über Rundfunk-Verteilnetze vertreiben. ${ }^{397}$ Für die Nutzung von digitalem interaktivem Fernsehen werden Zusatzgeräte für Empfang, Entschlüsselung und Interaktion benötigt. Charakteristisch für die Nutzung

\footnotetext{
392 Vgl. Olderog/Skiera (1998), S. 7

$393 \mathrm{Vgl}$. Olderog/Skiera (1998), S. 25.

$394 \mathrm{Vgl}$. Skiera (2001), S. $250 \mathrm{f}$.

$395 \mathrm{Vgl}$. Kuhlen (1995), S. $82 \mathrm{ff}$.

396 Vgl. Kuhlen, S. $86 \mathrm{ff}$.

397 Vgl. Breunig (1997), S. 59.
} 
von digitalem interaktivem Fernsehen ist die zusammenhängende Abfolge von Datenkommunikation eines Empfängers und einer individuellen Rückkopplung des Senders an den Empfänger. ${ }^{398}$ Dadurch wird es dem Rezipienten ermöglicht, zum einen beim Sender gespeicherte Inhalte über einen Rückkanal auszuwählen und zum anderen eigene Informationen mit anderen Menschen auszutauschen. Ein Pilotangebot für ein interaktives digitales Fernsehprogramm bildet der Video-on-demand Dienst „T-Online Vision“ der deutschen Telekom. ${ }^{399}$ Zur Nutzung des Dienstes sind ein spezielles Empfangsgerät sowie ein A-DSL Anschluss notwendig. Der Dienst repräsentiert einen zusätzlichen kostenpflichtigen Verwertungskanal für Kinofilme mit Nutzungsgebühren von EUR 1,90 pro Film. Der Mehrwert besteht zum einen in einem zeitlichen Vorteil gegenüber der Erstausstrahlung des Films im kostenfreien Rundfunk sowie einen Bequemlichkeitsgewinn durch freie Gestaltung des Programms.

Wie in Kapitel 2 bereits erläutert wurde, werden als mobiles Internet im Allgemeinen Datenkommunikationsanwendungen verstanden, die Mobilfunktechniken zum Verbindungsaufbau nutzen. Dem mobilen Internet werden Mehrwerteigenschaften beschieden, die eng mit den technischen Merkmalen der Mobilfunktechnik in Zusammenhang stehen. Diese Merkmale werden auch als Technologiewertbeiträge ${ }^{400}$ bezeichnet. Eine solche Mehrwerteigenschaft wird insbesondere zwei Aspekten der Mobilfunktechnik beschieden: die allgegenwärtige und vor allem ortsunabhängige Verfügbarkeit mobiler Endgeräte und der damit abrufbaren Dienste sowie die jederzeit eindeutige Identifizierbarkeit mobiler Anwender.

Ortsunabhängigkeit repräsentiert aufgrund der Portabilität mobiler Endgeräte ein definitionsgemäßes Kriterium des mobilen Internet. Der Mehrwert für einen Nutzer im Rahmen der Inhalterezeption beruht insbesondere auf der Aktualität und der Schnelligkeit des Inhaltezugriffs. Identifizierbarkeit bildet dagegen ein spezielles Kriterium für Anwendungen in flächendeckenden Kommunikationsnetzen des kommerziellen Mobilfunks wie GSM oder UMTS. Ein Endgerät wird sowohl im GSM als auch UMTS Standard über eine spezielle Chipkarte, die so genannte SIM (Subscriber Identification Module)-Karte, identifiziert. Anhand dieser eindeutigen Identifikation eines Teilnehmers nimmt das Mobilfunknetz im Vermittlungs-Subsystem eine Lokalisierung des Teilnehmers und dann eine Signalvermittlung vor. ${ }^{401}$ Die permanente Identifizierbarkeit und Lokalisierbarkeit eines Mobilfunknutzers eröffnet neue Potenziale in Bezug auf die Möglichkeiten von Marktforschung und Identifikation individueller Nachfragerbedürfnisse. Die Teilnehmeridentifizierbarkeit erhöht also das ressourcenwerte Beitragspotenzial eines Kunden an der Wertschöpfung.

\footnotetext{
$398 \mathrm{Vgl}$. Dahm/Rössler/Schenk (1998), S. 35.

$399 \mathrm{Vgl}$. T-Online (2004).

400 Vgl. Zobel (2001), S. 63.

401 Vgl. Schiller (2002), S. 380 und Abschnitt 5.2.2.1.
} 


\subsection{Mehrfachverwendung von Inhalten und Individualisierung von Mediengütern}

Eine kernproduktorientierte Erzeugung von Mediengütern stößt in der Regel auch bei mehrfacher Verwertung auf der Wertschöpfungsstufe der Distribution an kostenbedingte Varietätsgrenzen. Wie bereits in Kapitel 2 dargelegt wurde, bilden Differenzierung und Kostenführerschaft im traditionellen Strategieverständnis zwei streng zu trennende Alternativen. Einem Unternehmen, dem es nicht gelingt, seine Strategie in eine dieser Richtungen zu entwickeln, sitzt sinnbildlich zwischen zwei Stühlen, da für Kostenführerschaft Skaleneffekte einer Massenproduktion ausgenutzt werden müssen, deren Ausbringungsvolumina bei einer hohen Leistungsvarietät jedoch nicht erreicht werden können. ${ }^{402}$ Das Prinzip der Massenkommunikation von Inhalten in der Medienbranche mit einem geringen Varietätsgrad trägt diesem Strategieverständnis Rechnung.

Der vor allem im industriebetrieblichen Bereich diskutierte Ausweg aus dem Varietätsdilemma bildet eine Reduktion der internen Produktionskomplexität einer hohen Angebotsvarietät durch Modularisierung der Fertigung. Wie in Abschnitt 3.3.1.3 dargestellt wurde, kann eine solche Modularisierung in der Medienbranche in begrenzter Form durch verwertungsneutrale Auszeichnung von Inhalteformaten ermöglicht werden, indem bspw. bei unterschiedlichen Verwertungsformaten nur bestimmte Formatbereiche des Inhalts verwertet werden.

Für eine über diese begrenzte Modularisierung auf der Distributionsebene hinausgehende Modularisierung bieten sich in der Medienbranche datenbankgestützte Lösungen für eine Modularisierung und dadurch mehrfachen Verwendung von Inhalten auf der Wertschöpfungsstufe der Erzeugung und Bündelung von Inhalten. Solche, auch als "Content-Management-Systeme“ bezeichneten Lösungen trennen die Erzeugung und Speicherung von Inhalten strikt von einerseits von ihrer Verwendung im Rahmen der Produktbündelung und andererseits ihrer Verwertung im Rahmen der Distribution. Dazu werden Inhalte zunächst in Datenbanken gespeichert und dynamisch in bestimmten Bündelungsformaten, die als Formatvorlagen, sog. „Templates“, vorproduziert werden können, referenziert (Vgl. Abbildung 3-18). Durch die Verwendung von Formatvorlagen auf der Grundlage verwertungsneutraler Formattypdefinitionen, können diese Templates wiederum in verschiedenen Distributionskanälen (bspw. als Druckvorlage für eine Zeitschrift, als Online- oder mobiles Ausgabeformat) verwertet werden.

402 Vgl. Porter (1980), S. 79 . 


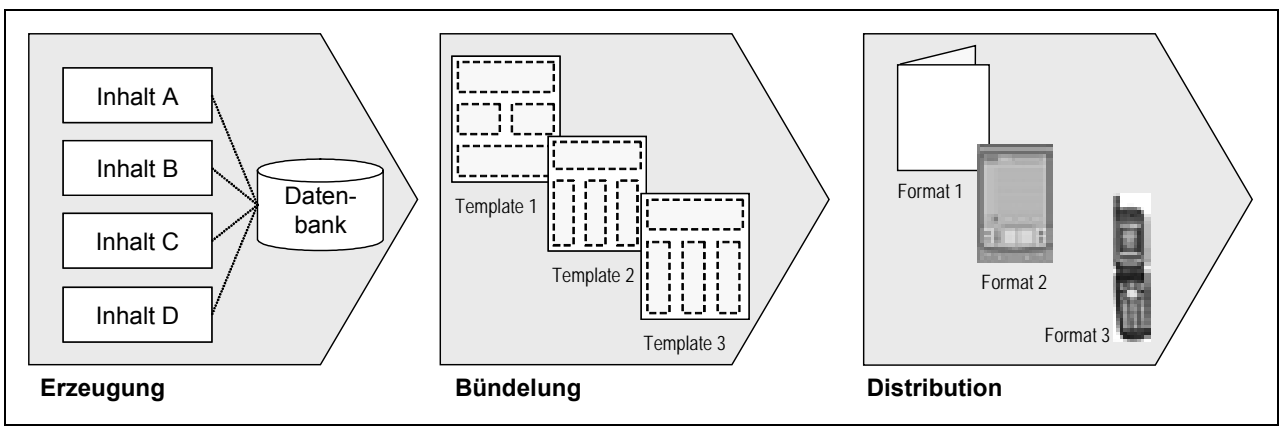

Abbildung 3-18: Mehrfachverwendung von Inhalten

Wie bereits in Abschnitt 3.3.1.2.2 dargestellt, repräsentieren Mediengüter Produktbündel gekoppelter Informationsgüter. Diese Kopplung erfolgt traditionell aus Gründen von Größen- und Verbundeffekten des Vertriebs. Eine Zeitung repräsentiert bspw. ein Bündel aus redaktionellen Artikeln, Werbe- und Kleinanzeigen. Im Gegensatz zum „linearen“ bzw. statischen Aufbau herkömmlicher Mediengüter können digital erzeugte Inhalte als modulare Konfigurationen von einzelnen Informationsgüterobjekten interpretiert werden. Entsprechend erlauben digitale Mediengüter eine zweifache strukturelle Variation: zum einen die interessensspezifische Konfiguration der Kombination verschiedener Inhalte innerhalb eines Produktbündels; zum anderen die Konfiguration der inhaltlichen Aggregationstiefe einzelner Beiträge. Sind Medienunternehmen in der Lage, inhaltliche Nachfragebedürfnisse auf individueller Ebene zu identifizieren, kann durch Mehrfachverwendung von Inhalten ein Produkt individuell auf beiden Ebenen entsprechend dieser Bedürfnisse konfiguriert und angeboten werden.

\subsubsection{Bewertung der strategischen Handlungsmöglichkeiten in der Medienbranche}

Vor dem Hintergrund des einleitend in Abschnitt 3.3 aufgespannten Strategiefächers können zusammenfassend drei strategische Handlungsmöglichkeiten für die Medienbranche identifiziert werden: eine Ausweitung des Leistungsumfangs auf traditionell Medien-untypische Marktbereiche wie den physischen und logischen Netzwerkzugang (1); eine Erhöhung des Standardisierungsgrads im Rahmen der Erzeugung und Formatierung von Inhalten zur Schaffung crossmedialer Inhaltesynergien (2); eine radikale Fokussierung auf den individuellen Gebrauchswert von Mediengütern durch Adressierung individueller und situationsbezogener Bedürfnisse (3).

(1) Wie bereits angesprochen wurde, macht die Digitalisierung von Kommunikationssignalen und publizistischen Inhalten Mediengüter und Kommunikationsdienstleistungen zu potenziellen Systemgütern. Diese Systemleistung besitzt sowohl für Medien- als auch für Telekommunikationsunternehmen eine hohe strategische Bedeutung: Deutsche Telekommunikationsnetze besitzen durch Glasfasertechnik im Festnetz dem Aufbau von flächendeckenden UMTSNetzen im Mobilfunk bis 2005 einen hohen technischen Standard mit hohen 
Übertragungsbandreiten. Dieser technische Fortschritt ist geknüpft an hohe Investitionskosten für Lizenzerwerb und Netzaufbau in der Vergangenheit. Entsprechend hoch ist aus Sicht der Telekommunikationsbranche die Notwendigkeit, die aufgebauten Netzkapazitäten zu vermarkten. Besondere Hoffnung wird dabei auf die Nachfrage nach großvolumigen Onlineinhalten gesetzt, wodurch dem digitalen Angebot der Medienbranche die Funktion eines Erfolgsfaktors für die Telekommunikationsbranche zukommt. Die Onlinedistribution von Inhalten ist aus Sicht der Medienbranche jedoch mit einem dreifachen Risiko verbunden: Onlinemedien repräsentieren kein exklusives Distributionsmedium, denn die Nachfrage nach Onlineinhalten erfordert nur die Kenntnis der logischen Inhalteadresse, wobei die Konkurrenz sprichwörtlich nur einen Mausklick entfernt ist; die Realisierung von Mehrwertpotenzialen wie eine Adressierung individueller Kundenbedürfnisse erfordert Informationen über das Onlinenutzungsverhalten des Kunden, die üblicherweise dem Netzzugangsbetreiber vorbehalten sind; in der Funktion des Netzzugangsdienstleisters besteht eine Schlüsselfunktion, die traditionell von Telekommunikationsunternehmen eingenommen wird. Diese birgt die Gefahr einer Konzentration von Nachfragermacht seitens der Telekommunikationsunternehmen. Die aus Wettbewerbsperspektive sicher dominante Strategie einer Diversifikation von Medienunternehmen in den Bereich des Netzzugangs mindert dieses Risiko des Onlinevertriebs nur scheinbar, da eine solche Diversifikation den Erwerb zusätzlicher Fähigkeiten von Medienunternehmen sowohl im technischen Bereich des Zugangsangebots als auch auf der Ebene der Kundebetreuung und der Leistungsabrechnung voraussetzt.

(2) Die technische Entwicklung im Bereich der Medienwertschöpfung ist gekennzeichnet durch die Entwicklung standardisierter Inhalteformate. Eine solche Standardisierung birgt für die Medienbranche ein zweifaches Wettbewerbspotenzial. Durch die Standardisierung der Inhalteerzeugung und -bündelung können Synergien zwischen verschiedenen Produktvarianten und -distributionsformen genutzt werden, indem dieselben Inhalte mehrfach verwendet und verwertet werden. Dies kann zum einen den Ressourcenverzehr eines hoch integrierten Medienunternehmens reduzieren. Zum anderen kann die Reichweite eines Inhalts ohne hohe Zusatzkosten cross-medial erhöht werden. Die Standardisierung der Erzeugung und Bündelung von Inhalten schafft folglich für ein Medienunternehmen nicht nur ein Diversifikationspotenzial, sondern besitzt darüber hinaus wertbezogene Implikationen. Insofern ist die in Kapitel 3.3 vorgenommene Unterscheidung zwischen Diversifikationsstrategien und wertbezogenen Strategieformen zwar methodisch sauber, in der Praxis jedoch nicht so eindeutig vorzunehmen. Vor dem Hintergrund inhaltebezogener Synergiepotenziale durch verwendungsneutrale Formatierungen sehen es einige Autoren als zentrale Aufgabe des strategischen Medienmanagements, durch eine integrierte Planung, Implementierung und Steuerung medienübergreifender Vermarktungskonzepte vorhandene Marken, Inhalte und Kundenbeziehungen wertsteigernd cross-medial zu nutzen.

(3) Aufgrund der Standardisierbarkeit von Inhalten auf Modulebene besitzt die traditionelle Alternativhypothese in Bezug auf Kostenoptimierung und Varietätsgrad des Angebots in der Medienbranche nur noch bedingt Gültigkeit. Ins- 
besondere gilt der durch hohe First-Copy-Costs begründete Zwang zu Massenkommunikation für Medienunternehmen nicht mehr uneingeschränkt. Wenn Medienunternehmen die Wünsche ihrer Kunden individuell bestimmen können, dann kann eine individuelle Produktvariante auf der Grundlage standardisierter Module zu einem Kostenniveau erzeugt werden, das dem der Massenfertigung entspricht. Diese Veränderungen der Erzeugungsmöglichkeiten von Mediengütern besitzt annähernd das Potenzial für einen radikalen Paradigmenwechsel in der Medienbranche: War bislang die absolut generierbare Reichweite das dominante Zielkriterium des strategischen Medienmanagements, kann nun der individuelle Kundenwertbeitrag als strategisches Ziel verfolgt werden, wobei der Endkunde individuell den Wert der wirtschaftlichen Aktivitäten bestimmt. Hebel für eine solche strategische Neuausrichtung bilden eine Verlängerung der Kundenbeziehungsdauer durch Steigerung der Kundenloyalität sowie eine crossmediale Erfassung und Berücksichtigung der Kundenbedürfnisse.

\subsection{Zusammenfassung der strategischen Herausforderun- gen von Medienunternehmen}

In Kapitel 3.2 wurde dargelegt, dass Medienunternehmen im Zuge konvergenzgeprägter Wettbewerbsbedingungen mit sinkenden durchschnittlichen Reichweiten ihrer Produkte und Dienstleistungen konfrontiert werden. Der Rückgang der Durchschnittsreichweite wird verursacht durch ein Überangebot an Information und Unterhaltung bei einem begrenzten zeitlichen und monetären Budget sowie einer begrenzten Wahrnehmungsaufmerksamkeit der Rezipienten. Diese Entwicklung birgt die Gefahr negativer Anzeigen-Auflagen-Effekte, als Umkehrung der in Abschnitt 2.1 erläuterten Anzeigen-Auflagen-Spirale.

In Kapitel 3.3 wurde dargestellt, dass für Medienunternehmen zwei Strategieoptionen existieren, um dieser Entwicklung zu begegnen: eine Diversifikation des Leistungsspektrums oder eine Steigerung des relativen Kundenwertbeitrags. In Abschnitt 3.3.1 wurde dargelegt, dass, zum einen durch funktionale Überschneidungen der Branchenwertschöpfung von Telekommunikations- und Medienbranche, zum anderen durch Synergien in der Bündelung und Distribution auf Grundlage einer standardisierten Formatierung digital erzeugter Inhalte, zusätzliche Handlungsmöglichkeiten für Medienunternehmen entstehen, im Zuge derer durch eine Diversifikation des Leistungsspektrums zusätzliche Einnahmen erzielt werden können. Diversifikationsentscheidungen sind jedoch unabhängig vom Verwandtschaftsgrad von Ziel- und Ausgangsbranche durch ein hohes Maß an Risiko gekennzeichnet. Bei Diversifikationsentscheidungen mit geringem Verwandtschaftsgrad, wie bspw. das Angebot von Netzzugangsdiensten durch Medienunternehmen, müssen in der Regel zum einen zusätzliche Unternehmensressourcen wie bspw. Abrechnungsmechanismen für Übertragungskapazitäten aufgebaut werden. Bei Diversifikationsentscheidungen mit hohem Verwandtschaftsgrad, wie bspw. eine cross-mediale Distribution von Inhalten, können zum anderen die Kosten der Formatstandardisierung nur dann durch das Unternehmen kompensiert werden, wenn dadurch eine ausreichende zusätzliche 
Aufmerksamkeit und dadurch zusätzliche Einnahmen erzeugt werden. Dies lässt auf die Notwendigkeit schließen, dass Medienunternehmen ihren Kunden einen Zusatznutzen bieten müssen, um im Bereich der digitalen Inhalteverwertung einen monetären Mehrwert für das Unternehmen zu schaffen.

Neben bereits hinreichend bekannten Differenzierungsformen wie Windowing, Timing oder Versioning wurden die Möglichkeiten einer Individualisierung von Mediengütern und der Inhaltevertrieb im mobilen Internet als potenzielle Quellen eines Kundennutzens identifiziert. Diese beiden Strategieoptionen für Medienunternehmen werden im Weiteren im Detail untersucht. 


\section{Individualisierung von Mediengütern}

Die Frage der optimalen Sortenvarietät bildet von je her ein zentrales Problem der strategischen Planung, nicht nur für die Medienbranche. Im Zuge der Verbreitung des Internet hat die Varietätsplanung für die Medienbranche jedoch eine neue Qualität erfahren: Zum einen erlaubt das Internet einen interaktiven Dialog mit den Rezipienten von Onlinemedien. Zum anderen ermöglicht die Verbreitung digitaler Informations- und Kommunikationstechnik in Verbindung mit dem Internet die nahtlose Integration von Interaktions- und Feedbackdaten aus diesem Dialog in den Prozess der Inhaltebündelung und -distribution. Die Folge ist eine potenziell höhere Transparenz bzgl. des Verhaltens und der Bedürfnisse eines Kunden bei gleichzeitiger verbesserter Effizienz der Integration kundenbezogener Daten. Aus diesen Potenzialen der Internettechnologien leitet sich eine Reihe theoretischer und jüngst auch erster praktischer Konzepte ab, mit dem Ziel, diese Potenziale im Sinne eines kundenindividuellen Varietätsgrads auszuschöpfen.

In Abschnitt 2.2.2 wurde dargelegt, dass sowohl im Bereich der Industriebetriebslehre als auch in der Marketingforschung besondere Wettbewerbspotenziale darin gesehen werden, Produktangebote für individuelle Nutzer maßzuschneidern. In Kapitel 3 wurde gezeigt, dass in der Medienbranche digital erzeugte Inhalte durch die Möglichkeiten einer modularen, formatneutralen Inhaltespeicherung grundsätzlich beliebig und ohne erhebliche Mehrkosten zu vielfältigen Produktvarianten gebündelt werden können. Die Bereitstellung maßgeschneiderter Mediengüter bildet insofern eine zweifache Problemstellung: Aus einer marketingorientierten Perspektive ist die Bereitstellung maßgeschneiderter Mediengüter in erster Linie eine Frage der Identifikation individueller Rezipientenbedürfnisse und deren Integration in den Prozess der Inhaltebündelung. Aus Sicht der Wirtschaftsinformatik repräsentieren maßgeschneiderte Mediengüter dagegen eine Frage der Automatisierung des Prozesses der Individualisierung.

Vor dem Hintergrund dieser zweigeteilten Problemstellung wird in Abschnitt 4.1 zunächst ein Überblick über die Möglichkeiten und Verfahren gegeben, die eingesetzt werden können, um individuelle Bedürfnisse eines Rezipienten von Onlineinhalten zu identifizieren. In Abschnitt 4.2 werden Softwarekonzepte vorgestellt, die automatisch oder zumindest teilautomatisiert eine Bündelung von Inhalteangeboten entsprechend individuell identifizierbarer Bedürfnisse vornehmen können. Des Weiteren wird in Abschnitt 0 in ökonomischer Hinsicht geprüft, inwiefern eine solche Individualisierung einen Beitrag leisten kann, um einen Mehrwert im Rahmen der Vermarktung von Mediengütern zu bieten. In Abschnitt 4.4 werden Ergebnisse einer empirischen Erhebung zur Vorteilhaftigkeit der Individualisierung von Mediengütern, die anhand von zwei prototypisch realisierten Individualisierungsverfahren durchgeführt wurde, vorgestellt. Die Ergebnisse des Kapitels werden in Abschnitt 4.5 abschließend zusammengefasst. 


\subsection{Individuelle Identifikation von Onlinerezipienten}

Die traditionelle Marktforschung zielt darauf ab, über Umfragen und Beobachtungen der potenziellen Käuferschaft, statistische Aussagen über die Struktur relevanter Kundenbedürfnisse zu ermitteln. ${ }^{403}$ Die Interaktivität digitaler luK-Technik eröffnet dagegen die Möglichkeit, Kunden, ihre Bedürfnisse und ihr Verhalten auf individueller Ebene mit einer Präzision zu identifizieren, die weit über traditionelle statistische Methoden hinausgeht. ${ }^{404}$ Damit individuelle Merkmale eines Rezipienten von Onlineinhalten jedoch dazu verwendet werden können, das inhaltliche Angebot individuell für diesen Rezipienten maßzuschneidern, müssen seine Präferenzen erstens identifiziert und zweitens so gespeichert werden, dass der Rezipient anhand dieser Merkmale bei jedem Wiederholungsbesuch wieder erkannt wird.

In Abschnitt 4.1.1 werden Möglichkeiten der Erstellung von Kundenprofilen auf der Grundlage von freiwilligen Angaben im Zuge einer - entweder eindeutigen oder pseudonymen - Registrierung von Kundenprofilen vorgestellt. In Abschnitt 4.1.2 werden Verfahren gezeigt, um Onlinerezipienten anhand von Verhaltensbeobachtungen zu charakterisieren. Da Kundeninteressen in Bezug auf Profilinformationen denen eines Online-Anbieters diametral gegenüberstehen, ${ }^{405}$ werden in Abschnitt 4.1.3 datenschutzrechtliche Anforderungen der Identifikation von Onlinerezipienten zusammengefasst. Abschnitt 4.1.4 fasst die Möglichkeiten der Identifikation der Präferenzen der Rezipienten von Onlineinhalten zusammen.

\subsubsection{Nutzerprofile auf Grundlage von Befragung}

In Abschnitt 4.1.1.1 werden zunächst die Mechanismen der Ermittlung von kundenindividuellen Profilen auf der Basis freiwilliger Angaben des Inhalterezipienten und die damit verbundenen Probleme erläutert. In Abschnitt 4.1.1.2 werden Mechanismen zur Wiedererkennung eines Rezipienten auf der Grundlage dieser Angaben vorgestellt. In Abschnitt 4.1.1.3 werden die Möglichkeiten der Identifikation von Rezipienten anhand expliziter Befragungen zusammenfassend bewertet.

\section{1.1.1 Erhebung von Präferenzen durch Befragung}

Die Interaktivität digitaler Onlinemedien als Distributionskanal für digitale Güter und Informationsgüter vereinfacht aus Sicht der empirischen Marktforschung die Möglichkeit der Kundenkommunikation sowohl in Bezug auf die technische Abwicklung als auch in Bezug auf die Reichweite der Erhebung von Kundeninformationen im Rahmen von Markt- und Präferenzanalysen. ${ }^{406}$ Dadurch erschließt sich nicht nur die Möglichkeit, stichprobenhafte Marktdaten für statistische Rückschlüsse auf die Marktstruktur zu erheben, sondern darüber hinaus ein umfassendes Kun-

\footnotetext{
$403 \mathrm{Vgl}$. Meffert (2000), S. 93 und $147 \mathrm{f}$.

404 Vgl. Merz (2000), S. 234.

405 Für Onlineanbieter stellen Profilinformationen zwar wertvolle Markt- und Kundeninformationen dar.

Deren automatische Erhebung birgt jedoch die Gefahr eines Eingriffs in die geschützten Persönlichkeitsrechte des Kunden.
}

406 Vgl. Bensberg (2001), S. 2. 
denmanagement in Form der Speicherung von Einzelkundenprofilen auf der Grundlage von demografischen oder präferenzbezogenen Angaben zu entwickeln. ${ }^{407}$

Die im Internet mittlerweile geläufigste Form der Erhebung von individuellen Kundenprofilen bildet die explizite Befragung von Inhalterezipienten in Verbindung mit einer zwar kostenfreien, jedoch häufig obligatorischen Registrierung eines individuellen Nutzerkontos für Informationsangebote. ${ }^{408}$ Bereits Shapiro und Varian erkannten allerdings das Dilemma im Rahmen der Registrierung von Personenund Präferenzprofilen: Mangels Kontrollierbarkeit durch den Anbieter ist die Qualität freiwilliger Angaben von Kunden in der Regel sehr schlecht und in hohem Grade abhängig von ihrem kalkulierten Nutzen aus der Preisgabe ihrer Daten. Eine Untersuchung des Internetnutzungsverhaltens durch die amerikanische Vanderbilt University deckte auf, dass $94 \%$ der beobachteten Internetnutzer generell keine persönlichen Informationen in einer Internetseite hinterließen, und $40 \%$ der registrierten Informationen falsche Angaben enthielten. ${ }^{409}$

Die Qualität und Verbindlichkeit von Profilinformationen ist jedoch, wie Abbildung 4-1 zeigt, abhängig von der Tiefe der Interaktion zwischen Rezipient und Inhalteanbieter und kann durch die Schaffung dauerhafter Transaktionsbeziehungen gesteigert werden:

\begin{tabular}{|c|c|c|c|}
\hline & $\begin{array}{l}\text { Beobachtungsgestützte } \\
\text { Profile }\end{array}$ & $\begin{array}{l}\text { Befragungsgestützte } \\
\text { Profile }\end{array}$ & \\
\hline Qualität der Daten & Information: & Interaktion: & Transaktion: \\
\hline Datenquelle & $\begin{array}{l}\text { - Page Impressions } \\
\text { - AdClicks } \\
\text { - Visits } \\
\text { - Produktauswahl } \\
\text { - Produkte im } \\
\text { Einkaufskorb } \\
\text { - Suchanfrage }\end{array}$ & $\begin{array}{l}\text { - Suchanfrage } \\
\text { - E-Mails } \\
\text { - Nost } \\
\text { Rewsletter- } \\
\text { Registrierung }\end{array}$ & $\begin{array}{l}\text { - Bestellung } \\
\text { - Bezahlung }\end{array}$ \\
\hline Datenverwendung & Log-Dateien & Pseudonyme Profile & $\begin{array}{l}\text { - Kundendaten } \\
\text { - Transaktionsdaten } \\
\text { - Bonität } \\
\text { - Kreditkartendaten }\end{array}$ \\
\hline \multirow[t]{3}{*}{$\begin{array}{l}\text { Identifikation } \\
\text { des Kunden }\end{array}$} & Anonym & Pseudonym & Identifikation \\
\hline & \multicolumn{3}{|c|}{ Handelstransaktion } \\
\hline & Information & Verhandlung & Abwicklung \\
\hline
\end{tabular}

Abbildung 4-1: Qualität von Benutzerprofilen ${ }^{410}$

407 Vgl. Wehleit (2004); Chamoni/Dürsing/Stock (2004).

${ }^{408}$ Ein Beispiel für eine solche Onlineregistrierung bildet etwa das Onlineangebot der Süddeutschen Zeitung (vgl. http://www.sueddeutsche.de).

409 Vgl. Shapiro/Varian (1999), S. 35.

410 In Anlehnung an Merz (2000), S. 235. 
Bei der unregistrierten Informationsnachfrage auf einer Internetseite kann ein Nutzer in der Regel lediglich anonym bspw. anhand von Verhaltensauffälligkeiten in der Protokolldatei des Webservers identifiziert werden. Als Kennzahlen zur Charakterisierung des Besuchs dient bspw. die Anzahl der Seiten- und Anzeigenaufrufe oder eine Analyse betrachteter Seiteninhalte. Interaktionsfunktionen wie Email, Diskussionsforen oder Newsletter erfordern darüber hinaus eine zumindest pseudonyme Authentifizierung eines Nutzers, bspw. anhand eines selbst gewählten Nutzernamens. Die Bestellung und Bezahlung von Produkten setzt schließlich die eindeutige und nachgewiesene Identifikation des Kunden auf Grundlage von Transaktions-, Adress- oder Bezahlungsangaben voraus.

Der Online Buchhändler Amazon verwendet bspw. ein zweistufiges Registrierungsverfahren: Zur Teilnahme an den verschiedenen Formen des Produktempfehlung kann sich ein Nutzer zunächst ein Profil seiner Präferenzen anlegen. Dazu muss eine gültige E-Mailadresse angegeben sowie eine zunächst nicht weiter geprüfte Namensangabe gemacht werden. Die Präferenzen werden dann in einer vierseitigen Auswahlliste von den bevorzugten Produktbereichen auf themen- und schließlich produktspezifischer Ebene eingeschränkt (vgl. Abbildung 4-2). Zum Abschluss einer Transaktion müssen im zweiten Schritt dann eine gültige Rechnungs- und Lieferanschrift bzw. eine Einzugsermächtigung für ein überprüftes Kreditkarten- oder Bankkonto hinterlegt werden.

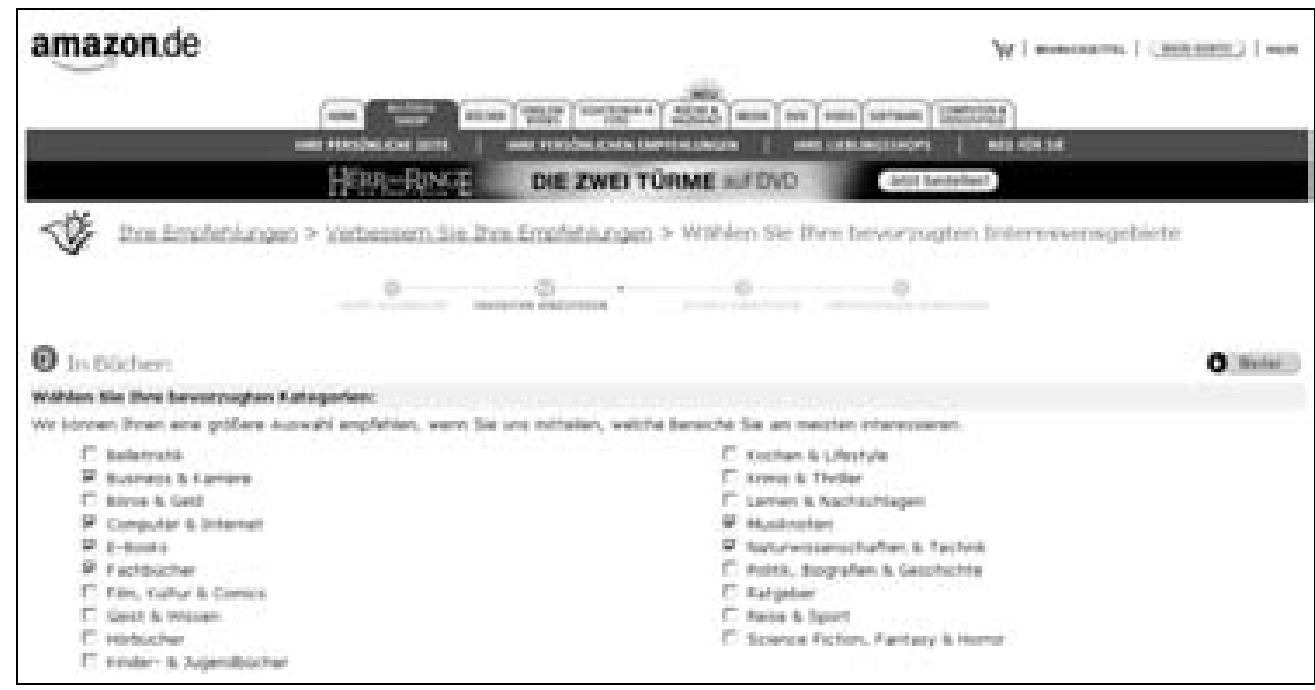

Abbildung 4-2: Registrierung bei Amazon

Neben dem angesprochenen Problem der mangelnden Überprüfbarkeit der Validität von Kunden- und Präferenzinformationen, die im Rahmen von derartigen Befragungsformen erhoben werden, begründen sich weitere Probleme in Bezug auf die Datenqualität in der Natur der Befragung als Erhebungsform: Der Vorteil einer Individualisierung der Kundenbeziehung im Sinne eines "One-to-One“Marketingansatzes besteht darin, aus der Menge potenzieller Kunden diejenigen zu identifizieren, die eine starke Produkt- oder Dienstleistungszufriedenheit und 
damit eine hohe Wiederkaufswahrscheinlichkeit aufweisen. ${ }^{411}$ Durch die freie Zugänglichkeit einer mediengestützen Befragung im Falle einer freiwilligen Dienstregistrierung können jedoch zum einen Selbstselektionseffekte entstehen, die bspw. aufgrund der erforderlichen technischen Bedienkompetenz des Mediums potenziell produktaffine Kunden aus dem Teilnehmerkreis ausschließen und somit die Aussagekraft der Daten des registrierten Kundenstamms verfälschen. ${ }^{412}$ Zum anderen sind die im Zuge einer einmaligen Befragung und Registrierung ermittelten Profile im Zeitablauf statisch. ${ }^{413}$ Lebenslaufbedingte Änderungen der Präferenzen, etwa bei einem Wechsel des Arbeitsplatzes oder Veränderungen in der familiären Situation eines Kunden, bzw. Veränderungen des verfügbaren Angebots, bspw. durch Produktinnovationen mit bislang unbekannten Ausprägungen der Produktmerkmale, können daher nicht ausreichend berücksichtigt werden.

\section{1. 1.2 Identifikation von Rezipienten anhand von Profilen}

Um eine dauerhafte Beziehung mit einem Rezipienten, u.a. zur Förderung der Produkttreue, zu gewährleisten, muss es möglich sein, dass der Rezipient bei jedem Besuch auf der Onlineseite des Inhalteanbieters wiedererkannt wird. Die Gesamtheit der einen Onlinenutzer identifizierenden Merkmale werden im Bereich der Datenverarbeitung häufig auch als „Identität“ bezeichnet. ${ }^{414} \mathrm{Um}$ einen Nutzer im Rahmen einer Dienstsitzung identifizieren zu können, werden die erhobenen Nutzermerkmale seiner Identität in der Regel als individuelles Profil auf dem Server des Dienstanbieters gespeichert. Derzeit existiert kein einheitlicher Standard zur Speicherung von Profilen. Ein Profil enthält jedoch in der Regel Identifikatoren wie z.B. X.500-Verzeichnisnamen oder digitale Zertifikate des Nutzers, demographische Informationen wie Email-Adresse oder Postanschrift, Zahlungsinformation und Transaktionshistorie sowie Bewertungen, Interessen, Qualifikationen oder persönliche Präferenzen. ${ }^{415}$

Die Verwaltung von Nutzerprofilen erfolgt üblicherweise in Form einer ausschließlich serverseitigen Speicherung beim Dienstanbieter. Dies ist unproblematisch, sofern sich der Kunde freiwillig bei jedem Besuch durch Eingabe einer Nutzerkennung gegenüber dem Anbieter einer Onlinepräsenz authentifiziert. Darüber hinaus bieten sich technisch vier denkbare Alternativen einer automatisierten Nutzerauthentifizierung an: die Identifikation über die IP-Adresse des verwendeten Endgeräts (1), die Verwendung clientseitiger Cookies (2), die Wiedererkennung des anbieterseitig gespeicherten Kundenprofils durch eine clientseitige Erweiterungssoftware des Browsers (3).

(1) Die Identifizierung zwischen zwei Kommunikationspartnern im Internet erfolgt grundsätzlich über den 32-Bit Zahlencode der IP-Adresse. Während eines Kommunikationsvorgangs muss jedem Sender und Empfänger eine eindeutige

\footnotetext{
$411 \mathrm{Vgl}$. Kapitel 2.

412 Vgl. Bensberg (2001), S. 35.

$413 \mathrm{Vgl}$. Mobasher (2002), S. 2.

$414 \mathrm{Vgl}$. Köhntopp (2000).

415 Vgl. Wörndl (2003), S. 9.
} 
Adresse zugeordnet sein. Speziell privaten Anwendern, die über einen Dienstanbieter auf das Internet zugreifen, wird allerdings in der Regel für jede Zugangssitzung eine dynamische IP-Adresse aus einem Adresspool des Zugangsdienstleisters zugeordnet, wodurch diese Anwender sitzungsübergreifend nicht mehr eindeutig allein anhand der IP-Adresse identifiziert werden können.

(2) Unter dem Begriff "Cookie“ wird eine Erweiterung der HTTP-Spezifikation zum Austausch von Statusinformationen verstanden, standardisiert als „Request for Comment (RFC) 2109“ im Rahmen der HTTP-Spezifikation. ${ }^{416}$ Cookies sind kleine Textdateien, die auf der Festplatte des Kunden gespeichert werden. Typischerweise enthält ein Cookie lediglich jeweils eine Identifikationsnummer zur Wiedererkennung eines Benutzers und einer Sitzung oder Transaktion. ${ }^{417}$ Weitere standardisierte Attribute im Rahmen der Spezifikation RFC 2109 bilden der Verfallszeitpunkt eines Cookies, der Domainnamen des Webservers des Anbieters und ggf. der Pfadname der Dateireferenz durch die die Sendung des Cookie auslöst wird. ${ }^{418}$ Ein Cookie wird durch den Webserver des Anbieters an den Browser des Nutzers, vor dem eigentlichen HTML-Dokument, zur Speicherung übertragen und entweder bei Abfragen unterhalb des referenzierten Dateipfads oder alternativ, falls dieser nicht angegeben ist, bei jedem HTTP-Befehl des Browsers an den Server gesendet. Aus datenschutzrechtlichen Gründen legt die Cookie-Spezifikation jedoch fest, dass der Benutzer die Cookie-Funktionalität zur Wahrung seiner Anonymität jederzeit ausschalten kann. Deshalb bilden Cookies kein zuverlässiges Instrument für eine eindeutige Identifizierbarkeit eines Nutzers.

(3) Zur Speicherung von Kundenprofilinformationen beim Dienstanbieter reichten Netscape, Firefly und VeriSign 1997 beim World Wide Web Consortium (W3C) die Spezifikation des "Open Profiling Standard“ (OPS) als Träger der Profilinformation als Vorschlag für einen weltweiten Kundenprofilstandard ein. OPS legt Datenformate und Übertragungsverfahren fest, die die OnlineRegistrierung vereinfachen und das Problem der ungeschützten Ablage von Profilinformationen lösen sollen. ${ }^{419}$ Die Verwendung von OPS setzt eine zusätzliche Softwarekomponente im Internetbrowser voraus. Mit dieser Software kann ein Rezipient einmalig ein individuelles Profil anlegen. Die Profilsoftware kontrolliert dann automatisch die vom Kunden einzeln bestimmbare Freigabe jeder Profilinformationskategorie für jeden abgefragten Dienst.

Eine Weiterentwicklung des OPS bildet das „Platform for Privacy Preferences Protocol“ (P3P), das im April 2002 als Spezifikation P3P 1.0 vom W3C veröffentlicht wurde. ${ }^{420}$ Das P3P repräsentiert eine auf XML basierende Protokollfamilie, die sich aus verschiedenen Teilstandards zur Beschreibung und Aus-

\footnotetext{
416 Vgl. Kristol/ Montulli (1997).

$417 \mathrm{Vgl}$. Amberg/Wehrmann (2001), S. 15 f.

418 Vgl. Merz (1999), S. 237.

419 Vgl. Hensley/Metral/Shardanand/Converse/Myers(1997) und Merz (1999), S. 249.

420 Vgl. Cranor/Langheinrich/Marchiori/Presler-Marshall/Reagle (2002).
} 
handlung von Profildaten zusammensetzt. ${ }^{421} \mathrm{P} 3 \mathrm{P}$ zielt in erster Linie darauf ab, Nutzer und deren eindeutig identifizierende Merkmale automatisch gegenüber Dienstanbietern bereitzustellen - und zwar in Abhängigkeit ihrer jeweiligen Datenschutzpräferenzen und dem jeweils vom Dienstanbieter gebotenen Datenschutzniveau. P3P umfasst einerseits eine Sprache, um sowohl nutzerseitige Datenschutzpräferenzen als auch anbieterseitige Datenschutzerklärungen in einem maschinenlesbaren Format zu definieren, andererseits ein Protokoll für den Austausch von Datenschutzpräferenzen, -erklärungen und Profilangaben des Nutzers. Die Profilangaben des Nutzers werden durch ein persönliches Profilrepository im Webbrowser gespeichert. Beim Aufruf einer Webseite erkennt der P3P-Agent im Browser des Agenten automatisch die P3P-lesbare Datenschutzerklärung des Seitenanbieters und stellt ggf. solche Profildaten, zu deren Herausgabe die Datenschutzpräferenzen des Nutzers berechtigen, bereit. Als Kontrollmechanismus für die Richtigkeit der in der Datenschutzerklärung des Seitenanbieters gemachten Angaben können dabei Zertifikate von Drittinstanzen, die das Vertrauen des Nutzers besitzen, dienen. Bislang besitzt das Aushandeln von Profilangaben auf der Grundlage von P3P allerdings noch keine praktische Bedeutung.

\section{1. 1.3 Bewertung freiwilliger Profilangaben}

Demografische Angaben des Kunden zu Person, Alter, Wohnsitz, Beruf und ggf. Bankverbindung bilden grundsätzlich eine umfangreiche Grundlage zur Identifikation eines Kunden. Allerdings kann auf diese Angaben ohne freiwillige Informationspreisgabe des Kunden nicht anderweitig rückgeschlossen werden. Mangels Überprüfbarkeit sind die von Kunden in der Praxis im Internet gemachten Angaben nur von geringer Aussagekraft für einerseits die Identifikation, andererseits auch - wie gezeigt - für die langfristige Entwicklung seiner Bedürfnisse. Ein weiteres Problem in Verbindung mit der Verwendung freiwilliger Angaben des Kunden entsteht zudem im Zusammenhang mit seiner sitzungsübergreifenden Wiedererkennung. Aufgrund der Deaktivierbarkeit im Browser des Nutzers bildet der Cookie-Mechanismus kein verlässliches Verfahren zur Kundenidentifikation. Alternative Verfahren zur Wiedererkennung konnten sich jedoch demgegenüber bislang noch nicht durchsetzen. Standardisierungen wie die P3P-Familie stellen zwar ein ambitioniertes Vorhaben dar, stehen jedoch absehbar vor datenschutzrechtlichen Umsetzungshürden: P3P ist zum einen eine Standardisierung, die im Wesentlichen auf die regulatorischen Bedingungen der USA abzielt und daher einen sehr liberalen Ansatz darstellt. Im Hinblick auf die Geschäftspraktiken der USA, wo praktisch jede Form von Information frei gehandelt wird, stellt P3P dennoch eine regulatorische Einschränkung für Onlineanbieter und somit eine wesentliche Akzeptanzhürde dar. Demgegenüber ist es fraglich, ob sich P3P an den, in Europa im Vergleich zu den USA verordneten Mindeststandards für den Datenschutz anpassen lässt. Zudem unterliegt die Nutzung von P3P in hohem Maße dem Vertrauen der Nutzer in die tatsächlich praktizierte Einhaltung der offiziell bekundeten Datenschutzvereinbarungen durch Zertifizierungsstel-

${ }^{421}$ Vgl. Cranor/Weitzner (2002). 
le und Anbieter. Mangels detaillierter Rechtsvorschriften in diesem Bereich ist es zukünftig vor allem Aufgabe der Zertifizierungsinstanz, zunächst ein solches Nutzervertrauen aufzubauen und durch Möglichkeiten der Einhaltungskontrolle bei Anbietern auch langfristig zu bestätigen.

Aufgrund der genannten Unzulänglichkeiten der individuellen Identifikation von Nutzern auf der Grundlage freiwilliger Angaben, wächst die Bedeutung von Verfahren zur Nutzeridentifikation auf der Grundlage von Beobachtungen des Nutzerverhaltens. Insbesondere wird die Anwendung von Methoden des Data Mining auf Verhaltensdaten eine hohe methodische Qualität zur Ableitung von Nutzungsprofilen und auf deren Grundlage zur Ableitung von individuellen Kundenbedürfnissen beschieden. ${ }^{422}$ Im Folgenden werden diese Verfahren vorgestellt.

\subsubsection{Nutzerprofile auf der Grundlage von Verhaltens- beobachtungen}

Bei jedem Seitenaufruf im Internet hinterlässt ein Nutzer Spuren auf den Webservern der verschiedenen Anbieter. Jeder Webserver verfügt über eine Protokollkomponente, ein softwaretechnisches System, das sämtliche Serveraktivitäten registriert und chronologisch in meist unterschiedlichen anwendungsorientierten Protokolldateien, den so genannten „Server Logfiles“, abspeichert. Zum Standardumfang eines jeden Webservers gehört dabei das sog. "Zugriffsprotokoll“, das sämtliche HTTP-Elementaroperationen dokumentiert. ${ }^{423}$ Diese Zugriffsprotokolle können im Sinne indirekter Beobachtungen als Gegenstand systematischer Analysen des Nutzerverhaltens verwendet werden, über die Rückschlüsse auf die Präferenzen gezogen werden können. In Abschnitt 4.1.2.1 wird zunächst der Inhalt des Zugriffsprotokolls näher erläutert. In Abschnitt 4.1.2.2 werden Analysemöglichkeiten in den Inhalten des Zugriffsprotokolls vorgestellt.

\subsubsection{Protokolldateien als Grundlage einer Beobachtung des Rezipien- tenverhaltens}

Abbildung 4-3 zeigt den Zeilenausschnitt eines typischen Zugriffsprotokolls im Beispiel des Internetauftritts der Universität Göttingen. ${ }^{424}$ Im Zugriffsprotokoll werden folgende Angaben protokolliert: Die IP-Adresse des anfragenden Nutzers, die ggf. übertragene Anmelde- und Authentifizierungskennung des Nutzers (ausgenommen spezielle Anmeldeoperationen bleibt dieses Feld üblicherweise

422 Vgl. u.a. Markellou/Rigou/Sirmakessis (2005); Holland/Kießling (2004); Holland/Ester/Kießling (2002); Ko/Lee (2002).

${ }^{423}$ Darüber hinaus existieren anbieterabhängig weitere Protokollanwendungen wie das sog. „Browserprotokoll", das die Typenbezeichnung des Browsers und des Betriebssystems des zugreifenden Clients dokumentiert, das sog. „Referentenprotokoll“, das die URL-Adresse der Ressource, die der Client in der vorhergehenden Interaktion angefordert hat aufzeichnet und das Fehlerprotokoll, in dem ggf. Uhrzeit, Zieladresse des Verbindungsversuchs und Art eines aufgetretenen Verbindungsfehlers gespeichert werden. Diese werden jedoch im Weiteren nicht berücksichtigt. Vgl. Schockert (2003); Bensberg (2001), S. $43 \mathrm{f}$.

$424 \mathrm{http}: / /$ www.uni-goettingen.de 
leer), der Zugriffszeitpunkt der Serveranfrage, mit Angaben zu Datum (Tag, Monat, Jahr) und Uhrzeit (Stunde, Minute, Sekunde, Zeitzone) und die HTTPAnforderung des Zugriffs. Aufgezeichnet werden zudem die Zugriffsmethode („GET“ für Downloads, „POST“ für Uploads oder „HEAD“ für Anfragen, ob sich die Datei geändert hat), der Zugriffspfad zur übertragenen Datei, das Serverprotokoll und der Status der Dateiübertragung (,200“ für ordnungsgemäß abgeschlossene Übertragung OK, „304“ für die Dateiübertragung bereits besuchter Seiten aus dem Browsercache des Nutzers) sowie der Umfang der übertragenen Datei in Byte.

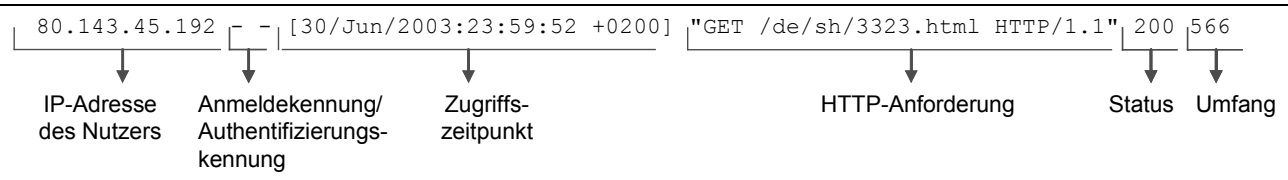

Abbildung 4-3: Inhalt eines Protokolleintrags

Protokolldateien erlauben umfangreiche Analysen des Rezeptionsverhaltens im Sinne einer Beobachtung, da sie sämtliche manipulativen Handlungen des Nutzers auf dem Server lückenlos protokollieren. Dabei sind grundsätzlich zwei verschiedene Analysemethoden denkbar: Erstens, berichtsbasierte Analysen, die auf der Grundlage von Häufigkeitskennzahlen aggregierte statistische Aussagen generieren. Gängige Protokollauswertungen beschäftigen sich dabei unter anderem mit der Ableitung von Interaktivitätsmaßen, bspw. in Bezug auf die Anzahl bestimmter Bereiche im Informationsangebot einer Webseite oder die durchschnittlichen Verweildauer eines Nutzers, sowie der Ableitung von technischen Kennzahlen in Bezug auf den Auslastungsgrad des Servers. ${ }^{425}$ Zweitens, multivariate Methoden speziell aus dem Bereich des Data Mining zur Überprüfung vorformulierter oder automatisch generierter Hypothesen. ${ }^{426}$ Für die Identifikation von individuellen Kundenpräferenzen spielen insbesondere letztgenannte Verfahren eine wichtige Rolle zur Entwicklung und Überprüfung von Hypothesen in Bezug auf individuelle Verhaltensmuster im Rahmen der Gesamtheit der protokollierten Aktivitäten. Im Folgenden werden deshalb Methoden des Data Mining in der Anwendung auf Server Protokolldateien vorgestellt.

\section{1.2.2 Verhaltensanalyse im Zugriffsprotokoll durch Data Mining}

Unter Data Mining ist ein Prozess zu verstehen, „der aus einer Datenmenge implizit vorhandene, aber bisher unentdeckte, nützliche Informationen extrahiert. “427 Data Mining dient dazu, im Zuge der wachsenden Datenflut komplexe Zusammenhänge in großvolumigen Datenbeständen zu untersuchen, die traditionelle statistische Verfahren nur unzureichend durchdringen. Im Bereich des Internet ergeben sich zwei Einsatzfelder für das Data Mining (vgl. Abbildung 4-4): ${ }^{428}$

$425 \mathrm{Vgl}$. Meyer/Wingärtner/Jahke/Lieven (2001), S. 1.

426 Vgl. u.a. Mobasher (2002); Spilinopoulou (2001); Meyer/Wingärtner/Jahke/Lieven (2001).

427 Vgl. Mertens (1994), S. 793.

428 Vgl. Spiliopoulou (2001), S. 491. 
zum einen die strukturelle und inhaltliche Analyse von Internetseiten und verweisen, das so genannte "Web Content Mining“; zum anderen die Analyse von Nutzeraktivitäten in Bezug auf die angebotenen Seiteninhalte, das so genannte „Web Usage Mining“. Im Bereich des hier im Mittelpunkt stehenden Web Usage Mining lassen sich wiederum zwei Anwendungsbereiche unterscheiden: zum einen die nicht personalisierte Nutzungsanalyse zur Identifikation von Verhaltensmuster zur Bildung von Rückschlüssen aus dem Nachfrageverhalten über die inhaltliche Qualität einer Webseite; zum anderen die personalisierte Nutzungsanalyse zur Identifikation individueller Verhaltensmuster zur Erhöhung von Kundenzufriedenheit und Kundenbindung durch maßgeschneiderte Angebote.

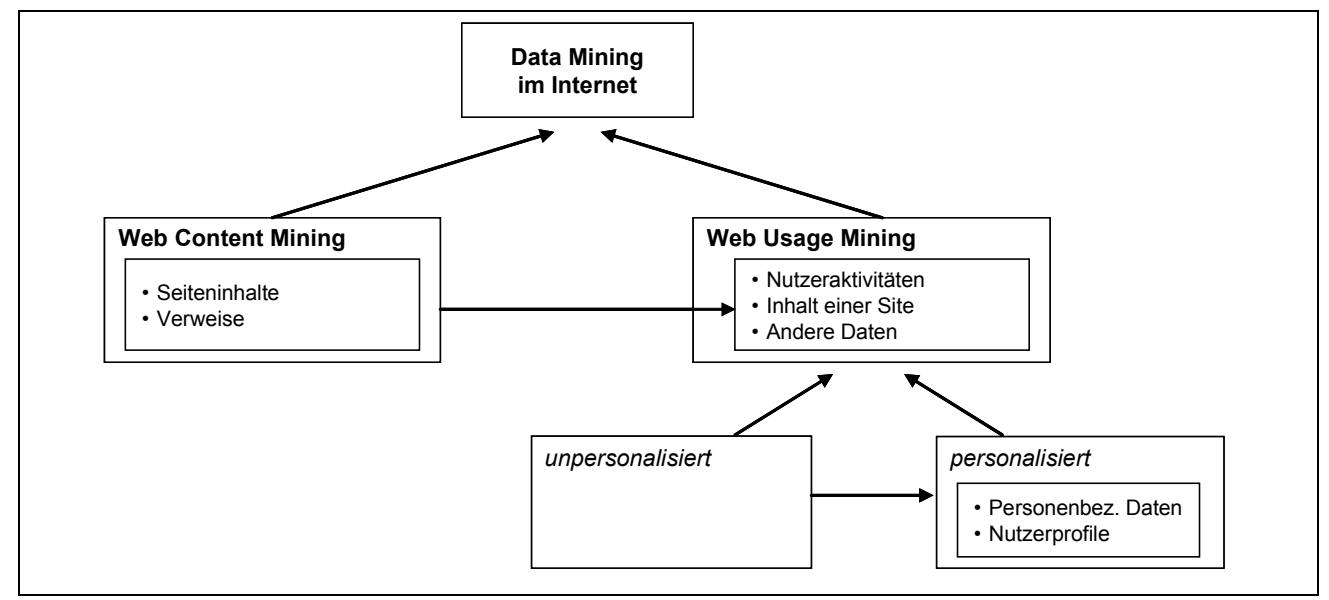

Abbildung 4-4: Anwendungsbereiche von Data Mining im Internet 429

In Abschnitt 4.1.2.2.1 wird zunächst allgemein das Vorgehen des Data MiningVerfahren bei der Identifikation Datenmustern vorgestellt. Im Rahmen der Identifikation von Verhaltensmustern in Protokolldateien werden zwei Teiloperationen unterschieden: die Vorbereitung und Transformation der Daten des Zugriffsprotokolls (vgl. Abschnitt 4.1.2.2.2) und die Erkennung von Verhaltensmustern in den transformierten Protokolldaten (vgl. Abschnitt 4.1.2.2.3).

\subsection{Datenanalyse durch Data Mining}

Data Mining basiert im Wesentlichen auf unterschiedlichen statistischen Verfahren zur Datenaufbereitung. Zum Instrumentarium gehören Klassifikationen bspw. auf der Grundlage von Diskriminanzanlysen oder induktiven Lernverfahren, lineare und nichtlineare Regressionen, Segmentierungen durch Gruppenbildung auf der Grundlage von statistischen Verfahren oder maschinellem Lernen sowie Abhängigkeits- und Abweichungsanalysen. ${ }^{430}$ Data Mining dient zur Ableitung von Informationen aus einer Menge von Daten, bspw. zum Zwecke der Ermittlung von Gestaltungsempfehlungen der Website oder der Kontrolle

${ }^{429} \mathrm{Vgl}$. Spiliopoulou (2001), S. 491.

$430 \mathrm{Vgl}$. Bensberg (2001), S. 96. 
der Seitenqualität. ${ }^{431}$ Durch Anwendung von Methoden des Data Mining erfolgt aus Sicht der Semiotik eine Verdichtung von syntaktisch beschriebenen Daten, wie hier im Fall einer Webserver Protokolldatei, zu sachfeldorientierten Informationen und problembezogenem Wissen. Dieser Vorgang wird allgemein auch beschreiben als Prozess der Wissensidentifikation in Datenbanken („Knowledge Discovery in Databases“, KDD). ${ }^{432}$ Dieser Prozess untergliedert sich in mehrere Teilschritte (vgl. Abbildung 4-5): die Selektion und Transformation von Daten, die Ableitung von Datenmustern, deren Evaluation, Präsentation und Interpretation vor dem Hintergrund der zugrunde gelegten Hypothese.

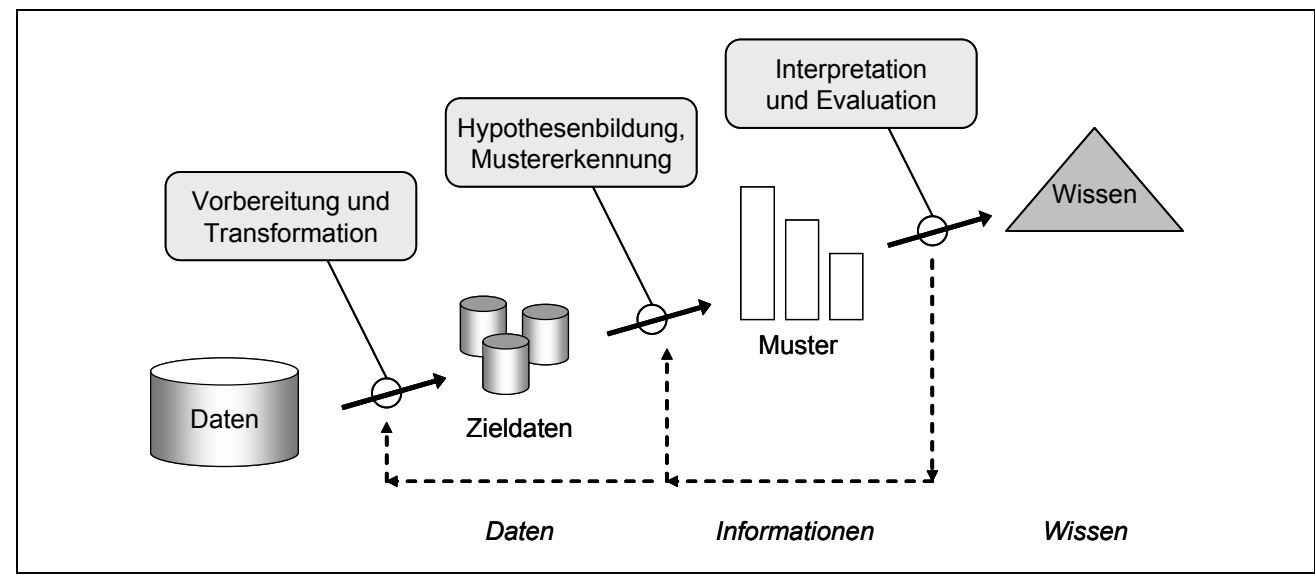

Abbildung 4-5: KDD

Für die Analyse von Webserver-Protokolldateien zur Identifikation individueller Verhaltensmuster müssen im Zuge eines solchen analytischen Prozesses der Wissensidentifikation im Rahmen der Vorbereitungsphase die protokollierten Daten zum einen von Aktivitäten bereinigt werden, die für die Unterscheidung individuellen Verhaltens nicht notwendig sind; zum anderen müssen die Aktivitäten unterschiedlicher Nutzer getrennt und nach Aggregaten virtueller Kundenbesuche wie Sitzungen oder Transaktionen gruppiert werden. ${ }^{433}$ Zur Mustererkennung im nächsten Schritt können dann auf Grundlage vorgegebener oder automatisch generierter Hypothesen über das Nutzerverhalten durch Data Mining Algorithmen signifikante individuelle Verhaltensmuster identifiziert werden. Die Ergebnisse werden im letzten Schritt, bspw. anhand von Kriterien wie Validität, Neuheit oder Verständlichkeit in Bezug auf ihren Wissensbeitrag zum sog. „Domänenwissen“, also der allgemeinen Sachfeldkompetenz eines Inhalteanbieters über seinen Zielmarkt, bewertet. ${ }^{434}$

Insofern in der vorliegenden Untersuchung weniger die Frage einer (ggf. automatisierten) Ergebnismessung, als vielmehr die grundsätzliche Generierung

431 Vgl. Meyer/Wingärtner/Jahke/Lieven (2001), S. 3.

432 Vgl. Frawley/Piatetsky-Shapiro/Matheus (2001) und Fayyad/Piatetsky-Shapiro/Smyth (1996).

433 Vgl. Spiliopoulou (2001), S. 493.

434 Vgl. Fayyad/Piatetsky-Shapiro/Smyth (1996), S. 50 und Bensberg (2001), S. 72. 
solcher Ergebnisse (in Bezug auf die Identifikation individueller Verhaltensmuster) von Interesse ist, wird im Folgenden lediglich auf die Voraussetzungen und Möglichkeiten des Data Mining in den beiden erstgenannten Prozessteilschritten der Datenvorbereitung und -transformation (vgl. Abschnitt 4.1.2.2.2) sowie der Mustererkennung (vgl. Abschnitt 4.1.2.2.3) eingegangen.

\subsection{Datenvorbereitung und -transformation von Webserver- Protokolldateien}

Im Rahmen der Datenvorbereitung können aufgezeichnete Aktivitäten in einer Protokolldatei in zweifacher Hinsicht untersucht werden: Im Zuge einer vertikalen Datenselektion werden verschiedene Datensätze auf ihre Relevanz in Bezug auf den zu prüfenden Zusammenhang hin verglichen (1). Demgegenüber werden im Zuge einer horizontalen Selektion relevante Daten innerhalb jeweils einer protokollierten Elementaroperation des Webservers gefiltert (2). ${ }^{435}$

(1) Der Webserver protokolliert generell sämtliche Aktivitäten, die eine Nutzeranfrage auslöst. Dazu gehören neben den tatsächlichen Seitenaufrufen auch bspw. die Ausführung von grafischen oder verdeckten funktionalen Seitenelementen, Seitenzugriffen von Suchmaschinen oder fehlerhafte Seitenübertragungen. Sofern solche Aktivitäten nicht bewusst von einem Nutzer aufgerufen werden, können sie im Rahmen der vertikalen Datenselektion zur Reduktion des Umfangs der zu analysierenden Daten herausgefiltert werden. Abbildung 4-6 zeigt am Beispiel eines Ausschnitts aus der Protokolldatei des Webservers für den Internetauftritt der Universität Göttingen drei Formen solcher filterbaren Aktivitäten: eine automatisch in Verbindung mit einer Seite aufgerufene JavascriptDatei für funktionale Seitenbedienelemente (1); der automatische Aufruf einer eingebundenen Grafikdatei (2) sowie eine Transkriptionsregel des Webservers für die Umsetzung dynamischer Seitenadressen in der Gestalt statischer HTML-Links (3).

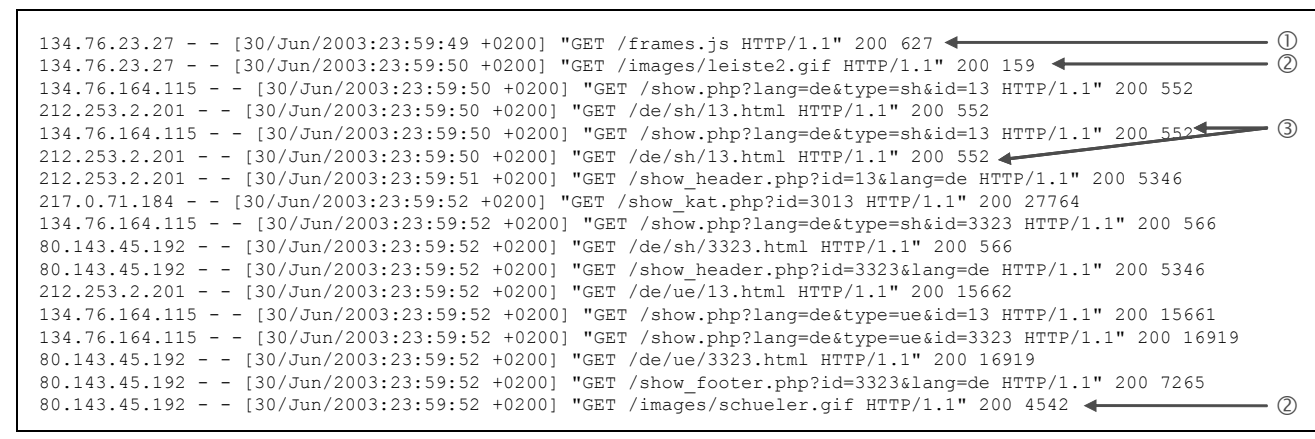

Abbildung 4-6: Datenbereinigung im Zugriffsprotokoll

(2) Nach der vertikalen Datenbereinigung müssen dann die übrig gebliebenen Elementaroperationen horizontal hinsichtlich der interessierenden Daten zerlegt werden, bspw. durch Selektion der Dateiadresse aus dem zusammengesetzten

435 Vgl. Bensberg (2001), S. 134-137. 
Attribut der HTTP-Abfragen. Aufgrund der bereits angesprochenen Akzeptanzprobleme in Verbindung mit Browserregistrierung per Cookie oder Benutzerauthentifizierung per Registrierung repräsentieren die fehlenden Anmeldungsund Authentifizierunskennungen eines Nutzers Fehldaten in Bezug auf die individuelle Identifikation eines Nutzers. Deshalb müssen für die Identifikation einzelner Nutzersitzungen oder Transaktionen sachliche oder zeitliche Zusammenhänge zwischen den protokollierten Elementaroperationen der Protokolldatei hergestellt werden. ${ }^{436}$ Eine Möglichkeit zur Transaktionsableitung auf der Grundlage zeitlicher Abgrenzung bietet sich durch Gruppenbildung in Form einer Clusteranalyse über die zeitliche Distanz zwischen Operationen, ausgelöst von derselben Internetadresse. ${ }^{437}$ Als Proximitätsmaß zur Gruppenbildung kann vom Beobachter und Analysten bspw. eine kritische Referenzdauer auf der Basis der durchschnittlichen Sitzungsdauer einer beobachteten Testgruppe festgelegt werden. Eine andere Form der Transaktionsableitung liegt in der rückwärtigen Pfadrekonstruktion zwischen verschiedenen Abfragen von derselben Zugriffsadresse bis hin zum Einstiegspunkt einer Webseite. Als Analysegrundlage können, neben Internetadresse und Zugriffszeitpunkt aus dem Zugriffsprotokoll, die aufgezeichneten Navigationspfade im Referentenprotokoll herangezogen werden. ${ }^{438}$

Im Rahmen der Datentransformation werden die gruppierten Daten schließlich in Form einer parametrisierten oder binären Vektorisierung für die Anwendung der Algorithmen zur Mustererkennung aufbereitet. Dieser Teilschritt hängt maßgeblich von der gewählten Data Mining-Methodik ab, wobei Assoziationsund Sequenzanalysen die gruppierten Transaktionsdaten direkt, Cluster- und Entscheidungsbaumanalysen dagegen Transaktionsdaten nur in Form aufbereiteter Datenmodelle verarbeiten können. ${ }^{439}$

\subsection{Mustererkennung in Webserver Protokolldateien}

Im Mittelpunkt der folgenden Beschreibung der Methoden im Data Mining zur Mustererkennung in Webserver-Protokolldateien stehen solche Verfahren, die eine automatische Generierung von Hypothesen in Bezug auf die Identifikation individueller Verhaltensmuster erlauben. Dazu gehören insbesondere die Assoziationsanalyse (1) und die Clusteranalyse (2).

(1) Durch Assoziationsanalysen können inhaltliche Abhängigkeiten zwischen Teilmengen eines Datenbestands identifiziert werden. ${ }^{40}$ Die Beschreibung der Assoziationsregeln erfolgt über Signifikanzen auf der Grundlage der Häufigkeit einer identifizierten Regel, dem sog. „Supportfaktor“, in Verbindung mit der Zu-

\footnotetext{
436 Vgl. Bensberg (2001), S. 138.

$437 \mathrm{Vgl}$. Wu/Yu/Ballman (1998).

$438 \mathrm{Vgl}$. Wu/Yu/Ballmann (1998), S. $92 \mathrm{f}$.

$439 \mathrm{Vgl}$. Bensberg (2001), S. 143.

$440 \mathrm{Vgl}$. Agrawal//mielinski/Swami (1993).
} 
verlässigkeit bzgl. des Auftretens dieser Regel in der Grundgesamtheit der analysierten Daten, dem sog. „Konfidenzfaktor“. ${ }^{441}$

Eine Assoziationsanalyse zur Identifikation von Abhängigkeiten zwischen verschiedenen Seitenabfragen auf einem Webserver erfordert zunächst die Bestimmung gruppierter Navigationspfade einzelner Nutzersitzungen. Über den Vergleich der Ähnlichkeit in den Pfadmustern verschiedener Transaktionen können dann signifikante Abhängigkeiten zwischen den Seitenabfragen dieser Transaktionen gebildet werden. Der Supportfaktor (sup) einer Assoziationsregel A.html $\rightarrow$ B.html für die Abfragen zweier HTML-Seiten A und B kann entsprechend Ausdruck 4.1.2.2.3-1 bestimmt werden. Der Konfidenzfaktor (conf) ergibt sich aus der bedingten Wahrscheinlichkeit des Seitenaufrufs B.html gegeben den Seitenaufruf $A . h t m l$ (vgl. Ausdruck 4.1.2.2.3-2). Die Notation der Abhängigkeiten zwischen zwei Seitenabfragen A und B, die bspw. bei einer Konfidenz von $90 \%$ der Aufrufe beider Seiten festgestellt wurde und bei einem Support von 20\% aller Transaktionen auftritt, erfolgt wie abgebildet in Ausdruck 4.1.2.2.3-3.

$$
\begin{aligned}
& \sup (A . h t m l \rightarrow B . h t m l)=p(A \cdot h t m l \cup B . h t m l) \\
& \operatorname{conf}(A . h t m l \rightarrow B . h t m l)=\frac{\sup (A \cdot h t m l \rightarrow B . h t m l)}{\sup (A . h t m l)} \\
& A . h t m l \rightarrow B . h t m l^{0,9 ; 0,2}
\end{aligned}
$$

Eine Variante der Assoziationsanalyse bildet die sog. Pfadanalyse, die Transaktionen auf gleichförmige lineare Pfadmuster hinsichtlich der Häufigkeit ihres Auftretens untersucht. ${ }^{442}$ Ein signifikantes Pfadmuster für eine Seite A, von der mit einer Häufigkeit von $40 \%$ in allen Transaktionen immer die Seite B aufgerufen wird, wird formal notiert entsprechend Ausdruck 4.1.2.2.3-4.

$$
\text { A.html } \rightarrow \text { B.html } l^{0,4}
$$

(2) Clusteranalysen dienen allgemein zur Bestimmung möglichst homogener Gruppen in den Teilmengen eines Datenbestands. ${ }^{443}$ Zur Ermittlung der Ähnlichkeit zwischen den Teilmengen ist zunächst die Bestimmung eines Proximitätsmaßes notwendig, über das dann im nächsten Schritt die einzelnen Daten zu Gruppen fusioniert werden. ${ }^{444}$ Metrisch skalierte Daten können dabei unmittelbar über die quantitative Distanz ihrer Werte verglichen werden. Ein Beispiel für ein Distanzmaß für metrisch skalierte Daten bildet die Minkowski-Metrik in Abhängigkeit eines Distanzgewichtungsfaktors $r$ in Ausdruck 4.1.2.2.3-5. Je höher $r$ gewählt wird, desto höher wird die Differenz zwischen verschiedenen Seitenaufrufen $x_{i}$ und $x_{j}$ eines Nutzers $k$ gewichtet und damit der Abstand $z w i-$ schen Gruppen ähnlicher Distanzwerte größer:

\footnotetext{
441 Vgl. Bensberg/Weiß (1999), S. 430.

442 Vgl. Bensberg (2001), S. 149.

443 Vgl. Grimmer/Mucha (1998), S. 109; Kaufmann/Pape (1996); Steinhausen/Langer (1977). Einen Ü-

berblick über die verschiedenen Verfahren der Clusteranalyse geben Jain/Murty/Flynn (1999).

$444 \mathrm{Vgl}$. Backhaus/Erichson/Plinke/Weiber (2003).
} 


$$
d_{i j}=\left[\sum_{k=1}^{n_{M}}\left|x_{i k}-x_{j k}\right|^{r}\right]^{\frac{1}{r}}
$$

Die protokollierten Aktivitäten eines Webservers werden allerdings als nominalskalierte Daten gespeichert. Um ihre Ähnlichkeit über verschiedene Merkmalsausprägungen zu quantifizieren, muss zuvor eine Binärcodierung für diese Merkmale wie dargestellt in Abbildung 4-7 vorgenommen werden. ${ }^{445}$

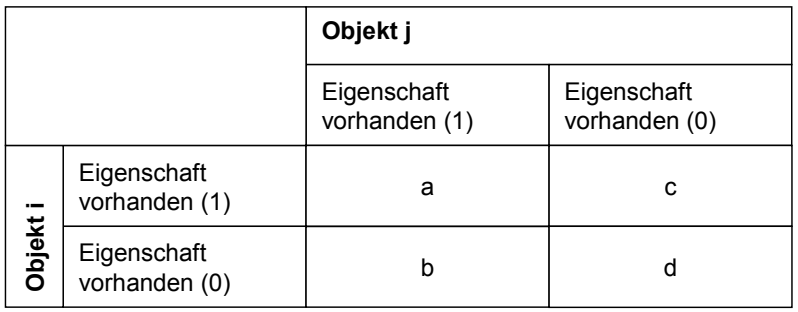

Abbildung 4-7: Binärcodierung zur Ähnlichkeitsbestimmung

Anhand der Häufigkeiten der Merkmalsübereinstimmungen bzw. -abweichungen in den Ausprägungen a-d wird die Ähnlichkeit zweier Objekte i und j bspw. anhand einer Ähnlichkeitsfunktion s gemäß Ausdruck 4.1.2.2.3-6 bestimmt. Die Werte $\lambda$ und $\delta$ repräsentieren darin Gewichtungsfaktoren für die Bedeutung fehlender gemeinsamer Merkmale für die Objektähnlichkeit:

$$
s_{i j}=\frac{a+\delta \cdot d}{a+\delta \cdot d+\lambda \cdot(b+c)}
$$

\section{1.2.3 Bewertung des Web Usage Mining zur Bestimmung von Präfe- renzen}

Der Vorteil der Verwendung beobachtungsgestützt ermittelter Verhaltensmuster im Vergleich zu befragungsgestützten Präferenz- und Kundenprofilen liegt einerseits in der Lückenlosigkeit der Beobachtbarkeit des Nutzerverhaltens, zum anderen in der Flexibilität in Bezug auf die Identifizierbarkeit momentaner individueller Bedürfnisse. Im Falle des Fehlens einer Benutzer- und Authentifizierungskennung ist demgegenüber jedoch allein aus der Verhaltensbeobachtung keine eindeutige Identifizierbarkeit eines Rezipienten gewährleistet. Die konsolidierten Verhaltensmuster entsprechend der dargestellten Methoden ermöglichen lediglich eine Kategorisierung individueller Aktivitäten in stereotype Gruppen, erlauben jedoch keine eindeutige Wiedererkennung eines bestimmten Nutzers im Falle unterschiedlichen Nutzungsverhaltens im Zuge verschiedener Sitzungen.

Die nahe liegende Lösung des Problems der eindeutigen Identifizierbarkeit bei gleichzeitiger Wahrung der situativen Flexibilität, die Kopplung zwischen personenbezogenen und verhaltensbezogenen Daten, unterliegt in Deutschland allerdings rechtlichen Restriktionen. Die derzeitigen rechtlichen Rahmen-

445 Vgl. Bensberg (2001), S. 115 f. 
bedingungen für die Verwendung von personenbezogenen Daten durch kommerzielle Anbieter werden deshalb im Folgenden kurz dargestellt.

\subsubsection{Rechtliche Aspekte der Identifikation von Onlinerezipienten}

Im Voranstehenden wurden die Möglichkeiten zur Identifikation von Onlinenutzern vor dem Hintergrund einer Bereitstellung maßgeschneiderter Angebote als sowohl aus Anbieter- als auch Nachfragersicht wünschenswertes Potenzial dargestellt. Die verbesserten Möglichkeiten der Speicherung, Verarbeitung und Übermittlung personenbezogener Daten bergen jedoch insbesondere aus Nachfragersicht ein erhebliches Missbrauchspotenzial in Form materieller oder immaterieller Nachteile, die Betroffene durch die falsche, unvollständige oder unzulässige Speicherung, Verknüpfung oder Übermittlung erfahren können. ${ }^{446}$ Ein Beispiel für die Missbrauchsrisiken im Umgang mit personenbezogenen Daten bildet die wachsende Spam-Problematik im Internet. ${ }^{447}$ "Spam" bezeichnet unerwünschte Massen-Emails, meist mit Werbeinhalt. Diese ungefragte Massenreklame ist nicht nur lästig, sondern stellt auch ein wirtschaftliches Problem dar, da Serverkapazitäten blockiert werden und für den Adressaten ein Zeitaufwand für das Lesen oder Löschen entsteht. Schätzungen besagen, dass bereits jede zweite E-Mail unerwünscht zugestellt wird, was einem weltweiten Gesamtaufkommen von monatlich zehn Milliarden Nachrichten entspricht. ${ }^{448}$

Der Umgang mit personenbezogenen Daten wird nach deutschem Recht durch den Datenschutz, als juristischer Schutzgegenstand des Individuums vor ungewünschten Eingriffen in die Privatsphäre, reglementiert. Nach deutscher Rechtsauffassung bezeichnet der Begriff des "Datenschutzes“ entgegen seiner wörtlichen Interpretation nicht nur den Schutz von Daten, sondern vielmehr den „Schutz persönlicher Belange bzw. des Persönlichkeitsrechts sowie des Rechts auf informationelle Selbstbestimmung desjenigen, der durch die Daten abgebildet wird. “449 Es handelt sich also in erster Linie um ein juristisches Konzept, mit dem der unbefugte Zugriff sowie die unerlaubte Kenntnisnahme, Verarbeitung und Nutzung von Daten verhindert werden soll. ${ }^{450}$ Insbesondere betrifft dies alle „Einzelangaben über persönliche oder sachliche Verhältnisse einer bestimmten oder bestimmbaren natürlichen Person.“451

Die Verarbeitung personenbezogener Daten sowohl durch öffentliche Stellen des Bundes und der Länder als auch durch nicht-öffentliche Stellen wie privatwirtschaftliche Unternehmen ist reglementiert durch das Bundesdatenschutzgesetz (BDSG). Das BDSG dient dem Schutz des Einzelnen davor, „dass er durch den Umgang mit seinen personenbezogenen Daten in seinem Persönlichkeitsrecht beeinträchtigt wird“ ( $§ 1$, Abs. 2 BDSG). Als personenbezogene

\footnotetext{
446 Vgl. Taeger (1995), S. 18.

$447 \mathrm{Vgl}$. Karzauninkat (2003).

$448 \mathrm{Vgl}$. Braun/Kocovski/Rickert/Waldhauser (2004), S. 11.

449 Vgl. Hobert (1998), S. 80.

$450 \mathrm{Vgl}$. Hobert (1998), S. 81.

451 Vgl. Enquete (1998), S. 219.
} 
Daten gelten dabei alle Einzelangaben „über persönliche oder sachliche Verhältnisse einer bestimmten oder bestimmbaren Person" (§ 3 BDSG). Als spezielle Rechtsnorm wird das Datenschutzrecht seit 1997 durch das Gesetz über die Nutzung von Telediensten (TDG) und das Teledienste-Datenschutzgesetz (TDDSG) für das Internet konkretisiert. Als Teledienste gelten solche elektronischen Informations- und Kommunikationsdienste, „die für eine individuelle Nutzung von kombinierbaren Daten wie Zeichen, Bilder oder Töne bestimmt sind, und denen eine Übermittlung mittels Telekommunikation zugrunde liegt" ( $§ 2$, Abs. 1 TDG). Dies betrifft insbesondere Angebote im Bereich der Individualkommunikation wie Telebanking, Datendienste wie bspw. Verkehrs- oder Wetterdaten, Angebote zur Nutzung des Internet, zur Nutzung von Telespielen oder Transaktionsformen für Waren und Dienstleistungen im E-Commerce (§ 2, Abs. 2 TDG).

Für die Erhebung, Verarbeitung und Nutzung personenbezogener Daten legen BDSG ( $\S 4$, Abs. 1) und TDDSG ( $\S 3$, Abs.1) ein Verbot mit Erlaubnisvorbehalt fest: Ausgenommen einer ausdrücklichen Erlaubnis durch andere Rechtsnormen dürfen personenbezogene Daten nur im Falle einer Einwilligung des Nutzers erhoben, verarbeitet und vom Dienstanbieter genutzt werden ( $\S 3$, Abs. 2 TDDSG). Die Einwilligung durch den Nutzer muss als eindeutige, bewusste Handlung erfolgen, als solche vom Dienstanbieter protokolliert und vom Nutzer sowohl jederzeit abgerufen als auch widerrufen werden können (§ 4, Abs. 2 TDDSG). In der Gesetzesfassung von 2001 hat der Anbieter die Pflicht, den Nutzer zwar über Art, Umfang und Zweck der Erhebung, Verarbeitung und Nutzung seiner Daten zu unterrichten und seine Einwilligung abzuholen, jedoch erst ab Beginn der Nutzung dieser Daten im Rahmen seines Dienstangebots (§ 4, Abs. 1 TDDSG).

Hinsichtlich des Verwendungszecks personenbezogener Daten unterscheidet das TDDSG explizit zwischen Bestands- und Nutzungsdaten. Bestandsdaten sind personenbezogene Daten, die für die Begründung, inhaltliche Ausgestaltung und Änderung eines Vertragsverhältnisses über die Nutzung von Telediensten erforderlich sind (§ 5 TDDSG). Bestandsdaten dürfen zweckgebunden auch ohne Einwilligung des Nutzers erhoben, verarbeitet und genutzt werden. Ihre Verwendung zum Zwecke jeglicher Form der Marktforschung bedarf jedoch der Einwilligung. Nutzungsdaten umfassen dagegen solche Daten, die zur Ermöglichung der Inanspruchnahme und der Abrechnung von Telediensten dienen. Dazu gehören insbesondere Merkmale zur Identifikation eines Nutzers, Angaben über Beginn, Ende und Umfang der Nutzung sowie Angaben über die in Anspruch genommenen Teledienste ( $\S 6$, Abs. 1 TDDSG). Nutzungsdaten dürfen zum Zwecke von Inanspruchnahme und Abrechnung eines Dienstes auch ohne Einwilligung des Nutzers erhoben und bis zu einer Frist von 6 Monaten gespeichert werden. Für solche Daten, die nicht zur Abrechnung dienen, besteht jedoch über das Ende des Nutzungsvorgangs hinaus ein Löschgebot (§ 6, Abs. 4 TDDSG). Im oben genannten Beispiel des Buchhändlers Amazon sind abrechnungsbezogene Nutzungsdaten, die ohne Einwilligung des Nutzers erhoben und über den Nutzungsvorgang hinaus gespeichert werden dürfen, die zur Transaktionsabwicklung notwendigen Zahlungsangaben eines Kunden. Die im voranstehenden Abschnitt beschriebenen Protokolldaten 
dürfen dagegen nur im Rahmen der Dauer der Inanspruchnahme eines Dienstes erfasst werden. Eine Speicherung über die Nutzungsdauer hinaus bedarf dagegen der Anonymisierung der Daten. Dabei gilt ein explizites Kopplungsverbot zwischen allgemeinen Einzelangaben und solchen Merkmalen, die mit denen Einzelangaben einer bestimmten oder bestimmbaren natürlichen Person zugeordnet werden können.

\subsubsection{Bewertung der Möglichkeiten zur Identifikation von Onlinerezipienten}

In Abschnitt 4.1.1 wurde gezeigt, dass Onlinemedien tendenziell die Befragung von Rezipienten im Hinblick auf ihre Präferenzen erleichtern, jedoch ein grundsätzliches Problem einerseits in Bezug auf die Überprüfung des Wahrheitsgehalts der gemachten Angaben und andererseits in Bezug auf die Möglichkeiten zur Wiedererkennung im Falle eines wiederholten Konsums von Onlineinhalten eines Anbieters besteht. in Abschnitt 4.1.2 wurde gezeigt, dass das Problem des Wahrheitsgehalts von freiwilligen Rezipientenangaben durch eine analytische Auswertung von Protokolldateien gemindert werden kann. In Abschnitt 4.1.3 wurde jedoch erläutert, dass die Auswertung der in einem Serverprotokoll gespeicherten Verhaltensdaten im Falle eines Wiederholungskonsums nicht einem individuellen Rezipienten eindeutig zugeordnet werden darf. Abbildung 4-8 fasst die Merkmale der vorgestellten Identifikationsverfahren abschließend zusammen.

\begin{tabular}{|l|l|l|}
\hline $\begin{array}{l}\text { Eigenschaften } \\
\text { des Nutzerprofils }\end{array}$ & \multicolumn{1}{|c|}{ Explizite Befragung } & \multicolumn{1}{|c|}{ Verhaltensbeobachtung } \\
\hline Quellen der Profildaten & $\begin{array}{l}\text { - Registrierung eines Nutzerkontos } \\
\text { - Zufriedenheitsbefragung } \\
\text { - Abwicklung getätigter Transaktionen }\end{array}$ & $\begin{array}{l}\text { Dokumentation einzelner Requests (bspw. } \\
\text { Page-Impressions, Ad-Clicks, etc.) in } \\
\text { Serverprotokollen }\end{array}$ \\
\hline Identifikation durch... & $\begin{array}{l}\text { - IP-Adresse } \\
\text { - Cookies } \\
\text { - OPS/P3P }\end{array}$ & $\begin{array}{l}\text { - Bestimmung eindeutiger Sitzungen in } \\
\text { zuvor bereinigten Protokolldaten } \\
\text { Bestimmung stereotyper Verhaltensmuster } \\
\text { anhand von Sitzungsvergleichen }\end{array}$ \\
\hline Bewertung & $\begin{array}{l}\text { - Statische Natur erhobener Profile } \\
\text { - Fehlende Kontrolle der Datenqualität } \\
\text { - Unzuverlässigkeit bestehender Identifkations- } \\
\text { instrumente }\end{array}$ & $\begin{array}{l}\text { - Keine eindeutige Nutzeridenfikation } \\
- \text { Zusammenführung von Verhaltensprofilen } \\
\text { und expliziter Befragung datenschutz- } \\
\text { rechtlich untersagt }\end{array}$ \\
\hline
\end{tabular}

Abbildung 4-8: Zusammenfassung der vorgestellten Identifikationsverfahren

Vor dem Hintergrund der insbesondere datenschutzrechtlichen Unvereinbarkeit von expliziter Befragung und Verhaltensbeobachtung zur Identifikation von Inhalterezipienten muss im Rahmen der im Weiteren untersuchten Möglichkeiten einer Bereitstellung individueller Mediengüter zwischen Verfahren unterschieden werden, die Angebote für einen eindeutig bestimmbaren Nutzer maßschneidern und solchen, die lediglich eine verhaltensbezogene Anpassung vornehmen. Diese Unterscheidung wird daher im Rahmen der im Weiteren dargestellten Verfahren zu individuellen Bereitstellung von Mediengütern aufgegriffen. 


\subsection{Bereitstellung individueller Mediengüter}

Gemäß der Theorie des in Kapitel 3 beschriebenen „Uses and Gratifications Approach" erwirbt ein Käufer dasjenige Mediengut, von dem er sich zum Kaufzeitpunkt den höchsten Nutzen verspricht. ${ }^{452}$ Aufgrund der beschriebenen Identifizierbarkeit individueller Merkmale im Rahmen eines rechnergestützten Onlinedialogs eröffnen sich dabei Möglichkeiten, den Identifikationsvorteil gegenüber dem Käufer in einer individuellen Gestaltung des Produktangebots auszunutzen und dadurch den Kaufanreiz für inn zu erhöhen.

Maßgeschneiderte Angebote von Mediengütern liefern, wie in Kapitel 0 noch eingehender untersucht wird, nur dann einen Mehrwert gegenüber standardisierten Angebotsformen, wenn das Inhalteangebot automatisch entsprechend wahrgenommener Handlungen oder Profilmerkmale eines Nutzers angepasst wird und dadurch der Auswahl- und Nutzungsaufwand des Rezipienten sinkt. Vor dem Hintergrund der bereits in Kapitel 2 angestellten Vorüberlegungen zu den DV-technischen Möglichkeiten einer Individualisierung von Beratungssystemen im Handel, kann das dort beschriebene Prinzip sog. Empfehlungssysteme eingesetzt werden, um automatisch eine solche Inhalteselektion durchzuführen. Abschnitt 4.2.1 stellt die grundsätzlichen Eigenschaften von Systemen zur Bereitstellung individueller Mediengüter dar. In Abschnitten 4.2.2 und 0 werden unterschiedliche Varianten von Systemen zur Individualisierung von Mediengütern vorgestellt. Die Möglichkeiten der Bereitstellung individueller Mediengüter werden in Abschnitt 4.2.4 zusammenfassend bewertet.

\subsubsection{Eigenschaften von Systemen zur Individualisierung von Inhalteangeboten}

Anknüpfend an die in Kapitel 3 angestellten Überlegungen zur Bereitstellung von Mediengütern als Inhaltebündel werden in Abschnitt 4.2.1.1 zunächst grundsätzliche Möglichkeiten der Individualisierung im Angebot von Mediengütern erläutert. In Abschnitt 4.2.1.2 wird das Konzept der Individualisierung vorgestellt, auf deren Grundlage Mediengüter automatisch nach individuellen Kundenbedürfnissen maßgeschneidert werden können.

\subsection{1 Formen der Individualisierung im Angebot von Mediengütern}

Wie bereits in Kapitel 3 erläutert wurde, werden Inhalte in der Medienbranche üblicherweise in gebündelter Form angeboten, um einerseits Distributionskosten zu senken und andererseits Synergien zwischen den Zahlungsbereitschaften der Rezipienten zu erzeugen. Insofern hat ein Rezipient, der einen bestimmten Inhalt rezipieren möchte, ein zweifaches Auswahlproblem: Er muss erstens aus der Vielfalt angebotener und ggf. konkurrierender Angebote ein für inn passendes Mediengut auswählen. Ist die Kaufentscheidung für ein bestimmtes Mediengut gefallen, werden aus den im Distributionsformat dieses

452 Vgl. Runte (2000), S. 1. 
Produkts gebündelten Inhalten dasjenige (oder diejenigen) gezielt rezipiert, für die sich der Rezipient interessiert.

Vor diesem Hintergrund können Empfehlungssysteme im Rahmen des Angebots von Mediengütern in zweifacher Hinsicht zum Einsatz kommen (vgl. Abbildung 4-9.): als Angebotsfilter im Zuge der Auswahl konkurrierender Mediengüter (1) und als Inhaltefilter im Rahmen der Bündelung von Inhalten in einem Mediengut.

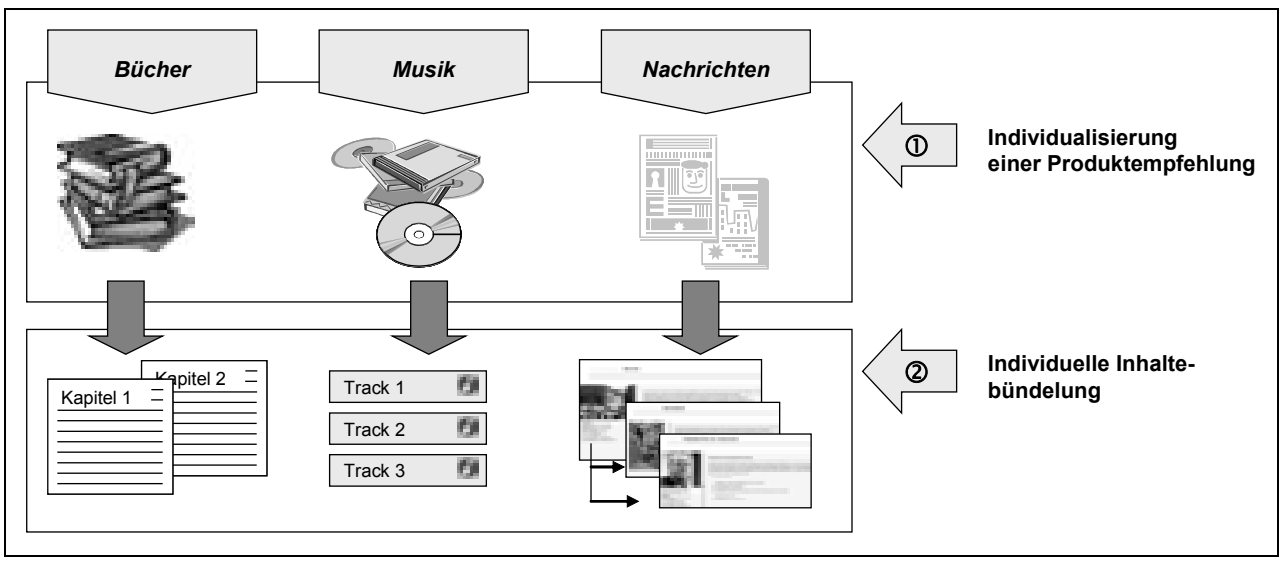

Abbildung 4-9: Individualisierungsformen im Angebot von Mediengütern

(1) Systeme zur individuellen Empfehlung von Produktangeboten sind kein rein medienspezifisches Phänomen. Wenn auch der Onlinebuchhandel Amazon mit einem Empfehlungssystem für Mediengüter eine gewisse Vorreiterrolle im E-Commerce spielte, stellen mittlerweile eine ganze Reihe von Internetshops ähnliche Empfehlungssysteme bereit. Das Prinzip solcher Empfehlungssysteme beruht darauf, zu einem jeweils ausgewählten Produkt Kaufempfehlungen durch andere Käufer oder durch den jeweiligen Seitenbetreiber anzubieten. ${ }^{453}$ Insofern derartige Empfehlungssysteme keine medienspezifischen Besonderheiten besitzen, wird im Folgenden auf eine eingehende Untersuchung verzichtet. ${ }^{454}$

(2) Um ein Mediengut individuell aus verschiedenen Inhalten für einen Rezipienten maßzuschneidern, muss nicht nur eine Kaufempfehlung ausgesprochen werden, sondern automatisch eine individuelle Bündelung verfügbarer In-

453 Vgl. Kapitel 2.

${ }^{454}$ Einen über die bereits in Kapitel 2 angestellten Vorüberlegungen hinausgehenden Überblick über Empfehlungssysteme bieten Konstan/Schafer/Riedl (2001). Neben Empfehlungssystemen wurden in den späten 1990er Jahren unter dem Stichwort "selektive Informationsverteilung" oder "Selektive Dissemination of Information" (SDI) auch die Anwendung von Individualisierungssystemen für industrielle Informationssysteme vorgeschlagen. SDI-Verfahren zielen darauf ab, dynamische Informationen im Rahmen von industriellen Planungs- und Kontrollsystemen gezielt an Interessenten zu verteilen (vgl. Mertens/Griese (2002), S. 55). Ein Anwendungsgebiet von SDI-Verfahren in Industriebetrieben bilden bspw. Früherkennungs- und Frühwarnsysteme. Die technischen Konzepte zur individuellen informationsbereitstellung beruhen im Kern ebenfalls auf den nachstehend erläuterten Prinzipien und werden daher nicht gesondert untersucht. 
halte vorgenommen werden. Eine solche aktive Produktbündelung erfordert entsprechend eine vergleichsweise detaillierte Analyse von einerseits den Bedürfnissen des Rezipienten und andererseits der Merkmale der verfügbaren Inhalte. Bereits in den 1980er Jahren wurden am amerikanischen MIT erste Konzepte entwickelt, um Inhalte individuell in einem speziellen Bündelungsformat, ähnlich einer Zeitungstitelseite, anzubieten. ${ }^{455}$ Für die in diesem Zusammenhang angestellten Überlegungen wurde zusammenfassend der Begriff "Daily Me" - sinnbildlich für eine individuelle elektronische Tageszeitung - entwickelt. Abbildung 4-10 zeigt den grundsätzlichen Prozess der Bereitstellung einer solchen individuellen Zeitung. ${ }^{456}$

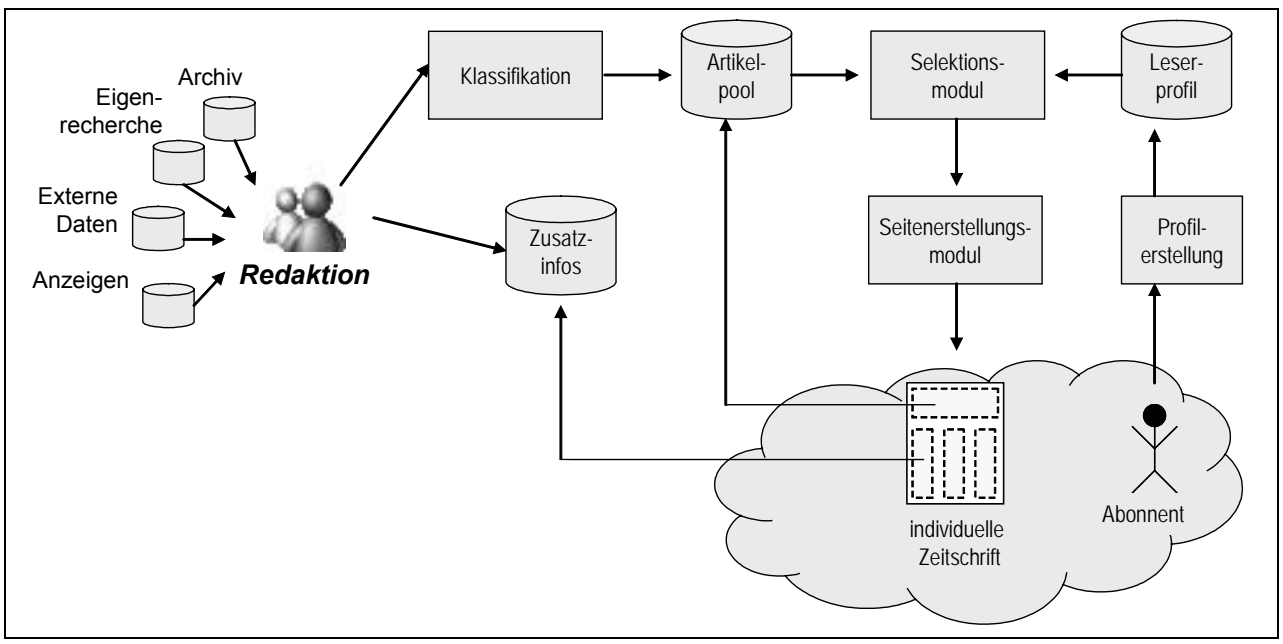

Abbildung 4-10: Grundkonzept eines individuellen Medienguts

Die im Zusammenhang mit Überlegungen zu „Daily Me"-Konzepte beruhen einerseits auf Klassifikationsmechanismen der verfügbaren Inhalte eines Artikelpools und andererseits auf gespeicherten Leserprofilen. Es wurden eine Reihe von Softwaremechanismen vorgeschlagen, um Inhalte individuell bereitzustellen, darunter Suchmechanismen ${ }^{457}$, gruppenbezogene Empfehlungen ${ }^{458}$ und Verfahren zur Integration unterschiedlicher Inhaltequellen ${ }^{459}$. Allerdings verzichten diese Pionierkonzepte grundsätzlich auf eine explizite Modellierung individueller Rezipientenpräferenzen. Vor dem Hintergrund der in Abschnitt 4.1 dargelegten Entwicklungen der letzten Jahre, die eine Identifikation der Präferenzen von Inhalterezipienten auf individueller Ebene erlauben, werden daher im Folgenden Konzepte erläutert, die eine Inhaltebündelung automatisch auf der Grundlage individueller Rezipientenmerkmale vornehmen.

$455 \mathrm{Vgl}$. Bender (2002) und Bender/Cehsnais/Elo/Shaw/Shaw (1996).

$456 \mathrm{Vgl}$. Mertens et al. (2005), S. 140.

457 Vgl. Kay (1991).

458 Vgl. Chesnais/Mucklo/Sheena (1995).

$459 \mathrm{Vgl}$. Bender/Cehsnais/Elo/Shaw/Shaw (1996). 


\subsection{2 Aufbau von Individualisierungssystemen für Mediengüter}

Empfehlungssysteme (i.w.S.) verwenden digitalisierte Inhalte, die ein Produkt oder eine Dienstleistung beschreiben, um daraus eine Kaufberatung für einen individuellen Kunden abzuleiten. Das Empfehlungssystem unterstützt dadurch die Leistungsspezifikation und -zuordnung im Rahmen der Handelstransaktion. ${ }^{460}$ Bilden digitale Inhalte selbst die zu vermarktende Güterleistung, kann ein Empfehlungssystem darüber hinaus dafür eingesetzt werden, um eine Individualisierung im Bereich der Leistungserbringung zu ermöglichen. In Anlehnung an Grimm/Jüstel/Klotz (2002) sollen solche Empfehlungssysteme, die eine individualisierte Leistungserbringung digitaler Güter ermöglichen, im Weiteren als Individualisierungssysteme bezeichnet werden.

Den Kern eines Individualisierungssystems bildet eine regelbasierte Software, die die Art und Weise festlegt, wie Informationen und Angebote dem Kunden präsentiert werden. ${ }^{461}$ Ein Individualisierungssystem, das digitale Inhalte individuell für einen Rezipienten entsprechend seiner Bedürfnisse bündelt, muss über drei Komponenten verfügen (vgl. Abbildung 4-11): ein Nutzermodell, in dem die Bedürfnisse und Präferenzen des Rezipienten gespeichert sind (1); ein Ressourcenmodell, das verfügbare Inhalte auf der Grundlage von Inhalteeigenschaften kategorisiert (2); ein automatisches Verfahren, einen sog. „Inferenzmechanismus", der die individuelle Zuordnung zwischen Ressourcen und Kunden vornimmt (3).

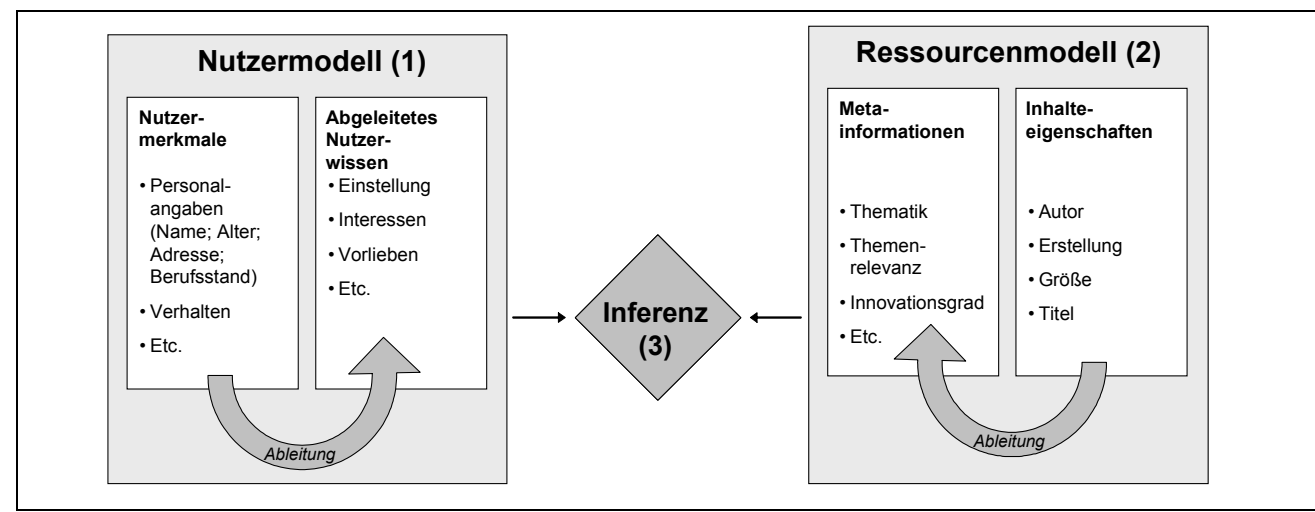

Abbildung 4-11: Aufbau eines Individualisierungssystems

(1) In der Regel kann ein einzelnes identifiziertes Nutzermerkmal, wie bspw. eine dem Nutzer zugeordnete Handlung in der Protokolldatei eines Webservers, nicht automatisch in ein maschinenverständliches Ereignis umgewandelt werden, anhand dessen die individuelle Anpassung eines Medienguts vorgenommen werden kann. Das zentrale Problem von Individualisierungssystemen im Rahmen der Nutzermodellierung besteht vielmehr darin, die maschinell erfassbaren nutzerbezogenen Rohdaten zu Bedürfnissen und Präferenzen in Be-

\footnotetext{
460 Vgl. Kapitel 2.

461 Vgl. Grimm/Jüstel/Klotz (2002), S. 181 und Schackmann/Link (2001).
} 
zug auf die verfügbaren Inhalte zu verdichten. Ein Individualisierungssystem erzeugt daher auf der Grundlage maschinell erfassbarer Nutzermerkmale Annahmen über den Nutzer in Bezug auf seine Einstellungen, Interessen oder Vorlieben, die in einem Nutzermodell gespeichert werden. Auf der Grundlage der Annahmen des Nutzermodells wird die weitere Interaktion des Nutzers an dessen individuelle Bedürfnisse und Präferenzen angepasst. Das Nutzermodell enthält somit das gesammelte spezifische Wissen über einen individuellen Nutzer für das jeweilige Mediengut. ${ }^{462}$ Die Rohdaten der Nutzermodellierung können, wie auch schon in Kapitel 4.1 erläutert wurde, aus Beobachtungen der unmittelbaren manipulativen Interaktionen eines Nutzers, aus expliziten Nutzerbefragungen oder aus Analysen der durch den Nutzer nachgefragten Informationen gewonnen werden. Die Umsetzung der Nutzermodellierung kann in expliziter Form durch ein gekapseltes Programmmodul vorgenommen werden, oder lediglich implizit als integraler, eingebetteter Bestandteil des Individualisierungssystems erfolgen. Wenngleich auch der Fokus der im Weiteren vorgestellten Systemkonzepte weniger auf einer DV-technischen Umsetzung als vielmehr auf der algorithmischen Funktionsweise der Individualisierung liegt, ist in erster Linie von impliziten Nutzermodellierungsformen auszugehen. ${ }^{463}$

(2) Damit ein Individualisierungssystem eine individuelle Auswahl an Inhalten für einen Rezipienten vornehmen kann, müssen zu den in der Datenbank verfügbaren Inhalten Metainformationen als Auswahlkriterien auf Grundlage vordefinierter Kategorien gespeichert werden. ${ }^{464}$ Eine solche Inhaltekategorisierung kann entweder im einfachsten Fall durch den Autor, bspw. im Zuge der Inhalteerstellung erfolgen oder - technisch anspruchsvoller - automatisch durch Inhaltsanalyse und -klassifikationsinstrumente gebildet werden. Beispiel für Verfahren zur Analyse von textbasierten Inhalten bilden Techniken zur Erkennung von Textstrukturen, die Erstellung von Stopplisten sowie Wortanalysen durch Stammformbildung, durch Analyse des Bedeutungskontextes bei Mehrdeutigkeiten oder durch Ermittlung von Mehrworttermen. Auf der Grundlage solcher Textanalysen können Inhalte in logisch zusammengehörige Partitionen gruppiert werden. ${ }^{465} \mathrm{Im}$ Weiteren werden insbesondere im Rahmen der ersten Systemvariante Verfahren einer automatischen Inhalteanalyse erläutert.

(3) Als Inferenzmechanismen verwenden Individualisierungssysteme in der Regel statistische Klassifikationsalgorithmen, um Inhalte entsprechend den jeweiligen Nutzermodellen zu gruppieren. ${ }^{466}$ Zum Einsatz kommen dabei Vektorraummodelle, ${ }^{467}$ Verfahren auf Grundlage kombinierter Wahrscheinlichkeiten, ${ }^{468}$

\footnotetext{
462 Vgl. Fink/Kobsa (2000).

463 Beispiele für gekapselte Nutzermodellierungskomponenten finden sich bspw. bei Finin (1989), Brajnik/Tasso (1992), Kay (1995), Kobsa/Pohl (1995), Orwant (1995) und Konstan et al. (1997)

$464 \mathrm{Vgl}$. Hess (2004).

$465 \mathrm{Vgl}$. Frants/Shapiro/Voiskunskii (1997).

$466 \mathrm{Vgl}$. van Merteren/van Sommeren (2000).

467 Vgl. Salton/Wong/Yang (1975).

$468 \mathrm{Vgl}$. Billsus/Pazzani (1999).
} 
Entscheidungsbäume oder neuronale Netze. ${ }^{469}$ Inferenzmechanismen von Individualisierungssystemen werden üblicherweise nach der Quelle der Daten, auf denen die Empfehlung beruht, unterschieden. Im Zuge der einführenden Überlegungen zu anpassungsfähigen DV-Systemen in Kapitel 2 wurden bereits zwei grundsätzliche Varianten von Inferenzmechanismen zur Auswahl individueller Angebote vorgestellt: „Empfehlungssysteme (i.e.S.)“, die eine Zuordnung zwischen Produkteigenschaften und Profilmerkmalen vornehmen und „Empfehlersysteme“, die Empfehlungen auf der Grundlage von Ähnlichkeiten zwischen Profileigenschaften verschiedener Nutzer ableiten. Wie in Kapitel 4.1 erläutert wurde, muss zudem in Bezug auf die Datengrundlage der durch den Inferenzmechanismus verwendeten Profile unterschieden werden zwischen Systemen auf der Grundlage expliziter Nutzerangaben und solchen auf der Grundlage von Verhaltensbeobachtungen. Vor dem Hintergrund dieser zwei Klassifikationsmerkmale lassen sich idealtypisch vier Systemvarianten von Individualisierungssystemen unterscheiden (vgl. Abbildung 4-12).

\begin{tabular}{|c|c|c|}
\hline \multicolumn{1}{c|}{$\begin{array}{c}\text { Empfehlungs- } \\
\text { systeme }\end{array}$} & \multicolumn{1}{c|}{$\begin{array}{c}\text { Empfehler- } \\
\text { systeme }\end{array}$} \\
\cline { 2 - 3 } Explizite & $\begin{array}{c}\text { Selbst- } \\
\text { selektions- } \\
\text { systeme }\end{array}$ & $\begin{array}{c}\text { Collaborative } \\
\text { Filtering } \\
\text { Systeme }\end{array}$ \\
\cline { 2 - 3 } Beobachtung & $\begin{array}{c}\text { Eigenschafts- } \\
\text { basierte Filter- } \\
\text { systeme }\end{array}$ & $\begin{array}{c}\text { Beobachtungs- } \\
\text { basierte Filter- } \\
\text { systeme }\end{array}$ \\
\hline
\end{tabular}

Abbildung 4-12: Varianten von Individualisierungssystemen

Bei Selbstselektionssystemen wird die individuelle Auswahl an Inhalten durch den Rezipienten selbst vorgenommen. Bei eigenschaftsbasierten Filtersystemen wird die Auswahl an Inhalten durch Auswertungen der vom Rezipienten in der Vergangenheit konsumierten Inhalte ermittelt. Empfehlersysteme ermitteln demgegenüber systematische Ähnlichkeiten in den Merkmalen verschiedener Nutzer und bilden darüber Annahmen über gemeinsame Produktpräferenzen. Dabei verwenden sog. „Collaborative Filtering Systeme“ explizit vom Rezipienten gemachte Bewertungen in Bezug auf konsumierte Inhalte während beobachtungsbasierte Filtersysteme Aktivitäten eines Rezipienten spezifischen Stereotypen gespeicherter Verhaltensmuster und den diesen Mustern beigeordneten Empfehlungen zuordnen.

Im Folgenden werden diese vier Systemvarianten im Einzelnen erläutert. In Abschnitt 4.2.2 werden zunächst die beiden Varianten von Empfehlungssystemen erläutert. In Abschnitt 0 erfolgt eine Darstellung der Funktionsweise von Empfehlersystemen im Rahmen von Individualisierungssystemen.

${ }^{469} \mathrm{Vgl.} \mathrm{Yang/Liu} \mathrm{(1999).}$ 


\subsubsection{Individualisierung auf Grundlage von Empfehlungs- systemen}

Der einfachste Fall der Individualisierung eines Medienguts bildet die Selbstselektion als relevant erachteter Inhaltemerkmale oder -eigenschaften durch den Nutzer. Selektionsgrundlage bilden Auswahllisten, die der Anbieter anhand von kategorisierten Merkmalen und Eigenschaften verfügbarer Inhalte bereitstellt. Die jeweilige Auswahl eines Nutzers wird in einem Profil gespeichert. Auf der Grundlage dieses Profils wird die Inhalteauswahl beim nächsten Dienstaufruf des Nutzers auf diejenigen Angebote mit den entsprechenden Eigenschaften und Merkmalen reduziert. In Abschnitt 4.2.2.1 wird ein einfaches Konzept eines auf Selbstselektion beruhenden Individualisierungsmechanismus vorgestellt.

Eine Variante der Selbstselektion bildet das Verfahren des sog. „eigenschaftsbasierten Filterns", das eine Angebotsindividualisierung lediglich auf der Grundlage Eigenschaften der vom Rezipienten in der Vergangenheit konsumierten Inhalte, jedoch ohne spezielle Kategorisierung inhaltlicher Merkmale, vornimmt. Ziel des eigenschaftsbasierten Filterns bildet die Ableitung einer Klassifikationsregel für vom Nutzer ungesehene Objekte hinsichtlich ihrer Passgenauigkeit zu seinen Präferenzen anhand von signifikanten Korrelationen zwischen Inhalten und Präferenzprofil. ${ }^{470}$ In Abschnitt 4.2.2.2 wird ein eigenschaftsbasiertes Individualisierungssystem auf Grundlage eines eigenschaftsbasierten Filteralgorithmus erläutert. ${ }^{471}$

\subsubsection{Individualisierung durch Selbstselektion}

Damit ein Inhalterezipient selbst Auswahlkriterien für ein maßgeschneidertes Angebot an Inhalten bestimmen kann, müssen ihm Merkmale bekannt sein, nach denen die beim Anbieter verfügbaren Inhalte unterschieden werden können. Solche Unterscheidungsmerkmale können je nach Art der angebotenen Inhalte zeitbezogener Natur (bspw. das Erstellungsdatum oder der Erstellungszeitpunkt), typbezogener Natur (bspw. Text-, Musik- oder Filmdateien), urheberbezogener Natur (bspw. der Autor von Texten, der Komponist von Musiktiteln oder der Regisseur von Filmen) oder inhaltebezogener Natur (bspw. das redaktionelle Ressort einer Nachricht, die Musikgattung eines Musiktitels oder das Genre eines Films) sein. Anhand derartiger Merkmale kann der Rezipient beim ersten Aufruf des Inhalteangebots eines Anbieters ein Präferenzprofil definieren, indem er die gelisteten Merkmale in der Reihenfolge entsprechend seiner Präferenzen anordnet. Ein Beispiel für eine derartige Form der Selbstselektion bietet das individuelle Dienstangebot des Onlineportals Yahoo!. Abbildung 4-13 zeigt die Konfigurationsseite des zugehörigen Individualisierungssystems mit den entsprechenden Auswahllisten. Darin können Inhalte, die nach inhaltlichen Merkmalen vorkategorisiert wurden, jeweils bestimmten Anzeigebereichen des durch die Portalseite vorgegebenen Distributionsformats zugeordnet werden.

\footnotetext{
470 Vgl. van Meteren/van Someren (2000) und Runte (2000), S. 10 f.

$471 \mathrm{Vgl}$. van Meteren/van Someren (2000).
} 


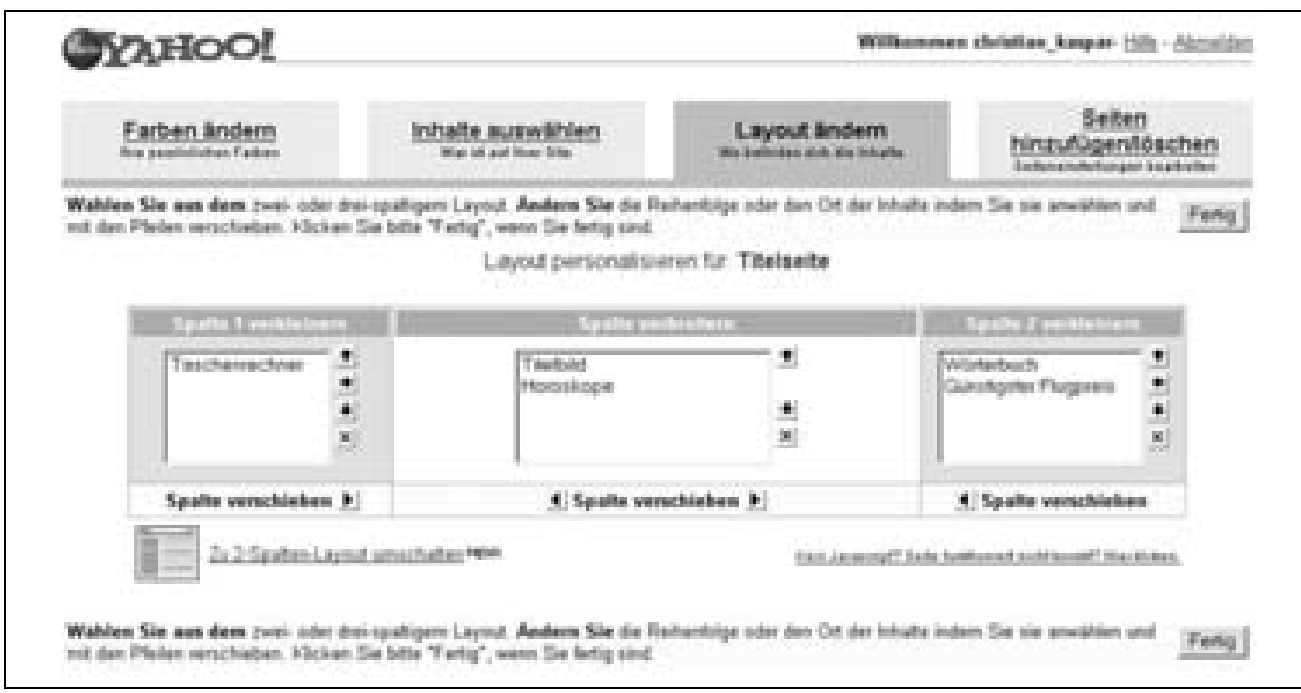

Abbildung 4-13: Individualisierung durch Selbstselektion im Onlineportal Yahoo!

Damit das Individualisierungssystem eines auf Selbstselektion basierenden Inhalteangebots eine Auswahl an Inhalten entsprechend der durch den Rezipienten vorgegebenen Merkmalspräferenzen vornehmen kann, wird im Profil des Nutzers für das jeweilige Inhaltemerkmal $\left(C_{i}\right)$ ein Klassenwahrscheinlichkeitswert $\left.\left(0 \leq P\left(C_{i}\right) \leq 1\right)\right)$ gespeichert. ${ }^{472}$ In Abhängigkeit der Anzahl $(n)$ der maximal durch das jeweilige Distributionsformat des Inhalteanbieters vorgegebenen Anzeigebereiche kann ein solcher Klassenwahrscheinlichkeitswert bspw. für das am meisten präferierte Inhaltemerkmal als $P\left(C_{1}\right)=1$ bzw. für das am wenigsten präferierte Merkmal als $P\left(C_{n}\right)=1 / n$ berechnet werden. Ruft der Rezipient nach der Definition seiner Präferenzen das Inhalteangebot auf, ordnet das Individualisierungssystem automatisch diejenigen Inhalte, die der jeweils präferierten Kategorie angehören, dem jeweiligen Anzeigebereich des Bündelungsformats zu.

Existieren mehr Inhalte eines präferierten Merkmalstyps als im jeweiligen Anzeigebereich des Bündelungsformats darzustellen sind, muss die Auswahl der jeweiligen Inhalte weiter, bspw. anhand ihrer Aktualität, eingeschränkt werden. Dafür kann der jeweilige Klassenwahrscheinlichkeitswert mit einem zeitlichen Gewichtungsfaktor $0<\beta \leq 1$ multipliziert werden. Bspw. bleiben tagesaktuelle Inhalte ungewichtet, Inhalte vom Vortag erhalten den Gewichtungsfaktor 0,95 usw. Die Zuordnungswert (REC) eines Inhalts im Themenbereich $j$ des Distributionsformats des Inhalteanbieters wird damit berechnet gemäß Ausdruck 4.2.2.1-1.

$$
R E C=\beta \cdot P\left(C_{j}\right) \cdot{ }^{473}
$$

472 Vgl. Kaspar/Hagenhoff (2004).

$473 \mathrm{Vgl}$. Kaspar/Hagenhoff (2004). 


\subsubsection{Individualisierung durch eigenschaftsbasiertes Filtern}

Die Selbstselektion von Inhaltemerkmalen als Grundlage eines individuellen Inhalteangebots setzt zum einen voraus, dass die verfügbaren Inhalte des Anbieters entweder bereits entsprechend bestimmter Merkmale vorkategorisiert sind oder eine solche Kategorisierung leicht durchzuführen ist. Zum anderen erfordert eine Selbstselektion zwangsläufig ein gewisses Maß an Interaktionsbereitschaft seitens des Rezipienten. Um den Interaktionsaufwand für den Nutzer zu reduzieren, können alternativ sog. eigenschaftsbasierte Filterverfahren eingesetzt werden, um Präferenzen eines Rezipienten automatisch anhand von inm in der Vergangenheit konsumierter Inhalte, bzw. anhand von Beurteilungen, die er über inm bekannte Inhalte abgibt, abzuleiten. Eigenschaftsbasierten Filterverfahren liegt die Annahme zugrunde, dass die Eigenschaften von Inhalten, die ein Rezipient in der Vergangenheit konsumiert hat, wesentliches Indiz für seine Präferenzen darstellt und er auch in Zukunft Inhalte mit ähnlichen Eigenschaften konsumieren wird. Die Anwendung eigenschaftsbasierter Filterverfahren setzt voraus, dass das maßzuschneidernde Inhalteangebot maschinell analysierbar ist, weshalb sich insbesondere textbasierte Inhalte für dieses Verfahren eignen.

Die Funktionsweise des eigenschaftsbasierten Filterverfahrens kann vereinfachend anhand des sog. Vektorraummodells erläutert werden: Bspw. kann ein Inhalt $X$ durch Indizierung der Wortbausteine des zugrunde liegenden Texts beschrieben werden als Vektor $\vec{x}$ seiner Worteigenschaften im n-dimensionalen Merkmalsraum entsprechend Ausdruck 4.2.2.2-1 bzw. als Ortsvektor durch den Punkt $x=\left(x_{1}, \ldots, x_{n}\right)$.

$$
\vec{x}=\left(\begin{array}{c}
x_{1} \\
\vdots \\
x_{n}
\end{array}\right)
$$

Zur Reduktion des Merkmalsraums auf sprachliche Besonderheiten können im Rahmen einer Datenvorbereitung irrelevante Textbausteine wie Stoppwörter oder HTML-Tags gelöscht werden und die verbleibenden Wörter auf ihre Wortstämme reduziert werden. ${ }^{474}$ Als Ergebnis dieser Reduktion kann ein Dokument $D$ beschrieben werden als gewichteter m-dimensionaler Vektor $D=\left(w_{i} t_{i}, \ldots, w_{m} t_{m}\right)$ signifikanter Wortstammmerkmale $t_{i}$. Der Gewichtungsfaktor $w_{i}$ kann dabei über das sog. tf-idf Schema ${ }^{475}$ anhand der Merkmalshäufigkeiten $t f_{i}$ bestimmt werden gemäß Ausdruck 4.2.2.2-2, wobei n die Anzahl der Dokumente im Produktangebot und $d f_{i}$ die Anzahl von Dokumenten mit mindestens einem Merkmal $t_{i}$ darstellen.

474 Eine solche sprachliche Merkmalsreduktion von Inhalten basiert auf der Annahme, dass Merkmale, die häufig in verschiedenen Dokumenten verwendet werden, weniger geeignet sind, Merkmale, die seltener in verschiedenen Dokumenten verwendet werden, dagegen besser geeignet sind, einen konkreten Inhalt zu beschreiben. Letztere werden daher im Rahmen entsprechender Reduktionsverfahren stärker gewichtet. Das dabei zur Anwendung kommende Prinzip wird auch als „inverse Dokumentenhäufigkeit" bezeichnet (vgl. Rechenberg/Pomberger (2002), S. 924).

475 term-frequency - inverse document frequency 


$$
w_{i}=t f_{i} \cdot \log \left(\frac{n}{d f_{i}}\right)
$$

Das Präferenzprofil eines Rezipienten kann (in Analogie zur Vektorisierung der Produktinhalte) als Vektor der Eigenschaften von solchen Inhalten abgebildet werden, die er in der Vergangenheit konsumiert hat. Somit lässt sich das Profil des Nutzers notieren als Vektor $\vec{p}$ präferierter Merkmalsausprägungen durch den Punkt $P=\left(u_{1}, \ldots, u_{n}\right)$, wobei $u_{i}$ die jeweils präferierte Ausprägung des Nutzers in Bezug auf das Merkmal $i$ darstellt. Der Vektor $\vec{p}$ wird allgemein bezeichnet als „Rating" eines Nutzers über die betrachteten Merkmalsdimensionen. ${ }^{476}$

Damit kann schließlich die Ähnlichkeit eines Dokuments mit den Präferenzen des Nutzers anhand der Vektorähnlichkeit über den geometrischen Abstand bestimmt werden. Eine solche Ähnlichkeitsmetrik bildet das sog. "Cosinus-Maß" für die Verhältnis zweier Vektoren mit dem Zwischenwinkel $\alpha$ in Bezug auf die betrachteten Merkmalsdimensionen, wie als Beispiel für einen dreidimensionalen Merkmalsraum dargestellt in Abbildung 4-14. ${ }^{477}$

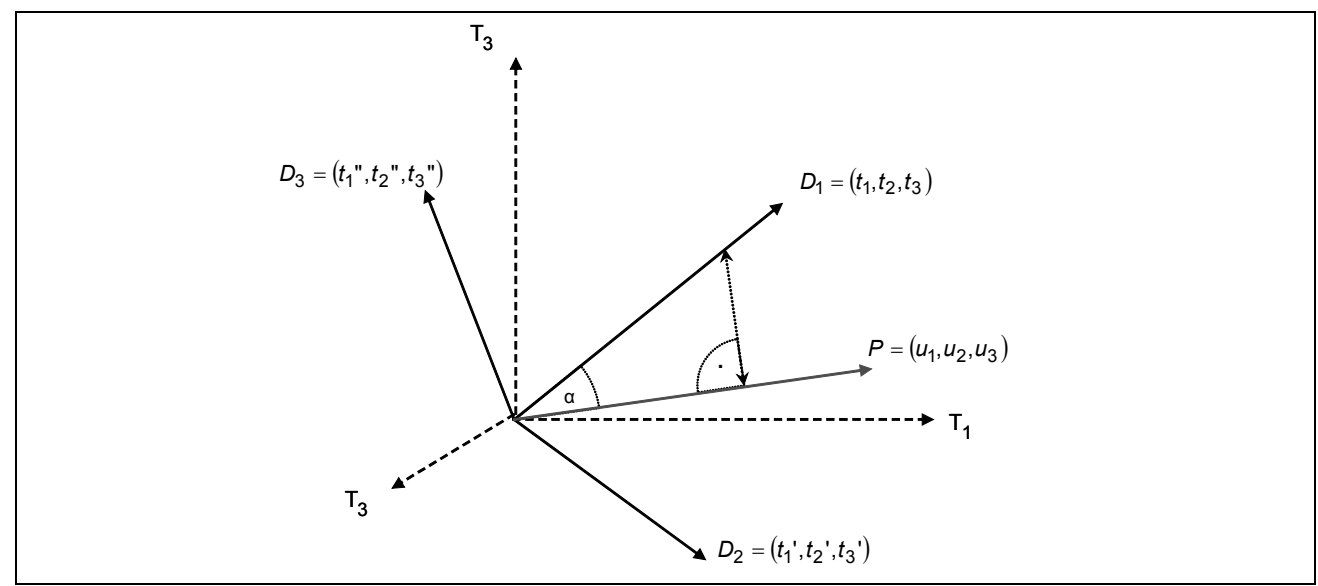

Abbildung 4-14: Ähnlichkeitsbestimmung durch eigenschaftsbasiertes Filtern ${ }^{478}$

Das Maß für die Ähnlichkeit $\operatorname{sim}(D, P)$ zwischen einem Dokument $D$ und dem Präferenzprofil $P$ eines Nutzers kann berechnet werden über das Skalarprodukt der beiden Vektoren, auf den Einheitskreis normiert um das Produkt ihrer Beträge ${ }^{479}$ entsprechend Ausdruck 4.2.2.2-3.

476 Vgl. Runte (2000), S: 35 f.

477 Wenn Vektoren ähnlich ausgerichtet sind, ist der Winkel zwischen ihnen entsprechend klein und der Cosinus des Winkels bewegt sich gegen eins. Sind zwei Vektoren genau gleich ausgerichtet, ist der Winkel zwischen innen 0 und der Cosinus genau 1. Es ist anzumerken, dass das Cosinus-Maß keine Bestimmung von Vektoridentitäten erlaubt (was eine Überprüfung von Ausrichtung und Länge erfordern würde), sondern lediglich Ähnlichkeiten zwischen Vektoren anhand ihrer Ausrichtung feststellt.

478 In Anlehnung an Salton/Wong/Yang (1975), S. 614.

479 Vgl. Hess/Schneider (2003): Der Abstand zwischen zwei Punkten $D_{1}=\left(t_{1}, t_{2}, t_{3}\right)$ und $P=\left(u_{1}, u_{2}, u_{3}\right)$ ist definiert durch ihren sog. „euklidischen Abstand“ als $\sqrt{\left(u_{1}-t_{1}\right)^{2}+\left(u_{2}-t_{2}\right)^{2}+\left(u_{3}-t_{3}\right)^{2}}$. Darüber hinaus ist das 


$$
\operatorname{sim}(D, P)=\cos (\alpha)=\frac{D \cdot P}{\|D\| \cdot\|P\|}=\frac{\sum_{k} u_{k} \cdot w_{k}}{\sqrt{\sum_{k} u_{k}^{2} \cdot \sum_{k} w_{k}^{2}}}
$$

\subsubsection{Kombination von Selbstselektion und eigenschaftsbasiertem Filtern}

Beide Varianten von Empfehlungssystemen, Selbstselektion und eigenschaftsbasiertes Filtern, erfordern ein gewisses Maß an Interaktion des Rezipienten. Im Falle der Selbstselektion gibt der Rezipient allerdings eine Anzahl von Selektionskriterien aktiv vor, im Falle des eigenschaftsbasierten Filters leitet dagegen das System automatisch Selektionskriterien aus zuvor bewerteten Inhalten ab. Dabei besteht das Problem eines Selbstselektionssystems darin, dass die von Rezipienten abgegebene Präferenzordnung im Zeitablauf statisch ist, während ein eigenschaftsbasierter Filter bei jeder abgegebenen Bewertung eine neue Ordnungsskala berechnet. Insofern ist ein eigenschaftsbasierter Filter einem Selbstselektionssystem immer dann überlegen, wenn die automatische Ableitung von Selektionskriterien in der Lage ist, eine größere Anzahl von Inhalteeigenschaften, die für den Rezipienten relevante Auswahlkriterien für seine Bewertung darstellen, zu erfassen. Allerdings ist ein eigenschaftsbasierter Filter auch immer dem Risiko einer gewissen Fehlauswahl unterworfen. Beispielsweise bewertet ein fußballinteressierter Rezipient einen Artikel über die Kritik des Grünen-Politikers Daniel Cohn-Bendit an der Kandidatur Franz Beckenbauers als UEFA-Präsident mit hohem Interesse. ${ }^{480}$ Durch Worthäufungen im Zusammenhang mit EU-Politik - Cohn-Bendit ist Abgeordneter des Europaparlaments - kann ein eigenschaftsbasierter Filter eine mögliche Fehlinterpretation des Interesses des Rezipienten an Europapolitik vornehmen.

Um die Probleme beider Systeme - die statische Natur von Präferenzordnungen eines Selbstselektionssystems und das Fehlerrisiko der Präferenzenermittlung eines eigenschaftsbasierten Filters - zumindern, erscheint eine Kombination beider Verfahren sinnvoll. Dafür definiert ein Rezipient zunächst mit Hilfe eines Selbstselektionssystems eine Präferenzordnung (vgl. Abschnitt 4.2.2.1). Um eine langfristige Verbesserung der statischen Präferenzordnung zu erreichen, kann bei wiederholtem Dienstaufruf durch einen Nutzer die anfängliche Klassifikation der Präferenzen mit Hilfe einer naiven Bayesschen Textklassifikation verfeinert werden. Es wird dafür angenommen, dass jeder Aufruf eines Inhalts ein Signal für ein besonderes Interesse des Nutzers am jeweiligen Thema darstellt. Es werden signifikante Wortmerkmale eine semantische Textanalyse als Bestandteile des individuellen Nutzerprofils gespeichert.

Skalarprodukt (oder innere Produkt) der Ortsvektoren dieser Punkte proportional zum Cosinus des Winkels zwischen den beiden Produkten bzw. gleich dem Produkt von Cosinus des Zwischenwinkels und den Beträgen der beiden Vektoren: $\vec{d} \cdot \vec{p}=\cos (\alpha) \cdot|\vec{d} \cdot \cdot| \vec{p} \mid$. Das Skalarprodukt wird häufig zur

Abstandsbestimmung vorgezogen, da es sich in der Regel leichter berechnen lässt. 480 Vgl. Spiegel-Online (2005). 
Enthält ein neu erzeugter Inhalt im Inhaltearchiv des Anbieters ähnliche Merkmale - signalisiert durch eine signifikante Häufigkeit ähnlicher Begriffe - wird das ursprüngliche Präferenzmaß durch die sog. „a posteriori Wahrscheinlichkeit" gewichtet. Die a posteriori Wahrscheinlichkeit zeigt an, dass ein Inhalt $X$, der die Merkmale $x_{i}$ enthält, in der Präferenzklasse $C_{j}$ eingeordnet wurde. Nach der (naiven) Bayesschen Annahme, die besagt, dass alle Merkmale $x_{i}$ eines Inhalts $X$ unabhängig sind, kann die a posteriori Wahrscheinlichkeit berechnet werden gemäß Ausdruck 4.2.2.3-1 (wobei D die Anzahl der Merkmale in Klasse $C_{j}$ bestimmt). Die Zuordnungswert (REC) eines Inhalts im Themenbereich $j$ des Distributionsformats des Inhalteanbieters wird (in Erweiterung zu Ausdruck 4.2.2.1-1) berechnet gemäß 4.2.2.3-2.

$$
\begin{gathered}
P\left(C_{j} \mid x\right)=\frac{P\left(C_{j}\right)}{P\left(x_{i}\right)} \cdot \prod_{i=1}^{\mid D} P\left(x_{i} \mid C_{j}\right) \\
R E C=\beta \cdot\left[P\left(C_{j}\right)+P\left(C_{j} \mid C_{i}\right)\right]
\end{gathered}
$$

Übersteigt bspw. aufgrund einer hohen semantischen Passgenauigkeit eines neu erstellten Inhalts dessen kalkulierter Empfehlungswert dem ursprünglichen Klassifikationsschwellenwert, wird das zugehörige Themenfeld für die Dauer der Sitzung in die jeweils höhere Präferenzklasse und damit einer höher liegende Darstellungsebene eingeordnet. Bei einem neuen Aufruf des Inhalteangebots wird wieder die ursprüngliche Klassifikationsreihenfolge verwendet, die Selektion einzelner Nachrichtenbeiträge folgt also dem Prinzip „Ziehen mit Zurücklegen“. Abbildung 4-15 zeigt den Ablauf des Individualisierungsverfahrens.

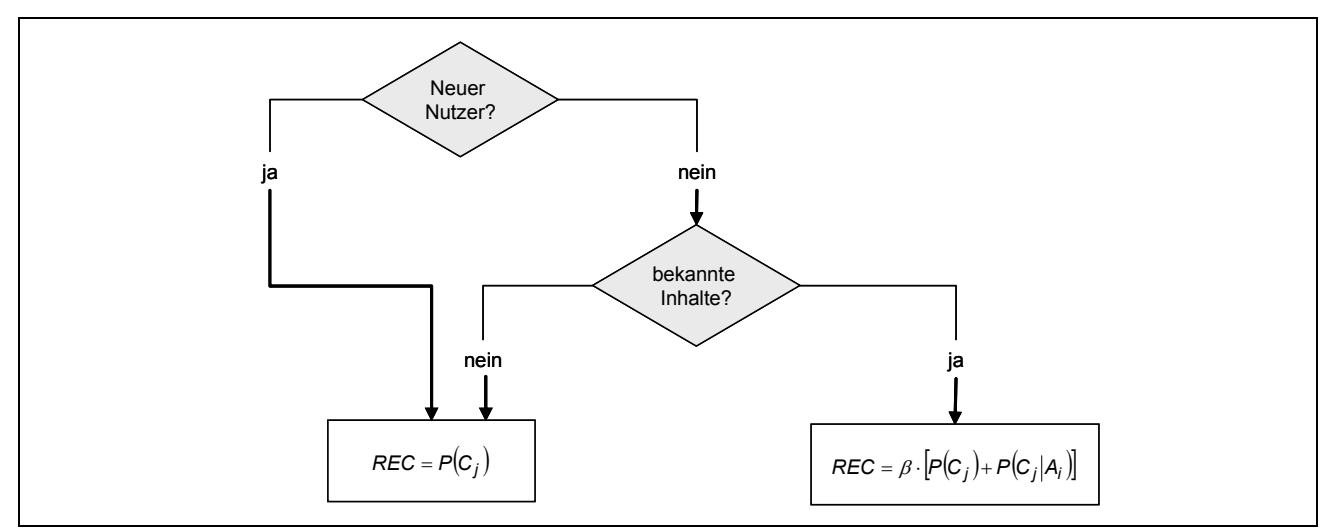

Abbildung 4-15: Kombination aus Selbstselektion und eigenschaftsbasiertem Filtern

\subsubsection{Individualisierung auf Grundlage von Empfehlersystemen}

Die Verwendung des eigenschaftsbasierten Filterverfahrens zur Individualisierung des Produktangebots besitzt in der Praxis zwei Schwachstellen: ${ }^{481}$ Erstens kann das Verfahren nur bei solchen Produkten angewandt werden, deren Inhalt einfach rechnergestützt analysierbar ist. Es versagt jedoch bei Produkten, die

481 Vgl. Melville/Mooney/Nagarajan (2002), S. 1. 
nur einen geringen Textanteil besitzen, wie Software, Bilder oder Ideen. Zweitens können solche Produkte generell nicht empfohlen werden, die keine semantischen Übereinstimmungen mit dem Präferenzprofil des Nutzers aufweisen. Eine zweite Gruppe von Empfehlungsalgorithmen die im Rahmen sog. Empfehlersysteme eingesetzt werden, verwendet daher anstelle einer direkten Ähnlichkeitsüberprüfung zwischen Produkteigenschaften und Nutzerpräferenzen strukturelle Ähnlichkeiten zwischen den Präferenzprofilen verschiedener Nutzer als Grundlage einer individuellen Produktempfehlung. Dabei kann anhand des Grades, in dem ein Nutzer seine Präferenzen durch explizite Angaben selbst aufdeckt oder diese anhand von Beobachtungen abgeleitet werden, zwischen zwei Systemtypen, sog. „Collaborative Filtering“ (CF) Verfahren (vgl. Abschnitt 4.2.3.1) auf der einen Seite und beobachtungsbasierten Filtern (vgl. Abschnitt 4.2.3.2) auf der anderen Seite, unterschieden werden.

\subsubsection{Individualisierung durch Collaborative Filtering}

Die Verwendung des eigenschaftsbasierten Filterverfahrens zur Individualisierung des Produktangebots besitzt in der Praxis zwei Schwachstellen: ${ }^{482}$ Erstens kann das Verfahren nur bei solchen Produkten angewandt werden, deren Inhalt einfach rechnergestützt analysierbar ist. Es versagt jedoch bei Produkten, die nur einen geringen Textanteil besitzen, wie Software, Bilder oder Ideen. Zweitens können solche Produkte generell nicht empfohlen werden, die keine semantischen Übereinstimmungen mit dem Präferenzprofil des Nutzers aufweisen. Eine zweite Gruppe von Empfehlungsalgorithmen, sog. „Collaborative Filtering“ (CF) Verfahren, verwenden daher anstelle einer direkten Ähnlichkeitsüberprüfung zwischen Produkteigenschaften und Nutzerpräferenzen strukturelle Ähnlichkeiten zwischen den Präferenzprofilen verschiedener Nutzer als Grundlage einer individuellen Produktempfehlung. In Abschnitt 4.2.3.1.1 wird das dem Collaborative Filtering zugrunde liegende Prinzip erläutert. Folgend wird in Abschnitt 4.2.3.1.2 eine Erweiterung des Collaborative Filtering Grundprinzips speziell für die Individualisierung von inhaltebezogenen Gütern erläutert.

\subsection{Grundform von Collaborative Filtering-Algorithmen}

Ebenso wie eigenschaftsbasierte Filterverfahren verwenden CF-Algorithmen gespeicherte Produktbewertungen als Präferenzprofile. Grundlage des Filterverfahrens bildet die Datenmatrix $(U)$ sämtlicher Ratingvektoren $\left(u_{1} \ldots u_{M}\right)$ relevanter Nutzer (M) innerhalb der betrachteten Gruppe von Objekten ( $\mathrm{N}$; vgl. Fehler! Verweisquelle konnte nicht gefunden werden.). 


$$
U=\left(\begin{array}{c}
u_{1} \\
\ldots \\
u_{i} \\
\ldots \\
u_{M}
\end{array}\right)=\left[\begin{array}{ccccc}
u_{11} & \ldots & u_{1 j} & \ldots & u_{1 N} \\
\ldots & \ldots & \ldots & \ldots & \ldots \\
u_{i 1} & \ldots & u_{i j} & \ldots & u_{i N} \\
\ldots & \ldots & \ldots & \ldots & \ldots \\
u_{M 1} & \ldots & u_{M j} & \ldots & u_{M N}
\end{array}\right]
$$

Abbildung 4-16: Ratingmatrix beim Collaborative Filtering

In der Realität ist die Datenmatrix aufgrund des bereits angesprochenen Informationsproblems hinsichtlich der Erhebung von Nutzerpräferenzen unvollständig. Das Ziel der CF-Methodik besteht deshalb darin, die fehlenden Werte im Rating-Vektor jeweils eines Kunden über Ähnlichkeiten zu anderen in der Datenmatrix gespeicherten Ratings durch Prognosewerte zu ersetzen. Die Ableitung von individuellen Produktempfehlungen umfasst beim CF drei Prozessschritte: ${ }^{483}$

(1) Systematische Ähnlichkeiten zwischen verschiedenen Kunden werden anhand von Distanz- oder Korrelationsanalysen identifiziert. Eine der gebräuchlichsten Maßzahlen, um für - wie in diesem Fall - metrische Daten die Stärke des Zusammenhanges zu ermitteln, ist der Korrelationskoeffizient nach Bravais-Pearson. Der Koeffizient der Präferenzen ( $r$ ) zweier Nutzer a und $u$, mit den durchschnittlichen Standardabweichungen $s_{a}$ und $s_{b}$ von den mittleren Produktbewertungen in der Datenmatrix, ist definiert gemäß Ausdruck 0-1.

$$
P_{a, u}=\frac{\operatorname{cov}(a, u)}{s_{a} \cdot s_{u}}
$$

Die Ähnlichkeit zwischen den Nutzerprofilen in der Datenmatrix kann daher berechnet werden entsprechend Ausdruck 0-2.

$$
P_{a, u}=\frac{\sum_{i=1}^{m}\left(r_{a, i}-\overline{r_{a}}\right) \cdot\left(r_{u, i}-\overline{r_{u}}\right)}{\sqrt{\sum_{i=1}^{m}\left(r_{a, i}-\overline{r_{a}}\right)^{2} \cdot \sum_{i=1}^{m}\left(r_{u, i}-\overline{r_{u}}\right)^{2}}}
$$

(2) Ähnliche Benutzer N werden als „Mentoren“ bzw. „Nachbarn“ selektiert und die jeweils fehlenden Werte der Rating-Vektoren ergänzt.

(3) Die konkrete Angebotsindividualisierung erfolgt schließlich durch eine Auswahl derjenigen Objekte mit den höchsten individuellen Prognosewerten. Die Prognosewerte eines Produkts $i$ werden berechnet als Summe aus der mittleren Präferenz $(\bar{r})$ des Konsumenten a und der Abweichungen der Präferenzen der Nachbarn $u$ für dieses Objekt von ihren Durchschnittspräferenzen (vgl. Ausdruck 0-3).

$$
p_{a, i}=\overline{r_{a}}+\frac{\sum_{u=1}^{n}\left(r_{u, i}-\overline{r_{u}}\right) \cdot P_{a, u}}{\sum_{u=1}^{n} P_{a, u}}
$$

483 Vgl. Melville/Mooney/Nagarajan (2002), S. 3 f. 
Die Genauigkeit der Prognosewerte eines CF-Systems ist zum einen in hohem Maße abhängig von der Anzahl der Benutzer, die das System verwenden, zum anderen von der Anzahl verfügbarer Produktbewertungen. Probleme ergeben sich jedoch, wenn das Verhältnis von Produkten und Nutzern sehr groß ist, wodurch die Wahrscheinlichkeit der Existenz signifikant ähnlicher Bewertungsprofile sinkt. Eine weitere Schwachstelle bildet das sog. „Erstbewerterproblem“ bzw. „first rater problem“: Produkte können überhaupt dann erst individuell empfohlen werden, wenn sie von mindestens einem Nutzer bewertet wurden. ${ }^{484}$

\subsection{Inhaltebezogenes Collaborative Filtering}

Zur Erhöhung der Prognosegenauigkeit von CF-Verfahren vor dem Hintergrund dieser Probleme schlagen Melville/Mooney/Nagarajan (2002) die Kopplung von eigenschaftsbasierten und kollaborativen Filtern in Form eines "inhaltebezogenen“ oder "content-boosted" Collaborative Filtering (CBCF) vor. Als Beispiel dient ein Empfehlungssystem für Kinofilme. Wie in Abbildung 4.17 dargestellt, wird die lückenhafte Datenmatrix der verfügbaren Produktbewertungen aus den Nutzerprofilen zunächst durch ein eigenschaftsbasiertes Filterverfahren vervollständigt. Als Grundlage für die eigenschaftsbasierte Vervollständigung dient im Beispielfall der kommerzielle Internetdienst InternetMovieDatabase (IMDb), der für jeden Film textbasierte Angaben bspw. zu Handlung, Ensemble oder Kritiken bereitstellt. Über die vervollständigte Datenmatrix $V$, die für jeden Nutzer ein eigenschaftsbasiert gefilterten „Pseudo-Ratingvektor ${ }^{\text {‘485 }}$ enthält, wird dann wiederum über CFAlgorithmen eine Empfehlung berechnet.

$484 \mathrm{Vgl}$. Melville/Mooney/Nagarajan (2002), S. 1.

485 Die Vektorkoordinaten von $\mathrm{V}$ werden notiert als $v_{u, i}=\left\{\begin{array}{l}r_{u, i}, \text { fallsivonu bewertet } \\ c_{u, i}, \text { andernfalls }\end{array}\right.$. 


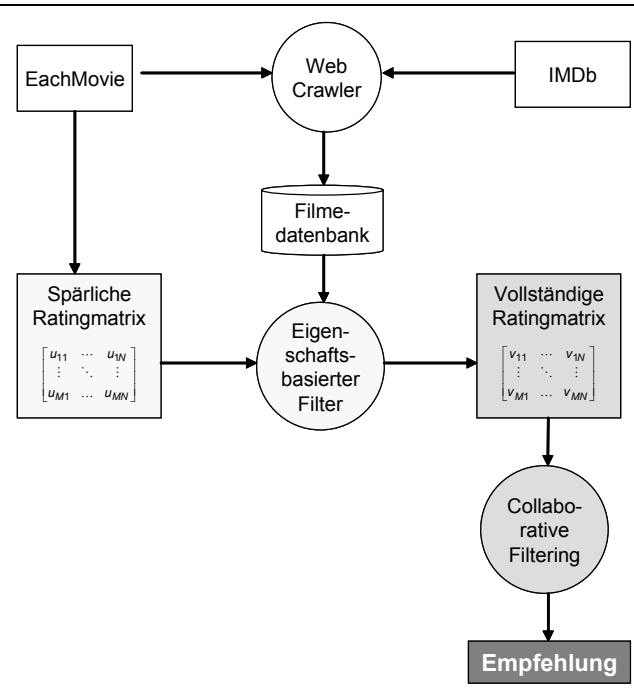

Abbildung 4-17: Content-boosted Collaborative Filtering

In einer empirischen Testreihe deckten Melville/Mooney/Nagarajan (2002) drei weitere Abhängigkeiten in Bezug auf die Genauigkeit der Empfehlungsergebnisse auf:

(1) Eine Beeinflussung der Korrelation $P_{a, u} z$ wischen den Ratings zweier Nutzer $a$ und $u$ durch die Anzahl $n_{i}$ der jeweils von beiden überlappend bewerteten Produkte, wobei hoch korrelierte Mentoren mit jedoch nur wenigen überlappenden Produkten das Empfehlungsergebnis negativ beeinträchtigen. Zur Abwertung dieser Korrelationen wird ein Signifikanzgewicht (significance weighting factor, sg) entsprechend Ausdruck 0-4 vorgeschlagen.

$$
s g_{u, i}=\left\{\begin{array}{l}
r_{u, i}, \text { für } n_{i}<50 \\
1, \text { für } n_{i} \geq 50
\end{array}\right.
$$

(2) Eine Beeinflussung der Qualität des eigenschaftsbasierten Filterverfahrens durch die Anzahl insgesamt bewerteter Produkte durch einen Nutzer, wobei eine geringe Zahl von Bewertungen durch einen Nutzer a zu einer verfälscht hohen Korrelation zum Profil eines anderen Nutzer $u$ führt. Zur Verringerung der Fehlerwahrscheinlichkeit wird der sog. „harmonisierende Mittelwertsgewichtungsfaktor" (harmonic mean weighting factor, hm) entsprechend Ausdruck 0-5 eingeführt.

$$
h m_{a, u}=\frac{2 m_{a} m_{u}}{m_{a}+m_{u}}
$$

Signifikanzgewicht und harmonisches Mittelwertgewicht beziehen sich beide auf die Korrelation $P_{a, u}$ zwischen den Ratings zweier Nutzer $a$ und $u$ und kön-

486 Es gilt: $m_{a / u}=\left\{\begin{array}{l}\frac{n_{a / u}}{50}, f u ̈ r n_{a / u}<50 \\ 1, \text { für } n_{a / u} \geq 50\end{array}\right.$. 
nen daher zusammengefasst werden zu einem „hybriden Korrelationsgewicht“ (hybrid correlation weight, hw) entsprechend Ausdruck 0-6.

$$
h w_{a, u}=h m_{a, u}+s g_{a, u}
$$

(3) Es wird unterstellt, dass die Ergebnisse des eigenschaftsbasierten Filterverfahrens zu Ergänzung der Profillücken, da sie auf den ursprüngliche vom Nutzer spezifizierten Bewertungen beruhen, insgesamt eine höhere Übereinstimmung mit seinen Präferenzen als kollaborative, im Vergleich mit anderen Nutzern ermittelte Prognosewerte. Daher werden die über eigenschaftsbasiertes Filtern ermittelten Koordinaten im Pseudo-Rating eines Nutzers gegenüber Mentorenpräferenzen im Rahmen der Prognosewertberechnung höher gewichtet, durch einen „Selbstgewichtungsfaktor“ entsprechend Ausdruck 0-7.

$$
s g_{u, i}=\left\{\begin{array}{l}
r_{u, i}, \text { für } n_{i}<50 \\
1, f u ̈ r \\
n_{i} \geq 50
\end{array}\right.
$$

Unter Einbeziehung der drei Gewichtungsfaktoren lässt sich somit eine Empfehlung für den Nutzer a für ein Produkt $i$ berechnen entsprechend Ausdruck 08.

$$
p_{a, i}=\overline{v_{a}}+\frac{s w_{a}\left(c_{a, i}-\overline{v_{a}}\right)+\sum_{u=1}^{n}\left(v_{u, i}-\overline{v_{u}}\right) \cdot h w_{a, u} P_{a, u}}{s w_{a}+\sum_{u=1}^{n} h w_{a, u} P_{a, u}}
$$

\subsubsection{Individualisierung durch beobachtungsbasiertes Filtern}

Der Vorteil der Verwendung expliziter Präferenzprofile in Form von nutzerspezifischen Produktbewertungen im Rahmen der Produktindividualisierung besteht darin, dass die Profildaten bereits als metrisch skalierte Produktgewichtungen vorliegen. Jedoch ist, wie gezeigt, die Qualität der Prognosewerte eines Empfehlungssystems im hohen Maße vom Umfang der angegebenen Produktpräferenzen im Verhältnis zum Umfang des Produktkatalogs abhängig. Wie jedoch bereits erörtert wurde, ist die Bereitschaft von Nutzern, aktiv Informationen über sich preiszugeben, in der Regel sehr gering. Wie Runte (2000) feststellt, setzen sowohl eigenschaftsbasierte als auch kollaborative Filterverfahren nicht, wie in den gezeigten Beispielen, zwangsläufig die Existenz explizit spezifizierter Präferenzen voraus, sondern können auch in Verbindung mit impliziten, aus Verhaltensbeobachtungen abgeleiteten Nutzerprofilen angewendet werden. ${ }^{487}$

487 Vgl. Runte (2000), S. 24. 


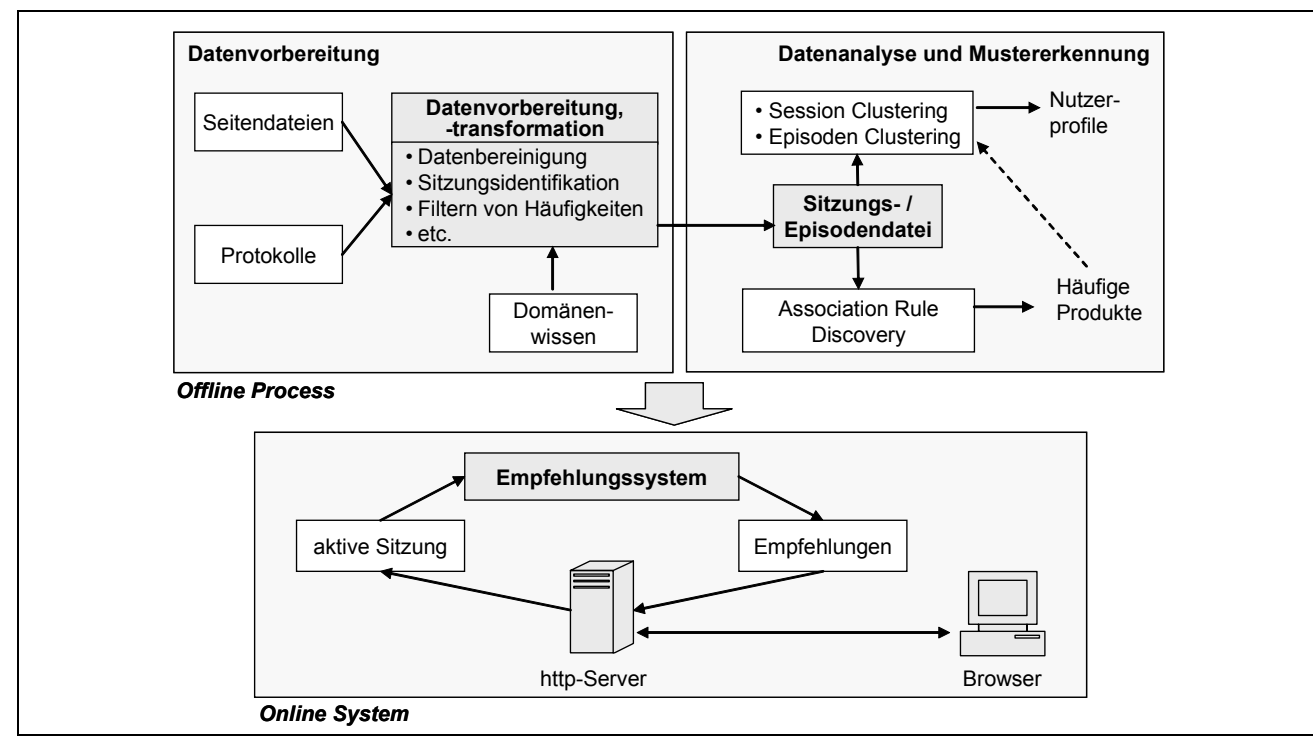

Abbildung 4-18: Aufbau eines beobachtungsbasierten Filtersystems ${ }^{488}$

Ein beobachtungsbasiertes Empfehlungssystem besteht, wie in Abbildung 4-18 dargestellt, in der Regel aus zwei Komponenten: einem Offlinesystem, das über analytische Auswertungen der protokollierten Aktivitäten eines Webservers typische Verhaltensmuster ableitet und speichert; einem Onlinesystem, das das aktive Verhalten eines Nutzers mit den gespeicherten Verhaltensmustern vergleicht und zuordnet. Im Folgenden werden die Verfahren der Profilaggregation im Rahmen der Offlinekomponente und die Ableitung einer nutzerindividuellen Empfehlung im Rahmen der Onlinekomponente am Beispiel eines beobachtungsbasierten Empfehlungssystems vorgestellt. ${ }^{489}$

\subsection{Profilaggregation im Rahmen eines beobachtungsbasierten Empfehlungssystems}

Wie bereits angesprochen, ist die Auswahl der Verfahren zur Datenvorbereitung ebenso wie die Anwendbarkeit des Prognosealgorithmus selbst in hohem Maße abhängig vom gewählten Verfahren zur Mustererkennung von Nutzerprofilen im Zuge der Datenanalyse. Mobasher/Dai/ Luo/Nakagawa (2002) schlagen ein beobachtungsbasiertes Empfehlungssystem vor, das Nutzerprofile in Form von gewichteten Gruppen häufig besuchter URL-Kombination als Grundlage eines eigenschaftsbasierten Filterverfahrens verwendet. Für die Ableitung individueller Nutzerprofile werden zwei Verfahrensweisen vorgeschlagen, die Transaktionsclusterung (1) und die Pageview-Clusterung (2):

(1) Wie in Abbildung 4-19 dargestellt, werden im Rahmen der Transaktionsclusterung zunächst - im Zuge der Datenvorbereitung - die von nicht relevanten Aktivitäten gesäuberten Protokolldaten $(P)$ des Webservers durch Pfad- und

488 In Anlehnung an Mobasher (2002); Mobasher/Cooley/Srivasta (1999); Mobasher/Dai/Luo/Nagakawa (2001). 489 Vgl. Mobasher (2002); Mobasher/Cooley/Srivasta (1999); Mobasher/Dai/Luo/Nagakawa (2001). 
Assoziationsanalysen zu Cluster individueller Transaktionen und Nutzersitzungen ( $T$ ) gruppiert (1), (2)). Die einzelnen Transaktionen werden als Vektoren gewichteter Objektaufrufe (Pageviews) dargestellt. Als Gewichtungsfaktoren können bspw. die Häufigkeit des Aufrufs eines Objekts, dessen Betrachtungsdauer oder domänenspezifische Kriterien wie die unterschiedliche Gewichtung zwischen Navigations- und Inhaltsseiten verwendet werden. Die Transaktionen $t_{i}$ werden anhand der Ähnlichkeit ihrer Pageviews wiederum in Cluster (TC) gruppiert (3). Im letzten Schritt werden diese Transaktionscluster hinsichtlich ihrer Besonderheiten zu individuellen Profilen (pr) aggregiert (4)). Dabei werden durch Mittelwertsbildung der Gewichtungsfaktoren der Pageviews innerhalb eines Transaktionsclusters diejenigen Seitenaufrufe herausgefiltert, deren Gewichtungsfaktor unter dem jeweiligen Transaktionsmittelwert liegt.

\begin{tabular}{|c|c|}
\hline $\begin{array}{l}134.76 .23 .27--[30 / \text { Jun } / 2003: 23: 59: 49+. \\
134.76 .23 .27--[30 / \text { Jun } / 2003: \ldots \\
\ldots\end{array}$ & $\begin{array}{l}\text { Webserver- } \\
\text { Zugriffsprotokoll }\end{array}$ \\
\hline$P=\left\{p_{1}, p_{2}, \ldots, p_{n}\right\}$ & Datenbereinigung \\
\hline 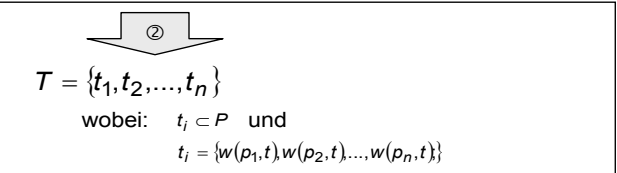 & $\begin{array}{l}\text { Transaktions- } \\
\text { ableitung }\end{array}$ \\
\hline $\begin{array}{c}T C=\begin{array}{c}\left\{c_{1}, c_{2}, \ldots, c_{k}\right\} \\
\text { wobei: } \quad c_{i} \subset T\end{array} \\
\end{array}$ & $\begin{array}{l}\text { Transaktions- } \\
\text { clusterung }\end{array}$ \\
\hline $\begin{aligned} p r_{c_{i}}= & \left\{\left\langle p, \text { weight }\left(p, p r_{c_{i}}\right)\right\rangle \mid p \in P, \text { weight }\left(p, p r_{c_{i}}\right) \geq \mu\right\} \\
& \text { wobei: weight }\left(p, p r_{c_{i}}\right)=\frac{\left.\sum w(p, t)\right)}{|c|}\end{aligned}$ & $\begin{array}{l}\text { Mittelwertsbildung / } \\
\text { Clusteraggregation }\end{array}$ \\
\hline
\end{tabular}

Abbildung 4-19: Profilaggregation durch Profil-Clusterung

(2) Im Rahmen der Transaktionsclusterung werden gemeinsam aufgetretene Pageviews in ähnlichen Transaktionen zu Profilen gruppiert. Im Gegensatz dazu werden im Rahmen der Pageview-Clusterung häufige Pageviews unabhängig von der Ähnlichkeit der zugrunde liegenden Transaktionen gruppiert, wodurch auch überlappende Interessen unterschiedlicher Nutzer abgebildet werden können. Dazu wird ein Assoziationsregelverfahren angewendet, dem der Hypergraf der Objekte einer Internetseite, als Repräsentationsform des Beziehungsnetzwerks der Objekte und der sie verbindenden Links, zugrunde gelegt wird. ${ }^{490}$ 


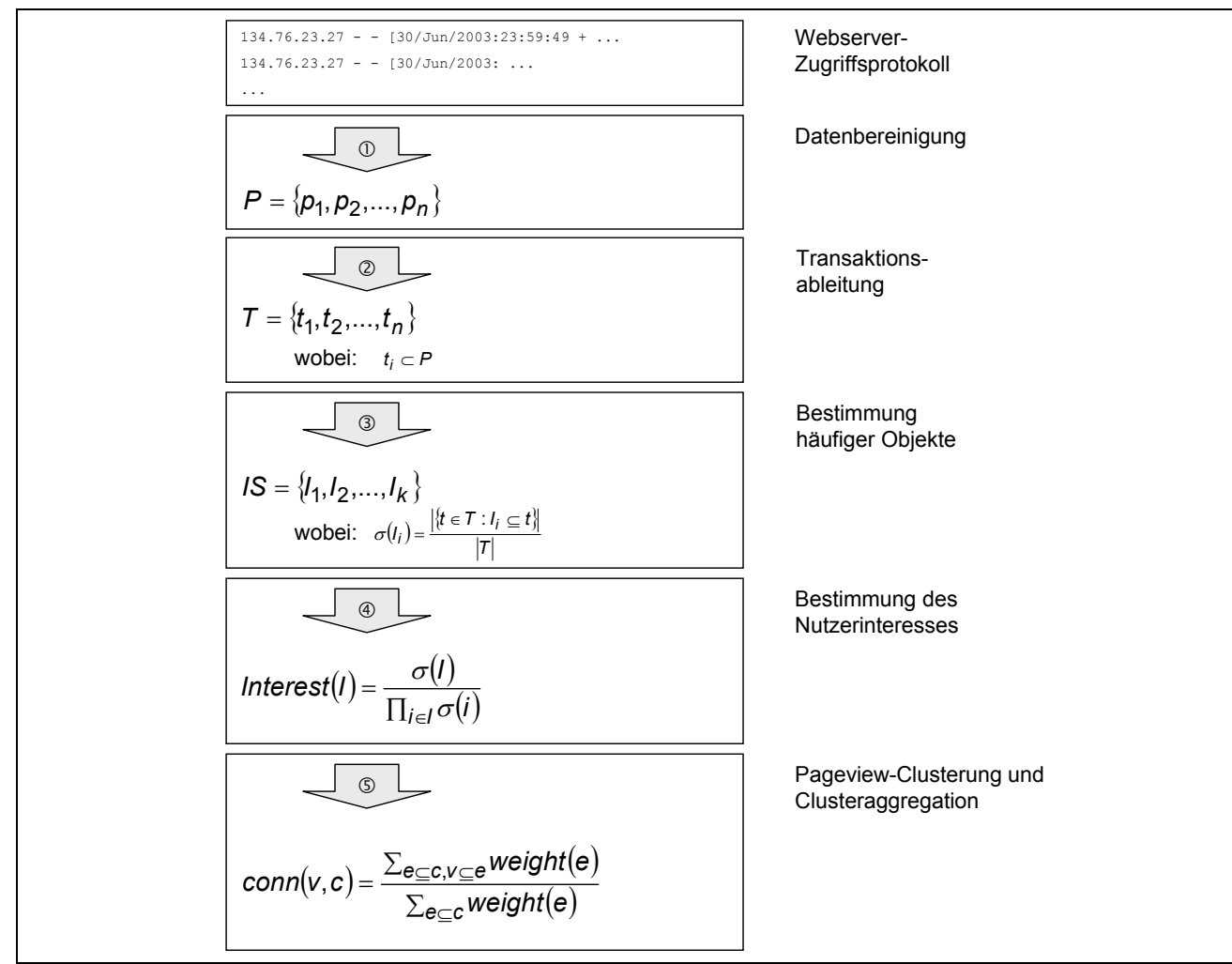

Abbildung 4-20: Profilaggregation durch Pageviewclusterung

Wie in Abbildung 4-20 dargestellt ist, werden innerhalb der aus den bereinigten Daten (1) aggregierten Transaktionsgruppen $T$ (2) häufig aufgerufene Objekte IS gefiltert (3). Als Maß $\sigma$ der Häufigkeit eines Seitenaufrufs $I_{i}$ innerhalb eines Transaktionsclusters $c$ wird die Anzahl der Seitenaufrufe eines Objekts innerhalb des Transaktionsclusters im Verhältnis zur Mächtigkeit sämtlicher Transaktionscluster bestimmt. Diese häufig aufgerufenen Objekte werden im ARHPVerfahren als Eckpunkte $E$ eines Hypergrafen $H=\langle V, E\rangle$ interpretiert, wobei die Knoten $V$ des Grafen $H$ Elemente der gesäuberten Datenbasis $P$ darstellen. Dabei werden diejenigen Knoten des Hypergrafen herausgefiltert, die eine geringe Verbindung zur den jeweiligen Eckpunkten besitzen. Dazu wird zunächst das Interesse Interest $(I)$ eines individuellen Nutzers an einem häufig aufgerufenen Objekt anhand der Häufigkeit dieses Objektaufrufs im Verhältnis zur Gesamtzahl des Auftretens aller häufigen Objekte $l_{i}$ in sämtlichen Clustern bestimmt(4)).

\subsection{Ermittlung einer individuellen Empfehlung im Rahmen eines beobachtungsbasierten Empfehlungssystems}

Die Onlinekomponente eines Empfehlungssystems zeichnet für die aktive Sitzung eines Nutzers die zuletzt besuchten Seiten über ein Sitzungskontrollprogramm auf. Bspw. können die aufgezeichneten Seiteneinträge in einem $n$-zeiligen verborgenen Fensterbereich protokolliert werden. Anhand von Seiteneigenschaften, wie der Un- 
terscheidung zwischen Inhalts- und Navigationsseiten, kann jeder der besuchten Seiten ein Signifikanzgewicht in Bezug auf die vom Seitenbetreiber für eine Nutzersitzung als bedeutsam erachteten Seiteneigenschaften zugeordnet werden. Ebenso wie die gespeicherten Profile der Offlinekomponente kann damit die aktive Nutzersitzung als $n$-dimensionaler Vektor $S=\left\{s_{1}, s_{2}, \ldots, s_{n}\right\}$ der Gewichtungsfaktoren $s_{i}$ eines aufgerufenen Objekts $p_{i}$ abgebildet werden, wobei gilt, dass

$S_{i}=\left\{\begin{array}{ll}1+s_{i} \text { für } p_{i} \in S \\ 0 \quad \text { sonst }\end{array}\right.$.

Die entsprechende $n$-dimensionalen verfügbaren Profile sind wie voran stehend beschrieben bestimmt als

$C=\left\{w_{1}{ }^{c}, w_{2}{ }^{c}, \ldots, w_{n}{ }^{c}\right\}$, wobei $w_{i}^{c}=\left\{\begin{array}{cc}\text { weight }\left(p_{i}, C\right) \text { für } p_{i} \in C \\ 0 & \text { sonst }\end{array}\right.$.

Zur Auswahl des der aktiven Sitzung ähnlichsten Profils wird das bereits bekannte Cosinus-Maß verwendet:

$\operatorname{sim}(S, C)=\frac{\sum_{k} w_{k}^{c} \cdot s_{k}}{\sqrt{\sum_{k} s_{k}^{2} \cdot \sum_{k} w_{k}^{c^{2}}}}$.

Die Auswahl von Seiten aus dem ähnlichsten Nutzungsmuster der Onlinekomponente kann abschließend anhand der Gewichtungsfaktoren der Objekte im Profilmuster und dem Übereinstimmungsgrad zwischen Profilmuster und aktiver Sitzung anhand eines Empfehlungsgrads berechnet werden: $\operatorname{Re} c(S, n)=\sqrt{\text { weight }(n, C) \cdot \operatorname{match}(S, C)}$.

\subsubsection{Zusammenfassende Bewertung der dargestellten Ver- fahren}

Selbstselektionsverfahren und eigenschaftsbasierte Inhaltefilter lassen sich wie dargestellt nur auf solche Inhalte anwenden, deren Merkmale automatisch computergestützt analysierbar sind. Sie repräsentieren einerseits zwar vergleichsweise einfach anzuwendende Verfahren zu Überprüfung semantischer Ähnlichkeiten zwischen den Präferenzen eines Nutzers und den Merkmalen einer Ressource, das (ausgenommen der Wortstammanalyse der Ressourcen) ohne aufwendigen analytischen Vorlauf eingesetzt werden kann. Es wird andererseits allerdings die Bereitschaft des Rezipienten vorausgesetzt, seine Präferenzen über Produktbewertungen zu spezifizieren. Zudem werden nur solche Produkte von der Filterregel erfasst, deren auffällige Merkmale spezifizierten Präferenzen des Nutzers entsprechen. Solche Ressourcen, die zwar eine sinnvolle sachliche Anknüpfung an die Präferenzen eines Nutzers bieten würden, jedoch keine sprachlichen Gemeinsamkeiten aufweisen, werden von der Filterregel nicht erfasst. Ein Beispiel für einen solchen Fall im Rahmen eines individuell gefilterten Nachrichtenportals wäre ein Bericht über eine Gesetzesentwurf zur Verschärfung des Dopingstrafrechts, der unter Umständen einem sportinteressierten Leser mangels signifikanter sprachlicher Übereinstimmungen mit spezifizierten Präferenzen im Bereich Fußball und Tennis nicht angeboten wird. 
Genau hier liegen die Stärken von Collaborative Filtering-Verfahren: Anstelle eines direkten Vergleichs zwischen den Eigenschaften eines Produkts und den Präferenzen eines Nutzers werden solche Ressourcen individuell gefiltert, denen andere Nutzer mit grundsätzlich ähnlich strukturierten Präferenzen eine hohe Relevanz beigemessen haben. Dadurch werden unter Umständen auch solche Ressourcen von der Filterregel erfasst, die wie im oben genannten Fall keine signifikanten sprachlichen Gemeinsamkeiten mit den Präferenzen eines Nutzers besitzen, aber eventuell einen sachlichen Zusammenhang aufweisen. Dabei können auch solche Ressourcen gefiltert werden, deren Inhalte computergestützt nicht automatisch analysiert werden können. Der Nachteil von Collaborative Filtering-Verfahren liegt im langen Vorlauf zum Aufbau eines repräsentativen Umfangs an Referenzprofilen für den Ähnlichkeitsvergleich der Präferenzen eines Nutzers. Problematisch ist der Einsatz von Collaborative Filtering-Verfahren zudem bei solchen Angeboten, deren Ressourcen eine hohe Innovationsdynamik aufweisen. Liegen noch keine Bewertungen einer Ressource durch mindestens einen Nutzer vor, ist diese Ressource auch für keinen anderen Nutzer filterbar.

Gegenwärtig werden in der Literatur vorwiegend solche Individualisierungssysteme diskutiert, deren Nutzermodell auf explizit durch den Nutzer spezifizierten Präferenzmerkmalen basieren. Dennoch setzen grundsätzlich wie bereits angesprochen weder eigenschaftsbasierte Filterverfahren noch das Collaborative Filtering ein solches explizites Präferenzprofil voraus. In neueren Veröffentlichungen wird stattdessen die Möglichkeit einer Modellierung signifikanter Nutzermerkmale auf der Grundlage beobachtbarer und vom Webserver protokollierter Verhaltensaktivitäten hervorgehoben. Neben dem Wegfall des bereits an anderer Stelle mehrfach thematisierten Problems der in der Regel mangelnden Bereitschaft von Nutzern, im Internet persönliche Angaben zu machen, haben beobachtungsgestützten Verfahren den Vorteil, dass nur die aktuellen situative Bedürfnisse eines Nutzers unabhängig von seinen demografischen Angaben oder seinen Präferenzen in der Vergangenheit die Grundlage des Filterverfahrens bilden. Bewegt sich bspw. der Nutzer eines Informationsdienstes mit einer generellen Vorliebe für klassische Literatur im Rahmen seiner Urlaubsvorbereitung auffällig lange auf den Seiten für Reiseinformationen über eine bestimmte Urlaubsregion, werden unabhängig von seinen sonstigen Vorlieben auch Inhalte für die entsprechende Reiseregion von der Filterregel erfasst. Im Vergleich zu den bereits dargestellten Filterverfahren auf Grundlage expliziter Präferenzangaben erhöht sich jedoch durch eine Verwendung beobachtungsbasiert aggregierter Profile die Komplexität des Filterverfahrens und dadurch die Vorlaufsdauer für dessen Einsatz. Diese Komplexität wird insbesondere beeinflusst durch die Länge der aufgezeichneten Aktivitäten der jeweils aktuellen Nutzersitzung und dadurch der Länge der notwendigen Referenzcluster der Offlinekomponente des Empfehlungssystems. Neben der verfahrensimmanenten Komplexität der Profilaggregation liegt ein weiteres Problem solcher Systeme, die ausschließlich auf der Verwendung beobachtungsbasierter Nutzungsprofile beruhen, in der mangelnden eindeutigen Identifizierbarkeit des Nutzers. Sollen andere Merkmale als die durch das situative Verhalten innerhalb der aktuellen Sitzung rückschließbaren (wie bspw. Alter, Geschlecht, Wohnort, etc.) zur Individualisie- 
rung herangezogen werden, muss das beobachtungsbasierte Verfahren zwangsläufig mit expliziten Angaben des Nutzers kombiniert werden. Abbildung 4-21 fasst die Bewertung der dargestellten Verfahren im Überblick zusammen. ${ }^{49}$

\begin{tabular}{|c|c|c|c|}
\hline Systemtyp & \multicolumn{2}{|c|}{ Systemkomponente } & Merkmalsausprägung \\
\hline \multirow{5}{*}{$\begin{array}{l}\text { 1. Eigenschaftsbasiertes } \\
\text { Filtern }\end{array}$} & \multicolumn{2}{|c|}{ Ressourcenmodellierung } & $\begin{array}{l}\text { Reduktion einer textbasierten Ressource auf den Vektor ihrer signifikant } \\
\text { häufigen Wortstamm-Merkmale }\end{array}$ \\
\hline & \multicolumn{2}{|c|}{ Nutzermodellierung } & $\begin{array}{l}\text { Präferenzprofile als vektorisierte Produktratings, erstellt auf der Basis von } \\
\text { Trainingsdaten }\end{array}$ \\
\hline & \multicolumn{2}{|c|}{ Matching } & $\begin{array}{l}\text { Verhältnisähnlichkeit von Rating- und Ressourcenvektor } \\
\text { (Cosinus-Maß) }\end{array}$ \\
\hline & \multirow[t]{2}{*}{ Fazit } & negativ: & $\begin{array}{l}\text { - Rechnergestützte Analysierbarkeit von Inhalten vorausgesetzt } \\
\text { - Matching nur bei semantischen Übereinstimmung zwischen Ressourcen- } \\
\text { und Nutzerprofilen }\end{array}$ \\
\hline & & positiv: & $\begin{array}{l}\text { - Ohne Vorlauf einsetzbar } \\
\text { - Gute Empfehlungsqualität für bewertete Produktmerkmale }\end{array}$ \\
\hline \multirow[t]{5}{*}{ 2. Collaborative Filtering } & \multicolumn{2}{|c|}{ Ressourcenmodellierung } & entfällt \\
\hline & \multicolumn{2}{|c|}{ Nutzermodellierung } & $\begin{array}{l}\text { Präferenzprofile als vektorisierte Produktratings, erstellt auf der Basis von } \\
\text { Trainingsdaten }\end{array}$ \\
\hline & \multicolumn{2}{|c|}{ Matching } & $\begin{array}{l}\text { Mentorenselektion anhand von Merkmalskorrelationen und Produktprognose } \\
\text { anhand von Mittelwertsvergleichen zwischen Nutzer und Mentor }\end{array}$ \\
\hline & \multirow[b]{2}{*}{ Fazit } & negativ: & $\begin{array}{l}\text { - Startfristproblem und typische Dürftigkeit der Ratingmatrix } \\
\text { - First-Rater-Problem }\end{array}$ \\
\hline & & positiv: & $\begin{array}{l}\text { - Auch für nicht-rechnergestützt analysierbare Ressourcen } \\
\text { - Möglichkeit der Empfehlungen relevanter Ressourcen ohne semantische } \\
\text { Profilübereinstimmungrn }\end{array}$ \\
\hline \multirow{5}{*}{$\begin{array}{l}\text { 3. Beobachtungsbasiertes } \\
\text { Filtern }\end{array}$} & \multicolumn{2}{|c|}{ Ressourcenmodellierung } & Vektorisierung der Pageviews der aktuellen Nutzersitzung \\
\hline & \multicolumn{2}{|c|}{ Nutzermodellierung } & $\begin{array}{l}\text { - Gewichtete Pageview-Transaktionscluster } \\
\text { - Assoziierte überlappende Pageview-Cluster }\end{array}$ \\
\hline & \multicolumn{2}{|c|}{ Matching } & $\begin{array}{l}\text { Verhältnisähnlichkeit der Vektoren der aktuellen Sitzung und der abgeleiteten } \\
\text { Profile und Auswahl des Objekts mit der höchsten Ähnlichkeitsrelevanz }\end{array}$ \\
\hline & \multirow{2}{*}{ Fazit } & negativ: & $\begin{array}{l}\text { - Zeitlicher analytischer Vorlauf zur Clusterbildung } \\
\text { - Abhängigkeit der Empfehlungsqualität von der Aufzeichnungstiefe der } \\
\text { aktuellen Sitzung impliziert hohen Rechenaufwand für gute Empfehlungen }\end{array}$ \\
\hline & & positiv: & $\begin{array}{l}\text { - Trennung zwischen demografischem Profil und situativen Bedürfnissen } \\
\text { - Keine expliziten Profilangaben notwendig }\end{array}$ \\
\hline
\end{tabular}

Abbildung 4-21: Bewertung

\subsection{Nutzen individueller Mediengüter}

Einleitend wurde unterstellt, dass eine Individualisierung von Mediengütern einen Mehrwert für Rezipienten bewirken kann. Im Folgenden wird aus Sicht eines Medienunternehmens erläutert, inwiefern Konsumenten durch Berücksichtigung individueller Konsumpräferenzen ein Kaufanreiz für ein Mediengut geboten werden kann. In Abschnitt 4.3.1 werden Bedingungen der Entschei-

491 Der Überblick zeigt einen zusammenfassenden Vergleich zwischen den vorgestellten Systemvarianten anhand der drei in Abschnitt 4.2.1.2 eingeführten Komponenten eines Individualisierungssystems (Ressourcenmodellierung, Nutzermodellierung und Matching). Ein Vergleich der Wirkungen des Einsatzes der verschiedenen Systeme auf den Rezipientennutzen wird an dieser Stelle noch nicht vorgenommen, da ein solcher Wirkungsvergleich eines Tests konkreter Implementierungen dieser Systeme, wie er beispielhaft in Abschnitt 4.4 vorgenommen wird, bedarf. Als Bewertungskriterien für die Systemwirkung können bspw. Qualitätsmaße, die aus dem Bereich der Forschung zu Dokumentensuche und -erschließung bekannte sind, wie die Abdeckung (Recall) oder die Präzision (Precision), herangezogen werden (vgl. Abschnitt 4.3.4). 
dung eines Rezipienten für den Konsum von Inhalten erläutert und ein ökonomisches Nutzenkalkül formuliert. Auf dieser Grundlage wird in Abschnitt 4.3.2 untersucht, welche Auswirkungen eine Individualisierung der Bestandteile eines Medienguts auf die Zahlungsbereitschaft des Rezipienten besitzt.

\subsubsection{Nutzenkalkül eines Rezipienten}

Ein Mediengut wird in erster Linie wegen seines Inhalts nachgefragt. ${ }^{492}$ Ein Rezipient wählt im Rahmen seiner Konsumentscheidung immer dasjenige Mediengut aus, von dessen Inhalten er sich den höchsten Nutzen verspricht. Dabei ist ein Rezipient bereit, für ein Mediengut nur gerade den Differenzwert aus dem für inn erwarteten Nutzwert der Inhalte dieses Medienguts und den für inn durch Auswahl und Rezeption entstehenden Kosten zu bezahlen (vgl. Abbildung 4-22).

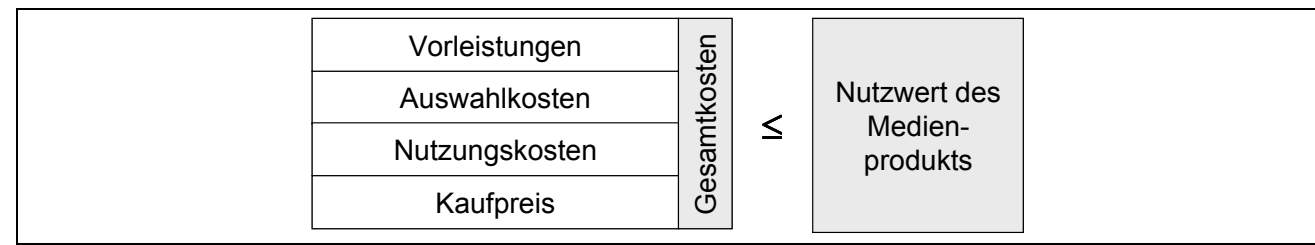

Abbildung 4-22: Notwendige Bedingung des Medienkonsums

Die Digitalisierbarkeit von Inhalten schafft - wie in Kapitel 2 erläutert wurde zwar neue Möglichkeiten im Rahmen der Inhaltebündelung und -distribution, der kreative Prozess im Rahmen der Inhalteerzeugung und dadurch der objektive Nutzwert der resultierenden Inhalte bleibt jedoch weitgehend unverändert. Sollen digitalisierte Inhalte einen Kaufanreiz für Rezipienten bieten, muss daher ihr Bündelungs- oder Distributionsformat gegenüber bestehenden Formaten einen geringeren Rezeptionsaufwand verursachen oder, bspw. durch höhere Aktualität, einen Mehrwert stiften. Dabei lassen sich im Rahmen der Rezeption von Mediengütern vier Aufwandsarten unterscheiden: ${ }^{493}$ der nominelle Kaufpreis des Medienguts (1) sowie Aufwendungen für Vorleistungen (2), Auswahl (3) und Nutzung (4).

(1) Der Kaufpreis eines Medienguts kann einmaliger oder wiederkehrender Natur sein. Einmalige Kaufpreise bilden bspw. Abonnementzahlungen für Zeitungen oder Zeitschriften. Wiederkehrende Kaufpreise hängen von der Nutzungsintensität nachgefragter Inhalte ab. Neben nutzungsabhängigen nominellen Kaufpreisen, wie sie bspw. bei Tageszeitungen im Straßenverkauf anfallen, können beim Erwerb von Onlineinhalten weitere wiederkehrende Kosten bspw. durch den Aufwand der Zahlungsabwicklung anfallen.

(2) Neben Aufwendungen für den Erwerb von Mediengütern können, bspw. im Falle von Onlineinhalten, für die Inhalterezeption Vorleistungen in Form von

492 Vgl. Brandtweiner (2000), S 41; Sjurts (2002a), S.8; Schumann/Hess (2002), S. 39.

$493 \mathrm{Vgl}$. Fehr (2003), S. 29 f. 
Sach- und Lerninvestitionen zur Gewährleistung der Nutzung des Mediums anfallen. Derartige Sachinvestitionen umfassen bspw. die Anschaffung eines PCs sowie der Unterhalt eines Internetanschlusses. Lerninvestitionen bestehen demgegenüber bspw. in der Aneignung von Kenntnissen, die zum Umgang mit $\mathrm{PC}$ und Internet erforderlich sind.

(3) Der Aufwand im Zuge der Auswahl eines Medienguts ist zweifacher Natur: Die Auswahl bedürfnisgerechter Inhalte erzeugt durch die Dauer der Suche erstens einen zeitlichen Aufwand. Bereits 1961 stellte der später u.a. für diese Untersuchung mit dem Nobelpreis geehrte amerikanische Ökonom George Stigler fest, dass die Marktpreise selbst bei homogenen Gütern wie Steinkohle zum Teil erheblich streuen können. ${ }^{494}$ Ursache dieser Preisstreuung bilden Informationsasymmetrien zwischen den Marktnachfragern, die entstehen, weil der Vergleich von konkurrierenden Preisen und Eigenschaften unterschiedlicher Angebote einen zeit- und damit kostenintensiven Vorgang darstellt. Dieses allgemeine Auswahlproblem wird im Falle von Mediengütern zweitens dadurch verstärkt, dass ein Rezipient den genauen Produktnutzen angebotener Inhalte vor dem Kauf bestenfalls unvollständig kennt. ${ }^{495} \mathrm{Um}$ das resultierende Rezeptionsrisiko zu minimieren, entsteht für den Rezipienten ein zusätzlicher Suchaufwand im Rahmen der Nutzung von Bewertungssubstituten für ein Mediengut, bspw. in Form von Kaufempfehlungen oder redaktionellen Kritiken.

(4) Die Inhalterezeption selbst kann durch ihren Zeitverzehr einen Aufwand für den Rezipienten darstellen. Der Nutzungsaufwand wird zum einen beeinflusst durch performative und nutzungsökonomische Merkmale des jeweiligen Distributionsformats. Üblicherweise erfolgt die Vermittlung kurzer, stark aggregierter Inhalte tendenziell in elektronischen, bspw. audio-visuellen Formaten, die Vermittlung von detaillierten Hintergrundinformationen in textbasierten Formaten. Digitale Distributionsformate wie Webseiten oder DVDs erlauben potenziell eine Senkung des durchschnittlichen Nutzungsaufwands durch die Verknüpfung audio-visueller und textbasierter Vermittlung. Ob tatsächlich der Nutzungsaufwand für ein Mediengut reduziert werden kann, hängt dabei insbesondere von der Gestaltung der Inhaltepräsentation, bspw. in Form einer intuitiven Bedienbarkeit, sowie insbesondere bei Onlineinhalten von einer akzeptablen Bereitstellungsdauer und Darstellungsqualität ab. Zum anderen können speziell bei werbefinanzierten Inhalten Werbeelemente den Nutzungsaufwand erhöhen, besonders dann, wenn Werbung parallel mit den rezipierten Inhalten wahrgenommen wird. Je mehr Werbebanner bspw. auf einer Seite platziert sind, desto zeitaufwändiger ist die Rezeption des gewünschten Inhalts, da die Nutzeraufmerksamkeit abgelenkt wird.

Zusammenfassend kann das Entscheidungskalkül eines Rezipienten (j) als Budgetrestriktion seines Einkommens $(E)$ in Abhängigkeit vom nutzungsunabhängigen Aufwand für Kaufpreis $\left(\mathrm{p}_{\mathrm{NOM}}\right)$, Auswahl $\left(\mathrm{p}_{\mathrm{INF}}\right)$ und Vorleistungen $\left(p_{A D P}\right)$ sowie vom nutzungsabhängigen Aufwand für Einzelpreise von Inhalten

\footnotetext{
494 Vgl. Stigler (1961).

495 Vgl. Heinrich (2001a), S. 99.
} 
( $q_{\text {NOM }}$ ) und dem inhaltebezogenen Nutzungsaufwand (quSE) in Abhängigkeit von der Nutzungsintensität ( $v$ ) eines Inhalts (i), sowie seinen Ausgaben für alle anderen Konsumgüter $(H)$ formuliert werden gemäß Ausdruck (4.3.1-1): ${ }^{496}$

$$
E=H+\underbrace{\left(p_{N O M}+p_{I N F}+p_{A D P}\right)}_{p}+\underbrace{\left(q_{N O M}+q_{U S E}\right)}_{q} \cdot \sum_{i=1}^{n} v_{i}
$$

Im Modellfall einer Cobb-Douglas Nutzenfunktion lässt sich der von einem Rezipienten aus Inhalte- und sonstigem Güterkonsum erzielbare Nutzen (U) des Einkommens in Abhängigkeit von der relativen Präferenz des Medienkonsums $(\alpha)$ im Vergleich zu sonstigen Konsummöglichkeiten formulieren als:

$$
U=H^{\left(1-\sum_{i=1}^{n} \alpha_{i}\right)} \cdot \prod_{i=1}^{n} v_{i}^{\alpha_{i}}
$$

bzw. als deren monotone Transformation:

$$
U=\left(1-\sum_{i=1}^{n} \alpha_{i}\right) \ln (H) \cdot \sum_{i=1}^{n} \alpha_{i} \ln \left(v_{i}\right)
$$

\subsubsection{Konsumentscheidung eines Rezipienten}

Die Konsumentscheidung eines Inhalterezipienten repräsentiert ein zweistufiges Optimierungskalkül in Bezug auf die Nutzenfunktion in Ausdruck 4.3.1-2b unter der Bedingung der Budgetrestriktion in Ausdruck 4.3.2-1. ${ }^{497}$ Zunächst muss ein Konsumplan für die optimale Aufteilung des verfügbaren Einkommens zwischen der Nachfrage (v) nach Inhalten (i) und allen übrigen Konsummöglichkeiten. Die zugehörige Lagrangefunktion für das Optimierungsproblem der Budgetaufteilung lautet:

$$
L=\underbrace{\left(1-\sum_{i=1}^{n} \alpha_{i}\right) \ln (H) \cdot \sum_{i=1}^{n} \alpha_{i} \ln \left(v_{i}\right)}_{\text {Nutzenfunktion }}+\lambda \underbrace{\lambda\left(E-H-p-q \sum_{i=1}^{n} v_{i}\right)}_{\text {Budgetrestriktion }} \rightarrow \max !
$$

Die Lösung der Lagrangefunktion liefert die bedingte optimale Nachfragefunktion für die Menge (v) eines Inhalts (i) in Abhängigkeit der Konsumpräferenzen $(\alpha)$ des Nutzers für den jeweiligen Inhalt, seinem Einkommen sowie dem Rezeptionsaufwand ( $p$ bzw. q):

$$
v_{i}=\frac{\alpha_{i}(E-p)}{q}
$$

Im zweiten Schritt muss überprüft werden, ob der optimale Konsumplan in Ausdruck 4.3.2-2 die in Abbildung 4-21 dargestellte Bedingung eines positiven Nutzwerts erfüllt. Diese Bedingung ist dann erfüllt, wenn der Nutzen aus der Aufteilung des Budgets in Inhaltekonsum und dem Konsum aller anderen Güter gemäß Aus-

\footnotetext{
496 Vgl. Fink (2002), S. 71 f.

$497 \mathrm{Vgl}$. Fink (2002), S. 74 ff.
} 
druck 4.3.2-2 einen höheren Nutzwert erzeugt, als die Verwendung des gesamten Budgets ausschließlich für andere Güter. Diese Bedingung eines positiven Nutzwerts spiegelt sich formal in der Ungleichung in Ausdruck 4.3.2-3 wieder:

$$
\underbrace{\left(1-\sum_{i=1}^{n} \alpha_{i}\right) \ln (H)}_{\begin{array}{c}
\text { Nutzen des reduzierten } \\
\text { Konsums anderer Güter }
\end{array}}+\underbrace{\sum_{i=1}^{n}\left(\alpha_{i} \cdot \ln \left(v_{i}\right)\right)}_{\begin{array}{c}
\text { Nutzen des } \\
\text { Inhaltekonsums }
\end{array}}-\underbrace{\left(1-\sum_{i=1}^{n} \alpha_{i}\right) \cdot \ln (E)}_{\begin{array}{c}
\text { Nutzen des ausschl. } \\
\text { Konsums anderer } \\
\text { Güter }
\end{array}} \geq 0
$$

Durch Einsetzen des optimalen Konsumplans in Ausdruck 4.3.2-2 in die Konsumbedingung in Ausdruck 4.2-3 kann ein positiver Nutzwert (S) als Bedingung aus der nutzungsabhängigen Zahlungsbereitschaft und dem nutzungsunabhängigen Aufwand der Inhalterezeption formuliert werden, wie dargestellt in Ausdruck 4.3.2-4.

$$
S_{j}=\sum_{i=1}^{n}\{\underbrace{\alpha_{i}\left[\ln \left(\frac{\alpha_{i}}{q}\right)-1\right]}_{\begin{array}{c}
\text { Bruttonutzen eines } \\
\text { Innalts } i
\end{array}}\}-p \stackrel{!}{\geq 0}
$$

Aus der Konsumbedingung in Ausdruck 4.3.2-4 können zwei Schlussfolgerungen gezogen werden: Zum einen kann ein positiver Nutzwert nur dann entstehen, wenn der nutzungsabhängige Aufwand (q) der Inhalterezeption möglichst klein ist. Zum anderen steigt der kumulierte Nutzwert eines Nutzers (j) in Abhängigkeit von seinen inhaltebezogenen Präferenzen $(\alpha)$ mit der Anzahl $(n)$ der rezipierten Inhalte (i).

\subsubsection{Einfluss von Individualisierung auf die Konsum- entscheidung eines Rezipienten}

Wie in Abschnitt 2.2 dargestellt, werden Mediengüter üblicherweise aus anbieterseitigen Kostengründen als Bündel verschiedener Inhalte zu einem nominellen Einheitspreis ( $p_{\text {NOM }}$ ) ohne zusätzliche Gebühren für die Einzelinhalte ( $q_{\text {NOM }}$ $=0$ ) angeboten. Vor dem Hintergrund der Schlussfolgerungen aus Ausdruck 4.3.2-4 erzeugt eine Bündelung von Inhalten jedoch nicht nur anbieterseitige Skaleneffekte des Vertriebs, sondern kann zudem zu einer nachfrageseitigen Senkung des Rezeptionsaufwands führen und damit durch den Anbieter als Preisgestaltungsinstrument eingesetzt werden. Unter der Annahme normalverteilter Reservationspreise verschiedener Rezipienten (ausgedrückt durch den Präferenzparameter $\alpha$ ) nähert sich im Falle einer standardisierten Bündelung von Inhalten für den Massenmarkt die durchschnittliche Zahlungsbereitschaft für das Inhaltebündel an den Erwartungswert der gemeinsamen Reservationspreisverteilung aller Rezipienten an. ${ }^{498}$

Wie in Kapitel 3 erläutert wurde, fungiert ein Individualisierungssystem als Filtermechanismus, der von einem Medienunternehmen dafür eingesetzt wer-

498 Vgl. Adams/Yellen (1976); Schmalensee (1984); Olderog/Skiera (1998). 
den kann, für einen Rezipienten aus verfügbaren Inhalten diejenigen auszuwählen, die am besten zu seinen Interessen passen. Indem nur solche Inhalte angeboten werden, die den Interessen des Rezipienten entsprechen, kann ein Individualisierungssystem, das eingesetzt wird, um ein bspw. im Rahmen einer Onlinezeitung ein individuelles Inhaltebündel zu konfigurieren, dazu beitragen, den Nutzungsaufwand im Rahmen der Rezeption im Vergleich zu einem unkonfigurierten Bündel zu senken (quSE $\downarrow$ ). Darüber hinaus kann ein individuelles Empfehlungssystem den nutzungsunabhängigen Suchaufwand nach wunschgemäßen Mediengütern senken und gleichzeitig dazu beitragen, das angesprochene Auswahlrisiko in Bezug auf die Inhaltequalität zu senken ( $p_{\text {INF }} \downarrow$ ). Allerdings setzt eine effektive Senkung des Auswahlaufwands voraus, dass der Rezipient mit der Funktionsweise des der Empfehlung zugrunde liegenden Filtermechanismus vertraut ist und seiner Empfehlung Vertrauen schenkt. Dies erfordert einen gewissen Lernaufwand sowohl in Bezug auf die Erweiterung der Fähigkeiten des Rezipienten als auch ggf. in Bezug auf eine gewisse Trainingsdauer des verwendeten Filteralgorithmus $\left(p_{A D P} \uparrow\right)$.

Vor dem Hintergrund der einleitenden Fragestellung, inwiefern Individualisierungssysteme einen Beitrag leisten, um die Zahlungsbereitschaft von Rezipienten zu steigern, kann der Einfluss einer Individualisierung auf die Konsumentscheidung des Rezipienten gemäß der Ungleichung in Ausdruck 4.3.3-1 zusammenfasst werden:

$$
p_{\text {NOM }} \leq \sum_{i=1}^{n}\left\{\alpha_{i}\left[\ln \left(\frac{\alpha_{i}}{q_{\text {USE }} \downarrow}\right)-1\right]\right\}-\left(p_{\text {INF }} \downarrow+p_{\text {ADP }} \uparrow\right)
$$

Ein Rezipient wird nur dann bereit sein, für individuelle Inhalteangebote zu bezahlen (ausgedrückt durch $\mathrm{p}_{\mathrm{NO}}>0$ ), wenn die Summe des Nutzens aus den nachgefragten Inhalten seinen Such- und Lernaufwand übersteigt. Die Bedingung der relativen Vorteilhaftigkeit individualisierter Angebote gegenüber nichtindividualisierten Inhaltebündeln kann dementsprechend gemäß der Ungleichung in Ausdruck (4.3.3-2) formuliert werden.

$$
\sum_{i=1}^{n}\left\{\alpha_{i}\left[\ln \left(\frac{\alpha_{i}}{q_{U S E_{B}}}\right)-1\right]\right\}-\left(p_{I N F_{B}}+p_{A D P_{B}}\right) \leq \sum_{i=1}^{m}\left\{\alpha_{i}\left[\ln \left(\frac{\alpha_{i}}{q_{U S E_{I}}}\right)-1\right]\right\}-\left(p_{I N F_{I}}+p_{A D P_{I}}\right)
$$

Individualisierung ist im Vergleich zu einer standardisierten Bündelung immer dann von Vorteil, (1) wenn entweder die nutzungsunabhängigen Kosten eines individuell angebotenen Bündels $\left(p_{l}\right)$ kleiner sind als im Falle des Massenmarktprodukts $\left(p_{B}\right)$, oder (2), wenn ein individuelles Bündel beim Nutzer einen höheren Bruttonutzen $\left(S_{l, b r}\right)$ als ein nichtindividualisiertes Bündel $\left(S_{B, b r}\right)$ erzeugt. 
(1) Anhand von Ausdruck 4.3.3-1 wurde ausgeführt, dass ein individuelles Empfehlungssystem zwar die nachfrageseitigen Suchkosten $\left(p_{\mathrm{INF}, \mathrm{I}}\right)$ reduzieren kann, jedoch $z u$ einem höheren Lernaufwand $\left(p_{A D P, I}\right)$ führt. Die Vorteilhaftigkeitsbedingung in Ungleichung 4.3.3-2 ist ceteris paribus immer dann erfüllt, wenn die nutzungsunabhängigen Kosten eines individuellen Bündels $\left(p_{l}\right)$ geringer ausfallen als die des Massenmarktprodukts $\left(p_{B}\right)$. Dies ist immer dann der Fall, wenn die Differenz der Suchkosten von individuellem und Massenmarktbündel höher ist als die Differenz der Lernkosten (vgl. Ausdruck 4.3.3-3).

$$
p_{I N F B}+p_{A D P B}>p_{I N F I}+p_{A D P I} \Longleftrightarrow p_{I N F B}-p_{I N F I}>p_{A D P I}-p_{A D P B}
$$

(2) Der nutzungsbezogene Bruttowert eines Inhaltebündels ist abhängig vom Präferenzparameter $(\alpha)$ eines Nutzers und den Opportunitätskosten (quSE) des Konsums der im Bündel enthaltenen Inhalte. Vor dem Hintergrund der vereinfachenden Annahme normalverteilter Konsumpräferenzen kann der Präferenzparameter als nutzerinvariant und dadurch sowohl im Falle individualisierter als auch nichtindividualisierter Bündelung vereinfachend als gleichförmig verteilt angenommen werden. Insofern Konsumenten in der Regel nur einen kleinen Teil ihres Budgets in den Medienkonsum investieren, kann der Erwartungswert für das Präferenzmaß als klein angenommen werden. Ebenso bilden die Opportunitätskosten der Inhalterezeption eines Inhalteaggregats unabhängig von dessen Individualisierungsgrad üblicherweise lediglich einen kleinen Aufwand im Cent-Bereich. Somit ergibt die anhand von Ausdruck 4.3.3-1 gezogene Schlussfolgerung einer Reduzierung der Opportunitätskosten der Inhalterezeption durch Individualisierung der Aggregation einzelner Elemente eines Inhaltebündels vor dem Hintergrund der getroffenen Annahmen ein zwingendes Vorteilskriterium. Abbildung 4-23 veranschaulicht den Verlauf der Bruttonutzenfunktion für ein individualisiertes Inhaltebündel $\left(S_{l_{b r}}\right)$ mit $n=8$ enthaltenen Elementen bzw. für ein nichtindividualisiertes Inhaltebündel $\left(S_{B_{b r}}\right)$ mit $n=10$ Elementen. ${ }^{499}$

499 Die Abbildung dient zur Veranschaulichung des Verlaufs der zuvor im Modell verwendeten logarithmischen Funktionen. Als Zahlenbeispiel wurden angenommen: $E(\alpha)=0,1 ; q_{U S E, B}=0,3 ; q_{U S E, I}=0,2$. 


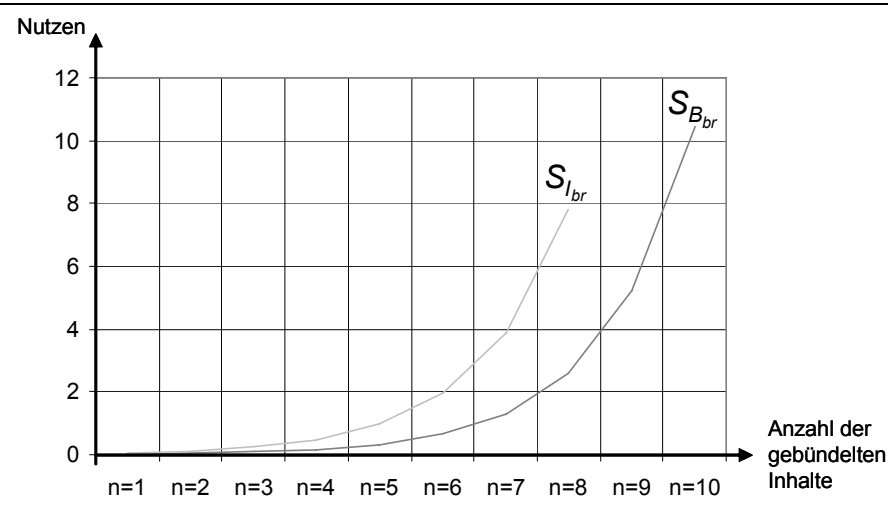

Abbildung 4-23: Bruttonutzenfunktionen für ein individualisiertes und ein nichtindividualisiertes Bündel

Beide abgebildeten Nutzenfunktionen besitzen unter den in Ausdruck 4.2-4 zugrunde gelegten Annahmen eine streng monoton steigende Form. Die Vorteilhaftigkeit individueller Inhaltebündel wird gemeinhin jedoch insbesondere dann unterstellt, wenn das Überangebot an Inhalten ein Auswahlproblem für den Nutzer erzeugt. Vor diesem Hintergrund ist davon auszugehen, dass bei einem individuellen Inhaltebündel ab einer gewissen Bündelgröße $(m)$ der durch das Vorselektieren relevanter Inhalte geschaffene Mehrwert konterkariert wird. Es ist demnach ab einer gewissen Bündelgröße von einem sinkenden Grenznutzen zusätzlicher Elemente eines individuellen Inhaltebündels auszugehen.

\subsubsection{Bedingungen der Vorteilhaftigkeit individueller Me- diengüter}

Das Ergebnis der Vorteilhaftigkeitsprüfung von Individualisierungsformen in der Medienbranche wurde im Voranstehenden unter stark vereinfachenden Annahmen ermittelt. Bspw. wurden in der Praxis sicher existierende Effekte wie ein sinkender Grenznutzen der Rezeption zusätzlicher Inhalte bei steigender Zahl gemeinsam angebotener Inhalte oder nachfrageseitige Budgetrivalitäten zwischen Mediengütern unterschiedlicher Distributionsformate vernachlässigt. Dadurch konnte gezeigt werden, dass eine Individualisierung des Angebots von Inhalten unter gewissen Bedingungen, wie hohen Such- und Selektionskosten, für Rezipienten einen Mehrwert bieten kann. Durch zwei sich anknüpfende Überlegungen können die Aussagen des Modells weiter verfeinert werden:

Wie beschrieben, ist zum einen das Vorteilhaftigkeitskriterium einer hohen Suchkostendifferenz zwischen individuellen und nichtindividualisierten Mediengütern abhängig von der Zahl der von einem Rezipienten erwünschten Inhalte im Verhältnis zur Gesamtzahl angebotener Inhalte. Die Vorteilhaftigkeit der Individualisierung erscheint daher umso wahrscheinlicher, je größer dieses Verhältnis ausfällt. Der Umfang des Verhältnisses ist dabei von zwei Faktoren ab- 
hängig: den individuellen Präferenzen eines Rezipienten ${ }^{500}$ und der jeweiligen Inhaltedomäne. ${ }^{501}$ Es ist daher zusammenfassend davon auszugehen, dass eine Individualisierung von Inhalteangeboten tendenziell in spezialisierten Themengebieten mit breitem Inhalteangebot den größten Mehrwert liefert.

Zum andern ist das in Abschnitt 4.3.3 eingeführte Vorteilhaftigkeitskriterium reduzierter Suchkosten bei der Rezeption eines individualisierten Medienguts gemäß den Modellannahmen von Ausdruck 4.3.3-2 unabhängig von den zuvor in Kapitel 4.2 beschriebenen Typen unterschiedlicher Individualisierungssysteme. Diese Annahme ist zwar für eine abstrakte Untersuchung der Vorteilhaftigkeit einer Individualisierungsstrategie tragfähig, sollte jedoch im Hinblick auf die notwendige Auswahlentscheidung eines Inhalteanbieters für eine (oder mehrere) konkrete Individualisierungstechnik(en) weiter verfeinert werden. Zu diesem Zweck ist die Ergebnisqualität der Empfehlungen des verwendeten Individualisierungssystems als Qualitätskriterium in Abhängigkeit des individuellen Nutzens eines Rezipienten zu überprüfen. Der individuelle Rezipientennutzen wurde bereits in Ausdruck 4.3.2-2 als Faktor $\alpha$ berücksichtig. Wie Schackmann anhand eines informationsökonomischen Suchmodells erläutert, repräsentiert der individuelle Rezipientennutzen jedoch keinen deterministischen Faktor sondern - im Hinblick auf die grundsätzliche Heterogenität der Rezipientenbedürfnisse - eine stochastische Verteilung. ${ }^{502}$ Der erwartete Rezipientennutzen eines individualisierten Medienguts kann daher formal beschrieben werden als Verteilungsfunktion $\mathrm{F}^{\mathrm{P}}(\alpha \mid \Theta)$ in Abhängigkeit von der Ergebnisqualität $(\Theta)$ des Systems und der themenbezogenen Verteilung des Rezipientennutzens $(\alpha)$. Die Ergebnisqualität des Individualisierungssystems repräsentiert dabei eine domänenabhängige Funktion aus dem relativen Anteil zwischen richtigen und falschen Empfehlungen des Systems. Zur Bemessung der Qualität der Empfehlungen eines Individualisierungssystems können bekannte Standardmaße für die Evaluierung von Dokumentensuchsystemen herangezogen werden. ${ }^{503}$ Die Qualität eines Suchsystems wird üblicherweise anhand von drei Kennzahlen bestimmt: der Genauigkeit der Suche (Precision), der Vollständigkeit des Suchergebnisses (Recall) und der Effektivität des Suchvorgangs (Fallout). ${ }^{504}$ Bewertungsgegenstand dieser drei Maße bilden Paare aus einer Suchanfrage und dem jeweils durch das System zurück gelieferten Suchergebnis. Das Suchergebnis wird dahingehend geprüft, ob die enthaltenen Empfehlungen relevant oder nicht relevant für die Suchabfrage sind, sowie ggf. mit der in der durchsuchten Menge an Dokumenten enthaltenen Anzahl an insgesamt relevanten

500 Bspw. wünscht der typische Leser einer Tageszeitung ein möglichst breites Angebot tagesaktueller Inhalte und besitzt daher eine geringe Suchkostenrelation, während ein Jugendlicher, der nur selektiv an tagesaktuellen Nachrichten interessiert ist, eine größere Suchkostenrelation besitzt.

501 Bspw. entsteht gegenüber dem tagesaktuellen Angebot an weltpolitischen Nachrichten über die weltweiten Finanzmärkte ein deutlich breiteres und detaillierteres Informationsangebot, das selbst für professionalisierte Informationsnachfrager nur in bestimmten Teilbereichen von Interesse ist.

502 Vgl. Schackmann (2004), S. 89.

$503 \mathrm{Vgl}$. Konstan et al. (1997).

504 Vgl. Rechenberg/Pomberger (2002), S. 926 f. 
und nicht relevanten Elemente verglichen. Dadurch können für ein Anfrage/Ergebnis-Paar vier Prüfkriterien gebildet werden (vgl. Abbildung 4-24).

\begin{tabular}{|c|c|c|c|c|c|}
\hline \multirow[b]{3}{*}{ relevant } & enthalten & nicht enthalten & & & \\
\hline & \multirow{3}{*}{$\begin{array}{c}\text { Treffer }(\mathbf{T}) \\
\text { (relevante } \\
\text { empfohlene Inhalte) }\end{array}$} & \multirow{3}{*}{$\begin{array}{l}\text { Versäumnis (V) } \\
\text { (relevante nicht- } \\
\text { empfohlene Inhalte) }\end{array}$} & Precision: & $T$ & Treffer \\
\hline & & & & $P=\overline{T+B}$ & Anzahl empfohlener Inhalte \\
\hline & & & & $T$ & Treffer \\
\hline \multirow{3}{*}{$\begin{array}{r}\text { nicht } \\
\text { relevant }\end{array}$} & \multirow{3}{*}{$\begin{array}{c}\text { Ballast (B) } \\
\text { (nicht-relevante } \\
\text { empfohlene Inhalte) }\end{array}$} & \multirow{3}{*}{$\begin{array}{c}\text { Korrekte } \\
\text { Zurückweisung (Z) } \\
\text { (nicht-relevante nicht- } \\
\text { empfohlene Inhalte) }\end{array}$} & Recall: & $R=\frac{T+V}{T+V}$ & Anzahl relevanter Inhalte \\
\hline & & & Fallout: & $F=B$ & Ballast \\
\hline & & & rallout: & $F=\overline{B+Z}$ & Anzahl nichtrelevanter Inhalte \\
\hline
\end{tabular}

Abbildung 4-24: Prüfkriterien für die Individualisierungsqualität

Als „richtige Empfehlungen“ werden solche Empfehlungen gewertet, die relevante Inhalte enthalten $(T)$, oder solche, die keine falschen Inhalte enthalten (Z). Als falsche Empfehlungen gelten dagegen solche, die entweder unerwünschte Inhalte enthalten (B) oder erwünschte Inhalte nicht empfehlen (V). Die Genauigkeit eines Suchsystems kann quantitativ anhand des Quotienten aus Treffern und der Summe aus Treffern und Ballast berechnet werden. ${ }^{505}$ Die Vollständigkeit des Ergebnisses kann demgegenüber als Quotient aus Treffern und der Anzahl der insgesamt relevanten Inhalte bestimmt werden. Dabei verhalten sich Genauigkeit und Vollständigkeit eines Systems üblicherweise gegenläufig. Die Effektivität eines Systems kann bestimmt werden durch den Quotienten aus dem Ballast in den Empfehlungen und der Zahl der insgesamt nicht relevanten Dokumente.

Wie bereits in Abschnitt 4.2 gezeigt wurde, ist die der Individualisierungsqualität zugrunde liegende Frage, welche Inhalte durch das Regelwerk des Individualisierungssystems selektiert bzw. vernachlässigt werden, hochgradig von der jeweils eingesetzten Individualisierungstechnik abhängig. Zwar existieren bereits in der Literatur vergleichende Untersuchungen der Qualität individualisierter und nichtindividualisierter Güter, nach Kenntnisstand des Autors existiert jedoch noch keine Gegenüberstellung der Individualisierungsqualität verschiedener Techniken. Eine solche Gegenüberstellung wird im folgenden Abschnitt am Beispiel prototypischer Systemrealisierung für den Internetauftritt der Universität Göttingen vorgenommen. Insofern davon auszugehen ist, dass unter den in Abschnitt 4.2 dargelegten Systemvarianten die methodische Distanz zwischen Selbstselektionssystemen (mit einem hohen Maß an Nutzerinteraktion) und beobachtungsbasierten Filtern (ohne explizite Nutzerinteraktion) am Größten ist, wird die weitere Untersuchung aus Umfangsgründen auf eine Gegenüberstellung dieser beiden Varianten reduziert.

505 Als Referenzwert: Griesbaum/Rittberger/Bekavac (2002) ermitteln für die deutsche Ausgabe der Onlinesuchmaschine Google einen Präzisionsgrad von knapp 59\%. 


\subsection{Wirkungsvergleich prototypischer Individualisierungs- systeme}

Im folgenden Abschnitt wird geprüft, inwiefern Individualisierung eine empirisch nachweisbare Wirkung auf den Nutzen eines Medienguts hat und inwiefern sich Unterschiede in Bezug auf diese Wirkung durch Einsatz unterschiedlicher Individualisierungsverfahren ergeben. ${ }^{506} \mathrm{Zu}$ diesem Zweck wurden im Internetauftritt der Universität Göttingen, der - wie in Abschnitt 4.4.1 gezeigt werden wird - Eigenschaften eines Medienguts besitzt, zwei Individualisierungsverfahren implementiert, die auf ihren gestifteten Nutzen bei typischen Nutzern hin untersucht wurden. Abschnitt 4.4.2 erläutert die Implementierungsform der eingesetzten Verfahren. Abschnitt 0 stellt die Ergebnisse eines im Juni 2005 unter studentischen Nutzern der Universitätsseiten durchgeführten Experiments im Zusammenhang mit der Nutzung der implementierten Verfahren dar. Die Erkenntnisse aus dem Systemvergleich werden in Abschnitt 4.4.4 zusammengefasst.

\subsubsection{Der Internetauftritt der Universität Göttingen als Me- diengut}

Der Internetauftritt der Universität Göttingen (http://www.uni-goettingen.de) beherbergt sämtliche Informationen zu zentralen Lehr-, Forschungs- und Verwaltungseinrichtungen der Universität. Dazu gehören die Internetpräsenzen sämtlicher Abteilungen der Universitätsverwaltung, einschl. Präsidium, Senat und Stiftungsrat, das zentrale Veranstaltungsregister der Universität, ein Archiv der Pressemitteilungen der Universität sowie der Internetauftritt von insgesamt 56 Fakultäten, Instituten und Forschungszentren. ${ }^{507}$ Die bereitgestellten Inhalte werden entweder von einer zentralen Redaktion erzeugt oder von dezentralen Stellen der Universität zur Publikation freigegeben. Zielgruppen der bereitgestellten Informationen bilden Studiumsinteressierte, Studierende, Universitätsmitarbeiter, ehemalige Universitätsangehörige und Interessenten aus Presse und Wirtschaft.

Die Informationen im zentralen Internetauftritt der Universität Göttingen werden durch ein datenbankgestütztes Content-Management-System (CMS) bereitgestellt. Das eingesetzte CMS ist eine Eigenentwicklung der Universität Göttingen, die im Jahr 2002 in Betrieb genommen wurde. Technisch beruht das CMS auf der Serversprache PHP und kostenfrei verfügbaren Systemkomponenten wie dem Apache Web Server ${ }^{508}$, der MySQL-Datenbank ${ }^{509}$ und der

\footnotetext{
506 Zum Begriff der Wirkung vgl. Abschnitt 5.3.1.1.

507 Stand: 2005-06-30. Die Zahl der beherbergten Einrichtungen variiert deshalb, weil die dezentralen Universitätseinrichtungen wie Fakultäten oder Institute auch eigene Internetpräsenzen unterhalten können, auf die von der zentralen Universitätsseite lediglich verwiesen wird.

$508 \mathrm{Vgl}$. http://httpd.apache.org.

$509 \mathrm{Vgl.} \mathrm{http://www.mysql.com.}$
} 
Suchmaschinentechnik MNoGoSearch ${ }^{510}$. Der Aufbau des CMS folgt einem 3Schichten-Modell: Die zugrunde liegende MySQL-Datenbank verfügt über 40 Tabellen, darunter ein Bild-, ein Dokumenten- und ein Textarchiv. Die Datenbank verwaltet über 25.000 Seitenelemente, die als Mikrobündel aus den jeweils archivierten Bild-, Dokument- oder Textelementen gebildet werden können. Die einzelnen Seitenelemente sind hierarchisch als Makrobündel mit einer Baumstruktur angeordnet. Der Zugriff auf die Datenbank erfolgt über eine webbasierte Administrationsoberfläche. Die Inhaltebereitstellung im Internet erfolgt dynamisch auf der Grundlage eines Templatemechanismus mit insgesamt 35 Formatvarianten.

Vor dem Hintergrund der in Abschnitt 2.3 verwendeten Kriterien zur Charakterisierung von Mediengütern - Immaterialität des Inhalts, materielles oder digitales Trägermedium, Informations- oder Unterhaltungscharakter, Adressierung eines Massenpublikums und kommerzielle Verbreitung - besitzen die Internetseiten der Universität Göttingen Merkmale eines Medienguts: Die präsentierten Inhalte dienen Informationszwecken und werden über das Internet an ein Massenpublikum transportiert. Derzeit werden im Internetauftritt der der Universität durchschnittlich knapp drei Mio. Seitenaufrufe (Pageviews) pro Monat verzeichnet, womit die Internetseiten die Reichweite eines kleinen Publikumsmediums besitzen. Allerdings ist - abgesehen vom Anwerben neuer Studierender - kein kommerzielles Interesse mit der Inhaltedistribution verbunden. Des Weiteren ist einschränkend davon auszugehen, dass lediglich ein Teil der präsentierten Inhalte nach redaktionellen Prinzipien erzeugt wird, wobei diesen redaktionellen Inhalten eine große Zahl an universitätstypischen Informationen in Veranstaltungs- und Personenverzeichnissen gegenübersteht. Von diesen Einschränkungen abgesehen besitzen die Internetseiten zumindest annähernd den Charakter von Mediengütern und werden im Weiteren als Anwendungsbeispiel für Individualisierungsverfahren in der Medienbranche herangezogen.

\subsubsection{Implementierungsform prototypischer Individualisie- rungssysteme}

Zur Prüfung der Akzeptanz von Individualisierungsverfahren bei Mediengütern wurde der Templatemechanismus der Internetseiten der Universität Göttingen mit zwei Individualisierungsverfahren ausgestattet: einer persönlichen Seite (MyUni), die von Nutzern individuell durch Selbstselektion von zu präsentierenden Inhalten konfiguriert werden kann (1) und einem beobachtungsbasierten Filter (MyBestBets), der für jeden aufgerufenen Inhalt Vorschläge für wahrscheinliche nächste Navigationsschritte anhand statistischer Auswertungen eines Sitzungsprotokolls ermittelt (2).

(1) Die inhaltliche Struktur des zentralen Internetauftritts der Universität Göttingen folgt wie bereits angesprochen einer Baumhierarchie, an deren Wurzel die Äste „Fakultäten“, „Studium“, „Forschung“, „Einrichtungen“ und „Service“ ste-

510 Vgl. http://search.mnogo.ru. 
hen. Jeder dieser Äste verzweigt sich in bis zu zehn Unterinhalte, die wiederum zwischen zwei und fünf Detailebenen besitzen. Ein Navigieren in dieser granularen Baumstruktur kann deshalb für einen Informationssuchenden zeitaufwändig sein, weil jede Anzeigeseite zwar Verweise auf die Inhalte der jeweils nächsten Detailebene beinhaltet, hierarchisch nachgeordnete Inhalte desselben Astes oder Inhalte anderer Äste jedoch verborgen bleiben. Vor dem Hintergrund dieses strukturbedingten Navigationsaufwands wurde zur Vereinfachung eines wiederholten Zugriffs auf Informationen verschiedener Äste eine Auswahlseite entwickelt, in der alle insgesamt 32 Verzweigungsknoten der ersten und zweiten Baumstruktur individuell ausgewählt werden können. Abbildung 4-25 zeigt die implementierte Auswahlseite.

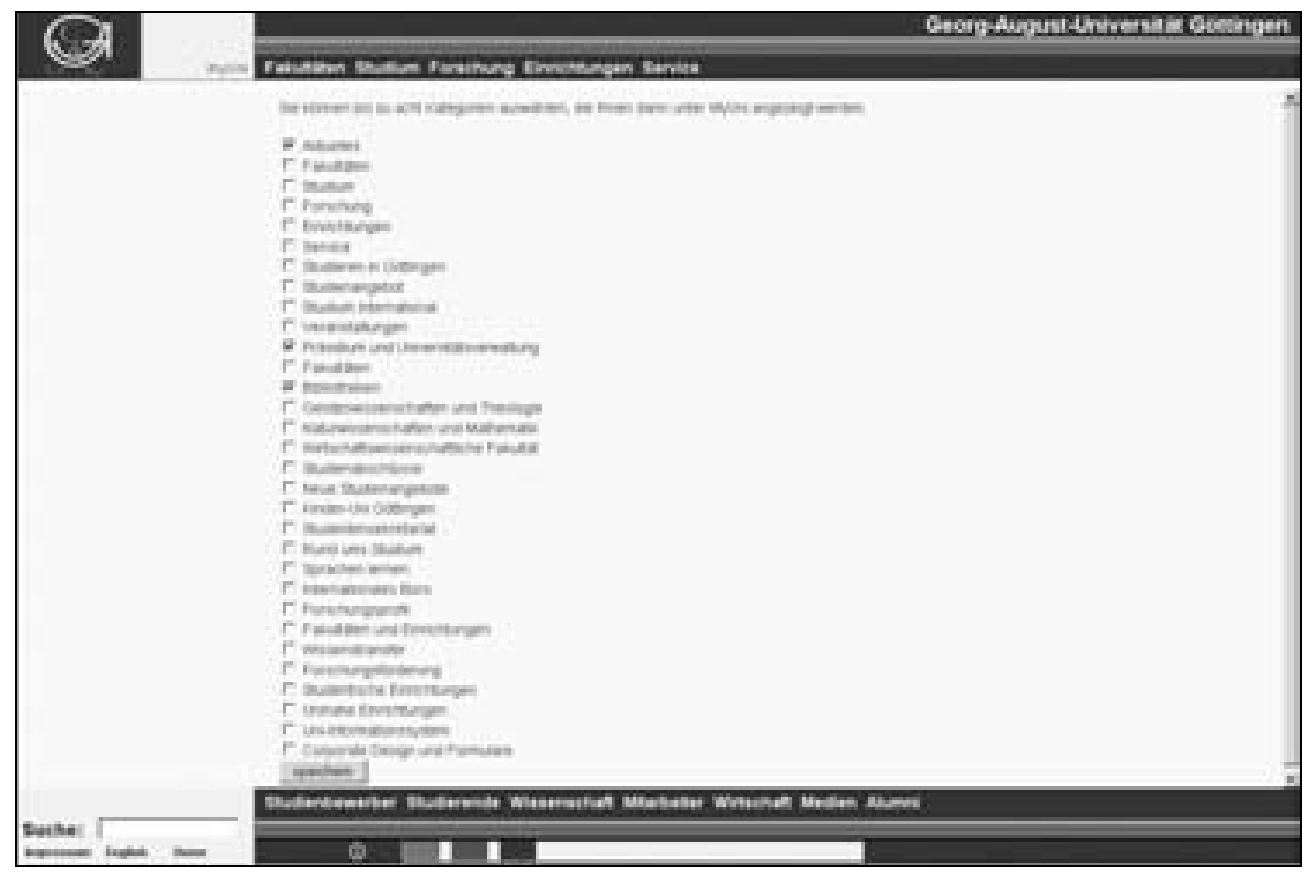

Abbildung 4-25: Auswahlseite des Instruments MyUni

Durch Speichern der Auswahl wird ein Cookie erzeugt, in dem die Datenbankschlüssel der ausgewählten Inhalte in der Datenbank gespeichert werden (Vgl. Abbildung 4-26). 


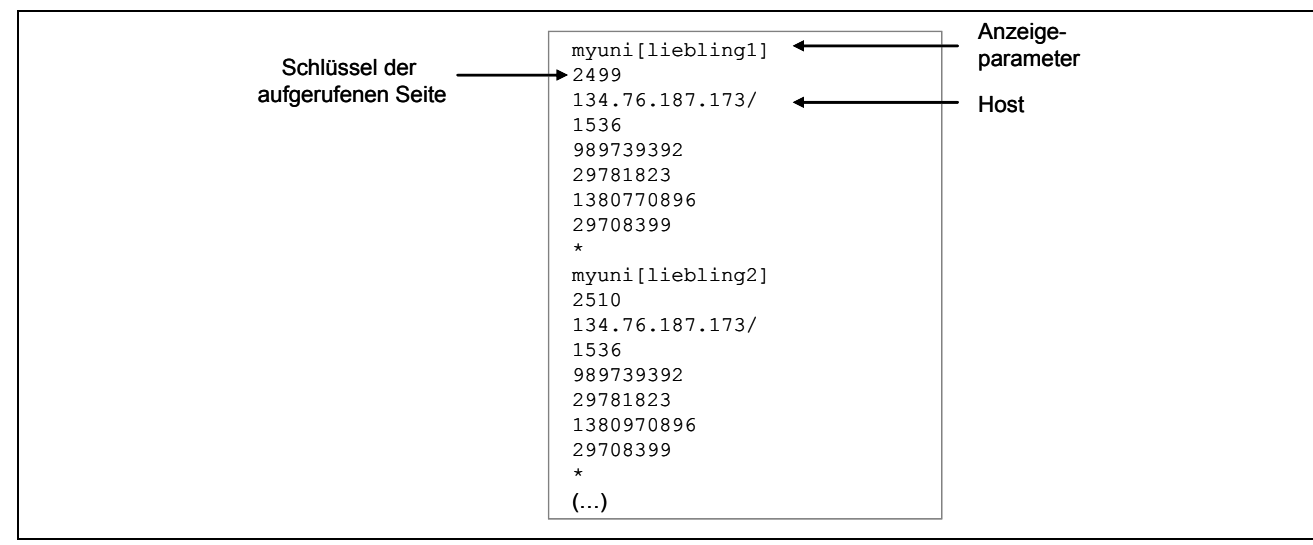

Abbildung 4-26:Kodierung des Nutzerprofils per Cookie

Eine spezielle Ausgabeseite, die durch einen Verweis in der (statischen) Kopfzeile von jeder Internetseite der Universität aufgerufen werden kann, liest dieses Cookie aus und zeigt die ausgewählten Inhalte sowie die unmittelbar diesen Inhalten folgenden Verknüpfungen auf einer einzigen Seite in tabellarischer Form an. Abbildung 4-27 zeigt die Ausgabeseite der drei in Abbildung 4-25 ausgewählten Inhalte.

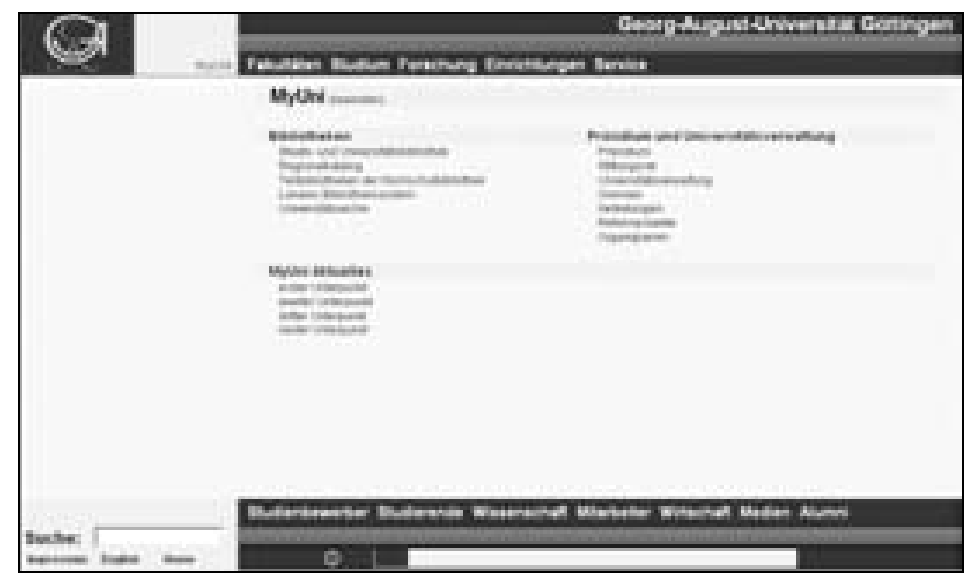

Abbildung 4-27: Ausgabeseite von MyUni

(2) In grundsätzlichen Zügen dem in Abschnitt 4.2.2 vorgestellten Verfahren des beobachtungsbasierten Filterns folgend, wurde ein Filter implementiert, der anhand der in der Vergangenheit vollzogenen Sitzungen für jede aufgerufene Seite Vermutungen über das Navigationsziel eines Nutzers anstellt. Zur Bestimmung dieser Vermutungen werden für jeden durch einen Nutzer aufgerufenen Seiteninhalt jeweils jene Seiteninhalte ausgewählt, die in vergangenen Sitzungen, in denen der aktuell aufgerufene Inhalt enthalten ist, am Sitzungsende betrachtet wurden. Da PHP, die Entwicklungssprache des zugrunde liegenden CMS der Internetseiten der Universität Göttingen, bereits standardmäßig mit einer Sitzungsverwaltung ausgeliefert wird, wurde auf der Grundlage dieser Sitzungsverwaltung, anstelle der vergleichsweise aufwändigen Sitzungsbestimmung 
im Webserverprotokoll, ein anwendungsspezifisches Sitzungsprotokoll entwickelt. Dieses Sitzungsprotokoll vergibt jeder durch einen Nutzer initiierten Sitzung einen eindeutigen Schlüssel und speichert jeden aufgerufenen Seiteninhalt zusammen mit dem zugehörigen Sitzungsschlüssel in einer Sitzungstabelle der Datenbank. Abbildung 4-28 zeigt einen Ausschnitt dieser Sitzungstabelle.

\begin{tabular}{|c|c|c|c|c|}
\hline 360 & 620485c1bfbe31b3d2749a27c5789737 & 12 & /show.php?lang=de\&type=sh\&id=2925 & 2005-04-15 12:15:20 \\
\hline 361 & abc41e9139a5b532b594aebaa3684aa1 & 4 & /show.php?lang=de\&type=sh\&id=13567 & 2005-04-15 12:15:20 \\
\hline 362 & c3ab74d1d85687e8cdeec964767a6850 & 10 & /show.php?lang=de\&type=sh\&id=19259 & 2005-04-15 12:15:20 \\
\hline 363 & f7ffc1e533497ca9a4086fd32f632d60 & 10 & /show.php?lang=en\&type=sh\&id=2925 & 2005-04-15 12:15:20 \\
\hline 364 & c3ab74d1d85687e8cdeec964767a6850 & 11 & /show.php?lang=de\&type=sh\&id=22665 & 2005-04-15 12:15:22 \\
\hline 365 & 8876d6e26bbb788b4dd6460804b72c5e & 6 & /show.php?lang=de\&type=sh\&id=14119 & 2005-04-15 12:15:23 \\
\hline 366 & 8876d6e26bbb788b4dd6460804b72c5e & 7 & /show.php?lang=de\&type=sh\&id=916 & 2005-04-15 12:15:23 \\
\hline & Sitzungsschlüssel & eihe & Seitenaufruf & Uhrzeit \\
\hline
\end{tabular}

Abbildung 4-28: Auszug des Sitzungsprotokolls von MyBestBets

Die Auswertung der Sitzungstabelle erfolgt datenbankgestützt durch Datenselektion auf Grundlage des in Abbildung 4-29 abgebildeten SQL-Statements. Um Übersichtlichkeit der Filterergebnisse zu wahren, wird die Datenselektion auf die fünf häufigsten Navigationsziele innerhalb der betrachteten Sitzungen beschränkt. Des Weiteren erfolgt die Datenselektion des Filters zur Erhöhung der Filterperformanz nicht unmittelbar jeweils im Zuge des Aufrufs einer Seite, sondern wird in periodischen Abständen einmalig für sämtliche Seiteninhalte der Datenbank durchgeführt. Das Ergebnis dieser Datenselektion wird in einer Ergebnistabelle gespeichert.

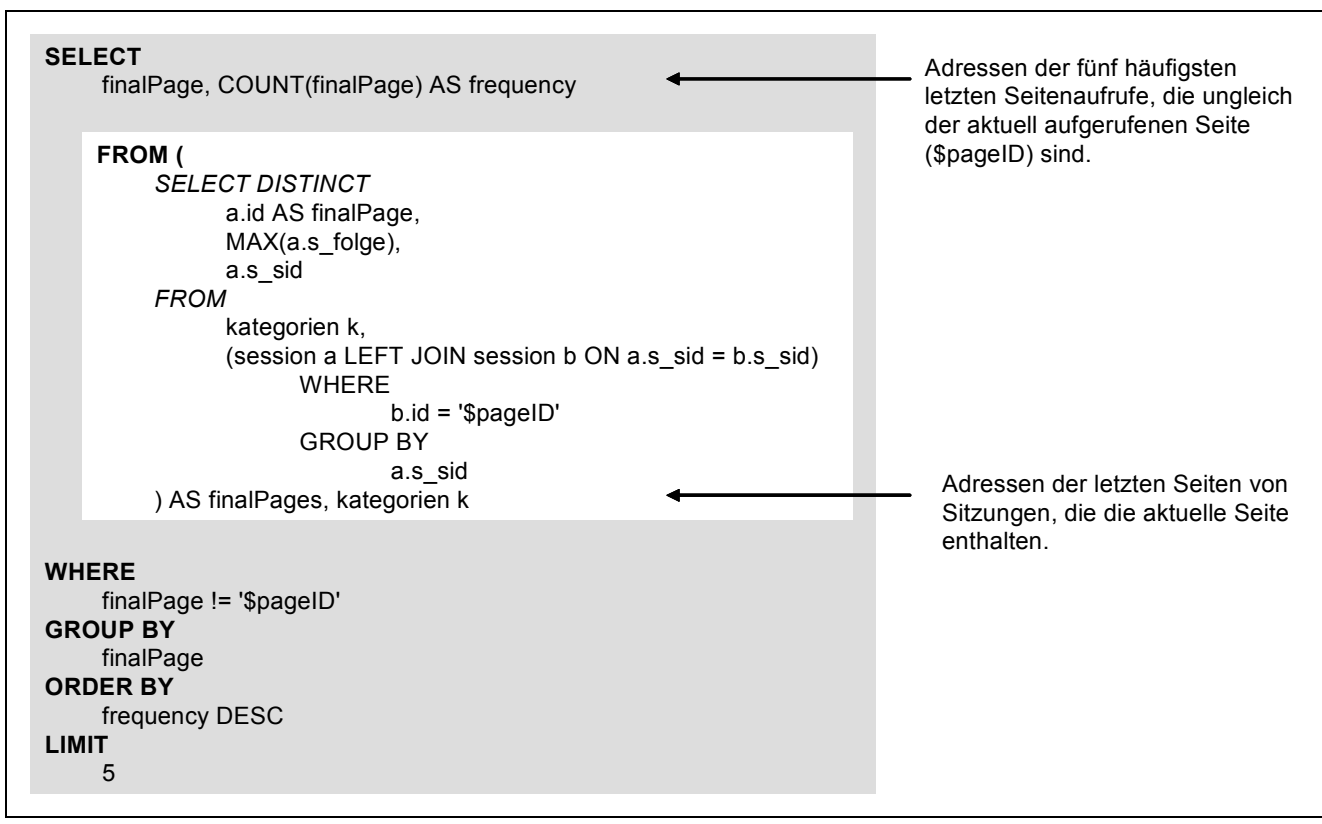

Abbildung 4-29: Datenselektion des MyBestBets-Filters 
Ein spezieller Fensterbereich, der in jedes Template der Universitätsseiten integriert wurde, liest in Abhängigkeit des jeweils aktuellen Seiteninhalts (\$pagelD) die Einträge der Ergebnistabelle aus und stellt die Filterergebnisse als Verweisliste im Anzeigebereich des jeweiligen Templates dar. Abbildung 4-30 zeigt beispielhaft das dunkel hinterlegt Filterfenster im Anzeigebereich der Ausgabeseite für Kurzinformationen zum Studium in Göttingen.

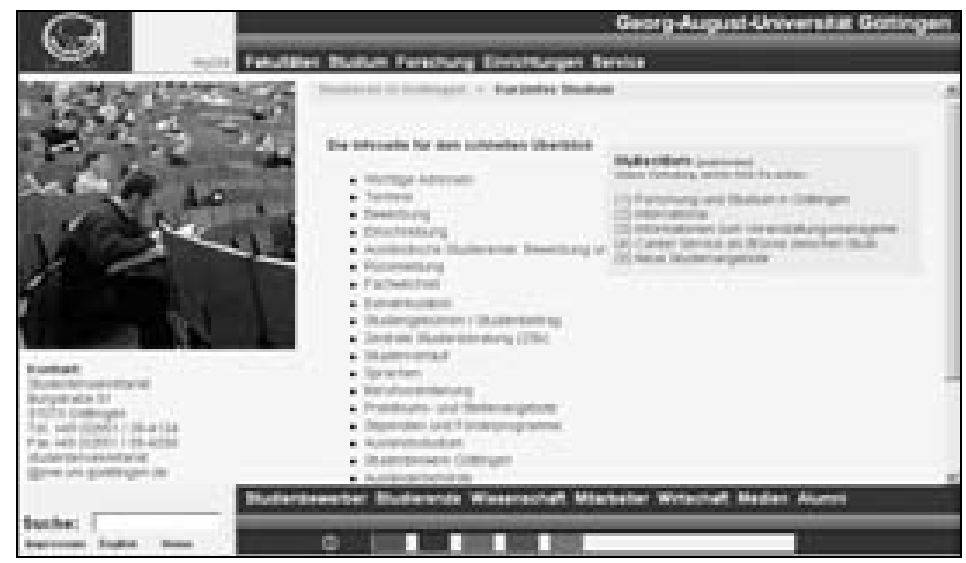

Abbildung 4-30: Ausgabeformat des MyBestBets-Filters

\subsubsection{Ergebnisse einer experimentellen Anwendung der implementierten Verfahren}

Zur Überprüfung des durch Individualisierungssysteme gestifteten Nutzens wurde im Juni 2005 eine Untersuchung durchgeführt, in der die beiden implementierten Verfahren erstmalig in einer kontrollierten Anwendungssituation an potenziellen zukünftigen Nutzern aus dem studentischen Umfeld getestet wurden. Das Ziel der Untersuchung bestand darin, die Auswirkungen und die Beurteilung des Einsatzes der implementierten Individualisierungssysteme in qualitativer Hinsicht zu prüfen sowie die Nutzerbeurteilung der eingesetzten Verfahren zu ermitteln. Die Untersuchung wurde in Form einer quasi-experimentellen Laboruntersuchung durchgeführt. ${ }^{511}$ Im Rahmen der Untersuchung waren von den Teilnehmern, zunächst ohne und später mit Zuhilfenahme der implementierten Individualisierungsverfahren, verschiedene Informationen zu recherchieren. Die Beurteilung der angebotenen Individualisierungssysteme wurde im Anschluss durch Befragung der Teilnehmer ermittelt. Abschnitt 4.4.3.1 gibt einen Überblick

511 Laboruntersuchungen unterscheiden sich von herkömmlichen Felduntersuchungen durch Ausschaltung von Störvariablen. Eine solche Störungsreduktion wurde im Falle der vorliegenden Untersuchung zum einen durch eine aufgabengestützte Erhebungsform und zum anderen durch ein kontrolliertes Erhebungsumfeld, einem PC-Labor mit gleichförmig konfigurierten Internet-PCs, gewährleistet. Der quasi-experimentelle Charakter der Untersuchung beruht auf der Rekrutierungsform der Teilnehmergruppen, die auf Grundlage von Lehrveranstaltungen erfolgte. Zur Methodik quasiexperimenteller und Laboruntersuchungen vgl. Bortz/Döring (2003), S. 57 und 60. 
über Aufbau und Stichprobe der Erhebung. Abschnitt 4.4.3.2 stellt die Ergebnisse der durchgeführten Befragungen vor.

\subsubsection{Aufbau und Stichprobe der Erhebung}

Die Erhebung der Beurteilungen der Untersuchungsteilnehmer erfolgte durch Kombination strukturierter schriftlicher Befragung mit leitfadengestützten (halbstrukturierten) mündlichen Gruppengesprächen. ${ }^{512}$ Im Rahmen der Erhebung wurde die Wirkung der beiden implementierten Verfahren sowohl auf die gezielte Informationssuche als auch auf ungerichtetes Informationsverhalten untersucht. Die schriftliche Befragung bestand aus drei Teilen: Die Teilnehmer mussten zunächst gezielt Inhalte in den Internetseiten der Universität Göttingen recherchieren. Die Rechercheaktivitäten wurden durch schriftlich vorgegebene Aufgaben, die typische Szenarien aus dem studentischen Alltag widerspiegeln, angeleitet. Beispielhafte Rechercheaufgaben bilden die Suche nach Ausgabemodalitäten des Studentenausweises oder die Suche nach Hinweisen zu individuell interessierenden Veranstaltungen auf dem Campusgelände. Nach Erledigung dieser Aufgaben wurde zunächst ohne nähere Information per Fragebogen geprüft, ob das Individualisierungsinstrument MyBestBets wahrgenommen oder sogar verwendet wurde. ${ }^{513}$ Daran anschließend mussten abermals Rechercheaufgaben - dieses Mal unter Zuhilfenahme des MyBestBetsFilters - bearbeitet werden. In einer zweiten, schriftlichen Befragung wurde geprüft, inwiefern die Empfehlungen des Filters bei der Durchführung der Recherche hilfreich waren. Abschließend wurde - ohne zusätzliche Rechercheaufgaben - ein Hinweis auf die Verfügbarkeit des MyUni-Konfigurators gegeben, der in einem dritten Fragebogen bewertet werden konnte. In den sich unmittelbar an die schriftliche Befragung anschließenden Gruppengesprächen wurden die abgegebenen Bewertungen im Zuge einer moderierten Diskussion erörtert. Die Konservierung der Gesprächsinhalte erfolgte mit der Zustimmung der Untersuchungsteilnehmer durch Bild- und Tonaufzeichnung.

Die Durchführung der Untersuchung erfolgte in Kleingruppen von 2-6 Personen. Jede Untersuchungsrunde dauerte einschließlich Gruppengespräch ca. 60 Minuten. Insgesamt nahmen 35 Studenten der Universität Göttingen an der zweitägigen Erhebung teil. Die Teilnehmer wurden in Lehrveranstaltungen der Universität Göttingen rekrutiert, der Aufwand für die freiwillige Teilnahme am Experiment wurde finanziell entschädigt. Die Teilnehmer der Untersuchung hatten ein Durchschnittsalter von knapp 23 Jahren, 60\% der Teilnehmer waren Frauen. Die Teilnehmer waren hochgradig internetaffin, $77 \%$ der Teilnehmer gaben an, das Internet täglich zu nutzen, insgesamt $97 \%$ nutzen das Internet mindestens einmal pro Woche. Keinem der Teilnehmer waren die untersuchten Individualisierungssysteme vor Beginn des Experiments bekannt, da beide Instrumente erst zum Untersuchungsbeginn erstmalig freigegeben wurden. Die

512 Zur Methodik der Befragung vgl. Abschnitt 5.3.2.1.2. Der Aufbau und die Ergebnisse der Befragung sind im Detail in Anhang, Abschnitt 7.1, abgebildet.

513 Die beobachtungsgestützten Empfehlungen des MyBestBets-Filters waren während des gesamten Experiments aktiviert. 
Ergebnisliste des MyBestBets-Filters wurde auf der Grundlage der Auswertung einer Sitzungstabelle der vorangegangenen sechs Wochen gebildet. Die Mächtigkeit der Sitzungstabelle betrug 2,9 Mio. Einträge.

\subsubsection{Ergebnisse der Teilnehmerbefragung}

Entsprechend der getrennten Untersuchung der Wirkung des MyBestBetsFilters (1) und des MyUni-Konfigurators (2) im Zuge der Erhebung, werden im Folgenden die Ergebnisse der Bewertungen der Teilnehmer für die beiden Instrumente zunächst getrennt voneinander erläutert. Eine Gegenüberstellung der Bewertungen beider Verfahren erfolgt in Abschnitt 4.4.4.

(1) Die Teilnehmer gaben eine insgesamt geringe Erfahrung mit automatischen Filtern an, lediglich drei Untersuchungsteilnehmer wussten Filterfunktionen zu nennen, die mit dem MyBestBets-Instrument vergleichbar sind. 93\% der Teilnehmer der Untersuchung nahmen den implementierten MyBestBets-Filter ohne explizite Hinweise wahr, knapp 75\% der Befragten lasen unaufgefordert die darin enthaltenen Vorschläge. Anfangs verwendeten nur 25\% der Befragten die vorgeschlagenen Verweise im Rahmen der gestellten Rechercheaufgaben. Nach entsprechenden Erläuterungen der Filterfunktion stieg die Zahl der Anwender auf knapp 63\%.

Die technische Handhabung der Empfehlungen des MyBestBets-Filters wurde zwar von $80 \%$ der Teilnehmer als unproblematisch empfunden. Dennoch bewerteten nur etwa $40 \%$ der Teilnehmer die Filterempfehlungen als hilfreich, weitere $37 \%$ empfanden die Empfehlungen wenigstens als nicht störend. Im Hinblick auf die Präzision der Empfehlungsergebnisse des Filters beurteilten $80 \%$ der Teilnehmer zumindest vereinzelte Verweise als hilfreich (wobei allerdings nur $5 \%$ viele der empfohlenen Verweise als hilfreich beurteilten). Im Rahmen der Gruppengespräche wurde deutlich, dass die wahrgenommene Trefferquote (Precision) des Filters zwischen den Befragten zum Teil erheblich, mit einer Spannweite zwischen $2 \%$ und $60 \%$, schwankte. Eine hohe Präzision empfanden vor allem jene Teilnehmer, die an das Instrument nicht den Anspruch eines vollwertigen Ersatzes einer Suchmaschine richteten, sondern die Filterergebnisse als Quelle von Zusatzinformationen betrachteten. Die Befragten stimmten weitgehend darin überein, dass das Instrument in erster Linie einen Sekundärnutzen in Ergänzung zur Suchmaschine und dem gezielten Blättern in den Seiten stiftet, ${ }^{514}$ jedoch nicht den Primärnutzen der Seite steigert. Die Fehlertoleranz gegenüber den Empfehlungen des Filters war daher überwiegend sehr hoch. Dies spiegelt sich darin wieder, dass trotz der nur mäßigen Individualisierungsqualität dennoch rund $77 \%{ }^{515}$ der Teilnehmer das MyBestBetsInstrument auch zukünftig wieder nutzen würden.

Im Rahmen der Gruppengespräche konnten drei wesentliche Problemquellen im Hinblick auf die Verwendung des MyBestBets-Filters identifiziert werden:

514 Im Rahmen ihrer Suchstrategien empfanden 74\% der Teilnehmer die Suchmaschine der Seite, 71\% das Blättern in Seiteninhalten und 31\% den MyBestBets-Filter als wichtige Unterstützung.

515 Rund 29\% der Teilnehmer gaben an, dass sie den Filter in Zukunft mit Bestimmtheit nutzen würden und weitere $49 \%$, dass eine zukünftige Nutzung für sie zumindest wahrscheinlich ist. 
Zum einen traten Redundanzen zwischen dem Filterergebnis und den Verweisen in den jeweils aufgerufenen Seiteninhalten auf, wodurch die wahrgenommene Filterpräzision gemindert wurde. Zum anderen hatten negative Erfahrungen mit als störend wahrgenommenen maschinellen Assistenten wie dem Microsoft-Office-Assistenten einen schmälernden Einfluss auf das Vertrauen in die Ergebnisqualität des Filters. Schließlich weckte die grafische Einblendung der Filterergebnisse Assoziationen mit Bannerwerbung, weshalb einige Teilnehmer das Instrument z.T. während der gesamten Untersuchung ignorierten.

(2) Sowohl der inhaltliche Nutzen als auch die technische Handhabung des MyUni-Konfigurators wurde gleichmäßig von über $80 \%$ der Teilnehmer als gut empfunden. Neben der naturgemäß hohen Präzision eines Selbstselektionsverfahrens wurde im Rahmen der Gruppengespräche ein weiterer Nutzen des Instruments darin gesehen, dass dadurch eine Hilfe für die Orientierung in der Inhaltestruktur der Seite geboten wird. Der Konfigurationsaufwand des MyUniKonfigurators wurde von allen Teilnehmern als vernachlässigbar empfunden. Entgegen dem in Abschnitt 4.1 thematisierten Problem einer geringen Bereitschaft zur Selbstidentifikation von Onlinenutzern gaben einige Teilnehmer an, für einen höheren Individualisierungsgrad des MyUni-Konfigurators und für Möglichkeiten zur Interaktion mit anderen Nutzern (bspw. durch Foren) auch detaillierte persönliche Angaben auf der Seite hinterlassen zu wollen. Als problematisch wurde hingegen die bei Nutzung des Konfigurators fehlende Möglichkeit bewertet, neben den ausgewählten Inhalten auch zu solchen Inhalten abzuschweifen, die dem jeweiligen Tagesinteresse entsprechen. Dennoch gaben $94 \%$ der Teilnehmer an, ein derartiges System auch ggf. in Zukunft nutzen zu wollen.

\subsubsection{Zusammenfassung}

Die Untersuchung der Wirkung der beiden implementierten Individualisierungssysteme lieferte zusammenfassend das Ergebnis, dass in beiden Fällen jeweils ein wahrgenommener Zusatznutzen durch Individualisierung festzustellen ist. Dieser Nutzen ist dabei zumindest teilweise unabhängig von der Präzision des Individualisierungsergebnisses: Im Rahmen des MyBestBets-Filters werden auch solche Empfehlungen, die zwar für ein konkretes Suchproblem keine Lösung bieten, jedoch sinnvolle Zusatzinformationen zu dem jeweils aktuell betrachteten Inhalt enthalten, als nützlich empfunden. Es ist anzunehmen, dass dieser Nutzen steigt, je diffuser das Informations- oder Unterhaltungsbedürfnis eines Konsumenten ist. Des Weiteren wurde bei der Anwendung des MyUni-Systems nicht nur der Konfigurationsvorgang, sondern bereits der Konfigurator selbst zum Zwecke der Orientierung als nützlich empfunden. Es ist zu vermuten, dass der Nutzen eines Konfigurators grundsätzlich mit dem Umfang angebotener Inhalte und dem Fokussierungsgrad des Informations- oder Unterhaltungsbedürfnisses eines Konsumenten steigt.

Im Vergleich zwischen den beiden implementierten Verfahren bewirkt der MyUni-Konfigurator einen nachweislich höheren Wiedernutzungsgrad. Die wahrgenommene Vorteilhaftigkeit des Konfigurators gegenüber dem MyBestBets-Filter kann auf zwei Ursachen zurückgeführt werden: Erstens stellten die Teilnehmer eine höhere Ergebnispräzision im Rahmen der individuellen Konfiguration fest. Neben den bereits von den Teilnehmern aufgezeigten 
ration fest. Neben den bereits von den Teilnehmern aufgezeigten Verbesserungsmöglichkeiten des MyBestBets-Filtersystems kann dessen Präzision ggf. dadurch erhöht werden, dass nicht nur der jeweils aktuell aufgerufene Inhalt, sondern sämtliche zuvor in einer Sitzung aufgerufenen Inhalte sowie ggf. die Reihenfolge dieser Aufrufe als Grundlage der Filterempfehlung herangezogen werden. Zweitens wirkte sich die geringe intuitive Nachvollziehbarkeit des Zustandekommens der Filterergebnisse negativ auf das Vertrauen der Teilnehmer in die Individualisierungsergebnisse aus, wobei der Nutzungsgrad der Filterergebnisse nach entsprechenden Erläuterungen zur Funktionsweise nachweisbar anstieg.

Die Ergebnisse der Untersuchung speziell aus den Gruppengesprächen legen zusammenfassend die Vermutung nahe, dass die Wirkung des eingesetzten Individualisierungsverfahrens stark vom jeweiligen Zweck der Inhaltenachfrage abhängt. Es ist insbesondere zwischen fokussierter Inhaltenachfrage und einem Blättern in Inhalten mit tendenziell offenem Informations- oder Unterhaltungsziel zu unterscheiden. Diese Vermutung ist konsistent mit etablierten Theorien zum allgemeinen Kaufverhalten, wonach zwischen fokussierteren „Purchasern“ und tendenziell weniger zielgerichteten „Browsern“ unterschieden wird. ${ }^{516}$ Vor diesem Hintergrund kann geschlussfolgert werden, dass ein individueller Konfigurator, wie das dargestellte System MyUni, tendenziell einen höheren Nutzen bei fokussierter Informationsnachfrage bewirkt, während ein beobachtungsgestützter Filter ein diffuses Blättern in Inhalten unterstützt.

\subsection{Zusammenfassung der Möglichkeiten einer Individuali- sierung von Mediengütern}

In Kapitel 3 wurde dargelegt, dass die traditionelle Alternativhypothese in Bezug auf Kostenoptimierung und Varietätsgrad, durch die Standardisierbarkeit digital und medienneutral erzeugter Inhalte, in der Medienbranche nur noch bedingt Gültigkeit besitzt. Insbesondere gilt der durch hohe First-Copy-Costs begründete Zwang zur Massenkommunikation für Medienunternehmen nicht mehr uneingeschränkt. Wenn Medienunternehmen die Wünsche ihrer Kunden individuell bestimmen können, dann kann eine individuelle Produktvariante auf der Grundlage standardisierter Module zu einem Kostenniveau erzeugt werden, das dem der Massenfertigung entspricht. Verfahren zur Bestimmung individueller Kundenmerkmale wurden in Abschnitt 4.1 erläutert. Die Individualisierung von Mediengütern erfordert den Einsatz von Individualisierungssystemen, die überblicksartig in Abschnitt 4.2 vorgestellt wurden. Die Individualisierung des Angebots von Mediengütern besitzt immer dann gegenüber alternativen Inhalteangeboten einen relativen Mehrwert, wenn dadurch der für inn mit der Rezeption verbundene Aufwand gesenkt werden kann. Wie in Abschnitt 0 gezeigt wurde, ist die Vorteilhaftigkeit von Individualisierungsformen immer dann gegeben, wenn zum einen im Rahmen der Auswahl der betreffenden Inhalte ein hoher Suchaufwand besteht und zum anderen der Aufwand der Rezeption einzelner

516 Vgl. Büttner/Rauch/Silberer (2005) und Gollwitzer (1990). 
Inhalte gesenkt wird. Am Gegenstand zweier prototypischer Implementierungen von Individualisierungssystemen konnte in Abschnitt 4.4 durch eine empirische Nutzererhebung nachgewiesen werden, dass Individualisierungssysteme grundsätzlich unabhängig vom jeweiligen Verfahren neben anderen Hilfsmitteln wie bspw. Suchfunktionen einen Mehrwert im Rahmen der Nutzung von Onlineinformationen stiften. Dabei konnten neben der Präzision des Empfehlungsergebnisses ein Einfluss des Zwecks der jeweiligen Informationsnachfrage eines Nutzers und ein Einfluss der Transparenz des Individualisierungsvorgangs für den Nutzer auf die Wirkung des Individualisierungssystems nachgewiesen werden.

Somit kann der Möglichkeit zur Individualisierung von Mediengütern, vor dem Hintergrund der einschränkenden Vorteilhaftigkeitsbedingungen, das Potenzial für einen radikalen Paradigmenwechsel in der Medienbranche beschieden werden: War bislang die absolut generierbare Reichweite das dominante ökonomische Zielkriterium des Medienmanagements, kann nun der individuelle Kundenwertbeitrag als strategisches Ziel verfolgt werden, wobei der Endkunde individuell den Wert der wirtschaftlichen Aktivitäten bestimmt. Denkbare Hebel für eine solche strategische Neuausrichtung bilden eine Verlängerung der Kundenbeziehungsdauer durch Steigerung der Kundenloyalität sowie eine crossmediale Erfassung und Berücksichtigung der Kundenbedürfnisse. 



\section{Inhalteverwertung im mobilen Internet}

In Kapitel 3 wurde dargelegt, dass Medienunternehmen zwei Strategien verfolgen können, um zusätzliche Einnahmen zu erzielen: durch Diversifikation in neue Geschäftsfelder, wie bspw. in den Bereich des technischen Onlinezugangs oder durch Erhöhung der Zahlungsbereitschaft ihrer Konsumenten. Es wurde gezeigt, dass die Zahlungsbereitschaft von Inhaltekonsumenten nicht nur von der Qualität bereitgestellter Inhalte, sondern außerdem von ihrem situativen Gebrauchswert und der Qualität des Inhalteformats abhängen. Neben der bereits beschriebenen Individualisierung von Mediengütern wurden dabei mobile Dienste als Träger potenzieller Mehrwertformate für den Inhaltevertrieb identifiziert. Das folgende Kapitel untersucht vor diesem Hintergrund im Detail die Möglichkeiten einer mehrwertstiftenden Vermarktung von Inhalten auf der Grundlage von mobilen Diensten.

Insofern in Kapitel 2.2 bislang lediglich begriffliche und technische Grundlagen mobiler Dienste erläutert wurden, wird in Abschnitt 5.1 zunächst ein Überblick über den aktuellen Stand der Forschung im Bereich des Geschäfts mit mobilen Diensten gegeben. In Abschnitt 5.2 werden im Zuge einer theoriegeleiteten Deduktion Rahmenbedingungen einer Inhalteverwertung von Medienunternehmen im mobilen Internet identifiziert und Beispiele bestehender Geschäftsmodelle für inhalteorientierte mobile Dienste analysiert. In Abschnitt 5.3 erfolgt eine empirische Untersuchung der Akzeptanz mobiler Inhalteformate und eine Analyse der Nutzungsmotive inhalteorientierter mobiler Dienste. Auf der Grundlage der empirischen Untersuchungsergebnisse werden in Abschnitt 5.4 Konzepte für eine mehrwertstiftende Bereitstellung von Inhalten im mobilen Internet vorgestellt. Das Kapitel schließt in Abschnitt 5.5 mit einer Zusammenfassung.

\subsection{Stand der Forschung zum Geschäft mit mobilen Diensten}

Die wissenschaftliche Untersuchung des kommerziellen Geschäfts, das sowohl mit als auch durch Telekommunikationstechnologien wie dem digitalen Mobilfunk ermöglicht wird, repräsentiert eine vergleichsweise junge Forschungsrichtung der Ökonomie. Bis vor einigen Jahren standen anstelle ökonomischer Aspekte des Telekommunikationsgeschäfts in erster Linie die Entwicklung und die Erörterung technologischer Innovationen der Netztechnik und der Signalübertragung im Mittelpunkt der Aufmerksamkeit. ${ }^{517}$ Die wenigen verfügbaren öko-

517 Vgl. bspw. Schiller (2003); Müller/Eymann/Kreutzer (2003). 
nomischen Beiträge beschäftigten sich entweder mit nachfrageorientierten Aspekten der Diffusion von Telekommunikationstechnik ${ }^{518}$, mit organisatorischen Problemen hochintegrierter Monopolisten der Telekommunikationsbranche ${ }^{519}$ oder mit dem Systemgutcharakter von Telekommunikationsdienstleistungen ${ }^{520}$. Seit Ende der 1990er Jahre ist jedoch im Bereich der ökonomischen Wissenschaften ein verstärktes Interesse am Telekommunikationsgeschäft und insbesondere den Geschäftsmöglichkeiten, die sich im Zusammenhang mit dem digitalen Mobilfunk ergeben, zu beobachten. Die steigende Popularität ist abzulesen an einer wachsenden Anzahl von Beiträgen in den Organen der Wissenschafts- und Fachkommunikation. Die in den letzten Jahren zu diesem Thema erschienen Beiträge können dabei grob in drei Gruppen unterteilt werden: praxisnahe Grundlagen- und Zukunftsstudien (1), wissenschaftliche Konferenzbeiträge und Tagungsbände (2) sowie Herausgeberbände und Lehrwerke (3).

(1) Grundlagen- und Zukunftsstudien zum Geschäft auf der Grundlage des digitalen Mobilfunks entstanden vor allem in den Jahren zwischen 1999 und 2001 und wurden zumeist von kommerziellen Informationsdienstleistern wie Marktforschungsunternehmen, Unternehmensberatungen oder Investmentbanken in Auftrag gegeben oder verfasst. ${ }^{521}$ Gegenstand dieser Studien bilden zum einen die Erläuterung von neuen und als relevant erachteten Mobilfunktechnologien sowie zum anderen die Beschreibung von deren Erfolg versprechenden Anwendungsfeldern. Als Forschungsmethodik kommen vorwiegend Technologieanalysen und Expertenbefragungen zum Einsatz.

(2) Mobile Dienste haben sich in den letzten Jahren stetig zu einem festen Bestandteil des Themenkanons von nationalen und internationalen Wissenschaftskonferenzen sowohl im Bereich der Betriebswirtschaftslehre als auch insbesondere der Wirtschaftsinformatik entwickelt. ${ }^{522}$ Gegenstand der wissenschaftlichen Diskussion ist dabei „die Entwicklung, Standardisierung und Integration mobiler und multimedialer Dienste und Anwendungen in differenzierten Märkten sowie die damit verbundenen nachhaltigen Veränderungen in Wirtschaft und Gesellschaft". ${ }^{523}$ An einen solchen Forschungsgegenstand anknüpfende Fragestellungen bilden bspw. die Lösung von Problemen im Bereich der Datensicherheit mobilfunkgestützter Kommunikationsvorgänge, die Verbesserung der Mensch-zu-Maschine-Interaktion auf der Grundlage mobiler Endgeräte, Geschäftsmodelle von mobilen Multimediadiensten sowie Möglichkeiten der betrieblichen Anwendungs- und Prozessintegration mit Mobilfunktechnologien.

\footnotetext{
518 Vgl. Weiber (1992); Weiber 1995; Schenk/Dahm/Sonje (1996); Krafft/Litfin (2002).

519 Vgl. Grunwald/Schwellbach (1999).

$520 \mathrm{Vgl}$. bspw. Taschner (2001).

521 Vgl. bspw. Müller-Verse (1999); Day/Daly/Sheedy/Christiansen (2000); Ericsson Consulting (2000); Ovum (2000); TIMElabs (2002).

522 Vgl. bspw. Mobile HCl (2003); MobiSys (2003); ACM MobiCom (2003); WI (2003); AMOC (2004); MoMM (2004); MCTA (2005)

$523 \mathrm{Vgl}$. Uhr/Esswein/Schoop (2003).
} 
(3) Aufsatzsammlungen und Herausgeberwerke beschäftigen sich mit den Anwendungsformen mobiler Dienste entweder aus einer betrieblich-funktionalen oder aus einer branchenbezogenen Perspektive. ${ }^{524}$ Im Rahmen funktionaler Beiträge werden u.a. Einsatzmöglichkeiten von mobilen Diensten als Werbeträger im Rahmen des betrieblichen Marketings und der kommerziellen Werbewirtschaft beschrieben sowie Aspekte der Preispolitik und der Gestaltung von Benutzeroberflächen mobiler Dienste thematisiert. Aus Branchensicht werden u.a. Einsatzfelder für mobile Dienste im Bereich des Tourismus, der Finanzdienstleistungen oder der Logistikbranche erörtert. Neben derartigen Herausgeberwerken entstanden erste Lehrwerke, die ausschließlich das Geschäft mit mobilen Diensten zum Gegenstand haben und einen Überblick über den aktuellen Stand technischer Entwicklungen und ökonomischer Anwendungsformen geben. ${ }^{525}$

Trotz der wachsenden Popularität der ökonomischen Erforschung mobiler Dienste ist das Forschungsfeld des mobilen Internet erst in Ansätzen erschlossen. Zum einen ist die Beschreibung der branchenbezogenen Anwendungsmöglichkeiten mobiler Dienste lückenhaft. ${ }^{526}$ Insbesondere existiert bislang für die Medienbranche keine umfassende Analyse der Rahmenbedingungen und Handlungsmöglichkeiten im Zusammenhang mit der Inhalteverwertung im mobilen Internet. Zum anderen identifizierte eine interdisziplinäre Forschungsgruppe aus Wissenschaftlern der Fachbereiche Informatik, Soziologie, Betriebswirtschaftslehre und Wirtschaftsinformatik, vor dem Hintergrund des bislang mäßigen kommerziellen Erfolgs existierender Pionieranwendungen für Endkunden im mobilen Internet, in einem Grundsatzbeitrag zu offenen Forschungsfragen im Zusammenhang mit mobilen Diensten drei grundsätzlich zu lösende Kernprobleme: ${ }^{.527}$

1. Die Identifikation tragfähiger Geschäftsmodelle im Rahmen der Vermarktung mobiler Dienste.

2. Die Untersuchung von Akzeptanz und Nutzungsmotiven von Konsumenten in Bezug auf mobile Dienste.

3. Die Entwicklung von Technologien und Dienstformaten zum Schaffen eines kundenseitigen Mehrwerts.

Vor dem Hintergrund der genannten Forschungslücken wird im Weiteren versucht, diese Lücken aus Sicht der Medienbranche zu verkleinern. Der Aufbau der weiteren Untersuchung folgt dabei den drei genannten Kernproblemen: In Abschnitt 5.2 werden die Rahmenbedingungen der Inhalteverwertung im mobilen Internet aus Geschäftsmodellperspektive untersucht. Zur Bestimmung der Akzeptanz mobiler Inhalteformate werden in Abschnitt 5.3 die Ergebnisse zweier empirischer Studien, die im Zusammenhang mit der vorliegenden Arbeit durchgeführt wurden, erläutert, und Schlussfolgerungen in Bezug auf die An-

524 Vgl. u.a. Nicolai/Petersmann (2001); Gora/Röttger-Gerigk (2002); Reichwald (2002); Silberer/Wohlfahrt/Wilhelm (2002).

525 Vgl. Lehner (2003);Tourowski/Pousttchi (2004).

526 Vgl. Wohlfahrt (2004), S. 6 und S. 43.

$527 \mathrm{Vgl}$. Hess et al. (2005). 
forderungen mehrwertstiftender Dienstformate abgeleitet. In Abschnitt 5.4 werden anhand dieser Anforderungen Konzepte zur Bereitstellung inhalteorientierter mobiler Mehrwertdienste vorgestellt. Abschnitt 5.5 fasst die Ergebnisse des Kapitels zusammen.

\subsection{Geschäftsmodelle für das Angebot von Mediengütern im mobilen Internet}

In diesem Kapitel werden Möglichkeiten der Vermarktung von Mediengütern in Form von inhalteorientierten Onlineangeboten im mobilen Internet untersucht. Zunächst werden in Abschnitt 5.2.1 allgemeine Untersuchungskriterien für Geschäftsmodelle als Analyseeinheit innovativer Geschäftsvorhaben bestimmt. Darauf aufbauend werden in Abschnitt 5.2.2 Rahmenbedingungen für Geschäftsmodelle von Medienunternehmen im mobilen Internet anhand der zuvor in Abschnitt 5.2.1 abgeleiteten Kriterien erläutert. Schließlich werden in Abschnitt 5.2.3 drei konkrete Fallbeispiele der Geschäftsmodelle von Anbietern für Inhalte im mobilen Internet erläutert. Abschnitt 5.2.4 fasst die Ergebnisse des Kapitels zusammen.

\subsubsection{Das Konzept des Geschäftsmodells als Analyseeinheit}

Das Geschäftsmodell ist weder ein fachlich fundiertes Analyseinstrument, noch ist es begrifflich klar abgegrenzt. Dennoch hat es sich in der Praxis bewährt, insbesondere in den technologieaffinen, innovativen Marktbereichen der Internetökonomie, die betriebswirtschaftlichen Ziele in Verbindung mit einem Geschäftsvorhabens aus mehr als nur einer einzigen Perspektive zu charakterisieren, um das gesamte Spektrum an Abhängigkeiten eines solchen Vorhabens zu berücksichtigen. ${ }^{528}$ Als Analyseeinheit für ein solches Geschäftsvorhaben und den damit einhergehenden Abhängigkeiten wird seit einigen Jahren das Konzept des sog. „Geschäftsmodells“ diskutiert. ${ }^{529}$ Ein Geschäftsmodell kann dabei allgemein verstanden werden als eine Beschreibung der Strategie eines Unternehmens, um potenziellen Investoren die Sinnhaftigkeit ihres Engagements zu verdeutlichen. ${ }^{530}$ Allerdings existiert in der betriebswirtschaftlichen Literatur eine gewisse Vielfalt an Vorschlägen unterschiedlicher Kriterien für eine solche Strategiebeschreibung. ${ }^{531}$ Nachfolgend wird ein Überblick über die verschiedenen diskutierten Geschäftsmodellaspekte gegeben und ein Rahmenmodell für die weitere Analyse von Geschäftsvorhaben im mobilen Internet abgeleitet.

528 In Anlehnung an Bettis (1998) nennt Stähler (2001) als „übliche Verdächtige" der traditionellen Analyse von Geschäftsvorhaben die Geschäftseinheit, die Industrie und das Unternehmen. Vgl. Stähler (2001); S. $32 \mathrm{ff}$.

$529 \mathrm{Vgl}$. Bieger/Bickhoff/von Knyphausen-Aufseß (2002), S. 3.

530 Vgl. von Knyphausen-Aufseß/Meinhardt (2002), S. 64.

531 Eine ausführliche Übersicht über verschiedene Konzepte zum Thema Geschäftsmodelle finden sich bei Stähler (2001), Bieger et al. (2002) und Hass (2002). 
Trotz seiner engen Verbundenheit mit der Beschreibung kommerzieller Aktivitäten im Internet stammt der Begriff des Geschäftsmodells ursprünglich aus dem Bereich des Informationsmanagements, wo Geschäftsmodelle als Referenzmodelle für die Geschäftsprozesse, Funktions- oder Datenstrukturen von Unternehmen verstanden werden. ${ }^{532}$ Diese ursprünglich eng mit der Beschreibung der Architektur der Informationssysteme eines Unternehmens verknüpfte Begriffskonotation hat seit Ende der 1990er Jahre eine deutliche Erweiterung erfahren. Anstelle einer Beschreibung des Informationssystems des bestehenden Unternehmens werden Geschäftsmodelle vermehrt dafür verwendet, darzustellen, wie das Unternehmen zukünftig auszusehen hat, um gewisse Kundenbedürfnisse zu befriedigen. ${ }^{533}$ Abbildung 5-1 zeigt einige Beispiele von Geschäftsmodelldefinitionen neueren Datums in chronologischer Abfolge.

\begin{tabular}{|c|c|}
\hline Autor & Das Geschäftsmodell beschreibt ein Geschäftsvorhaben anhand... \\
\hline $\begin{array}{l}\text { Timmers (1998), S. 4; } \\
\text { Ähnlich: Selz (1999), S. } 106\end{array}$ & $\begin{array}{l}\text {... der Architektur für Produkt, Service und Informationsfluss, einschließlich der involvierten Akteure und } \\
\text { ihrer Rollen } \\
\ldots \text { seinem Nutzen für die verschiedenen involvierten Akteure } \\
\ldots \text { seinen Erlösquellen }\end{array}$ \\
\hline $\begin{array}{l}\text { Venkatraman/Henderson } \\
\text { (1998) }\end{array}$ & $\begin{array}{l}\text {... der erforderlichen Kundeninteraktion } \\
\ldots \text { der zugrunde liegenden Ressourcenkonfiguration } \\
\ldots \text { dem zu erzielenden Gewinn an Wissen }\end{array}$ \\
\hline $\begin{array}{l}\text { Tomczak/Schögel/Birkhofer } \\
\text { (1999) }\end{array}$ & $\begin{array}{l}\text {... der involvierten Akteure } \\
\ldots \text { der Konzeption der Mehrwertaktivitäten } \\
\ldots \text { der Verbindungen dieser Aktivitäten } \\
\ldots \text { der Koordination der Wertkette }\end{array}$ \\
\hline Amit/Zott (2000) & $\begin{array}{l}\text {... der Konfiguration einer Architektur von Transaktionskomponenten, wobei } \\
\ldots \text { als "Transaktionskomponenten" Informationen, Dienste oder Produkte zu verstehen sind, die an einer } \\
\text { Transaktion ebteiligt sind und } \\
\ldots \text { eine "Konfiguration der Architektur" die Verbindung zwischen Transaktionskomponenten und deren } \\
\text { Abfolge repräsentiert }\end{array}$ \\
\hline Hamel (2000), S. 65 f. & $\begin{array}{l}\text {... seiner Kernstrategie (d.h. die Geschäftsvision, die Produkt/Markt-Kombination und die } \\
\text { Differentierungsgrundlage) } \\
\ldots \text { kritischer strategischer Ressourcen (Kernkompetenzen und strategische Vermögenswerte) } \\
\ldots \text { der Kundenschnittstelle (Preisstruktur, Untersützungsleistungen, Erkenntnisse und Beziehungsdynamik) } \\
\ldots \text { des zugrunde liegenden Werschöpfungsnetzwerks (Zulieferer, Partner und Koalitionen) }\end{array}$ \\
\hline Mahedevan (2000) & $\begin{array}{l}\text {... des Prozesses der Wertgenerierung (value stream) für die Geschäftspartner und Kunden } \\
\ldots \text { des Prozesses der Umsatzgenerierung (revenue stream) } \\
\ldots \text { des zugrunde liegenden logistischen Prozesses (logistical stream) }\end{array}$ \\
\hline Rüegg-Stürm (2000) & $\begin{array}{l}\text {... der relativen Anspruchsgruppen } \\
\ldots \text { des Leistungsangebots } \\
\ldots \text { des dafür verantwortlichen Wertschöpfungsbereich } \\
\ldots \text { denkbarer Kooperationsfelder } \\
\ldots \text { dafür notwendiger Kernkompetenzen }\end{array}$ \\
\hline Stähler (2001), S. 41 & $\begin{array}{l}\text {... seiner Value Proposition } \\
\ldots \text { der Architektur seiner Wertschöpfung } \\
\ldots \text { seines Ertragsmodell }\end{array}$ \\
\hline Hass (2002), S. $90 \mathrm{f}$. & $\begin{array}{l}\text {... seiner Produktarchitektur } \\
\ldots \text { seiner Wertschöpfungsstruktur } \\
\ldots \text { seines Erlösmodells }\end{array}$ \\
\hline
\end{tabular}

Abbildung 5-1: Literaturbeispiele für Analysekriterien von Geschäftsmodellen

Einen ersten Schritt in Richtung der Erweiterung des Verständnisses gegenüber dem Konzept des Geschäftsmodells im Informationsmanagement unter-

532 Vgl. Stähler (2001), S 38; Penker/Eriksson (2000), S. 3.

533 Vgl. Stähler (2001), S. 39. 
nahm Timmers (1998) mit seinem Vorschlag, das Geschäftsvorhaben eines Unternehmen anhand von drei Kriterien zu beschreiben: der Architektur des erzeugten Produkts bzw. der zu erbringenden Dienstleistung; dem Nutzen, den die Vermarktung dieser Leistung verschiedenen involvierten Akteuren bietet; und den Erlösquellen, durch die im Zuge der Vermarktung der Leistung Einnahmen erzeugt werden. Timmers Vorschlag impliziert zum einen die Notwendigkeit, dass durch ein innovatives Geschäftsvorhaben ein Zusatznutzen oder Mehrwert geschaffen werden muss, der noch von keinem Konkurrenzangebot adressiert wird. Zum anderen zielt Timmers Konzept auf Geschäftsvorhaben $a b$, denen eine in besonderer Weise arbeitsteilige und vernetzte Wertschöpfung zugrunde liegt. Daher kommt einer Beschreibung der Architektur dieser Wertschöpfung eine besondere Bedeutung zu. Mehrwertgenerierung und vernetzte Wertschöpfung werden auch von anderen Autoren wie Selz (1999), Tomzcak/Schögel (1999) und Mahedevan (2000) als zentrale Wesensmerkmale von Geschäftsmodellen betrachtet und erfahren in deren Modellen explizite Berücksichtigung.

An den Geschäftsmodellkonzeptionen von Timmers und den ihm nachfolgenden Autoren ist jedoch zu kritisieren, dass diese zwar die Outputseite (bzw. die „Außenperspektive“) eines geplanten Geschäftsvorhabens darstellen, jedoch keine Aussagen über die dafür notwendige Inputseite (die „Binnenperspektive" des Vorhabens) erlauben. ${ }^{534}$ Insbesondere vor dem Hintergrund der großen Zahl von Misserfolgen der Geschäftsvorhaben in der Internetökonomie, die teilweise durch eine mangelnde Berücksichtigung der Inputseite (bspw. im Hinblick auf Geschäftsfinanzierung oder Umsatzgenerierung) bedingt waren, berücksichtigen neuere Ansätze zur Grundkonzeption von Geschäftsmodellen verstärkt inputbezogene Aspekte. Dabei nehmen bspw. Hamel (2000) und RüeggStürm (2000) einen ressourcenorientierten Betrachtungswinkel ein, während bspw. Venkatram/Henderson (1998) und Amit/Zott (2000) eine transaktionskostentheoretische Betrachtungsweise wählen.

In Anbetracht der Heterogenität dieser Vorschläge entwickeln Bieger/RüeggStürm/von Rohr (2002) ein zusammenfassendes Synthesemodell für die Beschreibung eines Geschäftsvorhabens, das insgesamt acht Konzeptbeschreibungen enthält: den Zweck der erzeugten Leistung (Leistungskonzept), das Konzept der Kommunikation dieser Leistung (Kommunikationskonzept), die erwarteten Erträge (Ertragskonzept) und deren geplantes Wachstum (Wachstumskonzept), die Konfiguration der im Rahmen des Vorhabens benötigten Ressourcen (Kompetenzkonfiguration) und deren Organisation (Organisationsform), das Konzept notwendiger Kooperation (Kooperationskonzept) sowie das Konzept für deren Koordination (Koordinationskonzept). In Abgrenzung zu dieser vergleichsweise detaillierten Synthese entwickeln Stähler (2001) und Hass (2002) jeweils ein stärker aggregiertes Konzept des Geschäftsmodells mit lediglich drei Partialmodellen: dem Produkt- oder Nutzenmodell (1), dem Wert-

534 Vgl. Anding (2004), S. 155 f. und Böning-Spohr/Hess (2000), S. 4: Anding unterscheidet als Betrachtungsdimensionen eines Geschäfts dessen Outputseite und Inputseite. Eine ähnliche Unterscheidung, allerdings mit anderen Begriffen, findet sich bei Böning-Spohr/Hess mit einer Differenzierung zwischen Außensicht und Binnensicht. 
diglich drei Partialmodellen: dem Produkt- oder Nutzenmodell (1), dem Wertschöpfungsmodell (2) und dem Ertragsmodell (3):

(1) Das Produkt- und Nutzenmodell (bzw. die sog. „Value Proposition“) beschreibt die im Rahmen des Geschäftsvorhabens erzeugte Leistung anhand von Produkt-/Marktkombinationen. Das Partialmodell legt dar, welchen Zweck die Leistung für welchen Adressatenkreis erfüllen soll und inwiefern durch dieses Leistungsversprechen ein Mehrwert adressiert werden kann. Das Produktoder Nutzenmodell deckt damit im Syntheseansatz nach Bieger et al. (2002) sowohl das Leistungs- als auch das Kommunikationskonzept ab.

(2) Das Wertschöpfungsmodell beschreibt die Organisation der Leistungserstellung. ${ }^{535}$ Es wird zum einen die unternehmensinterne Wertkette des jeweils betrachteten Unternehmens als auch zum anderen die Einbettung dieser Wertkette in die unternehmensübergreifende Wertschöpfungskette der Branche berücksichtigt. Damit deckt das Wertschöpfungsmodell im Syntheseansatz die Aspekte der Kompetenzenkonfiguration, der Organisationsform sowie des Kooperations- und Koordinationskonzepts ab.

(3) Das Erlösmodell beschreibt die Zahlungsströme im Zuge der Vermarktung der Leistung. Dabei enthält das Erlösmodell sowohl die geplanten Erlösformen und -quellen als auch die Mechanik der Erlösverteilung zwischen den im Rahmen des Geschäftsvorhabens involvierten Akteuren. Damit deckt das Erlösmodell das Ertrags- und das Wachstumskonzept im Syntheseansatz ab.

Vor dem Hintergrund der hier angestrebten allgemeinen Charakterisierung der Handlungsmöglichkeiten von Inhalteanbietern im mobilen Internet wird aufgrund des insgesamt höheren Abstraktionsniveaus der Unterscheidung von lediglich drei Partialmodellen eine Abgrenzung der Geschäftsmodelloptionen herangezogen, die sich an der Geschäftsmodellkonzeption nach Hass und Stähler anlehnt. ${ }^{536}$

\subsubsection{Rahmenbedingungen für Geschäftsmodelle inhalteorien- tierter Angebote im mobilen Internet}

Im folgenden Abschnitt werden Rahmenbedingungen einer konkreten Ausgestaltung mobiler Dienstangebote aus Sicht der Medienbranche beschrieben. Dazu werden jeweils anhand spezifischer Aspekte des Produkt- und Nutzenmodells, des Wertschöpfungsmodells und des Erlösmodells potenzielle Erfolgsfaktoren der Inhalteverwertung im mobilen Internet abgeleitet. In Abschnitt 5.2.2.1 werden im Rahmen des Produktmodells Bedingungen der Schaffung eines Kundenmehrwerts durch mobile Dienste erläutert. Im Zusammenhang mit dem Wertschöpfungsmodell werden in Abschnitt 5.2.2.2 die Besonderheiten des Angebots inhalteorientierter Onlineangebote im Wertschöpfungssystem des mobilen Internet beschrieben. In Abschnitt 5.2.2.3 werden für das Erlösmodell Bedingungen des Preismodells mobiler Dienste gegenüber Endkunden un-

$535 \mathrm{Vgl}$. Hass (2004), S. 39

536 Vgl. Stähler (2002), S. 41 f.; Hass (2002), S. 80; Hass (2004), S. 37. 
tersucht. Die Rahmenbedingungen von Geschäftsmodellen für inhalteorientierte mobile Dienste werden in Abschnitt 5.2.2.4 abschließend zusammengefasst.

\subsubsection{Rahmenbedingungen des Produkt- und Nutzenmodells inhalte- orientierter mobiler Dienste}

In Kapitel 2.2 wurde eine Klassifikation mobiler Dienste vorgenommen, die zwischen informationsorientierten, kommunikationsorientierten, transaktionsorientierten, und anwendungsorientierten Diensten unterscheidet. Eine Verwertung von Inhalteangeboten im mobilen Internet, wie die in erster Linie aktuell informierenden Erzeugnisse der hier im Mittelpunkt stehenden Verlage oder Rundfunkanstalten, gehören naturgemäß in erster Linie zur Klasse informationsorientierter Dienste. Inhalte oder Inhaltebündel, die in einem verwendungsneutralen Format (bspw. einer Datenbank oder einer XML-Syntax) gespeichert sind, können bspw. einem Distributionsformat zugewiesen werden, das durch Mobilfunk übertragen und von mobilen Endgeräten dargestellt werden kann (bspw. einem WML-Template). Neben den Möglichkeiten des Abrufs von Inhalten in WAP-kompatiblen Formaten können Inhalte jedoch des Weiteren entweder an kommunikationsorientierte Dienstformen wie bspw. SMS oder MMS geknüpft werden oder als downloadfähige Datei- oder Programmformate wie Audio-, Video oder Spieledateien bereitgestellt werden. Insofern können inhalteorientierte Angebote im mobilen Internet sowohl kommunikationsorientierte als auch applikationsorientierte Dienstmerkmale besitzen.

In Kapitel 3 wurde erläutert, dass Medienunternehmen und insbesondere Verlage den stagnierenden Einnahmen und Absatzzahlen u.a. dadurch begegnen können, dass sie ihre Inhalte in Formaten anbieten, die ihren Kunden gegenüber den traditionellen gedruckten Vertriebsformaten einen Mehrwert bieten. Inhalteorientierte Onlineangebote im mobilen Internet müssen daher gegenüber alternativen Inhaltevertriebsformen einen Mehrwert besitzen, um Konsumenten einen Anreiz für ihre Nachfrage zu geben. Nachfolgend wird aufgezeigt, dass mobile Dienstformate einen solchen Mehrwert in dreifacher Hinsicht adressieren können: durch die Ortsunabhängigkeit ihrer Nachfrage (1); durch die Ortsspezifität inrer Bereitstellung (2) und auf der Grundlage von Netzeffekten im Zuge ihrer Distribution (3).

(1) Mobile Distributionsformate zeichnen sich dadurch aus, dass Inhalte im Empfangsbereich eines Mobilfunknetzes über mobile Endgeräte und dadurch ohne räumliche Beschränkung abzurufen sind. ${ }^{537}$ Die Ortsunabhängigkeit mobiler Dienste besitzt gegenüber der Inhaltedistribution im leitungsgebundenen Internet dadurch Vorteile, dass mobile Dienste an jedem Ort des Sendeempfangsbereichs des Netzes verfügbar sind, und ihre Nutzung nicht auf diskrete Netzzugangspunkte beschränkt ist. Es ist allerdings kritisch zu prüfen, ob der Vorteil an Bewegungsfreiheit bereits zwangsläufig eine höhere Zahlungsbereitschaft bei Rezipienten erzeugt, da traditionelle Medienformate wie bspw. Zeitungen oder Zeitschriften ebenfalls transportierfähig und somit in gewisser Hin-

$537 \mathrm{Vgl.} \mathrm{Abschnitt} \mathrm{2.3.}$ 
sicht ortsunabhängig sind. Der Ortsunabhängigkeit mobiler Dienste wird daher von einigen Autoren lediglich die Eigenschaft eines "Hygienefaktors" beschieden, der zwar zum Zeitpunkt der Dienstnutzung erfüllt sein muss, jedoch keine direkten Auswirkungen auf die Zahlungsbereitschaft des Nutzers hat. ${ }^{538}$

(2) Eine Begleiterscheinung der Ortsunabhängigkeit besteht in der sog. "Ortsspezifität“" mobiler Dienste. Der Begriff der Ortsspezifität kann sowohl aus einem technischen als auch aus einem soziologischen Blickwinkel interpretiert werden. Aus technischer Sicht bedeutet Ortsspezifität, dass ein Mobilfunknutzer bzw. ein mobiles Endgerät immer anhand bestimmter Merkmale des Aufenthaltsorts, an dem ein Dienst abgerufen wird, identifiziert (bzw. lokalisiert) werden muss. ${ }^{539}$ Bspw. muss ein Mobilfunknetzbetreiber jederzeit die Mobilfunkzelle kennen, in der sich der Dienstnutzer befindet, um einen Kommunikationsvorgang mit dem Dienstanbieter aufbauen zu können. ${ }^{540}$ Abbildung 5-2 stellt den Vorgang der Teilnehmeridentifikation und Lokalisierung schematisch am Beispiel eines Anrufs vom Festnetz in ein GSM-Netzes dar: ${ }^{541}$

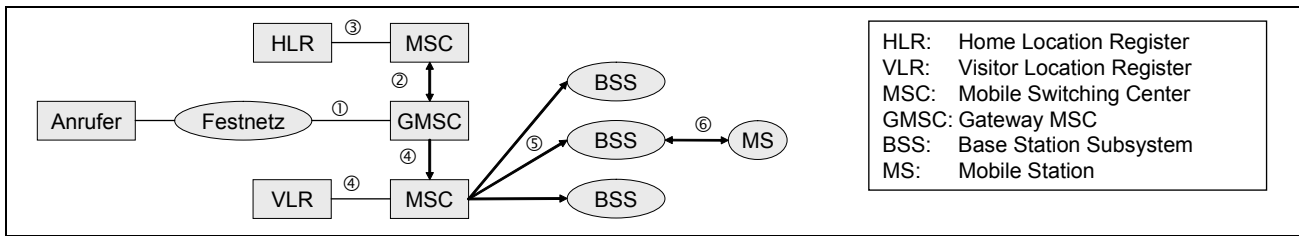

Abbildung 5-2: Teilnehmerlokalisierung im GSM-Netz

Anhand der Netzvorwahl wird im Festnetz erkannt, dass das Signal an ein Gateway Mobile Switching Center (GMSC) im Mobilfunknetz geleitet werden muss (1). Durch die Rufnummernkennzahl wird das Teilnehmerspezifische Home Location Register (HLR; (2/3) identifiziert und von diesem das aktuelle Visitor Location Register (VLR; (4) ausgelesen. Durch so genanntes „Paging“, einem Suchsignal, das die Basisstation (BSS) im Zuständigkeitsbereich des VLR zur Lokalisierung des Teilnehmers aussendet (5), wird die aktuell zuständige Basistransferstation (BTS) erkannt. Zu dieser wird vom Mobile Switching Center (MSC) das Signal vermittelt und mit der Mobile Station, dem Endgerät des Empfängers, verbunden (๑)).

538 Vgl. Röttger-Gerigk (2002), S. 22; Zobel (2001), S. 117: Als weitere Hygienefaktoren mobiler Dienste nennt Röttger-Gerigk die Bequemlichkeit, die Verfügbarkeit, die Kostengünstigkeit und die Sicherheit der Angebotsnutzung. Eine ähnliche Argumentation findet sich bei Zobel, der statt Hygienefaktoren den Begriff Muss-Faktoren einführt.

539 Der Begriff der Ortsspezifität steht in engem Zusammenhang mit dem bereits in Kapitel 2. diskutierten Besonderheiten mobiler Dienste in Bezug auf ihre systemtechnische Verteilungstransparenz. Vgl. auch Coulouis/Dollimore/Kindberg (2002), S. 42.

540 Das Verfahren der zellbasierten Ortung wird auch als "Cell of Origin" (COO)-Verfahren bezeichnet. Vgl. auch Abschnitt 5.4.1.1.

541 Vgl. Schiller (2002), S. 380. 
Orte sind dagegen in einem soziologischen Verständnis nicht nur geografisch, sondern auch sozial definiert. ${ }^{542}$ Mit Orten verbinden sich unterschiedliche soziale Kontexte, die einen dort Anwesenden in spezifische Rollenerwartungen, soziale Regeln und Strukturen einbinden. ${ }^{543}$ Ein Ort, bzw. die Bewegung zu diesem Ort hin oder von diesem Ort weg, bindet daher entweder partiell die Aufmerksamkeit eines Nutzers bzw. determiniert bestimmte (bspw. rollenbezogene) Bedürfnisse. Ortsspezifität bedeutet daher in einem soziologischen Begriffsverständnis die zumindest teilweise räumliche und soziale Determinierung von Informations- und Dienstleistungsbedürfnissen. Abbildung 5-3 zeigt beispielhaft Ausprägungen solcher räumlich bedingten Bedürfnisse im Zuge der Anreise zu einer Verabredung für ein Geschäftsessen.

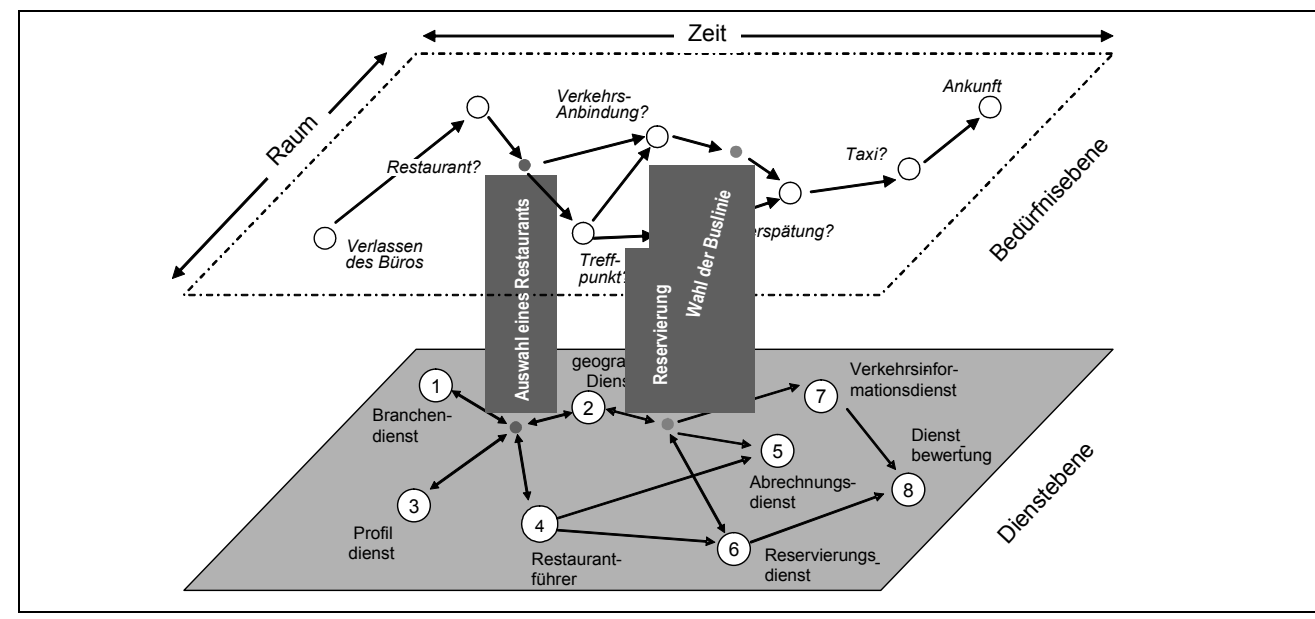

Abbildung 5-3: Orts- und Zeitspezifität von Bedürnissen

Vor dem Hintergrund ihrer Ortsspezifität können mobile Dienste einem Konsumenten immer dann einen Mehrwert bieten, wenn die transportierten Inhalte oder Dienstleistungen so bereitgestellt werden, dass sie den Bedürfnissen des Konsumenten im Zusammenhang mit seinem jeweiligen Aufenthaltsort bzw. seiner Mobilität zwischen zwei Orten gerecht werden. Um Hinweise auf mögliche mobilitätsbezogene Informationsbedürfnisse zu erhalten, ist aus soziologischer Sicht in Verbindung mit einem physischen Ortswechsel zwischen verschiedenen Formen der Mobilität zu differenzieren: ${ }^{.44}$ Zum einen kann anhand der Wiederholungen derselben Wegstrecke zwischen der sog. "gerichteten"

542 Vgl. Franz (1984); Giddens (1992), S. 185 f.

$543 \mathrm{Vgl}$. Hess et al. (2005).

544 Hiermit wird bereits eine Einengung der in der Soziologie vertretenen Begriffsauffassung zum Thema Mobilität vorgenommen. Neben physischer oder geografischer Personenmobilität werden in der Soziologie des Weiteren unterschieden: die physische Mobilität von Sachen, die soziale Mobilität von Individuen in der Gesellschaft sowohl in vertikaler als auch horizontaler Hinsicht sowie die Mobilität von Informationen entweder durch individuelle geistige Entwicklung oder durch mediengebundene zwischenmenschliche Kommunikation. Einen Überblick über die Dimensionen des soziologischen Begriffsverständnisses geben u.a. Zängler (2000), Eckhoff (2001) und Hess et al. (2005). 
und der „zirkulären Mobilität unterschieden werden. ${ }^{545}$ Gerichtete Mobilität hat die einmalige Bewältigung eines speziellen Ortswechsels wie bspw. die Anreise zum Urlaubsort oder die Anfahrt zu einem Konzertbesuch zum Ziel. Dagegen ist die zirkuläre Mobilität wiederkehrender Natur und dient zur Bewältigung des Alltags, bspw. in Form des Pendelverkehrs zum Arbeitsplatz, den Fahrten zum Einkaufen oder zur Freizeitgestaltung. Zum anderen kann anhand des im Vordergrund stehenden Bedürfnisses zwischen originären und sekundären Mobilitätsformen unterschieden werden. ${ }^{546}$ Bei originärer Mobilität findet die Bewegung in erster Linie um inrer selbst willen statt, bspw. bei einer Spazierfahrt mit dem Auto oder einem Spaziergang. Bei der sekundären Mobilität ist die Bewegung eines Individuums dagegen die Folge der Befriedigung anderer Bedürfnisse.

Untersuchungen des Mobilitätsverhaltens deutscher Bürger zeigen, ${ }^{547}$ dass im Alltag Formen der zirkulären, sekundären Mobilität überwiegen mit kurzen bis mittleren Distanzen überwiegen. Es ist daher zu schlussfolgern, dass sich Informationsbedürfnisse im Zuge einer solchen Mobilität seltener aus dem Anlass der Mobilität bzw. des Aufenthaltsorts selbst herleiten, sondern vielmehr lediglich unter der einschränkenden Rezeptionsbedingung der Mobilität, bspw. zur Überbrückung der Reisezeit, geschehen. Beispiele solcher einschränkender Bedingungen bilden etwa kleine Anzeigebildschirme und eingeschränkte Eingabemöglichkeiten mobiler Endgeräte sowie ggf. die angesprochene Beeinträchtigung der Aufmerksamkeit des Rezipienten. ${ }^{548}$ Ausnahmen, in denen sich ein Informationsbedürfnis unmittelbar aus dem Anlass der Mobilität herleitet, bilden dagegen bspw. die Mediennutzung zur Orientierung im Raum und zur Unterstützung bei den damit verbundenen Tätigkeiten. ${ }^{549}$ Dienste die zur Befriedigung derartiger Mobilitätsbedürfnisse dienen, werden auch als „Mobilitätsdienste" bezeichnet. ${ }^{550}$ Beispiele für Mobilitätsdienste bilden Parksysteme, Taxi-, Chauffeur- oder Reiseplaninformationsdienste sowie Dienste für PKWMehrfachnutzungssysteme.

(3) Multilaterale kommunikationsorientierte Dienstleistungen wie Sprachtelefonie oder E-Mail, bzw. speziell im Mobilfunk Dienstformen wie SMS und MMS, unterliegen besonderen interaktions- und verbreitungsabhängigen Effekten der Nutzengenerierung. Diese Effekte beruhen, wie bereits angesprochen wurde, darauf, dass der Nutzen eines Netzwerks bzw. darüber erbrachter Dienstleistungen oder Produkte mit der Anzahl der Netzteilnehmer ansteigt. ${ }^{551}$ Netzeffek-

545 Vgl. Forcher (1996), S. 15; Eckhoff (2001), S. 23.

546 Vgl. Zängler (2000), S. $36 \mathrm{f}$.

547 Vgl. u.a. Hradil (2002) und Zängler (2000). Bspw. stellte Zängler (2000) bei einer Untersuchung des Mobilitätsverhaltens von insgesamt 986 Haushalten fest, dass die durchschnittliche Distanz der von den Haushaltsmitgliedern zurückgelegten Wegstrecken 10,8km betrug, was einer durchschnittlichen Reisezeit von 21 Minuten entspricht. Dabei sind 40\% der Wege dem Freizeitbereich, 30\% der Warenbeschaffung und 30\% der Beschättigung zuzurechnen.

${ }^{548} \mathrm{Vgl}$. Billsus et al. (2000); Smyth/Cotter (2003).

$549 \mathrm{Vgl}$. Hess et al. (2005).

$550 \mathrm{Vgl}$. Eckhoff (2001), S. $25 \mathrm{ff}$.

551 Vgl. Abschnitt 2.3.2.2. 
te dieser Art sind für das Angebot von inhalteorientierten Diensten im mobilen Internet in zweifacher Hinsicht von Bedeutung:

- Inhalteanbieter können zum einen Netzeffekte im Rahmen des Inhaltekonsums erzeugen, indem sie Inhalte in solchen Formaten bereitstellen, die Konsumenten gegenseitig austauschen können. Beispiele solcher Angebotsformen bilden downloadfähige Audio-, Video- und Textformate oder Inhalte, die über Dienstformen wie SMS oder MMS bereitgestellt werden. Durch die Wiederverwendbarkeit solcher Vertriebsformate muss ein Dienstanbieter allerdings in Kauf nehmen, dass inm unter Umständen ein Teil seiner Einnahmen bei solchen Konsumenten entgeht, die Inhalte nicht vom Anbieter sondern von anderen Konsumenten erhalten.

- Zum anderen ist jedoch die Adoption von Gütern mit Netzeffekten grundsätzlich durch ein verstärktes Startproblem gekennzeichnet. Als Adoption wird dabei im Allgemeinen der Prozess einer intraindividuellen Annahme einer Innovation verstanden. ${ }^{552}$ Das Startproblem der Güteradoption eines Netzeffektguts beruht darauf, dass Konsumenten in der frühen Phase der Güteradoption grundsätzlich ein geringerer Nutzen gestiftet wird als allen späteren Konsumenten. Als eine Lösung dieses Startproblems wird in der ökonomischen Adoptionstheorie die Möglichkeit hervorgehoben, innovative Güter im Rahmen ihrer Einführung stärker an die Bedürfnisse solcher Nutzergruppen anzupassen, die einerseits (bspw. aufgrund rollenbezogener oder demografischer Merkmale) eine besonders hohe Kaufwahrscheinlichkeit und die andererseits in ihrem sozialen Umfeld eine Schlüsselfunktion in Bezug auf die Meinungsbildung für die dem Umfeld angehörenden Individuen besitzen. ${ }^{553}$ Indem diese Schlüsselpersonen durch ein bedürfnisgerechtes Angebot zum Konsum motiviert werden, können sich durch ihre Rolle als Meinungsführer zukünftig Multiplikationseffekte im Rahmen der Gütereinführung ergeben. Im Zusammenhang mit inhalteorientierten mobilen Diensten wird dabei insbesondere der Gruppe männlicher, technikaffiner und tendenziell gebildeter Konsumenten mit höherem Einkommen eine Vorreiterrolle bei der Akzeptanz neuer mobiler Dienste zugesprochen.

\subsubsection{Rahmenbedingungen des Wertschöpfungsmodells mobiler Dienste}

Als Wertschöpfung wird der um Vorleistungen verminderte Gesamtwert bezeichnet, den ein Unternehmen für seine Abnehmer schafft. ${ }^{554}$ Die Höhe der Wertschöpfung wird bestimmt durch die Gewinnspanne des Unternehmens und den Wert der sog. Wertschöpfungsaktivitäten des Unternehmens. Wertschöp-

\footnotetext{
552 Vgl. Gierl (1987), S. 29; Weiber (1992), S. 4.

553 Personen, die einen hohen Einfluss auf die Meinungsbildung ihres Umfelds besitzen, werden auch als

Meinungsführer oder Promotoren bezeichnet. Vgl. u.a. Nieschlag et al. (1997), S. 571 ff.; Trommsdorf (1993), S. 215; Meffert (1998), S. 124.
}

554 Vgl. Heinen (1991), S. 49. 
fungsaktivitäten können dabei in primäre Aktivitäten, wie Beschaffung, Produktion und Vertrieb, und in sekundäre Aktivitäten wie Organisation, Personal- oder Kapitalwirtschaft, differenziert werden. Die Wertschöpfungsaktivitäten eines Unternehmens bilden ein System, das als „Wertkette“ oder "Wertschöpfungskette“ bezeichnet wird. Die Wertkette ist ein ursprünglich auf Michael Porter zurückgehendes Analyseinstrument, um einzelne Merkmale der Wertschöpfungsaktivitäten eines Unternehmens bewerten und mit dem Hauptkonkurrenten vergleichen zu können. ${ }^{555} \mathrm{Im}$ Falle kooperativ vernetzter Wertschöpfungsaktivitäten kann die Wertschöpfungskette auch zur Beschreibung der Abfolge unternehmensübergreifender Wertschöpfungsaktivitäten herangezogen werden. Die Verknüpfung der Wertketten verschiedener Unternehmen einer Branche wird dabei auch als Wertschöpfungssystem bezeichnet. ${ }^{556}$

Damit ein Unternehmen in seinem Wettbewerbsumfeld Vorteile erringen kann, ist es notwendig, dass seine Wertkette effizient in das Wertschöpfungssystem seiner Branche eingebunden ist. Um mögliche Einbindungsformen der Wertkette von Inhalteanbietern im Wertschöpfungssystem des mobilen Internet zu bestimmen, ist es erforderlich, die Struktur dieses Wertschöpfungssystems zu identifizieren. In der betriebswirtschaftlich geprägten Literatur zum mobilen Internet existiert dabei derzeit eine Kontroverse in Bezug auf die zukünftige Struktur der Wertschöpfung im Geschäft mit mobilen Diensten. Diese Kontroverse entspringt in ihrem Kern der Fragestellung, ob die Anwendungsfelder des mobilen Internet lediglich als eine Erweiterung der traditionellen Wertschöpfung der Telekommunikationsindustrie anzusehen sind, oder ob mit dem mobilen Internet eine Verschmelzung von Wertschöpfungsbereichen aus Telekommunikations-, Medien und IT-Branche erfolgt und somit ein vollkommen neues Wertschöpfungssystem entsteht. ${ }^{557}$ Anhaltspunkte zur Beantwortung dieser Fragestellung können, wie nachstehend gezeigt wird, aus drei verschiedenen Blickwinkeln entwickelt werden: aus dem Blickwinkel der Wertschöpfung der Telekommunikationsbranche (1), aus dem Blickwinkel der bereits angesprochenen Konvergenztheorie ${ }^{558}$ (2) sowie aus einem nachfrageseitigen Blickwinkel im Hinblick auf die Spezifika der Geschäftsabwicklung mobiler Dienste (3).

(1) Die traditionelle Wertschöpfungskette der Telekommunikationsbranche umfasst vier Stufen: ${ }^{559}$ Die Bereitstellung und der Betrieb einer Netzinfrastruktur, die Bereitstellung von Basisdiensten zur Sprachkommunikation, die Bündelung und Vermarktung von Produktpaketen und -tarifen sowie die Abrechnung geleisteter Dienste gegenüber dem Kunden. Durch die Möglichkeiten der Datenkommunikation sind im Rahmen der traditionellen Wertschöpfung der Telekommunikationsbranche insbesondere durch das Angebot von Basisdiensten für Datenübertragung sowie durch Leistungsbündelung, bspw. in Form von da-

\footnotetext{
555 Vgl. Porter (2000), S. 64 f.

556 Vgl. Heinen (1991), S. 52.

557 Vgl. Zobel (2001), S. 32.

$558 \mathrm{Vgl}$. Kapitel 3.1.

559 Vgl. Dengler (2000), S. 92 ff.
} 
tenvolumenabhängigen Tarifen, Veränderungen aufgetreten. Darüber hinaus ermöglichen die Verknüpfung von Daten- und Sprachtechnologien und die $\mathrm{He}-$ terogenität von Datennetzstandardisierungen neue zusätzliche Wertschöpfungsaktivitäten in Form der Systemintegration von einerseits Sprach- und Datendiensttechniken als auch zwischen unterschiedlichen Datennetztechniken (vgl. Abbildung 5-4).

Durch die Liberalisierung des deutschen Telekommunikationssektors ist im Bereich der Mobilkommunikation ein enges Oligopol aus vier vergleichsweise hochintegrierten Mobilfunkbetreibern entstanden. ${ }^{560}$ Der Wettbewerb im Mobilfunkmarkt wird dabei durch zwei Faktoren beeinflusst: Die Wettbewerbsintensivierung nach dem Abbau nationalstaatlicher Wettbewerbsschranken und hohe Lizenzkosten bspw. durch die Ersteigerung der UMTS Frequenzen ${ }^{561}$ haben zu einer Überschuldung der deutschen Telekommunikationsunternehmen geführt. ${ }^{562}$ Durch den kostenintensiven Ausbau der sowohl leitungsgebundenen als auch funkgestützten Übertragungswege sind außerdem im Bereich der Datenübertragung Überkapazitäten entstanden. Um diese Überkapazitäten durch zusätzlichen Datenverkehr abzubauen und mit den dadurch geschaffenen Einnahmen die Schuldenlast zu mindern, entsteht auf Seiten von Telekommunikationsunternehmen die Notwendigkeit, neue Dienste im Bereich des Datenverkehrs anzubieten. ${ }^{563}$ Diese Wettbewerbsveränderungen haben zwei Auswirkungen auf das Wertschöpfungssystem der Telekommunikationsbranche: Erstens konnten sich neben den etablierten Oligopolisten zusätzliche Anbieter auf dem Markt etablieren, die von Netzbetreibern Übertragungskontingente einkaufen und diese in eigener Rechnung vermarkten. Derartige Anbieter, die über keine eigenen Netzinfrastrukturen verfügen, werden auch als "Virtuelle Mobilfunkbetreiber" bezeichnet. ${ }^{564}$ Zweitens bieten alle derzeit am Markt befindlichen Mobilfunkbetreiber Plattformen für den Zugang zu mobilen

560 Die vier deutschen Mobilfunkbetreiber sind die Firmen T-Mobile, Vodafone, E-Plus und $\mathrm{O} 2$ bedienten 2004 insgesamt 71,32 Mio. Kunden. Auf T-Mobile entfiel ein Marktanteil von 38,5\%, auf Vodafone $37,8 \%$, auf E-Plus 13,3\% und auf O2 10,4\%. Vgl. Sydow/Beckmann (2004).

561 Im August 2000 wurden insgesamt 6 UMTS-Lizenzen zu einem Preis von durchschnittlich ca. 8 Mrd. EUR pro Lizenz versteigert. Zusätzlich zu den Aufwendungen der Lizenzgebühren fallen darüber hinaus für jeden Lizenzerwerber durch den Netzaufbau Kosten in Höhe von ca. 4-5 Mrd. EUR an. Vgl. Ziegler/Adam (2001), S. $44 \mathrm{f}$.

562 Vgl. bspw. Neudorfer/Simonitsch (2003), S. 64; Müller/Preissner/Schwarzer (2002), S. 170 f.

$563 \mathrm{Vgl}$. Feldmann (2002), S. 353. In einer beispielhaften Investitionskalkulation für das Engagement eines Mobilfunkbetreibers im UMTS-Geschäft, unter der (aus heutiger Sicht sicherlich sehr optimistischen) Annahme von ersten betriebsbedingten Rückflüssen bereits im Jahr 2003, errechneten Ziegerl/Adam (2001), S. 11 f., dass Mobilfunkbetreiber bereits ab dem 3. Betriebsjahr des UMTS-Netzes eine Steigerung des Jahresumsatzes pro Kunde auf 235\% gegenüber dem Referenzjahr 2001 erreichen müssen, um ein Amortisationsziel der Investitionsausgaben bis zum Jahr 2013 zu erreichen. Aufgrund der bereits annähernd erreichten Marktsättigung im Bereich der Sprachkommunikation kann eine solche Steigerung nur durch einen deutlichen Anstieg im Bereich der Datenkommunikation auf einen Umsatzanteil von über $50 \%$ erreicht werden.

564 Vgl. Pescatore (2005). Der deutsche Markt für den virtuellen Mobilfunkbetrieb umfasst derzeit $20 \mathrm{An}$ bieter. Eine permanent aktualisierte Liste ist einzusehen unter Takashi Mobile (2005). 
Mehrwertdiensten an. Beispiele solcher Dienstplattformen bilden das Dienstportal "T-Zones" des Mobilfunkbetreibers T-Mobile oder das Portal Vodafone Life. Abbildung 5-4 fasst die Entwicklung der Wertschöpfungskette der Telekommunikationsbranche am Beispiel des Übergangs von der ersten (bspw. C-Netz) zur zweiten Mobilfunkgeneration (GSM, GPRS) zusammen.

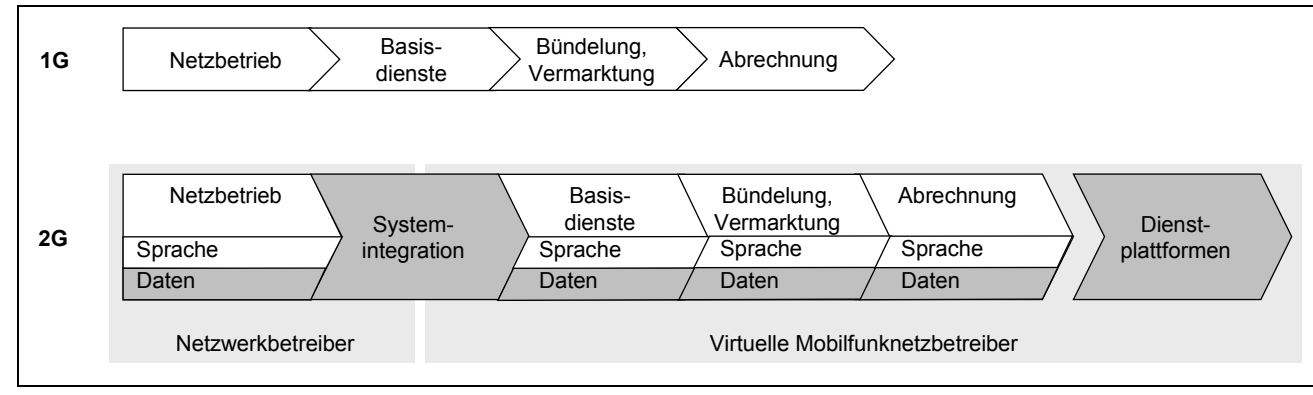

Abbildung 5-4: Veränderungen der Wertschöpfungskette von Mobilfunkbetreibern

(2) Die Mehrzahl der Beiträge zur Diskussion der zukünftigen Struktur des Wertschöpfungssystems für das mobile Internet untersucht die Möglichkeiten neuer Wertschöpfungsaktivitäten vor dem Hintergrund der bereits angesprochenen Konvergenz der Branchen Telekommunikation, Informationstechnologie und Medien. Wie bereits in Kapitel 3.1 erläutert wurde, ist unter Konvergenz die Entstehung neuer Zulieferer-/Abnehmer-Beziehungen zwischen den drei genannten Branchen zu verstehen. Dadurch entsteht im Zusammenhang mit der Bereitstellung inhalteorientierter mobiler Dienste ein neues Wertschöpfungssystem. Dieses Wertschöpfungssystem enthält in seiner Grundform drei Systemelemente: Die Bereitstellung von Endgeräten und Komponenten einer Mobilfunkinfrastruktur (IT), den Betrieb und die Abwicklung von Dienstleistungen eines Mobilfunknetzes und das mobilitätsgerechte Angebot von Inhalten und nicht-sprachorientierten Mehrwertdiensten.

Neben den bereits genannten allgemeinen Auslösern der Konvergenz wie bspw. die Miniaturisierung der Informationsverarbeitungstechnik und die digitale Gerätevernetzung wird die Entwicklung eines Wertschöpfungssystems im Bereich des mobilen Internet aus Sicht von Inhalteanbietern durch einige spezielle Aspekte beeinflusst: Wie bereits in Kapitel 2 gezeigt wurde, repräsentieren inhalteorientierte mobile Dienstformate wie bspw. WAP-kompatible Inhalteformate oder an einen SMS- oder MMS-Dienst geknüpfte Angebotsformen in erster Linie Telekommunikationsdienste. Ein mobilitätsgerechtes Inhalteangebot erfordert, stärker als bspw. Inhalteangebote im leitungsgebundenen Internet, spezielle Kenntnisse im Bereich der Formatkodierung und der nachrichtentechnischen Besonderheiten der mobilen Distribution von Inhalten. Um eine solche mobilitätsgerechte Inhalteformatierung gewährleisten zu können, müssen sowohl Inhalteanbieter als auch Telekommunikationsunternehmen zusätzliche Wertschöpfungsaktivitäten wahrnehmen, die nicht Bestandteil der traditionellen Wertkette dieser Branchen sind. Die Wahrnehmung solcher Aktivitäten ist daher sowohl für Medien- als auch Telekommunikationsunternehmen mit zusätzlichen Investitionen verbunden. Die durch geringe Einnahmen geprägten Erfah- 
rungen im Bereich des Internetgeschäfts und die zögerliche kommerzielle Entwicklung einer Nachfrage im mobilen Internet haben jedoch sowohl in Telekommunikations- als auch in Medienbranche zu einer allgemeinen Risikoaversion im Hinblick auf zusätzliche Investitionsvorhaben geführt. ${ }^{565}$ Diese Risikoaversion schlägt sich nieder in einer derzeit zu beobachtenden Intermediationstendenz durch Unternehmensausgründungen und Kooperationen in solchen Geschäftsbereichen, die, wie die Entwicklung mobilitätsgerechter Inhalte- und Dienstformate, nicht zum traditionellen Kerngeschäft gehören. Einige Autoren fassen dabei alle Wertschöpfungsaktivitäten, die zu einer mobilitätsgerechten Inhalte- und Diensteformatierung beitragen, unter der Bezeichnung des sog. „Wireless Application Service Providing“ (WASP) zu einem gemeinsamen Systemelement des Wertschöpfungssystems im mobilen Internet zusammen. Hinter dieser gemeinsamen Klassifikation verbergen sich jedoch z.T. stark divergierende Geschäftsmodelle, darunter einerseits Unternehmen wie die Dortmunder Materna $\mathrm{GmbH}$, deren Geschäft in erster Linie der technische Betrieb und die Abwicklung von SMS- und MMS-Diensten darstellt, und andererseits Unternehmen wie die Hamburger Arvato Mobile $\mathrm{GmbH}$, einem Tochterunternehmen des Bertelsmann Konzerns, deren Kerngeschäft die redaktionelle Selektion und Bündelung mobilitätsgerechter Inhalte ist. ${ }^{566}$

Trotz einiger Gemeinsamkeiten in Bezug auf die Einschätzung der Rolle von Telekommunikations-, IT- und Medienunternehmen im Wertschöpfungssystem des mobilen Internet existiert zwischen den bestehenden konvergenzgeprägten Diskussionsbeiträgen zur Wertschöpfung im mobilen Internet eine gewisse Heterogenität in Bezug auf die Klassifikation der Wertschöpfungsaktivitäten neuer Intermediäre. Abbildung 5-5 gibt einen Überblick über drei Beispiele, deren Modelle des Wertschöpfungssystems eine Spanne von vier bis maximal acht Wertschöpfungsstufen umfassen.

565 Vgl. Feldmann (2002), S. 356.

566 Einen Überblick über verschiedene Ausprägungsformen von Geschäftsmodellen für Wireless Application Service Provider bietet Simonitsch (2003), S. 53. 


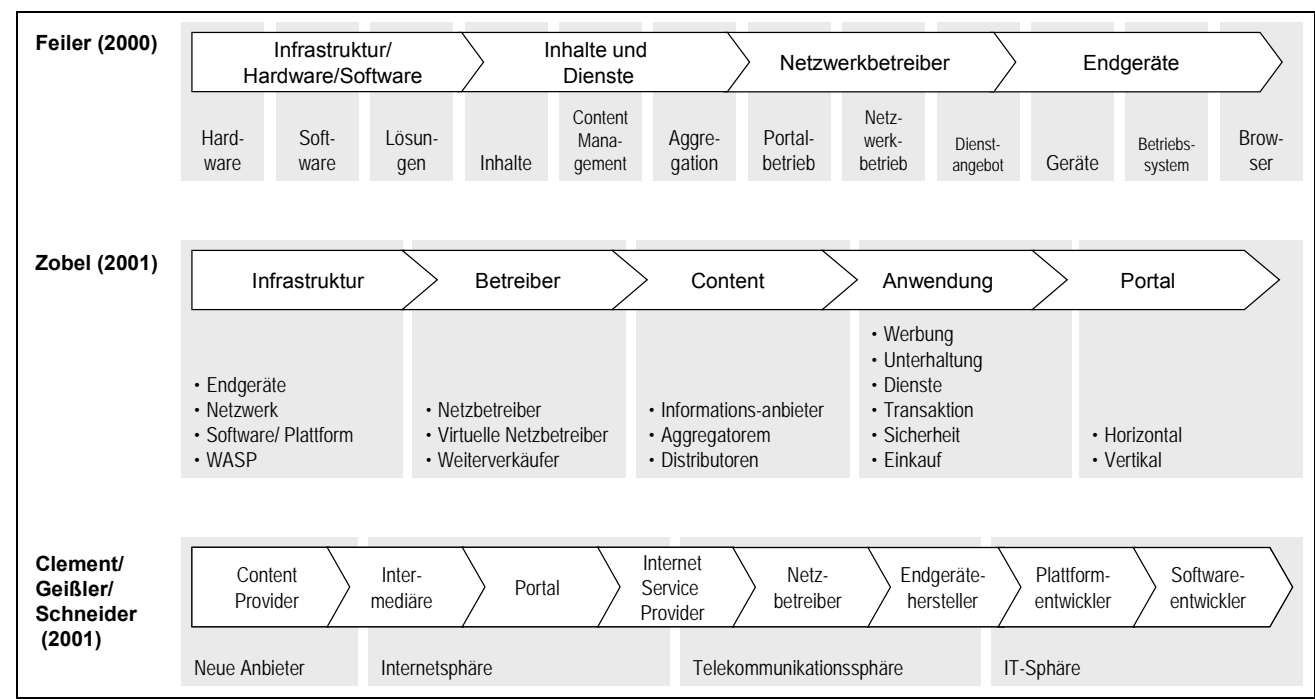

Abbildung 5-5: Beispiele für Modelle des Wertschöpfungssystems im mobilen Internet

Die Ursache der Heterogenität der verschiedenen Ansätze besteht darin, dass - wie Hamel anmerkt - die Konvergenzthematik nicht automatisch die Identifikation dauerhaft erfolgreicher Geschäftsmodelle erlaubt. ${ }^{567}$ Die geringe Aussagekraft konvergenzorientierter Ansätze für die praktische Identifikation von Geschäftsmodellen rührt daher, dass Konvergenz immer Ausdruck von Marktimitationen bereits existierender Erfolgsmodelle darstellt. Aufgrund dieses Imitationsdrucks müssen Unternehmen, die durch innovative Geschäftskonzepte einen Wettbewerbsvorteil suchen, den am Markt vorherrschenden Geschäftsmodellen „vorauseilen“. Tatsächlich, so stellte eine Erhebung unter Geschäftsmodellen im mobilen Internet der Universität Regensburg fest, konnten 66 von insgesamt 98 untersuchten Geschäftsmodelle nicht oder nur unter Zuhilfenahme weiterer Betrachtungskriterien in die Wertschöpfungsschemata der genannten Beiträge eingeordnet werden. ${ }^{568}$

(3) Um einen mobilen Dienst nutzen zu können, muss ein Nutzer neben diesem Dienst gleichzeitig weitere Produkte und Dienstleistungen in Anspruch nehmen: ein Endgerät, das mit einem Sendeempfangsgerät für den Mobilfunk ausgestattet ist und zur Anzeige des jeweiligen Dienstes dient oder Kapazitäten eines Mobilfunknetzes zur Übertragung des Dienstes. Diese Leistungen müssen zueinander komplementär sein, damit das Endgerät das jeweilige Inhalteformat des Dienstes bzw. das Kommunikationsprotokoll des Mobilfunknetzes verstehen muss, bzw. der Dienst in einem durch das Mobilfunknetz transportierfähigen Format bereit stehen muss. Ein Nutzer nimmt somit im Zuge des Abrufs eines mobilen Dienstes nicht nur jeweils diesen Dienst sondern ein ganzes Bündel an weiteren Leistungen in Anspruch.

567 Vgl. Hamel (2000), S. 48.

$568 \mathrm{Vgl}$. Lehmann/Lehner (2003), S. 6. 
Ein solches Bündel aus verschiedenen komplementären Produkten und Dienstleistungen wird auch als "Leistungssystem" bezeichnet. ${ }^{569}$ Ein solches Leistungssystem zeichnet sich dadurch aus, dass durch die Interaktion der Systemelemente ein Nutzen entsteht, der nicht durch seine Elemente oder Teilsysteme erzeugt werden kann. ${ }^{570}$ Leistungssysteme werden üblicherweise in Form sog. „Systemgeschäfte" vermarktet. Systemgeschäfte sind solche Geschäfte, bei denen ein Anbieter oder mehrere Kooperationspartner ein neues komplexes Leistungssystem entwickeln, betreiben und vertreiben. ${ }^{571}$ Die Systemelemente eines in Form eines Systemgeschäfts vermarkteten Leistungssystems beruhen auf einer gemeinsamen Architektur. ${ }^{572}$ Die Systemarchitektur sorgt zum einen systemintern für Schnittstellen, die eine Interaktion der Systemelemente und deren Integration zu einem Gesamtsystem erlauben. Zum anderen verringert die Systemarchitektur die vom Kunden wahrgenommene Komplexität des Leistungssystems, indem es eine gemeinsame externe Schnittstelle für die Systemelemente schafft, wodurch das System vom Kunden als integrierte Einheit wahrgenommen wird. Beispiele für unterschiedliche Systemarchitekturen im mobilen Internet bilden die Architekturen der 2G- und 3G-Mobilfunknetze oder der jeweils verwendete Standard für lokal begrenzte Funknetzinfrastrukturen (Vgl. Abbildung 5-6).

Die Vermarktung von einzelnen Gütern in einem Systemgeschäft ist immer dann von Vorteil, wenn zwischen den Systemelementen Netzeffekte bestehen, die so beschaffen sind, dass die einzelnen Systemelemente keinen originären Nutzen stiften, sondern nur gemeinsam einen derivativen Nutzen erzeugen. Güter mit solchen Eigenschaften der Nutzenstiftung werden auch als Systemgüter bezeichnet. ${ }^{573}$ Wie bereits dargelegt, besitzen mobile Dienste einen Systemgutcharakter in Verbindung mit Mobilfunknetzen und mobilen Endgeräten. Dies ist jedoch nicht damit gleichzusetzen, dass mobile Dienste zwangsläufig und ausschließlich in Form von Systemgeschäften gemeinsam mit Netzzugangsverträgen und Endgeräten vermarktet werden müssen. Vielmehr existieren für das Geschäft mit mobilen Diensten verschiedene Formen von Systemgeschäften. Abbildung 5-6 zeigt drei Beispiele für Ausprägungsformen von Systemgeschäften im mobilen Internet.

\footnotetext{
569 Vgl. Eckhoff (2001), S. 15.

570 Vgl. Weiber (1992), S. 30; Backhaus et al. (1994), S. 9.

571 Vgl. Eckhoff (2001), S. 19.

572 Vgl. Weiber (1992), S. 32.

573 Vgl. bspw. Stoetzer/Mahler (1995), S. 8.
} 


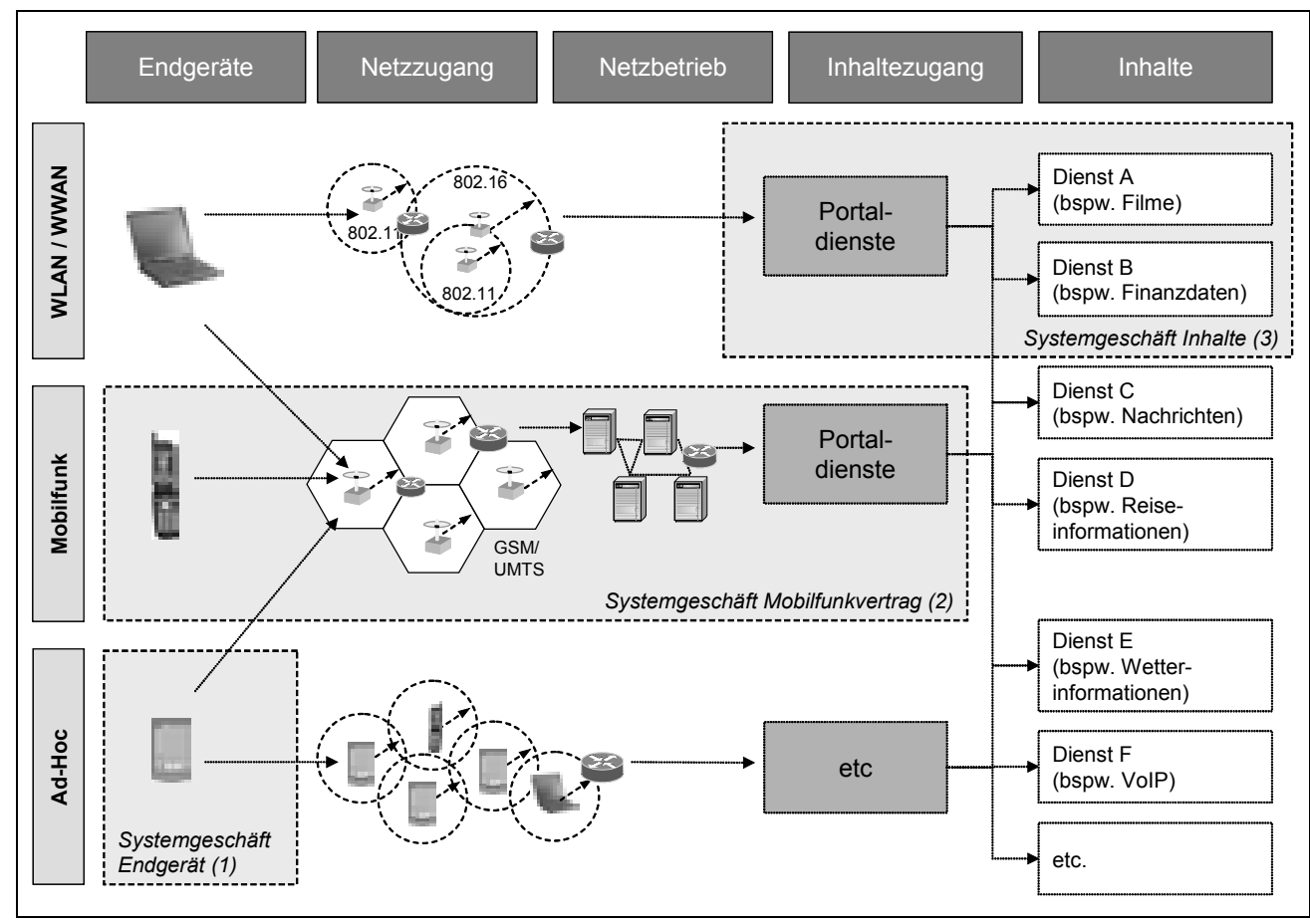

Abbildung 5-6: Systemgeschäfte im mobilen Internet

(1) Im Handel erhältliche mobile Endgeräte werden üblicherweise als betriebsfähige Leistungsbündel angeboten. Das Leistungsbündel besteht einerseits aus der Gerätehardware, bspw. ein Mobiltelefon, ein PDA oder ein Notebook, und andererseits der darauf vorinstallierten Software. Die vorinstallierte Software umfasst das Betriebssystem, die gerätespezifische Basissoftware zur Steuerung einzelner Gerätefunktionen und ggf. zusätzlich Anwendungssoftware.

(2) Während Netzzugang und mobile Endgeräte für lokal begrenzte Netzinfrastrukturen aufgrund der betreiberunabhängigen Standardisierungen der Systemarchitekturen in der Regel getrennt vermarktet werden, erfolgt im Mobilfunk üblicherweise eine gemeinsame Vermarktung der beiden Leistungen Endgerät und Netzzugang im Leistungssystem des Mobilfunkvertrags durch Mobilfunkbetreiber. Der Vertrag beinhaltet daher in der Regel zusätzlich die Nutzungsberechtigung der jeweils betreiberspezifischen Plattform für mobile Mehrwertdienste.

(3) Ebenso wie das leitungsgebundene Internet erlaubt das mobile Internet grundsätzlich eine direkte Adressierung eines Dienstes durch einen Nutzer anhand der IP- oder DNS-Adresse seines Speicherorts. Allerdings sind solche Adressen für mobile Dienste aufgrund der geringen Marktreife bei Konsumenten bislang wenig bekannt, weshalb den Plattformdiensten eines Mobilfunkbetreibers eine besondere Rolle der Aufmerksamkeitskanalisierung zukommt. Mobile Angebote werden daher häufig exklusiv für die jeweiligen 
Plattformen der Mobilfunkbetreiber produziert und im System mit diesen vermarktet.

\subsubsection{Rahmenbedingungen des Erlösmodells inhalteorientierter - mobiler Dienste}

Bei der Gestaltung des Geschäftsmodells für eine inhalteorientierten mobilen Dienst müssen im Rahmen des Erlösmodells drei Aspekte berücksichtigt werden: die Quellen der beabsichtigten Einnahmen (1), die für diese Einnahmen verantwortlichen Tarifstrukturen (2) und die Art der Abrechnung der tatsächlich erzielten Einnahmen (3).

(1) Digitale Mediengüter besitzen potenziell drei verschiedene Erlösquellen: Einnahmen aus den transportierten Inhalten, Einnahmen aus der Vermarktung von Werberaum, der durch die Vermittlung von Kundenkontakten an die Adresse von Werbetreibenden auf der Basis der inhaltlichen Attraktivität des Informationsguts entsteht und ggf. Einnahmen aus der Vermarktung von Profilinformationen individuell identifizierter Rezipienten unabhängig vom Mediengut. ${ }^{574}$ Die Erlöse können dem Unternehmen direkt, entweder abhängig oder unabhängig von der Nutzungsintensität, zufließen oder indirekt, bspw. durch Lizenzeinnahmen entstehen. Abbildung 5-7 gibt einen Überblick über die grundsätzlich denkbaren Erlösformen.

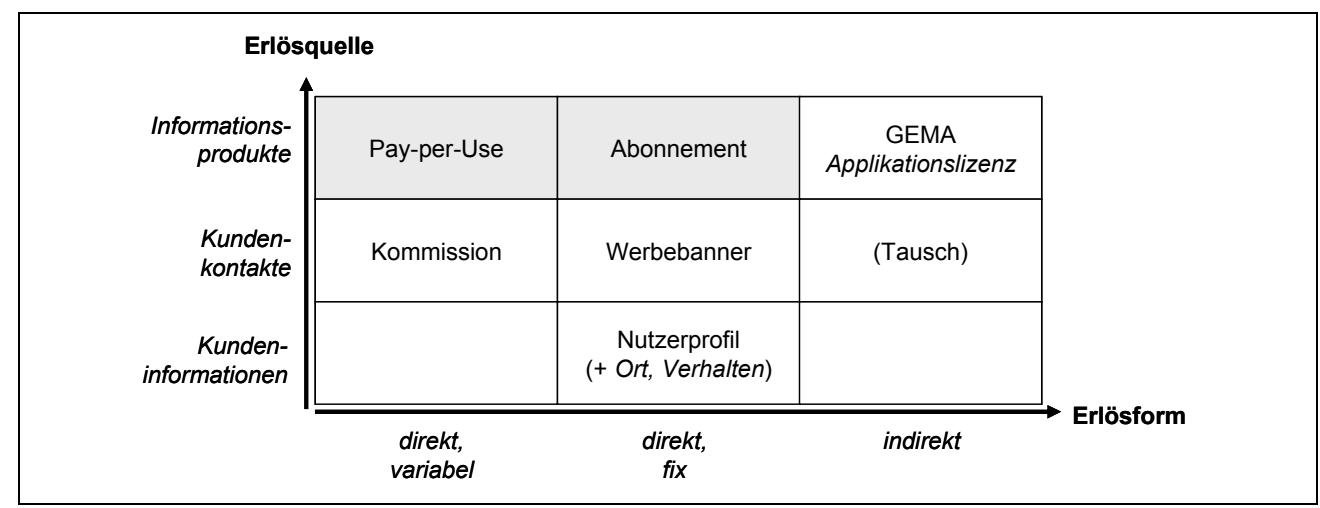

Abbildung 5-7: Varianten des Erlösmodells digitaler Medienprodukte

Aufgrund des frühen Marktstadiums des mobilen Internet und den vor allem über Dienstplattformen vermittelten Kundenkontakten besitzen Anbieter inhalteorientierter Dienste tendenziell weniger Informationen über ihre Kunden als Anbieter vergleichbarer Dienste im leitungsgebundenen Internet. Des Weiteren können mobile Inhalteformate aufgrund der bislang geringen Darstellungskapazitäten mobiler Endgeräte nicht oder zumindest nicht im selben Umfang wie im leitungsgebundenen Internet mit Werbeinhalten gekoppelt werden. Insofern ist für Inhalteanbieter die Möglichkeit, Kundeninformationen und Kundenkontakte aus dem mobilen Internet erlöswirksam zu vermarkten, grundsätzlich eingeschränkt.

574 Vgl. Heinrich (1999a), S. 236 ff. 
Aus Sicht von Inhalteanbietern besteht dagegen in Bezug auf das mobile Internet die Hoffnung, dass Mobilfunknutzer tendenziell eher bereit sind, für in Anspruch genommene Dienste zu bezahlen, insofern eine gewachsene Gratismentalität wie im Internet keine Vertriebsbarriere für Bezahlinhalte und -dienste darstellen. ${ }^{575}$ Daher stehen im Rahmen des Erlösmodells inhalteorientierter mobiler Dienste in erster Linie direkte oder indirekte Erlöse aus der Inhaltevermarktung gegenüber Endkunden (in Abbildung 5-7 grau hinterlegt) im Vordergrund.

(2) Preismodelle für Bezahlinhalte und -dienste im mobilen Internet befinden sich bislang in einem experimentellen Stadium und beruhen auf Schätzungen der Zahlungsbereitschaft potenzieller Nutzer. Die Schätzung der Zahlungsbereitschaft wird dabei insbesondere dadurch erschwert, dass mobile Dienste (ebenso wie Bezahlinhalte und -dienste im leitungsgebundenen Internet) in der Regel unabhängig von den für ihre Nutzung notwendigen Datentransfervolumina abgerechnet werden. Dies ist selbst dann der Fall, wenn inhalteorientierte Dienstangebote als Systemgeschäft mit der Dienstplattform eines Mobilfunkbetreibers vermarktet werden. Während allerdings im leitungsgebundenen Internet die bestehenden Überkapazitäten der Datennetze zu vernachlässigbaren Übertragungspreisen geführt haben, sind die Kapazitäten der Luftschnittstelle eines Mobilfunknetzes prinzipiell knapp. Dadurch ist der Betreiber eines Mobilfunknetzes in der Lage, seine Preise für den Datentransfer auf Grundlage einer quasi-monopolistischen Kalkulation zu bestimmen. Um eine optimale Abschöpfung der Konsumentenrente zu erreichen, verwenden Telekommunikationsunternehmen üblicherweise mehrteilige Tarifstrukturen, die sich aus einem nutzungsabhängigen Preis und einer nutzungsunabhängigen Grundgebühr zusammensetzen. ${ }^{576}$ Ein gewinnmaximierender Mobilfunknetzbetreiber wird dabei seine Tarifstruktur so wählen, dass zwar seine nutzungsabhängigen Gebühren deutlich unter dem Preis bei einem einteiligen Tarif liegen ${ }^{577}$, es wird jedoch eine Grundgebühr erhoben, die die Abschöpfung der Konsumentenrente maximiert. Vor dem Hintergrund einer solchen Tarifwahl des Netzbetreibers kann geschlussfolgert werden, dass das Geschäft mit dem Datentransfer auf einer Quersubventionierung durch den mit mobilen Diensten erzeugten Kundenmehrwert beruht. Aufgrund der geringen Marktmacht von Inhalteanbietern im mobilen Internet wird diese Quersubventionierung jedoch bislang nicht oder nur zum Teil

\footnotetext{
$575 \mathrm{Vgl}$. Mings/White (2000), S.64 f.

576 Mehrteilige Tarifstrukturen wurden erstmals 1941 durch Lewis für Marktsituationen untersucht, in denen der neoklassische Gleichgewichtspreis nicht zustande kommt, weil die Kosten des Anbieters mit zunehmender Kundenzahl abnehmen. Für den Fall des gewinnmaximierenden Monopolisten wurde die Theorie zweiteiliger Tarife erweitert von Faulhaber/Panzar (1977), Oi (1979) und Finsinger (1980). Vgl. auch Spremann (1985).

577 Im Extremfall entsprechen die nutzungsabhängigen Gebühren den Grenzkosten der Dienstbereitstellung.
} 
durch entsprechende Rückvergütungen ${ }^{578}$ von Seiten der Mobilfunkbetreiber an die Anbieter inhalteorientierter Dienste kompensiert. ${ }^{579}$

(3) In Bezug auf die Abrechnung mobiler Inhalteangebote können aus Sicht eines Inhalteanbieters drei Varianten von Abrechnungsmodellen unterschieden werden (vgl. Abbildung 5-8): $:$ :80

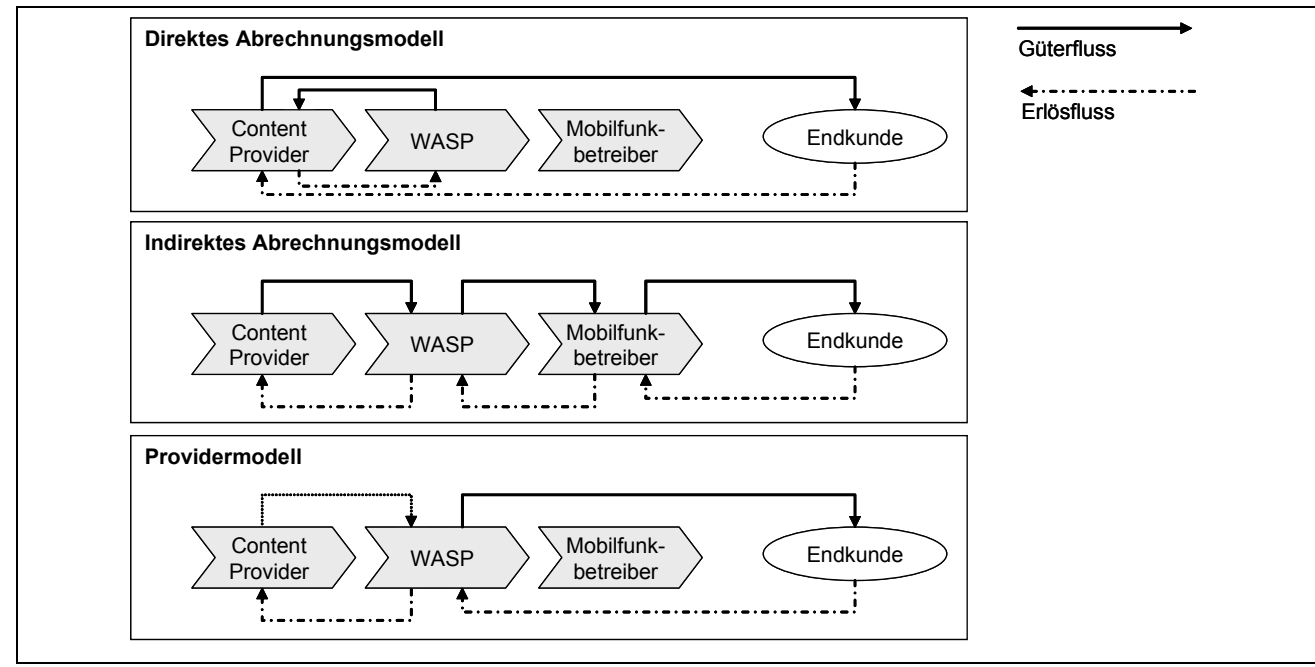

Abbildung 5-8: Abrechnungsmodelle für mobile Dienste aus Sicht von Inhalteanbietern

- Beim direkten Abrechnungsmodell lässt ein Inhalteanbieter von WASPUnternehmen mobilitätsgerechte Dienstformate entwickeln und vermarktet diese gegenüber Endkunden auf eigene Rechnung. Beispiel solcher Vermarktungs- und Abrechnungsformen bildet die Bewerbung mobiler Dienste in den leitungsgebundenen Angeboten von Onlineinhalten, wie bspw. die Vermarktung von FTD-mobil auf den Seiten von FTD-Online. Der wesentliche Erfolgsfaktor eines solchen Abrechnungsmodells ist dabei die Auswahl oder Entwicklung eines aus Kundensicht einfach zu nutzenden Bezahlverfahrens.

- Beim indirekten Abrechnungsmodell werden Einkünfte aus inhalteorientierten Diensten nicht vom Inhalteanbieter selbst, sondern vom Dienstentwickler abgerechnet. Der Dienstanbieter vergütet dabei die von ihm in Anspruch genommenen Inhalte gegenüber dem Inhalteanbieter. Aus Sicht des Inhalteanbieters entspricht dieses Abrechnungsmodell weitgehend dem bereits in Kapitel 3 dargelegten Prinzip der Inhaltesyndizierung.

578 Derartige Formen der Rückvergütung werden auch als „Airtime-Sharing" bezeichnet. Unter AirtimeSharing ist die Partizipierung der Inhalteanbieter an den Verbindungserlösen (entweder pro Zeiteinheit oder pro Datenpaket) des Mobilfunkbetreibers zu verstehen. Vgl. Ziegler/Adam (2001), S 16.

579 Mobilfunkbetreiber haben bisher noch keine grundsätzliche Diskussionsbereitschaft signalisiert, um über eine Teilhabe von Inhalteanbietern an Verbindungserlösen zu sprechen. Vgl. Ziegler/Adam (2001), S. 64 und Seidenfaden/Kamrich/Kaspar/Ortelbach (2005).

580 Vgl. Simonitsch (2003), S. 56. 
- Beim sog. Providermodell besteht eine direkte Abrechnungsbeziehung lediglich zwischen Kunden und Mobilfunkbetreibern. Die Abrechnung der Inhaltenutzung erfolgt durch den Mobilfunkbetreiber entweder direkt in Abhängigkeit der Dienstnutzung oder indirekt in Abhängigkeit von Einnahmen aus dem Datenverkehr. Der Einzug des Rechnungsbetrags beim Kunden kann dabei über die Mobiltelefonrechnung erfolgen. Als Pionier für die Entwicklung eines erfolgreichen Providermodells für die Abrechnung von Nutzungsgebühren inhalteorientierter mobiler Dienste mit Drittanbietern gilt der japanische Mobilfunkanbieter NTT Docomo für seine Dienstplattform i-mode. ${ }^{581}$

\subsubsection{Zusammenfassung}

In Abschnitt 5.2.2.1 wurde festgestellt, dass die Bedingung eines erfolgversprechenden Produkt- und Nutzenmodells inhalteorientierter mobiler Dienste darin besteht, einen kundenseitigen Mehrwert zu erzeugen. Es wurden zwei Möglichkeiten zur Erzeugung eines solchen Mehrwerts identifiziert: die Inhaltebereitstellung in mobilitätsgerechten und ortspezifischen Dienstformaten sowie die Schaffung nachfrageseitiger Netzeffekte durch die Bündelung von Inhalten und kommunikationsorientierten Dienstformaten. Im Zusammenhang mit der netzeffektbezogenen Mehrwertgenerierung wurde ein grundsätzliches Kundenadoptionsproblem angesprochen, das dadurch gemindert werden kann, indem in einer frühen Marktentwicklungsphase in erster Linie Bedürfnisse technikaffiner und mobilfunkinteressierter Nutzergruppen angesprochen werden. Des Weiteren zeigte die Untersuchung der Rahmenbedingungen der Wertschöpfung im mobilen Internet in Abschnitt 5.2.2.2, dass Inhalteangebote im mobilen Internet zusätzlicher Wertschöpfungsaktivitäten im Bereich des sog. Wireless Application Service Providing bedürfen, um eine mobilitätsgerechte Inhaltekodierung vorzunehmen. Neben der Kooperation mit Dienstentwicklern ergeben sich für Inhalteanbieter Möglichkeiten einer Einbindung in die Wertschöpfungskette des mobilen Internet durch die Zusammenarbeit mit solchen Partnern, wie bspw. Mobilfunkbetreibern und virtuellen Mobilfunkbetreibern, die über eine eigene Dienstplattform verfügen. In Abschnitt 5.2.2.3 wurde schließlich gezeigt, dass für Inhalteanbieter zusätzliche Erlösquellen in erster Linie im Bereich der Inhaltevermarktung bestehen. Eine solche Inhaltevermarktung kann entweder gegenüber Endkunden, gegenüber Dienstentwicklern oder gegenüber Mobilfunkbetreibern und virtuellen Mobilfunkbetreibern, die über eine eigene Dienstplattform verfügen, erfolgen.

In einer Studie der Handlungsmöglichkeiten von Medienunternehmen im Bereich des mobilen Internet identifizierten Ziegler/Adam (2001) drei grundsätzliche Strategieoptionen: die Syndizierung von Inhalten an Drittanbieter mobiler Dienste ohne eigenes Dienstangebot, das eigene oder zumindest partnerschaftlich entwickelte Angebot mobiler Mehrwertdienste oder das Angebot einer eigenen Diensteplattform in Form eines mobilen Portals. ${ }^{582}$ Die

581 Vgl. Funk (2000) und NTT (2004).

582 Vgl. Ziegler/Adam (2001), S. 104 f.; Ähnlich auch Feldmann (2002). 
Inhaltesyndizierung an Drittanbieter stellt dabei aufgrund des dargestellten Bedarfs an Inhalten zur Entwicklung von Mehrwertdiensten ebenso wie im PC-basierten Onlinegeschäft einen viel versprechenden zusätzlichen Absatzkanal für die Inhalte eines Medienunternehmens dar. Die Inhaltesyndizierung im Bereich mobiler Dienste besitzt jedoch keine untersuchenswerten Besonderheiten gegenüber den bereits in Kapitel 3 erläuterten Handlungsmöglichkeiten und werden daher im Weiteren vernachlässigt. Anders als im Syndikationsmarkt besitzen Medienunternehmen dagegen im Markt für mobile Portale aus heutiger Sicht lediglich geringe Marktzutrittschancen. Ursache dafür bildet die dominierende Marktstellung von Mobilfunkbetreibern im Markt für mobile Portale, die aufgrund des beschriebenen Systemgeschäfts von Netzbetrieb, Endgerätevermarktung und Plattformangeboten, bspw. als voreingestellte Startseite des Endgerätebrowsers, ausgeliefert werden können. ${ }^{583}$ Vor dem Hintergrund der geringen Spezifität des Syndikationsgeschäfts und der geringen Erfolgsaussichten mobiler Portalangebote durch Medienunternehmen wird im Weiteren in erster Linie das Angebot eigener oder zumindest partnerschaftlich mit unabhängigen Service Providern entwickelter und betriebener Dienste fokussiert.

\subsubsection{Ausprägungsformen von Geschäftsmodellen für inhalte- orientierte mobile Dienste}

In Abschnitt 5.2.2.1 wurde gezeigt, dass Mediengüter, die über Mobilfunknetze vertrieben werden, in Abhängigkeit von ihrem jeweiligen Distributionsformat zum einen den Charakter informationsorientierter Dienste (wie bspw. WAPkompatibel formatierte Onlineseiten) und zum anderen unter Umständen Merkmale kommunikationsorientierter Dienste (bspw. durch den Inhalteversand auf der Grundlage eines MMS-Dienstes) besitzen können. Kommunikationsorientierte Dienstformate unterliegen dabei wie dargestellt der Besonderheit, dass im Rahmen der Inhaltedistribution nachfrageseitige Netzeffekte entstehen können. Darüber hinaus wurden zwei weitere potenzielle Mehrwerteigenschaften mobiler Inhalteformate identifiziert: die Ortsunabhängigkeit der Inhaltebereitstellung und die Ortsspezifität des Inhaltekonsums. Durch kreuzweise Paarung dieser vier Diensteigenschaften (Informations- und Kommunikationsorientierung, Ortsunabhängigkeit und Ortsspezifität) entsteht ein vierteiliger Merkmalsraum, der zur Klassifikation Erfolg versprechender Anwendungsgebiete inhalteorientierter mobiler Dienste herangezogen werden kann (vgl. Abbildung 5-9).

583 Als einziger Konkurrent zu den Plattformangeboten der Mobilfunknetzbetreiber aus dem mediennahen Anbieterumfeld konnte sich im Segment mobiler Portaldienste das Unternehmen Jamba mit einem eigenen Portalangebot etablieren. Den Erfolgsfaktor der Marktzutrittsstrategie von Jamba bildet eine strategische Allianz mit den Handelsunternehmen der Media-Saturn-Holding, in deren Elektrofachmärkten Mobilfunkgeräte mit dem Jamba-Portal als voreingestellte Browserstartseite ausgeliefert werden. 


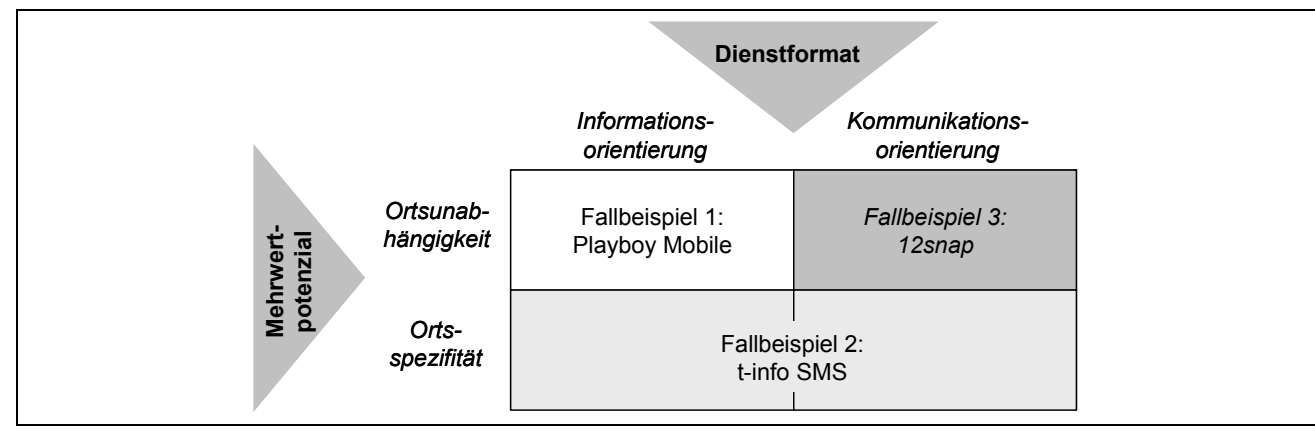

Abbildung 5-9: Klassifikationsraster inhalteorientierter mobiler Dienste

Im folgenden Abschnitt werden auf der Grundlage des Klassifikationsrasters in Abbildung 5-9 drei Geschäftsmodellbeispiele für das Angebot inhalteorientierter mobiler Dienste vorgestellt, die sich jeweils in unterschiedlichen Anwendungsgebieten der Matrix positionieren. Das Ziel der Untersuchung dieser Beispiele bildet die Konkretisierung der zuvor lediglich in allgemeiner Form diskutierten Gestaltungsmöglichkeiten der Geschäftsmodelle mobiler Dienste auf den Ebenen der Nutzengenerierung, der Einbindung in die Wertschöpfungskette des mobilen Internet und des Erlösmodells. Die Auswahl der Beispiele richtet sich in erster Linie nach dem Unternehmenserfolg: In Abschnitt 5.2.3.1 wird das Geschäftsmodell für den Dienst „Playboy Mobile“ der Firma Tomorrow-Focus beschrieben. Playboy Mobile repräsentiert eines der wenigen überhaupt existierenden Beispiele für eine erfolgreiche Vermarktung von Inhalten aus dem Mediensegment des Zeitschriftenmarkts im mobilen Internet. In Abschnitt 5.2.3.2 wird das Geschäftsmodell für die mobilen Verzeichnisdienste der Firma t-info erläutert. t-info gilt als marktführend im Bereich ortsbezogener mobiler Dienste, wobei das vorgestellte Beispiel „tinfo SMS“ ein kommunikationsorientiertes Distributionsformat verwendet. Schließlich wird in Abschnitt 5.2.3.3 das Geschäftsmodell des Dienstes „VCard" der Firma 12snap erläutert. Anders als die beiden zuvor präsentierten Beispiele ist allerdings der Dienst $\mathrm{V}$-Card bislang noch nicht praktisch eingeführt. Die Rechtfertigung der Präsentation dieses Beispiel begründet sich durch die im Vergleich zu den zuvor präsentierten Geschäftsmodellen besondere Form der kommunikationsorientierten Nutzenadressierung dieses Dienstes, wodurch sich eine Vervollständigung der zukünftigen Handlungsperspektiven für Inhalteanbieter im mobilen Internet ergibt.

Alle Angaben zu den drei Geschäftsmodellbeispielen wurden in Form von Einzelgesprächen mit verantwortlichen Mitarbeitern der jeweiligen Unternehmen erhoben. Der Aufbau der Fallstudien folgt im Wesentlichen dem in Abschnitt 5.2.2 eingeführten Rahmenkonzept zur Untersuchung von Geschäftsmodellen anhand der Partialmodelle für Produkt und Nutzen, Wertschöpfung und Erlösmechanik. 


\subsubsection{Fallstudie 1: Playboy Mobile}

Playboy Mobile repräsentiert die kommerziell erfolgreiche Realisierung eines mobilen Datendienstes durch den Geschäftsbereich "next media“ des deutschen Unternehmens Tomorrow Focus AG. Tomorrow Focus ist eine Aktiengesellschaft nach deutschem Recht mit Firmensitz in München. Das Unternehmen erzielt einen Jahresumsatz von 42,9 Mio. EUR und beschäftigt insgesamt 180 Mitarbeiter. Die Tomorrow Focus AG entstand im Dezember 2001 als Fusion aus der Focus Digital AG und der Tomorrow Internet AG. Die Focus Digital AG war im Mai des Vorjahres als Zusammenschluss der Internetaktivitäten der Hubert Burda $\mathrm{GmbH} \&$ Co. KG und der Focus Magazin-Verlag $\mathrm{GmbH}$ gegründet worden. Bereits im Juli 1999 erfolgte die Gründung der Tomorrow Internet AG, hervorgegangen aus den Internetaktivitäten der Verlagsgruppe Milchstraße.

Die Tomorrow Focus AG gliedert sich in die Geschäftsbereiche: Portal, Sales, Technologies und next media (TFNM). Sie hält Beteiligungen an der Bellevue and more AG (Immobilien-Magazine) sowie der Playboy Deutschland Publishing $\mathrm{GmbH}$. Aufgabe von TFNM ist es, die Inhalte der Verlagsmarken der Hubert Burda AG und der Verlagsgruppe Milchstraße wertsteigernd in zusätzlichen digitalen Vertriebsformaten, insbesondere im mobilen Internet, zu verwerten. Dabei liegt der Fokus derzeit auf modernen, unterhaltungsorientierten (sog. „Lifestyle"-orientierten) Inhalten. Den Kundenkreis von TFNM bilden in erster Linie Telekommunikationsunternehmen mit eigener Dienstplattform für das mobile Internet. Im Mittelpunkt der Geschäftsaktivitäten stehen drei Produktgruppen: die mobilgerätetaugliche Zweitvermarktung der redaktionellen Onlineinhalte der beteiligten Verlagshäuser; die Verknüpfung der redaktionellen Onlineinhalte der beteiligten Verlagshäuser mit kommunikationsorientierten Zusatzfunktionen wie Chat oder Instant Messaging; die Verknüpfung der redaktionellen Onlineinhalte der beteiligten Verlagshäuser mit ortsbezogenen Informationen.

Auf den Geschäftsbereich next media entfiel im Geschäftsjahr 2004 ein Umsatzanteil von ca. 2 Mio. EUR. Im Bereich Next media sind am Standort Hamburg 10 Mitarbeiter beschäftigt. Dieser Bereich ist dem Unternehmen derzeit als Profit Center angegliedert. Im laufenden Jahr wird der Bereich jedoch mit dem Berliner Unternehmen iconmobile $\mathrm{GmbH}$ zur Cellular $\mathrm{GmbH}$ mit Firmensitz in Berlin und Hamburg fusioniert werden. Für das Joint Venture sind zwei Tätigkeitsschwerpunkte vorgesehen: Technologie- und Dienstleistungsprojekte im Bereich mobiler Anwendungen sowie Entwicklung und Betrieb von mobilen Inhalteangeboten. TFNM ist Anbieter von derzeit rund 80 mobilen Inhalteangeboten in allen vier deutschen Mobilfunknetzen (D1, D2, O2, E-Plus), in den österreichischen Netzen Mobilkom Austria und one.at sowie im schweizerischen Mobilfunknetz der Swisscom. Die Dienstangebote von TFNM werden entweder als Lizenz an Mobilfunkbetreiber verkauft, oder über Revenue Sharing mit Mobilfunkbetreibern selbst betrieben.

\subsection{Produkt- und Nutzenmodell von Playboy Mobile}

Der Medienmarkt für qualitativ hochwertige, überregionale redaktionelle Inhalte in Deutschland ist trotz der bereits in Kapitel 3 dargestellten Angebots- 
vielfalt auf Zeitungs- und Zeitschriftenmarkt vergleichsweise hoch konzentriert. Während jedoch nahezu alle Marktteilnehmer Zusatzangebote im stationären Internet bieten, beschränken sich Verlagsaktivitäten im mobilen Internet derzeit noch auf wenige Pionierangebote. Durch Etablierung kostenpflichtiger Inhalteverwertungsformen soll bereits im frühen Marktstadium dem Problem der existierenden Gratismentalität von Kunden gegenüber Onlineinhalten entgegen gewirkt werden. Aufgrund der frühen Entwicklungsaktivitäten in Bezug auf mobile Angebote profitiert TFNM derzeit von einem zeitlichen Vorsprung seiner Entwicklung einer technischen Plattform zur Bereitstellung mobiler Anwendungen. Insofern repräsentiert das Angebot eines mobilen Dienstes unter der Marke Playboy einerseits den Versuch, den Markt für zahlungspflichtige Inhalte mit einem derzeit deutlich von erotischen Inhalten dominierten Pionierangebot zu erschließen, andererseits, durch Wahrung eines jugendfreien (FSK 16) und qualitativ hochwertigen Niveaus, eine ausbaufähige Markierung im mobilen Internet zu erzeugen.

Die deutsche Ausgabe der Zeitschrift Playboy erreichte 2004 eine verkaufte Auflage von 870.000 Stück. Anders als der amerikanische Lizenzgeber besitzt die Zeitschrift Playboy in Deutschland neben dem erotischen Aspekt aufgrund von Inhalten bspw. zu Mode und Technik eine am modernen Lebensstil orientierte Markierung. Der Playboy richtet sich in erster Linie an eine junge, überwiegend männliche, spaßorientierte Mittelschicht im Alter zwischen 20 und 39 Jahren mit überdurchschnittlichem Einkommen. ${ }^{584}$ Die Käuferschaft weist eine überdurchschnittlich hohe Technik- und Onlineaffinität sowohl im beruflichen als auch im privaten Umfeld sowie eine überdurchschnittlich hohe Verwendung von Mobilfunktelefonen auf.

Die Käuferstruktur des Playboy-Magazins besitzt eine hohe Kongruenz mit jenen gesellschaftlichen Leitmilieus, denen eine hohe Affinität für die Nutzung kostenpflichtiger unterhaltungsorientierter mobiler Datendienste beschieden wird. ${ }^{585}$ Playboy Mobile stellt insofern den als durchaus erfolgreich zu bewertenden Versuch dar, die Markierung des Zeitschriftentitels auf den Bereich des mobilen Internet zu übertragen. Dabei wird in erster Linie ein Mehrwert durch den ortsungebundenen und dadurch zeitnahen Zugriff auf die Kerninhalte des Heftes in Form von erotischen Ablichtungen prominenter Personen des öffentlichen Lebens sowie allgemeinen erotischen Inhalten erzeugt. Der Kontext der Inhaltenutzung spielt aufgrund der besonderen erotischen Natur der Inhalte der Marke Playboy für die Nutzung der mobilen Anwendung insofern eine Rolle, als die Inhalte auf dem mobilen Endgerät stärker noch als die stationäre Onlinenutzung durch

584 Vgl. TdW (2004): Entsprechend der Nutzertypologie der sog. Sinus Milieus, einem etablierten Instrument der qualitativen Konsumforschung, rekrutieren sich 30\% der Playboyleser aus den tendenziell hedonistisch geprägten Milieus der sog. „Experimentalisten“ und Hedonisten, 15\% aus dem Milieu der sog. „modernen Performer“. Alle drei Milieutypen werden Merkmalseigenschaften junger, technikaffiner und unkonventioneller Avantgardisten zugeschrieben (vgl. Blödorn/Gerhards (2004)).

585 Vgl. TdW (2004): Bspw. gehören derzeit 35,5\% aller Mobilfunknutzer, die eine Nutzungsbereitschaft im Bereich von WAP-gestützten Diensten aufweisen, den Milieus der modernen Performer und der Hedonisten an. 
ihre private Natur gekennzeichnet ist. Spezifische räumliche Kontexte spielen jedoch keine Rolle.

Das mobile Inhalteangebot der Marke Playboy erfolgt ausschließlich für Vertragskunden deutschsprachiger Mobilfunkbetreiber. Personen- und Endgerätemobilität werden im Rahmen der verfügbaren Netztechnologien GSM und UMTS geboten, Dienst- und Sitzungsmobilität werden jedoch nicht adressiert. Die Bereitstellung von Diensten für das mobile Internet erfolgt bei TFNM in einem dreistufigen Prozess (vgl. Abbildung 5-10).

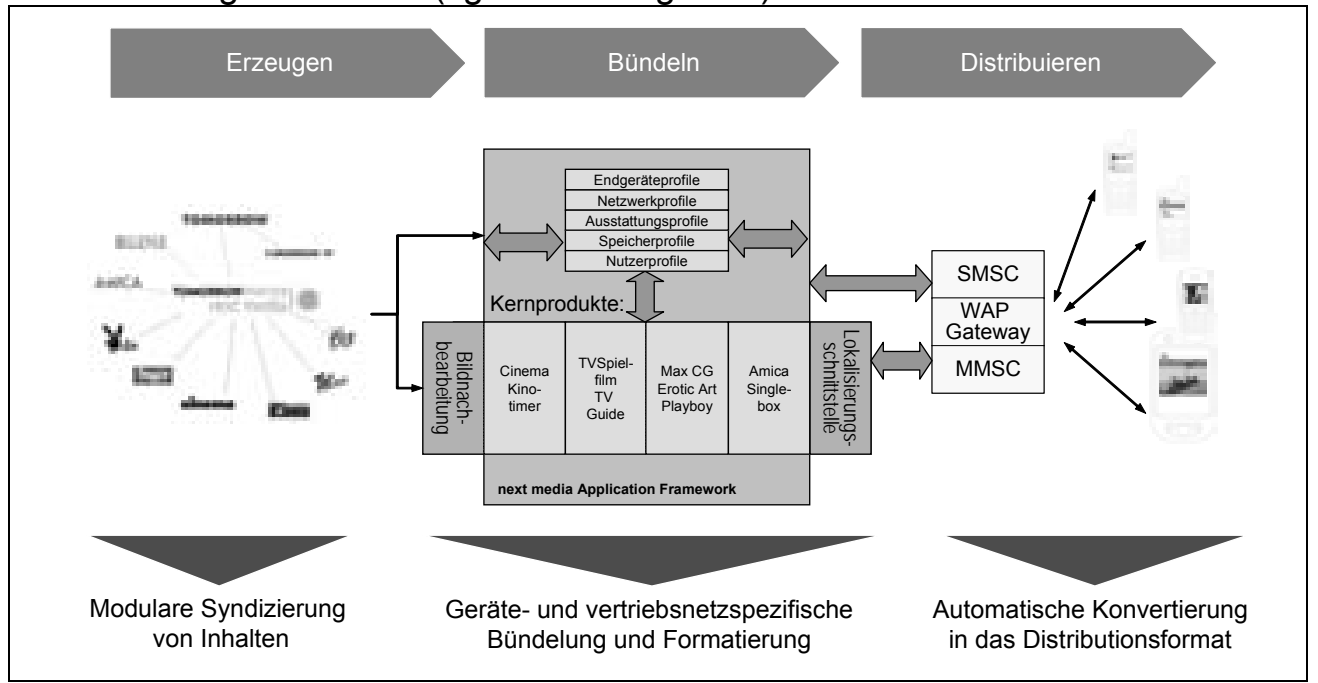

Abbildung 5-10: Technische Bereitstellung mobiler Dienste durch TFNM

- TFNM erhält einen Datenbank-Export aus den Onlinedatenbanken der kooperierenden Verlage. Die Inhalte für Playboy Mobile werden durch den deutschen Lizenznehmer der Marke Playboy, die Playboy Deutschland Publishing $\mathrm{GmbH}$, einem Joint Venture der Tomorrow Focus AG und dem Focus Magazin Verlag bereitgestellt. Der Inhaltezugriff auf die Datenbank wird prinzipiell einmal monatlich freigegeben. Die Verwertungslizenz von TFNM erstreckt sich auf die Verwendung der Marke, der Onlineinhalte des deutschen Lizenznehmers und der Onlineinhalte des amerikanischen Lizenzgebers. Der Umfang der Zugriffsrechte kann durch den Lizenzgeber eingeschränkt werden. Insbesondere Verwertungsrechte an Bildern von Prominenten und Nacktmodelle werden für jedes Verwertungsformat einzeln verhandelt. Ingesamt stehen TFNM über 30.000 Bilder durch den deutschen und weitere 30.000 Bilder durch den amerikanischen Lizenzgeber zur Verfügung.

- Die Inhalte werden auf einer eigenen Datenbank gespeichert, auf Aktualität geprüft, gekürzt und ggf. neu kombiniert. Neben Aufgaben in Bezug auf die redaktionelle Auswahl besteht die Hauptaufgabe bei der Aufbereitung von Onlineinhalten der Marke Playboy, aufgrund der Dominanz von Bildformaten, in der Vorformatierung der jeweiligen Abbildungen zur optimalen Darstellung für unterschiedliche Endgeräte. Es erfolgt eine An- 
passung für jedes der derzeit insgesamt 65 unterstützten unterschiedlichen Endgeräteprofile. Die Anpassung umfasst einerseits das Rendering der Bilddateien in ein jeweils geräteoptimiertes Format sowie andererseits die Zuweisung des jeweiligen Distributionsformats, bspw. in WML, XHTML, HTML, MMS- oder SMS-Format. Zudem werden Sonderformate für mobile Endgeräte wie Hintergrundbilder oder Java-Anwendungen produziert.

- Das Ausgabeformat wird entsprechend den mobilen Endgeräten bzw. den jeweiligen Partnerplattformen optimiert. Das Hosting der Anwendungen erfolgt derzeit auf Servern der Firma MediaWays. Jeder Mobilfunkbetreiber erhält eine eigene Adresse für die jeweils im Design der Betreiberplattform formatierten Anwendungen. Die verschiedenen betreiberspezifischen Darstellungsformate sind als Profilklassen im ContentManagement-System von TFNM hinterlegt.

Die operative Auslieferung von Inhalten in Form mobiler Anwendungen erfolgt auf Grundlage des sog. „next media application framework“. Diese Diensteplattform ist eine javabasierte eigenentwickelte Individualsoftware. Die Plattform erlaubt eine individuelle Typerkennung des abfragenden mobilen Endgeräts und sorgt für eine gerätespezifische Anpassung des Ausgabeformats des jeweils abgerufenen Dienstes. Die Plattform besteht im Wesentlichen aus drei Komponenten: eine Inhaltedatenbank, auf der die jeweiligen Inhalte der kooperierenden Verlage gespeichert werden; Dienstmodule für Pulldienste, die die jeweiligen Inhalte bündeln und endgerätespezifisch sowie ggf. ortsbezogen anpassen; einem Nachrichtensystem für mobile Push-Dienste, das spezifische Inhalte betreibergerecht versendet und abrechnet.

\subsection{Wertschöpfungsmodell von Playboy Mobile}

An der Wertschöpfung von Playboy Mobile sind mindestens drei und maximal insgesamt fünf Partner beteiligt:

- Die Playboy Deutschland Publishing $\mathrm{GmbH}$ als Lizenzgeber für die mobile Verwertung der Inhalte und Marke von Playboy übernimmt drei Aufgaben: Sie stellt Inhalte zur Verfügung, stimmt die Rechteverfügbarkeit mit dem amerikanischen Lizenzgeber ab und steuert Marketingmaßnahmen in Bezug auf die mobile Anwendung.

- TFNM ist verantwortlich für die technische Aufbereitung der verfügbaren Inhalte, vermarktet die Anwendung gegenüber Geschäftskunden und steuert die Abrechnung gegenüber Endkunden. Es erfolgt ein eigenes Monitoring der verschiedenen Inhaltezugriffe sowie eine Steuerung der Werbemaßnahmen für die eigenen Anwendungen auf der jeweiligen Betreiberplattform. Bspw. hat TFNM im Rahmen einer regelmäßigen Teaser-Rotation Einfluss auf die Art und das Ziel des Teasering ihrer Anwendungen auf der Startseite des Portals Vodafone Live.

- Mobilfunkbetreiber wie die Unternehmen T-Mobile, Vodafone, O2 oder E-Plus stellen die Playboy Anwendungen in ihren Dienstportalen (z.B. "T-Zones" oder „Vodafone Live") bereit und sorgen für die Abrechnung 
von Leistungen, die durch Kunden in Anspruch genommen wurden. Die Abrechnung gegenüber TFNM erfolgt auf Grundlage eines monatlich erstellten Operatorreporting, das sämtliche erfolgreich abgeschlossenen Transaktionen aufführt. Auf die Position und die Bewerbung ihrer Anwendungen hat TFNM nur im oben aufgeführten Umfang Einfluss.

- Optional: Medienpartner des deutschen Lizenzgebers sorgen für eine Bewerbung der angebotenen mobilen Anwendung. Dazu gehören zum einen zur Burda Gruppe gehörende Print Titel, zum anderen Partnerschaften mit Fernsehsendern wie RTL 2. Abgesehen von der oben genannten Einflussnahme auf das Teasering im Rahmen der Dienstplattform führt TFNM keine eigene Bewerbung der angebotenen Anwendungen gegenüber Endkunden durch.

- Optional: Technische Dienstleister wie die Unternehmen Materna $\mathrm{GmbH}$ als technischer Servicedienstleister des Mobilfunkbetreibers Vodafone zur Abwicklung von MMS Diensten oder die Convisual AG als MMS Servicedienstleister von T-Mobile. Das Angebot solcher Dienstleister wird immer dann benötigt, wenn eine sensitive Technikinfrastruktur, bspw. bei umfangreicheren Downloadinhalten in Bezug auf die Gewährleistung vollständiger und fehlerfreier Übertragungen, in Anspruch genommen werden muss.

Das Dienstangebot lässt sich in vier Bereiche gliedern:

- Im Bereich „Browsable Applications“ können zahlungspflichtige Text-, Bild- und Videoinhalte über eine WAP-gestützte Oberfläche direkt von einem Nutzer abgerufen werden. Das Angebot umfasst überwiegend Unterhaltungs- und Erotikinhalte, darunter Bildergalerien, Witze und Einkaufshinweise.

- Im Bereich „MMS Dienste“ können regelmäßig per Push-Nachricht versandte Inhalte kostenpflichtig abonniert werden. Zum Angebot gehören Witze per SMS und - kommerziell wichtiger - Erotikbilder per MMS.

- Im Bereich „Mobile Fun Download“ können Spiele, Bildbetrachtungssoftware, Grußkarten, Hintergrundbilder oder Layoutmotive für mobile Endgeräte kostenpflichtig herunter geladen werden.

- Im Bereich „Videos“ können Kurzfilme auf mobile Endgeräte geladen werden. Die Inhalte stammen vorwiegend aus dem Bestand des amerikanischen Markenlizenzgebers. Derzeit erfolgt die Vermarktung durch Netzbetreiber in Form von preisgünstigen Pauschaltarifen, die Abrechnung gegenüber TFNM erfolgt in Form von Mindestumsätzen.

Das Angebot im Bereich „Browsable Applications“ ist zum einen in den mobilen Inhalteportalen der genannten Mobilfunkbetreiber integriert und kann zum anderen direkt über eine unabhängig beworbene Onlineadresse angesteuert werden. Demgegenüber können alle übrigen Dienstformen ausschließlich über das Dienstportal des jeweiligen Mobilfunkbetreibers in Anspruch genommen werden. Entsprechend ist der Erfolg aller vier Angebotsbereiche in hohem Maße abhängig von Attraktivität der Dienstportale der jeweiligen Netzbetreiber und 
der Positionierung des Dienstes innerhalb des jeweiligen Portals, wodurch der Erfolg je nach Dienstplattform des Netzbetreibers unterschiedlich ausfällt. Im Portal "Vodafone Live“ des umsatzwirksamsten Partners Vodafone, der bereits früh eine werbewirksame Produktplatzierung eines browsergestützt navigierbaren Inhalteangebots betrieb, bilden die Pull-Anwendungen der Angebotsbereiche „Browsable Applications“ und „Fun Download“ die erlöskräftigsten Einnahmequellen. Im Portal "T-Zones“ von T-Mobile, das zugunsten von MMSgestützten Push-Angeboten vergleichsweise spät im Markt eingeführt wurde, bilden dagegen die Angebotsbereiche „Browsable Applications“ und „MMSDienste" die umsatzstärksten Einnahmequellen.

\subsection{Erlösmodell von Playboy Mobile}

Die Erfolgs- und Umsatzmessung von TFNM erfolgt einerseits auf der Grundlage des monatlichen Operatorreporting und andererseits über das Monitoring der Inhaltezugriffe auf den Anwendungsservern. Zu den erfolgreichsten Anwendungen gehören WAP-gestützte Inhaltezugriffe mit monatlich maximal 160.000 Seitenzugriffen (Page Impressions) pro Mobilfunkbetreiber. ${ }^{586}$ Diese Zugriffe verteilen sich auf insgesamt 11.000 Einzelbesuche (Visits) mit durchschnittlich ca. 15 Seitenaufrufen pro Besuch. Für das Blättern in Seiteninhalten wird ein dreistufiges Abonnementmodell mit unbegrenzter Nutzungsmöglichkeit während der jeweiligen Abonnementfrist angeboten: Das Abonnement einer einstündigen Seitennutzung kostet derzeit (exklusive Gebühren für den Datentransfer) 0,49 EUR, ein einwöchiges Abonnement 1,49 EUR und das Monatsabonnement 3,99 EUR (womit das Monatsabonnement 0,61 EUR günstiger als die gedruckte Ausgabe des Playboy Magazins ausfällt). Wallpaper und Spiele wurden im selben Zeitraum ca. $32.000 \mathrm{Mal}$, Videos dagegen derzeit lediglich ca. 1.200 Mal abgefragt. Für den Download eines Wallpapers werden 1,99 EUR berechnet, ein ca. 90 KByte großer Videoclip kostet 2,49 EUR.

Playboy Mobile erwirtschaftete im Kalenderjahr 2004 einen Jahresumsatz von ca. 800.000 EUR und repräsentiert damit derzeit den größten Umsatzträger im Dienstportfolio von TFNM. Üblicherweise vereinbaren Mobilfunkbetreiber und TFNM eine Gesamtlizenz für alle vier Produktbereiche von Playboy Mobile in Form einer Mindestumsatzgarantie. Für nationale Exklusivlizenzen wie derzeit im Falle des österreichischen Mobilfunkbetreibers H3G wird eine zusätzliche Lizenzgebühr berechnet. Mit der Mindestlizenzgebühr werden tatsächlich in Anspruch genommene Leistungen verrechnet. Überinanspruchnahmen werden zusätzlich an TFNM vergütet während eine Unterschreitung der Garantiesumme aus Sicht von TFNM folgenlos bleibt. Die Umsätze verteilen sich auf Wertschöpfungspartner und Kunden dem Grundsatz nach wie folgt:

- Der Mobilfunkbetreiber zahlt einen Umsatzanteil von ca. $50 \%$ an TFNM aus. Davon verbleiben i.d.R. 40\% Umsatzanteil bei TFNM selbst, 60\% werden an den Lizenzgeber Playboy Deutschland ausgezahlt. Dadurch verbleibt ein Nettoerlös von $20 \%$ des erzielten Umsatzes bei TFNM, wo-

586 Die Zahl bezieht sich auf Januar 2005 im Netz von Vodafone. Im Mobilfunknetz von 02 wurden im selben Monat knapp 60.000 Zugriffe, verzeichnet, bei T-Mobile 45.000 und bei E-Plus 6.000 . 
durch im Regelbetrieb eine Gewinnmarge von ca. 15\% erwirtschaftet wird.

- Mehr als 50\% der derzeitigen Umsätze werden mit Kunden des Mobilfunkbetreibers Vodafone erzielt, die weiteren Umsatzanteile verteilen sich zu gleichen Teilen auf die Mobilfunknetze von $\mathrm{O} 2$ und T-Mobile sowie ein kleinerer Anteil auf das Mobilfunknetz von E-Plus.

Neben den aus dem operativen Geschäft stammenden Umsätzen erzielt TFNM mit aktionsbezogenen Demonstrationsanwendungen, bspw. Show Cases für Telekommunikationsunternehmen auf Großmessen, zusätzliche Einnahmen in Höhe von jeweils 80.000-100.000 EUR p.a.

\subsection{Ausblick}

Bereits in der Vergangenheit testete TFNM mehrfach eine breitere Positionierung der mobilen Playboy Angebote, bspw. durch das verstärkte Angebot textgestützter Inhalte in Form von Auszügen aus Reportagen oder Interviews, jedoch mit vergleichsweise geringem Erfolg. Dies führte zu der Schlussfolgerung, dass die derzeitige Nutzerschaft mobiler Anwendungen stabil entsprechend der derzeit erfolgreichen inhalteorientierten Anwendungen im mobilen Internet um aktuelle Nachrichten, Unterhaltungsangebote (insbesondere Klingeltöne) und Erotik-/Lifestyleangebote gruppiert ist. Ausgehend von der Annahme einer gewissen zeitlichen Stabilität der Nachfrage in diesen Bereichen zielen zukünftige Strategien von TFNM für Playboy Mobile auf zwei Bereiche: zum einen, der Ausbau des Angebots im Bereich von Download- und Streaminginhalten und zum anderen, der Erhöhung der Kundenverweildauer durch Community Angebote wie Chat, Foren und Instant Messaging. Zudem erwartet TFNM einen mittelfristigen Rückzug der Mobilfunkbetreiber aus dem Geschäft für mobile Inhalteangebote, wodurch die Notwendigkeit entsteht, eigene Bezahlungs- und Abrechnungslösungen zu entwickeln. Besondere Bedeutung wird in diesem Zusammenhang dem zukünftigen Angebot sog. „Downloadable Clients“ beigemessen. Als Downloadable Client wird die Installation von Sofwareanwendungen über das Internet verstanden, die selbst Inhalte automatisch über selbständig aufgebaute Internetverbindungen beziehen können, wodurch ein geschützter und für den Kunden in Bezug auf die technische Komplexität unsichtbarer Inhaltedistributionskanal unabhängig von bestehenden WAP- und Downloadlösungen entsteht.

\subsubsection{Fallbeispiel 2: t-info SMS}

T-info SMS ist ein ortsbezogener Verzeichnisdienst, der durch das deutsche Unternehmen t-info angeboten wird. Das Unternehmen t-info repräsentiert einen der Pioniere im Angebot ortsbezogener Dienste und gilt als marktführend im Angebot von Verzeichnisdiensten. Verzeichnisdienste repräsentieren eine Variante informationsorientierter mobiler Dienste. t-info ist eine Tochtergesellschaft der Deutschen Telekom AG. Das Unternehmen wurde im Mai 2001 als $\mathrm{GmbH}$ mit Sitz in München gegründet. Derzeit werden 24 Mitarbeiter beschäftigt. Geschäftsgegenstand von t-info bildet die online- und speziell mobilitätsge- 
rechte Vermarktung von Informationen aus Branchen- und Personenverzeichnissen. Die Quelle der vermarkteten Verzeichnisinformationen sind Inhalte der Verzeichnisse „Gelbe Seiten“, „Das Örtliche“ und „Das Telefonbuch“ sowie Informationen von spezialisierten Informationsdienstleistern. t-Info bearbeitet ausschließlich den deutschen Sprachraum. Zum Leistungsumfang des Unternehmens im mobilen Internet gehören einerseits Dienste, die unter eigener Marke in allen bestehenden Mobilfunknetzen vertrieben werden und andererseits die Vermarktung von Dienstleistungen in Zusammenhang mit ortsbezogenen Angeboten sowie der Betrieb fremder Portaldienste für Dritte. Im vergleichsweise jungen Marktsegment ortsbezogener Dienste werden Verzeichnisinformationen in verschiedenen Formaten angeboten, wobei der ortsbezogene Verzeichnisdienst t-info SMS das derzeit erfolgreichste Angebot darstellt. Dieser Dienst ist Gegenstand der nachfolgenden Fallstudie. Der Aufbau der Fallstudie folgt dem bereits bekannten Muster: In Abschnitt 5.2.3.2.1 werden Nutzenund Produktmodell von t-info SMS untersucht. In Abschnitt 5.2.3.2.2 werden Besonderheiten des Wertschöpfungsmodells dargestellt. In Abschnitt 5.2.3.2.3 wird das Erlösmodell erläutert. Abschnitt 5.2.3.2.4 enthält einen Ausblick.

\subsection{Nutzen- und Produktmodell von t-info SMS}

Das Leistungsangebot von t-info im mobilen Internet hat zwei Schwerpunkte: Zum einen werden Dienste unter eigener Marke betrieben, mit dem Ziel, mobilen Kunden einen Mehrwert durch Informationen über Gegebenheiten im geografischen Umkreis des Aufenthaltsorts zu bieten. Zum anderen fungiert t-info als Dienstleister zur Bereitstellung ortsbezogener Marketingdienste. Ein realisiertes Anwendungsszenario bildet ein Filialsuchdienst, der für Handelsunternehmen mit verzweigtem Niederlassungsnetz bereitgestellt und betrieben wird, den diese Unternehmen in eigener Verantwortung bewerben. Vor dem Hintergrund der Klassifikation mobiler Dienste in Kapitel 2 ist das Leistungsportfolio von t-info im mobilen Internet daher sowohl im Bereich des Endkundengeschäfts als auch der Transaktionsunterstützung angesiedelt. Im Folgenden wird ausschließlich das Angebot von t-info im Bereich des Endkundengeschäfts am Beispiel der Anwendung t-info SMS untersucht.

t-info SMS repräsentiert eine Pionieranwendung im Bereich ortsbezogener mobiler Dienste. Der Dienst wurde im Oktober 2004 in den Markt eingeführt. Aus Sicht von t-info werden mit dem Angebot ortsbezogener Verzeichnisdienste zwei Ziele verfolgt: Zum einen soll frühzeitig im Marktsegment ortsbezogener Anwendungen eine vertrauenswürdige Marke aufgebaut werden. Zum anderen repräsentiert das mobile Angebot von Verzeichnisinformationen eine crossmediale Mehrfachverwertung der im Konzern der Deutschen Telekom im Zusammenhang mit Branchen- und Telefonverzeichnissen bestehenden Verzeichniskompetenzen. Mit dem Dienst werden zwei Informationsbedürfnisse mobiler Nutzer adressiert: die Bestimmung des aktuellen Aufenthaltsorts und die Identifizierung nahe gelegener Einrichtungen von besonderem Interesse. Beispiele solcher potenziell interessanter Einrichtungen bilden etwa Tankstellen, Apotheken, Geldautomaten, besondere Gebäude oder Veranstaltungen. Derzeit werden insgesamt 17 verschiedene Inhaltekategorien zur Auswahl gestellt, anhand 
derer eine ortsbezogene Suche vorgenommen werden kann. Eine Transaktion des t-info SMS dienst ähnelt dabei einer Suchmaschinenabfrage, erweitert jedoch das Interaktionsmodell einer konventionellen Onlinesuchabfrage einerseits um die Lokalisierung des Kunden und andererseits um die Integration SMS-Nachrichten als Trägerformat für Anfragen und Antworten. Insgesamt umfasst eine Transaktion sieben Teilschritte (vgl. Abbildung 5-11):

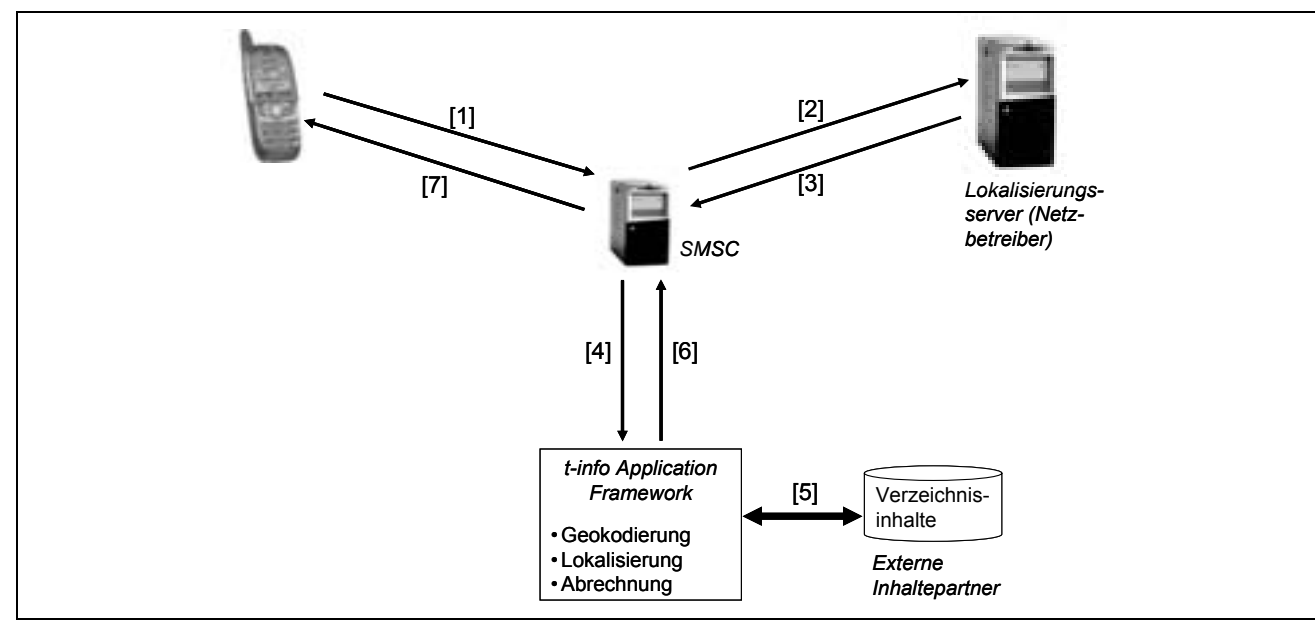

Abbildung 5-11: Transaktionsmodell von t-info SMS

Ein Kunde sendet eine SMS-Nachricht mit einem Schlüsselbegriff, bspw. „Apotheke", an die Kurzwahlnummer 84636, die in allen deutschen Mobilfunknetzen eingerichtet wurde [1]. Die Nachricht wird durch einen spezialisierten Dienstleister, ein sog. SMS-Center (SMSC), empfangen und eine Lokalisierungsabfrage an den jeweiligen Netzbetreiber des Kunden gesendet [2]. Die empfangenen Koordinaten [3] werden zusammen mit dem Suchbegriff des Kunden in anonymisierter Form an einen speziellen Anwendungsserver von t-info gesendet [4]. Anhand der Aufenthaltskoordinate prüft ein Anwendungssystem die Geokodierung der jeweils für den Suchbegriff relevanten Verzeichnisinformationen kooperierender Inhalteanbieter [5], wählt das am nahesten gelegene Objekt aus und sendet die Verzeichnisangaben zurück an das SMSC. Dieses versendet eine Antwort-SMS an den Kunden.

Die zentrale Mehrwert stiftende Komponente in einem jeden Transaktionsvorgang bildet das von t-info betriebene Application Framework. Das Framework repräsentiert eine Individualsoftware, die mit einer insgesamt zweijährigen Entwicklungsdauer fremdentwickelt wurde. Das Framework besteht aus drei Schichten (vgl. Abbildung 5-12): In der Diensteschicht werden Lokalisierungsdaten von Nutzeranfragen verarbeitet und geokodierte Verweise für Verzeichnisinhalte von Drittanbietern gebildet und zugeordnet. Auf der Anwendungsebene werden die jeweiligen Dienstfunktionen für eigen- oder fremdvermarktete Endkundenanwendungen vorgehalten. Auf der Distributionsebene werden die abgerufenen Dienste entsprechend den gewünschten Distributionsformaten kodiert und für das jeweilige Endgerät gerendert. 


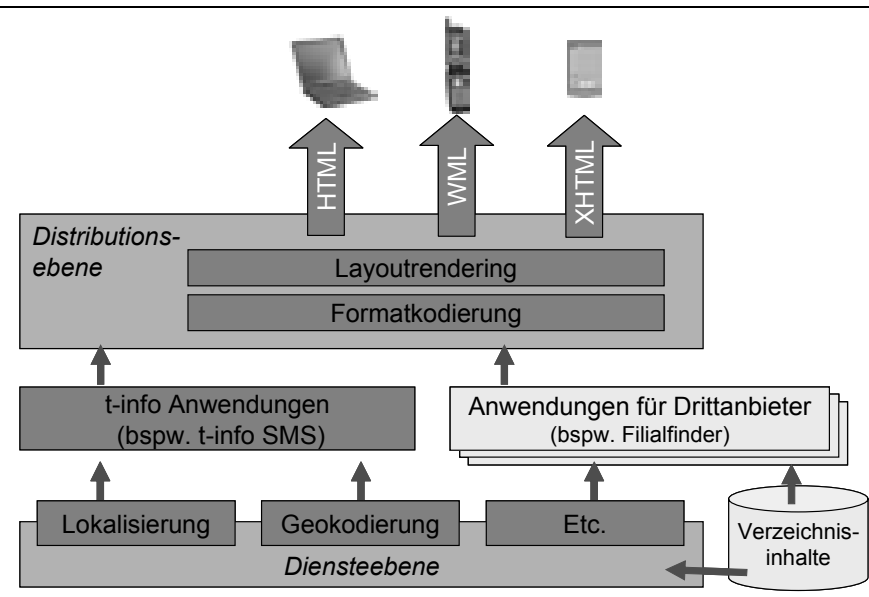

Abbildung 5-12: Architekturmodell des Anwendungsystems von t-info

\subsection{Wertschöpfungsmodell von t-info SMS}

An einer Transaktion von t-info SMS sind insgesamt fünf Wertschöpfungspartner beteiligt:

- Der Mobilfunkbetreiber besitzt vier Funktionen: Er sorgt erstens für den Versand der Abfrage- und Antwortnachricht zwischen SMSC und Endkunde. Zweitens rechnet der Netzbetreiber den Preis der Abfragenachricht, die in Form einer sog. Premium-SMS zu einem deutlich höheren Tarif als herkömmliche Nachrichten zwischen Endkunden versendet wird, abzüglich seiner Abwicklungs- und Abrechnungsprovision mit t-info ab. Drittens stellt der Netzbetreiber in Abstimmung mit den übrigen deutschen Netzbetreibern eine für jedes Mobilfunknetz gültige Kurzwahlnummer für den SMS-Dienst bereit. Die Servicenummernverwaltung erfolgt dabei kooperativ zwischen allen Netzbetreibern, wobei jeder Netzbetreiber jeweils die Verantwortung für bestimmte Ziffern der Kurzwahlnummernkombination, sog. „Gassen“, die Verantwortung trägt. Viertens öffnet der Netzbetreiber eine Schnittstelle zu seinem Lokalisierungssystem für Abfragen durch den SMSC, deren Nutzung t-info transaktionsabhängig in Rechnung gestellt wird.

- Das SMS-Center ist ein spezialisierter Wireless Application Service Provider, der für die Abwicklung der SMS-Nachrichten sorgt. Im Rahmen einer Transaktion übernimmt dabei das SMSC vier Aufgaben: Es wickelt die SMS-gestützte Kommunikation mit dem Endkunden ab, führt die Abfrage der Lokalisierungsdaten beim Netzbetreiber durch, versendet Abfrage- und Lokalisierungsdaten in anonymisierter Form an den t-infoServer und kodiert die zurück erhaltenen Verzeichnisdaten in SMSgerechtem Format.

- Als Dienstbetreiber besitzt t-info ebenfalls vier Aufgaben. Das Unternehmen beauftragt den Betrieb des eigenen Anwendungssystems durch Rechenzentren (als Dienstleister für den Betrieb fungiert dabei die Konzern- 
schwester T-Systems), schließt Kooperationsverträge mit Anbietern von Verzeichnisinhalten und sorgt für Geokodierung und Qualitätssicherung dieser Inhalte.

- Die im Rahmen des Dienstes verwerteten Verzeichnisinhalte werden durch spezialisierte Inhalteanbieter bereitgestellt. Dabei lassen sich zwei Gruppen solcher Inhalteanbieter unterscheiden: die (insgesamt über 100) Verlage der herkömmlichen Verzeichnisdruckwerke wie „Das Telefonbuch“, „Gelbe Seiten“ oder „Das Örtliche“ sowie Anbieter von Spezialinhalten wie Adressen von Geldautomaten oder Notapotheken. Die Inhalteanbieter ermöglichen t-info entweder Zugriff auf die eigene Datenbank oder stellen Inhalte in Form von Datenbankexporten zur Verfügung. Die Abrechnung der Inanspruchnahme erfolgt transaktionsabhängig.

\subsection{Erlösmodell von t-info SMS}

t-info SMS wird gegenüber Endkunden als sog. „Premium-SMS“ angeboten, deren Tarif von EUR 0,69 die Kalkulationsgrundlage des Erlösmodells bildet. Dem Grundsatz nach verteilt sich der Tarif auf die jeweiligen Wertschöpfungspartner wie folgt:

- Für die Abwicklung des Nachrichtenverkehrs und Abrechnung der Transaktion erheben Mobilfunkbetreiber einen Umsatzanteil zwischen EUR 0,30 und 0,40 je Transaktion. Darüber hinaus ist die Einrichtung der exklusiven Servicekurzwahl an eine Mindestabsatzmenge geknüpft, im Falle von deren Unterschreitung die Differenz durch den Dienstanbieter ausgeglichen werden muss. Eine derartige vertragliche Regelung ist dabei von t-info mit allen der insgesamt zehn deutschen virtuellen und faktischen Mobilfunknetzbetreiber geschlossen.

- Für die Inanspruchnahme der Lokalisierungsinformationen der Netzbetreiber werden je Transaktion ca. EUR 0,10 an den jeweiligen Netzbetreiber entrichtet. Eine entsprechende Vereinbarung ist mit allen vier (faktischen) Mobilfunkbetreibern geschlossen.

- Die SMS-gestützte Antwortnachricht, die die gewünschten Verzeichnisinformationen enthält, kostet je nach Netzbetreiber zwischen EUR 0,07 und EUR 0,10.

- Abzüglich der Verrechnungspreise aller Vorprodukte verbleibt im Unternehmen ein Bruttodeckungsbeitrag zwischen EUR 0,12 und EUR 0,17 je Transaktion, wovon der operative Anwendungsbetrieb und die von externen Anbietern zur Verfügung gestellten Verzeichnisinhalte finanziert werden müssen.

Die t-info $\mathrm{GmbH}$ unterliegt derzeit keiner externen Berichtspflicht, weshalb vor dem Hintergrund des derzeit sehr kompetitiven Wettbewerbsumfelds im Bereich mobiler Verzeichnisdienste keine Umsatz- oder Absatzzahlen bekannt gegeben werden. In der gesamten Gattung der mobilen Verzeichnisdienste ist bei t-info allerdings von einem Transaktionsvolumen in Höhe von mehreren Millionen Einzeltransaktionen pro Monat auszugehen. 


\subsection{Ausblick}

Für den Telekommunikationskonzern Deutsche Telekom repräsentiert das Segment mobiler Verzeichnisdienste einen zukunftsweisenden Markt, von dem zukünftig ein signifikantes Wachstum erwartet wird. Als Treiber dieser Entwicklung werden vor allem in Zusammenhang mit der Technikevolution von GSM zu UMTS zu erwartende Preissenkungen, insbesondere aufgrund einer Ablösung des transaktionsbezogenen Preismodells durch ausschließlich volumenbezogene Datentarife eingeschätzt. Als Fristigkeit für die Ablösung des SMS-gestützten Dienstmodells durch weiterentwickelte Zugangsund Distributionsformate wird ein Zeitraum von maximal vier Jahren erwartet. Dabei verfolgt t-info langfristig die Vision einer Integration verschiedener Assistenzdienstleistungen, die komplementär zur Lokalisierbarkeit des Mobilfunknutzers sind, zu einem intelligenten Dienstleistungsprogramm. Denkbare Szenarien bilden etwa die Kombination von individuellen Informationen zu Boden- oder Luftbelastung, Vitalfunktionen, Kleinanzeigen, Routenplanung oder Verkehrsinformationen. Als Wettbewerber im Hinblick auf die Entwicklung einer signifikanten Akzeptanz solcher Assistenzdienste gelten vor allem Suchmaschinenbetreiber aus dem stationären Internet, die wie bspw. das amerikanische Unternehmen Google, das laut Presseberichten ${ }^{587}$ ebenfalls den Aufbau von Kompetenzen im Bereich intelligenter, ortsbezogener Suchfunktionen planen.

\subsubsection{Fallbeispiel 3: V-Card (12snap)}

Die Firma 12snap ist ein Dienstleister im Bereich mobiler Datendienste. 12snap wurde 1999 als $\mathrm{GmbH}$ in Deutschland mit Firmensitz in München gegründet. Weitere Niederlassungen wurden in Italien, Großbritannien, Österreich, Schweden und den USA eröffnet. Nach Ablösung des ursprünglichen Geschäftsmodells auf der Grundlage eines mobilen Auktionsdienstes im Jahr 2001 positioniert sich 12snap heute als Agentur für mobile Werbekampagnen. Das Unternehmen beschäftigt in Deutschland 45 und weltweit insgesamt 60 Mitarbeiter. Zu den Kunden gehört u.a. Mcdonald's, Wrigley, Adidas, Sony und L'Oréal. 12snap gilt als marktführend im Bereich mobiler Werbedienste.

\subsection{Produkt- und Nutzenmodell von V-Card}

Das V-Card Projekt ist ein gemeinsames Forschungsvorhaben der Firmen 12snap und Lucent Technologies. Die Entwicklung des V-Card Projekts wird nach einer deutschlandweiten Ausschreibung zum Thema Mobilmedia des Bundesministeriums seit Oktober 2002 vom Bundesministerium für Wirtschaft und Technologie gefördert. Förderungsziel ist es dabei, durch attraktive und leicht zugängliche Inhalte und Dienste eine schnelle Verbreitung und Verwertung mobiler Multimediadienste in Deutschland zu erreichen. Der Schwerpunkt von Lucent Technologies im V-Card Projekt liegt in der Integra-

587 Vgl. Heise (2005). 
tion und dem Test und der funktionalen Optimierung der Applikation und der zugrunde liegenden Infrastruktur. 12snap tritt als Entwickler der V-Card Applikation auf.

Die Applikation V-Card ist ein multimedialer Veredlungsdienst für textbasierte mobile Kurznachrichten zwischen verschiedenen Endnutzern eines Mobilfunknetzes. Dabei wird entweder eine Textnachricht per SMS oder eine Bildnachricht per MMS oder über das Internet an die Adresse des Dienstanbieters versendet. Der Absender gibt dabei die Telefonnummer des Endempfängers an und wählt ein Veredelungsverfahren aus. Denkbare Beispiele einer solchen Veredelung bilden das Verknüpfen einer Textbotschaft in Verbindung mit einem Audio- oder Videoclip. Kern der Applikation bildet das so genannte V-Card Hub, in dem die Nachricht des Absenders mit den ausgewählten multimedialen Inhalten wie Klänge, Animationen oder Sprachbotschaften kombiniert und schließlich an den Empfänger versendet wird.

Die Infrastruktur der V-Card Applikation besteht im Wesentlichen aus drei Komponenten (vgl. Abbildung 5-13): einem Short Message Service Center (SMSC), dem Applikationshub und einem Multimedia Message Service Center (MMSC). Das SMSC ist ein GSM-typisches Vermittlungsgerät, das innerhalb oder außerhalb der vom Netzbetreiber verwalteten Netzinfrastruktur betrieben wird und das die Vermittlung von Kurznachrichten an die jeweiligen Adressaten vornimmt. Anhand einer speziellen Servicenummer für den VCard Dienst wird das SMSC des Dienstanbieters erkannt. Dieses überträgt die Daten der Textnachricht weiter an das Applikationshub. Dort wird die Veredlung der Nachricht in Form einer Ergänzung der Textnachricht um audio-visuell Elemente wie einer Animation oder einem persönlichen Sprachgruß. Die Auslieferung der Nachricht an den Empfänger erfolgt auf der Grundlage der GPRS-Infrastruktur des Netzbetreibers. Zwei Varianten für Gestaltung des Dienstes sind dabei grundsätzlich denkbar: Die Auslieferung erfolgt als Pull-Dienst durch den Empfänger auf der Basis einer WAPAbfrage oder als Push-Dienst in Form einer MMS. Aufgrund der in Verbindung mit den derzeit noch hohen Gebühren in Mobilfunknetzen absehbaren Anreizproblematik für potenzielle Empfänger in Bezug auf einen Pulldienst, wird bei 12snap primär ein Push-Konzept fokussiert. Dazu ist ein sog. Multimedia Message Service Center (MMSC) erforderlich, ein Vermittlungsgerät ähnlich einem SMSC, das die veredelte Nachricht an die Empfängerrufnummer übermittelt. Ein MMSC steht typischerweise mit einem WAP-Server in Verbindung, da bspw. Konfigurationseinstellungen und Abfragen im WAPDialog vollzogen werden. Gegenüber reinen GSM-Infrastrukturen erfolgt die Auslieferung in GPRS-fähigen Netzen über zwei weitere Vermittlungsgerätetypen, einem Serving GPRS Support Node (SGSN) und einem Gateway GPRS Support Node (GGSN), die als Router der Datenpakete dienen. 


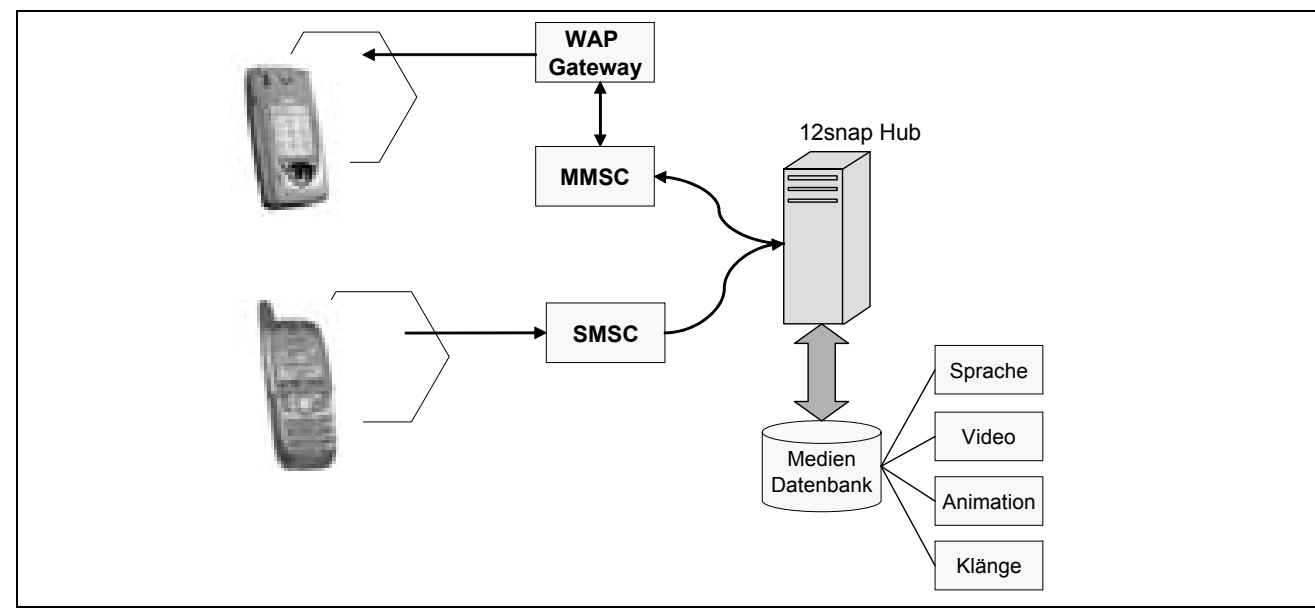

Abbildung 5-13: Technische Architektur von V-Card

\subsection{Wertschöpfungsmodell der V-Card}

Für den Betrieb der V-Card Applikation sind generell zwei Varianten denkbar: Die Lizenzierung der Applikation an Netzbetreiber oder firmenfremde Dienstanbieter und der Eigenbetrieb. Insofern 12snap die Auswahl interessanter und für Empfänger attraktive Inhalte als kritisch für den Erfolg der V-Card erachtet, wird in Bezug auf das Angebot der V-Card primär das Ziel eines Full-Service Angebots in Eigenbetrieb verfolgt. An einem solchen Eigenbetrieb sind dennoch mindestens drei Kooperationspartner beteiligt:

Der Mobilfunknetzbetreiber sorgt für die ordnungsgemäße Adressierung von Nachrichten, die von seinen Mobilfunkkunden oder an diese versendet werden. Die Ausführung der Versende- und Empfangsoperationen wird von einem oder mehreren spezialisierten Anbietern (SMSC/MMSC) durchgeführt und überwacht. 12snap übernimmt schließlich im Rahmen des Angebots der V-Card eine zweifache Funktion: Erstens, die Bündelung der Nachricht und einer Werbebotschaft im Sinne eines Marketingdienstleisters für Kunden aus dem Konsumgüterbereich. Aufgrund der multimedialen Veredelung kann die V-Card unter anderem als Träger einer mit der Nachricht verknüpften Werbebotschaft dienen. Als denkbare Vermarktungsformen wird dabei ein Sponsoring des Dienstes durch Werbetreibende gesehen. Zweitens, die Bündelung von Nachricht und Veredelungsinhalten. 12snap wird dabei sowohl eigene Inhalte, bspw. Animationen oder Geräusche, als auch fremde Inhalte, bspw. Musikclips, anbieten.

\subsection{Erlösmodell der V-Card}

Kalkulationsgrundlage des Erlösmodells der V-Card bildet ein Premiumtarif von EUR 1,36 je Transaktion. Diese Transaktionserlöse verteilen sich auf die Wertschöpfungspartner wie folgt: Für den Nachrichtenversand von SMS- oder MMSNachricht (kleiner als $30 \mathrm{kB}$ ) durch den jeweiligen Transaktionsinitiator erhebt der Mobilfunkbetreiber einen Erlösanteil von EUR 0,34 und für den Versand der 
veredelten Botschaft (kleiner als 100kB) erneut einen Erlösanteil EUR 0,45. Zusätzlich muss an den SMS/MMS-Dienstleister ein Erlösanteil in Höhe von EUR 0,12 entrichtet werden. Somit verbleibt ein Bruttoerlösanteil je Transaktion in Höhe von EUR 0,45 im Unternehmen.

Grundsätzlich sind zwei unterschiedliche Abrechnungsverfahren des Dienstes denkbar: ein direktes und ein indirektes Modell. Im Falle des direkten Abrechnungsmodells wird der vollständige Tarifumfang dem jeweils transaktionsauslösenden Kunden in Rechnung gestellt. Vor dem Hintergrund des hohen Preisniveaus scheint dieses Konzept für ein rein unterhaltungsorientiertes Angebot jedoch in der Breite des Marktes schwierig durchsetzbar. Erfolgversprechender erscheint dagegen ein indirektes Abrechnungsmodell. Dabei wird eine von Endkunden initiierte Transaktion entweder in vollem Umfang oder zumindest anteilig am Gesamttarif von Werbekunden finanziert. Denkbar ist eine Integration von Werbeinhalten in die veredelte Nachricht, bspw. in Form eines Verweises auf den Sponsor. Die Abrechnung mit dem Werbekunden erfolgt dabei ebenso wie beim direkten Abrechnungsmodell transaktionsabhängig.

\subsection{Ausblick}

Ziel der V-Card ist das Einführungsangebot eines multimedialen Dienstes für MMS-fähige Endgeräte. Wesentliche Erfolgsfaktoren bilden einerseits die geringen Nutzungshürden insofern Standardhandsets ohne zusätzliche Clients verwendet werden. Andererseits lässt die Kernkompetenz von 12snap im mobilen Marketing sinnvolle Sponsoring-Kooperationen und damit für Endkunden attraktive Erlösmodelle erwarten, die eine hohe Nutzungswahrscheinlichkeit garantieren. Das Kooperationsprojekt, im Rahmen dessen der Dienst V-Card entwickelt wurde, wurde im Juli 2004 mit einem erfolgreichen Feldtest abgeschlossen. Vor einer geplanten Markteinführung werden derzeit rechtliche Fragen des praktischen Einsatzes der V-Card erörtert. Derartige rechtliche Fragestellungen sind etwa die Anwendung des Trennungsgebotes von Werbung und Inhalt auf die V-Card und die Gewährleistung einer Möglichkeit des Empfängers zur Einwilligung gegenüber einem V-CardEmpfang zur Vermeidung von SPAM.

\subsubsection{Zusammenfassung der Geschäftsmodellanalyse}

In Abschnitt 5.2.1 wurden drei Partialmodelle, die im Mittelpunkt einer Analyse von Geschäftsmodellen stehen, identifiziert: Das Produkt- und Nutzenmodell, das Wertschöpfungsmodell und das Erlösmodell. Abbildung 5-14 gibt einen zusammenfassenden Überblick über die erläuterten Besonderheiten in den drei Partialmodellen der vorgestellten Geschäftsmodellbeispiele. 


\begin{tabular}{|c|c|c|c|}
\hline $\begin{array}{r}\text { Fallbeispiel } \\
\text { Partialmodell }\end{array}$ & Playboy Mobile & t-info SMS & 12snap V-Card \\
\hline Nutzenmodell & $\begin{array}{l}\text { Ortsunabhängige Erotikinhalte für } \\
\text { technikaffine Zielgruppe }\end{array}$ & Ortsbezogener Auskunftdienst & $\begin{array}{l}\text { Kommunikationsorientierter } \\
\text { Multimediadienst }\end{array}$ \\
\hline Wertschöpfungsmodell & $\begin{array}{l}\text { Wertschöpfungskooperation mit: } \\
\text { - Mobilfunkbetreibern } \\
\text { - Playboy Deutschland } \\
\text { - Optional: Technische Dienstleister } \\
\text { und Werbepartner }\end{array}$ & $\begin{array}{l}\text { Wertschöpfungskooperation mit: } \\
\text { - Mobilfunkbetreibern } \\
\text { - SMS-Center } \\
\text { - Verlage von Verzeichnisdrucken } \\
\text { - Anbieter von Spezialinhalten } \\
\text { (Banken, Apothekerverband, etc.) }\end{array}$ & $\begin{array}{l}\text { Wertschöpfungskooperation mit: } \\
\text { - Mobilfunkbetreibern } \\
\text { - SMS-/MMS-Center }\end{array}$ \\
\hline Erlösmodell & $\begin{array}{l}\text { - Pay-per-Use } \\
\text { - Abonnement }\end{array}$ & Pay-per-Use & $\begin{array}{l}\text { - Pay-per-Use } \\
\text { - Anteilige Werbefinanzierung }\end{array}$ \\
\hline
\end{tabular}

Abbildung 5-14: Zusammenfassender Überblick über die vorgestellten Geschäftsmodelle

Als zentrale Bedingung des Erfolgs eines Inhalteangebots im mobilen Internet wurde die Notwendigkeit bestimmt, durch das Dienstangebot im Rahmen des Nutzenmodells eine Form des Kundennutzens zu schaffen, der bislang von keinem anderen Inhaltedistributionsformat geboten wird. Es wurden drei potenzielle Auslöser für die Schaffung eines derartigen Kundenmehrwerts im mobilen Internet identifiziert: Ortsunabhängigkeit, Ortsspezifität und Kommunikationsorientierung von Inhalten. Es wurde für jeden dieser Mehrwertfaktoren jeweils ein Beispiel für eine erfolgreiche praktische Ausschöpfung des Mehrwertpotenzials gegeben:

- Das Fallbeispiel Playboy Mobile macht deutlich, dass die ortsunabhängige Inhaltenutzung gewissen Konsumentengruppen wie Erotikinteressierten einen Mehrwert bietet, der einen kommerziellen Erfolg von Angeboten im mobilen Internet ermöglichen kann. Vor dem Hintergrund des bislang fehlenden kommerziellen Erfolgs mobiler Dienste im Massenmarkt wirft das Beispiel allerdings die Frage auf, ob und ggf. welchen weiteren Konsumentengruppen mit einem jeweils bedürfnisgerechten Inhalteangebot ein Mehrwert geboten werden kann.

- Das Fallbeispiel t-info SMS zeigt des Weiteren, dass ortsbezogene Auskunftsdienste ein gewisses Marktpotenzial besitzen. Es ist allerdings die Frage aufzuwerfen, inwiefern etablierte Inhalteanbieter im Medienmarkt, deren Angebot traditionell tendenziell allgemeininformierender Natur ist, am Erfolg dieses Segments des mobilen Internet beteiligt sein können. Es ist daher zu prüfen, welche der typischen Inhalteangebote der Publikumsmedien das Potenzial besitzen, durch ortsbezogene Distribution einen Mehrwert zu stiften.

- Das Fallbeispiel V-Card zeigt einerseits einen Lösungsansatz für die medientypische Bündelung von Werbe- und Redaktionsinhalten im mobilen Internet. Andererseits besteht das Mehrwertversprechen des vorgestellten Dienstes V-Card darin, asynchrone Kommunikationsvorgänge, wie typischerweise SMS-Nachrichten, mit multimedialen Inhalten anzureichern und dadurch interessanter zu gestalten. Darüber hinaus erlaubt das Erlösmodell der V-Card aufgrund der Tarifsubventionierung durch Werbetreibende einen deutlich geringeren Endkundenpreis als vergleichbare, ausschließlich durch direkte Erlöse finanzierte Angebote im mobilen Internet. Insofern allerdings das Unternehmen 12snap als Anbieter 
der V-Card selbst kaum eigene verwertbare Veredelungsinhalte besitzt, besteht einerseits die Gefahr einer Abhängigkeit von Inhaltelieferanten und andererseits die Gefahr einer Imitation des Dienstkonzepts durch konkurrierende Marktteilnehmer.

Aus wissenschaftsmethodischer Sicht wird zusammenfassend deutlich, dass eine theoriegeleitete Geschäftsmodellanalyse bei der Aufdeckung potenziell erfolgreicher Angebote im mobilen Internet an Grenzen stößt. Zwar konnten Mehrwertpotenziale und Beispiele für deren erfolgreiche Ausschöpfung bestimmt werden. Dennoch bleiben hinsichtlich der Übertragbarkeit und der praktischen Validierung der Analyseergebnisse offene Fragen zurück, die aus dem Unvermögen einer Theoriededuktion herrühren, offene Bedürfnisse und existierende Handlungsmotive von Konsumenten zu identifizieren. Die Frage, ob und inwiefern welche Inhalte für welche Kunden im mobilen Internet mit einem erstrebenswerten Nutzen verbunden sind, für den diese Kunden einen Preis zu zahlen bereit sind, ist daher Gegenstand des folgenden Kapitels.

\subsection{Akzeptanz inhalteorientierter mobiler Dienste}

Die zentrale Bedingung dafür, dass innovative Produkte oder innovative Dienstleistungen - wie die hier fokussierten mobilen Dienste - einen kundenseitigen Mehrwert schaffen, besteht darin, dass die Innovation entweder beim Kunden neue Bedürfniskategorien adressiert oder zumindest bestehende Bedürfnisse auf neuartige oder bessere Weise bedient. Damit ein Medienunternehmen im Zusammenhang mit der Inhalteverwertung im mobilen Internet tatsächlich zusätzliche Einnahmen erzeugen kann, müssen deshalb nicht nur Dienstformate mit technischen Mehrwertpotenzialen entwickelt, sondern auf der Grundlage dieser Formate Inhalte bereitgestellt werden, die existierende Kundenbedürfnisse adressieren. Ob und inwiefern mit derartigen innovativen Anwendungen tatsächlich ein praktischer Mehrwert für einen Konsumenten erzielt werden kann, ist dabei ex-ante für den Anbieter solcher Dienste zwangsläufig mit Unsicherheit behaftet.

Die Identifikation bedürfnisgerechter Anwendungsfelder mobiler Dienste ist für Medienunternehmen in der Praxis mit Problemen verbunden, insofern die inhaltlichen Bedürfnisse, die ein potenzieller Dienstkonsument im Zuge seiner Mobilität entwickelt, bzw. die Motive, die einen Konsumenten zur Nutzung bestehender mobiler Dienstformen veranlassen, bislang nur rudimentär erforscht sind. Der - mit Ausnahme der bereits dargestellten Geschäftsmodellbeispiele in der Masse der Anwendungen bislang ausgebliebene kommerzielle Erfolg inhalteorientierter mobiler Dienste legt allerdings die Vermutung nahe, dass die Mehrheit der Mobilfunknutzer und Inhalterezipienten (noch) keinen Mehrwert durch die Nutzung des mobilen Internet für sich erkennen konnte.

Die Untersuchung von Annahme und Ablehnung innovativer Anwendungen im Allgemeinen sowie die Akzeptanz der hier fokussierten inhalteorientierten mobilen Dienste im Speziellen ist Gegenstand der sog. „Akzeptanzforschung“. Abschnitt 5.3.1 gibt einen Überblick über den derzeitigen Stand der Akzeptanzforschung im Zusammenhang mit kommerziellen Diensten im mobilen Internet. 
Vor dem Hintergrund einer aufgedeckten Forschungslücke im Bereich der Akzeptanz inhalteorientierter Dienste werden in Abschnitt 5.3.2 die Ergebnisse zweier Onlinebefragungen zur Nutzung und zur Akzeptanz mobiler Dienste, die im Zusammenhang mit dieser Arbeit entstanden sind, vorgestellt. Abschnitt 5.3.3 fasst die Ergebnisse der beiden Akzeptanzanalysen zusammen.

\subsubsection{Stand der Forschung zur Akzeptanz mobiler Dienste}

Der folgende Abschnitt gibt einen einführenden Überblick über den Stand der Akzeptanzforschung für mobile Dienste und leitet bestehende Forschungslücken im Zusammenhang mit inhalteorientierten mobilen Diensten ab. Vor dem Hintergrund der existierenden Unschärfe des Akzeptanzbegriffs in der sozialwissenschaftlichen Forschung wird zunächst in Abschnitt 5.3.1.1 eine begriffliche Einführung in die Akzeptanzforschung gegeben. In Abschnitt 5.3.1.2 werden bestehende Forschungsbeiträge zur Analyse der Akzeptanz mobiler Dienste vorgestellt. In Abschnitt 5.3.1.3 werden die Ergebnisse der Akzeptanzforschung für das mobile Internet sowie bestehende Erklärungslücken zusammengefasst.

\subsubsection{Ausprägungen der Akzeptanz als Forschungsgegenstand}

Der Begriff der Akzeptanz wird in der sozialwissenschaftlichen Forschung zur Untersuchung des Erfolgs von Innovationen herangezogen. ${ }^{588}$ Je nach Wissenschaftsdisziplin wird der Akzeptanzbegriff dabei allerdings sehr variantenreich verwendet. ${ }^{589}$ Die Gemeinsamkeit dieser unterschiedlichen Begriffsverwendungen besteht darin, dass Akzeptanz eine Form von zustimmendem Verhalten oder zumindest einer zustimmenden Einstellung von Betroffenen gegenüber einer Innovation zum Ausdruck bringt. Die betriebswirtschaftliche Akzeptanzforschung ist daher als ein Forschungsansatz zu verstehen, der auf der Anwenderseite einer Innovation ansetzt, um Gründe für deren Annahme oder Ablehnung zu identifizieren. Die Akzeptanzforschung unterscheidet in diesem Zusammenhang zwei Aspekte einer Innovation: die Bedingungen ihrer Akzeptanz und ggf. ihre Wirkung (1) sowie die Ausprägungsdimensionen der Akzeptanz beim Kunden (2).

(1) In Bezug auf die Erforschung der Determinanten und der Wirkungen der Annahme einer Innovation existieren drei Grundmodelle zur Erklärung von Akzeptanz: ${ }^{590}$ statische Inputmodelle, Rückkopplungsmodelle und dynamische Modelle. Statische Inputmodelle bzw. Input/Outputmodelle stellen einen Ursache-Wirkungszusammenhang zwischen dem Erfolg einer Innovation und bestimmten Gestaltungsfaktoren her. Als bekannteste Input/Outputmodelle gelten das sog. „Technology Acceptance Model“ von Davis (1989), das Modell von Nielsen (1993) und die Adoptionsfaktoren von Rogers (1995). Davis beschreibt zwei Determinanten der Akzeptanz einer technischen Innovation: ihre Nützlich-

\footnotetext{
588 Vgl. Anstadt (1994); Pressmar (1982); Reichwald (1978).

$589 \mathrm{Vgl}$. Wohlfahrt (2004), S. 27.

$590 \mathrm{Vgl}$. Wohlfahrt (2004), S. 32.
} 
keit und die Einfachheit ihrer Bedienung. Nielsen (1993) erweitert Davis Modell, indem Nützlichkeit und Bedienbarkeit zur Determinante der „Brauchbarkeit“ vereint werden, die neben Kosten, Kompatibilität und Zuverlässigkeit einen Aspekt der praktischen Akzeptanz eines technischen Systems darstellen. ${ }^{591}$ Rogers erweitert wiederum den Aspekt der Brauchbarkeit zur Bedingung eines „relativen Vorteils“ in Relation zur Bedürfnisbefriedigung durch bisher verwendete Produkte oder Leistungen. Zusätzlich identifiziert Rogers die Erprobbarkeit und Wahrnehmbarkeit der neuartigen Eigenschaften einer Innovation als Einflussfaktoren für deren Akzeptanz. Darüber hinaus erkennt Nielsen soziale Einflussfaktoren der Akzeptanz einer Technologie an, bspw. im Zusammenhang mit dem bestehenden gesellschaftlichen Wertesystem. Auf der Grundlage einer dreistufigen Delphistudie konkretisieren Schildhauer et al. (2003) derartige soziale Determinanten der Technologieakzeptanz im Bereich des Internet. Als soziale Einflussfaktoren der Akzeptanz von Internettechnologien werden die allgemeine Faszination der Technik, die Neugier gegenüber konkreten Diensten, die bspw. beruflich bedingte Gewohnheit und die Möglichkeit der Interaktivität mit anderen Individuen ermittelt.

Rückkopplungsmodelle verfolgen zwar ebenso wie Input/Outputmodelle einen statischen Messungsansatz, berücksichtigen jedoch außerdem die Rückwirkung einer Innovationsakzeptanz auf die ursprünglichen Akzeptanzkriterien. ${ }^{592}$ Dynamische Akzeptanzmodelle erweitern schließlich den Messungsrahmen der Akzeptanz in statischen Modellen um die Berücksichtigung zeitlicher Veränderungen. ${ }^{593}$

(2) Im Hinblick auf die Ausprägungsdimensionen der Akzeptanz kann zwischen eindimensionalen und mehrdimensionalen Konzepten unterschieden werden. ${ }^{594}$ Im Rahmen eindimensionaler Akzeptanzkonzepte ist wiederum zwischen verhaltensorientierten und einstellungsorientierten Konzepten zu differenzieren. Beiträge, denen eine verhaltensorientierte Akzeptanzkonzeption zugrunde liegt, unterstellen, dass sich der Grad der Akzeptanz einer Innovation aus dem unmittelbar beobachtbaren Konsumverhalten ablesen lässt. ${ }^{595}$ Daher kann die Akzeptanz einer Innovation anhand des quantitativ messbaren Konsumverhaltens festgestellt werden. Einstellungsorientierte Konzepte gehen dagegen davon aus, dass Akzeptanz lediglich eine gewisse individuelle Reaktionsbereitschaft darstellt, die zwar dem jeweiligen Verhalten zugrunde liegt, sich jedoch nicht unmittelbar in diesem ausdrückt. ${ }^{596}$ Insofern müssen einstellungsorientierte Untersuchungen der Annahme einer Innovation zunächst quantitativ messbare Faktoren ableiten, die einen signifikanten Einfluss auf die jeweilige Verhal-

\footnotetext{
591 Die Bedienbarkeit eines Systems drückt sich dabei u.a. in einer einfachen Erlernbarkeit, effizienter und effektiver Aufgabenerfüllung, geringen Fehlerraten und subjektiver Befriedigung aus.

592 Vgl. Reichwald (1978), S. 32; Silberer/Hannecke (1999).

$593 \mathrm{Vgl}$. Kollmann (1998), S. 87 f.

594 Vgl. Wohlfahrt (2004), S. 27.

$595 \mathrm{Vgl}$. Venkatesh/Davis (2000), S. 186; Govindarajulu/Reithel/Sethi (2000), S. 38.

596 Vgl. Trommsdorff (1975), S. 8; Dierkes (1982), S. 12.
} 
tensbereitschaft besitzen. Vor dem Hintergrund einer gewissen fachlichen Kontroverse zwischen den Vertretern verhaltensorientierter und einstellungsorientierter Akzeptanzkonzepte erkennen mehrdimensionale Akzeptanzkonzepte von vorne herein an, dass für den Erfolg einer Innovation Einstellungs- und Verhaltensdimension der Akzeptanz gleichermaßen gegeben sein müssen, wobei eine auf konkretem Verhalten basierende Akzeptanzmessung nur bedingt auf eine positive Einstellung schließen lässt. ${ }^{597}$ Die Vertreter eines solcher Art verstandenen Akzeptanzbegriffs verwenden üblicherweise eine dreidimensionale Akzeptanzkonzeption (vgl. Abbildung 5-15). ${ }^{598}$ Danach erfordert die erfolgreiche Adoption einer Innovation zunächst die (kognitive) Bekanntheit ihrer potenziellen Nutzer. Die Innovation muss Interesse und eine positive (affektive) Verhaltenseinstellung hervorrufen und schließlich eine konkrete (konative) Kaufentscheidung auslösen. ${ }^{599}$

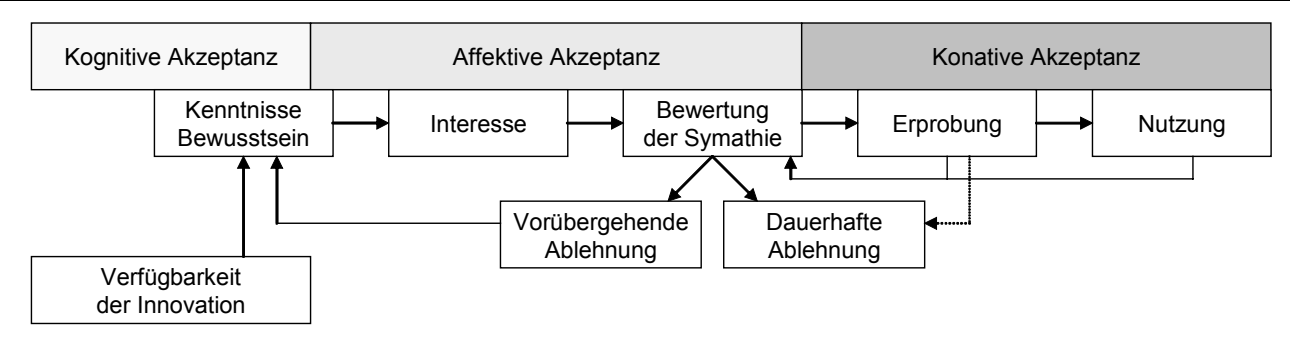

Abbildung 5-15: Mehrdimensionale Akzeptanzkonzeption

\subsection{2 Beiträge zur Akzeptanz mobiler Dienste}

Der folgende Abschnitt fasst die Ergebnisse der bereits existierenden Forschungsbeiträge zur Akzeptanz mobiler Dienste zusammen. Im Rahmen der Veröffentlichungen, die jeweils Erkenntnisse zur Akzeptanz mobiler Dienste enthalten, kommen zwei Untersuchungsmethoden zum Einsatz: Expertenbefragungen bzw. Delphistudien (1) und Kundenbefragungen (2).

(1) Bereits in der in Abschnitt 5.1 angesprochenen Gruppe kommerzieller Grundlagenstudien zur Entwicklung des Geschäfts im mobilen Internet wird das Thema der Nutzerakzeptanz mobiler Dienste vereinzelt angesprochen. Darin präsentierte Prognosen über die zukünftige Akzeptanz beruhen in erster Linie auf Gesprächen mit Fachleuten aus dem Telekommunikations-, IT- oder Medienbereich. Derartige Studien werden daher auch als „Expertenbefragungen“ oder "Delphistudien“ bezeichnet und repräsentieren Ausprägungsformen explorativer Erhebungen ${ }^{600}$, deren Zweck in erster Linie darin besteht,

597 Vgl. Wohlfahrt (2004), S. 30.

$598 \mathrm{Vgl}$. Wohlfahrt (2004), S. 92.

599 Vgl. Müller-Böhling/Müller (1986), S. 23.

600 Explorative Studien sind Ausprägungsformen qualitativer Erhebungen, die dazu dienen, Hypothesen auf induktivem Weg abzuleiten, die dann im Rahmen quantitativer Erhebungen geprüft werden kön- 
Trendaussagen im Zusammenhang mit einem Gegenstandsbereich zu formulieren. In Bezug auf die zukünftige Akzeptanz mobiler Dienste konzentrieren sich die genannten Studien auf die Identifikation sog. „Killerapplikationen“. Als Killerapplikationen werden solche Dienste bezeichnet, die aus Kundensicht besonders attraktiv erscheinen. Wie jedoch bereits Lehner (2003) feststellte, herrscht zwischen den verschiedenen Studien keineswegs Einigkeit darüber, welche Dienstformen das Potenzial einer Killerapplikation besitzen. ${ }^{601}$ Für das Endkundengeschäft werden kommunikationsorientierte Dienste (wie bspw. Email, SMS oder Instant Messaging) und Unterhaltungsdienste (wie Musik, Videos, Computerspiele, Ratgeber oder Glücksspiel) ebenso genannt wie informationsorientierte Dienstformen (bspw. lokale oder standortbezogene Inhalte) und aktuelle Nachrichten oder transaktionsunterstützende Dienste wie Bezahlungsmöglichkeiten und Einkaufsführer ${ }^{602}$ Neben der offensichtlichen Heterogenität benannter Killerapplikationen ist das Vorgehen dieser Studien wissenschaftsmethodisch vor allem deshalb anzuzweifeln, da in der Regel weder die zugrunde liegende Expertenrekrutierung noch die Befragungsergebnisse begründet werden, was die Vermutung einer gewissen Willkür nahe legt. ${ }^{603}$

(2) Vor dem Hintergrund der bislang geringen kommerziellen Bedeutung des mobilen Internet rücken Kundenbefragungen von der reinen Kombinatorik technologischer Mehrwertfaktoren und möglichen Anwendungsfeldern ab und untersuchen Motive von Mobilfunkkunden, die zu einer Dienstnutzung führen bzw. eine solche Nutzung verhindern. Anstelle der qualitativen Natur der voranstehend genannten Experten- und Delphistudien kommen dabei in erster Linie quantitative Analyseverfahren zum Einsatz. Nachfolgend werden die Ergebnisse der vier bekanntesten Kundenbefragungen zur Akzeptanz mobiler Dienste zusammengefasst:

- Eine der ersten Kundenbefragungen zum mobilen Internet führte der finnische Mobilfunktechnikausstatter Nokia (1999) im Hinblick auf die zu erwartende Nachfrage nach mobilen Mehrwertdiensten durch. Die Kundenbefragung, an der 155 finnischen Mobilfunkneukunden teilnahmen, fokussiert weniger die (kognitive) Bekanntheit oder das (konative) Nutzungsverhalten in Bezug auf konkrete Dienstformen, sondern in erster Linie das grundsätzliche (affektive) Interesse an derartigen Mehrwertdiensten. Nokia stellte fest, dass $86,8 \%$ der Befragten zumindest ein geringes Interesse an Mehrwertdiensten besitzen. Das größte Interesse beschieden die Befragten sog. "Infotainment"-Diensten wie Wetternachrichten, Wörterbüchern oder Navigationshilfen sowie transaktionsbezogenen Diensten wie Banktransaktionen, Email oder Pizzabestellung. Unterhaltungsorientierte Dienste wie Computerspiele, Witze oder Glücksspiel sind dagegen nur von geringem Interesse.

nen. Einen Überblick über die empirische Forschungsmethodik geben Martin (1989) und Bortz/Döring (2003).

$601 \mathrm{Vgl}$. Lehner (2003), S. 233.

602 Vgl. bspw. Müller-Veerse (1999); Ovum (2000); TIMElabs (2002).

603 Vgl. Wohlfahrt (2004), S. 47. 
- Ebenfalls auf das affektive Interesse an mobilen Diensten zielt eine Befragung der Unternehmensberatung BCG (The Boston Consulting Group) aus dem Jahr 2000 ab, an der 1633 Personen in Japan, Deutschland, Frankreich, Schweden und den USA in Bezug auf ihre Nutzungsbereitschaft mobiler Dienste teilnahmen. ${ }^{604}$ Der Fokus der Studie liegt auf der Hierarchisierung von Motiven, die eine Nutzung mobiler Dienste determinieren. Die Studie zeigt, dass vor allem rational-kognitive Motive wie die Zeitersparnis bei der Nachfrage nach aktuellen Informationen und der Durchführung von Kommunikationsvorgängen die wesentlichen Determinanten der Nutzung mobiler Dienste bilden. Unterhaltung zur Überbrückung von Nischenzeiten wird dagegen abermals nur geringe Bedeutung beigemessen.

- Im Zusammenhang mit der Erhebung des sog. „Mobinet Index“, einem jährlichen Panel, das die Akzeptanzentwicklung des mobilen Internet erforscht, untersucht die Unternehmensberatung A.T. Kearney neben dem affektiven Interesse zusätzlich einerseits das kognitive Bewusstsein mobiler Nutzer gegenüber der Technik und dem Angebot des mobilen Internet und andererseits die tatsächlich zu beobachtende (konative) Nutzung mobiler Dienste. Die dem Panel zugrunde liegende Befragung wird jährlich seit 2000 durchgeführt und adressierte zuletzt 5.600 Mobilfunkkunden in 15 Ländern. Die Studie belegt eine wachsende Bereitschaft der Nutzer, Onlinedienste über mobile Endgeräte zu nutzen, wobei MMSDienste die Beliebtheit dominieren. ${ }^{605}$ Die Ergebnisse der nachgewiesenen Nutzung unterscheiden sich dabei deutlich von jenen der zuvor genannten Studien, insofern das mobile Internet in erster Linie zu Unterhaltung, jedoch lediglich bestenfalls eingeschränkt zur Befriedigung von Informationsbedürfnissen und zur Durchführung von Transaktionen verwendet wird.

- Eine der ersten wissenschaftlichen Auseinandersetzungen mit dem Problem der Nutzerakzeptanz mobiler Dienste im deutschsprachigen Raum findet sich bei Wohlfahrt (2004). In einer mehrdimensionalen Faktoranalyse untersucht Wohlfahrt sowohl die Determinanten als auch die Wirkung der Nutzung mobiler Dienste. Dabei wird sowohl eine Anbieterperspektive anhand einer Stichprobe von 22 befragten Unternehmen als auch eine Nachfragersicht anhand einer Stichprobe von 1004 Teilnehmern geprüft. ${ }^{606}$ Auf Unternehmensebene wird gezeigt, dass sich zwar eine mangelnde Unternehmenserfahrung im Mobilfunk auf den Erfolg des Angebots mobiler Dienste auswirken kann, jedoch nur eine geringe Risikowahrnehmung festzustellen ist, was wiederum auf geringe Investitionshöhen der befragten Unternehmen im mobilen Internet hindeutet. Die Wirkung des Angebots mobiler Dienste sowohl auf Umsatz- oder Rationalisierungsergebnisse als auch auf die Qualität der Kundenkommunika-

\footnotetext{
604 Vgl. Sirkin/Dean (2000).

$605 \mathrm{Vgl}$. A.T. Kearney (2003).

606 Vgl. Wohlfahrt (2004), S. 174 und S. 205.
} 
tion wird bislang als gering bewertet. Auf Kundenseite ist die Nachfrage nach mobilen Diensten geprägt durch ein hohes Risikobewusstsein gegenüber einer Fehladoption sowie durch eine ausgeprägte KostenNutzen-Wahrnehmung. Weitgehend unbekannte Bedienungsbarrieren des mobilen Internet weisen auf ein frühes Adoptionsstadium hin. Entscheidende Kriterien einer positiven Wirkung bilden Individualisierung, Zeitnähe und Aktualität des Angebots.

\subsubsection{Zusammenfassung}

In Abschnitt 5.3.1.1 wurden verschiedene Betrachtungsdimensionen der Akzeptanzforschung vorgestellt. Akzeptanz kann demnach sowohl im Hinblick auf ihre Determinanten (Input) als auch im Hinblick auf ihre Wirkung (Output) in statischer oder dynamischer Form untersucht werden. Die Akzeptanz einer Innovation kann dabei drei Aspekte beinhalten: ihre Bekanntheit, ein positives Interesse und eine messbare Nutzung. In Abschnitt 5.3.2.1. wurde ein Überblick über bestehende Beiträge der Akzeptanzforschung für das mobile Internet gegeben. Es wurden Experten- und Kundenbefragungen vorgestellt und gezeigt, dass unterschiedliche Befragungsformen durchaus sehr unterschiedliche Ergebnisse liefern. Es ist somit einerseits festzustellen, dass die bestehenden Forschungsbeiträge zur Akzeptanz mobiler Dienste keiner gemeinsamen plausiblen Argumentationslinie angehören, die eine eindeutige Identifikation Erfolg versprechender mobiler Dienstformen ermöglicht. Stattdessen scheint die Akzeptanz mobiler Dienste im Einzelfall konkret empirisch oder experimentell nachgewiesen werden zu müssen. Andererseits ist der derzeitige Stand der Akzeptanzforschung für das mobile Internet im Hinblick auf die Chancen des Angebots mobiler Dienste durch telekommunikationsfremde Unternehmen, wie bspw. Medienunternehmen oder Finanzdienstleister, sehr unfokussiert oder besitzt - wie die dargestellten Beispiel der Expertenbefragungen - aufgrund kleiner Stichproben lediglich geringe Aussagekraft. Insofern ist im Zusammenhang mit einer Konkretisierung der Akzeptanzforschung für das Angebot von Medienunternehmen im mobilen Internet eine Forschungslücke festzustellen. Diese Forschungslücke wird im Weiteren adressiert.

\subsubsection{Analyse der Akzeptanz inhalteorientierter mobiler Dienste}

Im Folgenden wird ein Forschungsansatz zur Analyse der Akzeptanz der Inhaltenutzung im mobilen Internet vorgestellt. Des Weiteren werden Ergebnisse von Untersuchungen, die auf der Grundlage dieses Ansatzes durchgeführt wurden, dargestellt und erläutert. In Abschnitt 5.3.2.1 wird zunächst der gewählte Forschungsansatz erläutert und begründet. Es werden die zentralen Forschungsfragen der durchgeführten Untersuchungen abgeleitet. In Abschnitt 5.3.2.2 werden Aufbau und Ergebnisse einer Befragung vorgestellt, die als Vorstudie für eine breit angelegte Onlinebefragung unter den Konsumenten von Publikumsverlagen durchgeführt wurde. Ziel, Aufbau und Befunde der Hauptuntersuchung unter Verlagskunden werden in Abschnitt 5.3.2.3 erläutert. Ab- 
schnitt 5.3.3 fasst die Befunde der durchgeführten Erhebungen vor dem Hintergrund des zugrunde gelegten Forschungsansatzes zusammen.

\subsubsection{Konzeption des Forschungsansatzes}

Der folgende Abschnitt gibt einen Überblick über die Konzeption des zugrunde liegenden Forschungsansatzes der nachfolgenden Akzeptanzanalyse inhalteorientierter mobiler Dienste. In Abschnitt 5.3.2.1.1 werden Forschungsziel und Forschungsleitfragen der Analyse abgeleitet. In Abschnitt 5.3.2.1.2 wird das methodische Vorgehen dargestellt. Abschnitt 5.3.2.1.3 fasst die konzeptionellen Besonderheiten der Untersuchung zusammen.

\subsection{Forschungsziel und Forschungsleitfragen}

Vor dem Hintergrund der voluntaristisch geprägten Mehrwertkonzeption, die dieser Arbeit zugrunde liegt, besteht das Entscheidungsproblem eines Inhalteanbieters, der die Verwertung seiner Inhalte im mobilen Internet prüft, in erster Linie darin, die Bedürfnisse mobiler Kunden zu identifizieren und - sofern möglich - mit mobilitätsgerecht formatierten Inhalten zu befriedigen. Wie bereits in Abschnitt 4.1.2 gezeigt wurde, liefern jedoch einerseits bestehende Expertenbefragungen wenig sachdienliche Hinweise zu den tatsächlich bestehenden Bedürfnissen mobiler Nutzer. Gleichwohl ist andererseits auch die Identifikation von Rezipientenbedürfnissen auf der Grundlage von Kundenbefragungen mit Problemen verbunden, wie die Diskrepanz zwischen dem zwar bspw. in Nokia (1999) bekundeten Interesse an mobilen Mehrwertdiensten und dem aktuell dennoch geringen Nutzungsgrad solcher Dienste deutlich macht. Das Problem bestehender Kundenbefragungen zur Identifikation von Bedürfnissen und Präferenzen potenzieller Kunden mobiler Dienste mag dabei auf verschiedenen Ursachen beruhen: Einerseits scheinen mobile Dienste Eigenschaften aufzuweisen, die bei potenziellen Nutzern nur bedingt mit bewusst wahrgenommenen Bedürfnissen korrespondieren. ${ }^{607}$ Es sind daher neben kognitiven Motiven Aspekte einer affektiven Akzeptanz auf die Nutzung mobiler Dienste zu prüfen. Andererseits ist das Ausmaß der Bedürfniswahrnehmung, aufgrund des Systemgutcharakters mobiler Dienste im Zusammenspiel mit der Nutzung von mobilen Endgeräten und Mobilfunknetzen, an die Verbreitung und das Interesse an der Mobilfunktechnik geknüpft. Darüber hinaus besitzen inhalteorientierte mobile Dienste ebenso wie Mediengüter in anderen Distributionsformaten Erfahrungsguteigenschaften, wodurch ihr Mehrwert für einen Konsumenten im Vorfeld der Nutzung bestenfalls nur teilweise abgeschätzt werden kann.

Das Entscheidungsproblem eines Inhalteanbieters im Zusammenhang mit dem Angebot mobiler Dienste ist daher dreifacher Natur: Erstens sind im Kreise der potenziell erreichbaren Kunden jene zu identifizieren, die eine hohe Nutzungswahrscheinlichkeit in Bezug auf mobile Dienste besitzen (1). Es ist zweitens zu bestimmen, welche Inhalte eines Medienunternehmens das Potenzial besitzen, Bedürfnisse dieser mobilen Konsumenten zu befriedigen (2). Drittens

607 Vgl. Lehmann/Lehner (2003), S. 8. 
sind diejenigen Dienstformate zu bestimmen, die im jeweils zu adressierenden Nutzersegment die höchsten Präferenzen aufweisen (3). Vor dem Hintergrund dieses dreigeteilten Entscheidungsproblems im Zusammenhang mit dem Angebot inhalteorientierter mobiler Dienste ist es Zielsetzung der im Weiteren vorgenommenen Akzeptanzanalyse, einen Entscheidungsraum zu bestimmen, anhand dessen Medienunternehmen Erfolg versprechende Vermarktungsformen ihrer Inhalte im mobilen Internet identifizieren können. Zu diesem Zweck werden nachfolgend zunächst Leitfragen für die im Weiteren vorgestellte Analyse abgeleitet.

(1) Wie bereits dargelegt wurde, repräsentieren inhalteorientierte mobile Dienste Systemgüter, deren Verbreitung an die Nutzung von mobilen Endgeräten und Mobilfunknetzen geknüpft ist. Es ist daher zu prüfen, inwiefern der Akzeptanzgrad eines mobilen Dienstes nicht nur durch den ggf. von inm gebotenen inhaltebezogenen Mehrwertpotenzial beeinflusst wird, sondern darüber hinaus davon abhängt, inwiefern potenzielle Nutzer Kenntnis über und Interesse an der Mobilfunk- und Endgerätetechnik besitzen. Allgemeine Hinweise auf Unterschiede in der Technikadoption liefern aktuelle Untersuchungen der Konsumgewohnheiten, wie bspw. die bereits angesprochene „Typologie der Wünsche“, einem repräsentativen jährlichen Konsumpanel des Burda Verlags. ${ }^{608}$ Nachweisliche Determinanten der Adoptionsrate der Technik des mobilen Internet bilden etwa das Alter, das Geschlecht, die Ausbildung oder das Einkommen der jeweiligen Person. Abbildung 5-16 zeigt exemplarisch die Unterschiede des Nutzungsgrads von SMS, MMS und WAP-Diensten in Abhängigkeit von Alter und Geschlecht.

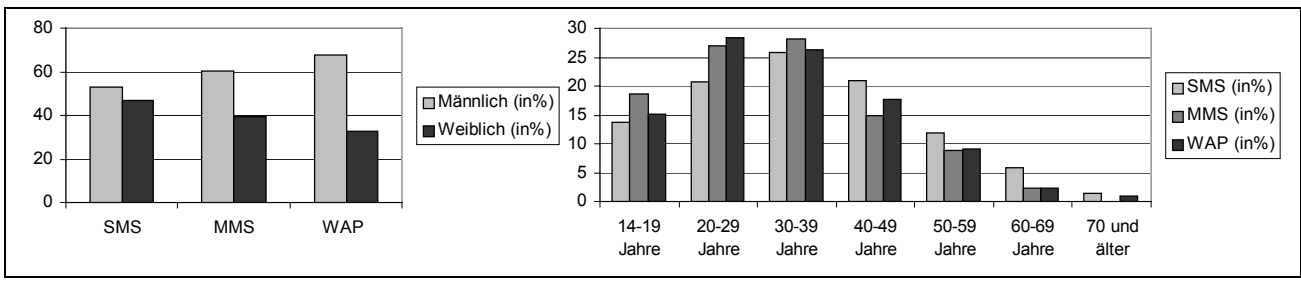

Abbildung 5-16: Nutzungsgrade von Mobilfunktechnik in Abhängigkeit von Geschlecht und Alter609

Vor dem Hintergrund der nachweisbaren demografischen Determinierung der Mobilfunkadoption lässt sich im Rahmen einer Akzeptanzanalyse inhalteorientierter mobiler Dienste aus Sicht eines Verlagsunternehmens als erste Forschungsfrage formulieren: Welchen Adoptionsgrad der Mobilfunktechnik besitzen die typischen Konsumenten von Publikumsmedien und wie schlagen sich ggf. nachweisbare Unterschiede der Technikadoption auf die Akzeptanz mobiler Dienste nieder [Leitfrage 1]?

(2) In einer Studie der Vermarktungsmöglichkeiten für Inhalte traditioneller Medienunternehmen und Verlage im mobilen Internet identifizieren Ziegler/Adam

$608 \mathrm{Vgl}$. TdW (2004).

609 Eigene Darstellung nach Zahlen aus dem TdW-Panel. 
(2001) die größten Potenziale für Anbieter solcher Inhalte, die einen hohen Spezialisierungsgrad besitzen. Beispiele solcher Spezialinhalte bilden Einkaufsführer, Programmhinweise, Rubrikenmärkte, Nachrichten über lokale oder in hohem Maße aktuelle Ereignisse sowie Unterhaltungsangebote wie Klingeltöne und Onlinespiele. Die vertretene Meinung wurde jedoch bislang ebenso wenig empirisch validiert wie auf ihre Gültigkeit für Konsumenten mit unterschiedlichen Bedürfnissen und Interessen geprüft. In Ermangelung einer solchen Validierung, stellt sich als zweite Forschungsfrage einer empirischen Akzeptanzanalyse mobiler Inhalteangebote: Welche Inhalte werden tatsächlich von welchen Nutzertypen in welchem Maße [Leitfrage 2] mobil genutzt? Dabei ist insbesondere zu prüfen: Welche Motive bewegen potenzielle Konsumenten zu einer Nutzung mobiler Dienste bzw. welche Motive unterbinden eine Nutzung [Leitfrage 3]?

(3) Konvergenzen im Bereich der Endgerätetechnik, bspw. die Anreicherung von Funktelefonen mit Komponenten zur Dokumenten-, Bild-, Musik- und Filmwiedergabe, sowie im Bereich der Funkübertragungstechnik, bspw. durch die Evolution von schmalbandigen GSM-Netzen zum breitbandigen UMTS-Standard, schaffen die Grundlage dafür, Inhalte unabhängig vom Inhalteformat (Text, Bild, Ton oder Film) über Mobilfunknetze zu übertragen und über Mobilfunkgeräte zu konsumieren. Wie bereits dargestellt wurde, können Inhalte dabei in Distributionsformaten übertragen werden, die jenen des stationären Internet ähneln (bspw. WAP-kompatible Onlineseiten der Dateidownloads) oder an mobilfunkspezifische Kommunikationsformate wie SMS oder MMS geknüpft werden. Aus Sicht eines Medienunternehmens muss daher die Frage beantwortet werden: Welche Distributionsformate besitzen bei potenziellen Nutzern die größte Akzeptanz [Leitfrage 4]? Abbildung 5-17 zeigt die vier abgeleiteten Forschungsleitfragen im Überblick.

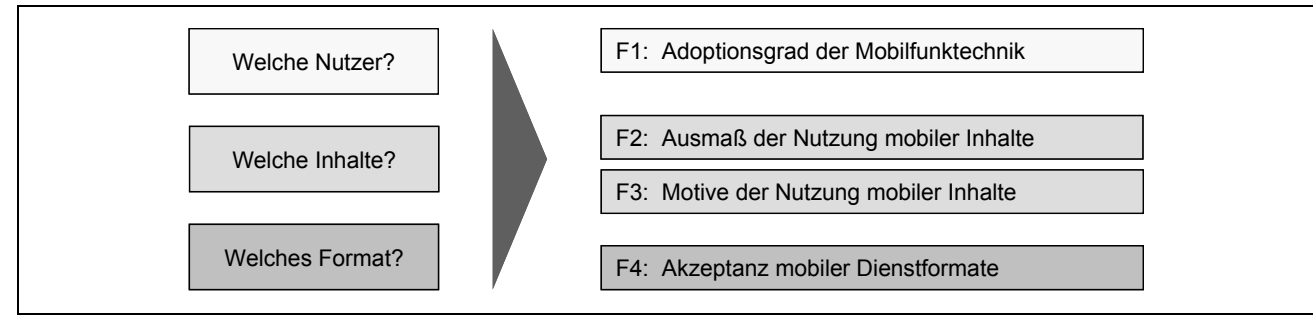

Abbildung 5-17: Forschungsleitfragen der Akzeptanzanalyse

\subsection{Methodisches Vorgehen}

Wie voranstehend deutlich wurde, stößt eine theoriegeleitete deduktive Forschungsmethodik, wie sie bspw. in Kapitel 5.2 verfolgt wurde, an Grenzen in Bezug auf ihre Möglichkeiten, Dienstformen zu bestimmen, die in der Breite der potenziellen Anwender eine nachweisliche praktische Akzeptanz besitzen. Die in Abschnitt 5.3.2.1.1 abgeleiteten Forschungsleitfragen adressieren in besonderem Maße Problemstellungen, die einer Validierung durch Erhebung von 
Marktdaten bedürfen. Datenerhebungen dieser Art repräsentieren Ausprägungsformen einer empirisch-induktiven Forschungsmethodik.

Empirische Forschung oder Empirismus fungiert als Sammelbezeichnung für ein breit gefächertes Instrumentarium. Klassischer Empirismus beruht auf der im Prinzip des sog. „logischen Positivismus“ wurzelnden Grundannahme, dass messbare Erfahrung Ursprung und Rechfertigung aller wissenschaftlichen Erkenntnis darstellt. ${ }^{610}$ Typische Kriterien zur Klassifikation empirischer Forschungsmethoden bilden die Art der Datenerhebung (1), der Grad des Einflusses des Forschers auf das Ergebnis der Datenerhebung und deren Standardisierungsgrad (2) sowie das formale Untersuchungsziel der Erhebung (3). ${ }^{611}$ Anhand dieser drei Kriterien wird die im Weiteren dargestellte Akzeptanzanalyse eingeordnet.

(1) In Anlehnung an die in Kapitel 4 vorgestellten Möglichkeiten einer Identifikation individueller Kundenbedürfnisse von Onlinerezipienten kann im Zusammenhang mit der Erhebungsform empirischer Daten zwischen Beobachtungsund Befragungsformen unterschieden werden. Als Grundlage einer Verhaltensbeobachtung mobiler Nutzer kann ebenso wie im stationären Internet das Protokoll des Webservers, durch den bspw. WAP-Seiten bereitgestellt werden, herangezogen werden. ${ }^{612}$ Inhaltliche Erhebungsziele einer solchen Beobachtung bilden etwa die Bestimmung auffällig häufig abgerufener WAP-Seiten eines Anbieters, wodurch auf eine ggf. hohe Akzeptanz des jeweiligen Inhalts im Zuge mobiler Nutzer geschlussfolgert werden kann. Für die jedoch hier zugrunde liegenden Fragestellungen im Zusammenhang mit einer anbieterübergreifenden Bestimmung der Akzeptanz inhalteorientierter mobiler Dienste ist das Verfahren jedoch wenig sachdienlich. Wie bereits dargestellt wurde, können Befragungsformen nach der Art der Befragten weiter klassifiziert werden in anbieterseitige bzw. Expertenbefragungen und Kundenbefragungen. ${ }^{613} \mathrm{Im}$ Hinblick darauf, dass das Unsicherheitsmoment von Inhalteanbietern in Bezug auf die Akzeptanz mobiler Inhalteformate das im Zusammenhang mit der Analyse zu lösende Problem darstellt, wurde die im Weiteren vorgestellte Untersuchung auf der Grundlage von Kundenbefragungen durchgeführt.

(2) Im Hinblick auf den Einfluss des Interviewers auf das Befragungsergebnis kann im Rahmen einer solchen Kundenbefragung zwischen mündlichen und schriftlichen Befragungsformen unterschieden werden. ${ }^{614}$ Mündliche Erhebungen werden in direkter Interaktion mit dem Befragten durchgeführt und zeichnen sich durch einen gewissen Spielraum in der Gestaltung dieser Interaktion aus. ${ }^{615}$ Daher werden mündliche Erhebungsformen vorwiegend zur Erforschung qualitativer Zusammenhänge des Befragungsgegenstandes durchge-

$610 \mathrm{Vgl}$. Wolf/Priebe (2001), S. 11.

611 Vgl. Bortz/Döring (2003), S. 295 ff. und Kromrey (2002), S. 377.

$612 \mathrm{Vgl}$. Bensberg (2002), S. 159.

$613 \mathrm{Vgl}$. Abschnitt 5.3.1.2.

$614 \mathrm{Vgl}$. Kromrey (2002), S. 377.

615 Vgl. Bortz/Döring (2003), S. 307 f. 
führt. ${ }^{616}$ Demgegenüber zielen schriftliche Befragungen anhand strukturierter Fragestellungen tendenziell auf quantitative Zusammenhänge ab. ${ }^{617}$ Dabei ist nach dem Standardisierungsgrad jeweils weiter zu unterscheiden zwischen standardisierten und nichtstandardisierten Befragungsformen. ${ }^{618}$ Bspw. verzichtet eine nichtstandardisierte Befragung vollkommen auf einen Interviewleitfaden, um bestmögliche Befragungsflexibilität zu gewährleisten, während bspw. eine standardisierte schriftliche Befragung lediglich eine Auswahl unter dezidiert vorgegebenen Antwortmöglichkeiten erlaubt. Insofern die der Analyse zugrunde liegenden Leitfragen in erster Linie quantitative Ausprägungsformen von Nutzungstatbeständen adressieren, die gut in Form von Produkt-/Marktkombinationen strukturierbar sind, wurden die im Weiteren vorgestellten Erhebungen in der Form standardisierter schriftlicher Befragungen durchgeführt.

(3) Die Grundlagendiskussion der Wissenschaftsmethodik empirischer Sozialforschung ist geprägt durch einen Dualismus, der auch als „Erklären/VerstehenKontroverse" bezeichnet wird. Die Kontroverse beruht auf einer gewissen Unvereinbarkeit der üblicherweise quantitativen Natur naturwissenschaftlicher Theoriebildung zum Zwecke der Erklärung ihres Gegenstandsbereichs und der tendenziell qualitativ geprägten Tradition geisteswissenschaftlicher Forschung zum Zwecke des Verstehens ihres Gegenstandsbereichs. Vor dem Hintergrund dieser Kontroverse können im Zusammenhang mit dem formalen Ziel empirischer Untersuchungen, entsprechend der Erklärungskraft der resultierenden Ergebnisse für ihren Gegenstandsbereich, vier Ausprägungsformen unterschieden werden: Explorative Untersuchungen, wie bspw. die bereits erläuterten Grundlagenstudien für das mobile Internet, dienen in erster Linie zu qualitativen Erkundung eines weitgehend unbekannten Forschungsgegenstands. Deskriptive Untersuchungen verwenden statistische Verfahren zur Quantifizierung von Sachzusammenhängen im Untersuchungsgegenstand. Erklärende Untersuchungen prüfen bereits vorgefertigte Theorien durch den Nachweis signifikanter Hypothesen und schließlich begründen evaluative Untersuchungen die Ursachen und Wirkungen solcher nachweisbaren Hypothesen und Theorien. Wie bereits dargelegt wurde, repräsentieren die im Voranstehenden abgeleiteten Forschungsfragen in erster Linie quantitative Problemstellungen. Aufgrund des bislang geringen Untersuchungsgrads der Akzeptanz inhalteorientierter mobiler Dienste trägt eine Prüfung von Hypothesen, die aufgrund des allgemein geringen Kenntnisstands zwangsläufig eine gewisse Willkür besäßen, kaum zum Forschungsziel einer Bestimmung Erfolg versprechender mobiler Inhalteverwertungsformen. Ziel der im Weiteren dargestellten Akzeptanzanalyse bildet daher in erster Linie die Deskription des Ausprägungsgrads nachweislicher Nutzung mobiler Dienste unter den Konsumenten von Medienunternehmen. Dennoch unterliegt die angestrebte Begründung sinnvoller Handlungsmöglichkeiten für Medienunternehmen, die beabsichtigen, ihren bestehenden Konsumenten im mobilen Internet einen zusätzlichen Nutzen zu stiften,

\footnotetext{
616 Vgl. von Saldern (1998), S. 50.

617 Vgl. Konrad (2001), S. 63.

618 Vgl. Kromrey (2002), S. 378.
} 
einer impliziten Vermutung eines sachlogischen Zusammenhangs zwischen der medientypischen Kundensegmentierung (anhand von Übertragungsmedium und Inhaltetyp) und der Nutzung mobiler Dienste. Diese Hypothese bedarf zunächst ihrer Evaluation. Die Leistung dieser Evaluation ist Gegenstand einer Voruntersuchung, die im Weiteren der Hauptuntersuchung der Akzeptanz mobiler Dienste unter den Konsumenten von Publikumsverlagen vorangestellt wird.

\subsection{Zusammenfassung}

Die zugrunde liegende Problemstellung der nachfolgenden Akzeptanzanalyse mobiler Dienste bildet die Suche von Medienunternehmen nach Möglichkeiten der Inhalteverwertung, die bestehenden Kunden einen zusätzlichen Nutzen stiften. An diese Problemstellung sind, wie in Abschnitt 5.3.2.1.1 abgeleitet wurde, vier Fragestellungen geknüpft. Die Beantwortung dieser Fragestellungen erfordert, wie in Abschnitt 5.3.2.1.2 dargelegt wurde, eine Validierung der medientypischen Nutzersegmentierung anhand einer empirischen Erhebung des derzeitigen Stands der Nutzung mobiler Dienste. Die Natur der Fragestellung legt dabei eine Erhebung in Form einer strukturierten Befragung nahe. Vor dem Hintergrund der in Abschnitt 5.3.1.1 eingeführten Akzeptanzdimensionen berührt eine solche Form der Nutzerbefragung, deren Ziel die Bestimmung des Nutzungsgrades mobiler Dienste ist, in erster Linie konative Akzeptanzaspekte. Des Weiteren werden allerdings auch im Zusammenhang mit der Untersuchung von Motiven der Nutzung mobiler Dienste (Leitfrage 3) die Wirkung affektiver und kognitiver Aspekte der Akzeptanz von Mobilfunk- und Endgerätetechnik erklärt. Abbildung 5-18 fasst die formale Abgrenzung der nachfolgenden Analyse zusammen.

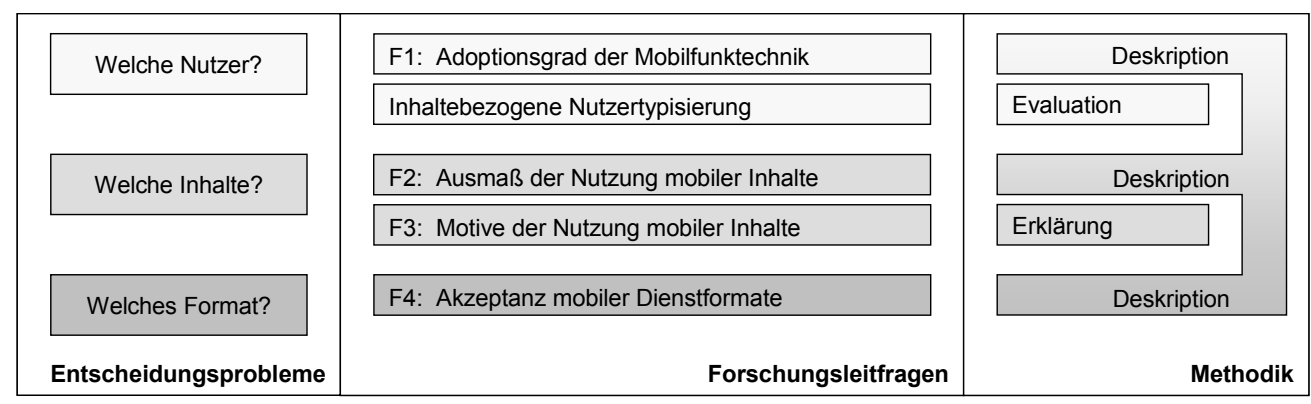

Abbildung 5-18: Formale Abgrenzung der durchgeführten Akzeptanzanalyse

\subsubsection{Evaluation einer inhaltebezogenen Segmentierung mobiler Nutzer}

Damit ein mobiler Dienst für den Konsumenten eines Publikumsmediums einen zusätzlichen Nutzen zu stiften im Stande ist, muss der Zweck der Nutzung des mobilen Internet eine gewisse Komplementarität zum Nutzungszweck etablierter Medien aufweisen. Dabei besitzen etablierte Publikumsmedien in der Wahrnehmung ihrer Konsumenten typischerweise ein ausgeprägtes Informationsoder Unterhaltungsprofil. Dieses Profil wird in der Medienwirkungsforschung 
auch als spezifische „Kompetenz“ eines Mediums bezeichnet. ${ }^{619}$ Des Weiteren ist vor dem Hintergrund der bereits in Kapitel 3 dargestellten wachsenden inhaltlichen Differenzierung der Medienmärkte eine zunehmend nutzergruppenspezifische Segmentierung der medienspezifischen Ausprägungen des jeweiligen inhaltlichen Profils festzustellen. ${ }^{60}$ Für die im Weiteren angestrebte Identifikation potenzieller Handlungsfelder aus Sicht eines Medienunternehmens im mobilen Internet ist daher zunächst zu prüfen, ob die medientypische Marktsegmentierung anhand inhaltlicher und zielgruppenbezogener Kriterien auf Onlinemärkten im Allgemeinen bzw. dem Markt für mobile Dienste im Speziellen zu übertragen ist. Im Folgenden werden Aufbau und Befunde einer zu diesem Zweck angestellten Voruntersuchung zur Nutzungsform mobiler Dienste vorgestellt. 5.3.2.2.1 gibt einen Überblick über Methodik und Aufbau der Untersuchung, Abschnitt 5.3.2.2.2 erläutert die Ergebnisse der Untersuchung und Abschnitt 5.3.2.2.3 fasst die Befunde für den Untersuchungsgegenstand der angestrebten Akzeptanzanalyse zusammen.

\subsection{Methodik und Aufbau der Untersuchung}

Ein zentrales Dilemma der Mediennutzungs- und -wirkungsforschung besteht darin, dass die Bedingungen, die zur Nutzung oder Nichtnutzung eines Mediums führen, häufig komplexer Natur sind, weshalb Veränderungen in den medialen Nutzungsformen nur schwer prognostizierbar oder nur ex-post anhand der wahrnehmbaren Veränderungen des Konsumverhaltens festzustellen sind. Ein etabliertes Hilfsmittel, um dennoch verhältnismäßig gesicherte Prognosen über mittelfristige Veränderungen der Mediennutzung machen zu können, besteht darin, das aktuelle Verhalten von solchen Konsumentengruppen zu beobachten, denen eine gewisse Vorreiterrolle auch für den Nutzungswandel der übrigen Konsumentengruppen zugesprochen wird. Als derartige Trendgruppen gelten dabei solche Konsumenten, die eine vergleichsweise hohe Adoptionsgeschwindigkeit von digitaler Medientechnik besitzen. Dazu gehören insbesondere die Gruppe der 14-19jährigen bzw. der 2039jährigen und die Gruppe der sog. „Onliner“, also Konsumenten, die Onlinemedien regelmäßig im Alltag nutzen. ${ }^{621}$

Vor dem Hintergrund dieser Trendgruppenbestimmung wurde im Zusammenhang mit der vorliegenden Arbeit im Sommer 2004 eine Erhebung des Nutzungsverhaltens mobiler Dienste durchgeführt, die in erster Linie eine junge Zielgruppe, vorwiegend aus dem universitären Umfeld, adressierte. Trotz etwaiger verzerrender Befragungseffekte durch den Ausschluss solcher Konsu-

619 Vgl. Gerhards/Klingler (2003); Blödorn/Gerhards (2004); Gerhards/Klingler (2004). Bspw. wird dem

Fernsehen im Bereich von Hintergrundinformationen zu Deutschland und der ganzen Welt eine hohe Informationskompetenz zugeschrieben, während Radio und Tageszeitungen Informationskompetenzen speziell für regionale Ereignisse besitzen. Gleichwohl besitzen Radio und Fernsehen neben ihrer Informationskompetenz zusätzlich eine ausgeprägte Entspannungs- und Unterhaltungsfunktion in

Verbindung mit der Verbreitung von Musik (Radio) oder Film und Serie (Fernsehen).

620 Vgl. Hickethier (1998); Schönbach/Preiser (1998); Gerhards/Klingler (2004).

621 Vgl. Gerhards/Klingler (2003) und Gerhards/Klingler (2004). 
menten, die über keine Onlineerfahrung oder -kenntnisse verfügen, wurde vor dem Hintergrund der besonderen Bedeutung der Konsumentengruppe der sog. "Onliner", auf das Verfahren einer standardisierten Onlinebefragung zurückgegriffen. Ziel der Befragung war einerseits die Exploration des Nutzungsgrads mobiler Dienste und der Bedingungen von deren Akzeptanz sowie andererseits die Evaluation systematischer Zusammenhänge zwischen der Nutzung traditioneller Publikumsmedien und mobiler Dienste. Befragt wurden drei Aspekte: Die Häufigkeit der Nutzung traditioneller elektronischer und gedruckter Mediengüter, die Häufigkeit der Nutzung mobiler Endgerätefunktionen und Dienstformate sowie das Interesse an Inhalten traditioneller Medienformate über mobile Dienste. Im Hinblick auf den letztgenannten Aspekt wurden konkret je ein unterhaltungsorientiertes Inhalteszenario aus dem Rundfunkbereich (die mobile Rezeption von Filmen und Fernsehserien) und ein informationsorientiertes Inhalteszenario aus dem Printbereich (die mobile Nutzung tagesaktueller Zeitungsnachrichten) geprüft.

An der Befragung nahmen insgesamt 557 Personen teil, wovon 462 Teilnehmer die Befragung vollständig zu Ende führten. ${ }^{622}$ Die Teilnehmer wurden einerseits passiv durch Verlinkung des Fragebogens auf der Startseite der Internetpräsenz des Instituts für Wirtschaftsinformatik II rekrutiert und andererseits aktiv per Email an die Adressen von Veranstaltungs- oder Mitarbeiterverteilerlisten der Universität Göttingen zur Teilnahme aufgefordert. Der Anteil der Studierenden aus dem Umfeld der Universität Göttingen an den Befragungsteilnehmern beträgt daher $63,6 \%$.

\subsection{Ergebnisse der Untersuchung}

Neben dem stationären Internet-PC gehört das Mobiltelefon bei den Befragten zu den am Häufigsten genutzten elektronischen Endgeräten, gefolgt von Fernseher, Radio, Musikanlage und Filmwiedergabegeräten. Allerdings werden am Mobiltelefon lediglich die Funktionen SMS, Kalender und KlingeltöneKonfiguration mit einer gewissen Regelmäßigkeit benutzt, inhaltebezogene Dienstformate oder Endgerätefunktionen wie das Abspielen von Musik oder das Abrufen von WAP-Seiten dagegen selten bis nie (vgl. Abbildung 5-19). Als Gründe der Nichtnutzung mobiler Inhalteformate auf Grundlage von WAP oder MMS wurden vor allem hohe Preise, geringe Formatqualität der übertragenen Inhalte sowie im Falle von WAP unübersichtliche Tarifstrukturen genannt. ${ }^{623}$

622 Der Aufbau der Befragung ist dargestellt im Anhang, Abschnitt 7.2.

623 Interessanterweise gaben zwar 62\% der Befragten an, dass WAP-Dienste zu teuer sind, allerdings konnten davon nur 8\% tatsächlich angeben, wie teuer die Datenübertragung ihres Mobilfunkbetreibers auf der Grundlage von WAP tatsächlich ist. 


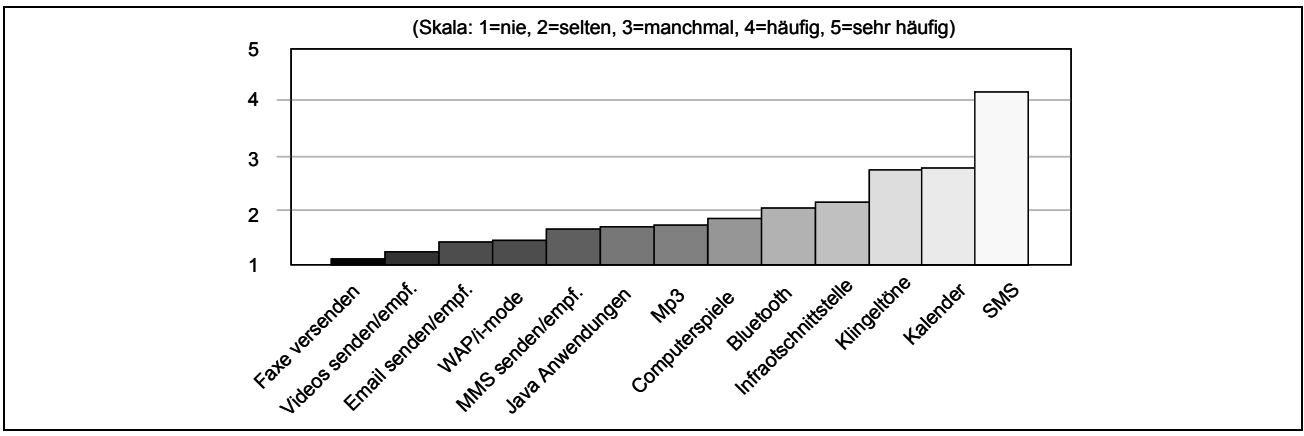

Abbildung 5-19: Mittlere Nutzungshäufigkeit der Funktionen des Mobiltelefons

Zur Evaluation des Einflusses der Kundensegmentierung etablierter Medienmärkte auf die Nutzung mobiler Dienste wurden den Teilnehmern zwei Szenarien der Nutzung von traditionellen Inhalteformaten (Videofilme und Zeitungsnachrichten) auf mobilen Endgeräten zur Auswahl gestellt. Abbildung 5-20 zeigt die Ausprägungen der Mittelwerte der jeweils mit Schulnoten von 1-5 skalierten Antwortmöglichkeiten. Es lässt sich ein nur vergleichsweise schwaches Interesse an den mobilen Dienstformaten bei einer gleichzeitig ausgeprägten Preissensibilität erkennen.

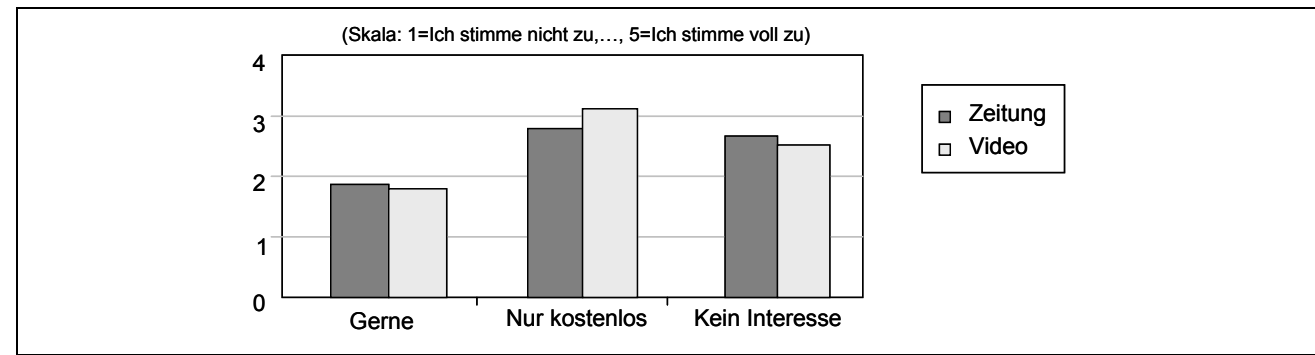

Abbildung 5-20: Nutzerinteresse an mobilen Dienstformaten für etablierte Inhalte

Zur Überprüfung eines systematischen Zusammenhangs zwischen der Nutzung von Filmen und Nachrichten in Fernsehen und Zeitung und der Nutzung mobiler Video- und Nachrichtendienste wurden Korrelationskoeffizienten und Kreuzhäufigkeiten der jeweiligen Antwortausprägungen berechnet. Mit einem Korrelationskoeffizienten von 0,34 besteht ein schwach positiver Zusammenhang zwischen dem Konsum von Fernsehfilmen und der Nutzung mobiler Videodienste. Im Rahmen der Berechnung der Kreuzhäufigkeiten zwischen der Nutzungsbereitschaft für mobile Videodienste zwischen Nutzern, die einen regelmäßigen Fernsehfilmkonsum angeben und solchen, die einen derartigen Konsum verneinen, zeigt der Vergleich der Mittelwerte der mit Schulnoten von 1-5 skalierten Antwortausprägungen eine deutlich ausgeprägtere Nutzungsbereitschaft in der Gruppe der Filmkonsumenten auf (vgl. Abbildung 5-21). Die Differenz ist im Rahmen des Z-Tests sowohl auf dem 95\%- als auch dem 99\%Niveau signifikant. Der Korrelationskoeffizient zwischen dem Konsum von Tageszeitungen und der Nutzungsbereitschaft mobiler Nachrichtendienste beträgt 
0,266. Die Differenz der Mittelwerte der Gruppe der regelmäßigen Tageszeitungsleser und der Gruppe der Nichtleser weist wiederum auf eine ausgeprägtere Nutzungsbereitschaft der Gruppe der Leser hin, wobei die Mittelwertdifferenz wiederum sowohl auf dem 95\%- als auch dem 99\%-Niveau signifikant ist (vgl. Abbildung 5-21).

\begin{tabular}{|c|c|c|}
\hline \multicolumn{2}{|r|}{ Zeitungsleser } & Nichtleser \\
\hline Mittlere Bewertung (SA) & $2,06(1,23)$ & $1,55(0,94)$ \\
\hline Kreuzhäufigkeiten & $n=235$ & $\mathrm{n}=237$ \\
\hline \multicolumn{2}{|r|}{ Filmezuschauer } & Nichtzuschauer \\
\hline Mittlere Bewertung (SA) & $2,41(1,56)$ & $1,58(1,04)$ \\
\hline Kreuzhäufigkeiten & $n=168$ & $n=307$ \\
\hline \multicolumn{3}{|c|}{ SA: Standardabweichung } \\
\hline
\end{tabular}

Abbildung 5-21: Mittelwertvergleich und Kreuzhäufigkeiten

\subsection{Zusammenfassung der Befunde}

Wie in Abschnitt 5.3.2.2.1 erläutert wurde, besitzen jüngere Konsumentengruppen mit Onlineerfahrung eine Indikatorfunktion im Hinblick auf die Veränderungen der Medienkonsumgewohnheiten in den nächsten 5-10 Jahren. Dennoch zeigen die in Abschnitt 5.3.2.2.2 dargestellten Ergebnisse der angestellten Befragung unter Onlinenutzern im universitären Umfeld einen lediglich geringen Nutzungsgrad inhalteorientierter mobiler Dienste, was auf eine nur langsame Entwicklung des Geschäfts mit Inhalten im mobilen Internet in den kommenden Jahren schlussfolgern lässt. Insbesondere die Diskrepanz zwischen der ausgeprägten Meinung hoher Preise und der gleichzeitig geringen Kenntnis tatsächlicher Preisstrukturen weist auf einen geringen kognitiven Akzeptanzgrad mobiler Dienste hin. Weiterhin stößt die ortsungebundene Nutzung von sowohl unterhaltungsorientierten als auch informationsorientierten Inhalteangeboten in den beiden beispielhaft untersuchten Szenarien in erster Linie auf affektive Akzeptanz. Auch wenn die beiden exemplarischen Szenarien kaum als repräsentativ für die typische Segmentierung eines traditionellen Medienmarkts einzuschätzen sind, weist die Zusammenhangsprüfung zwischen traditionellem Inhaltekonsum und dem Interesse an mobilen Inhalteformaten auf eine sich sowohl im Unterhaltungs- als auch im Informationsbereich etablierende Segmentierung des Interesses im mobilen Internet hin. Diese Erkenntnis segmentspezifischer Interessensunterschiede wurden zum Anlass genommen, eine erneute, breiter angelegte Befragung der Kunden von Publikumsmedien am Beispiel der Leser von Printtiteln durchzuführen, in der eine Nutzertypisierung auf der Grundlage der regelmäßigen Nutzung traditioneller Inhaltevertriebsformate angewandt wurde. Gegenstand, Aufbau und Ergebnisse dieser Befragung werden nachfolgend vorgestellt. 


\subsubsection{Akzeptanz mobiler Inhalteformate bei den Lesern von Printtiteln}

Bestehende Kundenbefragungen zur Akzeptanz mobiler Dienste erheben zwar üblicherweise Nutzungsgrade unterschiedlicher Dienstformen wie SMS, MMS oder WAP, nehmen jedoch in der Regel eine systematische Differenzierung weder nach dem Nutzungszweck einer Informations- oder Kommunikationsorientierung noch nach dem jeweils transportierten Inhalt vor. Eine solche differenzierte Auseinandersetzung mit der Akzeptanz des mobilen Internet ist erstmalig Gegenstand einer Erhebung, die im Rahmen der vorliegenden Arbeit im Frühjahr 2005 in Zusammenarbeit mit dem Verband deutscher Zeitschriftenverleger (VDZ) durchgeführt wurde. Im folgenden Abschnitt werden Form und Ergebnisse dieser Erhebung zusammengefasst. In Abschnitt 5.3.2.3.1 werden Ziel, Stichprobe und Aufbau der Erhebung erläutert. In Abschnitt 5.3.2.3.2 werden die Ergebnisse der Erhebung erläutert und in Abschnitt 5.3.2.3.3 die Befunde aus diesen Ergebnissen für die in Abschnitt 5.3.2.1.1 formulierten Leitfragen zusammengefasst.

\subsection{Ziel, Stichprobe und Aufbau der Untersuchung}

Ziel der Erhebung bildet die Identifikation auffälliger Zusammenhänge zwischen regelmäßiger Nutzung von Publikumsprinttiteln und mobilen Inhalteformaten, um für Zeitungs- und Zeitschriftenverlage Erfolg versprechende Inhalte-/Dienstformatkombinationen für ein zusätzliches Geschäft mit mobilen Diensten unter deren typischen Kunden zu bestimmen. Zu diesem Zweck wurde ein standardisierter Onlinefragebogen entwickelt, der vor dem Hintergrund größtmöglicher Befragungsrepräsentativität auf den Onlinepräsenzen von insgesamt 16 Publikumsprinttiteln beworben und verlinkt wurde. ${ }^{624}$ Von insgesamt 13.402 Abrufen des Fragebogens führten 7.178 Teilnehmer die Befragung vollständig zu Ende. Die Teilnehmer, die zu knapp drei Vierteln aus Männern bestanden, rekrutierten sich relativ gleichmäßig aus verschiedenen Alters- und Einkommensschichten (vgl. Abbildung 5-22).

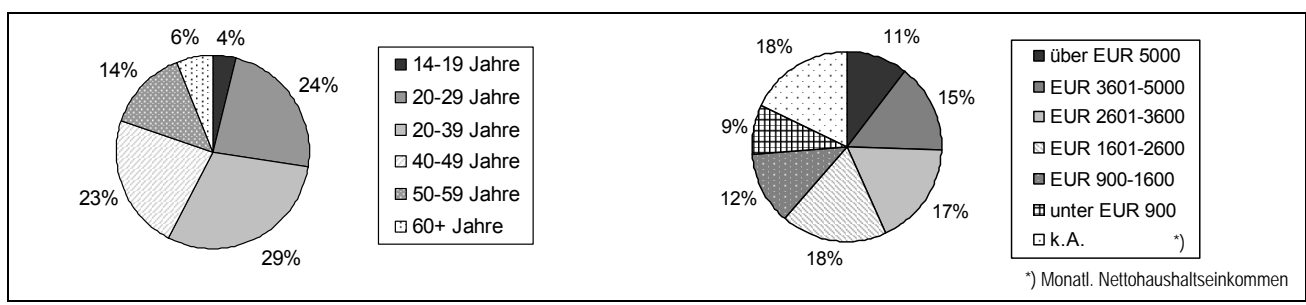

Abbildung 5-22: Alters- und Einkommensstruktur der Befragungsteilnehmer

$624 \mathrm{Zu}$ den teilnehmenden Anbietern gehörten in alphabetischer Reihenfolge: Auto-Motor-Sport.de, Bild.T-Online, Boerse-Online.de, Chip-Online.de, Family.de, Focus-Online.de, HamburgerAbendblatt.de, Handelsblatt.com, Handy.de, Immonet.de, Manager-Magazin.de, Maxim.de, Merian.de, MSN.de, Spiegel-Online.de und Stern.de. Auf allen Onlineseiten wurde der Fragebogen einer zufälligen Auswahl von Onlinerezipienten durch ein randomisertes Pop-Up-Fenster zur Teilnahme angeboten. Der Aufbau des Fragebogens ist dargestellt im Anhang, Abschnitt 7.3. 
Die Befragung gliederte sich in vier Bereiche: Vor dem Hintergrund der Ergebnisse der im voranstehenden Abschnitt dargestellten Voruntersuchung wurden zunächst häufig genutzte Printtiteltypen als Grundlage einer Nutzertypologie mobiler Inhaltenutzer abgefragt. Im Zusammenhang mit der in Abschnitt 5.3.2.1.1 formulierten Leitfrage des Einflusses der Akzeptanz der Mobilfunktechnologie auf die Nutzung des mobilen Internet wurden die im Besitz des Befragten befindlichen Endgeräte sowie die Art des bevorzugten Internetzugangs erhoben. Im Mittelpunkt der Befragung steht die Untersuchung der Inhaltenutzung im mobilen Internet. Geprüft wurden jeweils denkbare Inhaltevarianten für den Vertrieb in den Dienstformaten SMS, MMS und WAP. Des Weiteren wurde die mobile Nutzung von Musik- und Videoangeboten sowie von Spezialinhalten wie Ratgeberformate, Kleinanzeigen und ortsbezogene Dienste (Location Based Services, LBS) untersucht. Abschließend wurden Motive geprüft, die zur Nutzung bzw. ggf. Nichtnutzung der jeweiligen Dienstformate veranlasst hatten.

\subsection{Ergebnisse der Leserbefragung}

Zur besseren Übersicht wird die Ergebnispräsentation in drei Abschnitte untergliedert. In Abschnitt 5.3.2.3.2.1 werden zunächst die Häufigkeiten der jeweiligen Antwortausprägungen bezogen auf Grundgesamtheit und Lesertypologie vorgestellt. Abschnitt 5.3.2.3.2.2 beschreibt Details der Angaben zu den jeweils erhobenen Ausprägungsformen mobiler Dienste. In Abschnitt 5.3.2.3.2.3 werden die jeweils angegebenen Motive, die zur Nutzung oder Nichtnutzung der jeweiligen Dienstformen geführt haben, zusammenfasst.

\subsection{Nutzungshäufigkeiten je Lesersegment}

Zur Segmentierung der Nutzerschaft wurden 13 verschiedene Printgütertypen auf ihren regelmäßigen Konsum durch die Befragten hin geprüft. Übereinstimmend mit den in Kapitel 3 vorgestellten Daten zu den Medienteilsegmenten, wonach das Zeitungssegment in Auflage und Umsätzen deutlich vor den Zeitschriftenmärkten rangiert, werden auch unter den Befragten Tageszeitungen am häufigsten konsumiert, gefolgt von aktuellen Magazinen, Programm- und EDV-Zeitschriften (vgl. Abbildung 5-23).

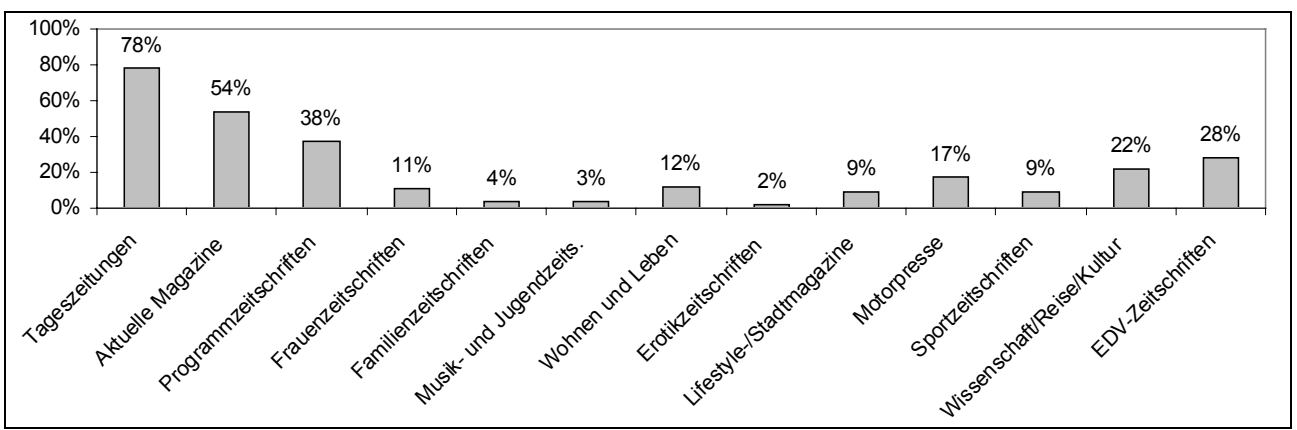

Abbildung 5-23: Anteile der Lesergruppen an der Stichprobe 
Unter den betrachteten Endgeräten besitzen stationäre PCs mit $87 \%$ die größte Verbreitung, gefolgt von Mobiltelefonen (79\%) und Notebooks (54\%). In Anteilen der jeweiligen Lesersegmentgröße ist dabei die größte Verbreitung von Mobiltelefonen in den Segmenten der Frauen, Jugend-, Musik- und Lifestylezeitschriften zu verzeichnen (vgl. Abbildung 5-24). Konvergente Endgeräte, wie bspw. „Smartphones“, das Ergebnis einer Verschmelzung von Minicomputer und Mobiltelefon, deren Besitz tendenziell auf eine hohe Mobilfunkakzeptanz hindeutet, werden lediglich von $4 \%$ der Befragten benutzt. Anteilig an der jeweiligen Lesersegmentgröße ist eine überdurchschnittliche Verbreitung solcher Geräte speziell in den Segmenten der Leser von Erotiktiteln und Motorpresse festzustellen.

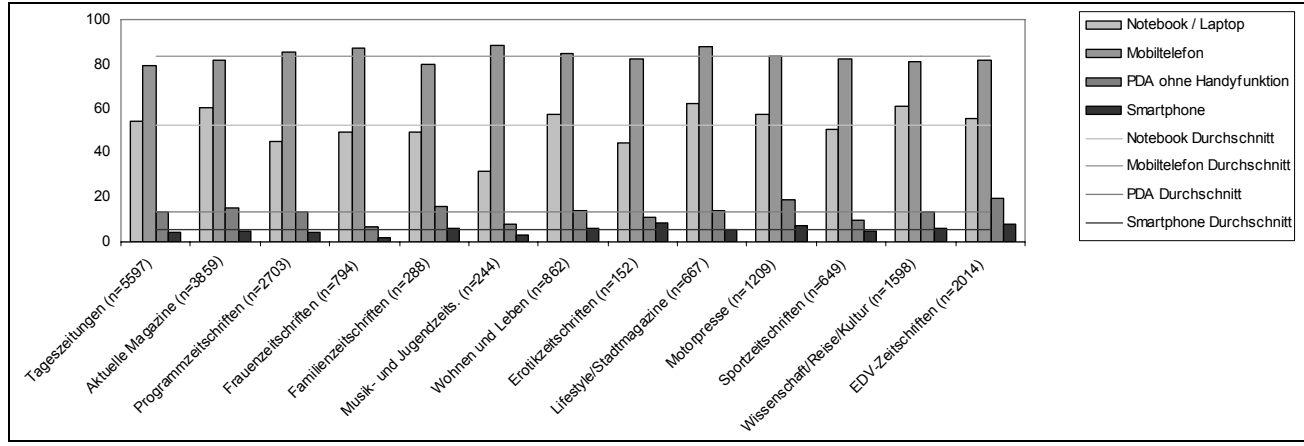

Abbildung 5-24: Endgerätehäufigkeiten anteilig je Lesersegment

Im Zusammenhang mit der Nutzung des mobilen Internet wurde ausschließlich der Konsum kostenpflichtiger Dienstformen erhoben. Zu den insgesamt am häufigsten genutzten Diensten gehören dabei die WAP-Dienste in den Portalen der Mobilfunkbetreiber, gefolgt von Inhalten, die entweder per SMS und MMS oder speziell ortsbezogen vertrieben werden. Von lediglich geringer Bedeutung im mobilen Internet erweisen sich Video, Musik, Kleinanzeigen und Ratgeberdienste (vgl. Abbildung 5-25).

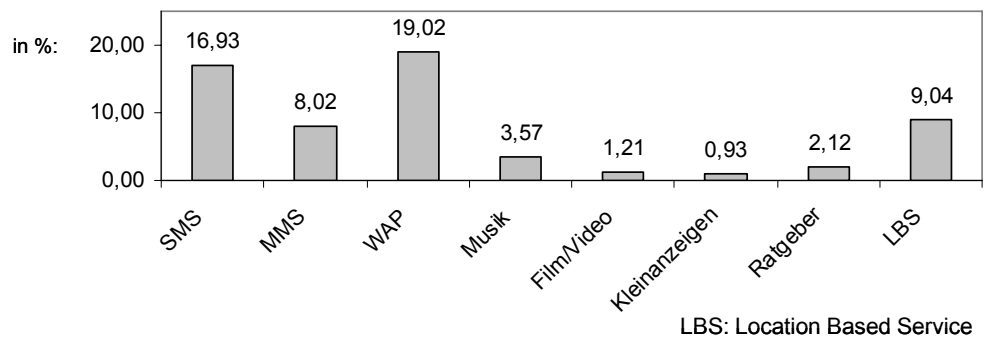

Abbildung 5-25: Segmentübergreifende Nutzung kostenpflichtiger Dienste im mobilen Internet

Relativ zur Grundgesamtheit besitzen die Massenmarktsegmente des Lesermarkts wie Tageszeitungen, Nachrichtenmagazine und Programmzeitschriften in allen Dienstkategorien überdurchschnittliche hohe Nutzungszahlen mobiler 
Dienste. ${ }^{625}$ Ein gegenteiliges Bild liefert dagegen eine Betrachtung der Dienstnutzung in Relation zur Größe des jeweiligen Lesersegments. Abbildung 5-26 zeigt den prozentuellen Anteil der fünf nutzungsintensivsten mobilen Dienstformate in Relation zur Lesersegmentgröße. Es wird deutlich, dass vor allem Leser in tendenziell auflagenschwächeren Marktnischen wie Musik- und Jugendzeitschriften, Erotik- und Stadtmagazine sowie Motor- und Sportpresse eine überdurchschnittlich hohe Nutzungsbereitschaft für kostenpflichtige mobile Inhalteformate besitzen. Bspw. nutzen gut $28 \%$ der Leser von Sportpresse zumindest gelegentlich kostenpflichtige SMS-Dienste und knapp 32\% der Erotikleser kostenpflichtige WAP-Dienste. Musikdienste weisen besonders in den Lesergruppen der Jugend- und Musikpresse (11,9\%) und Erotikpresse $(13,8 \%)$ überdurchschnittliche Anteile auf.

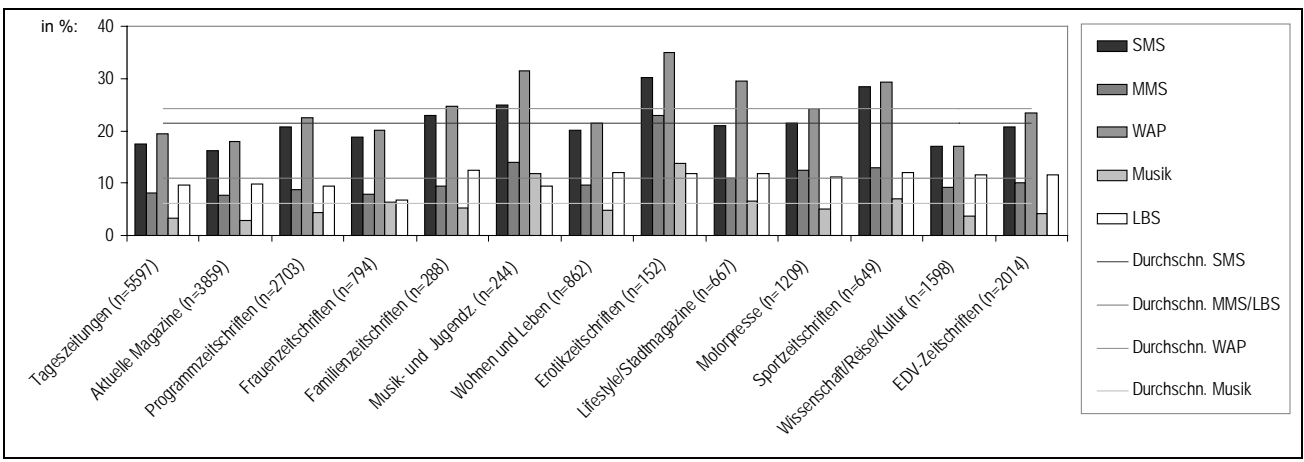

Abbildung 5-26: Nutzungsintensitäten je Dienstformat und Lesersegment

\subsection{Nutzungshäufigkeiten je Dienstkategorie}

Für ein detailliertes Verständnis über die Ausprägungen der jeweiligen Dienstakzeptanz durch die einzelnen Lesergruppen werden nachfolgend die jeweiligen Nutzungsintensitäten erhobener Ausprägungen der jeweils nutzungsstärksten Dienstformate SMS (1), MMS (2), WAP (3) und LBS (4) vertieft. Aufgrund geringer Nutzungshäufigkeiten werden die Ausprägungen von mobilen Video-, Kleinanzeigen- und Ratgeberdiensten im Detail vernachlässigt, ebenso wie Musikdienste, im Rahmen derer keine Detailbefragung vorgenommen wurde.

(1) Über alle Lesergruppen hinweg werden SMS-Dienste im Mittel von 17\% der Befragten genutzt. Das auffälligste Nutzungsverhalten zeigen die Leser von Erotiktiteln, von denen über $30 \%$ zumindest gelegentlich kostenpflichtige SMSDienste nachfragen, gefolgt von Sporttitellesern mit $28,3 \%$ sowie Leser von Musik- und Jugendzeitschriften mit 25\%. Im Detail wurden im Bereich kosten-

${ }^{625}$ Exemplarisch sei die Verteilung der nachgewiesenen Merkmalskombinationen von Printtiteln und mobilen Diensten am nutzungsstärksten Segment mobiler WAP-Dienste verdeutlicht: Im Einzelnen gaben 15,1\% der Befragten an, dass Sie regelmäßig eine Tageszeitung lesen und zumindest gelegentlich WAP-Dienste konsumieren. Die Kombination der Nutzung von WAP-Diensten mit dem regelmäßigen Konsum von aktuellen Magazinen trat bei 9,7\% und die Kombination mit Programmzeitschriften bei $8,5 \%$ der Befragten auf. 
pflichtiger SMS-Dienste sieben Ausprägungsvarianten von Diensten mit sowohl Informations- als auch Unterhaltungscharakter erhoben. Zu den im Mittel am häufigsten genutzten Dienstformen gehören Nachrichten zu Sportereignissen $(8,6 \%)$ und dem allgemeinen Weltgeschehen $(6,4 \%)$. Von geringer Bedeutung sind dagegen Finanznachrichten oder Horoskopdienste (2,5\% bzw. 2,4\%). Abbildung 5-27 zeigt die Antworthäufigkeiten in Relation zur jeweiligen Lesersegmentgröße. Neben der sicherlich auffälligsten Kreuzhäufigkeit der Nutzung von SMS-Nachrichten zu Sportereignissen durch Leser von Sportpresse (21\%) zeigt sich auch hier eine auffällige Nutzungsbereitschaft kostenpflichtiger SMSInhalte durch die Leser von Musik- und Jugendzeitschriften mit einem Nutzeranteil von $12 \%$ im Bereich von Unterhaltungsinhalten und durch die Leser von Erotik- und Lifestylemagazinen mit einem Nutzeranteil von $14 \%$ bzw. $8,5 \%$ im Bereich von Sportinhalten.

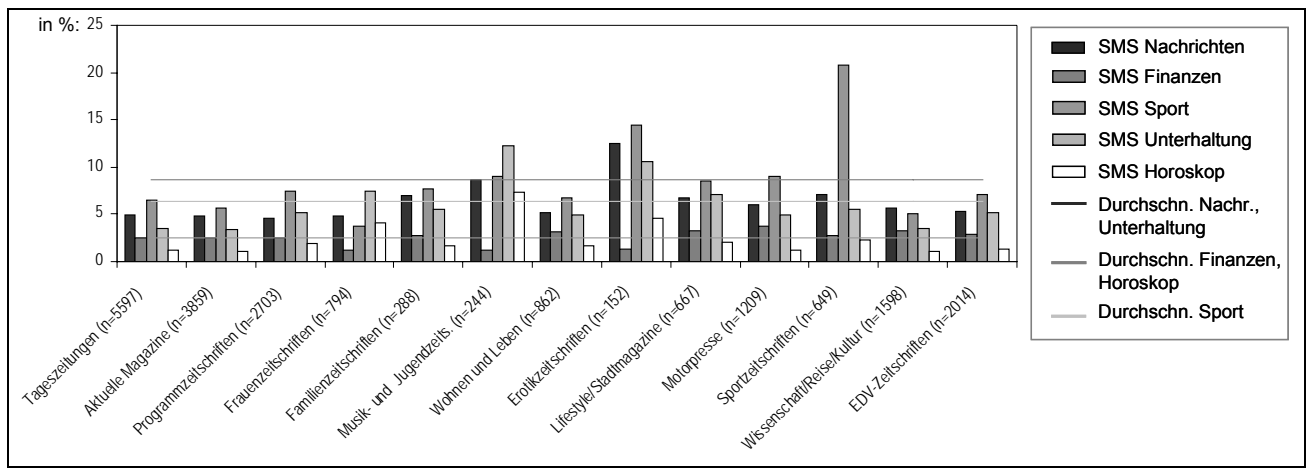

Abbildung 5-27: Nutzungsintensität von SMS-Diensten in Anteilen Lesergruppe

(2) Ausgehend von einer insgesamt niedrigeren Nutzungsintensität von durchschnittlich rund $8 \%$ der Befragten zeigt die Nutzung kostenpflichtiger MMSDienste ein zur Nutzung von SMS-Diensten vergleichbares Bild. Abbildung 5-28 zeigt die Anteile der Dienstnutzer am jeweiligen Lesersegment. Vergleichsweise intensive Nutzung von MMS-Diensten ist in den Segmenten der Leser von Erotiktiteln (23\%) sowie Musik- und Jugendtiteln (12,9\%) festzustellen. Erneut gehören Nachrichten zu Sport (knapp 4\%) und Weltgeschehen (3\%) zu den im segmentübergreifenden Mittel am häufigsten genutzten Diensten. Die Leser von Musik- und Jugendzeitschriften (14\%), Erotik- (23\%), Motor- und Sportpresse (je 12\%) besitzen inhalteübergreifend die ausgeprägteste Bereitschaft zur Nutzung von MMS-gestützten Inhalteformaten. Nach den Lesern von Sporttiteln (knapp 8\%) besitzen die Leser von Erotiktiteln $(7,8 \%)$ die ausgeprägteste Nutzungshäufigkeit MMS-gestützter Sportinhalte, MMSNachrichten zum politischen Weltgeschehen werden am häufigsten genutzt von Erotiklesern (knapp 6\%), gefolgt von Lesern der Musik- und Jugendtitel $(3,6 \%)$ und Sportpresse $(3,1 \%)$. 


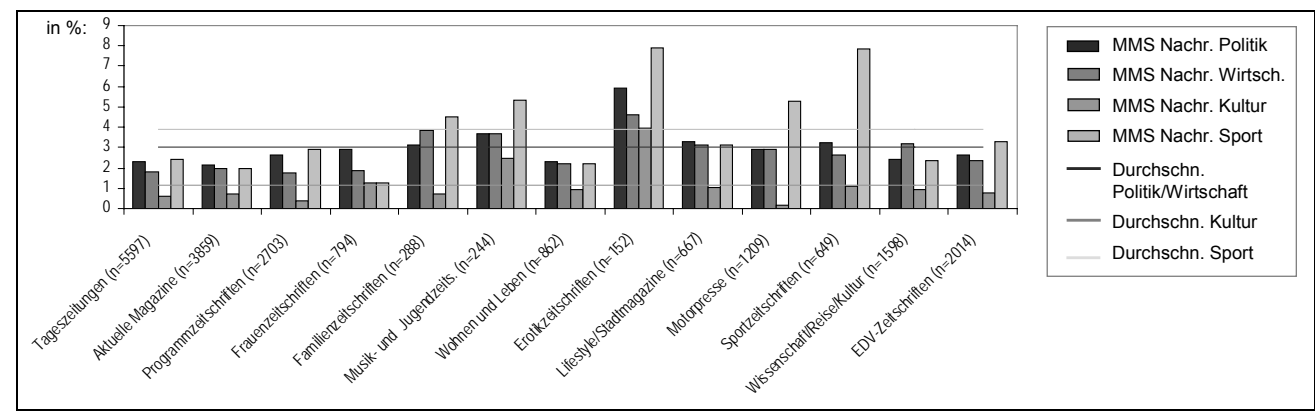

Abbildung 5-28: Nutzungsintensität von MMS-Diensten in Anteilen der Lesergruppe

(3) Wie bereits in Abbildung 5-25 ersichtlich wurde, bilden WAP-gestützte Portaldienste die nachfragestärkste Dienstform bei den Befragten. Insgesamt wurden 12 verschiedene WAP-typische Angebotsformen erhoben. Neben medientypischen Informations- und Unterhaltungsangeboten wie Nachrichten $(9,1 \%)$, Sportnachrichten $(8,9 \%)$, Freizeit- $(3,1 \%)$ und Reiseinformationen $(3,2 \%)$ bilden endgerätespezifische Unterhaltungs- und Kommunikationsangebote wie Klingeltöne $(9,4 \%)$, Email-Dienste $(7,1 \%)$ und Computerspiele $(6,7 \%)$ die segmentübergreifend am häufigsten von den befragten Onlinerezipienten genutzten Dienstformen. Abbildung 5-29 zeigt die die jeweilige Nutzungsintensität der sechs am häufigsten angegeben Dienstformen in Anteilen der Lesersegmente.

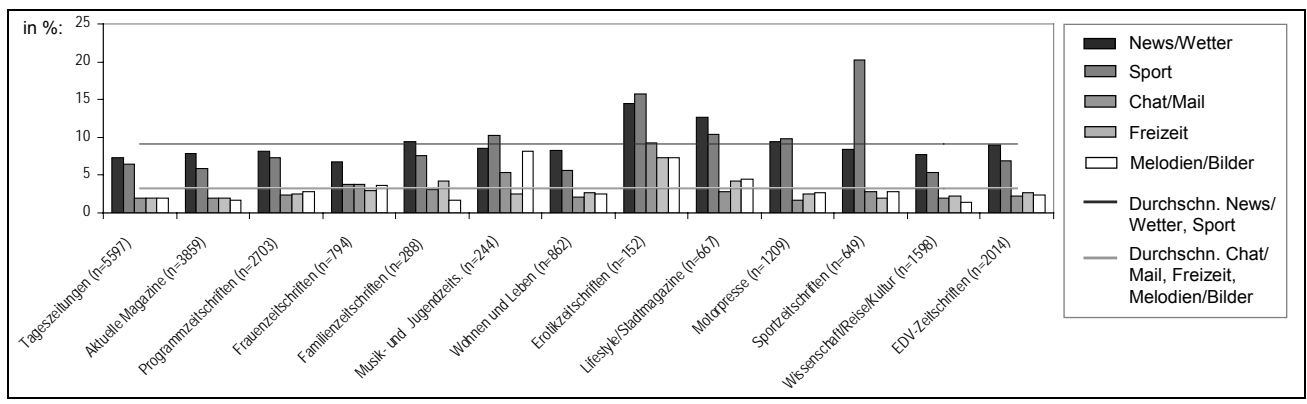

Abbildung 5-29: Nutzung von WAP-Diensten in Anteilen der Lesergruppe

Erwartungsgemäß werden unterhaltungsorientierte Inhalte wie Klingeltöne und Computerspiele in erster Linie im Lesersegment der Jugend- und Musikzeitschriften überdurchschnittlich $(8,2 \%)$ nachgefragt. Informationsorientierte Angebote wie Nachrichten zu Weltgeschehen und Sportereignissen werden erneut am häufigsten durch Leser der Erotik- (15,8\% bzw. 14,5\%), Lifestyle(12,6\% bzw. 10,3\%) und Sportpresse genutzt (8,5\% bzw. 20,2\%).

(4) Im Bereich ortsbezogener Anwendungen wurden vier bereits von einigen Anbietern realisierte Dienstformen zur Auswahl gestellt: Ortsbezogene Auskunft- oder Verzeichnisdienste, wie die in Abschnitt 5.2.3.2 dargestellten Anwendungen von t-info, sog. „Community-Dienste“ wie bspw. der unter der Marke der Frauenzeitschrift Amica von Tomorrow-Focus angebotene Personenlokalisierungsdienst „Friendfinder", Navigationsdienste, wie bspw. der von T- 
Mobile angebotene Verkehrsinformationsdienst „NaviGate“ und die Automatenbezahlung mit dem mobilen Endgerät, bspw. von Parkplatz- oder Beförderungsgebühren. Auf insgesamt geringem Nutzungsniveau werden davon segmentübergreifend vor allem Auskunfts- und Navigationsdienste $(3,6 \%$ bzw. $3,4 \%$ ) genutzt, von geringer Bedeutung sind bislang Community- und Bezahldienste $(1,6 \%$ bzw. $0,4 \%)$. Auffällig ist die insgesamt überdurchschnittliche Nutzung solcher Dienste bei den Lesern der Familienpresse (bspw. 6,3\% bei Auskunftsdiensten) und Einrichtungsmagazine (5\% bei Navigationsdiensten). Überdurchschnittliche Nutzungsbereitschaft ortsbezogener Dienste weise weiterhin die Leser der Musik- und Jugend-, Einrichtungs-, und Erotiktitel auf (vgl. Abbildung 5-30).

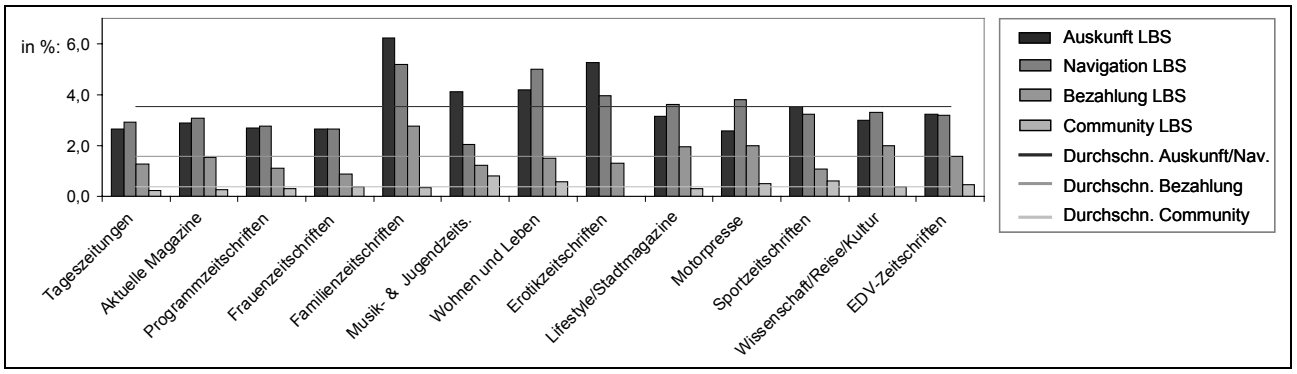

Abbildung 5-30: Nutzung ortsbezogener Dienste in Anteilen der Lesergruppe

\subsection{Motive der (Nicht-)Nutzung}

Im Falle einer Nichtinanspruchnahme der jeweils abgefragten Ausprägungsformen mobiler Dienste wurden die Bedingungen erhoben, unter denen für die jeweiligen Befragten eine zukünftige Nutzung in Frage käme. Geprüft wurden Aspekte des erwarteten Nutzens (Preis und Mehrwert), die wahrgenommene Inhaltequalität sowie Aspekte der technischen Brauchbarkeit (Geschwindigkeit, Darstellungsqualität und Gerätebedienbarkeit). Weder zwischen den jeweiligen Lesergruppen noch zwischen den einzelnen Dienstausprägungen konnten wesentliche Begründungsunterschiede festgestellt werden. Wie Abbildung 5-31 zeigt, dominieren deutlich ökonomische Bedingungen wie Preis und Mehrwert ${ }^{626}$.

\begin{tabular}{|l|r|r|}
\hline & Mittelwert & Standardabw. \\
\hline Günstigere Preise & $41,6 \%$ & 13,4 \\
\hline Gebotener Mehrwert & $18,5 \%$ & 6,4 \\
\hline Inhaltequalität & $18,1 \%$ & 7,0 \\
\hline Übertragungsgeschwindigkeit & $10,8 \%$ & 6,5 \\
\hline Displayqualität & $8,2 \%$ & 4,8 \\
\hline Bessere Gerätebedienbarkeit & $7,0 \%$ & 3,4 \\
\hline
\end{tabular}

Abbildung 5-31: Bedingungen der zukünttigen Nutzung mobiler Inhalte

${ }^{626}$ Als „Mehrwert" wurde der inhaltliche oder situationsbezogene Nutzen des Dienstes gegenüber etablierten Inhalteformaten erhoben. 
Zur Bestimmung der Motive, die die Nutzung der jeweils durch die Befragten angegebenen Dienstformen bestimmen, wurden vor dem Hintergrund der in Abschnitt 5.3.1.1 genannten Faktoren etablierter Inputmodelle Kriterien zur Auswahl gestellt, die in erster Linie Rückschlüsse auf die affektiven Nutzungsursachen geben sollen. Im Einzelnen wurden acht Kriterien geprüft, wovon vier Kriterien (Gebrauchswert, Neugier, Faszination und Gewohnheit) jeweils das zweckneutrale Interesse und vier Kriterien (Mobilität, Information, Unterhaltung und Interaktivität) jeweils zweckgebundene Gratifikationen prüfen. Abbildung 5-32 zeigt, dass das Innovationserlebnis im Zusammenhang mit dienstspezifischer Neugier und allgemeiner Technikfaszination das dominierende Nutzungsmotiv bildet. Dabei zeigen insbesondere WAP- und SMS-Dienste überdurchschnittlichen Gebrauchswert, was zudem deutlich stärker als bei MMS, LBS- oder Musikdiensten eine gewohnheitsmäßige Nutzung zur Folge hat.

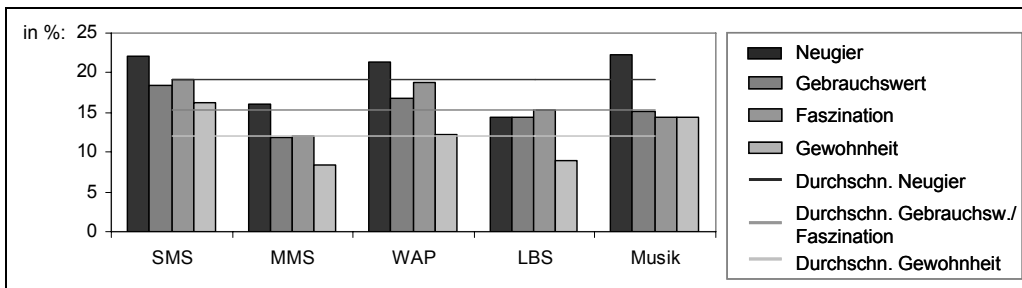

Abbildung 5-32: Ausprägungsgrade zweckneutraler Nutzungsmotive

Wie aus Abbildung 5-33 deutlich wird, bedienen bestehende mobile Dienste in erster Linie Bedürfnisse, die mit dem jeweiligen Mobilitätsgrund in Zusammenhang stehen. Während SMS und erstaunlicherweise auch Musikdienste in erster Linie Informationsbedürfnisse befriedigen, dienen MMS-, WAP- und ortsbezogene Dienste in erster Linie der Befriedigung eines Interaktivitätsbedürfnisses.

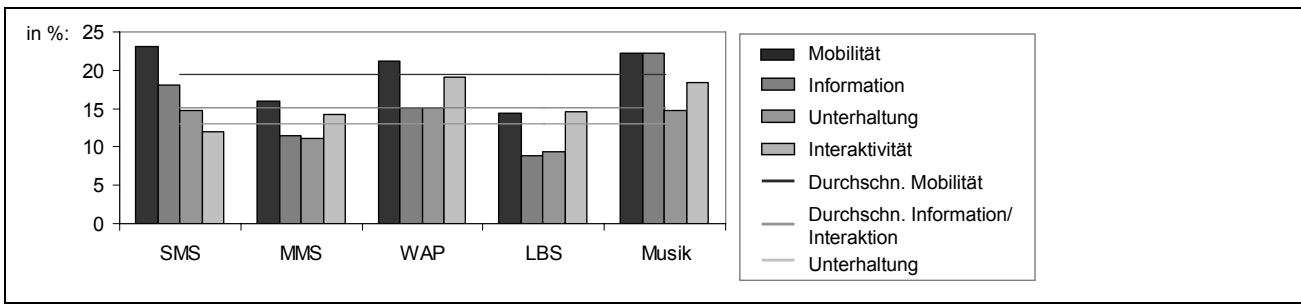

Abbildung 5-33: Inhaltebezogene Gratifikationen mobiler Dienste

\subsection{Befunde der Untersuchung}

Vor dem Hintergrund der in Abschnitt 5.3.2.1 abgeleiteten Forschungsleitfragen werden im Folgenden die Ergebnisse der Erhebung auf signifikante Zusammenhänge geprüft, die Schlussfolgerungen aus dem jeweiligen Nutzungsverhalten auf die segmentspezifischen Besonderheiten der Akzeptanz des mobilen Internet erlauben. Entsprechend dem voranstehend dargelegten Aufbau der Erhebung werden zunächst gemäß Leitfrage 1 die Befunde in Bezug auf die Auswirkungen der Technikadoption auf die Dienstakzeptanz zusammengefasst 
(1). Im Zusammenhang mit Leitfrage 4 werden daran anschließend die Unterschiede in der Nutzung der erhobenen Dienstformate für die differenzierten Lesergruppen auf systematische Abhängigkeiten analysiert (2). Schließlich werden vor dem Hintergrund der Leifragen 2 und 3 die Auffälligkeiten der Inhalteformate jeweils durch Lesergruppen nachgefragter mobiler Dienste auf Zusammenhänge mit den jeweiligen Nutzungsmotiven hin untersucht (3).

(1) Leitfrage 1 liegt die Vermutung zugrunde, dass - aufgrund der Systemguteigenschaften mobiler Dienste - Kenntnisse im Bereich von Mobilfunk- und Endgerätetechnik positive Auswirkungen auf die Akzeptanz und somit das messbare Nutzungsverhalten mobiler Dienste besitzen. Eine Prüfung der jeweiligen Kenntnisse kann dabei anhand der jeweils durch die Befragten verwendeten Endgeräte, insbesondere durch eine Differenzierung zwischen Mobiltelefonen und sog. Smartphones, erfolgen. Wie bereits angesprochen wurde, repräsentieren Smartphones das Ergebnis der Konvergenz von Computern und Funkgeräten, wobei sich Smartphones darin von einfachen Mobiltelefonen unterscheiden, dass sie in der Regel einerseits über größere Anzeigebildschirme, größere Rechen- und Speicherkapazitäten sowie eine umfangreichere funktionale Ausstattung (wie bspw. die Ausgabemöglichkeit versch. Video-, Audio- und Dokumentenformate) verfügen, andererseits jedoch außerdem im Anschaffungspreis teurer als einfache Mobiltelefone sind. Es ist daher anzunehmen, dass Smartphones tendenziell gezielt von solchen Konsumenten gewählt werden, die die reichere Geräteausstattung zumindest wahrnehmen und verstehen können. Damit die in Zusammenhang mit Leitfrage 1 formulierte Hypothese als wahr angenommen werden kann, sollten Smartphonenutzer einen höheren Nutzungsgrad mobiler Dienstformate besitzen. Abbildung 5-34 zeigt einen Mittelwertvergleich für die jeweils am häufigsten nachgefragten Ausprägungen unter den erhobenen mobilen Diensten von Mobiltelefon- und Smartphonenutzern.

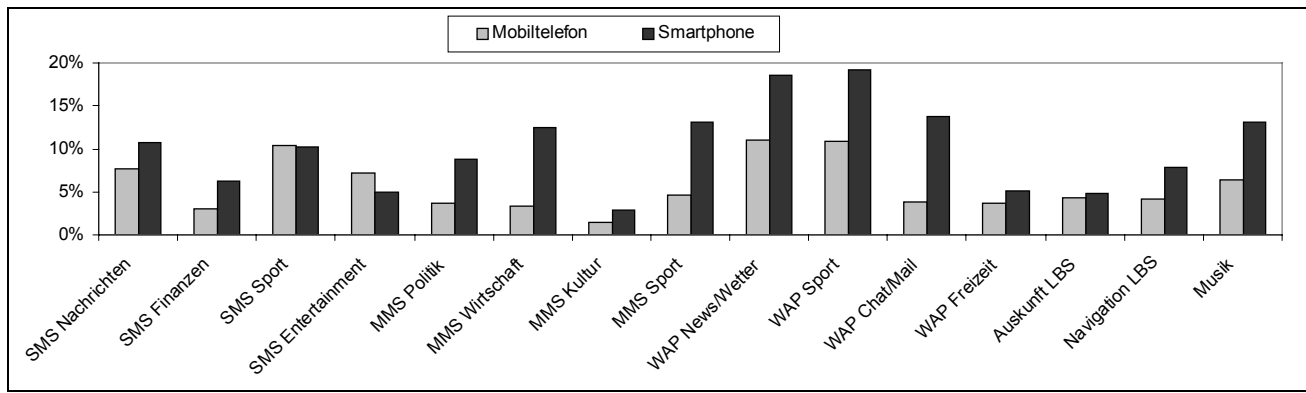

Abbildung 5-34: Vergleich der mittleren Nutzungsanteile von Mobiltelefon und Smartphone

Die Abbildung macht deutlich, dass die Annahme der Hypothese plausibel ist. Es ist daher davon auszugehen, dass jene Lesergruppen, die eine hohe Smartphoneverbreitung aufweisen (im Rahmen der Stichprobe sind dies die Leser von Familien-, Erotik-, Motor- und EDV-Titeln) tendenziell eine höhere Akzeptanz mobiler Dienste besitzen. Es ist allerdings anzumerken, dass der Verbreitungsgrad von Smartphones selbst in diesen Lesergruppen sehr gering 
ist, was eine konkrete Quantifizierung der Auswirkungen der Technikadoption auf das Nutzungsverhalten mobiler Dienste erschwert.

(2) Abbildung 5-26 legt die Vermutung nahe, dass zwischen den verschiedenen Lesergruppen Unterschiede in der Nutzungsbereitschaft mobiler Dienste existieren, die nicht alleine anhand von unterschiedlichen Endgeräteaustattungen zu begründen sind. Insbesondere zeigen die Leser von Musik- und Jugend-, Erotik-, Lifestyle-, Motor- und Sporttiteln eine überdurchschnittliche Bereitschaft zur Nutzung der erhobenen Dienstformate. Abbildung 5-35 zeigt im Überblick anhand der Ergebnisse von Unabhängigkeitstests zwischen Lesegewohnheiten und der Nutzung mobiler Dienstformate, dass dieser Zusammenhang nicht zufällig ist. Grau hinterlegt sind alle Abhängigkeiten, die mindestens auf dem 95\%-Niveau signifikant sind, dunkler hervorgehoben darunter jene, die auf dem 99\%-Niveau signifikant sind.

\begin{tabular}{|c|c|c|c|c|c|c|c|c|c|c|c|c|c|c|}
\hline & & \begin{tabular}{|l} 
Tages- \\
zeitungen
\end{tabular} & \begin{tabular}{|l} 
Aktuelle \\
Magazine
\end{tabular} & \begin{tabular}{|l} 
Programm- \\
zeitschr.
\end{tabular} & $\begin{array}{l}\text { Frauen- } \\
\text { zeitschr. }\end{array}$ & \begin{tabular}{|l} 
Familien- \\
zeitschr.
\end{tabular} & $\begin{array}{l}\text { Musik- \& } \\
\text { Jugendz. }\end{array}$ & \begin{tabular}{|l} 
Wohnen \& \\
Leben
\end{tabular} & \begin{tabular}{|l} 
Erotik- \\
zeitschr.
\end{tabular} & \begin{tabular}{|l|} 
Lifestyle- \& \\
Stadtm.
\end{tabular} & $\begin{array}{l}\text { Motor- } \\
\text { presse }\end{array}$ & $\begin{array}{l}\text { Sport- } \\
\text { zeitschr. }\end{array}$ & $\begin{array}{l}\text { Wissens./ } \\
\text { Reise/Kul. }\end{array}$ & \begin{tabular}{|l} 
EDV- \\
Zeitschr.
\end{tabular} \\
\hline \multirow[t]{2}{*}{ SMS } & \begin{tabular}{|l} 
Chi-Quadrat \\
(Signifikanz)
\end{tabular} & $\begin{array}{c}5,6 \\
(0,133)\end{array}$ & $\begin{array}{c}9,9 \\
(0,019)\end{array}$ & $\begin{array}{c}46,1 \\
(0,000)\end{array}$ & $\begin{array}{c}18,7 \\
(0,000)\end{array}$ & $\begin{array}{c}6,2 \\
(0,100)\end{array}$ & $\begin{array}{c}19,8 \\
(0,000)\end{array}$ & $\begin{array}{c}9,9 \\
(0,030)\end{array}$ & $\begin{array}{c}33,0 \\
(0,000)\end{array}$ & $\begin{array}{c}14,9 \\
(0,002)\end{array}$ & $\begin{array}{c}22,5 \\
(0,000)\end{array}$ & $\begin{array}{c}71,2 \\
(0,000)\end{array}$ & $\begin{array}{c}5,1 \\
(0,162)\end{array}$ & $\begin{array}{c}32,8 \\
(0,000)\end{array}$ \\
\hline & \begin{tabular}{|l} 
Kontingenzkoeff. \\
(Signifikanz)
\end{tabular} & $\begin{array}{c}0,028 \\
(0,133)\end{array}$ & $\begin{array}{c}0,037 \\
(0,019)\end{array}$ & $\begin{array}{c}0,080 \\
(0,000)\end{array}$ & $\begin{array}{c}0,051 \\
(0,000)\end{array}$ & $\begin{array}{c}0,030 \\
(0,100)\end{array}$ & $\begin{array}{c}0,053 \\
(0,000)\end{array}$ & $\begin{array}{c}0,036 \\
(0,030)\end{array}$ & $\begin{array}{c}0,068 \\
(0,000)\end{array}$ & $\begin{array}{c}0,046 \\
(0,002)\end{array}$ & $\begin{array}{c}0,056 \\
(0,000)\end{array}$ & $\begin{array}{c}0,100 \\
(0,000)\end{array}$ & $\begin{array}{c}0,027 \\
(0,162)\end{array}$ & $\begin{array}{c}0,068 \\
(0,000) \\
\end{array}$ \\
\hline \multirow[t]{2}{*}{ MMS } & $\begin{array}{l}\text { Chi-Quadrat } \\
\text { (Signifikanz) }\end{array}$ & $\begin{array}{c}2,4 \\
(0,502)\end{array}$ & $\begin{array}{c}11,2 \\
(0,011)\end{array}$ & $\begin{array}{c}6,2 \\
(0,100)\end{array}$ & $\begin{array}{c}5,6 \\
(0,134)\end{array}$ & $\begin{array}{c}8,1 \\
(0,044)\end{array}$ & $\begin{array}{c}13,8 \\
(0,003)\end{array}$ & $\begin{array}{c}5,2 \\
(0,156)\end{array}$ & $\begin{array}{c}48,3 \\
(0,000)\end{array}$ & $\begin{array}{c}14,2 \\
(0,003)\end{array}$ & $\begin{array}{c}37,2 \\
(0,000)\end{array}$ & $\begin{array}{c}36,8 \\
(0,000)\end{array}$ & $\begin{array}{c}7,8 \\
(0,050)\end{array}$ & $\begin{array}{c}28,3 \\
(0,000)\end{array}$ \\
\hline & \begin{tabular}{|l}
$\begin{array}{l}\text { Kontingenzkoeff. } \\
\text { (Signifikanz) }\end{array}$ \\
\end{tabular} & $\begin{array}{c}0,018 \\
(0,502)\end{array}$ & $\begin{array}{c}0,040 \\
(0,011)\end{array}$ & $\begin{array}{c}0,030 \\
(0,100)\end{array}$ & $\begin{array}{c}0,028 \\
(0,134)\end{array}$ & $\begin{array}{c}0,034 \\
(0,044)\end{array}$ & $\begin{array}{c}0,044 \\
(0,003)\end{array}$ & $\begin{array}{c}0,027 \\
(0,156)\end{array}$ & $\begin{array}{c}0,082 \\
(0,000)\end{array}$ & $\begin{array}{c}0,045 \\
(0,003)\end{array}$ & $\begin{array}{c}0,072 \\
(0,000)\end{array}$ & $\begin{array}{c}0,072 \\
(0,000)\end{array}$ & $\begin{array}{c}0,033 \\
(0,050)\end{array}$ & $\begin{array}{c}0,063 \\
(0,000)\end{array}$ \\
\hline \multirow[t]{2}{*}{ WAP } & $\begin{array}{l}\text { Chi-Quadrat } \\
\text { (Signifikanz) }\end{array}$ & $\begin{array}{c}6,7 \\
(0,083)\end{array}$ & $\begin{array}{c}7,7 \\
(0,052)\end{array}$ & $\begin{array}{c}37,4 \\
(0,000)\end{array}$ & $\begin{array}{c}1,5 \\
(0,683)\end{array}$ & $\begin{array}{c}6,4 \\
(0,094)\end{array}$ & $\begin{array}{c}34,7 \\
(0,000)\end{array}$ & $\begin{array}{c}5,8 \\
(0,123)\end{array}$ & $\begin{array}{c}29,5 \\
(0,000)\end{array}$ & $\begin{array}{c}52,6 \\
(0,000)\end{array}$ & $\begin{array}{c}26,7 \\
(0,000)\end{array}$ & $\begin{array}{c}59,0 \\
(0,000)\end{array}$ & $\begin{array}{c}5,6 \\
(0,131)\end{array}$ & $\begin{array}{c}35,4 \\
(0,000)\end{array}$ \\
\hline & \begin{tabular}{|l|}
$\begin{array}{l}\text { Kontingenzkoeff. } \\
\text { (Signifikanz) }\end{array}$ \\
\end{tabular} & $\begin{array}{c}0,031 \\
(0,083)\end{array}$ & $\begin{array}{c}0,033 \\
(0,052) \\
\end{array}$ & $\begin{array}{c}0,073 \\
(0,000) \\
\end{array}$ & $\begin{array}{c}0,015 \\
(0,683) \\
\end{array}$ & $\begin{array}{c}0,030 \\
(0,094)\end{array}$ & $\begin{array}{c}0,070 \\
(0,000) \\
\end{array}$ & $\begin{array}{c}0,029 \\
(0,123) \\
\end{array}$ & $\begin{array}{l}0,065 \\
(0,000) \\
\end{array}$ & $\begin{array}{c}0,086 \\
(0,000) \\
\end{array}$ & $\begin{array}{c}0,061 \\
(0,000) \\
\end{array}$ & $\begin{array}{c}0,091 \\
(0,000) \\
\end{array}$ & $\begin{array}{c}0,028 \\
(0,131) \\
\end{array}$ & $\begin{array}{c}0,071 \\
(0,000) \\
\end{array}$ \\
\hline \multirow[t]{2}{*}{ LBS } & $\begin{array}{l}\text { Chi-Quadrat } \\
\text { (Signifikanz) }\end{array}$ & $\begin{array}{c}11,2 \\
(0,011)\end{array}$ & $\begin{array}{c}11,8 \\
(0,008)\end{array}$ & $\begin{array}{c}4,9 \\
(0,183)\end{array}$ & $\begin{array}{c}7,5 \\
(0,058)\end{array}$ & $\begin{array}{c}4,1 \\
(0,253)\end{array}$ & $\begin{array}{c}2,8 \\
(0,427)\end{array}$ & $\begin{array}{c}21,2 \\
(0,000)\end{array}$ & $\begin{array}{c}2,3 \\
(0,511)\end{array}$ & $\begin{array}{c}18,9 \\
(0,000)\end{array}$ & $\begin{array}{c}8,5 \\
(0,036)\end{array}$ & $\begin{array}{c}12,9 \\
(0,005)\end{array}$ & $\begin{array}{c}19,9 \\
(0,000)\end{array}$ & $\begin{array}{c}20,6 \\
(0,000)\end{array}$ \\
\hline & $\begin{array}{l}\text { Kontingenzkoeff. } \\
\text { (Signifikanz) }\end{array}$ & $\begin{array}{c}0,040 \\
(0,011)\end{array}$ & $\begin{array}{c}0,041 \\
(0,008)\end{array}$ & $\begin{array}{c}0,026 \\
(0,183)\end{array}$ & $\begin{array}{c}0,033 \\
(0,058)\end{array}$ & $\begin{array}{c}0,024 \\
(0,253)\end{array}$ & $\begin{array}{c}0,020 \\
(0,427)\end{array}$ & $\begin{array}{c}0,055 \\
(0,000)\end{array}$ & $\begin{array}{c}0,018 \\
(0,511)\end{array}$ & $\begin{array}{c}0,052 \\
(0,000)\end{array}$ & $\begin{array}{c}0,035 \\
(0,036)\end{array}$ & $\begin{array}{c}0,043 \\
(0,005)\end{array}$ & $\begin{array}{c}0,053 \\
(0,000)\end{array}$ & $\begin{array}{l}0,051 \\
(0,000)\end{array}$ \\
\hline \multirow[t]{2}{*}{ Musik } & $\begin{array}{l}\text { Chi-Quadrat } \\
\text { (Signifikanz) }\end{array}$ & $\begin{array}{c}4,7 \\
(0,031)\end{array}$ & $\begin{array}{c}14,8 \\
(0,000)\end{array}$ & $\begin{array}{c}9,5 \\
(0,02)\end{array}$ & $\begin{array}{c}28,9 \\
(0,000)\end{array}$ & $\begin{array}{c}0,418 \\
(0,518)\end{array}$ & $\begin{array}{c}41,3 \\
(0,000)\end{array}$ & $\begin{array}{c}2,6 \\
(0,108)\end{array}$ & $\begin{array}{c}23,5 \\
(0,000)\end{array}$ & $\begin{array}{c}20,8 \\
(0,000)\end{array}$ & $\begin{array}{c}10,0 \\
(0,002)\end{array}$ & $\begin{array}{c}18,8 \\
(0,000)\end{array}$ & $\begin{array}{c}0,035 \\
(0,853)\end{array}$ & $\begin{array}{c}0,6 \\
(0,432)\end{array}$ \\
\hline & $\begin{array}{l}\text { Kontingenzkoeff. } \\
\text { (Signifikanz) }\end{array}$ & $\begin{array}{c}0,026 \\
(0,031)\end{array}$ & $\begin{array}{c}0,046 \\
(0,000)\end{array}$ & $\begin{array}{l}0,037 \\
(0,02)\end{array}$ & $\begin{array}{c}0,064 \\
(0,000)\end{array}$ & $\begin{array}{c}0,008 \\
(0,518)\end{array}$ & $\begin{array}{c}0,077 \\
(0,000)\end{array}$ & $\begin{array}{c}0,019 \\
(0,108)\end{array}$ & $\begin{array}{c}0,058 \\
(0,000)\end{array}$ & $\begin{array}{c}0,054 \\
(0,000)\end{array}$ & $\begin{array}{c}0,038 \\
(0,002)\end{array}$ & $\begin{array}{c}0,052 \\
(0,000)\end{array}$ & $\begin{array}{c}0,002 \\
(0,853)\end{array}$ & $\begin{array}{c}0,009 \\
(0,432)\end{array}$ \\
\hline
\end{tabular}

Abbildung 5-35: Abhängigkeiten zwischen Leseverhalten und Nutzung des mobilen Internet

Die Ergebnisse der Unabhängigkeitstest machen deutlich, dass der Erklärungszusammenhang zwischen Leserverhalten und Dienstnutzung ist allerdings segmentübergreifend schwach ausgeprägt, was durch die niedrigen Werte der Konfidenzkoeffizienten zum Ausdruck kommt. Dies legt die Schlussfolgerung nahe, dass selbst in den grau hinterlegten Segmenten des Lesermarkts zwar ein Potenzial für zusätzliche Einnahmen durch bestehende Konsumenten auf der Grundlage einer Vermarktung mobiler Dienste besteht, das Geschäft im mobilen Internet jedoch kaum Massenmarkt- sondern bestenfalls Nischencharakter besitzt.

Abbildung 5-36 zeigt die Ausprägungen der Kontingenzkoeffizienten für die jeweils am häufigsten genutzten Dienstformate SMS, MMS und WAP in den fünf Lesergruppen mit der auffälligsten Nutzungsbereitschaft mobiler Dienste sowie - zum Vergleich - in der Lesergruppe aktueller Magazine. Die Abbildung zeigt, dass das Lesen von Sporttiteln für die Nutzung der Formate SMS und WAP den jeweils ausgeprägtesten Erklärungszusammenhang aufweist, wohingegen das MMS-Format vor allem von Erotiklesern bevorzugt wird. 


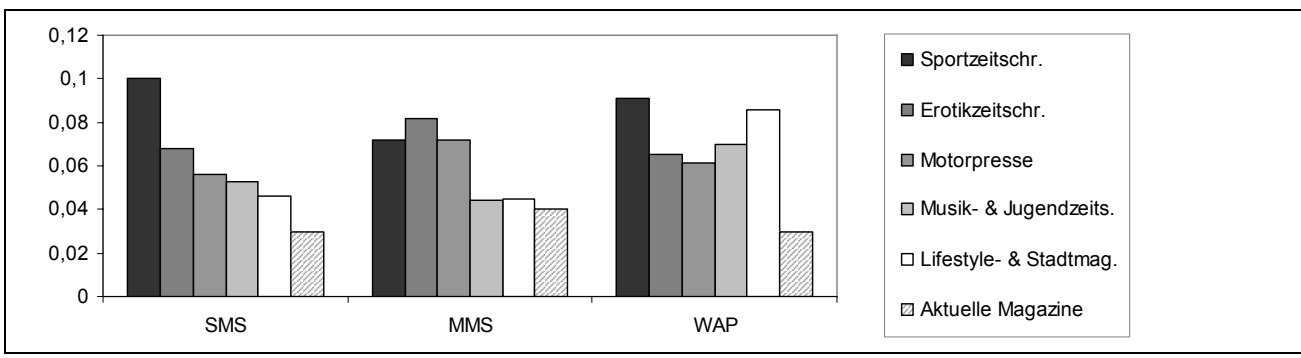

Abbildung 5-36: Zusammenhangsmaße für Leserverhalten und Nutzung mobiler Dienste

Die auf aggregierter Ebene des Dienstformats identifizierten Abhängigkeiten sind vor dem Hintergrund von Leitfrage 2 im Detail auf der Ebene inhaltebezogener Dienstvarianten nachzuweisen. Wie aus Abbildung 5-27 bis Abbildung 5-29 ersichtlich wird, weisen in den SMS-, MMS- und WAP-gestützten Dienstformaten jeweils nachrichten-, sport- und unterhaltungsorientierte Inhalte die größten Nutzungshäufigkeiten auf. Abbildung 5-37 zeigt vor diesem Hintergrund die Konfidenzkoeffizienten der jeweils drei nachfragestärksten Dienstformate für die vier Lesersegmente mit der höchsten Nutzungsbereitschaft für mobile Dienste. Die Abbildung macht deutlich, dass der Zusammenhang zwischen Leserverhalten und Inhaltenutzung von wenigen Ausnahmen abgesehen in allen drei Ausprägungen und für jedes Format signifikant ist. Abermals ist jedoch mit Ausnahme der Nutzung von Sportinhalten durch die Leser von Sporttiteln eine geringe Ausprägung der nachgewiesenen Abhängigkeit festzustellen.

\begin{tabular}{|c|c|c|c|c|c|}
\hline & & $\begin{array}{l}\text { Musik- \& } \\
\text { Jugendz. }\end{array}$ & $\begin{array}{l}\text { Erotikzeit- } \\
\text { schriften }\end{array}$ & $\begin{array}{l}\text { Lifestyle- \& } \\
\text { Stadtmag. }\end{array}$ & $\begin{array}{l}\text { Sportzeit- } \\
\text { schriften }\end{array}$ \\
\hline \multirow[t]{3}{*}{ SMS } & Sport & $0,026^{*}$ & $0,051^{* *}$ & $0,039^{\star *}$ & $0,205^{\star *}$ \\
\hline & Nachrichten & $0,038^{* *}$ & $0,053^{* *}$ & $0,034^{*}$ & $0,04^{* *}$ \\
\hline & Unterhaltung & $0,085^{\star \star}$ & $0,057^{\star *}$ & $0,058^{* *}$ & $0,029^{*}$ \\
\hline \multirow[t]{3}{*}{ MMS } & Sport & $0,038^{*}$ & $0,061^{* *}$ & 0,02 & $0,124^{* *}$ \\
\hline & Nachrichten & 0,018 & $0,037^{*}$ & 0,031 & $0,026^{*}$ \\
\hline & Unterhaltung & $0,042^{* *}$ & $0,059^{* *}$ & $0,025^{*}$ & $0,026^{*}$ \\
\hline \multirow[t]{3}{*}{ WAP } & Sport & $0,033^{*}$ & $0,061^{\text {** }}$ & $0,059^{\text {** }}$ & $0,183^{* *}$ \\
\hline & Nachrichten & 0,012 & $0,04^{* \star}$ & $0,071^{* *}$ & 0,016 \\
\hline & Unterhaltung & $0,087^{\star *}$ & $0,059^{\star *}$ & $0,058^{* *}$ & 0,017 \\
\hline & \multicolumn{5}{|c|}{$\begin{array}{l}\text { * signifikant auf dem 95\%-Niveau } \\
\text { ** signifikant auf dem 99\%-Niveau }\end{array}$} \\
\hline
\end{tabular}

Abbildung 5-37: Zusammenhangsmaße für Leserverhalten und Nutzung mobiler Inhalteformate

(3) in Abschnitt 5.3.2.3.2.3 wurde bereits gezeigt, dass derzeit in erster Linie das Innovationserlebnis, die Standortungebundenheit und die Befriedigung von Informationsbedürfnissen im Mittelpunkt der Nutzungsmotive bei mobilen Diensten stehen. Eine Untersuchung der Nutzungsmotive in Abhängigkeit der jeweiligen Lesergruppe zeigt nur in Nuancen Unterschiede. Während bspw. SMS- und MMS-Dienste für Leser von Jugend- und Musik- sowie Erotiktiteln tendenziell Unterhaltungscharakter besitzen, überwiegt bei den Lesern von Lifestyle- und Stadtmagazinen das Motiv der Befriedigung von Informationsbedürfnissen (vgl. Abbildung 5-38). 


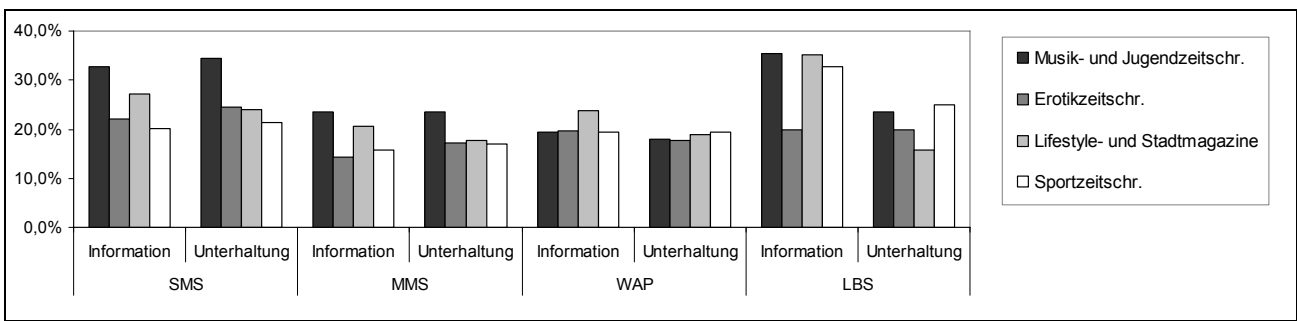

Abbildung 5-38: Verhältnis von Informations- und Unterhaltungsmotiv

Im Hinblick auf die Gewohnheitsmäßigkeit der Nutzung mobiler Dienste zeigen einzig die Leser von Erotiktiteln im Rahmen der Nutzung von WAP-Formaten ein gegenüber der Befriedigung spontaner Neugier zumindest ausgeglichenes Verhältnis (vgl. Abbildung 5-39).

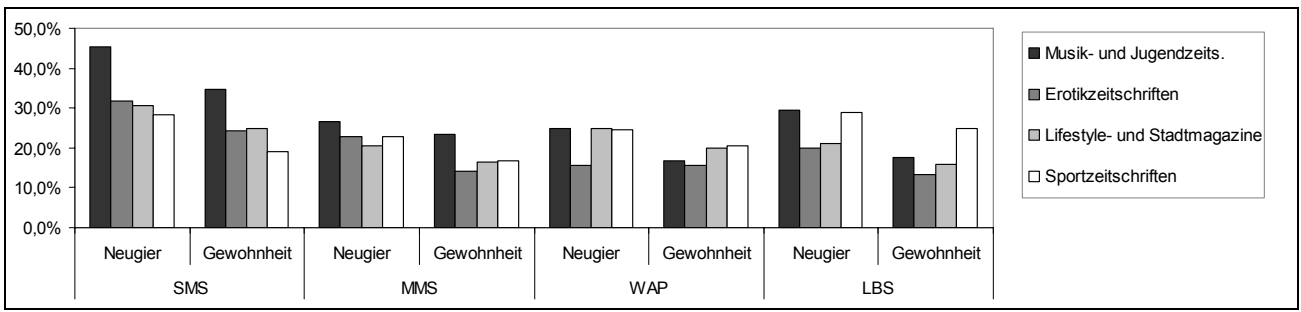

Abbildung 5-39: Verhältnis von Neugier und Nutzungsgewohnheit

Keinerlei signifikante Unterschiede konnten hingegen in den Ausprägungen des Motivs der Ortsungebundenheit, das in allen Lesergruppen insbesondere die Befriedigung des Interaktivitätsbedürfnisses dominiert, sowie in den Motiven der Technikfaszination und des Gebrauchswerts mobiler Dienste festgestellt werden.

\subsection{Zusammenfassung der Leserbefragung}

Die vorliegende Erhebung repräsentiert den Versuch einer verhaltensbezogenen Repräsentativbefragung zur Feststellung der Akzeptanz mobiler Dienste. Abbildung 5-40 zeigt die Ergebnisse der Erhebung im Überblick, geordnet nach den in Abschnitt 5.3.1.1 beschriebenen Kriterien und Dimensionen der etablierten Akzeptanzforschung. ${ }^{627}$

627 Entsprechend des in Abschnitt 5.3.1.1 beschriebenen mehrdimensionalen Akzeptanzverständnisses werden zur Klassifikation der Ergebnisse kognitive, affektive und konative Akzeptanzdimension unterschieden. Zusätzlich werden die Erhebungsergebnisse in Anlehnung an Nielsen (1993) und Rogers (1995) anhand der fünf Akzeptanzkriterien Wahrnehmbarkeit eines relativen Vorteils, Kosten, Kompatibilität und Komplexität strukturiert. 


\begin{tabular}{|c|c|c|c|c|}
\hline 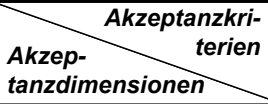 & $\begin{array}{l}\text { Relativer Vorteil, } \\
\text { Wahrnehmbarkeit }\end{array}$ & Kosten & Kompatibilität & $\begin{array}{l}\text { Komplexität, } \\
\text { Zuverlässigkeit }\end{array}$ \\
\hline $\begin{array}{l}\text { Kognitiv } \\
\text { (Kenntnis) }\end{array}$ & $\begin{array}{l}\text { Geringe Bekanntheit } \\
\text { des Dienstangebots }\end{array}$ & $\begin{array}{l}\text { Geringe } \\
\text { Bekanntheit der } \\
\text { Preisstrukturen }\end{array}$ & - & - \\
\hline $\begin{array}{c}\text { Affektiv } \\
\text { (Interesse) }\end{array}$ & $\begin{array}{l}\text { Nutzung bedingt } \\
\text { durch Neugier/ } \\
\text { Technikfaszination } \\
\text { - Mehrwert als Bedin- } \\
\text { gung zukünftiger } \\
\text { Nutzung }\end{array}$ & $\begin{array}{l}\text { Günstigere } \\
\text { Preise als } \\
\text { Bedingung } \\
\text { zukünftiger } \\
\text { Nutzung }\end{array}$ & $\begin{array}{l}\text { Bedingtheit der } \\
\text { Dienstnutzung } \\
\text { durch bestehende } \\
\text { Gewohnheiten der } \\
\text { Nutzung tradit. } \\
\text { Medien und } \\
\text { Geräte-Know-how }\end{array}$ & $\begin{array}{l}\text { - Wenig routiniertes, } \\
\text { aus Neugier } \\
\text { getriebenes } \\
\text { Nutzungsverhalten } \\
\text { - Formatqualität und } \\
\text { Bedienbarkeit als } \\
\text { Bedingungen } \\
\text { zukünft. Nutzung }\end{array}$ \\
\hline $\begin{array}{l}\text { Konativ } \\
\text { (Nutzung) }\end{array}$ & $\begin{array}{l}\text { - Insgesamt geringe } \\
\text { Nutzungsbereitschaft } \\
\text { nachweisbar } \\
\text { - Nutzung konzentriert } \\
\text { sich auf aktuell } \\
\text { informierende Inhalte }\end{array}$ & - & \multicolumn{2}{|c|}{$\begin{array}{l}\text { Nutzung konzentriert sich auf einfache } \\
\text { Dienstformate (insb. SMS, WAP) }\end{array}$} \\
\hline
\end{tabular}

Abbildung 5-40: Zusammenfassung der Erhebungsergebnisse

Die Erhebung diente in erster Linie dazu, das Interesse an mobilen Diensten (affektive Akzeptanz) und den gegenwärtigen Nutzungsgrad mobiler Inhalteformate (konative Akzeptanz) durch die Leser von Publikumsprinttiteln zu bestimmen. Kognitive Aspekte der Akzeptanz mobiler Dienste wurden nicht explizit adressiert, wobei bspw. anhand der jeweils durch die Befragten als bekannt angegebenen Dienste oder anhand der Kenntnisse aktueller Preisstrukturen Rückschlüsse auf eine insgesamt geringe Kenntnis der befragten Leser von dem Angebot mobiler Inhalteformate gebildet werden können. Die Erhebungsergebnisse machen deutlich, dass mobile Inhalteformate derzeit vor allem aus Neugier und zum Zwecke einer ortsungebundenen Inhalterezeption genutzt werden. Das Interesse an mobilen Inhalteformaten weist eine starke Abhängigkeit von den durch die Nutzung anfallenden Kosten, von der Qualität und Bedienbarkeit des jeweiligen mobilen Formats und von den Gewohnheiten der Mediennutzung eines Konsumenten auf.

Anders als bspw. Ziegler/Adam (2001) vermuten, werden im Rahmen der Nutzung mobiler Inhalteformate in erster Linie allgemeininformierende Inhalte wie Nachrichten zu Weltgeschehen und Sport nachgefragt. Dabei konnte die an anderer Stelle angestellte Vermutung einer fortbestehenden Ablehnung WAPorientierter Inhalteformate im Grundsatz widerlegt werden. Es sind insbesondere solche Rezipienten für mobile Dienste empfänglich, die im Bereich von Printtiteln ein differenziertes Leseverhalten zeigen. In den Massensegmenten des Lesermarkts wie dem Tageszeitungssegment ist dagegen keine signifikante Bereitschaft zur Nutzung des mobilen Internet festzustellen. Dieses Ergebnis ist aus drei Gründen ernüchternd: Erstens besitzen die angesprochenen Lesersegmente der Musik- und Jugend-, Erotik-, Lifestyle- und Stadt- sowie Sportpresse von Natur aus eine begrenzte Größe. Zweitens zeigt die Prüfung der Zusammenhangsstärke lediglich geringe Ausprägungen, was schlussfolgern lässt, dass selbst in den fünf genannten Lesernischen nur eine insgesamt geringe Nutzungsbereitschaft gegenüber dem mobilen Internet besteht. Drittens 
zeigt die Untersuchung der Nutzungsmotive ein wenig routiniertes, sondern vor allem durch Neugier und Technikinteresse getriebenes Verhalten, was keine Ableitung nachhaltiger Nachfragetendenzen erlaubt. Von besonderer Bedeutung für die in der Breite des Markts anhaltende Ablehnung des mobilen Internet scheint dabei ein durch Konsumenten wahrgenommenes Missverhältnis zwischen dem durch mobile Inhalte gebotenen Nutzen und dem dafür zu entrichtenden Preis zu sein.

\subsubsection{Zusammenfassung der Akzeptanzanalyse}

Die vorliegende Akzeptanzanalyse zielt auf eine Deskription des Nutzungsverhaltens mobiler Inhalteformate zur Exploration des Marktpotenzials des mobilen Internet aus Sicht der Medienbranche. Ebenso wie die Voruntersuchung in Abschnitt 5.3.2.2 weist auch die Hauptuntersuchung in Abschnitt 5.3.2.3 auf einen signifikanten Zusammenhang zwischen etablierter Mediennutzung und dem Interesse am mobilen Internet hin. Dennoch zeigen beide Untersuchungen von den Konsumenten wahrgenommene Missstände im Hinblick auf den erwarteten Mehrwert des mobilen Internet auf. Eine Erschließung des mobilen Internet als massentauglicher Inhaltemarkt bedarf daher Konzepte, um für mobile Inhalteformate über das bestehende Maß hinausgehende Mehrwerte zu schaffen. Der folgende Abschnitt stellt Möglichkeiten und Bedingungen der Bereitstellung derartiger Mehrwerte aus technischem Verständnis vor.

\subsection{Mobilitätsgerechte Bereitstellung von Mediengütern}

In Abschnitt 5.2.2.1 wurde dargelegt, dass die Rezeption von Onlineinhalten im mobilen Internet in der Regel als Nebentätigkeit im Zuge zirkulärer Mobilitätsformen des Alltags stattfindet. Eine solche Inhalterezeption erfolgt vor dem Hintergrund der derzeitigen Endgerätetechnik zwangsläufig unter eingeschränkten Rezeptionsbedingungen wie kleinen Anzeigebildschirmen, unbequemen Dateneingabe- und Interaktionsmöglichkeiten sowie einer (im Vergleich zum stationären Internet) langsamen Datenübertragung. ${ }^{628}$ Dabei weisen sowohl frem$\mathrm{de}^{629}$ als auch eigene Erhebungsergebnisse ${ }^{630}$ zur Akzeptanz mobiler Dienste einerseits auf eine ausgeprägte Kosten-Nutzen-Abwägung von Mobilfunkkunden im Hinblick auf in Anspruch genommene Datenübertragungskontingente hin. Andererseits gehören fehlender Mehrwert und schlechte Rezeptionsbedingungen (insbesondere die Übertragungsgeschwindigkeit, die Displayqualität und die Gerätebedienbarkeit) zu den am häufigsten genannten Gründen für eine Ablehnung des mobilen Internet.

Die genannten Ergebnisse der voranstehenden Untersuchungen machen deutlich, dass ein Erfolg der Inhalteverwertung im mobilen Internet nicht nur auf die Auswahl eines geeigneten Distributionsformats (wie SMS-, MMS- oder

\footnotetext{
628 Vgl. Passani (2002).

$629 \mathrm{Vgl}$. Abschnitt 5.3.1.2.

$630 \mathrm{Vgl}$. Abschnitte 5.3.2.2.2 und 5.3.2.3.2.3.
} 
WAP-gestützte Dienste) zurückzuführen ist, sondern in besonderer Weise davon abhängt, inwieweit es Inhalteanbietern gelingt, die bestehenden Beschränkungen der Rezeptionsbedingungen zu überwinden. Bereits in Abschnitt 4.3.4 wurde im Zusammenhang mit der Prüfung der Bedingungen einer Vorteilhaftigkeit individueller Mediengüter festgestellt, dass Individualisierung in solchen Fällen tendenziell vorteilhaft gegenüber einer standardisierten Inhaltedistribution ist, in denen ein breites Inhalteangebot einer kleinen Menge davon interessierender oder - wie im Falle des mobilen Internet - übertragbarer und darstellbarer Inhalte gegenüber steht. Die in Abschnitt 5.3.1.2 vorgestellten Ergebnisse der Akzeptanzanalyse von Wohlfahrt (2004) bestätigen die Vorteilhaftigkeitsvermutung von Individualisierungsformen für das mobile Internet. Neben dem nachweislichen Potenzial einer Anpassung des Inhalteangebots an individuelle Bedürfnisse und Präferenzen zeigt Abschnitt 5.2.2.1, dass im mobilen Internet darüber hinaus der jeweilige Aufenthaltsort eines Rezipienten ein sinnvolles Anpassungskriterium für angebotene Inhalte liefern kann.

In der Forschung zu mobilitätsgerechten Anwendungsformen hat sich die Diktion etabliert, individuelle und ortsbezogene mobile Dienste unter dem Begriff „anpassungsfähiger" oder „kontextadaptiver“ Anwendungen zusammenzufassen. In Abschnitt 5.4.1 wird der derzeitige Stand der Forschung in Bezug auf die Möglichkeiten einer derart kontextgerecht angepassten Inhaltedistribution dargestellt. Insofern eine kontextgerechte Anpassung der Inhaltedistribution in erster Linie auf den Fähigkeiten des Dienstanbieters beruht, die jeweils relevanten Bedingungen eines Abfragekontexts zu erfassen und zu verarbeiten, werden in Abschnitt 5.4.2 Besonderheiten und Lösungsansätze einer Kontextmodellierung für die Inhaltenachfrage im mobilen Internet vorgestellt. Vor dem Hintergrund der in der Systemgütereigenschaft wurzelnden Natur des mobilen Internet als verteiltes System beruht ein umfassendes Kontextmodell der mobilen Inhaltenachfrage zwangsläufig auf einer Vielzahl von Quellen für kontextbezogene Daten. Dies wirft Fragestellungen im Zusammenhang mit Datenschutz und Zugriffskontrolle auf. Abschnitt 5.4.3 zeigt einen Lösungsansatz für die datenschutzkonforme Zugriffskontrolle eines derartigen verteilten Kontextmodells. Die Ergebnisse des Kapitels werden in Abschnitt 5.4.4 zusammengefasst.

\subsubsection{Kontextadaptivität als Anforderung einer mobilitäts- gerechten Inhaltebereitstellung}

Ein Datenverarbeitungssystem wird immer dann als anpassungsfähig oder „kontextadaptiv“ bezeichnet, wenn es Informationen über die Situation der Systemabfrage einsetzt, um dem Benutzer relevante Informationen bzw. Dienste anzubieten. ${ }^{631} \mathrm{Um}$ die reale Abfragesituation eines Systems maschinell erfahrbar zu machen, muss diese Situation in Form eines abstrakten, maschinell verständlichen Ereignisses, einem so genannten „Kontext“, erfasst werden. ${ }^{632}$ Als

631 Vgl. Dey/Abowd (2000); Marmasse/Schmandt (2002); Chen/Finin/Joshi (2003); Salber/Dey/Abowd (1999); Chen/Kotz (2002).

632 Vgl. Kaspar/Diekmann/Hagenhoff (2005). 
Kontext wird dabei jegliche Information bezeichnet, die benutzt werden kann, um die für die Interaktion zwischen Benutzer und Anwendung relevante Situation einer Person, eines Orts oder eines Objekt zu charakterisieren. ${ }^{633}$ Adaptive Systeme setzen voraus, dass die Situation der Systemabfrage anhand solcher Kontexte maschinell wahrgenommen werden kann und das System entsprechend der wahrgenommenen Situation autonom Anpassungen seines Verhaltens vornehmen kann. Abschnitt 5.4.1.1 gibt einen Überblick über die Möglichkeiten einer maschinellen Situationswahrnehmung. Abschnitt 5.4.1.2 erläutert die Funktionsweise einer kontextbezogenen Systemadaption. Abschnitt 5.4.1.3 gibt einen Überblick über bestehende Forschungsbeiträge zu kontextadaptiven Systemen im Bereich mobiler Dienste. Die Ergebnisse des Teilkapitels werden in Abschnitt 5.4.1.4 zusammengefasst.

\subsubsection{Maschinelle Situationswahrnehmung}

Neben den bereits aus den voranstehenden Abschnitten bekannten Möglichkeiten der Wahrnehmung von individuellen Präferenzen auf Grundlage von Befragung oder Beobachtung und der Wahrnehmung des Aufenthaltsorts eines Mobilfunknutzers durch Lokalisierung anhand der Mobilfunkzelle, kann die Situation einer Systemabfrage darüber hinaus anhand der Abfragezeit, dem jeweils zur Abfrage verwendeten Endgerätetyp oder physikalischen Besonderheiten der Abfrage, bspw. der Umgebungstemperatur oder der Raumhelligkeit, erfasst werden. Eine der bekanntesten Klassifikationen für maschinell erfassbare Kontexte geht zurück auf Schilit/Adams/Want (1994). Diese unterscheiden den sozialen Kontext der Personen, die an der Systeminteraktion beteiligt sind (1), den technischen Kontext beteiligter oder verfügbarer Datenverarbeitungsressourcen (2) und den physikalischen Kontext des Aufenthaltsorts, an dem die Systeminteraktion stattfindet (3).

(1) Der soziale Kontext enthält Informationen über den oder die interaktionsbeteiligten Nutzer. Nutzerinformationen, wie Identität, Alter, Geschlecht und Vorlieben, können, wie bereits beschrieben, entweder explizit, d. h. durch Eingabe des Nutzers oder zum Teil auch implizit, d. h. durch Beobachtung des Nutzerverhaltens gewonnen werden. ${ }^{634}$

(2) Der Datenverarbeitungskontext beschreibt die verfügbare Netzwerkkonnektivität und Netzwerkbandbreite sowie in der Nähe befindliche Ressourcen wie Drucker, Bildschirme oder Computer. Kennt bspw. ein Multimediadienst die dem Nutzer im Zuge der Systemabfrage zur Verfügung stehende Netzwerkbandbreite, so kann er einen Videostream an diese anpassen und so gewährleisten, dass der Videostream störungsfrei aber mit einer möglichst hohen Auflösung angezeigt wird. Weiß der Dienst darüber hinaus, dass sich in der Nähe des Nutzers ein hochauflösendes Display befindet, kann dieses Display als Alternative für die Anzeige des Videostreams vorgeschlagen werden.

633 Vgl. Dey (2001).

$634 \mathrm{Vgl}$. Kapitel 4. 
(3) Informationen zu den physikalischen Bedingungen, unter denen eine Systemabfrage durchgeführt wird, können aus einer Vielzahl von Datenquellen wie Kontakt-, Thermo-, Feuchtigkeits-, Beschleunigungs-, Torsions- oder Fotosensoren, Kameras und Mikrofonen gewonnen werden. ${ }^{635}$ Mit Prozessoren ausgestattete Sensoren können Sensorenwerte nicht nur erfassen, sondern diese auch selbständig vorverarbeiten und gewisse Muster, z.B. Fingerabdrücke, erkennen. Wie bereits erläutert wurde, ist der geografische Aufenthaltsort, von dem aus ein Nutzer eine Systemabfrage ausführt, von besonderer Bedeutung zur Bestimmung des physikalischen Kontexts der Nutzung des mobilen Internet. Der geografische Aufenthaltsort eines Mobilfunknutzers kann entweder durch Selbstortung oder durch Fremdortung bestimmt werden. ${ }^{636}$ Bei der Selbstortung erfolgt die Positionsbestimmung durch Messungen, die ein speziell dafür ausgestattetes Endgerät selbst vornimmt. Die bekannteste Technologie zur Selbstortung ist das vom US-Militär betriebene Global Positioning System (GPS), bei dem GPS-Geräte anhand von Positionssignalen, die die GPSSatelliten ausstrahlen, durch Triangulation ihre momentane Position auf bis zu wenige Meter genau berechnen können. Bei der Fremdortung erfolgt die Lokalisierung der Endgeräteposition auf der Grundlage von Netzwerkinformationen. Neben dem in Abschnitt 5.2.2.1 beschriebenen Verfahren der Zellidentifikation (Cell of Origin), kann die Ortsbestimmung anhand von Funksignallaufzeitunterschieden von Endgeräten zu verschiedenen Basisstationen (Time Difference of Arrival), anhand der Einfallswinkel (Angle of Arrival) oder anhand der Signalstärke (Signal Attentuation) von Funksignalen, die an verschiedenen Basisstationen ankommen, erfolgen.

In Abgrenzung zur dreiteiligen Differenzierung von Kontextformen nach Schilit et al. unterscheidet Dey (2001) des Weiteren zwischen primären Kontexten und sekundären Kontexten. ${ }^{637}$ Dabei bilden der Aufenthaltsort, der Endgerätetyp, das wahrnehmbare Verhalten des Nutzers und der Abfragezeitpunkt jeweils primäre Abfragekontexte. Sekundäre Abfragekontexte werden dagegen aus Kombinationen von primären Kontextdaten gebildet. Auf die soziale Situation eines Nutzers (bspw. „mit Kollegen im Büro“ oder „mit Freunden auf einem Ausflug") kann bspw. geschlossen werden, indem aus Aufenthaltsort und in der Nähe befindlichen Personen ein sekundärer sozialer Kontext gebildet wird. Darüber hinaus differenzieren Chen/Kotz (2000) zwischen aktiven und passiven Kontexten. Ein aktiver Kontext determiniert zwingend eine Verhaltensänderung der zugrunde liegenden Anwendung (bspw. die Definition interessierender Ressorts für eine individuelle Onlinezeitung). Ein passiver Kontext zeigt die Änderung der Kontextbedingungen der Systemabfrage dagegen lediglich als Zusatzinformation für den Systemnutzer an, bspw. in Form von Kaufempfehlungen eines Empfehlungssystems oder durch Abbildung des Aufenthaltsorts auf ein Landkartensystem bei einem Auskunftsdienst.

635 Vgl. Chen/Kotz (2002); Kaspar/Diekmann/Hagenhoff (2005).

636 Einen Überblick über die verschiedenen Lokalisierungsverfahren gibt Lehner (2004).

637 Vgl. Conlan/Power/Barrett (2003). 


\subsubsection{Kontextbezogene Systemadaption}

Ein adaptiver Dienst setzt, wie beschrieben, wahrgenommene Informationen über den Abfragekontext ein, um für einen Benutzer relevante Informationen bzw. Dienste auszuwählen. Ein kontextadaptives System besitzt dabei in der Regel einen gewissen Grad an Autonomie bei der Erfüllung seiner Aufgaben. Adaptive Systeme werden daher auch als "Agentensysteme“ bezeichnet. ${ }^{638}$ Agenten sind Softwaresysteme, die ihre Umwelt über Sensoren als anwendungsbezogene Ereignisse wahrnehmen und anhand vordefinierter Filterregeln Handlungen entsprechend dieser Wahrnehmung auslösen (vgl. Abbildung 5-41). Die vom Agenten ausgelösten Handlungen können sowohl informationsbezogener Natur sein (bspw. die automatische Auswahl von Inhalten zu touristischen Besonderheiten in der Nähe des Aufenthaltsorts), als auch Steuerungsbefehle für andere Systeme (bspw. das Auslösen eines Bezahlvorgangs) beinhalten. ${ }^{639}$ Dabei werden die Handlungen entweder unmittelbar und autonom vom Agenten ausgeführt (aktives Kontextbewusstsein) oder besitzen lediglich einen Vorschlagscharakter für den Nutzer, bspw. in Form einer angepassten Auswahl (passives Kontextbewusstsein). ${ }^{640}$

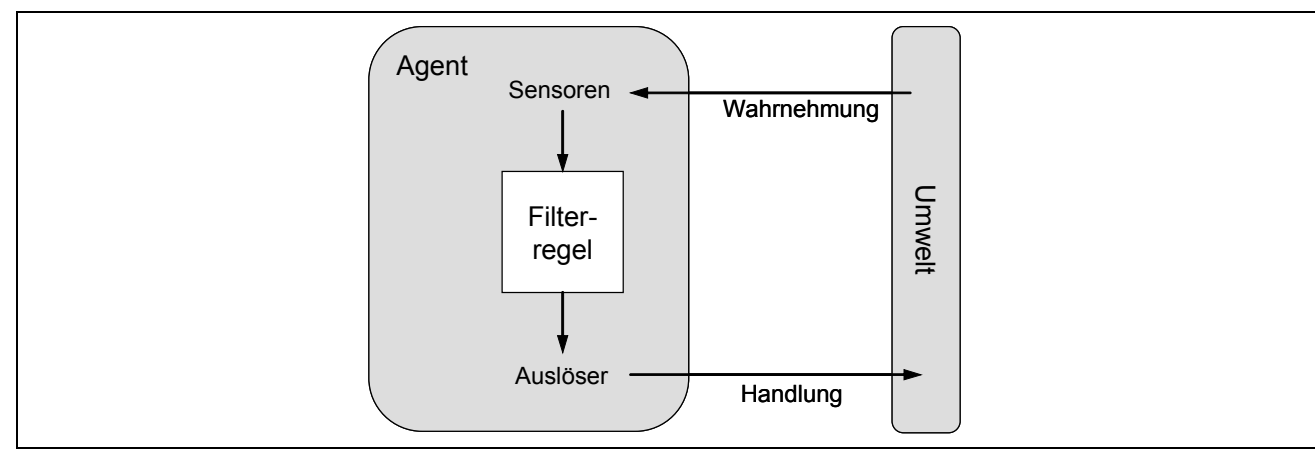

Abbildung 5-41: Aufbau eines Agentensystems

Eine adaptive Anwendung besteht aus Softwareobjekten, deren Aufruf durch ein Kontextereignis initiiert wird. Wird ein solches Schlüsselereignis durch die Anwendung wahrgenommen, werden das entsprechende Objekt bzw. darin enthaltene Methoden ausgeführt. Häufig ist es erforderlich, dass für ein solches Schlüsselereignis Rohdaten aus der sensorischen Umweltwahrnehmung, wie bspw. GPS-Koordinaten, Einträge in der Protokolldatei eines Webservers oder identifizierte, in der Nähe befindliche Netzressourcen, miteinander (zu einem sekundären Kontext) kombiniert werden, wenn ein einzelnes (primäres) Rohdatum alleine lediglich einen ungenügenden Hinweis auf die relevante Situation der Realität gibt. So benötigt ein adaptiver Verkehrsinformationsdienst Angaben über die aktuelle Tageszeit und den Wochentag (Laufzeituhr), den aktuellen Aufenthaltsort des Nutzers (bspw. GPS), das vom Nutzer angesteuerte Reise-

$638 \mathrm{Vgl}$. Russel/Norvig (2003).

$639 \mathrm{Vgl}$. Schilit/Adams/Want (1994).

$640 \mathrm{Vgl}$. Chen/Kotz (2000). 
ziel, seine bevorzugten Reiserouten und Verkehrsmittel (Nutzerprofil). Dabei können verschiedene Adaptionsverfahren in Abhängigkeit von Art und Umfang der für ein solches Schlüsselereignis kombinierten Kontextrohdaten unterschieden werden: ${ }^{641}$

- Systemnahe Kontextrohdaten wie Uhrzeit, Temperatur oder die Datenverarbeitungskapazität des Endgeräts, deren Wertigkeit ex-ante bekannt ist (closed-world-assumption), werden in der Regel unmittelbar an entsprechende Anwendungsereignisse geknüpft. Dazu werden bspw. dynamische Schlüssel-Wert-Paare gebildet, wobei das Softwareereignis den Schlüsselwert und das jeweils wahrgenommene Kontextdatum den Variablenwert enthält. ${ }^{642}$

- Bei systemfernen Kontextrohdaten wie Nutzungspräferenzen oder Angaben über den Aufenthaltsort müssen Ereignisse dagegen üblicherweise aus unterschiedlichen Kontextrohdaten aggregiert werden. Bspw. existiert derzeit kein standardisiertes Lokalisierungsverfahren, das sowohl in geschlossenen Räumen als auch im Freien eine exakte Aufenthaltsbestimmung liefert. ${ }^{643}$ Für eine exakte Bestimmung des Aufenthaltsortes eines Nutzers ist daher die Aggregation der Daten verschiedener Lokalisierungstechniken notwendig. Da verschiedene Lokalisierungssysteme unterschiedliche Maße zur Objektlokalisierung wie Distanz, Winkelabstand oder zugesicherte geometrische Position verwenden, erfordert eine Kombination der Rohdaten ihre Übersetzung in standardisierte Maße und ihre Überführung in ein gemeinsames Datenmodell. ${ }^{644}$ Dieser Vorgang wird auch als "Sensorfusion" bezeichnet. ${ }^{645}$

- Adaptive Systeme, die Kontexte verarbeiten, deren Wertigkeit einen hohen Veränderlichkeitsgrad besitzen (bspw. die Bewegung eines Nutzers innerhalb oder außerhalb von Räumen), benötigen eine große Anzahl von Schlüsselereignissen. Bei der Verarbeitung solcher Kontexte ist es in der Regel nicht sinnvoll, für jedes Ereignis ein konkretes Schlüssel-WertPaar zu modellieren. Stattdessen werden dafür Verfahren eingesetzt, die im Bereich der künstlichen Intelligenz u.a. zur Verarbeitung von Wissen entwickelt wurden. ${ }^{646}$ Ein Beispiel solcher Verfahren bilden künstliche neuronale Netze (KNN). Ein KNN ist ein System, das eine Vielzahl gleichförmiger, vernetzter Verarbeitungselemente besitzt. Dadurch können auch umfangreiche und stark veränderliche Rohdaten gleichzeitig effizient zu einem Kontext verarbeitet werden. ${ }^{647}$

\footnotetext{
641 Vgl. Kaspar/Diekmann/Hagenhoff (2005).

642 Vgl. Chen/Kotz (2000).

$643 \mathrm{Vgl}$. Chen/Kotz (2000).

$644 \mathrm{Vgl}$. Hightower/Brumitt/Borriello (2002).

$645 \mathrm{Vgl}$. Lin/Laddaga/Naito (2002).

646 Vgl. Mäntyjärvi/Seppänen (2002).

647 Vgl. Van Laerhoven/Aidoo/Lowette (2001).
} 


\subsubsection{Beiträge zu adaptiven mobilen Diensten}

Die ersten Beiträge zu adaptiven Systemen stammen aus dem Bereich der Forschung zu verteilten Anwendungen. Als Pioniere im Bereich kontextadaptiver Datenverarbeitung gelten die Forschungseinrichtungen der Reproduktionstechnologiehersteller Olivetti und XEROX, die Anfang der 1990er Jahre erste prototypische adaptive Anwendungen vorstellten. Zu den in dieser Zeit entwickelten Anwendungen gehören Systeme zur automatischen Anrufweiterleitung auf der Grundlage des Aufenthaltsorts eines Gesprächsempfängers in einem Bürokomplex, ${ }^{648}$ ortsbezogen anpassungsfähige Browsersoftware ${ }^{649}$ und ortsbezogene Einkaufsassistenten ${ }^{650}$. Als Lokalisierungsinstrument dieser Pionieranwendungen, die weniger vor dem Hintergrund mobilfunkgestützter Datenkommunikation sondern vor dem Hintergrund der Vision allgegenwärtiger Datenverarbeitung, des sog. „Ubiquitous“ bzw. „Pervasive Computing“, ${ }^{651}$ entwickelt wurden, dienen in erster Linie Infrarot- oder Funktransponder (sog. „Active Badges“), die von den jeweiligen Anwendungsnutzern mitgeführt und von speziell dafür entwickelten Raumsensoren erkannt werden. Weitere akademische Entwicklungen adaptiver Anwendungen späterer Jahre bilden touristische Reiseführer ${ }^{652}$ und Softwareassistenten für Konferenzteilnehmer ${ }^{653}$ und Feldforscher ${ }^{654}$.

Ende der 1990er Jahre entstanden erste Beiträge, die adaptive Dienstkonzepte für kommerzielle inhaltebezogene Mobilfunkanwendungen vorschlugen: Als Lösung des Problems partiell gebundener Aufmerksamkeit von mobilen Inhalterezipienten wurde in einem der ersten Beiträge das Konzept einer anpassungsfähigen Bildschirmanzeige für GSM-Endgeräte entwickelt, die bspw. in Abhängigkeit von Raumhelligkeit und der jeweiligen Aktivität des Benutzers die Anzeigegröße und -helligkeit anpassen. ${ }^{655} \mathrm{Im}$ Zuge der Freigabe der zellbasierten Teilnehmerortung in GSM-Netzen für die kommerzielle Nutzung im November 2001 entstand des weiteren eine Reihe von Vorschlägen für ortsbezogene mobile Dienste, ${ }^{656}$ darunter Tankstellensuchdienste, die Ortungs- und Fahrzeugdaten berücksichtigen, oder anpassungsfähige Fahrzeugmultimediaanwendungen, die in Abhängigkeit von der jeweiligen Empfangsqualität zwischen unterschiedlichen Empfangsvarianten wie GSM oder DVB wechseln. ${ }^{657}$ Neben der zellbasierten Ortung werden dabei auch Adaptionsformen auf der Grundlage einer Ortung durch GPS diskutiert. ${ }^{658}$ Einige Autoren schlagen speziell für

\footnotetext{
$648 \mathrm{Vgl}$. Want/Hopper/Falcao/Gibbons (1992); Wood/Richardson/Bennett/Harter/Hopper (1997).

$649 \mathrm{Vgl}$. Voelker/Bershad (1994).

$650 \mathrm{Vgl}$. Asthana/Cravatts/Krzyzanowski (1994).

$651 \mathrm{Vgl}$. Abschnitt 3.1.1.1.

652 Vgl. Bederson (1995); Long/Kooper/Abowd/Atkeson (1996); Davies/Cheverst/Mitchell/Friday (1999).

$653 \mathrm{Vgl}$. Dey/Futakawa/Salber/Abowd (1999).

654 Vgl. Pascoe (1998).

$655 \mathrm{Vgl.} \mathrm{Schmidt/Aidoo/Takaluoma/Tuomela/Van} \mathrm{Laerhoven/Van} \mathrm{de} \mathrm{Velde} \mathrm{(1999).}$

656 Vgl. Lehner (2004).

657 Vgl. Herden/Rautenstrauch/Zwanziger/Planck (2004).

658 Vgl. Diekmann/Gehrke (2003).
} 
die Besonderheiten mobiler Endgeräte angepasste Individualisierungskonzepte vor, die mobile Inhalteformate auf individuelle Präferenzen hin anpassen. Dabei ist zu unterscheiden zwischen Individualisierungskonzepten, die eine Anpassung auf der Aggregatsebene (bspw. eines mobilen Portals) vornehmen ${ }^{659}$ und solchen, die auf eine Individualisierung auf Ebene des jeweiligen Einzeldienstes abzielen. ${ }^{660}$ Als Individualisierungstechnik werden in erster Linie Verfahren auf der Grundlage expliziter Nutzerangaben und bedingter Wahrscheinlichkeiten diskutiert. ${ }^{661}$ Vor dem Hintergrund der wachsenden Vielfalt mobiler Endgeräte mit jeweils unterschiedlicher Hard- und Softwareausstattung untersuchen neuere Beiträge zudem die geräteunabhängige Inhaltedistribution durch MarkupTransformierung auf Basis von Schemabibliotheken für verschiedene Endgeräte und Auszeichnungsstandards wie WML, XHTML und HTML. Zur Identifikation des Endgerätetyps wurden verschiedene Geräteprofilstandards entwickelt, die im Zuge eines Datenkommunikationsvorgangs einen automatischen Austausch der jeweiligen Gerätekonfiguration zwischen Dienstbenutzer und anbieter erlauben. Zu den bekanntesten Geräteprofilstandards gehören die 2004 vom W3C spezifizierten "Composite Capabilities/Preference Profiles“ $(\mathrm{CC} / \mathrm{PP})^{662}$ oder deren bereits 2003 vom WAP-Forum vorgestellten Implementierung in Form des „User Agent Profile“663. Nach Identifikation der jeweiligen Endgerätekonfiguration erfolgt die geräteoptimale Anpassung der Auszeichnungssyntax typischerweise über bereits in Abschnitt 3.3.1.2.1 vorgestellte Verfahren wie bspw. auf der Grundlage der Stylesheet-Transformierungssprache XSLT. ${ }^{664}$

\subsubsection{Zusammenfassung}

In Abschnitt 5.1.1 wurde gezeigt, dass die Umstände einer Nutzung mobiler Dienste neben den Angaben zur Person und der Mobilfunkzelle, aus der die Nachfrage erfolgte, anhand weiterer Sensorsysteme wie Gerätedetektoren, GPS-Empfängern, Licht- oder Thermosensoren maschinell erfasst werden können. Diese maschinelle Situationswahrnehmung kann dazu verwendet werden, das Verhalten eines mobilen Dienstes situationsgerecht anzupassen und dadurch die Rezeptionsbequemlichkeit für den Nutzer zu steigern. Die Funktions-

659 Vgl. Smyth/Cotter (2003); Kaspar/Hagenhoff (2004).

$660 \mathrm{Vgl}$. Anderson/Domingos/Weld (2001).

$661 \mathrm{Vgl}$. Abschnitt 4.2.2.3.

$662 \mathrm{Vgl}$. W3C (2004b).

663 Vgl. OMA (2003): Das Vokabular von UAProf erlaubt die Charakterisierung der jeweiligen Konfiguration eines mobilen Endgeräts anhand von sechs Eigenschaften: HardwarePlatform, SoftwarePlatform, NetworkCharacteristics, BrowserUA, WaCharacteristics und PushCharacteristics.

664 Vgl. Jankowska (2005). Neben einstufigen Transformationsverfahren wie der XSLT, die Formatierungsvarianten eines Inhalts erzeugt, die immer dieselbe Struktur besitzen, werden vermehrt mehrstufige Verfahren zur Erzeugung von Formatvarianten diskutiert, die unterschiedliche Struktur und Gestalt besitzen. Derartige Transformationsverfahren werden auch als „Pipelined Processing“ bezeichnet, vgl. bspw. Pediaditakis/Shrimpton (2003). 
weise einer derartigen anpassungsfähigen Anwendung wurde in Abschnitt 5.1.2 am Modell sog. Agentensysteme, die in Informatik und Mathematik im Zusammenhang mit künstlicher Intelligenz erforscht werden, erläutert. Der Überblick über bestehende Forschungsbeiträge in Abschnitt 5.1.3 zeigt, dass im Bereich anpassungsfähiger Dienste bereits eine Vielfalt an plausiblen Umsetzungsvorschlägen existiert.

Allerdings zeigt ein Blick auf die Praxis, dass die akademischen Vorschläge bislang nur zum Teil realisiert wurden: In der praktischen Anwendung sind zwar auf der einen Seite bereits zahlreiche ortsbezogene Anwendungen zu finden. Neben dem in Abschnitt 5.2.3 vorgestellten Fallbeispiel t-info SMS umfassen derartige Beispiele unter anderem Verkehrsinformationsdienste, wie bspw. der bereits 2003 eingeführte Dienst Navi-Gate des Mobilfunkbetreibers T-Mobile, das ortsbezogene Auffinden von Freunden, wie bspw. der unter der Marke der Frauenzeitschrift Amica von Tomorrow-Focus angebotene Personenlokalisierungsdienst „Friendfinder" oder ortsbezogene Automatenbezahlung ${ }^{665}$. Des Weiteren zeigen die Beispiele t-info SMS und Playboy Mobile, dass das Problem der endgerätespezfischen Inhalteanpassung ebenso als gelöst betrachtet werden kann. Auf der anderen Seite sind jedoch im Bereich der Anpassung mobiler Dienste an individuelle Präferenzen (abgesehen von Abonnementkonfiguratoren wie bspw. die Dienstauswahl der in Japan entwickelten Plattform i-mode) keine praktischen Realisierungen festzustellen. ${ }^{666}$ Dieses Entwicklungsmanko deutet auf offene Forschungsfragen hin und kann insbesondere auf die verteilte Natur des Wertschöpfungssystems im mobilen Internet zurückgeführt werden: Wie bereits festgestellt wurde, besitzen mobile Inhalteangebote aufgrund der geringen Darstellungskapazitäten mobiler Endgeräte in der Regel eine geringere inhaltliche Breite und Tiefe als vergleichbare Angebote im stationären Internet. Dies führt dazu, dass Anbieter mobiler Inhalteformate weniger Bewegungsdaten über ihre Kunden aufzeichnen können und daher weniger Wissen über das Verhalten und die Präferenzen ihrer Kunden besitzen. Zudem verteilt sich dieses Wissen über Verhalten und Präferenzen mobiler Nutzer zwischen den jeweiligen Dienstanbietern, den Anbietern der Dienstplattform, über die ggf. der Dienstzugriff erfolgt und dem jeweiligen Mobilfunkbetreiber. Die erfolgreiche Implementierung von Individualisierungssystemen wirft daher Fragestellungen in Bezug auf eine semantische und dv-technische Integration verteilter Modelle der Präferenzen mobiler Inhaltenutzer auf. Eine solche Integration verteilter Nutzermodelle eröffnet wiederum Probleme hinsichtlich der datenschutzrechtlichen Kontrolle des damit verbundenen Austauschs personenbezogener Daten. Diese beiden Aspekte, die dienstübergreifende Modellierung inhaltebezogener Präferenzen mobiler Nutzer und die datenschutzrechtliche Kontrolle des damit verbundenen Austauschs von Personendaten, sind Gegenstand der beiden folgenden Abschnitte.

665 Vgl. Haneberg/Stenzel/Reif (2004).

666 Vgl. Figge/Albers (2005). 


\subsubsection{Mobilitätsgerechte Modellierung von Kontextdaten}

Im voranstehenden Abschnitt wurde gezeigt, dass Individualisierungsformen trotz anerkanntem Potenzial - in der Praxis lediglich einen geringen Verbreitungsgrad im mobilen Internet besitzen. Es wurde die Vermutung abgeleitet, dass der Grund für die geringe praktische Umsetzung im unzureichenden Kenntnisstand eines einzelnen Dienstanbieters über die Präferenzen seiner Kunden begründet ist. Im Folgenden wird ein Konzept vorgestellt, dass dieses Problem dadurch löst, indem die Daten zum Abfragekontext eines Dienstes im mobilen Internet über die verschiedenen von einem Nutzer abgerufenen Dienste hinweg einheitlich modelliert werden. Das Konzept bezieht sich in erster Linie auf die Modellierung inhaltebezogener Präferenzen, kann grundsätzlich jedoch auch auf andere Kontextdaten, die durch Dienstanbieter ermittelt werden können, wie etwa die Abfragezeit oder Ortungsdaten, angewendet werden.

Abschnitt 5.4.2.1 leitet die zugrunde liegenden Anforderungen an die Modellierung inhaltebezogener Präferenzen im mobilen Internet ab. Abschnitt 5.4.2.2 erläutert fachliche Aspekte einer derartigen Modellierung in erster Linie im Zusammenhang mit der Voraussetzung der Entwicklung einer einheitlichen Semantik verteilter Kundenprofile eines Nutzers im Wertschöpfungssystem des mobilen Internet. Abschnitt 5.4.2.3 enthält einen Lösungsvorschlag für die dvtechnische Integration verteilter Kundenprofile in einem gemeinsamen Modell. Abschnitt 5.4.2.4 fasst das vorgestellte Modellierungskonzept abschließend zusammen.

\subsubsection{Anforderungen der Modellierung von Inhaltepräferenzen für mobile Dienste}

Wie bereits ansatzweise aus Abbildung 5-6 in Abschnitt 5.2.2.2 deutlich wurde, beruht das Wertschöpfungssystem des mobilen Internet in Bezug auf die dvtechnische Bereitstellung mobiler Dienste auf einer verteilten Systemarchitektur. Die verteilte Architektur erlaubt zwar die logische Verknüpfung von Ressourcen über verschiedene verteilte Systemkomponenten hinweg, es findet jedoch keine systemweite Erfassung und Modellierung von Daten über das Verhalten und die Bedürfnisse von Kunden statt. Stattdessen entsteht über verschiedene Stellen im Wertschöpfungssystem verteiltes Wissen über die Nutzungskontexte mobiler Dienste (vgl. Abbildung 5-42): 


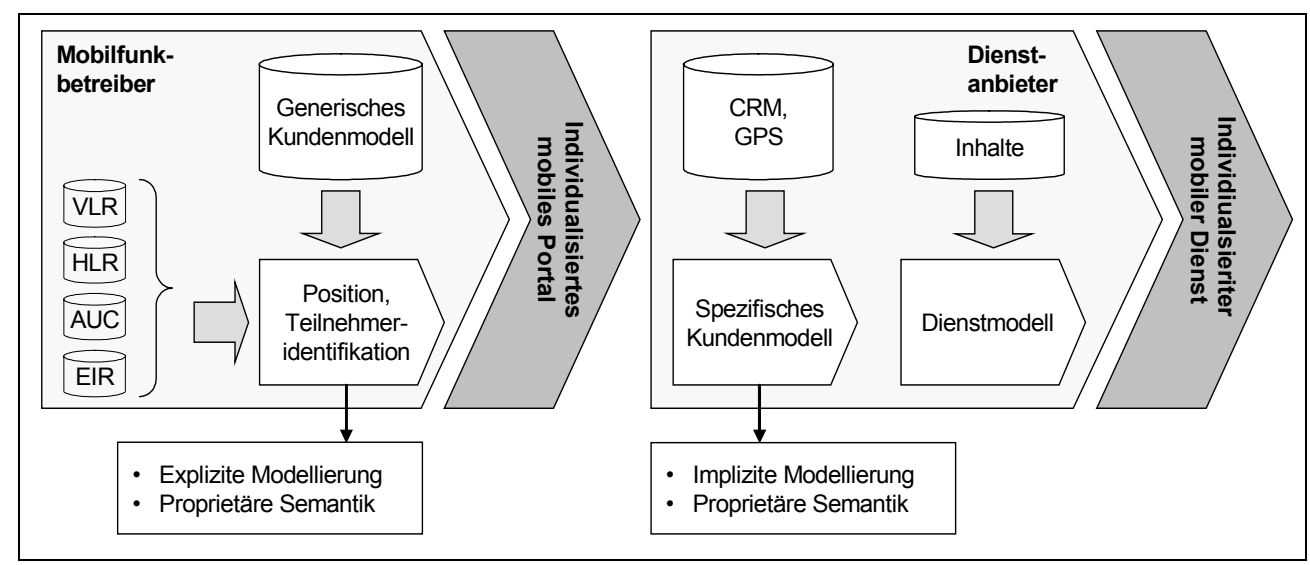

Abbildung 5-42: Kundenwissen im mobilen Internet667

Der Mobilfunkbetreiber eines GSM-Netzes kann die bei inm registrierten Teilnehmer im Rahmen einer typischen GSM-Architektur individuell anhand von vier Datenquellen identifizieren: Informationen über den aktuellen Aufenthaltsort eines Teilnehmers werden in den bereits angesprochenen Datenbanken von Visitor Location Register (VLR) und Home Location Register (HLR) gespeichert. ${ }^{668}$ Das Authentication Center (AUC), eine Datenbank, die u.a. den geheimen Schlüssel der SIM-Karten der Teilnehmerendgeräte enthält, erlaubt zudem eine individuelle Teilnehmerzuordnung einer Abfrage. Das Equipment Identity Register, eine Gerätedatenbank, ermöglicht des Weiteren die eindeutige Zuordnung einer Abfrage zu einem Endgerätetyp anhand eindeutiger Endgerätenummern (die sog. „International Mobile Equipment Identities“). Betreibt der Mobilfunkbetreiber darüber hinaus eine eigene Diensteplattform, die wie das zuvor genannte Beispiel i-mode über eine Abonnementkonfiguration verfügt, besitzt der Mobilfunkbetreiber auf aggregiertem Niveau Informationen über die von einem Kunden nachgefragten Dienste. Diese Informationen in Verbindung mit der individuellen Geräteidentifizierung und Teilnehmerlokalisierung ermöglichen es dem Mobilfunkbetreiber, im Sinne des bereits angesprochenen Individualisierungskonzepts von Smyth/Cotter (2003) eine individuelle Anpassung der Navigationslogik seiner Plattform vorzunehmen. Eine individuelle Auswahl kann jedoch nur auf Ebene der Dienstauswahl erfolgen, ein Mobilfunkbetreiber besitzt keine Kenntnisse über die auf Dienstebene konkret durch seine Kunden nachgefragten Inhalte. Demgegenüber kann ein Dienstanbieter auf der Grundlage inhaltlicher Analyseverfahren wie bspw. dem in Abschnitt 5.3.2.1.2 angesprochenen WAP Log Mining feststellen, welche Inhalte im Rahmen welcher Dienstsitzungen abgefragt wurden. Problematisch ist jedoch einerseits die Zuordnung einer solchen Sitzung zu einem individuellen Nutzer und - ausgenommen beim Einsatz von Selbstortungsverfahren wie dem GPS andererseits die Lokalisierung eines individuellen Nutzers. Eine ortsbezogene

667 In Anlehnung an Figge/Albers (2005).

$668 \mathrm{Vgl}$. Abschnitt 5.2.2.1. 
Dienstanpassung ist daher nur möglich, wenn der jeweilige Teilnehmer über ein GPS-fähiges Endgerät verfügt, eine Individualisierung auf Dienstebene kann darüber hinaus entweder nur verhaltensbezogen erfolgen oder erfordert eine individuelle Authentifizierung des Nutzers beim jeweiligen Dienstanbieter.

Es kann zusammengefasst werden, dass die verteilte Systemarchitektur des mobilen Internet und die typischerweise geringe inhaltliche Tiefe mobiler Dienste dazu führen, dass einerseits insgesamt nur wenig Wissen über die individuellen Präferenzen mobiler Nutzer existiert und dieses Wissen andererseits in zwischen Mobilfunkbetreiber und Dienstanbieter verteilten Nutzermodellen gespeichert ist. Neben der geringen Aussagekraft derartig verteilter Partialmodelle des Nutzerverhaltens für die inhaltlichen Präferenzen eines Nutzers ist eine verteilte Modellierung vor dem Hintergrund einer Individualisierung mobiler Dienste mit einer Reihe weiterer Probleme verbunden: ${ }^{69}$ Der Nutzer muss für die individuelle Authentifizierung beim Dienstanbieter persönliche Daten mehrfach explizit bei verschiedenen adaptiven Diensten angeben. Es entsteht zum einen ein erhöhter Aufwand durch die mehrfache Dateneingabe. Zum anderen kann der Nutzer den Überblick darüber verlieren, für welche Dienste welche Daten herausgegeben wurden. Insbesondere eine datenschutzrechtliche Überprüfung hinsichtlich einer Wahrung von Vertraulichkeit und Transparenz personenbezogener Daten wird häufig nicht gewährleistet. Für einen Dienstanbieter ist die Entwicklung adaptiver Anwendungen darüber hinaus mit einem hohen Implementierungsaufwand verbunden, da für jede Anwendung eine eigene Nutzermodellierungskomponente geschaffen werden muss. Zudem können dezentral erhobene Nutzerdaten keiner qualitativen Kontrolle bzgl. Aktualität oder Wahrheit unterzogen werden, wodurch die Aussagekraft der erhobenen Nutzermodelle auf den Inhaltebereich des jeweiligen Dienstes beschränkt ist.

Eine Lösung für das Problem bestünde theoretisch darin, die jeweiligen Partialmodelle eines Nutzers bei verschiedenen Diensten und ggf. sogar Diensteplattformen zu einem gemeinsamen Modell zu aggregieren. Eine derartige Aggregation besäße eine Reihe von Vorteilen: Durch Kombination der Partialmodelle können positive Synergieeffekte für die jeweiligen Dienste entstehen. Zudem wächst die Aktualität des in einem dienstübergreifend integrierten Nutzermodell beinhalteten Nutzerwissens für alle adaptiven Anwendungen. Aus Sicht des Nutzers wird darüber hinaus die Handhabung adaptiver Anwendungen vereinfacht, da redundante Dateneingaben entfallen. Schließlich besteht aus Entwicklersicht die Möglichkeit, Teile des Individualisierungsmechanismus mehrfach für verschiedene Dienste zu verwenden und damit den Implementierungsaufwand zu senken. Eine derartige dienstübergreifende Modellierung von Nutzerpräferenzen stellt allerdings erhöhte Anforderungen an die Repräsentation der Partialmodelle eines Nutzers durch die jeweiligen Dienste: Wie bereits in Abschnitt 4.2.1.2 erläutert wurde, erfolgt die Nutzermodellierung eines Individualisierungssystems üblicherweise in impliziter Form, als eingebetteter Bestandteil des Systems, bspw. in Form programmiersprachenspezifischer Softwareobjekte. Damit die jeweiligen Partialmodelle eines Nutzers je-

669 Vgl. Wörndl (2003). 
doch anwendungsübergreifend integriert werden können, ist es notwendig, diese Partialmodelle so zu kapseln, dass sie von verschiedenen Diensten verwendet werden können. Diese Form der Kapselung von Nutzermodellierungssystemen wird auch als „explizite Nutzermodellierung“ bezeichnet.

Bereits in den 1990er Jahren entstanden Beiträge zu gekapselten Nutzermodellierungssystemen. Erste sowohl akademische ${ }^{670}$ als auch kommerzielle $e^{671}$ Konzepte zielten darauf ab, ein gemeinsames Nutzermodellierungsprinzip anwendungsunabhängig in verschiedenen Zielanwendungen einzusetzen. Diese in den Beiträgen vorgeschlagenen Systeme sind überwiegend als zentralisierte Nutzermodellierungsserver für verschiedene Anwendungen innerhalb eines Netzwerks konzipiert und kommunizieren mit den jeweiligen Zielanwendungen über offene Netzwerkprotokolle wie bspw. TCP/IP, über Nachrichten oder unmittelbar über Funktionsaufrufe auf Grundlage einer Integrationsplattform. ${ }^{672}$ Ebenso wie bei dezentralen, gekapselten Nutzermodellierungssystemen besteht jedoch bei den derzeit existierenden kommerziellen Systemen zur dienstübergreifenden Nutzermodellierung eine starke Verschränkung zwischen adaptiven Zielsystemen und Nutzermodellierungsserver. Zudem findet auch bei zentralisierten Systemen die Nutzermodellierung überwiegend in impliziter Form, als integrierter Programmbestandteil, statt. Eine Erweiterbarkeit des Nutzermodells um externe, von Drittanwendungen erhobene Nutzerdaten, ist daher in der Regel nicht oder nur mit Programmieraufwand möglich.

Vor dem Hintergrund der beschriebenen Probleme bestehender Ansätze zur dienstübergreifenden Nutzermodellierung in Verbindung mit grundsätzlichen sicherheitstechnischen und datenschutzrechtlichen Problemen zentralisierter Systeme erscheint die Forderung nach spezialisierten, zentralen Dienstleistern zur anwendungsübergreifenden Sammlung und Modellierung von Nutzerdaten im mobilen Internet nicht zweckmäßig. Vielmehr erscheint eine Lösung wünschenswert, in der dezentral erhobene Nutzermodelle lediglich kompatibel repräsentiert werden, um individuelle Profildaten ggf. mit dem Einverständnis des Nutzers bilateral auszutauschen. An eine derartige kompatible Repräsentation dezentraler Nutzermodelle ist eine Reihe von Anforderungen zu stellen: Das jeweils verwendete Nutzermodell muss explizit modelliert werden. Dabei muss die Repräsentationsform eine Interoperabilität dezentraler Nutzermodelle in zweierlei Hinsicht ermöglichen: Zunächst muss ein einheitliches Datenformat verwendet werden, dass von verschiedenen Diensten gelesen werden kann. Dies stellt allerdings nur die korrekte Übertragung der Nutzerdaten sicher, nicht jedoch ihre Interpretation. Auf eine eindeutige Interpretation kann verzichtet werden, wenn das Profil lediglich aus Schlüsselwörtern besteht, die als einfache Zeichenketten gespeichert werden. Allerdings besteht dann die Gefahr, dass die unterschiedlichen Dienste Informationen, die an anderer Stelle gesammelt wurden, aufgrund der fehlenden semantischen Eindeutigkeit natürlicher Sprache falsch interpretieren. Sollen die Profilinhalte tatsächlich eindeutig sein,

670 Vgl. Finin (1989); Brajnik/Tasso (1992); Kay (1995).

$671 \mathrm{Vgl}$. Kobsa/Pohl (1995), Orwant (1995) und Konstan et al. (1997).

672 Vgl. Fink/Kobsa (2000). 
muss ihre Bedeutung in Form von kontrolliertem Vokabular eindeutig festgelegt werden. Es muss also auch auf der semantischen Ebene eine Einigung bezüglich der zu verwendenden Inhalte getroffen werden. Im Folgenden wird daher zunächst in Abschnitt 5.4.2.2 ein Konzept für ein einheitliches semantisches Vokabular im Rahmen der dezentralen Modellierung von Nutzerpräferenzen dargestellt. In Abschnitt 5.4.2.3 werden Lösungsansätze für eine datentechnische Integration verteilter Modelle mit gemeinsamer Semantik erläutert.

\subsubsection{Semantische Repräsentation von Nutzermodellen auf Grundlage von Ontologien}

Ein Nutzermodell zur Beschreibung inhaltlicher Präferenzen besteht in der Regel aus drei Komponenten: ${ }^{673}$ erstens, einer Sammlung von Rohdaten (Primärannahmen) aus Befragung oder Verhaltensbeobachtung; zweitens, Inferenzregeln zur Erweiterung der Primärdaten im Hinblick auf ihre Bedeutung in der jeweiligen Domäne zur Bestimmung individueller Nutzerpräferenzen; drittens, ergänzende Sekundärannahmen vor dem Hintergrund von Primärdaten und Inferenzregeln, bspw. auf Grundlage von Auswertungen der vom Nutzer nachgefragten Inhalte. Ein Nutzerprofil repräsentiert die konkrete Instanz des Nutzermodells für einen individuell identifizierbaren Nutzer. Wie in Abschnitt 4.2 gezeigt wurde, kann ein Nutzermodell in Bezug auf inhaltliche Präferenzen auf Grundlage von Beobachtungen automatisch erzeugt werden, indem gewichtete Schlagwortlisten gespeichert werden, die auf einer statistischen Auswertung der Seiteninhalte abgerufener Inhalte beruhen. Dazu können bspw. stereotype Verhaltensprofile in Form häufig besuchter URL-Kombinationen des WAPSeitenprotokolls (Primärannahmen) anhand von Inferenzregeln wie Clusteroder Assoziationsanalysen ermittelt werden. Diesen Verhaltensprofilen können dann auffällige Textmerkmale oder inhaltsbezogene Metadaten der besuchten Adressen zugeordnet werden, wodurch auf eine Präferenz des Nutzers von bestimmten inhaltlichen Kategorien geschlussfolgert werden kann (Sekundärannahmen). Das Ergebnis dieser Nutzermodellierung ist eine Sammlung stereotyper Nutzerprofile repräsentiert durch einen Vektorraum gewichteter Merkmalsvektoren. Ein individuelles Nutzerprofil repräsentiert die Auswahl eines oder mehrerer Profilstereotypen, die dem aktuellen Verhaltensmuster eines Nutzers am Ähnlichsten sind. Abbildung 5-43 zeigt das Klassenmodell der Beschreibungssemantik einer solchen Nutzermodellierungsform.

673 Vgl. Schreck (2000). 


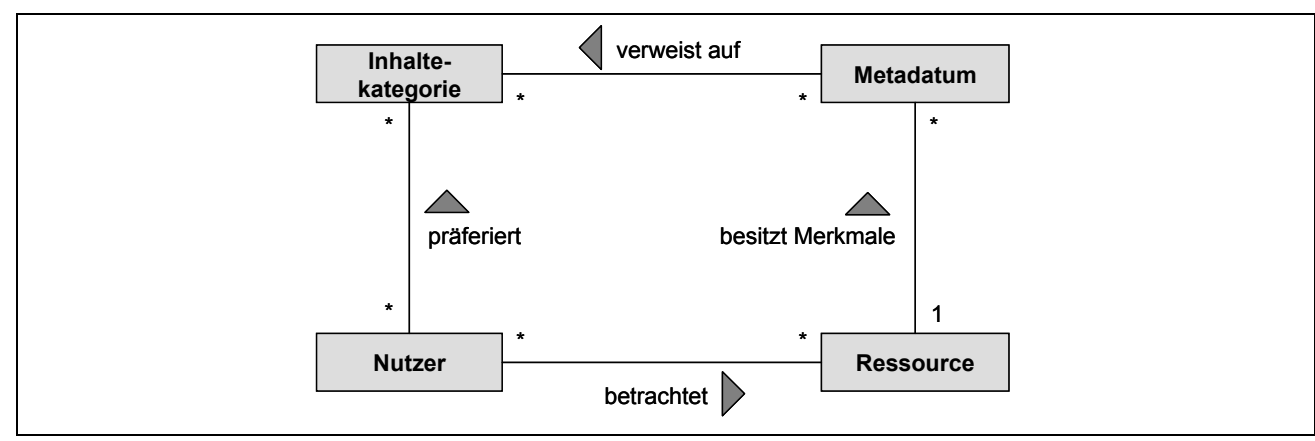

Abbildung 5-43: Klassenmodell einer inhaltebezogenen Nutzermodellierungssemantik

Im Bereich der Forschung zur Modellierung von Kontextinformationen für anpassungsfähige mobile Dienste werden insgesamt sechs unterschiedliche Repräsentationsformen für Kontextmodelle unterschieden: ${ }^{674}$ Schlüsselwertpaare, ${ }^{675}$ Schemamodelle für Markup-Sprachen, ${ }^{676}$ grafische Modelle, ${ }^{677}$ objektorientierte Modelle, ${ }^{678}$ logische Modelle ${ }^{679}$ und ontologiebasierte Modelle. Für die hier angestrebte explizite, formale Repräsentation von verteilten Nutzermodellen im mobilen Internet gelten dabei ontologiebasierte Kontextmodelle als am vielversprechendsten, da sie auf einer semantisch offenen, formalisierten und standardisierten Kontextrepräsentation beruhen. ${ }^{680}$

674 Vgl. Strang/Linnhoff-Popien (2004).

675 Schlüsselwertpaare bilden die einfachste Repräsentationsform für Kontextmodelle. Das Konzept der Schlüsselwertpaare beruht darauf, ein Kontextdatum bspw. einem Methodenaufruf als Schlüsselwert beizuordnen. Daher besitzen Schlüsselwertpaare einen hochgradig anwendungsproprietären Charakter und einen geringen Formalisierungsgrad. Vgl. auch Schilit/Adams/Want (1994).

676 Markupsprachen zur Kontextmodellierung bilden bspw. die bereits angesprochenen CC/PP oder das UAProf zur Repräsentierung des Datenverarbeitungskontextes (vgl. Abschnitt 5.4.1.3). Erweiterungen zur Beschreibung von Aspekten wie Ort oder Netzwerkeigenschaften bilden die Comprehensive Structure Context Profiles (CSCP, vgl. Held/Buchholz/Schill (2002)) oder die CC/PP Context Extension (vgl. Indulska et al. (2003)). Bestehende Markupsprachschemata formalisieren zwar Kontextdaten, beschränken sich aber auf die Repräsentierung einer kleinen Menge von Kontexten, deren Wertigkeit ex ante bekannt ist.

677 Graphische Modelle verwenden Modellierungssprachen wie die Unified Modeling Language (UML), um Kontextmodelle zu strukturieren (vgl. Halpin (2001) und Indulska/Hendricksen (2004)). Der Zweck der Modellierung ist dabei weniger die Schaffung einer implementierungsreifen Softwarekomponente, sondern vielmehr die Strukturierung des Modells, bspw. als Vorbereitung zur Entwicklung ER-Modells.

678 Objektorientierte Modelle zielen auf die Kapselung und Wiederverwendung von Kontextklassen ab (vgl. Schmidt/Beigl/Gellersen (1999) und Cheverst/Mitchell/Davies (1999)). Eine derartige Kapselung hat allerdings zur Folge, dass das zugrunde liegende Kontextmodell nach außen nicht sichtbar und sein Formalisierungsgrad nicht überprüfbar ist.

679 Logische Modelle formalisieren Kontextdaten als abstrakte mathematische Entitäten, die anhand von künstlicher Intelligenz (bspw. durch regelbasierte Systeme) weiterverarbeitet werden können (vgl. Bacon/Bates/Halls (1997)). Insofern ist die Anwendbarkeit auf solche Systeme eingeschränkt, die eine logische Regelverarbeitung, bspw. auf der Grundlage von PROLOG, erlauben.

680 Vgl. Strang/Linnhoff-Popien (2004). 
Als Ontologie wird das logische Modell eines Ausschnitts der Realität in Form einer formalen und expliziten Beschreibung der Begriffe, die für das Verständnis der Domäne notwendig sind, sowie ihre Bedeutungen und Beziehungen bezeichnet. Der Begriff der Ontologie steht in engem Zusammenhang mit der Entwicklung standardisierter Repräsentationsmöglichkeiten für eine maschinenlesbare Auszeichnung der Semantik individuell adressierbarer Internetressourcen im Zuge der sog. „Semantic Web“-Initiative. Das Semantic Web beruht auf einem Sprachen- und Protokollstapel zur Beschreibung und zum Austausch formalisierter Ressourcensemantiken. ${ }^{681}$ Dieser Stapel besteht aus eindeutigen Ressourcenidentifikatoren, der Auszeichnungssprache XML, dem Resource Description Framework (RDF), ${ }^{682}$ dem RDF-Schema (RDFS) ${ }^{683}$ und speziellen Sprachen zur Formalisierung von Ontologien wie DAML/OIL ${ }^{684}$ oder OWL ${ }^{685}$.

Ontologien kommen nicht nur zur Kategorisierung von Onlineressourcen zum Einsatz, sondern werden vermehrt auch zur Modellierung von Anwendungskontexten für adaptive Systeme diskutiert. Bspw. verwenden Labrou/Finin (1999) Ontologien auf Grundlage der Indizierungsstruktur des Onlinesuchdienstes Yahoo!, um Onlineressourcen entsprechend ihrer Seiteninhalte zu klassifizieren und zu kategorisieren. Der Kontextmakler eines intelligenten Veranstaltungskalenders in Chen/Finin/Joshi (2003) lokalisiert Personen und Ereignisse im Verlauf einer Veranstaltung auf Grundlage veranstaltungsbezogener Referenzontologien für Ortsangaben und Agentenrollen. Der ontologiebasierte Navigationsagent OBIWAN in Gauch/Chaffee/Pretschner (2004) erzeugt anhand von Auswertungen des Nutzerverhaltens individuelle Referenzontologien aus statistischen Analysen abgefragter Seiteninhalte, um dem Nutzer individuell relevante Seiteninhalte zu empfehlen.

Um ein Nutzermodell explizit in standardisierter Syntax und Semantik zu repräsentieren, muss die zugrunde liegende Ontologie des Modells in einer standardisierten Sprache formuliert werden. Die Repräsentation des Nutzermodells kann entweder auf der Grundlage einer semantischen Auszeichnung in RDF und RDFS oder in Ontologiesprachen wie DAML/OIL oder OWL erfolgen. OWL besitzt dabei gegenüber RDF/RDFS oder DAML/OIL eine Reihe von Vorteilen: OWL beruht zwar auf RDF oder RDFS, ist jedoch expressiver als diese; OWL wurde (anders als DAML/OIL) explizit als eine auf XML basierende Ontologiesprache entwickelt; die Spezifikation

$681 \mathrm{Vgl}$. Daconta/Obrst/Smith (2003).

682 Vgl. Manola/Miller (2004): RDF ermöglicht die Beschreibung von Ressourcen in Form logischer Tripel, bestehend aus Subjekt, Prädikat und Objekt. Das Subjekt repräsentiert die jeweils zu beschreibende Ressource, das Prädikat ein spezielles Attribut oder eine Beziehung des Subjekts, das Objekt den zugehörigen Wert.

683 Mit RDF-Schema ist es möglich, Klassen oder Attribute für die in RDF verwendeten Auszeichnungselemente zu bestimmen und dadurch einfache Ontologien in Form von Klassenhierarchien zu formulieren (vgl. Staab/Erdmann/Mädche (2001)).

684 DAML/OIL repräsentiert das Ergebnis der Zusammenlegung der amerikanischen DARPA (Defense Advanced Research Projects Agency) Agent Markup Language (DAML) und des europäischen Ontology Inference Layer (OIL). Vgl. van Harmelen/ Patel-Schneider/Horrocks (2001).

$685 \mathrm{Vgl}$. Smith/Welty/McGuinness (2004). 
sierende Ontologiesprache entwickelt; die Spezifikation von OWL repräsentiert einen allgemeinen, vom W3C verabschiedeten Standard. Der Sprachaufbau der OWL folgt dem Prinzip objektorientierter Programmiersprachen und enthält in erster Linie Klassen und Relationseigenschaften. Alle Instanzen der Ontologie sind dabei definitionsgemäß immer auch Instanzen der generischen Ressourcenklasse des RDF-Schemas (rdfs:Resource). Als Instanzen werden Klassen (owl:Class), Relationseigenschaften (owl:Property), Vererbungen (owl:subClassOf), Definitions- und Wertebereiche (owl:range, owl:domain) modelliert. Für Eigenschaften können zudem Typmerkmale (bspw. owl:inverseOf) und Restriktionen (bspw. owl:Cardinality) modelliert werden. Abbildung 5-44 gibt einen Überblick über die zur Repräsentation der Semantik eines Nutzermodells (wie dargestellt in Abbildung 5-43) notwendigen Modellinstanzen.

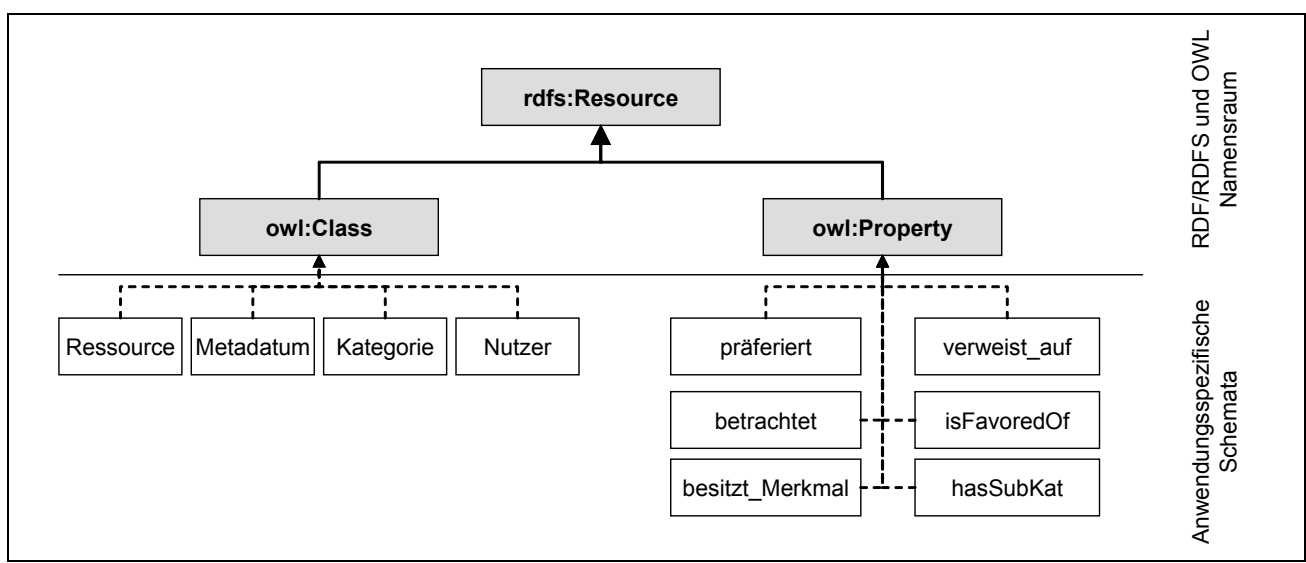

Abbildung 5-44: Instanzen eines Nutzermodells in OWL

Abbildung 5-45 zeigt beispielhaft das Codefragment für die Klasse „Nutzer“ mit den jeweils zugehörigen Relationseigenschaften „präferiert" und „betrachtet" der in OWL repräsentierten Ontologie des Nutzermodells. Die Repräsentation der Ontologie in einer standardisierten Repräsentationssprache wie OWL löst allerdings nur das Problem der formalen Kompatibilität jeweils durch Modellierungssysteme unterschiedlicher Dienstanbieter erstellter Profile. Damit jedoch verschiedene Partialmodelle der Präferenzen eines Nutzers zu einem gemeinsamen Modell aggregierbar sind, müssen die verschiedenen Modellierungssysteme darüber hinaus Profile erzeugen, die dasselbe semantische Vokabular verwenden. Vor dem Hintergrund des dargestellten Beispiels eines präferenzenbezogenen Nutzermodells muss zumindest in der Semantik auf Ebene von Klassen und Eigenschaften Übereinstimmung zwischen den dezentralen Modellierungsontologien bestehen. Standardisierungsbestrebungen, die eine Formalisierung der Modellierung von Kontextdaten zum Gegenstand haben, existieren bislang erst in Ansätzen. Beispiele zur Modellierung von Kontextmodellen bilden etwa die Point of Interest Exchange Language (POIX), ${ }^{686}$ eine XML-basierte Sprache, die über Schemata zur Beschreibung von

$686 \mathrm{Vgl.} \mathrm{Kanemitsu/Kamada} \mathrm{(1999).}$ 
Aufenthaltsdaten verfügt ${ }^{687}$ oder die CoBrA-ONT, ${ }^{688}$ eine akademische Ontologie, die Schemata zur Beschreibung von sowohl ortsbezogenen als auch personenbezogenen Kontextdaten wie Aktivitäten oder Eigenschaften besitzt.

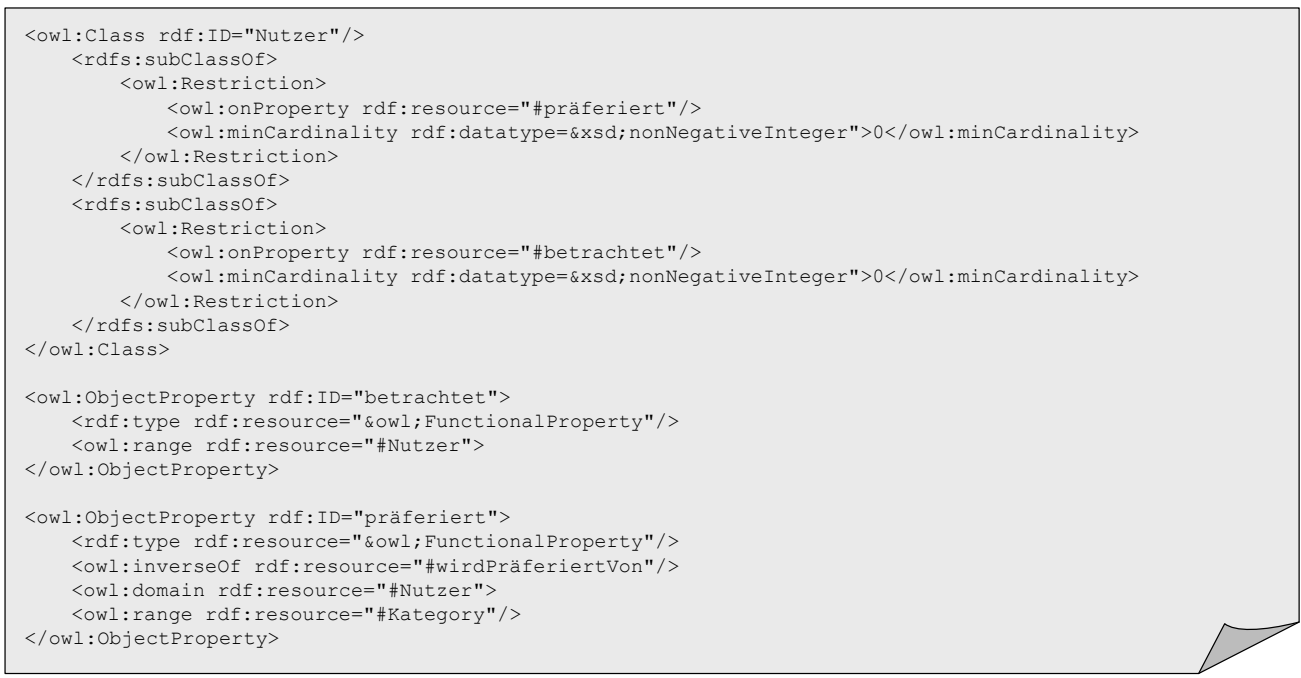

Abbildung 5-45: Codefragment eines Nutzermodells in OWL

\subsubsection{Datenintegration eines verteilten Kontextmodel/s}

Das mobile Internet repräsentiert ein Wertschöpfungssystem, in dem Leistungen und Dienste verschiedener Anbieter mit einer gewissen Dynamik und Flexibilität kombiniert werden. ${ }^{689}$ Um dieser Dynamik der Wertschöpfungsarchitektur Rechnung tragen zu können, ist es erforderlich, dass die jeweiligen Nutzermodellierungssysteme, die bei den verschiedenen Dienstanbietern zum Einsatz kommen, über Schnittstellen verfügen, die neben einer standardisierten Schnittstellensemantik eine flexible Kopplung erlauben. Aus der Sicht eines Dienstanbieters bietet die Bereitstellung von Profilinformationen über seine Kunden durch offene, flexibel koppelbare Schnittstellen zwei Vorteile: Ein Dienstanbieter kann zum einen auf dezentral gespeicherte Profile anderer Dienstanbieter zugreifen, diese fremden Profildaten automatisch mit seinen eigenen Kundendaten verknüpfen und somit umfangreichere Profile über Kunden aggregieren. Zum anderen können neben den konkreten Profilinhalten zusätzliche semantische Informationen der jeweiligen Modellierungsontologie über eine solche Schnittstelle beschrieben werden, wodurch die dienstübergreifende Aggregation einzelner Profile erleichtert wird. Dabei sind grundsätzlich zwei unterschiedliche Varianten einer dienstübergreifenden Aggregation von Kundenprofilen über standardisierte Schnittstellen denkbar:

$687 \mathrm{Vgl}$. Zadorozhny/Chrysanthis (2004).

$688 \mathrm{Vgl}$. Chen/Finin/Joshi (2003).

$689 \mathrm{Vgl}$. Figge/Albers (2005). 
- Die einzelnen Dienstanbieter stellen ihre jeweiligen Profildaten über eine Schnittstelle zur Verfügung, es existiert jedoch keine zentrale Aggregationsstelle sondern die jeweiligen Profildaten sind ausschließlich dezentral bei den jeweils profilerhebenden Diensten gespeichert. Zum Zwecke der Anpassung ihres Dienstangebots für einen konkreten Nutzer ermitteln die einzelnen Dienstanbieter jedoch strukturelle Profilähnlichkeiten für diesen Nutzer mit weiteren Profilen dieses Dienstes als auch mit Nutzerprofilen anderer Dienste. Eine denkbare Variante eines solchen Profilvergleichs bildet ein Collaborative Filtering, in dem Prognosewerte für den Nutzer eines Dienstes A anhand der Daten ähnlicher Profile in den Diensten B, C und D berechnet werden.

- Die Profilattribute von Nutzern in unterschiedlichen Diensten werden an zentraler Stelle aggregiert. Derartige Aggregatoren, deren Geschäftszweck in erster Linie darin besteht, Informationen über Kunden zu sammeln und zu speichern, werden auch als „Infomediäre“ bezeichnet. ${ }^{690}$ Für das Rollenverständnis eines solchen Aggregators sind zwei Alternativen denkbar: Im einfacheren Fall speichert der Aggregator für die jeweiligen Profilattribute lediglich parametrisierte Verweise auf die Schnittstelle der jeweiligen Dienstanbieter. Dabei wird anhand der semantischen Schnittstellenbeschreibung zwar eine logische Aggregation der dezentral erhobenen Attribute in ein dienstübergreifendes Ontologieschema durchgeführt, jedoch keine zentrale Aggregation der jeweiligen Werteausprägungen im Sinne einer Durchschnittsbildung vorgenommen. Ein Infomediär, der seine Aggregationsleistung als Dienstleistung anbietet, wird demgegenüber jedoch auch daran interessiert sein, nicht nur eine logische Aggregation von Attributklassen vorzunehmen, sondern die einzelnen Attributwerte selbst übergreifend zu aggregieren.

Für die dv-technische Umsetzung einer Systemarchitektur bestehend aus lose gekoppelten Diensten wird bereits seit den 1970er Jahren das Konzept der sog. „Service Orientierten Architektur" (SOA) diskutiert. Bei der technischen Umsetzung einer solchen lose gekoppelten Systemarchitektur kommen traditionell zwei alternative Verfahren zum Einsatz: ${ }^{691}$ die Kommunikation über entfernte Methodenaufrufe (Remote Procedure Calling, RPC) oder der Austausch von Nachrichten über nachrichtenorientierte Middleware (Message Oriented Middleware, MOM). Etablierte Technologien zur Umsetzung einer losen Kopplung wie bspw. das Distributed Common Object Model (DCOM) von Microsoft oder die Common Object Request Broker Architecture (CORBA) der Object Management Group (OMG) besitzen allerdings in der Regel Bindungen an bestimmte Programmiersprachen und Softwareplattformen oder verwenden proprietäre Übertragungsprotokolle und ermöglichen somit keine uneingeschränkte Flexibilität. Eine Entwicklung neueren Datums, die derartige Probleme beseitigen soll, bildet das Konzept sog. „Web Services“. Web Services repräsentieren selbstbeschreibende Softwarekomponen-

690 Vgl. Hagel/Rayport (1997).

691 Vgl. Burghardt (2005), S. 19-22. 
ten, die auf der Grundlage offener XML-basierter Standards sowie etablierter Internetprotokolle kommunizieren ${ }^{692}$ und deren Entwicklung und Nutzung programmiersprachen- und plattformunabhängig erfolgen kann. Abbildung 5-46 zeigt denkbare Einsatzmöglichkeiten der Web Service Technologien zur Gewährleistung eines dienstübergreifenden Austauschs von Kontextdaten. Der Zugriff eines Aggregators oder eines zweiten Dienstanbieters B auf die Profildaten eines Dienstanbieters A erfolgt dem Grundsatz nach wie folgt: ${ }^{693}$ Die Sitzungsdaten eines Nutzers werden anhand der Inhalteontologie des Dienstanbieters $A$ analysiert und auffällige Sitzungsmerkmale in einer Profildatenbank gespeichert. Die Ontologie der Profile wird gemeinsam mit der technischen Schnittstellenbeschreibung eines Web Service, der den Zugriff auf die Profildatenbank ermöglicht, in einem WSDL-Dokument in einem öffentlichen UDDI-Verzeichnis publiziert. Ein Aggregator oder ein zweiter dezentraler Dienstanbieter B greift auf der Grundlage eines generischen Web Service Proxy seines Anwendungsservers über diesen Web Service auf die Profildaten von Dienstanbieter A zu.

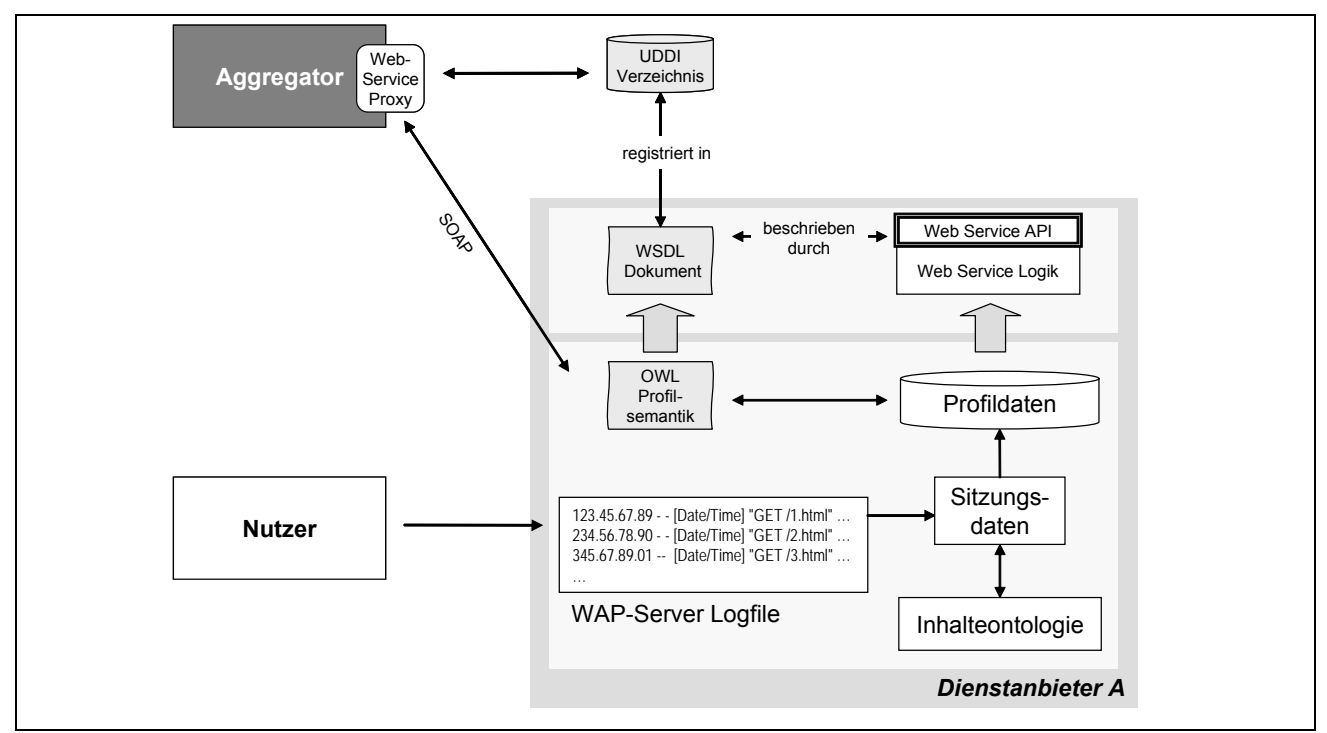

Abbildung 5-46: Austausch von Kontextdaten auf der Grundlage von Web Service-Technologien

692 Die Schnittstellensemantik eines Web Service wird auf der Grundlage der XML-Sprache WSDL (Web Service Description Language) in standardisierter Form beschrieben. Dabei kann das WSDLDokument bspw. als Träger von Informationen der zugrunde liegenden OWL-Ontologie verwendet werden. Optional kann ein Web Service in öffentlichen Verzeichnissen, der sog. Universal Description, Discovery and Integration (UDDI) registriert werden. Der Aufruf eines Web Service erfolgt über das Simple Object Access Protocol, einem XML-basierten Protokoll, das auszutauschende Inhalte in einem XML-Format überträgt, das bspw. innerhalb einer HTTP-Nachricht gekapselt ist. Vgl. Kaspar/Burghardt/Hagenhoff (2004).

$693 \mathrm{Vgl}$. Kaspar/Seidenfaden/Hagenhoff (2004). 


\subsubsection{Zusammenfassung}

In Abschnitt 5.4.2.1 wurde gezeigt, dass die Erfassung des Abfragekontextes im mobilen Internet durch dessen verteilte Architektur Probleme aufwirft. Um diese Probleme des verteilten Wissens über Abfragekontexte zu mildern wurde vorgeschlagen, die jeweils im Rahmen einzelner Dienste erhobenen Kontextdaten so bereitzustellen, dass das verteilte Wissen über Abfragekontexte in einem gemeinsamen Modell zusammengeführt werden kann. Als Anforderungen einer solchen Modellierung wurde die Schaffung einer formalisierten Sprache zur Kontextrepräsentation, eine standardisierte Semantik zur Kontextbeschreibung und eine dv-technische Integrationsarchitektur, die eine flexible Kopplung verschiedener Modellierungssysteme erlaubt, abgeleitet. Am Beispiel der semantischen Repräsentation von Kundenpräferenzen wurde in Abschnitt 5.4.2.2 gezeigt, dass sich für die formale Repräsentation von Kontexten die aus dem Bereich des Semantic Web bekannten Ontologiesprachen wie bspw. OWL eignen. Weiterhin wurde gezeigt, dass im Bereich der standardisierten Semantik auf der Grundlage von Ontologien repräsentierter Kontextmodelle noch weiterer Forschungs- und Standardisierungsbedarf besteht. Eine solche zukünftige Standardisierung vorausgesetzt wurde in Abschnitt 5.4.2.3 das Konzept für Datenverarbeitungskonzepte zur Aggregation formalisierter und standardisierter Kontextmodelle im mobilen Internet entwickelt.

Das vorgestellte Konzept zur dienstübergreifenden Modellierung von Kontextdaten liefert zusammenfassend zwar einen Lösungsansatz für die technische Umsetzbarkeit einer dienstübergreifenden Modellierung von Kontextdaten und damit zur Verbesserung der Anwendungsmöglichkeiten adaptiver Dienste im mobilen Internet. Die praktische Umsetzung eines solchen Konzepts ist allerdings in der Praxis mit zwei weiteren Problemen verbunden: Das Wissen eines Anbieters über seine Kunden repräsentiert einen kritischen Erfolgsfaktor für den Wettbewerb mit Konkurrenten, das ein Anbieter ohne entsprechende zugesicherte Gegenleistung nicht preiszugeben bereit sein wird. Es ist daher zum einen ein Anreizproblem im Hinblick auf die Preisgabe von Kontextinformationen durch Dienstanbieter zu lösen. Zum anderen repräsentiert jede Form von Kontextinformation aus rechtlicher Sicht ein personenbezogenes Datum, dessen Speicherung, Verarbeitung und vor allem dessen Weitergabe an Dritte ohne explizite Autorisierung durch die jeweils betreffende Person im eklatanten Widerspruch zur bestehenden Gesetzeslage im Datenschutz steht. ${ }^{694}$ Deshalb ist das Problem der Schaffung einer gesetzeskonformen Kontrolle des Zugriffs und der Weitergabe von Kontextdaten zu lösen. Im folgenden Abschnitt werden Lösungsansätze für die beiden Probleme der Anreizstiftung und der Zugriffskontrolle präsentiert.

\subsubsection{Zugriffskontrolle für ein aggregiertes Kontextmodell}

Die Tatsache, dass Käuferinformationen für Anbieter wertvolle Informationen im Rahmen der Produktentwicklung und -vermarktung darstellen, wurde ebenso

694 Vgl. Abschnitt 4.1.3. 
bereits erläutert, wie die generelle Datenschutzproblematik in Bezug auf persönliche Informationen im Internet. Dennoch zeigen die voranstehenden Ausführungen, dass eine mobilitätsgerechte Bereitstellung von Inhalten zwangsläufig eines Austauschs von Personendaten zwischen verschiedenen Inhalteanbietern bedarf. Neuere Untersuchungen zur Datenschutzproblematik zeigen, dass Onlinenutzer keineswegs den Austausch ihrer Daten zwischen verschiedenen Onlineanbietern vollkommen ablehnen, sich jedoch des ökonomischen Werts dieser Daten zunehmend bewusst sind und daher eine angemessene Entlohnung für die Preisgabe ihrer Informationen erwarten. Es entsteht somit ein Bedarf an Instrumenten, die eine Kontrolle über Art, Umfang, Adressat und Verwendungsform personenbezogener Daten ermöglichen. Im Folgenden wird ein Vorschlag für eine Dienstarchitektur entwickelt, die in der Lage ist, eine solche Kontrolle über personenbezogene Daten zu gewährleisten. In Abschnitt 5.4.3.1 wird ein einleitender Überblick über bestehende Ansätze zur Kontrolle von Personendaten gegeben. Abschnitt 5.4.3.2 stellt ein Konzept für den Austausch von Personendaten vor, in dem die Identität eines Nutzers pseudonymisiert werden kann, ohne die Möglichkeiten einer umfassenden Kontextmodellierung zu verhindern. Abschnitt 5.4.3.3 erläutert die Möglichkeiten einer anreizkompatiblen Zugriffskontrolle auf Personendaten im Rahmen dieses Austauschkonzepts. Abschnitt 5.4.3.4 fasst die Ergebnisse abschließend zusammen.

\subsubsection{Forschungsbeiträge im Bereich der Datenkontrolle}

In einer typischen Gesprächssituation im Alltag entscheidet ein Gesprächsteilnehmer in Abhängigkeit von der jeweiligen Gesprächssituation sowie seiner Beziehung zum Gesprächspartner in der Regel weitgehend intuitiv darüber, ob und wie viel seiner persönlichen Informationen er preisgeben wird. ${ }^{695}$ Eine solche intuitive Datenkontrolle existiert im Bereich der mobilen Datenkommunikation nicht: Der Verbindungsaufbau zum Datennetz erfordert protokollarisch eine zumindest für die Sitzungsdauer eindeutige logische Adresse; jeder Datenabruf wird in der Regel von mindestens einem Webserver, auf dem das abgerufene Datum gespeichert ist, protokolliert; um Nutzer darüber hinaus sitzungsübergreifend identifizieren zu können, werden entweder dienstspezifische Identifikationsdateien mehr oder weniger verdeckt auf dem Nutzerendgerät gespeichert, oder eine manuelle Authentifizierung des Nutzers in Form von Nutzername und Passwort verlangt. Das mobile Internet wird daher zwar nicht von einem "großen Bruder" kontrolliert, dafür gibt es jedoch zahlreiche „kleine Schwestern“, die persönliche Daten erheben und sammeln. ${ }^{696}$ Wie ebenfalls bereits erläutert wurde, ist die dezentrale Datensammlung mit einer Reihe von praktischen Problemen verbunden, wie einer mangelnden Kontrolle der Vertraulichkeit und einem erhöhten Aufwand durch Mehrfachangabe von persönlichen Daten auf Seiten des Kunden sowie einer mangelnden Kontrolle von Wahrheit und Aktualität erfasster Kundendaten durch Anbieter. ${ }^{697}$

$695 \mathrm{Vgl}$. Berthold/Köhntopp (2001).

696 Vgl. Schulzki-Haddouti (2000) und Abschnitt 5.4.2.1.

$697 \mathrm{Vgl}$. Abschnitt 5.4.2.1. 
Wie Untersuchungen des Konsumentenverhaltens im stationären Internet darlegen, ist die Folge der Intransparenz der Erhebung personenbezogener Daten ein grundsätzlicher Vertrauensmangel der Nutzer gegenüber dem Internet. ${ }^{698}$ Dieser Vertrauensmangel hat, wie bereits gezeigt wurde, die Konsequenz, dass Inhalteanbieter, die personenbezogene Daten erheben, generell gemieden werden oder falsche Personenangaben bei diesen Diensten gemacht werden. ${ }^{699}$ Dieser Vertrauensmangel muss jedoch nicht zwangsläufig ein unüberwindliches Dilemma für anpassungsfähige Dienstangebote im mobilen Internet, die auf personenbezogene Angaben angewiesen sind, darstellen. Wie Olivero/Lunt (2004) zeigen, führte der Zweifel am vertrauenswürdigen Umgang mit Personendaten im Internet bei Nutzern in den letzten Jahren zu einer zunehmend pragmatischen Evaluation von Kosten und Nutzen im Hinblick auf die Preisgabe personenbezogener Informationen, wodurch ein Bedarf an Kontrollmechanismen für Nutzer hinsichtlich der Verwendung ihrer Daten entstanden ist. In Ermangelung eines globalen rechtlichen Rahmens zur Kontrolle von Datenschutzpraktiken gelten vor allem technische Kontrollösungen im Zusammenhang mit diesen Bedarfsanforderungen als Erfolg versprechend. Derzeit werden vor diesem Hintergrund zwei verschiedene Möglichkeiten zur Verbesserung des Schutzes der Privatsphäre in Form einer vom Nutzer kontrollierbaren Handhabung personenbezogener Daten als Alternativen zur ausschließlich serverseitigen Profilspeicherung beim Dienstanbieter diskutiert: die vollkommene Anonymisierung der Onlinenutzung (1) und die ausschließliche Speicherung von Personendaten auf den Nutzerendgeräten (2).

(1) Die Grundlage einer Anonymisierung der Dienstnutzung in öffentlichen Netzen bildet einerseits die Verschleierung der individuellen Netzadresse durch den Zugriff auf den Dienst über den Umweg eines kommerziellen Anonymisierungs- oder Pseudonymisierungsproxies. Beispiel eines solchen Anonymisierungsproxies bildet der kommerzielle Dienst Anonymizer.com. Andererseits werden Verfahren diskutiert, um einzelne Dienstanfragen entweder in Gruppen verschiedener anderer Webanfragen ${ }^{700}$ oder durch eine Verkettung von Anfragen über verschiedene Netzwerkknoten ${ }^{701}$ hinweg zu verbergen. Unter der Voraussetzung, dass eine Verkettung der Zugriffsprotokolle des Anonymisierungsdienstes mit den Dienstzugriffsprotokollen verhindert wird, kann in diesem Fall weitestgehend eine vollkommen anonyme Dienstnutzung gewährleistet werden. Allerdings schließt eine solche vollkommene Anonymität auch die im Einzelfall ggf. vorteilhafte Nutzung adaptiver Dienste aus.

(2) Eine ausschließliche Speicherung von Profilen auf dem Nutzerendgerät kann dazu beitragen, die angesprochenen Überblicksprobleme eines Nutzers in Bezug auf die von ihm angelegten Profile zu mindern. ${ }^{702}$ Dennoch setzt auch

\footnotetext{
698 Vgl. CDT (2000).

699 Vgl. Abschnitt 4.1.1.3.

$700 \mathrm{Vgl}$. Reiter/Rubin (1999).

701 Vgl. Chaum (1985) und Syverson/ Goldschlag/Reed (1997).

702 Vgl. Richie/Brebner/Gittler (2001).
} 
die Profilspeicherung auf dem Endgerät weiterhin eine Übertragung personenbezogener Daten an den durch das Profil konfigurierten Dienst voraus, wodurch sich die übertragenen Daten der Kontrolle durch den Nutzer entziehen. Um zumindest das Transparenzproblem der Handhabung personenbezogener Daten durch einen Dienstanbieter zu mindern, wurde die bereits angesprochene „Platform for Privacy Preferences“ (P3P) als Austauschgrundlage von Datenschutzpraktiken und -präferenzen zwischen Dienstanbietern und Nutzern entwickelt. $^{703}$ P3P dient nicht dem Austausch der personenbezogenen Daten selbst, sondern lediglich dem transparenten Abgleich zwischen den Datenschutzpraktiken des Dienstanbieters und den allgemeinen Datenschutzpräferenzen des Dienstnutzers. Durch die strukturierte Überführung der Datenschutzpraktiken in ein maschinenlesbares XML-Format kann ein Nutzer beim Aufruf des betreffenden Dienstes mit einem P3P-fähigen Browser diese Praktiken per Dialog einsehen und über entsprechende Akzeptanz oder Ablehnung entscheiden. Um Vertrauen in die Datenschutzerklärung des Dienstanbieters zu erzeugen, kann dieser sich die Einhaltung der deklarierten Praktiken durch Anbieter von Gütesiegelprogrammen, sog. „Third-Trust-Parties“, zertifizieren lassen.

Um die Handhabung von personenbezogenen Daten in Onlineinteraktionsprozessen an die oben angesprochenen intuitiven Preisgabemechanismen menschlicher Akteure in alltäglichen Gesprächssituationen anzupassen, werden in neueren Arbeiten vermehrt Möglichkeiten eines vom Nutzer steuerbaren Managements rollenbasierter virtueller Identitäten diskutiert. Ein solches Identitätsmanagement kann grundsätzlich auf Grundlage von P3P erfolgen, bedarf jedoch einer umfassenden Erweiterung des derzeitigen P3P Vokabulars um eine Semantik zur Beschreibung von Rollen und zur erweiterten Deklaration rollenbezogener Inhaltsbereiche des Nutzerprofils. ${ }^{704}$ Demgegenüber beschreiben Dickinson et al. (2003) eine datenschutzgerechte Identitätskontrolle in einem agentenbasierten Framework auf Grundlage einer Public Key Infrastruktur. ${ }^{705} \mathrm{Im}$ Mittelpunkt dieses Ansatzes steht eine funktionale Differenzierung zwischen einem Manager lediglich pseudonymer Modelle eines Nutzers und einem vertrauenswürdigen Manager der Identifikationsdaten des Nutzers. Dem Ansatz von Dickinson et al. folgend, wird im nächsten Abschnitt eine Konzeptvariante zur aggregierten Modellierung verteilt erhobener Nutzerprofile auf Grundlage von Semantic Web Technologien in einer serviceorientierten Dienstarchitektur beschrieben.

\subsubsection{Konzeption eines pseudonymen Nutzermodellierungssystems}

Nachdem in Abschnitt 5.4.3.2 das grundlegende Konzept einer dienstübergreifenden Aggregation von Profildaten dargelegt wurde, sollen nun Aspekte einer datenschutzgerechten Weitergabe und Verwendung dieser Profildaten zwischen Dienstanbietern und dem Aggregator diskutiert werden. Dabei werden zwei Da-

\footnotetext{
$703 \mathrm{Vgl}$. Abschnitt 4.1.1.2.

$704 \mathrm{Vgl}$. Berthold/Köhntopp (2001) und Wörndl (2003).

705 Vgl. Dickinson/Reynolds/Banks/Cayzer/Vora (2003).
} 
tenschutzaspekte fokussiert: Die grundsätzliche Pseudonymität der verwendeten Personendaten und die durch den Nutzer gesteuerte Zugriffskontrolle auf die über inn erhobenen Daten.

Das in Abschnitt 5.4.2.2 dargelegte Prinzip der Nutzermodellierung auf der Grundlage von Verhaltensdaten erfordert prinzipiell keine explizite Nutzerinteraktion in Bezug auf eine freiwillige Preisgabe eindeutiger individueller Identifikationsdaten. Da die IP-Adresse des Nutzerendgeräts als einziges eindeutiges Identifikationskriterium in der Regel dynamisch durch den Netzzugangsprovider bei jeder Sitzung neu vergeben wird, ist keine unmittelbare Verkettung der Adressinformationen auf die Identität des Nutzers möglich. Die Identifikation erfolgt demnach notwendigerweise pseudonym, durch einen vom Dienstanbieter gewählten Identifikator. Um eine Aggregation der unterschiedlichen Profile, die verschiedene Dienste über einen konkreten Nutzer erheben, auf ein gemeinsames Modell eben dieses Nutzers abbilden zu können, ist es allerdings notwendig, dass die jeweils von den Dienstanbietern gewählten Identifikatoren durch den Aggregator auf eine einzige wiederum pseudonyme Identität abgebildet werden können. Abbildung 5-47 zeigt eine einfache Interaktionsstruktur, die eine solche pseudonyme Aggregation der Nutzermodelle zweier Dienstanbieter $A$ und $B$ durch einen Aggregator ermöglicht. ${ }^{706}$

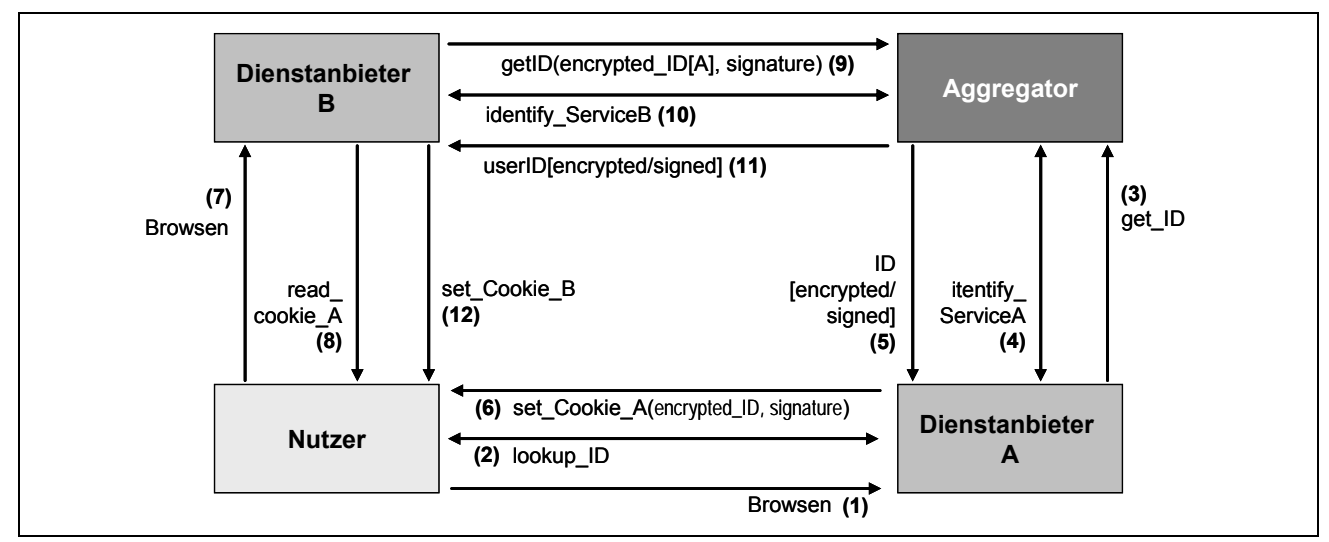

Abbildung 5-47: Kommunikationsmodell einer pseudonymen Aggregation von Nutzermodellen

Im Falle einer erstmaligen Inanspruchnahme (1) eines Dienstes A durch einen Nutzer überprüft der Dienst, ob bereits ein Identifikator in Form eines Cookies auf dem Nutzerendgerät existiert (2). Ist dies nicht der Fall, ruft der Dienst automatisch einen Dienst des Aggregators auf und fordert ein neues Pseudonym an (3). Der Aggregator überprüft durch Auslesen der Cookies von Dienst A, ob der Dienst bereits bei ihm bekannt ist. Ist dies nicht der Fall, legt der Aggregator ein verschlüsseltes Identifikations-Cookie bei Dienst $A$ ab (4). Der über eine Webschnittstelle von Dienst A angesprochene Dienst des Aggregators erzeugt dann einen eindeutigen Identifikator für den Nutzer von Dienst A. Um eine grundsätzlich denkbare betrügerische Zusammenarbeit verschiedener Dienste

706 Vgl. Kaspar/Seidenfaden/Hagenhoff (2004). 
A...N von vorn herein auszuschließen, verschlüsselt der Aggregator die herausgegebenen Identifikationsdaten mit seinem öffentlichen Schlüssel und signiert das Ergebnis mit seinem privaten Schlüssel (5). Dienst A legt die verschlüsselten und signierten Identifikationsdaten als Cookie auf dem jeweiligen Nutzerendgerät ab (6). Besucht der Nutzer einen weiteren Dienst B, der ebenfalls die Aggregationsleistung des Aggregators in Anspruch nehmen will (7), liest B alle vom Aggregator signierten Cookies auf dem Nutzerendgerät aus (8) und übergibt diese im Rahmen der Nutzeridentifikationsanfrage an die Schnittstelle des Aggregators (9). Nach erfolgter Identifikation von Dienst beim Aggregator (10) erzeugt der Aggregator einen neuen, vom Identifikator für Dienst A unterschiedlichen Identifikator für den Nutzer und übergibt diesen wiederum verschlüsselt und signiert an Dienst B. Dienst B speichert den Identifikator in einem Cookie auf den Endgerät des Nutzers ab (12).

\subsubsection{Zugriffskontrolle für Personendaten im Rahmen einer pseudo- nymen Nutzermodellierung}

Das Problem des Datenschutzes ist eng verknüpft mit der Frage, inwieweit der Erheber personenbezogener Daten Verwertungsrechte an diesen Daten ausüben oder sogar delegieren kann, bzw. ob ggf. unveräußerliche Schutzrechte im Sinne originärer „Urheberrechte“ der Person, über die Daten erhoben werden, zu wahren sind. Diese grundsätzliche Fragestellung besteht bei der Verwertung digitaler Inhalte nicht nur in Bezug auf personenbezogene Daten, sondern kann vor dem Hintergrund der Diskussion adäquater Lösungen für ein umfassendes Digital Rights Management auf sämtliche digitalisierbaren Inhalte erweitert werden. Die Architektur eines Systems zur Kontrolle von Verwertungsrechten an digitalen Inhalten besteht dabei im Kern aus drei Komponenten: ${ }^{707}$ einer Datenbank für die zu verwaltenden Inhalte, einem System zur Verwaltung von Lizenzen in Form von identitätsbezogenen Verwendungsrechten für die Elemente der Inhaltedatenbank und einem DRM-Controller, der auf dem Nutzerendgerät bzw. der Zielapplikation die Einhaltung der lizenzierten Rechte überwacht. Einem solchen allgemeinen Architekturmodell eines digitalen Rechtemanagement folgend sollen im Folgenden Möglichkeiten für eine Zugriffskontrolle insbesondere in Bezug auf mögliche Varianten der Lizenzgestaltung und Kontrolle für personenbezogene Profildaten erörtert werden. Die Problemstellung der Ausübung und Verwaltung von Kontrollrechten in Bezug auf die jeweils erhobenen und ausgetauschten Profildaten kann dabei in dem in Abbildung 5-47 dargestellten Interaktionsschema aus zweierlei Sicht interpretiert werden: aus Sicht der jeweils Daten erhebenden Dienste A...N (1) sowie aus Sicht des Nutzers, über den bei verschiedenen Diensten Profile erstellt werden (2):

(1) Da die verschiedenen Dienste, die mit einem Aggregator in Interaktion stehen, in der Regel direkt oder zumindest indirekt (in Bezug auf die Aufmerksamkeit potenzieller Nutzer) in einem Konkurrenzverhältnis stehen, existiert ein In-

707 Vgl. Rosenblatt/Trippe/Mooney (2002). 
teressenskonflikt hinsichtlich der Preisgabe der durch diese Dienste erhobenen Profilinformationen. Dieser Interessenskonflikt ist begründet in einem wettbewerbsstrategischen Dilemma, da ein Dienst zwar ein Interesse an dienstübergreifenden Informationen über das Verhalten eines Nutzers hat, die selbst erhobenen Profilinformationen jedoch als kritische Ressourcen nur ungern preisgegeben werden. Das Zugriffsproblem im Hinblick auf die Interaktion zwischen Aggregator und Dienstanbieter besteht in der Lösung dieses Dilemmas in Form einer in Bezug auf die Preisgabe von dienstspezifischen Profildaten anreizkompatiblen Gestaltung der Zugriffskontrolle. Die Bestimmung und Durchsetzung von Lizenzen in Form von Zugriffsrechten auf die aggregierten Profildaten des Aggregators kann im Rahmen des beschriebenen Interaktionsschemas an zwei Stellen erfolgen: beim Aggregator selbst oder auf Ebene der Schnittstelle zum Profilspeicher der jeweiligen Dienste.

Damit ein Anreiz existiert, dem Aggregator die eigenen Profildaten über eine Schnittstelle zugänglich zu machen, muss aus Sicht eines profilerhebenden Dienstes grundsätzlich die Möglichkeit bestehen, auf mehr als nur die selbst erhobenen Daten über das Verhalten eines Nutzers zugreifen zu können. Ein denkbares Schema für eine solche anreizkompatible Zugriffsberechtigung auf aggregierte Nutzerdaten besteht in der Vergabe von Zugriffsrechten auf Grundlage von Attributklassen des Nutzermodells durch den Aggregator. Wie bereits dargelegt, ordnet der Aggregator die über die einzelnen Dienste zugänglichen Profilattribute anhand einer dienstübergreifenden Referenzontologie in ein umfassendes Nutzermodell ein. Für einen Dienst, der Profildaten bereitstellt, ist es interessant, weitere Verhaltensinformationen in Bezug auf die Attributklassen des Nutzermodells einzusehen, für die von ihm Attributwerte beigesteuert werden. Die Umsetzung einer solchen Zugriffsberechtigung kann bspw. durch die Vergabe von Attributzertifikaten erfolgen, die Angaben über die jeweiligen Attributklassen enthalten, für die der Dienst Profildaten beisteuert.

Auf der Ebene des Dienstanbieters kann der Zugriff auf Profildaten nur dann gesteuert werden, wenn - wie in Abschnitt 5.4.2.3 dargelegt - die Attributwerte des Dienstprofis dem Aggregator nicht jeweils direkt zugänglich gemacht werden, sondern im Nutzermodell des Aggregators lediglich in Form parametrisierter Verweise auf die Schnittstelle des Dienstanbieters gespeichert werden. So kann ein Dienst B zwar beim Aggregator Informationen darüber beziehen, dass für die Klasse der Attribute, die dieser Dienst über seine Nutzer erhebt, weitere Attribute bspw. bei einem anderen Dienst A existieren, die Preisgabe der konkreten Werte wird jedoch direkt zwischen den beiden Diensten ausgehandelt.

(2) Eine individuelle Anpassung eines mobilen Inhalteangebots ist aus Nutzersicht immer nur dann vorteilhaft, wenn der Anpassung tatsächlich die jeweiligen Bedürfnisse des Nutzers zugrunde liegen. Solche Bedürfnisse derselben Person können jedoch im Rahmen der Ausübung verschiedener sozialer Rollen, die diese Person bspw. in der beruflichen oder privaten Lebenswelt innehat, variieren. Ein entsprechender rollenspezifischer Filter kann im Zuge einer ausschließlich in Form einer Verhaltensbeobachtung erfolgenden Nutzermodellierung nur schwer abgeleitet werden und muss daher vom Nutzer selbst in Form 
von rollenbezogenen Zugriffsregeln auf die über inn erhobenen Profildaten erstellt werden. Insofern die Definition solcher Filterregeln eine explizite Preisgabe von persönlichen Angaben durch den Nutzer erfordert, kann in Abhängigkeit vom Vertrauen des Nutzers in den Aggregator eine solche Regeldefinition wiederum an zwei Stellen erfolgen: beim Aggregator selbst oder bei einem Dienstleister, der im Auftrag des Nutzers Kontrollfunktionen über die beim Aggregator gesammelten Profildaten ausübt. Dieser Dienstleister wird im Weiteren als „Bedürfnismanager" bezeichnet.

Im Regelfall besteht die Dienstleistung des Aggregators darin, die Interessen von Dienstanbietern an dienstübergreifenden Verhaltensdaten von individuellen Nutzern zu bedienen und diese Daten als Dienstleistung zu vermarkten. Insofern stehen die Interessen des Aggregators nach umfangreicher Vermarktung von Nutzerdaten dem Interesse des Nutzers entgegen, den Datenverkehr einerseits in Bezug auf situative Bedürfnisse einzuschränken, andererseits eindeutige Identifikationsmerkmale weitest möglich geheim zu halten. Daher scheint eine zusätzliche Rolle im beschriebenen Interaktionsschema plausibel, die nutzerindividuelle Bedürfniskategorien kennt und auf deren Grundlage eine Kontrollfunktion im Austausch der Profildaten des Nutzers in dessen Interesse steuert. Abbildung 5-48 zeigt die Einordnung dieser Rolle eines Bedürfnismanagers im Rahmen des beschriebenen Interaktionsschemas.

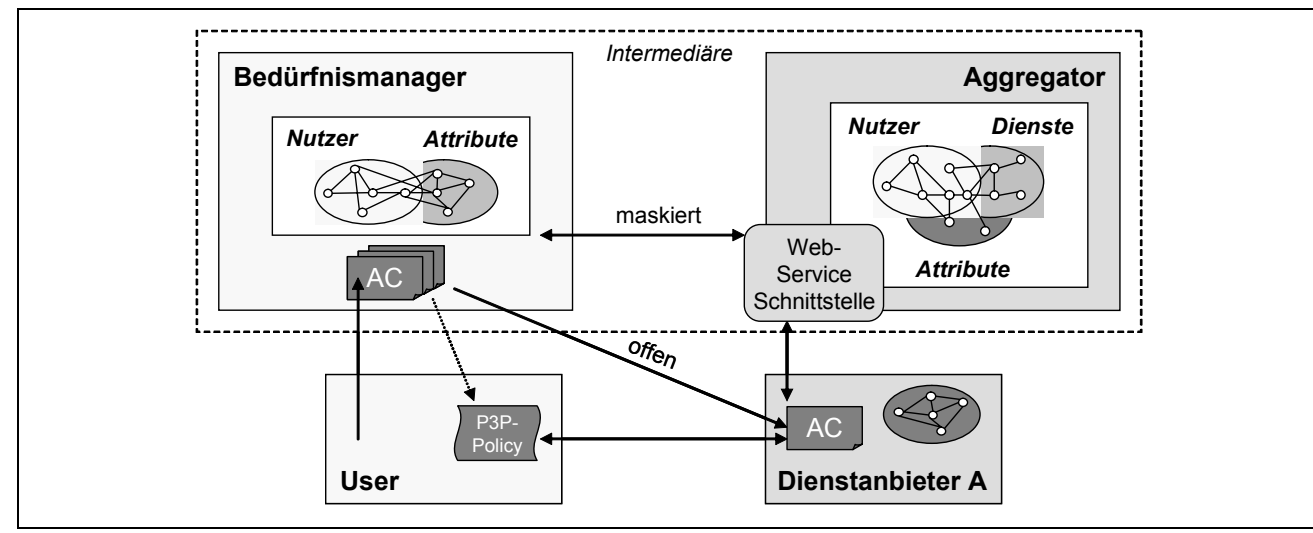

Abbildung 5-48: Intermediation mit Aggregator und Bedürnismanager

Der Bedürfnismanager kann gegenüber Aggregator und Dienstanbieter entweder maskiert oder offen auftreten. Im Falle einer maskierten Interaktion operiert der Bedürfnismanager als Scheindienst, indem die beim Aggregator gespeicherten Attribute durch Übergabe von Scheinattributen zusammen mit dem beim Nutzer gespeicherten Identifikatoren des Aggregators ausgelesen werden. Das Ziel der verdeckten Interaktion besteht darin, dem Nutzer Transparenz über Umfang und ggf. Inhalt der über inn erhobenen Daten zu geben. Eine Kontrollfunktion in Bezug auf die Verwendung von Profildaten durch Dienstanbieter kann jedoch nicht ausgeübt werden. Im Falle einer offenen Interaktion definiert der Nutzer beim Bedürfnismanager spezielle Verwendungsregeln für die beim Aggregator modellierten Attributklassen und -werte, Dies setzt eine 
Kenntnis der jeweiligen Referenzontologie des Aggregators durch den Bedürfnismanager voraus. Anhand dieser Verwendungsregeln können Dienstanbieter konkrete Attributzertifikate (AC) erwerben, die auf Seiten des Nutzerendgeräts bspw. im Rahmen einer erweiterten P3P-Policy im Browser hinterlegt werden. Auf Grundlage dieser Policies kann der Nutzer im Zuge der Interaktion mit einem Dienst bspw. in Abhängigkeit von der situativ ausgeübten Rolle durch Akzeptanz eines oder mehrerer Zertifikate entscheiden, welche Zugriffsregeln vom Dienst verwendet werden sollen.

\subsubsection{Zusammenfassung}

Der voranstehende Abschnitt beschäftigt sich mit der Fragestellung, wie ein dienstübergreifender Austausch von Profildaten zwischen Onlinediensten erfolgen kann, ohne datenschutzrechtliche Bedenken bei Nutzern hervorzurufen oder solche bestehenden Bedenken zu vermehren. Vor diesem Hintergrund wurde in 5.4.3.2 das Modell einer Interaktion zwischen Nutzern, Onlinediensten und möglichen Intermediären für den Austausch von Profildaten auf Grundlage einer zwar technisch offenen, im Hinblick auf die Vertraulichkeit personenbezogener Daten lediglich pseudonym identifizierender Techniken vorgestellt. In Abschnitt 5.4.3.3 wurden Möglichkeiten erörtert, um auf Grundlage dieser Interaktionsarchitektur sowohl aus Sicht von Profildaten erhebenden Diensten als auch aus Sicht von deren Nutzern anreizkompatible Zugriffsregeln in Bezug auf erhobene Profildaten zu definieren und durchzusetzen. Dabei kann eine solche Zugriffsbeschränkung aus Nutzersicht nur dann durchgesetzt werden, wenn Mechanismen existieren, die eine grundsätzlich freiwillige Selbstbeschränkung von Dienstanbietern ökonomisch - bspw. aufgrund einer rollenspezifischen Bedürfnisidentifikation - plausibel machen, und eine derartige Regelkonformität einzelner Dienste durch den Nutzer auch identifizierbar ist.

Wie bereits in Abschnitt 5.4.3.1 angedeutet wurde, existieren in Bezug auf den rechtlichen Schutzanspruch der individuellen Privatsphäre sowie personenbezogener Daten keine weltweit bindenden Gesetzesnormen oder Rechtsauslegungen. Legt man allerdings die 1980 verabschiedeten Richtlinien ${ }^{708}$ der OECD zugrunde, können sieben Prinzipien in Bezug auf datentechnische Schutzaspekte unterschieden werden: Offenheit und Transparenz von Datenerhebung und -haltung; Möglichkeiten einer individuellen Einsichtnahme und Partizipation; eine dem Erhebungszweck angemessene Datensammlung; die Wahrung aktueller und wahrheitsgemäßer Datenqualität; eine adäquate Zugriffssicherung der Daten und eine diesbezügliche Haftung der erhebenden Einrichtung. Die im vorliegenden Beitrag vertretene Konzeption einer datenschutzgerechten Handhabung personenbezogener Daten steht offensichtlich im Widerspruch zu einer grundsätzlichen Beschränkung von Erhebung und Austausch personenbezogener Daten. Dabei wird unterstellt, dass mangels geeigneter Durchgriffsmöglichkeiten im Zuge einer restriktiven Beschränkung des Datenverkehrs ein unbehinderter Datenaustausch langfristig zu einer ökonomisch motivierten Selbstbeschränkung durch Daten erhebende Dienste führt.

$708 \mathrm{Vgl.} \mathrm{OECD} \mathrm{(1980).}$ 
Um diese lediglich konzeptionell begründete These weiter zu fundieren, sind drei abhängige Problembereiche zu untersuchen, mit der sich zukünftige Forschung auseinandersetzen muss: erstens, das hier weitgehend vernachlässigte Problem der logischen Zusammenführung dezentral erhobener Profilmerkmale in einem gemeinsamen Modell; zweitens, das grundsätzliche Sicherheitsrisiko zentral gespeicherter Daten; drittens, Techniken, die eine weitgehend automatische Bedürfniserkennung, entweder in Form von Heuristiken der Verhaltensbeobachtung, oder durch technische Erweiterungen im Bereich der abschlieBend angesprochenen Kopplung von Zertifikationsstandards mit Browser integrierten Regelstandards wie P3P, erlauben.

\subsubsection{Zusammenfassung}

Das Ziel des voranstehenden Kapitels bestand darin, offene Fragestellungen im Zusammenhang mit der mobilitätsgerechten Bereitstellung von Inhalten zu identifizieren und Lösungsansätze für diese Fragen zu präsentieren. In Abschnitt 5.4.1 wurden zunächst bereits zuvor angesprochene Konzepte zu individuellen und ortsbezogenen Inhalteanpassung aufgegriffen und vor diesem Hintergrund Anforderungen an die Umsetzung kontextbezogener Dienste im mobile Internet abgeleitet. In Abschnitt 5.4.2 wurden konzeptionelle Überlegungen im Hinblick auf die Modellierung von Kontextdaten in verteilten Systemen am Beispiel der Modellierung von Inhaltepräferenzen angestellt. In Abschnitt 5.4.3 wurde ein Lösungsansatz für die datenschutzkonforme Kontrolle der Preisgabe persönlicher Daten im Zuge einer solchen verteilten Modellierung präsentiert.

Die in den Abschnitten 5.4.2 und 5.4.3 vorgestellten Konzepte mögen zwar in ihren Ausführungen akademischen Charakter besitzen, dennoch belegen Gespräche mit Verantwortlichen für die Entwicklung von kommerziellen Anwendungen im mobilen Internet eine immense Bedeutung der Entwicklung plausibler Lösungen für die Erfassung und den rechtskonformen Umgang mit Kontextdaten mobiler Nutzer aus verschiedenen Datenquellen. Ähnliche Konzeptentwicklungen sind derzeit insbesondere bei allen der vier deutschen Mobilfunkbetreiber zu beobachten, die die Entwicklung des Handels mit verdichteten Kontextdaten als viel versprechendes zukünftiges Geschäftsfeld erachten. ${ }^{709}$ Leider waren derartige Konzepte aus Geheimhaltungsgründen für eine eingehendere Analyse im Rahmen dieser Arbeit nicht zugänglich.

709 Ein Beispiel für die Entwicklung eines gekapselten dienstübergreifenden Kontextmodellierungssystems bildet etwa das Projekt der sog. „Personal Tracing and Tracking Platform“ (PTTP) der Firma T-Systems, die einerseits Lokalisierungsdaten aus verschiedenen Quellen für einen Nutzer kombiniert und den Zugriff auf diese Daten über ein Berechtigungssystem (das sog. Permission and Privacy Gateway, PPGW) steuert. Ein Projekt mit ähnlicher Zielsetzung wird unter dem Projektnamen „context awareness for mobile services" derzeit beim Konkurrenten Vodafone durchgeführt. 


\subsection{Zusammenfassung der Möglichkeiten der Inhalte- verwertung im mobilen Internet}

Anhand eines Überblicks über bestehende Forschungsbeiträge zum Geschäft mit mobilen Diensten wurden in Kapitel 5.1 offene Forschungsfragen im Zusammenhang mit der Inhalteverwertung im mobilen Internet anhand von drei Perspektiven strukturiert: der kaufmännischen Geschäftsmodellperspektive für die Verwertung von Inhalten im mobilen Internet, der Perspektive der Kundenakzeptanz mobiler Inhalteformate und der Perspektive der technischen Machbarkeit mobiler Mehrwertdienste:

- Im Rahmen der Untersuchung der Gestaltungsmöglichkeiten von Geschäftsmodellen inhalteorientierter mobiler Dienste wurden in Kapitel 5.2 mit Ortsunabhängigkeit, Ortsspezifität und Kommunikationsorientierung drei produktbezogene Erfolgsfaktoren mobiler Dienste identifiziert. Des Weiteren wurde gezeigt, dass ein Inhalteanbieter zur Verwertung von Inhalten im mobilen Internet Wertschöpfungskooperationen, etwa mit Dienstentwicklern sowie Plattform- und Mobilfunkbetreibern, eingehen muss. Im Zusammenhang mit den Möglichkeiten der Preisgestaltung mobiler Dienste wurde eine deutliche Abhängigkeit von Inhalteanbietern von den vorgegebenen Tarifstrukturen im Datenverkehr festgestellt.

- Die Analyse der Akzeptanz mobiler Inhalteformate in Kapitel 5.3 zeigte, dass insbesondere solche Rezipientengruppen überdurchschnittlich bereit sind, mobile Dienste zu nutzen, die eine hohe Bereitschaft der Nachfrage nach spezialisierten Inhalten besitzen. Dabei dient das mobile Internet in erster Linie als Medium für den zeitnahen Konsum allgemeininformierender Inhalte. Es wurde insgesamt ein ausgesprochen geringer Nutzungsgrad mobiler Inhalteformate festgestellt, was vermuten lässt, dass das mobile Internet auch in der mittleren Frist für die Medienbranche lediglich ein Nischengeschäft repräsentieren wird.

- Als eines der zentralen Mehrwertversprechen des mobilen Internet gilt die Wahrnehmungsfähigkeit der physischen und dv-technischen Rahmenbedingungen einer Abfragesituation. Zwar zeigt Abschnitt 5.2.3.2 das Beispiel einer bereits existierenden erfolgreichen Implementierung eines kontextbezogenen Dienstes, dennoch wird in Kapitel 5.4 deutlich, dass eine weitere Ausschöpfung des kommerziellen Potenzials kontextbezogener Anwendungen im mobilen Internet einer Lösung vergleichsweise komplexer technischer und rechtlicher Fragen bedarf. Diese Fragen betreffen eine Standardisierung der Modellierungssemantik von Abfragekontexten, die Schaffung flexibler Systemarchitekturen für den Austausch von Kontextinformationen und die Gewährleistung einer vertrauenswürdigen Kontrolle der individuellen Privatsphäre. 


\section{Schlussbetrachtung}

Das Ziel der vorliegenden Arbeit ist es, Handlungsmöglichkeiten für Medienunternehmen im Zusammenhang mit der Individualisierung von Mediengütern und dem Inhaltevertrieb im mobilen Internet abzuleiten sowie diese Handlungsmöglichkeiten auf ihren Beitrag zur Minderung der wirtschaftlichen Krise der Medienbranche hin zu bewerten. Der Gang der Arbeit orientiert sich - abgesehen von der Darstellung notwendiger Grundlagen in Kapitel 2 - an vier zentralen Forschungsfragen, die an dieser Stelle abschließend beantwortet werden sollen:

\section{Frage 1: Welche Handlungsmöglichkeiten bieten sich Medienunternehmen, um die derzeitige Krise der Branche zu bewältigen?}

Dieser Frage wurde schwerpunktmäßig in Kapitel 3 nachgegangen. Es wurden die Rahmenbedingungen (1) und die aktuelle Situation des Wettbewerbs in der Medienbranche (2) analysiert sowie Optionen für Wettbewerbsstrategien von Medienunternehmen im Onlinegeschäft abgeleitet (3).

(1) Der Wettbewerb in der Medienbranche wird nachhaltig beeinflusst von den Auswirkungen einer in erster Linie technologiegetriebenen Branchenveränderung. Diese Veränderung wird auch als Konvergenz bezeichnet. Unter Konvergenz ist die zunehmende Verflechtung der Märkte von Telekommunikations-, IT- und Medienbranche zu verstehen. Diese Verflechtungen haben für die Medienbranche zwei Auswirkungen: Erstens verbessern sich durch IT-Unterstützung die Möglichkeiten einer medienneutralen Erzeugung von Inhalten, wodurch Mediengüter ohne wesentliche Mehrkosten nahezu beliebig miteinander kombiniert und in verschiedene Distributionsformate transformiert werden können. Zweitens entstehen auf der Grundlage von Telekommunikationsnetzen neue digitale Distributionskanäle für Mediengüter.

(2) Anhand einer Analyse der Wettbewerbssituation in den deutschen Medienmärkten wurden einerseits hohe Marktsättigungsgrade und andererseits speziell in Märkten für Druckerzeugnisse - sinkende Durchschnittsreichweiten von Mediengütern dokumentiert. Zwar konnte keine signifikante Substitutionsbeziehung zwischen traditionellen und digitalen Medienmärkten nachgewiesen werden, eine Veränderung der intermedialen Wettbewerbssituation ist jedoch aufgrund einer zunehmenden Habitualisierung der Inhaltenutzung über Onlinemedien mittelfristig abzusehen.

(3) Zur Erschließung zusätzlicher Einnahmequellen können Medienunternehmen bei der Vermarktung von Mediengütern in Onlinemedien zwei Strategieoptionen verfolgen: Erstens die zusätzliche Verwertung von Mediengütern, die ursprünglich für ein traditioneller Vertriebsmedium erzeugt wurden, in weitgehend unveränderter Form über Onlinemedien. Zweitens die Schaf- 
fung zusätzlicher Gütervarianten auf Bündelungsebene, um Bedürfnissen von Konsumentengruppen oder individuellen Kunden besser zu entsprechen. Es wurde geschlussfolgert, dass nur eine Kombination beider Optionen langfristig zum Erfolg führen kann, unter der Bedingung, dass es Medienunternehmen sowohl gelingt, zusätzliche Vertriebskanäle zu erschließen, als auch ihren Kunden einen zusätzlichen Konsumnutzen zu stiften. Für eine potenziell nutzenstiftende Inhalteverwertung existieren zwei Handlungsoptionen: die Individualisierung von Mediengütern im Onlinegeschäft und die Verwertung von Inhalten auf der Grundlage von Mehrwertformaten, entweder im mobilen Internet oder im interaktiven Fernsehen.

Frage 2: Wie können Mediengüter für einen individuellen Kunden maßgeschneidert werden?

Gestaltungsempfehlungen für die Bereitstellung maßgeschneiderter Mediengüter wurden in Kapitel 4 gegeben. In den Abschnitten 4.1 und 4.2 wurden Prinzipien intelligenter Inhaltefilter erläutert (1), in Abschnitt 4.4.2 wurden praktische Implementierungsbeispiele für derartige Filter vorgestellt (2).

(1) Die Voraussetzung jeder Form der Einzelkundenorientierung besteht darin, individuelle Kundenmerkmale zu identifizieren. Vor diesem Hintergrund wurden zum Teil bereits aus dem Bereich der Marktforschung bekannte Verfahren zur Kundenidentifikation und zur Modellierung von Kundenmerkmalen vorgestellt. Es wurde zwischen befragungsgestützter und beobachtungsgestützter Identifizierung von Kundenmerkmalen unterschieden. Bezugnehmend auf sowohl fremde als auch eigene Konzepte für ein intelligentes Datenfiltern wurden des Weiteren vier Verfahrensweisen erläutert und bewertet, die eine möglichst automatisierte Bereitstellung individuell maßgeschneiderter Mediengüter erlauben: die Selbstselektion, das eigenschaftsbasierte Filtern, das kollaborative Filtern und das beobachtungsgestützte Filtern.

(2) Um einen Nachweis der praktischen Realisierbarkeit der vorgestellten Filterverfahren zu erbringen und um ihren Nutzen bewerten zu können, wurden im Content-Management-System der Internetseiten der Universität Göttingen zwei Individualisierungsinstrumente implementiert: der MyUni-Konfigurator für eine individuelle Startseite und der MyBestBets-Filter für individuelle Navigationsempfehlungen. Diese beiden Instrumente bildeten die Grundlage für eine empirische Überprüfung des durch Individualisierung gestifteten Nutzens bei Anwendern.

Frage 3: Welchen Nutzen stiften maßgeschneiderte Mediengüter aus Kundensicht?

Zur Beantwortung dieser Frage wurde zum einen in Kapitel 4.3 der Einfluss von Individualisierung auf die Kaufentscheidung eines Konsumenten modelliert (1). Zum anderen wurde experimentell der Nutzen der beiden in Kapitel 4.4. prototypisch implementierten Filterverfahren nachgewiesen (2).

(1) Zur Bestimmung des Einflusses von Individualisierung auf den Kundenwert eines Medienguts wurde in Anlehnung an die mikroökonomische Bünde- 
lungstheorie ein modifiziertes Modell der Kaufentscheidung eines Inhaltekonsumenten entwickelt. Das Modell liefert die Erkenntnis, dass ein Individualisierungssystem aus Kundensicht immer dann Vorteile bietet, wenn dadurch der Aufwand der Suche nach relevanten Inhalten gesenkt wird, und das Verfahren einfach und ohne erheblichen Lernaufwand anzuwenden ist.

(2) Zur empirischen Validierung der im Modell gewonnenen Erkenntnisse wurde eine experimentelle Untersuchung der beiden im Rahmen dieser Arbeit implementierten Individualisierungssysteme (MyUni und MyBestBets) durchgeführt. Die Untersuchungsergebnisse stützen die zuvor gewonnenen Modellerkenntnisse in zweierlei Hinsicht: Zum einen konnte insgesamt ein Zusatznutzen durch den Einsatz von Individualisierungsverfahren nachgewiesen werden. Zum anderen wurde gezeigt, dass eine höhere Individualisierungspräzision den durch Anwender wahrgenommenen Nutzen steigert. Darüber hinaus konnte anhand der Untersuchungsergebnisse ein Zusammenhang zwischen dem jeweiligen Ziel der Informationsnachfrage und dem eingesetzten Individualisierungsverfahren festgestellt werden.

\section{Frage 4: Wie können Medienunternehmen das mobile Internet als Absatzmarkt für Mediengüter erschließen?}

Zur Beantwortung dieser Frage wurden in Kapitel 5 zunächst die Rahmenbedingungen einer Inhalteverwertung im mobilen Internet anhand theoretischdeduktiver Geschäftsmodellüberlegungen untersucht (1). Anhand einer empirischinduktiven Erhebung wurden geeignete Dienstformate für den Inhaltevertrieb im mobilen Internet ermittelt (2) und es wurden technisch-konzeptioneller Gestaltungsempfehlungen für die Bereitstellung mobiler Mehrwertformate gegeben (3).

(1) Unter Zuhilfenahme des dreiteiligen Analyseschemas für Geschäftsmodelle wurden potenzielle Mehrwertformate für die Inhalteverwertung im mobilen Internet in Form von ortsungebundenen, ortsbezogenen und nachrichtenbasierten Dienstformen identifiziert. Allerdings wurde ein grundsätzliches Adoptionsproblem festgestellt, das einerseits aus dem Netzgutcharakter mobiler Dienste und andererseits aus der frühen Marktentwicklungsphase des mobilen Internet herrührt.

(2) Eine Ausschöpfung der theoretischen Mehrwertpotenziale mobiler Dienstformate konnte zwar an einzelnen Beispielen, nicht jedoch als Massenmarkt nachgewiesen werden. Diese Erkenntnis bestätigt eine empirische Kundenerhebung der Akzeptanz mobiler Inhalteformate bei Lesern von Publikumsprinttiteln. Die Erhebungsbefunde lassen die Schlussfolgerung zu, dass sich das mobile Internet in erster Linie für den schnellen Zugriff auf aktuelle, allgemeininformierende Inhalte eignet. Darüber hinaus gehende Nutzungsformen wie ortsbezogene Anwendungen sind lediglich in Ansätzen zu erkennen.

(3) Als Grund für die geringe Akzeptanz (ortsbezogen) anpassungsfähiger Inhalteformate wurden offene Fragen im Zusammenhang mit der Erhebung, der Modellierung und der datenschutzrechtlichen Kontrolle personenbezogener Daten identifiziert. Es wurden Konzepte zur Lösung dieser Probleme durch semantische und pseudonyme Kontextmodellierung vorgestellt. 
Zusammenfassend können Strategieoptionen der Individualisierung von Mediengütern und der Inhalteverwertung im mobilen Internet vor dem Hintergrund der Erkenntnisse dieser Arbeit in zweierlei Hinsicht bewertet werden: Es konnte einerseits ein durch Individualisierung von Mediengütern geschaffener Mehrwert nachgewiesen werden, was für Medienunternehmen einen verstärkten Einsatz dieser Verfahren vorteilhaft erscheinen lässt. Demgegenüber stellen die zögerliche Akzeptanz und offene technische Umsetzungsfragen den Erfolg mobiler Anwendungen zumindest als Massengeschäft vorerst in Frage.

Im Zusammenhang mit dem Ziel dieser Arbeit, einer Bewertung der ökonomischen Vorteilhaftigkeit des Engagements von Medienunternehmen im Zusammenhang mit Individualisierung und Inhalteverwertung im mobilen Internet, bleiben einige spezielle Aspekte unberührt, die im Rahmen der vorliegenden Arbeit nicht vertieft werden konnten.

- Als ein Grundproblem der Inhalteverwertung im stationären Internet wurde die historisch bedingte Gratismentalität der Inhaltenachfrage beschrieben. Im Zusammenhang mit der Option der Individualisierung wurde zwar ein Zusatznutzen individuell maßgeschneiderter Mediengüter im Vergleich zu standardisierten Mediengütern festgestellt. Das gewählte Implementierungsbeispiel der Universität Göttingen war jedoch nicht-kommerzieller Natur, weshalb etwaige Auswirkungen dieses Zusatznutzens auf die Zahlungsbereitschaft im Rahmen dieses Beispiels nicht quantifiziert werden konnten. Des Weiteren sind im Markt für Onlineinhalte mittlerweile zwar erste Pionierangebote maßgeschneiderter Mediengüter (wie bspw. der Dienst MyFAZ im Onlineangebot der Frankfurter Allgemeinen Zeitung) festzustellen, aufgrund des frühen Marktstadiums kann jedoch keine Auskunft über den finanziellen Erfolg oder Misserfolg dieser Angebote gegeben werden. Daher konnte die Frage nicht abschließend beantwortet werden, ob und in welcher Höhe eine Individualisierung von Medienprodukten in der Praxis tatsächlich eine nachweisliche Zahlungsbereitschaft bei Onlinerezipienten bewirkt.

- Mediengüter repräsentieren üblicherweise Kuppelprodukte aus informierenden oder unterhaltenden Inhalten und aus an diese Inhalte geknüpften Werbebotschaften. Der Fokus dieser Arbeit richtet sich in erster Linie auf Aspekte einer Mehrwerterzeugung gegenüber Kunden auf dem Lesermarkt. Dennoch gilt das in Individualisierungsverfahren wurzelnde Mehrwertversprechen kundenindividuell maßgeschneiderter Produkte im selben (wenn nicht sogar ausgeprägterem) Maße auch für den Werbemarkt. Die im Rahmen dieser Arbeit vorgestellten konzeptionellen Verfahren für die Individualisierung von Informationsgütern können zwar uneingeschränkt auch auf Werbeinhalte angewendet werden. Dennoch stellt sich in diesem Zusammenhang die Anknüpfungsfrage an die Ergebnisse dieser Arbeit, inwiefern ggf. das Geschäft mit Werberaum in der Medienbranche durch die Möglichkeiten zur Individualisierung von Werbebotschaften verändert wird.

- Sowohl für die Strategieoption der Individualisierung als auch für die Inhalteverwertung im mobilen Internet konnte gezeigt werden, dass der jeweils durch die beiden Strategieoptionen gestiftete Mehrwert immer dann am höchsten ist, wenn die angebotenen Inhalte oder zumindest die inhaltlichen 
Interessen potenzieller Konsumenten einen hohen Spezialisierungsgrad besitzen. Wenngleich auch in der vorliegenden Arbeit in erster Linie Publikumsinhalte mit tendenziell allgemeininformierendem Charakter im Mittelpunkt der Untersuchung standen, lässt sich schlussfolgern, dass sich Erfolgspotenziale für Formen von Individualisierung und mobilen Diensten auch oder sogar besonders im Bereich der Publizistik von spezialisierten Fach- und Wissenschaftsinhalten ergeben. Diese Schlussfolgerung ist im Zuge von Anknüpfungsuntersuchungen im Detail zu validieren.

- Neben der Individualisierung von Mediengütern und dem Inhaltevertrieb im mobilen Internet wurde in Kapitel 3 als dritte Handlungsoption für Medienunternehmen der Inhaltevertrieb im digitalen, interaktiven Fernsehen benannt. Diese Handlungsoption konnte umfangsbedingt im Rahmen der vorliegenden Arbeit nicht berücksichtigt werden, was jedoch keine Beurteilung der Erfolgsaussichten des interaktiven Fernsehens implizieren soll.

Die vorliegende Arbeit hat im Zusammenhang mit intelligenten Inhaltefiltern und mobilen Diensten Technologien zum Gegenstand, die vergleichsweise kurzen Innovationszyklen unterliegen. Daher kann die Untersuchung von Anwendungsmöglichkeiten dieser Technologien lediglich eine Momentaufnahme für den vorliegenden oder zumindest absehbaren Stand der Technologieentwicklung geben. Von besonderer Bedeutung im Rahmen der zukünftigen Technologieveränderungen im Gegenstandsbereich dieser Arbeit erscheinen für Medienbranche dabei zwei Entwicklungen zu sein:

- Die im Rahmen dieser Arbeit vorgestellten Individualisierungssysteme verwenden vergleichsweise einfache Filterverfahren, die eine gewisse zeitliche Stabilität und Eindeutigkeit der verwendeten Filterkriterien voraussetzen. Dadurch ist die Ergebnisqualität dieser Filtern bislang kaum mit der Qualität von Ergebnissen kommerzieller Suchmaschinen vergleichbar, die bspw. Mehrdeutigkeiten unterscheiden, Suchabfragen kombinieren und Eingabefehler korrigieren können. Insofern allerdings intelligentes Filtern und indexgestützte Suche naturgemäß ein hohes Maß an Komplementarität besitzen, ist zukünftig von einer stärkeren Verknüpfung dieser beiden Technologien auszugehen. Dies muss allerdings für die Medienbranche nicht zwangsläufig mit einem Wachstumsimpuls einhergehen. In Ihrer Vision „Epic2014“ beschreiben die amerikanischen Journalisten Sloan und Thompson eine Medienzukunft, in der typische Redaktionstätigkeiten, wie das Bündeln und selbst das Erzeugen von Inhalten durch intelligente Maschinen vollzogen wird, womit diese Tätigkeiten preis- und somit wertlos werden. ${ }^{710}$ In Ansätzen sind derartige Entwicklungen bspw. an dem bereits im Jahr 2002 gestarteten maschinell erzeugten Nachrichtenportal GoogleNews ${ }^{711}$ zu erkennen.

- Die bereits angekündigte Evolution des kommerziellen Mobilfunks von der dritten zur vierten Generation verspricht in erster Linie unkomplizierte Kombinationsmöglichkeit verschiedener Mobilfunktechniken wie WLAN und

710 Vgl. Stöcker (2005).

711 Vgl. http://news.google.de. 
UMTS. Im Rahmen der Fortentwicklungen einzelner Funktechnologien sind darüber hinaus enorme Kapazitätserweiterungen absehbar, etwa in Form der bereits kurz vor der kommerziellen Einführung stehenden drahtlosen Breitbandtechnik WIMAX (IEEE 802.16), die Übertragungsraten von bis zu $75 \mathrm{MBit} / \mathrm{s}$ bei einer Reichweite von bis zu $48 \mathrm{~km}$ ermöglichen soll. Neben diesen Weiterentwicklungen bestehender Funktechnologien steht mit dem für mobile Endgeräte optimierten digitalen Rundfunkstandard DVB-H eine alternative Trägertechnik für mobile Anwendungen kurz vor ihrer Marktreife. Alle diese Technologieentwicklungen lassen erhoffen, dass dadurch eine höhere Akzeptanz und Verbreitung vor allem von multimedialen Dienstformen etabliert wird. Vor diesem Hintergrund ist ein Erfolg versprechendes Geschäft mit dem Inhaltevertrieb im mobilen Internet in Zukunft auch für audiovisuelle Angebote aus der Rundfunk-, Musik- und Filmindustrie zu erwarten. 


\section{Anhang}

Im Folgenden werden Aufbau und ausgewählte Ergebnisse der im Rahmen der vorliegenden Arbeit durchgeführten Erhebungen im Überblick dargestellt. Abgebildet sind die jeweils erhobenen Fragen, die Antwortmöglichkeiten und ihre Kodierung sowie die absoluten Antworthäufigkeiten. Abschnitt 7.1 zeigt die Ergebnisse der Befragung, die im Rahmen des in Abschnitt 4.4 beschriebenen Tests prototypischer Individualisierungsverfahren durchgeführt wurde. An der Befragung nahmen 35 Personen teil. In Abschnitt 7.2 werden die in Abschnitt 5.3.2.2 erläuterten Ergebnisse einer Erhebung des Mediennutzungsverhaltens von 557 Teilnehmern vorgestellt. Abschnitt 7.3 enthält die Ergebnisse der in Abschnitt 5.3.2.3 beschriebenen Befragung unter Lesern von Printtiteln zur Akzeptanz mobiler Inhalteformate. Die Befragung wurde von 7178 Teilnehmern vollständig zu Ende geführt.

\subsection{Ergebnisse der Erhebung zur Akzeptanz prototypischer Individualisierungsverfahren}

\begin{tabular}{|l|l|r|r|}
\hline Fragebogen 1 & ja(=1) & nein $(=2)$ \\
\hline B1F1 & MyBestBets gesehen? & 33 & 2 \\
\hline B1F2 & Empfehlungen der MyBestBets gelesen? & 26 & 9 \\
\hline B1F3 & Empfehlungen der MyBestBets genutzt? & 9 & 26 \\
\hline
\end{tabular}

\begin{tabular}{|c|c|c|c|c|c|c|}
\hline \multicolumn{3}{|c|}{ Fragebogen 2} & $\begin{array}{l}\text { gut } \\
(=1)\end{array}$ & $\begin{array}{l}\text { erst aufwändig, } \\
\text { dann gut (=2) }\end{array}$ & $\begin{array}{c}\text { einigermaßen } \\
(=3)\end{array}$ & $\begin{array}{l}\text { unübersichtlich } \\
(=4)\end{array}$ \\
\hline B2F1 & Bearbeitungsaufwand & & 16 & 12 & 6 & 1 \\
\hline & & & & $\begin{array}{c}j a \\
(=1)\end{array}$ & $\begin{array}{l}\text { nein } \\
(=2)\end{array}$ & $\begin{array}{c}\text { grundsätzlich } \\
\text { nein }(=3)\end{array}$ \\
\hline $\mathrm{B} 2 \mathrm{~F} 2$ & MyBestBets genutzt? & & & 22 & 12 & 1 \\
\hline & & $\begin{array}{l}j a \\
(=1)\end{array}$ & $\begin{array}{c}\text { hin und wieder } \\
(=2)\end{array}$ & $\begin{array}{l}\text { schadet nicht } \\
(=3)\end{array}$ & $\begin{array}{c}\text { nicht hilfreich } \\
(=4)\end{array}$ & $\begin{array}{c}\text { nie hilfreich } \\
(=5)\end{array}$ \\
\hline B2F3 & MyBestBets hilfreich? & 2 & 13 & 13 & 5 & 2 \\
\hline & & $\begin{array}{l}\begin{array}{l}\text { viele } \\
(=1)\end{array} \\
\end{array}$ & $\begin{array}{l}\text { einige } \\
(=2)\end{array}$ & $\begin{array}{l}\text { wenige } \\
(=3)\end{array}$ & $\begin{array}{c}\begin{array}{c}\text { keiner } \\
(=4)\end{array} \\
\end{array}$ & $\begin{array}{c}\text { nicht beachtet } \\
(=5)\end{array}$ \\
\hline $\mathrm{B} 2 \mathrm{~F} 4$ & Wie viele Treffer? & 2 & 9 & 19 & 2 & 3 \\
\hline & & & & & $j a(=1)$ & $\operatorname{nein}(=2)$ \\
\hline B2F5 & Ähnliche Instrumente bekannt? & & & & 3 & 32 \\
\hline B2F6 & $\begin{array}{l}\text { Wichtigkeit einzelı er Instrumente für } \\
\text { das Auffinden? }\end{array}$ & $\begin{array}{c}\text { sehr wichtig } \\
(=1)\end{array}$ & $\begin{array}{l}\text { wichtig } \\
(=2)\end{array}$ & $\begin{array}{c}\text { weniger wichtig } \\
(=3)\end{array}$ & $\begin{array}{l}\text { unwichtig } \\
(=4)\end{array}$ & $\begin{array}{l}\text { nicht benutzt } \\
\quad(=5)\end{array}$ \\
\hline $\mathrm{B} 2 \mathrm{~F} 6 \mathrm{a}$ & MyBestBets & 1 & 5 & 12 & 7 & 5 \\
\hline $\mathrm{B} 2 \mathrm{~F} 6 \mathrm{~b}$ & Suchefunktion & 18 & 7 & 2 & 1 & 6 \\
\hline $\mathrm{B} 2 \mathrm{~F} 6 \mathrm{c}$ & Ausprobieren und anklicken & 8 & 17 & 7 & 1 & 2 \\
\hline B2F6d & Externe Suche & 2 & 1 & 0 & 2 & 27 \\
\hline B2F6e & Verstehen des Seitenaufbaus & 5 & 17 & 7 & 3 & 2 \\
\hline
\end{tabular}




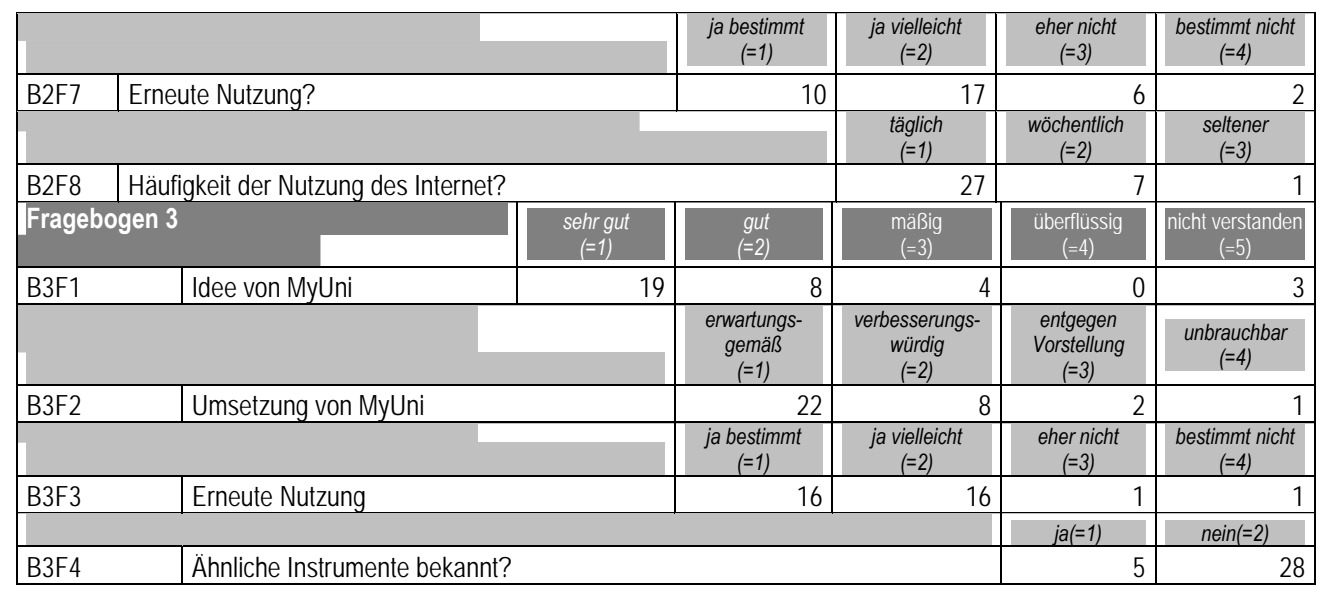

\subsection{Ergebnisse der Befragung zur Mediennutzung}

\begin{tabular}{|c|c|c|c|c|c|c|}
\hline \multicolumn{2}{|c|}{ 1. Wie häufig nutzen Sie elektro ische Medien? } & $\begin{array}{l}\text { nie } \\
(=1)\end{array}$ & $\begin{array}{l}\text { sellien } \\
(=2)\end{array}$ & $\begin{array}{l}\begin{array}{l}\text { manchmal } \\
(=3)\end{array}\end{array}$ & $\begin{array}{l}\text { häufig } \\
(=4)\end{array}$ & $\begin{array}{c}\text { regelmäßig } \\
(=5)\end{array}$ \\
\hline $1 \mathrm{a}$ & Fernsehen & 11 & 61 & 80 & 110 & 291 \\
\hline $1 b$ & Radio & 17 & 89 & 104 & 127 & 216 \\
\hline $1 \mathrm{c}$ & Audio CD & 20 & 72 & 128 & 175 & 158 \\
\hline $1 d$ & DVD & 59 & 172 & 182 & 104 & 36 \\
\hline $1 \mathrm{e}$ & Video & 130 & 269 & 108 & 32 & 14 \\
\hline 1f & Internet & 10 & 5 & 20 & 78 & 440 \\
\hline $1 \mathrm{~g}$ & Mobiltelefon & 12 & 27 & 73 & 106 & 335 \\
\hline $1 \mathrm{~h}$ & Spielekonsole & 383 & 101 & 31 & 22 & 16 \\
\hline
\end{tabular}

\begin{tabular}{|l|l|r|r|r|r|r|}
\hline \multicolumn{2}{|l|}{ 2. Welche Inhalte nutzen Sie im Rundfunk? } & \multicolumn{1}{c|}{$\begin{array}{c}\text { nie } \\
(=1)\end{array}$} & $\begin{array}{c}\text { selien } \\
(=2)\end{array}$ & $\begin{array}{c}\text { manchmal } \\
(=3)\end{array}$ & $\begin{array}{c}\text { häufig } \\
(=4)\end{array}$ & $\begin{array}{c}\text { regelmäßig } \\
(=5)\end{array}$ \\
\hline 2a & Informationssendungen & 10 & 43 & 170 & 198 & 111 \\
\hline 2b & Nachrichtensendungen & 8 & 64 & 131 & 169 & 160 \\
\hline 2c & Unterhaltungssendungen & 19 & 92 & 147 & 215 & 59 \\
\hline $2 \mathrm{~d}$ & Serien & 135 & 111 & 107 & 105 & 74 \\
\hline $2 \mathrm{e}$ & Spielfilme & 10 & 48 & 168 & 210 & 69 \\
\hline
\end{tabular}

\begin{tabular}{|l|l|r|r|}
\hline \multicolumn{2}{|l|}{ 3. Welche Printmedien lesen Sie regelmäßig? } & \multicolumn{1}{c|}{$\begin{array}{c}\text { ja } \\
(=1)\end{array}$} & \multicolumn{1}{c|}{$\begin{array}{c}\text { nein } \\
(=0)\end{array}$} \\
\hline 3a & Tageszeitung & 263 & 265 \\
\hline 3b & Programmzeitschrift & 136 & 392 \\
\hline 3c & Illustrierte & 109 & 419 \\
\hline 3d & Bücher & 388 & 140 \\
\hline 3e & Fachliteratur & 387 & 141 \\
\hline
\end{tabular}

\begin{tabular}{|c|c|c|c|c|c|c|}
\hline \multicolumn{2}{|c|}{$\begin{array}{l}\text { 2. Welche Funktionen Ihres Mol iltelefons nutzen } \\
\text { Sie? }\end{array}$} & $\begin{array}{l}\text { nie } \\
(=1)\end{array}$ & $\begin{array}{l}\text { seltien } \\
(=2)\end{array}$ & $\begin{array}{c}\operatorname{manchmal} \\
(=3)\end{array}$ & $\begin{array}{l}\text { häufig } \\
(=4)\end{array}$ & $\begin{array}{c}\begin{array}{c}\text { sehr häufig } \\
(=5)\end{array} \\
\end{array}$ \\
\hline $4 a$ & SMS senden/empfangen & 8 & 38 & 75 & 105 & 259 \\
\hline $4 \mathrm{~b}$ & MMS senden/empfangen & 155 & 64 & 33 & 5 & 7 \\
\hline $4 \mathrm{C}$ & WAP & 259 & 61 & 23 & 9 & 5 \\
\hline $4 d$ & Klingeltöne & 102 & 41 & 57 & 44 & 60 \\
\hline $4 \mathrm{e}$ & Java & 182 & 40 & 38 & 19 & 6 \\
\hline
\end{tabular}




\begin{tabular}{|c|c|c|c|c|c|c|}
\hline $4 f$ & Email & 274 & 35 & 21 & 9 & 11 \\
\hline $4 g$ & Bluetooth & 112 & 37 & 41 & 9 & 22 \\
\hline $4 \mathrm{~h}$ & Video senden/empfangen & 91 & 12 & 4 & 1 & 1 \\
\hline $4 i$ & Infrarotschnittstelle & 117 & 91 & 96 & 24 & 9 \\
\hline $4 j$ & Faxe versenden & 264 & 11 & 5 & 3 & 0 \\
\hline $4 \mathrm{k}$ & Kalender & 114 & 76 & 101 & 87 & 59 \\
\hline 41 & Mp3 empfangen/abspielen & 68 & 6 & 14 & 7 & 4 \\
\hline $4 \mathrm{~m}$ & Spiele & 212 & 134 & 69 & 21 & 13 \\
\hline
\end{tabular}

\begin{tabular}{|c|c|c|c|c|c|c|}
\hline \multicolumn{2}{|c|}{ 5. Warum nutzen Sie MMS nur $₫$ slten? } & $\begin{array}{c}\text { stimme nicht } \\
z u(=1)\end{array}$ & $(=2)$ & $(=3)$ & $(=4)$ & $\begin{array}{c}\text { stimme voll } \\
\mathrm{zu}(=5)\end{array}$ \\
\hline $5 a$ & Es ist zu teuer & 18 & 12 & 23 & 26 & 142 \\
\hline $5 b$ & Die Qualität ist zu schlecht & 37 & 32 & 60 & 48 & 44 \\
\hline $5 c$ & MMS finde ich überflüssig & 46 & 26 & 29 & 28 & 92 \\
\hline $5 d$ & Ich weiß nicht wie das funktioniert & 169 & 13 & 13 & 3 & 23 \\
\hline
\end{tabular}

\begin{tabular}{|c|c|c|c|c|c|c|}
\hline \multicolumn{2}{|c|}{ 6. Warum nutzen Sie WAP nur $s$ alten? } & \multirow{2}{*}{$\begin{array}{l}\begin{array}{c}\text { stimme nicht } \\
\text { zu (=1) }\end{array} \\
21\end{array}$} & \multirow{2}{*}{$\frac{(=2)}{13}$} & \multirow{2}{*}{$\begin{array}{l}(=3) \\
27\end{array}$} & \multirow{2}{*}{$\frac{(=4)}{56}$} & \multirow{2}{*}{$\begin{array}{c}\begin{array}{c}\text { stimme voll } \\
\mathrm{zu}(=5)\end{array} \\
198\end{array}$} \\
\hline $6 a$ & Es ist zu teuer & & & & & \\
\hline $6 \mathrm{~b}$ & $\begin{array}{l}\text { Es ist zu umständlich und unübersichtlich } \\
\text { zu bedienen }\end{array}$ & 31 & 20 & 59 & 83 & 122 \\
\hline $6 c$ & Die Inhalte sind uninteressant & 60 & 63 & 97 & 49 & 46 \\
\hline $6 \mathrm{~d}$ & Ich weiß nicht wie das funktioniert & 205 & 27 & 28 & 19 & 36 \\
\hline
\end{tabular}

\begin{tabular}{|l|l|r|r|r|r|r|}
\hline $\begin{array}{l}\text { 7. Würden Sie einen Film / eine jerie auf Ihrem } \\
\text { Mobiltelefon ansehen? }\end{array}$ & $\begin{array}{c}\text { stimme nicht } \\
\text { zu (=1) }\end{array}$ & (=2) & (=3) & stimme voll \\
zu (=5)
\end{tabular}

\begin{tabular}{|c|c|c|c|c|c|c|}
\hline \multicolumn{2}{|c|}{$\begin{array}{l}\text { 7. Würden Sie Zeitungsnachricl ten auf Ihrem } \\
\text { Mobiltelefon lesen? }\end{array}$} & \multirow{2}{*}{$\begin{array}{r}\begin{array}{c}\text { stimme nicht } \\
z u(=1)\end{array} \\
274\end{array}$} & \multirow{2}{*}{ (=2) } & \multirow{2}{*}{ (=3) } & \multirow{2}{*}{33} & \multirow{2}{*}{$\begin{array}{l}\begin{array}{l}\text { stimme voll } \\
\mathrm{zu}(=5)\end{array} \\
15\end{array}$} \\
\hline $7 a$ & Das würde ich gerne nutzen & & & & & \\
\hline $7 \mathrm{~b}$ & $\begin{array}{l}\text { Ich würde es nur nutzen, wenn es kosten- } \\
\text { los wäre }\end{array}$ & 106 & 63 & 69 & 88 & 119 \\
\hline $7 c$ & Das kann ich mir absolut nicht vorstellen & 195 & 62 & 69 & 39 & 84 \\
\hline
\end{tabular}

\subsection{Ergebnisse der Erhebung zur Akzeptanz mobiler Dienste}

\begin{tabular}{|l|l|c|c|}
\hline 1. Welche der folgenden Geräte benutzen Sie? & ja $(=1)$ & nein $(=2)$ \\
\hline G1F2 & PC & 6251 & 927 \\
\hline G1F3 & Notebook / Laptop & 3852 & 3326 \\
\hline G1F4 & Handy & 5696 & 1482 \\
\hline G1F5 & PDA ohne Handyfunktion & 957 & 6221 \\
\hline G1F6 & Smartphone & 321 & 6857 \\
\hline G1F7 & keines davon & 17 & 7161 \\
\hline
\end{tabular}

\begin{tabular}{|l|l|c|c|}
\hline 2. Welche der folgenden Printerzeugnisse lesen Sie regelmäßig? & ja(=1) & nein(=2) \\
\hline G2F1 & Tageszeitungen (z.B. Welt, Tagesspiegel, Bild) & 5597 & 1568 \\
\hline G2F2 & Zeitschriften/Magazine zum Zeitgeschehen (z.B. Spiegel, Bunte, Focus) & 3859 & 3306 \\
\hline G2F3 & Programmzeitschriften (z.B. Hörzu, TV-Spielfilm, Fernsehwoche) & 2703 & 4462 \\
\hline G2F4 & Frauenzeitschriften (z.B. Bild der Frau, Amica, Brigitte Young Miss, Für Sie) & 794 & 6371 \\
\hline
\end{tabular}




\begin{tabular}{|l|l|c|c|}
\hline G2F5 & Familienzeitschriften (z.B. Eltern, Leben \& Erziehen, Familie\&Co.) & 288 & 6877 \\
\hline G2F6 & Musik- und Jugendzeitschriften (z.B. Bravo, Mädchen, Popcorn) & 244 & 6921 \\
\hline G2F7 & Zeitschriften für Wohnen und Leben (z.B. Schöner Wohnen, Das Haus) & 862 & 6303 \\
\hline G2F8 & Erotik-Zeitschriften (z.B. Playboy, Praline, Coupé) & 152 & 7013 \\
\hline G2F9 & Lifestyle/Stadtmagazine (z.B. Fit For Fun, Max, PRINZ) & 667 & 6498 \\
\hline G2F10 & Motorpresse (z.B. Auto Bild, auto motor und sport, MOTORRAD) & 1209 & 5956 \\
\hline G2F11 & Sportzeitschriften (z.B. Kicker, Sport Bild, BRAVO Sport) & 649 & 6516 \\
\hline G2F12 & Wissenschaft/Reise/Kultur (z.B. GEO, MERIAN, P.M Magazin) & 1598 & 5567 \\
\hline G2F13 & EDV-Zeitschriften (z.B. TOMORROW, PC-Welt, Computer Bild) & 2014 & 5151 \\
\hline
\end{tabular}

\begin{tabular}{|c|c|c|c|c|c|}
\hline \multicolumn{2}{|c|}{ 3. Nutzung kostenpflichtiger SMS-Dienst : } & \multirow{2}{*}{ häufig (1) } & \multirow{2}{*}{$\frac{\text { manchmal (2) }}{323}$} & \multirow{2}{*}{$\frac{\operatorname{selien}(3)}{776}$} & \multirow{2}{*}{$\frac{n i e(4)}{5915}$} \\
\hline G3F1 & Nutzen Sie kostenpflichtige SMS-Dienste? & & & & \\
\hline \multicolumn{4}{|c|}{ 3.a Nachrichten (z.B. RTL aktuell) } & $j a(=1)$ & nein $=2)$ \\
\hline G3aF1 & \multicolumn{3}{|l|}{ Handy } & 320 & 6845 \\
\hline G3aF2 & \multicolumn{3}{|l|}{ Smartphone } & 36 & 7129 \\
\hline \multicolumn{4}{|c|}{ 3.b Finanzen (z.B. Börsenkurse) } & $j a(=1)$ & $\operatorname{nein}(=2)$ \\
\hline G3bF1 & \multicolumn{3}{|l|}{ Handy } & 162 & 7003 \\
\hline G3bF2 & \multicolumn{3}{|l|}{ Smartphone } & 17 & 7148 \\
\hline \multicolumn{4}{|c|}{ 3.c Sport (z.B. aktuelle Fußballergebnisse) } & $j a(=1)$ & nein( $=2)$ \\
\hline G3cF1 & \multicolumn{3}{|l|}{ Handy } & 408 & 6757 \\
\hline G3cF2 & \multicolumn{3}{|l|}{ Smartphone } & 30 & 7135 \\
\hline \multicolumn{4}{|c|}{ 3.d Entertainment (z.B. Spiele, Klingeltöne, Logos) } & $j a(=1)$ & nein $(=2)$ \\
\hline G3dF1 & \multicolumn{3}{|l|}{ Handy } & 268 & 6897 \\
\hline G3dF2 & \multicolumn{3}{|l|}{ Smartphone } & 17 & 7148 \\
\hline \multicolumn{4}{|c|}{ 3.e Horoskop } & $j a=1)$ & $\operatorname{nein}(=2)$ \\
\hline G3eF1 & \multicolumn{3}{|l|}{ Handy } & 94 & 7071 \\
\hline G3eF2 & \multicolumn{3}{|l|}{ Smartphone } & 2 & 7163 \\
\hline \multicolumn{4}{|c|}{ 3.f Wetter } & $j a(=1)$ & nein( $=2)$ \\
\hline G3fF1 & \multicolumn{3}{|l|}{ Handy } & 225 & 6940 \\
\hline G3fF2 & \multicolumn{3}{|l|}{ Smartphone } & 24 & 7141 \\
\hline \multicolumn{4}{|c|}{ 3.g Dating (z.B. Friend-Finder) } & $j a=1)$ & nein( $=2)$ \\
\hline G3gF1 & \multicolumn{3}{|l|}{ Handy } & 43 & 7122 \\
\hline G3gF2 & \multicolumn{3}{|l|}{ Smartphone } & 4 & 7161 \\
\hline \multicolumn{4}{|c|}{ 3.h Kleinanzeigen (z.B. Immobilien, Automobile) } & $j a(=1)$ & $\operatorname{nein}(=2)$ \\
\hline G3hF1 & \multicolumn{3}{|l|}{ Handy } & 58 & 7107 \\
\hline G3hF2 & \multicolumn{3}{|l|}{ Smartphone } & 8 & 7157 \\
\hline \multicolumn{4}{|c|}{$\begin{array}{l}\text { 3.i Unter welchen Voraussetzungen würden Sie innerhalb der nächsten 1-‘ Jahre das Ange- } \\
\text { bot kostenpflichtiger SMS nutzen? }\end{array}$} & $j a(=1)$ & nein $(=2)$ \\
\hline G3jF1 & günstigere Preise & & & 3823 & 3342 \\
\hline G3jF2 & höhere Qualität des Inhalts & & & 2084 & 5081 \\
\hline G3jF3 & gebotener Mehrwert & & & 1871 & 5294 \\
\hline G3jF4 & bessere Bedienbarkeit der Endgeräte & & & 823 & 6342 \\
\hline G3jF5 & bessere Darstellung des Inhalts (Displayqu & & & 1112 & 6053 \\
\hline G3jF6 & höhere verfügbare Geschwindigkeit der Da & & & 112 & 6045 \\
\hline
\end{tabular}

\begin{tabular}{|c|c|c|c|c|c|}
\hline \multicolumn{2}{|c|}{ 4. Nutzung kostenpflichtiger MMS-Diens e } & häufia $(=1)$ & manchmal $(=2)$ & seltien $(=3)$ & nie $(=4)$ \\
\hline G4F1 & Nutzen Sie kostenpflichtige MMS-Dienste? & 22 & 161 & 393 & 6527 \\
\hline \multicolumn{4}{|c|}{ 4.a Nachrichtendienste - Politik } & $j a=1)$ & nein(=2) \\
\hline G4aF1 & \multicolumn{3}{|l|}{ Handy } & 156 & 7009 \\
\hline G4aF2 & \multicolumn{3}{|l|}{ Smartphone } & 24 & 7141 \\
\hline \multicolumn{4}{|c|}{ 4.b Nachrichtendienste - Wirtschaft } & $j a=1)$ & nein(=2) \\
\hline G4bF1 & \multicolumn{3}{|l|}{ Handy } & 117 & 7048 \\
\hline G4bF2 & \multicolumn{3}{|l|}{ Smartphone } & 30 & 7135 \\
\hline
\end{tabular}




\begin{tabular}{|c|c|c|c|}
\hline \multicolumn{2}{|c|}{ 4.c Nachrichtendienste - Kultur } & \multirow{2}{*}{$\begin{array}{l}j a(=1) \\
42\end{array}$} & \multirow{2}{*}{$\begin{array}{c}\operatorname{nein}(=2) \\
7123\end{array}$} \\
\hline G4cF1 & Handy & & \\
\hline G4CF2 & Smartphone & 10 & 7155 \\
\hline \multicolumn{2}{|c|}{ 4.d Nachrichtendienste - Sport } & $j a=1)$ & nein(=2) \\
\hline G4dF1 & Handy & 163 & 7002 \\
\hline G4dF1 & Smartphone & 26 & 7139 \\
\hline \multicolumn{2}{|c|}{ 4.e Dating (z.B. Friend Finder) } & $j a(=1)$ & nein $(=2)$ \\
\hline G4eF1 & Handy & 37 & 7128 \\
\hline G4eF2 & Smartphone & 1 & 7164 \\
\hline \multicolumn{2}{|c|}{ 4.f Erotik } & $j a(=1)$ & $\operatorname{nein}(=2)$ \\
\hline G4fF1 & Handy & 24 & 7141 \\
\hline G4fF2 & Smartphone & 2 & 7163 \\
\hline \multicolumn{2}{|c|}{$\begin{array}{l}\text { 4.g Unter welchen Voraussetzungen würden Sie innerhalb der nächsten } 12 \text { Jahre das An- } \\
\text { gebot kostenpflichtiger MMS nutzen? }\end{array}$} & $j a=1)$ & $\operatorname{nein}(=2)$ \\
\hline G4gF1 & günstigere Preise & 4343 & 2822 \\
\hline G4gF2 & höhere Qualität des Inhalts & 2105 & 5060 \\
\hline G4gF3 & gebotener Mehrwert & 2014 & 5151 \\
\hline G4gF4 & bessere Bedienbarkeit der Endgeräte & 881 & 6284 \\
\hline G4gF5 & bessere Darstellung des Inhalts (Displayqualität) & 1268 & 5897 \\
\hline G4gF6 & höhere verfügbare Geschwindigkeit der Datenübertragung & 1356 & 5809 \\
\hline
\end{tabular}

\begin{tabular}{|c|c|c|c|c|c|}
\hline \multicolumn{2}{|c|}{ 5. Nutzung kostenpflichtiger standortbezogen :r Dienste } & \multirow{2}{*}{$\frac{24}{24}$} & \multirow{2}{*}{$\frac{\text { manchmal (2) }}{189}$} & \multirow{2}{*}{$\frac{\text { selien (3) }}{436}$} & \multirow{2}{*}{ nie (4) } \\
\hline G5F1 & Nutzen Sie kostenpflichtige standortbezogene Dienste? & & & & \\
\hline \multicolumn{4}{|c|}{ 5.a Auskunftsdienste (z.B. Shop Finder) } & $j a=1)$ & $\operatorname{nein}(=2)$ \\
\hline G5aF1 & \multicolumn{3}{|l|}{ Handy } & 179 & 6986 \\
\hline G5aF2 & \multicolumn{3}{|l|}{ Smartphone } & 16 & 7149 \\
\hline \multicolumn{4}{|c|}{ 5.b Community, Verabredung und Unterhaltung (z.B. Friendfinder) } & $j a=1)$ & $\operatorname{nein}(=2)$ \\
\hline G5bF1 & \multicolumn{3}{|l|}{ Handy } & 19 & 7146 \\
\hline G5bF2 & \multicolumn{3}{|l|}{ Smartphone } & 2 & 7163 \\
\hline \multicolumn{4}{|c|}{ 5.c Navigation } & $j a(=1)$ & nein $=2$ ) \\
\hline G5CF1 & \multicolumn{3}{|l|}{ Handy } & 184 & 6981 \\
\hline G5cF2 & \multicolumn{3}{|l|}{ Smartphone } & 28 & 7137 \\
\hline \multicolumn{4}{|c|}{ 5.d Sicherheit und Notfall (z.B. Notruf) } & $j a(=1)$ & nein $(=2)$ \\
\hline G5dF1 & \multicolumn{3}{|l|}{ Handy } & 211 & 6954 \\
\hline G5dF2 & \multicolumn{3}{|l|}{ Smartphone } & 10 & 7155 \\
\hline \multicolumn{4}{|c|}{ 5.e Bezahlfunktion vor Ort (z.B. öffentlicher Nahverkehr) } & $j a=1)$ & $\operatorname{nein}(=2)$ \\
\hline G5eF1 & \multicolumn{3}{|l|}{ Handy } & 87 & 7078 \\
\hline G5eF2 & \multicolumn{3}{|c|}{ Smartphone } & 5 & 7160 \\
\hline \multicolumn{4}{|c|}{$\begin{array}{l}\text { 5.f Unter welchen Voraussetzungen würden Sie innerhalb der nächsten } 12 \text { Jahre das An- } \\
\text { gebot kostenpflichtiger LBS-Dienste nutzen? }\end{array}$} & $j a=1)$ & $\operatorname{nein}(=2)$ \\
\hline G5fF1 & \multicolumn{3}{|c|}{ günstigere Preise } & 3672 & 3493 \\
\hline G5fF2 & \multicolumn{3}{|l|}{ höhere Qualität des Inhalts } & 1631 & 5534 \\
\hline G5fF3 & \multicolumn{3}{|l|}{ gebotener Mehrwert } & 1933 & 5232 \\
\hline G5fF4 & \multicolumn{3}{|l|}{ bessere Bedienbarkeit der Endgeräte } & 773 & 6392 \\
\hline G5fF5 & \multicolumn{3}{|l|}{ bessere Darstellung des Inhalts (Displayqualitätqualität) } & 753 & 6412 \\
\hline G5fF6 & \multicolumn{3}{|l|}{ höhere verfügbare Geschwindigkeit der Datenübertragung } & 1058 & 6107 \\
\hline
\end{tabular}

\begin{tabular}{|l|l|c|c|c|c|}
\hline 6. Nutzung kostenpflichtiger Portaldiens e & häufig $(=1)$ & manchmal $(=2)$ & selien $(=3)$ & nie $(=4)$ \\
\hline G6F1 & Nutzen Sie kostenpflichtige Portaldienste? & 71 & 390 & 904 & 5735 \\
\hline 6.a Nachrichten, Wetter & & $j a(=1)$ & nein(=2) \\
\hline G6aF1 & Handy & & 500 & 6665 \\
\hline G6aF2 & Smartphone & 67 & 7098 \\
\hline 6.b Sport & & $j a(=1)$ & nein(=2) \\
\hline
\end{tabular}




\begin{tabular}{|l|l|c|c|}
\hline G6bF1 & Handy & 433 & 6732 \\
\hline G6bF2 & Smartphone & 49 & 7116 \\
\hline
\end{tabular}

\begin{tabular}{|c|c|c|c|}
\hline \multicolumn{2}{|c|}{ 6.c Freizeit (Ausgehtipps, Kinoprogramm, Gastronomie) } & \multirow{2}{*}{$\begin{array}{c}j a(=1) \\
130\end{array}$} & \multirow{2}{*}{$\begin{array}{c}\text { nein( }=2) \\
7035\end{array}$} \\
\hline G6cF1 & Handy & & \\
\hline G6cF2 & Smartphone & 10 & 7155 \\
\hline \multicolumn{2}{|c|}{ 6.d Klingeltöne und Bilder } & $j a(=1)$ & $\operatorname{nein}(=2)$ \\
\hline G6dF1 & Handy & 142 & 7023 \\
\hline G6dF2 & Smartphone & 11 & 7154 \\
\hline 6.e Finanzen & & $j a(=1)$ & $\operatorname{nein}(=2)$ \\
\hline G6eF1 & Handy & 123 & 7042 \\
\hline G6eF2 & Smartphone & 28 & 7137 \\
\hline \multicolumn{2}{|c|}{ 6.f Nachschlagen } & $j a(=1)$ & $\operatorname{nein}(=2)$ \\
\hline G6fF1 & Handy & 59 & 7106 \\
\hline G6fF2 & Smartphone & 20 & 7145 \\
\hline 6.g E-Mail & & $j a(=1)$ & $\operatorname{nein}(=2)$ \\
\hline G6gF1 & Handy & 379 & 6786 \\
\hline G6gF2 & Smartphone & 84 & 7081 \\
\hline 6.h Erotik & & $j a(=1)$ & nein $(=2)$ \\
\hline G6hF1 & Handy & 28 & 7137 \\
\hline G6hF2 & Smartphone & 6 & 7159 \\
\hline \multicolumn{2}{|c|}{ 6.i Reisen \& Verkehr } & $j a(=1)$ & nein $(=2)$ \\
\hline G6iF1 & Handy & 173 & 6992 \\
\hline G6iF2 & Smartphone & 26 & 7139 \\
\hline \multicolumn{2}{|l|}{ 6.j Einkaufen } & $j a(=1)$ & $\operatorname{nein}(=2)$ \\
\hline G6jF1 & Handy & 55 & 7110 \\
\hline G6jF2 & Smartphone & 11 & 7154 \\
\hline \multicolumn{4}{|c|}{$\begin{array}{l}\text { 6.k Unter welchen Voraussetzungen würden Sie innerhalb der nächsten 1. 2 Jahre das Ange- } \\
\text { bot mobiler Portale nutzen? }\end{array}$} \\
\hline G6kF1 & günstigere Preise & 3309 & 3856 \\
\hline G6kF2 & höhere Qualität des Inhalts & 1518 & 5647 \\
\hline G6kF3 & gebotener Mehrwert & 1716 & 5449 \\
\hline G6kF4 & bessere Bedienbarkeit der Endgeräte & 597 & 6568 \\
\hline G6kF5 & bessere Darstellung des Inhalts (Displayqualität) & 677 & 6488 \\
\hline G6kF6 & höhere verfügbare Geschwindigkeit der Datenübertragung & 851 & 6314 \\
\hline
\end{tabular}

\begin{tabular}{|c|c|c|c|c|c|}
\hline \multicolumn{2}{|c|}{ 7.Nutzung kostenpflichtiger Musikdienst : } & häufig (=1) & manchmal (=2) & seltien $(=3)$ & nie $(=4)$ \\
\hline G7F1 & Nutzen Sie kostenpflichtige Musikdienste? & 216 & 929 & 1613 & 4389 \\
\hline \multicolumn{4}{|c|}{ 7.a Nutzungsgrade je Endgerätetyp } & $j a(=1)$ & nein(=2) \\
\hline G7aF1 & \multicolumn{3}{|l|}{ Nutzung per PC } & 2073 & 5092 \\
\hline G7aF2 & \multicolumn{3}{|l|}{ Nutzung per Notebook } & 919 & 6246 \\
\hline G7aF3 & \multicolumn{3}{|l|}{ Nutzung per Handy } & 233 & 6932 \\
\hline G7aF4 & \multicolumn{3}{|l|}{ Nutzung per Smartphone } & 23 & 7142 \\
\hline \multicolumn{4}{|c|}{$\begin{array}{l}\text { 7.b Unter welchen Voraussetzungen würden Sie innerhalb der nächsten 1. 2 Jahre kosten- } \\
\text { pflichtige Musik-Dienste nutzen? }\end{array}$} & $j a(=1)$ & nein $(=2)$ \\
\hline G7bF1 & \multicolumn{3}{|c|}{ günstigere Preise } & 2907 & 4258 \\
\hline G7bF2 & \multicolumn{3}{|l|}{ höhere Qualität des Inhalts } & 951 & 6214 \\
\hline G7bF3 & \multicolumn{3}{|l|}{ gebotener Mehrwert } & 753 & 6412 \\
\hline G7bF4 & \multicolumn{3}{|l|}{ bessere Bedienbarkeit der Endgeräte } & 437 & 6728 \\
\hline G7bF5 & \multicolumn{3}{|c|}{ bessere Darstellung des Inhalts (Displayqualität) } & 207 & 6958 \\
\hline G7bF6 & \multicolumn{3}{|c|}{ höhere verfügbare Geschwindigkeit der Datenübertragung } & 946 & 6219 \\
\hline
\end{tabular}




\begin{tabular}{|c|c|c|c|c|c|}
\hline \multicolumn{2}{|c|}{ 8. Nutzung kostenpflichtiger Film-\& Video-Ang؛ bote } & \multirow{2}{*}{$\begin{array}{c}\text { häufig } \\
(=1) \\
36\end{array}$} & $\begin{array}{c}\text { manchmal } \\
(=2)\end{array}$ & \multirow{2}{*}{$\begin{array}{c}\begin{array}{c}\text { sellien } \\
(=3) \\
558\end{array} \\
\end{array}$} & \multirow{2}{*}{$\begin{array}{l}\frac{n i e}{(=4)} \\
6319\end{array}$} \\
\hline G8F1 & Nutzen Sie kostenpflichtige Film- und Videoangebote? & & 225 & & \\
\hline \multicolumn{6}{|c|}{ 8.a Kurze Videoclips (z.B. Musik, News, Sport) } \\
\hline G8aF1 & \multicolumn{3}{|l|}{ Handy } & 36 & 7129 \\
\hline G8aF2 & \multicolumn{3}{|l|}{ Smartphone } & 11 & 7154 \\
\hline \multicolumn{6}{|c|}{ 8.b Video on Demand (z.B. Magazine, Reiseführer) } \\
\hline G8bF1 & \multicolumn{3}{|l|}{ Handy } & 5 & 7160 \\
\hline G8bF2 & \multicolumn{3}{|l|}{ Smartphone } & 2 & 7160 \\
\hline \multicolumn{6}{|c|}{ 8.c Video on Demand (z.B. Spielfilme) } \\
\hline G8cF1 & \multicolumn{3}{|l|}{ Handy } & 5 & 7160 \\
\hline G8cF2 & \multicolumn{3}{|l|}{ Smartphone } & 2 & 7163 \\
\hline \multicolumn{6}{|c|}{ 8.d mobiles Fernsehen über UMTS (z.B. Vodafone MobileTV) } \\
\hline G8dF1 & \multicolumn{3}{|l|}{ Handy } & 18 & 7147 \\
\hline G8dF2 & \multicolumn{3}{|l|}{ Smartphone } & 8 & 7157 \\
\hline \multicolumn{4}{|c|}{$\begin{array}{l}\text { 8.e Unter welchen Voraussetzungen würden Sie innerhalb der nächsten } 12 \text { Jahre kosten- } \\
\text { pflichtige Film-, Video-Angebote nutzen? }\end{array}$} & $j a(=1)$ & nein(=2) \\
\hline G8eF1 & \multicolumn{3}{|c|}{ günstigere Preise } & 4091 & 3074 \\
\hline G8eF2 & \multicolumn{3}{|l|}{ höhere Qualität des Inhalts } & 1627 & 5538 \\
\hline G8eF3 & \multicolumn{3}{|l|}{ gebotener Mehrwert } & 1171 & 5994 \\
\hline G8eF4 & \multicolumn{3}{|l|}{ bessere Bedienbarkeit der Endgeräte } & 553 & 6612 \\
\hline G8eF5 & \multicolumn{3}{|l|}{ bessere Darstellung des Inhalts (Displayqualität) } & 755 & 6410 \\
\hline G8eF6 & \multicolumn{3}{|l|}{ höhere verfügbare Geschwindigkeit der Datenübertragung } & 1734 & 5431 \\
\hline
\end{tabular}

\begin{tabular}{|l|l|r|r|r|r|}
\hline 9. Motive der Nutzung von SMS-Diensten & $\begin{array}{c}\text { keine } \\
\text { Angabe }(=0)\end{array}$ & $\begin{array}{c}\text { stimme nicht } \\
\text { zu }(=1)\end{array}$ & $\begin{array}{c}\text { stimme teil- } \\
\text { weise zu }(=2)\end{array}$ & $\begin{array}{c}\text { stimme zu } \\
(=3)\end{array}$ \\
\hline G9F1 & Neugier & 6873 & 36 & 111 & 158 \\
\hline G9F2 & Mobilität & 6875 & 23 & 72 & 208 \\
\hline G9F3 & Gebrauchswert im Alltag & 6897 & 56 & 119 & 106 \\
\hline G9F4 & Information & 6895 & 63 & 92 & 128 \\
\hline G9F5 & Faszination & 6908 & 37 & 77 & 156 \\
\hline G9F6 & Interaktivität & 6939 & 93 & 86 & 60 \\
\hline G9F7 & Unterhaltung & 6910 & 88 & 87 & 93 \\
\hline G9F8 & Gewohnheit/Akzeptanz & 6925 & 55 & 89 & 109 \\
\hline
\end{tabular}

\begin{tabular}{|l|l|r|r|r|r|}
\hline \multicolumn{1}{|l|}{ 10. Motive der Nutzung von MMS-Diensten } & $\begin{array}{c}\text { keine } \\
\text { Angabe }(=0)\end{array}$ & $\begin{array}{c}\text { stimme nicht } \\
\text { zu }(=1)\end{array}$ & $\begin{array}{c}\text { stimme teil- } \\
\text { weise zu }(=2)\end{array}$ & $\begin{array}{c}\text { stimme zu } \\
(=3)\end{array}$ \\
\hline G10F1 & Neugier & 7071 & 14 & 35 & 58 \\
\hline G10F2 & Mobiliät & 7075 & 11 & 25 & 67 \\
\hline G10F3 & Gebrauchswert im Alltag & 7075 & 11 & 25 & 67 \\
\hline G10F4 & Information & 7087 & 22 & 29 & 40 \\
\hline G10F5 & Faszination & 7082 & 14 & 35 & 47 \\
\hline G10F6 & Interaktivität & 7095 & 35 & 25 & 23 \\
\hline G10F7 & Unterhaltung & 7087 & 25 & 34 & 32 \\
\hline G10F8 & Gewohnheit/Akzeptanz & 7094 & 20 & 38 & 26 \\
\hline
\end{tabular}




\begin{tabular}{|l|l|r|r|r|r|}
\hline \multicolumn{2}{|l|}{ 11. Motive der Nutzung von ortsbezogenen Di nsten } & $\begin{array}{c}\text { keine } \\
\text { Angabe }(=0)\end{array}$ & $\begin{array}{c}\text { stimme nicht } \\
\text { zu }(=1)\end{array}$ & $\begin{array}{c}\text { stimme teil- } \\
\text { weise zu }(=2)\end{array}$ & $\begin{array}{c}\text { stimme zu } \\
(=3)\end{array}$ \\
\hline G11F1 & Neugier & 7065 & 20 & 46 & 47 \\
\hline G11F2 & Mobilität & 7073 & 12 & 35 & 58 \\
\hline G11F3 & Gebrauchswert im Alltag & 7071 & 14 & 47 & 46 \\
\hline G11F4 & Information & 7063 & 15 & 43 & 57 \\
\hline G11F5 & Faszination & 7068 & 15 & 47 & 48 \\
\hline G11F6 & Interaktivität & 7080 & 40 & 44 & 14 \\
\hline G11F7 & Unterhaltung & 7082 & 38 & 39 & 19 \\
\hline G11F8 & Gewohnheit/Akzeptanz & 7085 & 32 & 39 & 22 \\
\hline
\end{tabular}

\begin{tabular}{|l|l|r|r|r|r|}
\hline \multicolumn{1}{|l|}{ 12. Motive der Nutzung mobiler Portale } & $\begin{array}{c}\text { keine } \\
\text { Angabe }(=0)\end{array}$ & $\begin{array}{c}\text { stimme nicht } \\
\text { zu }(=1)\end{array}$ & $\begin{array}{c}\text { stimme teil- } \\
\text { weise zu }(=2)\end{array}$ & $\begin{array}{c}\text { stimme zu } \\
(=3)\end{array}$ \\
\hline G12F1 & Neugier & 6851 & 35 & 126 & 165 \\
\hline G12F2 & Mobilität & 6854 & 33 & 77 & 213 \\
\hline G12F3 & Gebrauchswert im Alltag & 6871 & 77 & 118 & 111 \\
\hline G12F4 & Information & 6864 & 57 & 112 & 144 \\
\hline G12F5 & Faszination & 6859 & 58 & 88 & 172 \\
\hline G12F6 & Interaktivität & 6901 & 110 & 110 & 56 \\
\hline G12F7 & Unterhaltung & 6881 & 91 & 108 & 97 \\
\hline G12F8 & Gewohnheit/Akzeptanz & 6890 & 81 & 138 & 68 \\
\hline
\end{tabular}

\begin{tabular}{|l|l|r|r|r|r|}
\hline \multicolumn{1}{|l|}{ 13. Motive der Nutzung von Musikdiensten } & $\begin{array}{c}\text { keine } \\
\text { Angabe }(=0)\end{array}$ & $\begin{array}{c}\text { stimme nicht } \\
\text { zu }(=1)\end{array}$ & $\begin{array}{c}\text { stimme teil- } \\
\text { weise zu }(=2)\end{array}$ & $\begin{array}{c}\text { stimme zu } \\
(=3)\end{array}$ \\
\hline G13F1 & Neugier & 6288 & 120 & 356 & 414 \\
\hline G13F2 & Mobiliät & 6338 & 108 & 258 & 474 \\
\hline G13F3 & Gebrauchswert im Alltag & 6469 & 304 & 239 & 166 \\
\hline G13F4 & Information & 6464 & 293 & 225 & 196 \\
\hline G13F5 & Faszination & 6404 & 135 & 253 & 386 \\
\hline G13F6 & Interaktivität & 6506 & 332 & 241 & 99 \\
\hline G13F7 & Unterhaltung & 6366 & 188 & 247 & 377 \\
\hline G13F8 & Gewohnheit/Akzeptanz & 6468 & 195 & 327 & 188 \\
\hline
\end{tabular}

\begin{tabular}{|l|l|r|r|r|r|r|}
\hline 14. Motive der Nutzung von mobilen Videos & $\begin{array}{c}\text { keine } \\
\text { Angabe }(=0)\end{array}$ & $\begin{array}{c}\text { stimme nicht } \\
\text { zu }(=1)\end{array}$ & $\begin{array}{c}\text { stimme teil- } \\
\text { weise zu }(=2)\end{array}$ & $\begin{array}{c}\text { stimme zu } \\
(=3)\end{array}$ \\
\hline G8F562 & Neugier & 7005 & 40 & 44 & 89 \\
\hline G8F565 & Mobiliät & 7020 & 38 & 49 & 71 \\
\hline G8F568 & Gebrauchswert im Alltag & 7031 & 59 & 50 & 38 \\
\hline G8F569 & Information & 7032 & 49 & 44 & 53 \\
\hline G8F570 & Faszination & 7020 & 28 & 45 & 85 \\
\hline G8F574 & Interaktivität & 7041 & 61 & 52 & 24 \\
\hline G8F576 & Unterhaltung & 7021 & 34 & 46 & 77 \\
\hline G8F579 & Gewohnheit/Akzeptanz & 7038 & 37 & 66 & 37 \\
\hline
\end{tabular}




\section{Quellenverzeichnis}

A.T. Kearney (2003): Mobinet 5: The New Mobile Mindset. Elektronisch veröffentlicht: http://www.atkearney.com/shared_res/pdf/Mobinet_Monograph_S.pdf; abgerufen am 2005-04-30.

ACM MobiCom (2003): Ninth Annual International Conference on Mobile Computing and Networking. Elektronisch veröffentlicht: http://www.sigmobile.org/mobicom/2003/; abgerufen am 2005-04-30.

Adams, William James; Yellen,Janet (1976): Commodity Bundling and the burden of monopoly. In: The Quaterly Journal of Economics, Bd. 90, Nr. 3 (Aug. 1976), S. 476-498.

Agrawal, Rakesh; Imielinski, Tomasz; Swami, Arun (1993): Mining Association Rules between Sets of Items in Large Databases. In: SIGMOD Record 21 (1993) Nr. 5, S. 207-216.

Amberg, Michael; Wehmann, Jens (2001): Situationsabhängige Dienste-Grundlagen ihrer Entwicklung. Elektronisch veröffentlicht: http://www.wi3.uni-erlangen.de/forschung/situation/SAD.pdf; abgerufen am 2004-12-01.

Amit, Raffid; Zott, Christoph (2000): Value Drivers of e-Commerce Business Models. In: Hitt, Michael; Amit, Raphael; Lucier, Charles; Nixon, Robert (Hrsg.): Creating Value: Winners in the New Business Environment. Blackwell Publishers, Oxford 2000, S. 15-47.

AMOC (2004): 3rd Asian International Mobile Computing Conference. Elektronisch veröffentlicht: http://amoc2004.cpe.ku.ac.th/; abgerufen am 2005-04-30.

Anderson, Corin; Domingos, Pedro; Weld, Daniel (2001): Personalizing Web Sites for Mobile Users. Elektronisch veröffentlicht: http://www.cs.washington.edu/ai/proteus/www10.pdf, 2001; abgerufen am 2004-05-31.

Anderson, David; Pine, Joseph (1997): Agile product development for mass customization. Irwing publishing, Chicago 1997.

Anding, Markus; Hess, Thomas (2000): Content Syndication - Basic Concept and Case Studies. Arbeitspapiere der Abt. Wirtschaftsinformatik II, Universität Göttingen, Nr. 11, Göttingen, 2001.

Anding, Markus (2004): Online Content Syndication. Deutscher Universitätsverlag, Wiesbaden 2004.

Ansoff, Igor (1979): Strategic Management. The Macmillan Press; London 1979.

Anstadt, Ulrich (1994): Determinanten der individuellen Akzeptanz bei Einführung neuer Technologien. Europäischer Verlag der Wissenschaften, Frankfurt a. M. 1994.

Aretz, Hans-Hürgen (2000): Individualisierung und Modernisierung. In: Kron, Thomas (Hrsg.): Individualisierung und soziologische Theorie. Leske + Budrich, Opladen 2000.

Arrow, Kenneth (1962): Economic Welfare and the Allocation of Resources for Invention in the Rate and Direction of Inventive Activity. Princeton University Press 1962, S. 609-626.

Asthana, Abhaya; Cravatts, Mark; Krzyzanowski. Paul (1994): An indoor wireless system for personalized shopping assistance. In: Proceedings of IEEE Workshop on Mobile Computing Systems and Applications, Santa Cruz 1994, S. 69-74. 
Backhaus, Klaus; Erichson, Bernd; Plinke, Wulff; Weiber, Rolf (2003): Multivariate Analysemethoden. Springer Verlag, Berlin 2003.

Bacon, Jean; Bates, John; Halls, David (1997): Location-orientied mutlimedia. In: IEEE Personal Communications, Bd. 4, Nr. 5.

Bain, Joe (1968): Industrial Organization. New York 1968.

Bakker, Dee; McMichael Gilster, Diane (2002): Bluetooth End to End. John Wiley and Sons, 2002.

Barney, Jay (2002): Gaining and sustaining competitive advantage. Prentice Hall, Upper Saddle River 2002.

Bartussek, Jörg (2001): Vom Newspaper zum Newsfilter. In: Vizjak, Andrej; Ringlstetter, Max (Hrsg.): Medienmanagement: Content gewinnbringend nutzen. Gabler, Wiesbaden 2001, S. 63-73.

Bea, Franz Xaver; Haas, Jürgen (1995): Strategisches Management. Gustav Fischer Verlag, Stuttgart 1995.

Bea, Franz; Dichtl, Erwin; Schweitzer, Marcel (2000): Allgemeine Betriebswirtschaftslehre, Band 1: Grundfragen. Fink, München 2000.

Beck, Hanno (2002): Medienökonomie. Springer, Frankfurt 2002.

Beck, Klaus (1999): Der Computer als Medium der Medienintegration: Ergebnisse und Erfahrungen einer internationalen online/ offline-Delphi-Befragung. elektronisch veröffentlicht: http://www.gor.de/tband99/pdfs/a_h/beck.pdf; abgerufen am 2004-12-01.

Beck, Ulrich (1983): Jenseits von Stand und Klasse? Soziale Ungleichheiten, gesellschaftliche Individualisierungsprozesse und die Entstehung neuer sozialer Formationen und Identitäten. In: Kreckel, Reinhard (Hrsg.): Soziale Ungleichheiten. Göttingen, S. 35-74.

Beck, Ulrich (1986): Risikogesellschaft. Auf dem Weg in eine andere Moderne. Suhrkamp, Frankfurt 1986.

Beck, Ulrich (1995): Die „Individualisierungsdebatte“. In: Schäfers, Bernhard (Hrsg.): Soziologie in Deutschland. Entwicklung, Institutionalisierung und Berufsfelder, theoretische Kontroversen. Opladen: Leske+Budrich, S.185-195.

Beck, Ulrich; Beck-Gernsheim, Elisabeth (1990): Freiheit oder Liebe. Gesellschaftliche Individualisierungsprozesse und soziale Lebens- und Liebesformen. In: Frankfurter Rundschau, 27.03.1990.

Becker, Jörg (2001): Internet \& Co: Historie, Technik und Geschäftsmodelle für den Handel. In: Ahlert, Dieter; Becker, Jörg; Kenning, Peter, Schütte, Reinhard (Hrsg.): Internet \& Co. im Handel. Springer Verlag, Berlin 2001.

Bederson, Benjamin (1995): Audio augmented reality: A prototype automated tour guide. In: Proceedings of Conference on Human Factors and Computing Systems 1995, Denver 1995, S. 210-211.

Beinlich, Georg (1995): Geschäftsbeziehungen - ein integrativer Überblick auf Basis der politischen Ökonomie. Arbeitspapier zur Marketingtheorie Nr. 5, Universität Trier, 1995.

Bender, Walter (2002): Twenty Years of Personalization. All about „Daily Me“. In: EDUCAUSE Review; Bd. 37, Nr. 5, September/Oktober 2002, S. 20-29.

Bender, Walter; Cehsnais, Pascal; Elo, Sara; Shaw, Alan; Shaw, Michelle (1996): Enriching communities: harbingers of news in the future. IBM Systems Journal archive Nr. 35, Band 3-4, 1996, S. 369 - 380. 
Bensberg, Frank (2001): Web Log Mining als Instrument der Marketingforschung: Ein systemgestaltender Ansatz für internetbasierte Märkte. Deutscher Universitätsverlag, Wiesbaden 2001.

Bensberg, Frank (2002): WAP Log Mining als Instrument der Marketingforschung für Mobile Commerce. In: Silberer, Günter; Wohlfahrt, Jens; Wilhelm, Thorsten (Hrsg.): Mobile Commerce. Grundlagen, Geschäftsmodelle, Erfolgsfaktoren. Gabler Verlag, Wiesbaden 2002.

Bensberg, Frank; Weiß, Thorsten (1999): Web Log Mining als Marktforschungsinstrument für das World Wide Web. In: Wirtschaftsinformatik 41 (1999) 5, S. 426-432.

Berger, Peter (1997): Individualisierung und sozialstrukturelle Dynamik; In: Beck, Ulrich; Sopp, Peter (Hrsg.): Individualisierung und Integration - Neue Konfliktlinien und neuer Integrationsmodus?; Leske+Budrich, Opladen 1997; S. 81-98.

Berry, Leonard (1983): Relationship Marketing. In: Berry Leonard et al. (Hrsg.): Emerging Perspectives on Service Marketing. Chicago 1983. S. 25-28.

Berry, Leonard (1995): Relationship Marketing of Services - Growing Interest, Emerging Perspectives. In: Journal of the Academy of Marketing Science. Vol. 23, Nr. 4, S. 236-245.

Berthold, Oliver; Köhntopp, Marit (2001): Identity Management based on P3P. In: Federrath, Hannes (Hrsg.): Designing Privacy Enhancing Technologies. Proceedings of the Workshop on Design Issues in Anonymity and Unobservability, Berkeley 2001, S. 141-160.

Best, Wolfgang (2002): Mobilfunk in der Semiometrie-Welt. TNS EMNID Telecommunication\&IT. Elektronisch veröffentlicht:

http://www.emnid.tnsofres.com/forschungsbereiche/doc_f_telekom/TNS_EMNID_MF_in_der_Semio metriewelt.pdf; abgerufen am 2004-12-01.

Bettis, Richard (1998): Commentary on "Redefining Industry Structure for the Information Age" by J.L. Sampler. In: Strategic Management Journal, Bd. 19, S. 357-361.

Bieger, Thomas; Bickhoff, Nils, von Knyphausen-Aufseß, Dodo (2002): Einleitung. In: Bieger, Thomas; Caspers, Rolf; zu Knyphausen-Aufseß, Reding (Hrsg.): Zukünftige Geschäftsmodelle. Springer Verlag, Berlin 2002, S. 1-11.

Bieger, Thomas; Rüegg-Stürm, Johannes; von Rohr, Thomas (2002): Strukturen und Ansätze einer Gestaltung von Beziehungskonfigurationen - Das Konzept Geschäftsmodell. In: Bieger, Thomas; Caspers, Rolf; zu Knyphausen-Aufseß, Reding (Hrsg.): Zukünttige Geschäftsmodelle. Springer Verlag, Berlin 2002, S. 35-61.

Billsus, Daniel; Pazzani, Michael; Chen, James (2000): A Learning Agent for Wireless News Access. Proceedings of the 5th international conference on intelligent user interfaces. ACM Press, New York, S. 33-36. Elektronisch veröffentlicht: http://www.fxpal.com/people/billsus/pubs/iuinews.pdf, 2000, abgerufen am 2004-05-31.

Bird, Adam; Künstner, Thomas; Vogelsang, Gregor (2003): Customer Centricity. Campus Verlag, Frankfurt 2003.

Bliemel, Friedhelm; Fassott, Georg (2000): Electronic Commerce und Kundenbindung. In: Bliemel, Friedhelm; Fassot, Georg; Theobald, Axel (Hrsg.): Electronic Commerce. Gybler Verlag, Wiesbaden 2000. S. 11-26.

Blödorn, Sascha; Gerhards, Maria (2004): Informationsverhalten der Deutschen. In: Media Perspektiven, Nr. 01/2004, S. 2-14. 
Bode, Jürgen (1997): Der Informationsbegriff in der Betriebswirtschaftslehre. In: ZfbF 49 (1997), S. 449-468.

Boening-Spohr, Patricia; Hess, Thomas (1999): Betriebswirtschaftliche Rahmenbedingungen für Medienunternehmen. Arbeitspapiere der Abt. Wirtschaftsinformatik II, Universität Göttingen, Nr. 2, Göttingen, 1999.

Bohn, Andreas (1993): Differenzierungsstrategien - Kritische Würdigung eines zentralen Konzeptes der strategischen Unternehmensführung. Verlag Barbara Kirsch, München 1993.

Böning-Spohr, Patricia; Hess, Thomas (2000): Geschäftsmodelle inhalteorientierter Online-Anbieter. In: Arbeitsberichte der Abt. Wirtschaftsinformatik II, Universität Göttingen, Nr. 01/2000.

Bortz, Jürgen; Döring, Nicola (2003): Forschungsmethoden und Evaluation für Human- und Sozialwissenschaftler. Springer Verlag, Heidelberg 2003.

Bourdieu, Pierre (1982): Der feine Unterschied. Kritik der gesellschaftlichen Urteilskraft. Frankfurt 1982.

Brack, Anke (2003): Das strategische Management von Medieninhalten. Deutscher Universitätsverlag, Wiesbaden 2003.

Brajnik, Giorgio; Tasso, Carlo (1994): A shell for developing non-monotonic user modeling systems. In: International Journal of Human Computer Studies 40, 1994, S. 31-62.

Brandtweiner, Roman (2000): Differenzierung und elektronischer Vertrieb digitaler Informationsgüter. Symposion Publishing, Düsseldorf 2000.

Braun, Dietmar; Kocovski, Jan; Rickert, Thomas; Waldhauser, Béla (2004): Anti Spam Task Force ASTF. Whitepaper des Verbands der deutschen Internetwirtschaft e.V.; elektronisch veröffentlicht: http://www.eco.de/servlet/PB/show/1455897/Whitepaper_Vollversion_100.pdf; abgerufen am 2004-12-01.

Braun, Hans (1989): „Helmut Schelskys Konzept der ,nivellierten Mittelstandsgesellschaft' und die Bundesrepublik der 50er Jahre“; In: Archiv für Sozialgeschichte, XXIX. Band 1989, S.199-223.

Braybrooke, David; Lindblom, Charles (1965): A Strategy of Decision: Policy Evaluation as a Social Process. Free Press, New York 1965.

Breunig, Peter (1997): Marktchancen des digitalen Fernsehens - eine Untersuchung zur Einführung digitaler Spartensender. Angewandte Medienforschung, Bd. 6., München 1997.

Bryan, Lowell (2002): Just-in-time strategy for a turbulent world. In: The McKinsey Quarterly, 2002 Nr. 2, Risk and resilience. Elektronisch veröffentlicht: http://www.mckinseyquarterly.c/article_page.asp?ar=1195\&L2=21\&L3=37; abg. am 01.06.2003.

Bücher, Karl (1926): Die Grundlagen des Zeitungswesens. In: Gesammelte Aufsätze zur Zeitungskunde. Tübingen 1926.

Bühler, Stefan; Jäger, Franz (2002): Einführung in die Industrieökonomik. Springer Verlag, Berlin u.a. 2002.

Büllingen, Franz; Stamm, Peter (2001): Entwicklungstrends im Telekommunikationssektor bis 2010 Studie im Auftrag des Bundesministeriums für Wirtschaft und Technologie. Elektronisch veröffentlicht: http://www.bmwi.de/Homepage/download/telekommunikation_post/Entwicklungstrends.pdf; abgerufen am 2004-12-01. 
Büttner, Oliver; Rauch, Manuel; Silberer, Günter (2005): Consumer Behavior and Cognitive Processes at the Point of Sale: An Integrated Process Tracing Methodology. In: Proceedings of the $12^{\text {th }}$ World Marketing Congress, S. 505-509.

Burghard, Werner; Kleinaltenkamp, Michael (1996): Standardisierung und Individualisierung - Gestaltung der Schnittstelle zum Kunden. In: Kleinaltenkamp, Michael et al. (Hrsg.): Customer Integration. Von der Kundenorientierung zur Kundenintegration. Gabler, Wiesbaden 1996, S. 163-176.

Burghardt, Markus (2005): Web Services. Aspekte von Sicherheit, Transaktionalität, Abrechnung und Workflow. Deutscher Universitätsverlag, Wiesbaden 2005.

Buse, Stephan (2002): Der mobile Erfolg - Ergebnisse einer empirischen Untersuchung in ausgewählten Branchen. In: Keuper, Frank (Hrsg.): Electronic Business und Mobile Business. Gabler Verlag, Wiesbaden 2002, S. 89-117.

CDT (2000): Privacy Survey Results. Center for Democracy \& Technology. Elektronisch veröffentlicht: http://www.cdt.org/privacy/survey/findings/; abgerufen am 2005-04-30.

Chamberlin, Edward Hastings (1954): Die Theorie der monopolistischen Konkurrenz. In: Herdzina, Klaus (Hrsg.), S. 76-89.

Chamoni, Peter; Dürsing, Roland; Stock, Steffen (2004): Customer Relationship Management auf der Grundlage von analytischen Informationssystemen im Handel. In: HMD - Praxis der Wirtschaftsinformatik. Nr. 235, 2004, S. 27-35.

Chaum, David (1985): Security without identification: transaction systems to make big brother obsolete. Communications of the ACM, Bd. 28, Nr. 10, 1985, S. 1030-1044.

Chen, Guanling; Kotz, David (2000). A survey of Context-Aware Mobile Computing Research. Dartmouth Computer Science Technical Report TR2000-381. Elektronisch veröffentlicht: http://www.cs.dartmouth.edu/ dfk/papers/chen:survey-tr.pdf; abgerufen am 2004-04-30.

Chen, Guanling; Kotz, David (2002): Solar: A pervasive-computing infrastructure for context-aware mobile applications. Dartmouth Computer Science Technical Reort TR2002-421.

Chen, Harry; Finin, Tim; Joshi, Anupam (2003): An Ontology for Context-Aware Pervasive Computing Environments, In: Knowledge Engineering Review. Special Issue on Ontologies for Distributed Systems, 2003.

Chesnais, Pascal; Mucklo, Matthew; Sheena, Jonathan (1995): The Fishwrap Personalized News System. In: Proceedings of the IEEE Second International Workshop on Community Networking Integrating Multimedia Services to the Home. Princeton (USA) 1995.

Cheverst, Keith; Mitchell, Keith; Davies, Nigel (1999): Design of an object model for a context sensitive tourist GUIDE. In: Computers and Graphics Bd. 23, Nr. 6, S. 883-891.

Conlan, Owen; Power, Ruaidhri; Barrett, Keara (2003): Next Generation Context Adaptive Services. In: Proceedings of the 1st international symposium on Information and communication technologies, Dublin, Ireland, S. 205.212.

Coulouris, George; Dollimore, Jean; Kindberg, Tim (2002): Verteilte Systeme. Konzepte und Design. Pearson Education, München 2002.

Cranor, Lorrie; Langheinrich, Marc; Marchiori, Massimo; Presler-Marshall, Martin; Reagle, Joseph (2002): The Platform for Privacy Preferences 1.0 (P3P1.0) Specification. W3C Recommendation, Apr. 2002. Elektronisch veröffentlicht: http://www.w3.org/TR/P3P/; abgerufen am 2004-12-01. 
Cranor, Lorrie; Weitzner, Daniel (2002): Summary Report - W3C Workshop on the Future of P3P. Elektronisch veröffentlicht: http://www.w3.org/2002/12/18-p3p-workshop-report.html; abgerufen am 2004-12-01.

Daconta, Michael; Obrst, Leo; Smith, Kevin (2003): The Semantic Web: A Guide to the Future of XML, Web Services, and Knowledge Management. Wiley Publishing, Indianapolis 2003.

Dahm, Hermann; Rössler, Patrick; Schenk, Michael (1998): Vom Zuschauer zum Anwender. Akzeptanz und Folgen digitaler Fernsehdienste. LIT Verlag, Münster 1998.

D'Aveni, Richard (1994): Hypercompetition: Managing the Dynamics of Strategic Maneuvering. Simon \& Schuster, New York 1994.

Davies, Nigel; Cheverst, Keith; Mitchell, Keith; Friday, Adrian (1999): Caches in the air: Disseminating tourist information in the GUIDE system. In: Proceedings of Second IEEE Workshop on Mobile Computing Systems and Applications, New Orleans 1999.

Davis, Fred (1989): Perceived usefulness, perceived ease of use, and user acceptance of information technology. In: MIS Quarterly, Vol. 13, Nr. 2, S. 319-340.

Davis, Stanley (1987): Future Perfect. Addison Wesley, New York 1987.

Day, Roseann; Daly, John; Sheedy, Tim; Christiansen, Chris (2000): Widening your secure eBusiness to Wireless. IDC White Paper. Elektronisch veröffentlicht: ftp://ftp.software.ibm.com/software/tivoli/analystreports/wireless_security.pdf; abgerufen am 2005-04-30.

Deitel, Harvey; Deitel, Paul; Nieto, Tem; Steinbuhler, Kate (2002): Wireless Internet \& Mobile Business How to Program. Prentice Hall, New Jersey 2002.

Dengler, Johannes (2000): Strategie integrierter Telekommunikationsanbieter. Deutscher Universitätsverlag, Wiesbaden 2000.

Dettki, Arne; Ferrari, Tilo; Resch, Bernhard (2001): Die Technologie: Breitband beschleunigt die Inhalte. In: Vizjak, Andrej; Ringlstetter, Max (Hrsg.): Medienmanagement: Content gewinnbringend nutzen. Gabler, Wiesbaden 2001, S. 26-36.

Dey, Anind (2001); Understanding and Using Context. In: Personal and Ubiquitous Computing Journal, Bd. 5 (1), 2001, S. 4-7.

Dey, Anind; Abowd, Gregory (2000): The Context Toolkit: Aiding the Development of Context-Aware Applications. In: Workshop on Software Engineering for Wearable and Pervasive Computing , Limerick, Ireland, Juni 2000.

Dey, Anind; Futakawa, Masayasu; Salber, Daniel; Abowd. Gregory (1999): The Conference Assistant: Combining Context-Awareness with Wearable Computing. In: Proceedings of the 3rd International Symposium on Wearable Computers (ISWC '99). San Francisco 1999, S. 21-28.

Dickinson, Ian; Reynolds, Dave; Banks, Dave; Cayzer, Steve; Vora, Poorvi (2003): User Profiling with Privacy: A Framework for Adaptive Information Agents. In: Klusch, Matthias et al. (Hrsg.): Intelligent Information Agents. Lecture Notes on Artificial Intelligence, Springer Verlag, Berlin 2003, S. 123-151.

Diederich, Bern; Lerner, Thomas; Lindemann, Roland; Vehlen, Ralf (2001): Mobile Business. Märkte, Techniken, Geschäftsmodelle. Gabler Verlag, Wiesbaden 2001.

Diekmann, Thomas; Gehrke, Nick (2003): Ein Framework zur Nutzung situationsabhängiger Dienste. In: Dittrich, K.; König, W.; Oberweis, A.; Rannenberg, K.; Wahlster, W. (Hrsg.): Lecture Notes in Informatics, Informatik 2003. Innovative Anwendungen, Band 1, Bonn, 2003, S. 217-221. 
Dierkes, Meinolf (1982): Akzeptanz und Akzeptabilität der Informationstechnologie. In: Technische Universität Berlin (Hrsg.): Wissenschaftsmagazin. Bd. 2, Nr. 1, S. 12-15.

Diller, Hermann (1995a): Beziehungs-Marketing. In: WiSt, 24. Jg., Nr. 9, S. 442-447.

Diller, Hermann (1995b): Kundenmanagement. In: Köhler, Richard; Tietz, Bruno; Zentes, Joachim (Hrsg.): Handwörterbuch des Marketing. Stuttgart 1995, S. 1363-1376.

Domizlaff, Hans (1929): Markentechnik - Die Gewinnung des öffentlichen Vertrauens. Hamburg 1982.

Drucker, Peter (1994): Knowledge Work and Knowledge Society - The Social Transformations of this Century. elektronisch veröffentlicht:

http://www.ksg.harvard.edu/ifactory/ksgpress/www/ksg_news/transcripts/drucklec.htm; abgerufen am 2004-12-01.

Eberspächer, Jörg; Vögel, Hans-Jörg; Bettstetter, Christian (2001): GSM - Switching, Services and Protocols. John Wiley and Sons, Chichester 2001.

Ecin (2002): Gute Perspektive für Paid Content. Elektronisch veröffentlicht: http://www.ecin.de/news/2002/12/16/05190/; abgerufen am 2004-12-01.

Ecin (2003): 3G: Preiskrieg der Anbieter; elektronisch veröffentlicht: http://www.ecin.de/news/2003/02/18/05393; abgerufen am 2004-12-01.

Eckhoff, Andreas (2001): Einführung innovativer Systemgeschäfte. Deutscher Universitätsverlag, Wiesbaden 2001.

Enquete (1998): Vierter Zwischenbericht der Enquete-Kommission „Zukunft der Medien in Wirtschaft und Gesellschaft - Deutschlands Weg in die Informationsgesellschaft" zum Thema Sicherheit und Schutz im Netz. BT-Drucksache 13/11002, 22.6.1998.

Ericsson Consulting (2000): Market Study UMTS - Perspectives and Potentials. Düsseldorf 2000.

Eschenbach, Rolf; Kunesch, Hermann (1996): Strategische Konzepte: Management-Ansätze von Ansoff bis Ulrich. Schäffer-Poeschel, Stuttgart 1996.

EU (1999): Grünbuch zur Konvergenz der Branchen Telekommunikation, Medien und Informationstechnologie und ihren ordnungspolitischen Auswirkungen. elektronisch veröffentlicht: http://europa.eu.int/scadplus/leg/de/lvb/l24165.htm; abgerufen am 2004-12-01.

Evans, Philip; Wursterer, Thomas (2000): Web Att@ck: Strategien für die Internet-Revolution. Carl Hanser Verlag, München 2000.

Faulhaber, Gerald; Panzar, John (1977): Optimal Two-Part Tariffs with Self-Selection. In: Economic Discussion Paper, Nr. 74, Bell Laboratories.

Fayyad, Usama; Piatetsky-Shapiro, Gregory; Smyth, Padhraic (1996): From Data Mining to Knowledge Discovery in Databases. Elektronisch veröffentlicht: http://www.kdnuggets.com/gpspubs/aimag-kddoverview-1996-Fayyad.pdf; abgerufen am 2004-12-01.

FAZ (2001): TIME to Survive? Strategien, Geschäftsmodelle und Management im digitalen Zeitalter. F.A.Z.-Institut für Management-, Markt- und Medieninformationen; Frankfurter Allgemeine Zeitung, Frankfurt 2001.

Fehr, Hannes (2003): Paid Content erfolgreich verkaufen. Ein Leitfaden zur Erstellung eines individuellen Konzepts zum Verkauf von digitalen Inhalten auf Online-Portalen. hansebuch Verlag, Hamburg 2003.

Feldmann, Valerie (2002): Competitive strategy for media companies in the mobile internet. In: Schmalenbach Business Review, Vol. 54, October 2002; S. 351-371. 
Ferber, Reginald (2003): Information Retrieval: Suchmodelle und Data-Mining-Verfahren für Textsammlungen und das Web. Dpunkt Verlag, Heidelberg 2003.

Figge, Stefan; Albers, Andreas (2005): Individualising M-Commerce Services by Semantic User Situation Modelling. In: Ferstl, Otto; Sinz, Elmar; Eckert, Sven; Isselhorst, Tilman (Hrsg.): Wirtschaftsinformatik 2005: eEconomy, eGovernment, eSociety, 7. Internationale Tagung Wirtschaftsinformatik 2005. Physica-Verlag, Heidelberg 2005.

Finin, Timothy (1989): GUMS. A general user modeling shell. In: Kobsa, Alfred; Wahlster, Wolfgang (Hrsg.): User Models in Dialog Systems. Springer-Verlag, Berlin 1989, S. 411-430.

Fink, Josef; Kobsa, Alfred (2000): A Review and Analysis of Commercial User Modeling Servers for Personalization on the World Wide Web. In: User Modeling and User-Adapted Interaction, Nr. 10, S. 209$249,2000$.

Fink, Lars-Rüdiger (2002): Economies of Aggregation - Internetportale als Informationsgüterbündel und die Zahlungsbereitschaft der Nachfrager, Dissertation, Universität Köln, Köln 2002.

Finsinger, Jörg (1980): Zur Anatomie von wohlfahrtsoptimalen Preisen für öffentliche Unternehmen, die unter zunehmenden Skalenerträgen produzieren. Zeitschrift für die gesamte Staatswissenschaft, Bd. 136, S. 136-160.

Fleisch, Elgar (2001): Das Netzwerkunternehmen. Springer, Berlin 2001.

Forcher, Ralf (1996): Entwurf einer Logistik-Dienstleistung zur Personenmobilität. Dissertation, Universität 1996.

Frants, Valery; Shapiro, Jacob; Voiskunskii, Vladimir (1997): Automated information retrieval: theory and methods. Academic Press Professional, San Diego 1997.

Franz, Peter (1984): Soziologie der räumlichen Mobilität. Campus Verlag, Frankfurt 1984.

Frawley, William; Piatetsky-Shapiro, Gregory; Matheus, Christopher (1991): Knowledge Discovery in Databases: An Overview. In: AAAI Press 1991, S. 1-27.

Fritz, Wolfgang (2000): Internet-Marketing und Electronic Commerce. Gabler Verlag, Wiesbaden 2000.

Funk Jeffrey (2000): The Mobile Internet Market: Lessons from Japan's i-mode System. Elektr. veröff.: http://e-conomy.berkeley.edu/conferences/9-2000/EC-conference2000_papers/Funk.pdf; abgerufen am 2004-05-31.

Gälweiler, Aloys (1987): Strategische Unternehmensführung. Campus Verlag, Frankfurt 1987.

Gauch, Susan; Chaffee, Jason; Pretschner, Alexander (2004): Ontology-Based Personalized Search and Browsing. In: Web Intelligence and Agent Systems: An International Journal, 2004. Elektronisch veröffentlicht: http://www.ittc.ku.edu/ sgauch/selectedpapers/WIAS2003.pdf; abgerufen am 2004-05-31.

Gehrke, Nick, Burghardt, Markus, Schumann, Matthias (2002): Strategien der Produktbündelung. In: WISU (2002) 3, S. 346-352.

Gerhards, Maria; Klingler, Walter (2003): Mediennutzung in der Zukunft. In: Media Perspektiven, Nr. 3/2003, S. 115-130.

Gerhards, Maria; Klingler, Walter (2004): Mediennutzung in der Zukunft - Konstanz und Wandel. In: Media Perspektiven, Nr. 10/2004, S. 472-482.

Gerpott, Thorsten (2003): Preispolitik für Online-Zeitungen auf dem Lesermarkt. In: information Management \& Consulting, 2003, 18. Jg., Nr. 1, S. 89-99. 
Giddens, Anthony (1992): Die Konstitution der Gesellschaft. Campus Verlag, Frankfurt 1992.

Gierl, Heribert (1987): Die Erklärung der Diffusion technischer Produkte. Duncker \& Humblot, Berlin 1987.

Gierl, Heribert (1989): Empirische Individualisierungsforschung. In: Jahrbuch der Absatz- und Verbrauchsforschung, 35. Jg., 1/1989, S. 4-22.

Gollwitzer, Peter (1990): Action Phases and Mind-Sets. In: Higgins, Edward; Sorrentino, Richard (Hrsg.): The Handbook of Motivation and Cognition: Foundations of Social Behavior (Vol. 2). Guilford press, New York 1990.

Gora, Walter; Röttger-Gerigk, Stefanie (2002): Handbuch Mobile Commerce. Springer Verlag, Berlin 2002.

Govindarajulu, Chittibabu; Reithel, Brian; Sethi, Vikram (2000): A model of end user attitudes and intentions toward alternative sources of support. In: Information \& Management, Bd. 37, Nr. 3, S. 77-86.

Graumann, Sabine; Köhne, Bärbel (2003): Monitoring Informationswirtschaft. 6 Faktenbericht 2003, im Auftrag des Bundesministeriums für Wirtschaft und Arbeit. Elektronisch veröffentlicht: http://www.bmwi.de/Redaktion/Inhalte/Downloads/6-faktenberichtvollversion,templateld=download.pdf; abgerufen am 2004-05-31.

Gregg, Stefanie (2001): Die Konvergenz: Telekommunikationsanbieter und Medienunternehmen - Wettbewerber oder Partner? In: Vizjak, Andre; Ringlstetter, Max (Hrsg.): Medienmanagement: Content gewinnbringend nutzen. Gabler, Wiesbaden 2001, S. 37-44.

Griesbaum, Joachim; Rittberger, Marc; Bekavac, Bernard (2002): Deutsche Suchmaschinen im Vergleich: AltaVista.de, Fireball.de, Google.de und Lycos.de. In: Proceedings des 8. Internationalen Symposiums für Informationswissenschaft. Konstanz 2002, S.201-223.

Grimm, Rudi; Jüstel, Matthias; Klotz, Michael (2002): Methoden zur Personalisierung im M-Commerce; In: Gora, Walter; Röttger-Gerigk, Stefanie (Hrsg.): Handbuch Mobile-Commerce; Springer, Berlin 2002; S. 177-190.

Grimmer, Udo; Mucha, Hans-Joachim (1998): Datensegmentierung mittels Clusteranalyse. In: Nakhaeizadeh, Gholamreza (Hrsg.): Data Mining. Theoretische Aspekte und Anwendungen. Physika-Verlag, Heidelberg 1998, S. 109-141.

Grunwald, Roman; Schwellbach, Uwe (1999): Strategien und Organisationsgestaltung in der Telekommunikationsbranche: Erfolgsfaktoren neuer und etablierter Anbieter. Gabler Verlag, Wiesbaden 1999.

GSM Association (2003): Membership and Market Statistics, March 2003. Elektronisch veröffentlicht: http://www.gsmworld.com/news/statistics/feb03_stats.pdf; abgerufen am 2004-05-31.

Guthery, Scott; Cronin, Mary (2003): Developing MMS Applications. McGraw-Hill, New York 2003.

Haartsen, Jaap; Allen, Warren; Inouye, Jon; Joeressen, Olaf; Naghshineh, Mahmoud (1998): Bluetooth: Vision, Goals, and Architecture. Mobile Computing and Communications Review. 2(4): 38-45, October 1998.

Habann, Frank (1999): Kernressourcenmanagement in Medienunternehmen. Josef Eul Verlag, Lohmar 1999.

Haertsch, Patrick (2000): Wettbewerbsstrategien für die Digital Economy - Eine kritische Überprüfung klassischer Strategiekonzepte. Josef Eul Verlag, Köln 2000.

Hagel, John; Rayport, Jeffrey (1997): The new Infomediaries. In: The McKinsey Quaterly, Nr. 4, 1997. 
Hagen, Lutz M. (2003): Riepls Gesetz im Online-Zeitalter. Eine Sekundäranalyse über die Grenzen der Substitution von Massenmedien durch das Internet. Elektronisch veröffentlicht: http://www.gor.de/tband99/pdfs/a_h/hagen.pdf; abgerufen am 2004-12-01.

Halpin, Terry (2001): Information Modeling and Relational Databases: From Conceptual Analysis to Logical Design. Morgan Kaufman Publishers, San Francisco 2001.

Hamel, Gary (2000): Leading the Revolution. Harvard Business School Press, Boston 2000.

Hammersley, Ben (2003): Content Syndication with RSS. O'Reilly, Sebastopol 2003.

Handelsblatt (2003): SWMH darf bei Süddeutschem Verlag einsteigen. Elektronisch veröffentlicht: http://uww.handelsblatt.com/hbiwwwangebot/fn/relhbi/sfn/buildhbi/cn/GoArt!200104,201200,602418/ SH/O/depot/0/; abgerufen am: 2004-12-01.

Haneberg, Dominik; Stenzel, Kurt; Reif, Wolfgang (2004): Electronic-Onboard-Ticketing: Software Challenges of an State-of-the-Art M-Commerce Application. In: In: Pousttchi, Key; Turowski, Klaus (Hrsg.): Mobile Economy - Transaktionen, Prozesse, Anwendungen und Dienste. Proceedings zum 4. Workshop Mobile Commerce, Augsburg 2004, S. 103-113.

Hartmann, S.; Dirksen, V. (2001): Effizienzsteigerung von unternehmensinternen Prozessen durch die Integration von Komponenten des M-Business. In: Information Management \& Consulting, 16.Jg, Nr. 2, 2001, Saarbrücken, S. 16-27.

Hass, Berthold (2002): Geschäftsmodelle von Medienunternehmen - Ökonomische Grundlagen und Veränderungen durch neue Informations- und Kommunikationstechnik. Deutscher Universitätsverlag, Wiesbaden 2002.

Hass, Berthold (2004): Desintegration und Reintegration im Mediensektor: Wie sich Geschäftsmodelle durch Digitalisierung verändern. In: Zerdick, Axel; Picot, Arnold; Schrape, Klaus, Burgelmann, JeanClaude; Silverstone, Roger (Hrsg.): E-Merging Media. Springer Verlag, Berlin 2004.

Hautsch, Gert (2002): Klappern gehört zum Handwer - Tendenzen in der Medienwirtschaft von Oktober 2001 bis März 2002. Elektronisch veröffentlicht: http://www.verdiverlage.de/branchen/inhalte/ueberblick.html; abgerufen am 2004-12-01.

Heinen, Edmund (1991): Industriebetriebslehre. Gabler Verlag, Wiesbaden 1991.

Heinrich, Jürgen (1999a): Medienökonomie - Bd.1, Mediensystem, Zeitung, Zeitschrift, Anzeigenblatt. Westdeutscher Verlag, Wiesbaden 1999.

Heinrich, Jürgen (1999b): Medienökonomie - Bd.2, Hörfunk und Fernsehen. Westdeutscher Verlag, Wiesbaden 1999.

Heinrich, Jürgen (2001a): Medienökonomie - Bd.1, Mediensystem, Zeitung, Zeitschrift, Anzeigenblatt. 2. Aufl., Westdeutscher Verlag, Wiesbaden 2001.

Heinrich, Jürgen (2001b): Medienökonomie - Bd.2, Hörfunk und Fernsehen. 2. Aufl., Westdeutscher Verlag, Wiesbaden 2001.

Heise (2005): „Google local" noch in diesem Jahr in Deutschland. Elektronisch veröffentlicht: http://www.heise.de/newsticker/meldung/57882; abgerufen am 2005-04-30.

Held, Albert; Buchholz, Sven; Schill, Alexander (2002): Modeling of context information for pervasive computing applications. In: Proceedings of SCI 2002/ISAS 2002. 
Hensley, Pat; Metral, Max; Shardanand, Upendra; Converse, Donna; Myers, Mike (1997): Implementation of OPS Over HTTP. W3C Submission Note, 1997-06-02. Elektronisch veröffentlicht: http://www.w3.org/TR/NOTE-OPS-OverHTTP; abgerufen am 2005-04-30.

Herden, Sebastian; Rautenstrauch, Claus; Zwanziger, André; Plack, Marco (2004): Personal information Guide - eine Plattform mit Location Based Services für Mobile Powered E-Commerce. In: Pousttchi, Key; Turowski, Klaus (Hrsg.): Mobile Economy - Transaktionen, Prozesse, Anwendungen und Dienste. Proceedings zum 4. Workshop Mobile Commerce, Augsburg 2004.

Herlocker, Jon; Konstan, Joseph; Borchers, Al; Riedl, John (1999): An algorithmic framework for performing collaborative filtering. In: Proceedings of the 22nd annual international ACM SIGIR conference, Berkeley 1999.

Hess, Thomas (2000): Netzeffekte - Verändern neue Informations- und Kommunikationstechnologien das klassische Marktmodell? In: WiSt 29 (2000), S. 96-98.

Hess, Thomas (2002): Implikationen des Internet für die Medienbranche - eine strukturelle Analyse, In: Keuper, F. (Hrsg.): Electronic Business und Mobile Business - Ansätze, Konzepte und Geschäftsmodelle, Wiesbaden, S. 569 - 602, 2002.

Hess, Thomas (2004): Medienunternehmen im Spannungsfeld von Mehrfachverwertung und Individualisierung - eine Analyse für statische Inhalte. In: Zerdick, Axel; Picot, Arnold; Schrape, Klaus; Burgelman, Jean-Claude; Silverstone, Roger; Feldmann, Valerie; Heger, Dominik; Wolff, Caroline (Hrsg.): EMerging Media - Kommunikation und Medienwirtschaft der Zukunft. European Communication Council Report; Springer Verlag, Berlin 2004.

Hess, Thomas; Anding, Markus (2002): Online Content Syndication - eine transaktionskostentheoretische. In Gabriel, Roland; Hoppe, Uwe (Hrsg.): Electronic Business - Theoretische Aspekte und Anwendungen in der betrieblichen Praxis. Physica-Verlag, Berlin 2002.

Hess, Thomas; Figge, Stefan; Hanekop, Heidemarie; Hochstatter, Iris; Hogrefe, Dieter; Kaspar, Christian; Rauscher, Barbara; Richter, Markus; Riedel, Andre; Zibull, Marco (2005): Technische Möglichkeiten und Akzeptanz mobiler Anwendungen - Eine interdisziplinäre Betrachtung, In: Wirtschaftsinformatik, 47. Jg., Sonderheft Nr. 1 2005, S. 6-16.

Hess, Thomas; Schulze, Bernd (2003): Die Mehrfachnutzung von Inhalten in der Medienindustrie. In: Das Wirtschaftsstudium, Nr. 10 2003., S. 1377-1382.

Heutmann, Thomas; Ewers, Thomas (2003): Aktuelle Aspekte der Frequenzregulierung durch die Regulierungsbehörde für Telekommunikation und Post (Reg TP). Elektronisch veröffentlicht: http://www.et.fh-osnabrueck.de/lhf/Mobilfunktagung/Heutmann.pdf; abgerufen am 2003-07-31.

Hickethier, Knuth (1998): Rezeptionsgeschichte des Fernsehens - Ein Überblick. In: Klingler, Walter; Rothers, Gunnar; Gerhards, Maria (Hrsg.): Medienrezeption seit 1945. Forschungsbilanz und Forschungsperspektiven. Baden-Baden 1999. S. 129-141.

Hightower, Jeffrey; Brumitt, Barry; Borriello, Gaetano (2002): The Location Stack: A Layered Model for Ubiquitous Computing. In: Proceedings of the 4th IEEE Workshop on Mobile Computing Systems \& Applications, Callicoon, New York, Juni 2002, S. 22-28.

Hildebrand, Volker (1997): Individualisierung als strategische Option der Marktbearbeitung: Determinanten und Erfolgswirkungen kundenindividueller Marketingkonzepte; Deutscher Universitätsverlag, Wiesbaden 1997.

Hinterhuber, Hans (1992): Strategische Unternehmensführung - I. Strategisches Denken. Verlag Walter de Gruyter, Berlin 1992. 
Hobert, Guido (1998): Datenschutz und Datensicherheit im Internet: Interdependenz und Korrelation von rechtlichen Grundlagen und technischen Möglichkeiten. In: Europäische Hochschulschriften, Reihe 2, Rechtswissenschaft; Bd. 2501; Frankfurt am Main, 1998.

Holland, Stefan; Ester, Martin; Kießling, Werner (2003): Preference Mining: A Novel Approach on Mining User Preferences for Personalized Applications. Proceedings of the 2003 European Conference on Principles of Data Mining and Knowledge Discovery (PKDD). CavtatDubrovnik, Croatia 2003, S. 204-216.

Holland, Stefan; Kießling, Werner (2004): User Preference Mining Techniques for Personalized Applications. In: Wirtschaftsinformatik Bd. 46 (2004), Nr. 6, S. 439-445.

Homburg, Christian (1995): Kundennähe von Industriegüterunternehmen: Konzeption - Erfolgsauswirkungen - Determinanten. Wiesbaden 1995.

Hörisch, Jochen (1998): Einleitung. In: Ludes, Peter (1998): Einführung in die Medienwissenschaft - Entwicklungen und Theorien. Erich Schmidt Verlag, Berlin 1998.

Hradil, Stefan (1999): Soziale Ungleichheit in Deutschland. Leske+Budrich, Opladen 1999.

Hradil, Stefan (2002): Mobilität. In: Endruweit, Günter; Trommsdorff, Gisela (Hrsg.): Wörterbuch der Soziologie. UTB, Stuttgart 2002.

Huldi, Christian (1992): Database-Marketing - Inhalt und Funktion eines Database-Marketing-Systems. St. Gallen 1992.

Hümmer, Bernd (2001): Strategisches Management von Kernkompetenzen im Hyperwettbewerb- Deutscher Universitätsverlag, Wiesbaden 2001.

IEEE (2001). Functional Requirements - IEEE Project 802. Elektronisch veröffentlicht: http://grouper.ieee.org/groups/802/802_archive/fureq6-8.html; abgerufen am 2004-05-31.

Indulska, Jadwiga; Hendricksen, Karen (2004): Modeling and Using Imperfect Context Information. in: Workshop Proceedings of the 2nd IEEE Conference on Pervasive Computing and Communications (PerCom2004), Orlando 2004, S. 33-37.

Indulska, Jadwiga; Robinson, Ricky; Rakatomirainy, Andry; Hendricksen, Karen (2003): Experiences in using cc/pp in context-aware systems. In: Proceedings of the 4th Internationale Conference on Mobile Data Management (MDM2003), Melbourne 2003, S. 247-261.

Intel (2003): Moore Optimistic on Moore's Law. elektronisch veröffentlicht: http://www.intel.com/labs/features/eml02031.htm; abgerufen am 2004-12-01.

IPTC (2004a): Documentation for NITF. IPTC International Press Telecommunications Council; elektronisch veröffentlicht: http://www.nitf.org/IPTC/NITF/3.2/documentation/nitf-documentation.html; abgerufen am 2004-12-01.

IPTC (2004b): NewsML DTD Overview document. IPTC; elektronisch veröffentlicht: http://www.newsml.org/IPTC/NewsML/1.2/documentation/NewsML_1.2-doc-DTDoverview_1.pdf; abgerufen am 2004-12-01.

ISC (2003): Internet Software Consortium - Internet Domain Survey, January 2000. elektronisch veröffentlicht: http://www.isc.org/ds/WWW-200001/report.html; abgerufen am 2004-12-01.

Jacob, Frank (1995): Produktindividualisierung. Gabler Verlag, Wiesbaden 1995.

Jacobson, Robert (1992): The „Austrian School of Strategy“. In: Academy of Management Review. Nr. 17 1992, S. 782-807. 
Jain, Anil; Murty, Narasimha; Flynn, Patrick (1999): Data Clustering: A Review. ACM Computing Surveys, Bd. 31, Nr. 3, S. 264-323.

Jankowska, Bożena (2005): Approaches for Device-Independent Content Delivery to Mobile Devices. In: Ferstl, Otto; Sinz, Elmar; Eckert, Sven; Isselhorst, Tilman (Hrsg.): Wirtschaftsinformatik 2005: eEconomy, eGovernment, eSociety, 7. Internationale Tagung Wirtschaftsinformatik 2005. Physica-Verlag, Heidelberg 2005.

Jüttner, Uta; Wehrli, Peter (1994): Relationship Marketing from a Value System Perspective. In: International Journal of Service Industry Management. Vol. 5, Nr. 5, S. 54-73.

Kaaranen, Heikki; Ahtiainen, Ari; Laitinen, Lauri; Naghian, Siamäk; Niemi, Valtteri (2001): UMTS Networks. John Wiley and Sons, Chichester 2001.

Kaas, Klaus Peter (1992): Kontraktgütermarketing als Kooperation zwischen Prinzipalen und Agenten. In: ZfbF, 44. Jg., Nr. 10, S. 885-901.

Kaas, Klaus Peter (1995): Marketing zwischen Markt und Hierarchie. In: zfbf Sonderheft 35/1995, S. $19-42$.

Kanemitsu, Hiroyuki; Kamada, Tomihisa (1999): POIX: Point Of Interest eXchange Language Specification. W3C Note, June 1999. Elektronisch veröffentlicht: http://www.w3.org/TR/poix; abgerufen am 2005-04-30.

Karmasin, Matthias; Winter, Carsten (2000): Grundlagen des Medienmanagements. Fink Verlag, München 2000.

Karzauninkat, Stefan (2003): Spam überschwemmt die Suchergebnisse. In: c't, Nr. 20, 2003, S. 88-91.

Kaspar, Christian; Burghardt, Markus; Hagenhoff, Svenja (2004): Web Services for Portlet Integration into Corporate Portals. In: Proceedings of the 2004 International Symposium on Web Services and Applications (ISWS 04), Las Vegas 2004.

Kaspar, Christian; Diekmann, Thomas, Hagenhoff, Svenja (2005): Kontextadaptive Datenverarbeitung. In: WiSt 34. Jahrgang, Heft 09, September 2005.

Kaspar, Christian; Hagenhoff, Svenja (2004): Individualization of a mobile news service - a simple approach. In: Jönsson, Sten (Hrsg.): Proceedings of the VIIth SAM/IFSAM World Congress, Göteborg, 2004.

Kaspar, Christian; Hagenhoff, Svenja (2005): An overview of mobile radio technologies. In: Pagani, Margherita (Hrsg.): Encyclopedia of Multimedia Technology and Networking, Hershey 2005.

Kaspar, Christian; Seidenfaden, Lutz; Hagenhoff, Svenja (2004): Privacy compliant intermediation for personalized online services. In: Proccedings of the first IMT Conference, Orlando 2004.

Kaufmann, Heinz; Pape, Heinz (1996): Clusteranalyse. In: Fahrmeir, Ludwig; Hamerle, Alfred; Tutz, Gerhard (Hrsg.): Multivariate statistische Verfahren. de Gruyter Verlag, Berlin 1996, S. 437-536.

Kay, Alan (1991): Computers, Networks and Education. In: Scientific American. Sept. 1991, S. 100-107.

Kay, Judy (1995): The um toolkit for cooparative user modelling. In: User Modeling and User-Adapted Interaction, Nr. 4, 1995, S. 149-196.

KEK (2003): Zuschaueranteile für das Jahr 2003. Kommission zur Ermittlung der Konzentration im Medienbereich (KEK). Elektronisch veröffentlicht: http://www.kek-online.de/cgi-bin/esc/zuschauer.html, Diagramm; abgerufen am 2004-12-01. 
Keller, Dieter (2004): Zur wirtschaftlichen Lage der deutschen Zeitungen 2004. In: BDZV (Hrsg.): Zeitungen 2004. Bundesverband Deutscher Zeitungsverleger e.V., Berlin 2004.

Kern, Egbert (1990): Der Interaktionsansatz im Investitionsgütermarketing, Berlin 1990.

Keuper, Frank (2001): Strategisches Management. Oldenbourg Verlag, München 2001.

Keuper, Frank (2002): Electronic Business und Mobile Business. Ansätze, Konzepte und Geschäftsmodelle. Gabler, Wiesbaden 2002.

Kiefer, Marie Luise (2001): Medienökonomik. Oldenbourg Verlag, München 2001.

Kieser, Alfred; Kubicek, Herbert (1992): Organisation. de Gruyer, Berlin 1992.

Kippele, Flavia (1998): Was heißt Individualisierung? Die Antworten soziologischer Klassiker. Opladen, Wiesbaden 1998.

Kirsch, Werner (1997a): Strategisches Management: Die geplante Evolution von Unternehmen. Verlag Barbara Kirsch, München 1997.

Kirsch, Werner (1997b): Wegweiser zur Konstruktion einer evolutionären Theorie der strategischen Führung. Verlag Barbara Kirsch, München 1997.

Kirsch, Werner (1998): Betriebswirtschaftslehre - Eine Annäherung aus der Perspektive der Unternehmensführung. Verlag Barbara Kirsch, München 1998.

Klages, Helmut (1984): Wertorientierungen im Wandel, Frankfurt 1984.

Klein, Christoph (2001): Online Service Providing - Herausforderungen im Massenmedium Internet; In: Vizjak, Andre; Ringlstetter, Max (Hrsg.): Medienmanagement: Content gewinnbringend nutzen; Gabler, Wiesbaden 2001; S. 99-108.

Kleinaltenkamp, Michael (1995): Standardisierung und Individualisierung, In: Köhler, Richard; Tietz, Bruno; Zentes, Joachim (Hrsg): Handwörterbuch des Marketing. Stuttgart 1995, S. 2354-2364.

Ko, Su-Jeong; Lee, Jung-Hyun (2002): User Preference Mining through Collaborative Filtering and Content Based Filtering in Recommender Systems. In: Proceedings of the Third International Conference in E-Commerce and Web Technologies (EC-Web 2002), Aix-en-Provence, France 2002, S. 244-253.

Kobsa, Alfred; Pohl, Wolfgang (1995): The User Modeling Shell System BGP-MS. In: User Modeling and User-Adapted Interaction, Nr. 4, 1995, S. 59-106.

Köhler, Richard (1994): Target Marketing. In: Die Betriebswirtschaft. 54. Jg., S. 121-123.

Köhntopp, Marit (2000): Identitätsmanagement. In: Bäumler, H.; Breinlinger A.; Schrader, H.-J. (Hrsg.): Datenschutz von A-Z, Luchterhand, Neuwied, 2000. Elektronisch veröffentlicht: http://marit.koehntopp.de/publikationen/idmanage/def/Koehn_00dldmanageDef.pdf; abgerufen am 2004-12-01.

Kollmann, Tobias (1998): Akzeptanz innovativer Nutzungsgüter und -systeme. Konsequenzen für die Einführung von Telekommunikations- und Multimediasystemen. Gabler Verlag, Wiesbaden 1998.

Konrad, Klaus (2001): Mündliche und schriftliche Befragung. Verlag Empirische Pädagogik, Landau 2001.

Konstan, Joseph; Miller, Bradley; Maltz, David; Herlocker, Jonathan; Gordon, Lee; Riedl, John (1997): GroupLens: Applying Collaborative Filtering to Usenet News. In: Communications of the ACM, 03/1997, Band 40, Nr. 3, S. 77-87.

Kondratjew, Nikolai (1984): The Long Wave Cycle. Richardson \& Snyder, 1984. 
Kotkamp, Stefan (2001): Electronic Publishing. Dissertation, Karlsruhe 2001. Elektronisch veröffentlicht: http://www.ubka.uni-karlsruhe.de/vvv/2001/wiwi/4/4.pdf; abgerufen am 2005-04-30.

Krafft, Manfred; Liftin, Thorsten (2002): Adoption innovativer Telekommunikationsdienste: Validierung der Rogers-Kriterien bei Vorliegen potenziell heterogener Gruppen. In: Zeitschrift für betriebswirtschaftliche Forschung, Nr. 2, Jg. 54, S. 64-83.

Krafft, Manfred; Marzian, Sieghard (1997): Dem Kundenwert auf der Spur. In: Absatzwirtschaft, 40. Jg., 6, 1997, S. 104-107.

Kreutzer, Ralf (1992): Zielgruppe-Management mit Kunden-Datenbanken. In Die Betriebswirtschaft, 52. Jg., Nr. 3, S. 325-340.

Kristol, David; Montulli, Lou (1997): HTTP State Management Mechanism. Elektronisch veröffentlicht: http://www.cse.ohio-state.edu/cgi-bin/rfc/rfc2109.html; abgerufen am 2004-12-01.

Kromrey, Helmut (2002): Empirische Sozialforschung: Modelle und Methoden der standardisierten Datenerhebung und Datenauswertung. Opladen 2002.

Kron, Thomas (2000): Individualisierung und soziologische Theorie. Leske + Budrich, Opladen 2000.

Krugman, Paul (1997): Speed Trap - The fuzzy logic of the „New Economy“. Elektronisch veröffentlicht: http://web.mit.edu/krugman/www/speed.html; abgerufen am 2004-12-01.

Kuhlen, Rainer (1995): Informationsmarkt. Chancen und Risiken der Kommerzialisierung von Wissen. Universitätsverlag Konstanz, 1995.

Kumar, Bala; Kline, Paul; Thompson Thimothy (2004): Bluetooth Application Programming with the JAVA APIs. The Morgan Kaufmann Series in Networking. Elsevier, San Francisco 2004.

Küpper, Axel; Reiser, Helmut; Schiffers, Michael (2004): Mobilitätsmanagement im Überblick - Von $2 \mathrm{G}$ zu 3.5G. In: Praxis der Informationsverarbeitung und Kommunikation (2004) 27, S. 68-73.

Labrou, Yannis; Finin, Tim (1999): Yahoo! as an ontology - Using Yahoo! Categories to Describe Documents. In: Proceedings of the 8th International Conference on Information and Knowledge Management, S. 180-187.

Lancaster, Kelvin (1979): Variety, Equity and Efficiency. Columbia University Press, New York 1979.

Le Bodic, Gwenäel (2003): Mobile Messaging Technologies and Services. John Wiley \& Sons, Weinheim 2003.

Leber, Martin (1988): Die Beziehung zwischen Mediennutzungsverhalten und nicht-medialen Freizeitaktivitäten. Verlag Peter Lang, Bern 1988.

Lehmann, Hans; Lehner, Franz (2003): Is there a ,Killer Application' in Mobile Technology? FORWINBericht-Nr.: FWN-2003- 004; FORWIN - Bayerischer Forschungsverbund Wirtschaftsinformatik, Bamberg, Bayreuth, Erlangen-Nürnberg, Regensburg, Würzburg 2003.

Lehner, Franz (2002): Mobile Business / Mobile Service. Elektronisch veröffentlicht: http://wwwmobile.uni-regensburg.de/freiedokumente/Berichte/MobileBusinessMobileServices.pdf; abgerufen am 2004-12-01.

Lehner, Franz (2003): Mobile und drahtlose Informationssysteme. Springer Verlag, Berlin 2003.

Lehner, Franz (2004): Lokalisierungstechniken und Location Based Services. In: WISU, Nr. 02/2004, S. 211.219. 
Leimeister, Jan Marco; Krcmar, Helmut (2002): Ubiquitous Computing. In: Das Wirtschaftsstudium 31 (2002) 10, S. 1284-1294.

Lewis, Arthur (1941): The Two-Part Tariff. Economica, Bd. 8, S. 249-270.

Lin, Justin; Laddaga, Robert; Naito, Hirohisa (2002): Personal Location Agent for Communicating Entities; In: Paternò, Fabio (Hrsg.): Human computer interaction with mobile devices; Springer, Berlin 2002.

Link, Jörg (1993): Merkmale und Einsatzmöglichkeiten des Database Marketing. In: WiSt, 22. Jg., Nr. 1, S. 23-28.

Link, Jörg; Hildebrand, Volker (1995): Mit IT zum Individual Marketing. In: Harvard Business Manager, 17. Jg., Nr. 3, S. 30-39.

Long, Sue; Kooper, Rob; Abowd, Gregory; Atkeson, Christopher (1996): Rapid prototyping of mobile context-aware applications: the Cyberguide case study. In: Proceedings of the Second Annual International Conference on Mobile Computing and Networking, White Plains 1996, S. 97-107.

Mahedevan, B. (2000): Business Models for Internet-Based E-Commerce: An anatomy. In: California Management Review. Sommer 2000, Bd. 42, Nr. 4, S. 55-69.

Manager-Magazin (2002): Deutsche Medien, ein durch und durch schwarzes Jahr. Elektronisch veröffentlicht: http://www.manager-magazin.de/ebusiness/artikel/0,2828,228865,00.html; abgerufen am 200412-01.

Manola, Frank; Miller, Eric (2004): RDF Primer. W3C Recommendation, February 2004. Elektronisch veröffentlicht: http://www.w3.org/TR/rdf-primer/; abgerufen am 2005-04-30.

Mäntyjärvi, Jani; Seppänen, Tapio (2002): Adapting Applications in Mobile Terminals Using Fuzzy Context Information. In: Paternò, Fabio (Hrsg.): Human computer interaction with mobile devices. Springer Verlag, Berlin 2002.

Markellou, Penelope; Rigou, Maria; Sirmakessis, Spiros (2005): Mining for Web Personalization. In: Scime, Anthony (Hrsg.): Web Mining: Applications and Techniques. Idea Group Publishing. Hershey (PA) 2005, S. 27-48.

Marmasse, Natalia; Schmandt, Chris (2002): A User-Centered Location Model; In: Personal and Ubiquitous Computing archive, Bd. 6, Nr. 5-6, S. 318-321.

Martin, Albert (1989): Die empirische Forschung in der Betriebswirtschaftslehre: Eine Untersuchung über die Logik der Hypothesenprüfung, die empirische Forschungspraxis und die Möglichkeit einer theoretischen Fundierung realwissenschaftlicher Untersuchungen. Schäffer-Poeschel Verlag, Stuttgart 1989.

Marx, Karl (1872): Das Kapital. Voltmedia Verlag, Paderborn 1972.

Mason, Edward (1939): Price and production policies of large-scale enterprises. In: American Economic Review, Nr. 29, S. 61-74.

Maturana, Humberto; Valera, Francisco (1987): Die biologische Wurzel des Menschlichen Erkennens. Scherz Verlag, Bern 1987.

Mayer, Rainer (1993): Strategien erfolgreicher Produktgestaltung. Individualisierung und Standardisierung. Deutscher Universitätsverlag, Wiesbaden 1993.

McCann, Joseph; Selsky, John (1984): Hyperturbulence and the Emergence of Type 5 Environments. In: Academy of Management Review, 9 (1984) 3, S. 460-470. 
McQuaid, J.D. (1992): Segment-of-One Marketing: Turning Customer Data into Actionable Information. In: The Journal of Serices Marketing. Vol. 6, Nr. 3, S. 75-78.

MCTA (2005): 5. Konferenz Mobile Commerce Technologien und Anwendungen. Elektronisch veröffentlicht: http://www.mcta.de; abgerufen am 2005-04-30.

Media Perspektive (2002): Basisdaten - Daten zur Mediensituation in Deutschland 2002; Arbeitsgemeinschaft der ARD-Werbegesellschaften, Frankfurt, 2002.

Media Perspektive (2003): Basisdaten - Daten zur Mediensituation in Deutschland 2002; Arbeitsgemeinschaft der ARD-Werbegesellschaften, Frankfurt, 2003.

Media Perspektive (2004): Basisdaten - Daten zur Mediensituation in Deutschland 2004; Arbeitsgemeinschaft der ARD-Werbegesellschaften, Frankfurt, 2004.

Meffert, Heribert (1995): Was versteht man unter dem Kundenwert? Welche Ansatzpunkte ergeben sich zur Verlängerung? In: Handelsblatt, Sonderheft „Berufsinformation und Stellenmarkt” 1995, S. 7-8.

Meffert, Heribert (1998): Marketing: Grundlagen marktorientierter Unternehmensführung: Konzepte Instrumente - Praxisbeispiele. Gabler Verlag, Wiesbaden 1998.

Meffert, Heribert (2000): Marketing - Grundlagen marktorientierter Unternehmenführung. Gabler, Wiesbaden 2000.

Meffert, Heribert (2001): Herausforderungen an das Marketing durch interaktive Medien. In: Ahlert, Dieter; Becker, Jörg; Kenning, Peter; Schütte, Reinhard (Hrsg.): Internet \& Co im Handel. Springer Verlage, Berlin u.a. 2001, S. 161-178.

Meffert, Heribert; Bruhn, Manfred (1997): Dienstleistungsmarketing. Gabler Verlag, Wiesbaden 1997.

Meier, Bernd (2003): Individualisierung - Ausführliche Begriffsbeschreibung. Elektronisch veröffentlicht: http://www.chancenfueralle.de/Lexikon///Individualisierung.html; abgerufen am 2005-04-01.

Meier, Roland (2002): Generierung von Kundenwert durch mobile Dienste. Deutscher Universitätsverlag, Wiesbaden 2002.

Melville, Prem; Mooney, Raymond; Nagarajan, Ramadass (2002): Content-Boosted Collaborative Filtering for Improved Recommendations. In: Eighteenth national conference on Artificial intelligence. Edmonton (Kanada), S. 187-192.

Mertens, Peter (1994): Datenmustererkennung in der Ergebnisrechnung mit Hilfe der Cluseranalyse; In: DBW, 54. Jg. 1994, Heft 6, S. 739-753.

Mertens, Peter (1995): Mass Customization (Massen-Maßfertigung). In: Die Wirtschaftsinformatik, 37. Jg., Nr. 5, S. 503-506.

Mertens, Peter (1997): Recommender Systems. In: Wirtschaftsinformatik, Nr. 39, Band 4 (1997), S. 401-404.

Mertens, Peter; Bodendorf, Freimut; König, Wolfgang; Picot, Arnold; Schumann, Matthias; Hess, Thomas (2005): Grundzüge der Wirtschaftsinformatik. Springer, Berlin u.a. 2005.

Mertens, Peter; Griese, Joachim (2002): Integrierte Informationsverarbeitung. Band 2: Planungs- und Kontrollsysteme in der Industrie. Gabler Verlag, Wiesbaden 2002.

Merz, Michael (2000): Electronic Commerce - Marktmodelle, Anwendungen und Technologien. dpunktVerlag, Heidelberg 2000. 
Meyer, Matthias; Weingärtner, Stefan; Lieven, Oliver; Jahke, Thilo (2001): Web Mining und Personalisierung in Echtzeit. Arbeitspapier Nr. 5 des Seminars für Empirische Forschung und Quantitative Unternehmensplanung der LMU München. Elektronisch veröffentlicht: http://www.efoplan.bwl.unimuenchen.de/pdf/AP0501_Web_Mining_und_Personalisierung.pdf; abgerufen am 2004-12-01.

Meyer-Lucht, Robin (2002): Zwischenzeit. Brand Eins Nr. 10/02, S. 144-148. Elektronisch veröffentlicht: http://www.brandeins.de/magazin/archiv/2002/ausgabe_10/schwerpunkt/artikel22.html; abgerufen am 2004-12-01.

Miller, George (1956): The Magical Number Seven, Plus or Minus Two: Some Limits on Our Capacity for Processing Information. In: The Psychological Review (1956), Nr. 63, S. 81-97. Elektronisch veröffentlicht: http://www.well.com/user/smalin/miller.html; abgerufen am 2005-04-01.

Mings, Susan; White, Peter (2000): Profiting from Online News: The Search for Viable Business Models. In: Kahin, Brian; Varian, Hal R. (Hrsg.): Internet Publishing and Beyond - The Economics of Digital Information and Intellectual Property. The MIT Press, Cambridge 2000; S. 62-96.

Mintzberg, Henry (1994): The rise and fall of strategic planning: reconceiving roles for planning, plans, planners. The Free Press, New York 1994.

Mintzberg, Henry (1999): Strategy Safari - Eine Reise durch die Wildnis des strategischen Managements. Ueberreuter, Wien 1999.

Mobasher, Bamshad (2002): WebPersonalizer: A Server-Side Recommender System Based on Web Usage Mining. Elektronisch veröffentlicht: http://facweb.cs.depaul.edu/research/TechReports/TR01004.pdf; abgerufen am 2004-12-01.

Mobasher, Bamshad; Cooley, Robert; Srivastava, Jaideep (1999): Creating Adaptive Web Sites through Usage-Based Clustering of URLs. In: Proceedings of the 1999 Workshop on Knowledge and Data Engineering Exchange, S. 19.

Mobasher, Bamshad; Dai, Honhua; Luo, Tao; Nakagawa, Miki (2001): Discovery and Evaluation of Aggregate Usage Profiles for Web Personalization. Elektronisch veröffentlicht: http://robotics.stanford.edu/users/ronnykMEBKDD-DMKD/Mobasher.pdf; abgerufen am 2004-12-01.

Mobile $\mathrm{HCl}$ (2003): Fifth International Symposium on Human Computer Interaction with Mobile Devices and Services. Elektronisch veröffentlicht: http://hcilab.uniud.it/mobilehci/index.html; abgerufen am 2005-04-30.

MobiSys (2003): The First International Conference on Mobile Systems, Applications, and Services. Elektronisch veröffentlicht: http://www.usenix.org/events/mobisys03/; abgerufen am 2005-04-30.

MoMM (2004): Second International Conference on Advances in Mobile Multimedia. Elektronisch veröffentlicht: http://www.iiwas.org/conferences/momm2004/; abgerufen am 2005-04-30.

Morgan, Rob; Hunt, Shelby (1994): The Commitment-Trust Theory of Relationship Marketing. In: Journal of Marketing. Vol. 58, Nr. 3, S. 20-38.

Mowshowitz, Abbe (1992): On the market Value of Information Commodities I-III. In: Journal of the Amercian Society for Information Science, Bd. 43, Nr. 3, S. 225-248.

Müller, Eva; Preissner, Anne; Schwarzer, Ursula (2002): Überleben in dünner Luft - Branchenanalyse Telekommunikation; In: Manager-Magazin 10/2002, S. 168-179.

Müller, Christian; Heitmann, Mark (2002): Methoden der Strategiefindung unter der Bedingung der Konvergenz von Branchen am Beispiel Mobile. Konzepte des Medien- und Kommunikationsmanagements, mcminstitute, University of St. Gallen, 2002. 
Müller, Günter; Eymann, Torsten; Kreutzer, Michael (2003): Telematik- und Kommunikationssysteme in der vernetzten Wirtschaft; Lehrbücher Wirtschaftsinformatik; Oldenbourg Verlag, München 2003.

Müller-Böhling, Detlef; Müller, Michael (1986): Akzeptanzfaktoren der Bürokommunikation. Oldenbourg, München 1986.

Müller-Kalthoff, Björn (2002): Cross-Media-Management. In: Müller-Kalthoff, Björn (Hrsg.): Cross-Media Management. Springer, Berlin 2002, S. 19-40.

Müller-Verse, Falk (1999): Mobile Commerce Report; Durlacher Research Report. Elektronisch veröffentlicht: http://www.durlacher.com/downloads/mcomreport.pdf; abgerufen am 2004-05-31.

Nash, Edward (1993): Database Marketing. New York 1993.

Nefiodow, Leo (2001): Der sechste Kondratieff. Rhein-Sieg Verlag, St.Augustin 2001.

Neudorfer, Reinhard; Simonitsch, Karoline (2003): Erfolgskritische Faktoren für Mobilfunk-Geschäftsmodelle: Kooperationen und Vertrauen. In: IM - Fachzeitschrift für Information Management \& Consulting. 02/2003, S. 63-68.

Nicolai, Alexander; Petersmann, Thomas (2001): Die Möglichkeiten des Mobile Business - eine qualitative Betrachtung. In: Nicolai, A.; Petersmann, T. (Hrsg.): Strategien im M-Commerce. SchäfferPoeschel Verlag, Stuttgart 2001.

Nicolai, Alexander; Petersmann, Thomas (2001): Strategien im M-Commerce. Grundlagen - Management - Geschäftsmodelle. Schäffer-Poeschel Verlag, Stuttgart 2001.

Nielsen, Jacob (1993): Usability Engineering. Academic Press, San Diego 1993.

Nieschlag, Robert; Dichtl., Erwin; Hörschgen, Hans (1997): Marketing. Duncker \& Humblot, Berlin 1997.

Nokia (1999): The demand for mobile Value-Added Services. Elektronisch veröffentlicht: http://www.telecomsportal.com/Assets_papers/Wireless/Nokia_mobile_vas.pdf; abgerufen am 200504-30.

Nokia (2003): Bluetooth Technology Overview. Version 1.0. April 4, 2003. Elektronisch veröffentlicht: http://ncsp.forum.nokia.com/downloads/nokia/documents/Bluetooth_Technology_Overview_v1_0.pdf; abgerufen am 2004-05-31.

Nokia (2004): Nokia Unveils the world's first NFC product, Nokia NFC shell for Nokia 3220 phone. Pressemitteilung vom 02.11.2004. Elektronisch veröffentlich: http://press.nokia.com/PR/200411/966879_5.html; abgerufen am 2004-12-01.

NTT (2004): i-mode business strategy. The official i-mode Website. Elektronisch veröffentlicht: http://www.nttdocomo.com/corebiz/imode/why/strategy.html; abgerufen am 2004-05-31.

Oebbeke, Alfons (2003): Internet-Zahlen (2003). Elektronisch veröffentlicht: http://www.glossar.de/glossar/1frame.htm?http\%3A//www.glossar.de/glossar/z_intrzahl.htm; abgerufen am 2004-12-01.

OeBF (2002): Open eBook Publication Structure Specification Version 1.2. Open eBook Forum. Elektronisch veröffentlicht: http://www.openebook.org/oebps/oebps1.2/index.htm; abgerufen am 2004-12-01.

OECD (1980): OECD Guidelines on the Protection of Privacy and Transborder Flows of Personal Data. Elektronisch veröffentlicht: http://www1.oecd.org/publications/e-book/9302011E.PDF; abgerufen am 2005-04-30.

Oehmichen, Ekkehardt; Schröter, Christian (2002): Zur Habitualisierung der Onlinenutzung. Media Perspektiven 08/2002; S. 376-388. 
Oesterdiekhoff, Brigitte (2004): Transcoding von Webinhalten. In: Informatik Spektrum, Band 27, Nr. 5, Oktober 2004, S. 448-452.

Oi, Walter (1971): A Disneyland Dilemma - Two-Part Tariffs for a Mickey Mouse Monopoly. In: Quarterly Journal of Economics, Bd. 85, Nr. 1, S. 77-96.

Olderog, Torsten; Skiera, Bernd (1998): Vorteilhaftigkeit von Preisbündelungsstrategien. Elektronisch veröffentlicht: http://www.wiwi.tu-freiberg.de/wi/courses/im/bundelung.pdf; abgerufen am 2004-12-01.

Olivero, Nadia; Lunt, Peter (2004): Privacy versus willingness to disclose in e-commerce exchanges: The effect of risk awareness on the relative role of trust and control. In: Journal of Economic Psychology, Nr. 25, 2004, S. 243-262.

OMA (2003): User Agent Profile. Open Mobile Alliance Spezifikation Nr. OMA-UAProf-v2_0-20030520-C. Elektr. veröffentlicht: http://www.openmobilealliance.org/release_program/docs/UAProf/OMA-UAProfV2_0-20030520-C.PDF; abgerufen am 2005-04-30.

Orwant, Jon (1995): Heterogeneous Learning in the Doppelgänger User Modelling System. In: User Modelling and User-Adapted Interaction, Nr. 4, 1995, S. 107-130.

Ovum (2000): Mobile E-Commerce: Market Strategies - An Ovum Report. London 2000.

P7S1 Holding (2004): Firmenhomepage. Elektronisch veröffentlicht: http://www.p7s1holding.com; abgerufen am 2004-12-01.

Palmgreen, Philip (1983): The uses and gratifications approach: a theoretical perspective. In: Media Panel, Report Nr. 30, November 1983.

Palmgreen, Philip (1984): Der uses and gratifications approach: Theoretische Perspektiven und praktische Relevanz. In: Rundfunk und Fernsehen, Nr. 32, 1984. S. 51-62.

Pascoe, Jason (1998): Adding generic contextual capabilities to wearable computers. In: Proceedings of the Second International Symposium on Wearable Computers. Pitt sburgh 1998.

Passani, Luca (2002); Building Usable Wireless Applications for Mobile Phones, In: Paternò, Fabio (Hrsg.): Human computer interaction with mobile devices. Springer Verlag, Berlin 2002.

Pazzani, Michael; Billsus, Daniel (1997): Learning and Revising User Profiles: The Identification of Interesting Web Sites. In: Machine Learning, Nr. 27, Kluwer Academic Publishers, 1997, S. 313-331.

Pediaditakis, Michael; Shrimpton, David (2003): Device neutral pipelined processing of XML documents. Elektronisch veröffentlicht: http://www.cs.kent.ac.uk/people/rpg/mp49/localPubs/ xmlPipeW3C2003.pdf; abgerufen am 2005-04-30.

Penker, Magnus; Eriksson, Hans-Erik (2000): Business Modeling With UML: Business Patterns at Work. Wiley Computer Publishing, New York 2000.

Peppers, Don; Rogers, Martha (1996): Strategien für ein individuelles Kundenmarketing - Die 1:1 Zukunft. Droemersche Verlagsanstalt, München 1996.

Pescatore, Paolo (2005): Opportunities for MVNOs in Western Europe. IDC Report Nr. \#HW58M, Juni 2005.

Petrison, Lisa; Blattberg, Robert; Wang, Paul (1993): Database Marketing. Past, Present, and Future. In: Journal of Direct Marketing, Vol. 7, Nr. 3, S. 27-43.

Phillips, Lee Anne (2000): Using XML. Macmillan, London 2000.

Picard, Robert (1989): Media Economics. Sage Publications, London 1989. 
Picot, Arnold; Dietl, Helmut; Franck, Egon (1997): Organisation - eine ökonomische Perspektive. Schäffer-Poeschel, Stuttgart 1997.

Picot, Arnold; Franck, Egon (1988): Die Planung der Unternehmensressource Information (I). In: WISU 10 (1988), S. 544-549.

Picot, Arnold; Reichwald, Ralf; Wigand, Rolf (2001): Die grenzenlose Unternehmung. Gabler Verlag, Wiesbaden 2001.

Pigou, Arthur Cecil (1950): The economics of welfare. Macmillan and co., London 1950.

Piller. Frank Thomas (2001): Mass Customization - Ein wettbewerbsstrategisches Konzept im Informationszeitalter. Deutscher Universitäts-Verlag, 2001.

Pine, Joseph (1993): Mass Customization - The New Frontier in Business Competition. Harvard Business School Press, Boston 1993.

Porter, Michael (1998): Wettbewerbsstrategie. Campus Verlag, Frankfurt 1998.

Pressmar, Dieter (1982): Zur Akzeptanz von computergestützten Planungssystemen. In: Krallmann, Hermann (Hrsg.): Unternehmensplanung und -steuerung in den 80er Jahren - eine Herausforderung an die Informatik. Springer Verlag, Berlin 1982, S. 324-348.

Rappaport, Alfred (1994): Shareholder Value: Wertsteigerung als Maßstab für die Unternehmensführung. Schäffer-Poeschel, Stuttgart 1994.

Rawolle, Joachim (2002): Content Management integrierter Medienprodukte - Ein XML-basierter Ansatz. Gabler, Wiesbaden 2002.

Rawolle, Joachim; Hess, Thomas (2001): Integrierte Medienprodukte - Grundlagen, Ausprägungen und Beispiele. Arbeitsbericht der Abteilung Wirtschaftsinformatik II der Universität Göttingen; Göttingen 2001.

Rechenberg, Peter; Pomberger Gustav (2002): Informatik-Handbuch. Hanser Verlag, München 2002.

Reichwald, Ralf (1978): Zur Notwendigkeit bei der Entwicklung neuer Systeme der Bürotechnik. In: Die Akzeptanz neuer Bürotechnologie, Band 1, München 1978.

Reichwald, Ralf (2002): Mobile Kommunikation. Wertschöpfung, Technologien, neue Dienste. Gabler Verlag, Wiesbaden 2002.

Reichwald, Ralf; Meier, R; Fremuth, Natalie (2002): Die mobile Ökonomie - Definition und Spezifika. In: Reichwald, Ralf (Hrsg.): Mobile Kommunikation- Wertschöpfung, Technologien, neue Dienste. Wiesbaden 2002, S. 5-13.

Reiter, Michael; Rubin, Aviel (1999): Crowds: Anonymity for Web Transactions. Communications of the ACM, Bd. 42, Nr. 2, 1999, S. 32-48.

Resnick, Paul; Varian, Hal R. (1997): Recommender Systems. In: Communications of the ACM; Band 40, Nr. 3 (03/1997), S. 56-58.

Richie, Stephanie; Brebner, Gavin; Gittler, Mickey (2001): Client-Side Profile Storage: A means to put the user in control. HP Laboratories Grenoble, 2001. Elektronisch veröffentlicht: http://www.hpl.hp.com/techreports/2001/HPL-2001-291.pdf; abgerufen am 2005-04-30.

Rieker, Stephen (1995): Bedeutende Kunden: Analyse und Gestaltung langfristiger Anbieter-NachfragerBeziehungen auf industriellen Märkten. Wiesbaden 1995. 
Roberts, Mary Lou (1992): Expanding the Role of the Direct Marketing Database. In: Journal of Direct Marketing, Vol. 6, Nr. 2, S. 51-60.

Rogers, Everett (1995): Diffusion of innovations. Free Press, New York 1995.

Rosenblatt, Bill; Trippe, Bill; Mooney, Stephen (2002): Digital Rights Management. M\&T Books, New York, 2002.

Röttger-Gerigk, Stefanie (2002): Mobile Dienste - Aber welche? In: Gora, Walter; Röttger-Gerigk, Stefanie (2002): Handbuch Mobie-Commerce. Technische Grundlagen, Marktchancen und Einsatzmöglichkeiten; Springer Verlag, Berlin 2002.

Rüegg-Sturm, Johannes (2000): Was ,ist' eine Unternehmung? Ein Unternehmensmodell zur Einführung in die Grundkategorien einer modernen Managementlehre. Diskussionsbeiträge Nr. 36, St. Gallen. IfBHSG.

Runte, Matthias (2000): Personalisierung im Internet - Individualisierte Angebote mit Collaborative Filtering. Deutscher Universitäts-Verlag, Wiesbaden 2000.

Russel, Stuart; Norvig, Peter (2003): Artificial Intelligence, a modern approach. Pearson Education, New Jersey 2003.

Rzesnitzek, Michael (2003): Das medienübergreifende Produktkonzept der Financial Times Deutschland. In: Habann, Frank (Hrsg.): Innovationsmanagement in Medienunternehmen. Gabler Verlag, Wiesbaden 2003.

Salber, Daniel; Dey, Anind; Abowd, Gregory (1999): The Context Toolkit: Aiding the Development of Context-Enabled Applications. In: Proceedings of the CHI'99, Pittsburgh 1999.

Salton, Gerald; Wong, A.; Yang, C. (1975): A vector space model for automatic indexing. Communications of the ACM, Bd. 18, Nr. 11, 1975, S. 613-620.

Saxer, Ulrich (1996): Medien- und Journalismusethik. In: Holzhey, Helmut; Schaber, Peter (Hrsg.): Ethik in der Schweiz. S. 109- 123.

Schackmann, Jürgen (2003): Ökonomisch vorteilhafte Individualisierung. Verlag Dr. Kovač, Hamburg 2003.

Schackmann, Jürgen; Link, Hubert (2001): Mass Customization of Digital Products in Electronic Commerce. Diskussionspapier WI-97, Lehrstuhl für Betriebswirtschaftslehre, Wirtschaftsinformatik \& Financial Engineering, Universität Augsburg 2001.

Schafer, Ben; Konstan, Joseph; Riedl, John (2001): E-Commerce Recommendation Applications. In: Data Mining and Knowledge Discovery. Bd. 5, Nr. 1-2 2001, S. 115-153.

Schäfer Henry (1995): Beziehungsmanagement durch Dialogmarketing: Bankloyalität aus institutionenökonomischer Sicht. In: zfbf-Sonderheft 35/1995, S. 119-137.

Scheer, August-Wilhelm (1990): CIM. Der computergesteuerte Industriebetrieb. Springer, Berlin u.a. 1990.

Scheer, August-Wilhelm; Feld, Thomas; Göbl, Mark; Hoffmann, Michael (2001): Das Mobile Unternehmen; In: Information Management\&Consulting, 16. Jg., Nr. 2, Saarbrücken 2001, S. 7-15.

Schelsky, Helmut (1979): Die Bedeutung des Klassenbegriffs für die Analyse unserer Gesellschaft. In: Helmut Schelsky (Hrsg.): Auf der Suche nach der Wirklichkeit. Gesammelte Aufsätze, München 1979, S. 350-392. 
Schenk, Michael; Hermann Dahm; Sonje, Deziderio (1996): Innovationen im Kommunikationssystem. LIT Verlag, Münster 1996.

Schildhauer, Thomas (2003): Das Internet als Medienplattform - Determinanten und Prognose ihrer Bedeutung für das Mediennutzungsverhalten aus Rezipientensicht. Studienband im Auftrag der AOL Deutschland $\mathrm{GmbH}$, Berlin 2003.

Schilit, Bill; Adams, Norman; Want, Roy (1994): Context-Aware Computing Applications. In: IEEE Workshop on Mobile Computing Systems and Applications, Dezember 1994.

Schiller, Jochen (2002): Mobilkommunikation. In: Krüger, Gerhard; Reschke, Dietrich (2002): Telematik. Netze - Dienste - Protokolle. Fachbuchverlag Leipzig, München 2002, S. 345-390.

Schiller, Jochen (2003): Mobile Communications. Addison-Wesley. Upper Saddle River 2003.

Schleuning, Christian (1995): Dialogmarketing. Theoretische Fundierung, Leistungsmerkmale und Gestaltungsansätze. Ettlingen 1994.

Schmalensee, Richard (1984): Pricing of Product Bundles. Elektronisch veröffentlicht: http://www.jstor.org/view/00219398/di993838/99p0133h/0; abgerufen am 2004-12-01.

Schmidt, Albrecht; Aidoo, Kofi; Takaluoma, Antti; Tuomela, Urpo; Van Laerhoven, Kristof; Van de Velde, Walter (1999): Advanced interaction in context. In: Proceedings of First International Symposium on Handheld and Ubiquitous Computing HUC'99. Karlsruhe 1999, S. 89-101.

Schmidt, Albrecht; Beigl, Michael; Gellersen, Hans-Werner (1999): There is more to context than location. In: Computers and graphics Bd. 23, Nr. 6, S. 893-901.

Schneider, Paul (1997): Produktindividualisieurng als Marketing-Ansatz. Dissertation Nr. 2010, Universität St. Gallen 1997.

Schockert, Sabine (2003): Datenspuren im Internet. Elektronisch veröffentlicht: http://alice.zfn.unibremen.de/ a02h/spuren/logfile-auswertung.htm; abgerufen am 2004-12-01.

Scholz, Christian; Stein, Volker; Eisenbeis, Uwe (2001): Die TIME-Branche: Konzepte - Entwicklungen Standorte. Rainer Hampp Verlag, München und Mering 2001.

Schönbach, Klaus; Preiser, Wolfram (1998): Was wird aus dem Zeitungslesen. In: Gerhards, Maria; Klingler, Walter; Roters, Gunnar (Hrsg.): Medienrezeption seit 1945. Forschungsbilanz und Forschungsperspektiven. Baden-Baden 1998.

Schreck, Jörg (2000): Security and Privacy in User Modeling. Dissertation, Essen 2000.

Schreyögg, Georg (1984): Unternehmensstrategie - Grundfragen einer Theorie der strategischen Unternehmensführung. de Gruyter, Berlin 1984.

Schulzki-Haddouti, Christiane (2000): Unsichtbar und raffiniert - die verdeckten Ermittlungen der kleinen Schwestern. In: Bäumler, Helmut (Hrsg.): E-Privacy. Verlag Vieweg, Wiesbaden 2000, S. 20-26.

Schumann, Jochen; Meyer, Ulrich; Ströbele, Wolfgang (1999): Grundzüge der mikroökonomischen Theorie. Springer, Berlin 1999.

Schumann, Matthias (2002): Betriebswirtschaftliche und technische Grundlagen von E-Commerce und M-Commerce. In: Keuper, Frank (Hrsg.): Electronic Business und Mobile Business, Gabler, Wiesbaden 2002, S. 3-26.

Schumann, Matthias (2003): Möglichkeiten der Mehrfachverwertung von Inhalten bei Medienunternehmen - technische, organisatorische und ökonomische Betrachtung. In: Ehrenberg, Dieter; Kaftan 
Hans-Jürgen (Hrsg.): Herausforderungen der Wirtschaftsinformatik in der Informationsgesellschaft, Leipzig, 2003.

Schumann, Matthias; Hess, Thomas (2002): Grundfragen der Medienwirtschaft. Springer Verlag, Berlin 2002.

Schumpeter, Joseph (1964): Theorie der wirtschaftlichen Entwicklung. Berlin 1964.

Schüring, Hendrik (1991): Database Marketing. Der Einsatz von Datenbanken für Direktmarketing, Verkauf und Werbung. Landsberg/Lech, 1991.

Schweikart, Rudolf (1994): Organisation, Individualität, Konsum. LIT Verlag, Münster 1994.

Seidenfaden, Lutz; Kahnwald, Nina; Kaspar, Christian; Ortelbach, Björn (2005): Mediennutzung im digitalen Leben: Active Content Interfaces, Paid Content und integrierte Geschäftsmodelle. Business Village, Berlin 2005.

Seller, Marianne; Gray, Paul (1999): A Survey of Database Marketing. Center for Research on Information Technology and Organizations, Paper 1999/147. Elektronisch veröffentlicht: http://repositories.collib.org/cgi/viewcontent.cgi?article=1132\&context=crito; abgerufen am 01.04.2005.

Selz, Daniel (1999): Value Webs: Emerging forms of fluid and flexible organizations: Thinking, organizing, communicating, and delivering value on the Internet. Dissertation, St. Gallen 1999.

Sennewald, Nicola (1998): Massenmedien und Internet - Zur Marktentwicklung der Pressebranche. Gabler, Wiesbaden 2002.

Shani, David; Chalasani, Sujana (1993): Exploiting Niches Using Relationship Marketing. In: Journal of Business and Industrial Marketing. Vol. 8, Nr. 4, S. 58-66.

Shapiro, Carl; Varian, Hal R. (1999): Information Rules - A strategic guide to the network economy. Harvard Business School Press, Boston 1999.

Shaw, Robert; Stone, Merlin (1988): Database Marketing. John Wiley \& Sons, 1988.

Shepard, Stephen (1997): The New Economy: What it really means. Business Week, 1997-11-17. Elektronisch veröffentlicht: http://www.businessweek.com/1997/46/b3553084.htm; abgerufen am 2004-12-01.

SIG (2004): The Official Bluetooth Membership Site. Elektronisch veröffentlicht: https://uww.bluetooth.org; abgerufen am 2004-05-31.

Sikora, Axel (2001): 802.11: Standard für drahtlose Netze. In: tecCHANNEL. Elektronisch veröffentlicht: http://www.tecchannel.de/hardware/680/index.html; abgerufen am 2003-07-31.

Silberer, Günter; Hannecke, Nicole (1999): Akzeptanz und Wirkung von multimedialen Kiosksystemen im Handel. In: Silberer, Günter (Hrsg.): Beiträge zur Marketingwissenschaft. Nr. 23, Universität Göttingen 1999.

Silberer, Günter; Wohlfahrt, Jens; Wilhelm, Thorsten (2002): Mobile Commerce. Grundlagen, Geschäftsmodelle, Erfolgsfaktoren. Gabler Verlag, Wiesbaden 2002.

Simmel, Georg (1900): Zur Psychologie des Geldes. Elektronisch veröffentlicht: http://www.digbib.org/Georg_Simmel_1858/Zur_Psychologie_des_Geldes_.pdf; abgerufen am 01.04.2005.

Simon, Herbert Alexander (1957): Administrative Behavior. Free Press, New York 1957. 
Simonitsch, Karoline (2003): Mobile Business. Geschäftsmodelle und Kooperationen. Josef Eul Verlag, Lohmar 2003.

Sirkin, Harold; Dean, David (2000): Mobile Commerce - Winning the on-air consumer. Elektronisch veröffentlicht: http://www.bcg.com/publications/files/M-Commerce_Nov_2000_Summary.pdf; abgerufen am 2005-04-30.

Sjurts, Insa (1996): Die deutsche Medienbranche; Gabler, Wiesbaden 1996.

Sjurts, Insa (2002a): Strategien in der Medienbranche - Grundlagen und Fallbeispiele. Gabler, Wiesbaden 2002.

Sjurts, Insa (2002b): Cross-Media Strategien; In: Müller-Kalthoff, Björn (Hrsg.): Cross-Media Management. Springer, Berlin 2002, S. 3-18.

Skiera, Bernd (2001): Preisdifferenzierung. In: Albers, Sönke; Clement, Michel; Peters, Kay; Skiera, Bernd (Hrsg.): Marketing mit interaktiven Medien - Strategien zum Markterfolg. F.A.Z.-Institut für Management-, Markt- und Medieninformationen, Frankfurt 2001.

Smith, Michael; Welty, Chris; McGuinness, Deborah (2004): OWL Web Ontology Language Guide. W3C Recommendation, February 2004. Elektronisch veröffentlicht: http://www.w3.org/TR/owlguide/; abgerufen am 2005-04-30.

Smyth, Barry, Cotter; Paul (2003): Intelligent Navigation for Mobile Internet Portals. In: Proceedings of the 18th International Joint Conference on Artificial Intelligence (IJCAI-03), Acapulco 2003. Elektronisch veröffentlicht: http://www.dimi.uniud.it/workshop/ai2ia/cameraready/smyth.pdf; abgerufen am 2004-12-01.

Sommerwerck, Marion (2004): Vom Kampf ums Wohnzimmer profitieren vor allem die Konsumenten. A.T. Kearney News Release vom 21.10.2004. Elektronisch veröffentlicht: http://www.atkearney.de/content/misc/wrapper.php/id/49250/name/pdf_at_kearney__kampf_ums_wohnzimmer_10983513052fca.pdf; abgerufen am 2004-12-01.

Sonntag, Michael (1999): Das verborgene des Herzens - Zur Geschichte der Individualität. Rowohlt Verlag, Hamburg 1999.

Spiegel-Online (2003): Brutales Minusjahr für die Musikbranche. Spiegel Online vom 26.02.2003. Elektronisch veröffentlicht: http://www.spiegel.de/wirtschaft/0,1518,237876,00.html; abgerufen am 200412-01.

Spiegel-Online (2004): Immer mehr illegal gebrannte DVDs. Spiegel Online vom 27.10.2004, Elektronisch veröffentlicht: http://www.spiegel.de/netzwelt/technologie/0,1518,325318,00.html. Abruf am 01.12.2004.

Spiegel-Online (2005): Allianz gegen Franz: Cohn-Bendit findet erste Mitstreiter. Elektronisch veröffentlicht: http://www.spiegel.de/sportfussball/0,1518,350213,00.html; abgerufen am 2005-04-30.

Spiesecke, Hartmut (2004): Inlandsabsatz 1. Halbjahr 2004. Bundesverband der Phonographischen Wirtschaft e.V.; Elektronisch veröffentlicht: http://www.ifpi.de/news/news-411.htm.

Spiliopoulou, Myra (2001): Web Usage Mining: Data Mining über die Nutzung des Web. In: Hippner, Hajo; Küsters, Ulrich; Meyer, Matthias; Wilde, Klaus (Hrsg.): Handbuch Data Mining im Marketing. Wiesbaden 2001, S. 489-510.

Spremann, Klaus; Klinkhammer, Margret (1985): Grundgebühren und zweiteilige Tarife. In: ZfB, 55. Jg., Heft 8, S. 790-820. 
Staab, Steffen; Erdmann, Michael; Mädche, Alexander (2001): Ontologies in RDF(S). In: Linköping Electronic Articles in Computer and Information Science, Bd. 6, Nr. 9, 2001.

Stähler, Patrick (2002): Geschäftsmodelle in der digitalen Ökonomie. Josef Eul Verlag, Lohmar 2002.

Stahmer, Frank (1995): Ökonomie des Presseverlages. Reinhard Firscher Verlag, München 1995.

Steimer, Fritz (2001): Mobile Business - Top oder Flop?. In: Absatzwirtschaft, 44. Jg., Nr. 5, 2001, S. 134-137.

Steinhausen, Detlef; Langer, Klaus (1977): Clusteranalyse: Einführung in Methoden und Verfahren der automatischen Klassifikation. de Gruyter Verlag, Berlin 1977.

Stigler, George Joseph (1961): The Economics of Information. In: The Journal of Political Economy, Vol. 69 , Nr. 3, S. 213-225. Elektronisch veröffentlicht:

http://www.jstor.org/view/00223808/di950876/95p0002d/0; abgerufen am 2004-12-01.

Stipp, Horst (1999): Convergence Now? In: The International Journal on MediaManagement, Volume 1, Nr. 1, 1999.

Stöcker, Christian (2005): Der Rechner als Redakteur. In: Spiegel Online, 26.06.2005. Elektronisch veröffentlicht: http://www.spiegel.de/netzwelt/netzkultur/0,1518,362087,00.html abgerufen am 2005-04-30.

Stoetzer, Matthias; Mahler, Alwin (1995): Die Diffusion von Innovationen in der Telekommunikation. Springer Verlag, Berlin 1995.

Strang, Thomas; Linnhoff-Popien, Claudia (2004): A Context Modeling Survey. Proceedings of the Sixth International Conference on Ubiquitous Computing, Nottingham 2004.

Suarez, Franciscus (1976): Über die Individualität und das Individuationsprinzip. In: Specht, Rainer (Hrsg.): Fünfte metaphysische Disputation. Hamburg 1976.

Sydow, Linda; Beckmann, Ilse (2004): Jahresbericht 2004 der Regulierungsbehörde für Telekommunikation und Post gem. § 122. Elektronisch veröffentlicht: http://www.regtp.de/imperia/md/content/aktuelles/jb2004_050308.pdf; abgerufen am 2004-04-30.

Syverson, Paul; Goldschlag, David; Reed, Michael (1997): Anonymous connections and onion routing. In: Proceedings of the IEEE Symposium on Security and Privacy, 1997, S. 44-54.

Taeger, Jürgen (1995): Datenschutzrecht in Europa. In: Europäisches Wirtschafts- und Steuerrecht (EWS) 1995, S. 69-80.

Takashi Mobile (2005): MVNO/SP List. Elektronisch veröffentlicht: http://www.takashimobile.com/mvno.html; abgerufen am 2005-04-30.

Taschner, Andreas (2001): Interaktive Medien als Systemgut. In: Albers, Sönke; Clement, Michael; Peters, Kay; Skiera, Bernd (Hrsg.): Marketing mit interaktiven Medien. F.A.Z.-Institut für Management-, Markt- und Medieninformationen. Frankfurt a. M. 2001, S. 83-100.

TdW (2004): Typologie der Wünsche Intermedia 2004/2005. Zweite Erhebungswelle (20.05.200415.07.2004). Burda Advertising Center $\mathrm{GmbH}$. Elektronisch veröffentlicht: http://uww.bac.de/de/std/tdw.html; abgerufen am 2004-12-01.

Thielmann, Bodo (2000): Strategisches Innovationsmanagement in konvergierenden Märkten: Medien und Telekommunikationsunternehmen in Online-Diensten und im digitalen Fernsehen. Gabler, Wiesbaden 2000.

TIMElabs (2002): Winning in Mobile eMarkets. Eschborn 2002. Elektronisch veröffentlicht: http://www.timelabs.de/pdf/TIMElabs_C_mB2B_d.pdf; abgerufen am 2005-04-30. 
Timmers, Paul (1998): Business Models for Electronic Markets. In: Electronic Markets - International Journal of Electronic Commerce \& Business Media. Bd. 8, Nr. 2, S. 3-8.

Tirole, Jean (1999): Industrieökonomik. Oldenbourg Verlag, München 1999.

Toffler, Alvin (1980): Die Zukunftschance: Von der Industriegesellschaft zu einer humaneren Zivilisation. Bertelsmann, München 1980.

Tomczak, Torsten; Schögel, Marcus; Birkhofer, Ben (1999): A Typology of Business Models in Electronic Commerce for Marketing Consumer Goods. In: Proceedings of the third international conference on telecommunications and information markets (COTIM-99), Rhode Island 1999.

T-Online (2004): T-Online Vision. Elektronisch veröffentlicht: http://www.t-online-vision.tv/; abgerufen am 2004-12-01.

Tourowski, Klaus; Pousttchi, Key (2004): Mobile Commerce. Grundlagen und Techniken. Springer Verlag, Berlin 2004.

Trommsdorff, Volker (1975): Die Messung von Produktimages für das Marketing. Grundlagen und Operationalisierung. Carl Heymanns, Köln 1974.

Uhr, Wolfgang; Esswein, Werner; Schoop, Eric (2003): Grußwort des Veranstalters zur 6. Internationalen Tagung Wirtschaftsinformatik 2003 in Dresden zum Thema „Medien - Märkte - Mobilität“. Elektronisch veröffentlicht: http://web.tu-dresden.de/wi2003/georg/kategorien/zurtagung/grusswort.html; abgerufen am 2005-04-30.

Van Harmelen, Frank; Patel-Schneider, Peter; Horrocks, lan (2001): Reference description of the DAML+OIL (March 2001) ontology markup language. Elektronisch veröffentlicht: http://www.daml.org/2001/03/reference.html; abgerufen am 2005-04-30.

Van Laerhoven, Kristof; Aidoo, Kofi; Lowette, Steven (2001): Real-time Analysis of Data from Many Sensors with Neural Networks. Proceedings of the 5th IEEE International Symposium on Wearable Computers, S. 115.

Van Meteren, Robin; Van Someren, Maarten (2000): Using Content-Based Filtering for Recommendation. Elektronisch veröffentlicht: www.ics.forth.gr/ potamias/mlnia/paper_6.pdf; abgerufen am 2004-12-01.

VDZ (2001): Branchendaten Zeitschriftenmarkt. Verband deutscher Zeitschriftenverleger. Elektronisch veröffentlicht: http://www.vdz.de/pages/static/12.aspx; abgerufen am 2004-12-01.

VDZ (2003): Brachendaten New Media 2002. Verband deutscher Zeitschriftenverleger. Elektronisch veröffentlicht: $\quad$ http://www.vdz.de/mediabase/documents/030123_BranchendatenNewMedia2002_ am.pdf; abgerufen am 2004-12-01.

Venkatesh, Viswanath; Davis, Fred (2000): A theoretical extension of the technology acceptance model. Four longitudinal field studies. In: Management Science, Bd. 46, Nr. 2, S. 186-203.

Venkatraman, Venkat; Henderson, John (1998): Real Strategies for Virtual Organizing. In: MIT Sloan Management Review, Bd. 40, Nr. 1, S. 33-48.

Vizjak, Andrej; Ringlstetter, Max (2001): Medienmanagement: Content gewinnbringend nutzen. Gabler, Wiesbaden 2001.

Voelker, Geoffrey; Bershad, Brian (1994): Mobisaic: An Information System for a Mobile Wireless Computing Environment. Proceedings of the First IEEE Workshop on Mobile Computing Systems and Applications, Santa Cruz 1994. 
Von Eimeren, Birgit; Gerhard, Heinz; Frees, Beate (2004): Internetverbreitung in Deutschland: Potenzial vorerst ausgeschöpft? ARD/ZDF-Onlinestudie 2004. In Media Perspektiven 08/2004, S. 350-370.

von Hayek, Friedrich August (1952): Sozialistische Wirtschaftsrechnung I: Natur und Geschichte des Problems. In: Individualismus und wirtschaftliche Ordnung; Eugen Rentsch Verlag, Zürich 1952.

von Knyphausen-Aufseß, Dodo; Meinhardt, Yves (2002): Revisiting Strategy: Ein Ansatz zur Systematisierung von Geschäftsmodellen. In: Bieger, Thomas; Caspers, Rolf; zu Knyphausen-Aufseß, Reding (Hrsg.): Zukünttige Geschäftsmodelle. Springer Verlag, Berlin 2002, S. 63-89.

von Reibnitz, Alexander (2004): Branchendaten New Media 2003. Verband Deutscher Zeitschriftenverleger e.V.; Elektronisch veröffentlicht:

http://www.vdz.de/mediabase/documents/37_BranchendatenNewMedia2003.pdf; abgerufen am 2004-12-01.

von Rosenstiel, Lutz (1993): Wandel in der Karrieremotivation - Neuorientierung in den 90er Jahren. In: Rosenstiel, Lutz von et al. (Hrsg.): Wertewandel. Herausforderungen für die Unternehmenspolitik in den 90er Jahren. 2. Auflage, Stuttgart 1993, S. 47-81.

von Saldern, Matthias (1998): Beobachtung und Befragung im Betrieb. Schneider Verlag, Hohengehren 1998.

W3C (1999): Cascading Style Sheets. W3C Recommendation 17 Dec 1996, revised 11 Jan 1999. Elektronisch veröffentlicht: http://www.w3.org/TR/REC-CSS1; abgerufen am 2004-12-01.

W3C (2001): Extensible Stylesheet Language (XSL), Version 1.0. W3C Recommendation 15 October 2001. Elektronisch veröffentlicht: http://www.w3.org/TR/xsl/; abgerufen am 2004-12-01.

W3C (2004a): Extensible Markup Language (XML) 1.0. Elektronisch veröffentlicht: http://www.w3.org/TR/REC-xml; abgerufen am 2004-12-01.

W3C (2004b): Composite Capability/Preference Profiles (CC/PP): Structure and Vocabularies 1.0. W3C Recommendation 15 January 2004. Elektronisch veröffentlicht: http://www.w3.org/TR/CCPP-structvocab/; abgerufen am 2005-04-30.

Walsh, Norman; Muellner, Leonard (2004): DocBook: The Definitive Guide. O'Reilly, Sebastopol 2004.

Want, Roy; Hopper, Andy; Falcao, Veronica; Gibbons, Jonathan (1992): The Active Badge Location System. ACM Transactions on Information Systems, Bd. 10, Nr. 1, S. 91-102.

Waterman, Robert (1982): Seven Elements of Strategic Fit. In: Journal of Business Strategy (1982), S. 71.

Webb, William (2001): The future of wireless communications. Artech House, Boston 2001.

Wehleit, Kolja (2004): Analytisches Customer Relationship Management. In: IO New Management, Nr. 782004 , S. 32-34.

Wehrli, Peter; Wirtz, Bernd (1996): Relationship Marketing: Auf welchem Niveau bewegt sich Europa? In: ZfbF, 47. Jg., Nr. 1, S. 43-65.

Weiber, Rolf (1992): Diffusion von Telekommunikation - Problem der Kritischen Masse. Gabler Verlag, Wiesbaden 1992.

Weiber, Rolf (1995): Systemgüter und klassische Diffusionstheorie - Elemente einer Diffusionstheorie für kritische Masse-Systeme. In: Stoetzer, Matthias; Mahler, Alwin (Hrsg.): Die Diffusion von Innovationen in der Telekommunikation. Springer Verlag, Berlin, S. 39-70. 
Weiber, Rolf; Kollmann, Tobias (2000): Wertschöpfungsprozesse und Wettbewerbsvorteile im Marketspace. In: Bliemel, Friedhelm; Fassot, Georg; Theobald, Axel (Hrsg.): Electronic Commerce. Gybler Verlag, Wiesbaden 2000. S.47-62.

Weitzel, Tim; Harder, Thomas; Buxmann, Peter (2001): Electronic Business und EDI mit XML. Dpunkt Verlag, Heidelberg 2001.

Welsch-Lehmann, Frank-Michael (2001): Personalisierung. In: Albers, Sönke; Clement, Michel; Peters, Kay; Skiera, Bernd (Hrsg.): Marketing mit Interaktiven Medien. Strategien zum Markterfolg. Frankfurt am Main (2001), S. 133-148.

Werbach, Kevin (1999): Syndication: The Emerging Model for Business in the Internet Era. Elektronisch veröffentlicht: http://www.edventure.com/conversation/articleprint.cfm?Counter=125457; abgerufen am 2004-12-01.

Werben \& Verkaufen (2002): Top 50 Domains im März 2002. Elektronisch veröffentlicht: http://uww.wuv.de/daten/studien/062002/546/1734.html; abgerufen am 2004-12-01.

WI (2003): 6. Internationale Tagung Wirtschaftsinformatik 2003. Medien - Märkte - Mobilität. Elektronisch veröffentlicht: http://www.wi2003.de/; abgerufen am 2005-04-30.

Wied-Nebbeling, Susanne (2004): Preistheorie und Industrieökonomik. Springer Verlag, Berlin u.a. 2004.

Wilde, Klaus (1992): Database-Marketing für Konsumgüter. In: Hermanns, A.; Flegel, V. (Hrsg.): Handbuch des Electronic Marketing, München 1992, S. 791-805.

Winger, Richard; Edelman, David (1989): Individualisierung oder Segment-of-One-Wettbewerb. In: von Öttinger, Bolko (Hrsg.): Das Boston Consulting Group Strategie Buch 2000. Econ Verlag, Düsseldorf.

Wirtz, Bernd (2001a): Medien- und Internetmanagement. Gabler, Wiesbaden 2001.

Wirtz, Bernd (2001b): Electronic Business. Gabler, Wiesbaden 2001.

Wiswede, Günter (1991): Der neue Konsument im Lichte des Wertewandels. In: Szallies, Rüdiger; Wiswede, Günter (Hrsg.): Wertewandel und Konsum. Fakten, Perspektiven und Szenarien für Markt und Marketing. Verlag Moderne Industrie, Landsberg a. L. 1991, S. 13-40.

Wohlfahrt, Jens (2004): Akzeptanz und Wirkung von Mobile-Business-Anwendungen. Verlag Dr. Kovač, Hamburg 2004.

Wohlrab-Sahr, Monika (1997): Individualisierung: Differenzierungsprozess und Zurechnungsmodus. In: Beck, Ulrich; Sopp, Peter (Hrsg.): Individualisierung und Integration - Neue Konfliktlinien und neuer Integrationsmodus? Leske+Budrich, Opladen 1997, S. 23-36.

Wolf, Bernhard; Priebe, Michael (2001): Wissenschaftstheoretische Richtungen. Verlag Empirische Pädagogik, Landau 2001.

Wood, Kenneth; Richardson, Tristan; Bennett, Frazer; Harter, Andy; Hopper, Andy (1997): Global Teleporting with Java: Toward Ubiquitous Personalized Computing. In: Computer. IEEE Computer Society Press, Bd. 30, Nr. 2, S. 53-59.

Wörndl, Wolfgang (2003): Privatheit bei dezentraler Profilverwaltung. Dissertation, Fakultät für Informatik, Technische Universität München, 2003. Elektronisch veröffentlicht: http://tumb1.biblio.tumuenchen.de/publ/diss/in/2003/woerndl.pdf; abgerufen am 2005-04-30.

Wössner, Mark (2001): Die Medien: Eine traditionsreiche Branche am Scheideweg. In: Vizjak, Andre; Ringlstetter, Max (Hrsg.): Medienmanagement: Content gewinnbringend nutzen. Gabler, Wiesbaden 2001, S. 19-25. 
Wu, Kun-Lung; Yu, Philip; Ballman, Allen (1998): SpeedTracer: A Web Usage Mining and Analysis Tool. In: IBM Systems Journal, Bd. 37, Nr. 1, S. 89-105.

Wyner, Gordon (1996): Customer Profitability: Linking Behavior to Economics. In: Marketing Research, Bd. 8, Nr. 2 1996, S. 36-38.

Yang, Yiming; Liu, Xin (1999): A Re-Examination of Text Categorization Methods. In: Proceedings of the 22nd Annual International ACM SIGIR Conference on Research and Development in Information Retrieval. 1999, S. 42-49.

Zadorozhny, Vladimir; Chrysanthis, Panos (2004): Location-Based Computing. In: Kamiri, Hassan (Hrsg.): Telegeoinformatics: Location-based computing and services. CRC Press LLC, Boca Raton 2004, S. 145-169.

Zängler, Thomas (2000): Mikroanalyse des Mobilitätsverhaltens in Alltag und Freizeit. Springer Verlag 2000.

ZAW (2001): Netto-Werbeeinnahmen erfaßbarer Werbeträger 2001. Zentralverband der deutschen Werbewirtschaft. Elektronisch veröffentlicht: http://www.interverband.com/u-img/184/NettoWerbeeinnahmen_2002.htm; abgerufen am 2004-12-01.

ZAW (2004): Werbung in Deutschland 2004.Verlag Edition ZAW; Zentralverband der deutschen Werbewirtschaft, Berlin 2004.

Zerdick, Axel et al. (2001): Die Internet-Ökonomie - Strategien für die digitale Wirtschaft. Springer Verlag, Berlin 2001.

Ziegler, Marc; Adam, Bernd (2001): Mastering Mobile Media Markets. Strategien zur Refinanzierung der UMTS-Investitionen. TIMElabs Studie. April 2001.

Zobel, Jörg (2001): Mobile Business and M-Commerce. Carl Hanser Verlag, München 2001.

Zöttl, Ines (2003): Er wollte immer der Erste sein. Über den Unternehmen Julius Reuter. DIE ZEIT Nr.21, 15.05.2003. 